W.

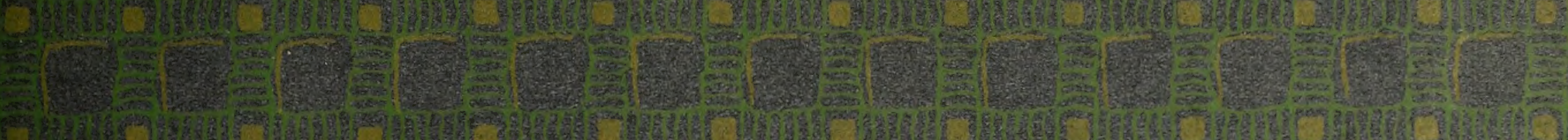

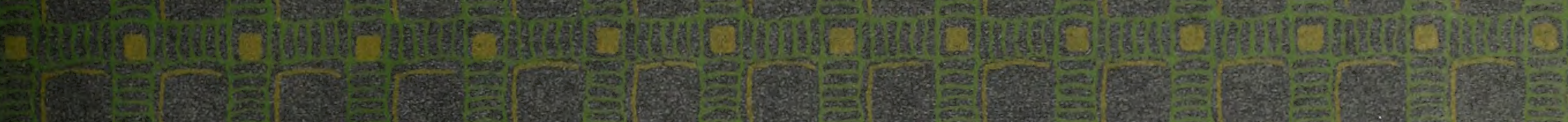

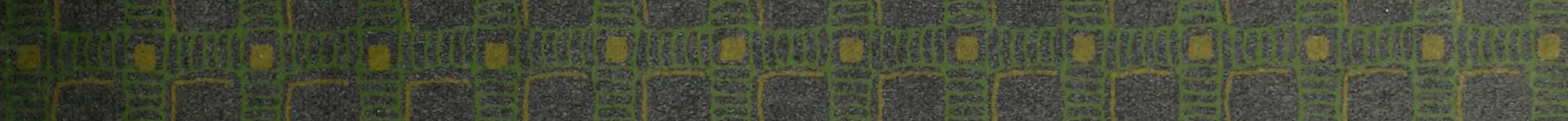

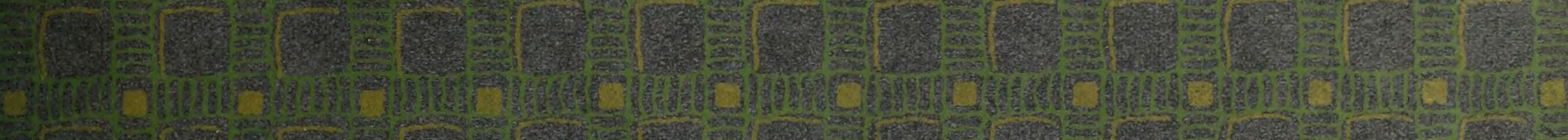
(5)

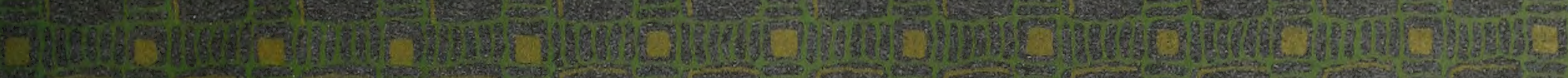
Whath

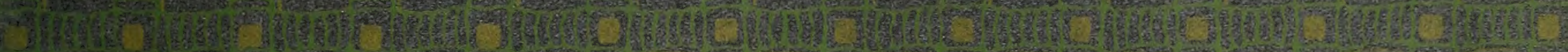

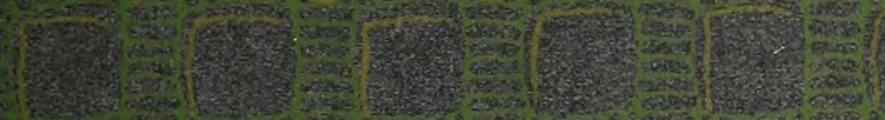

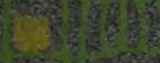
The

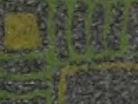

(1)

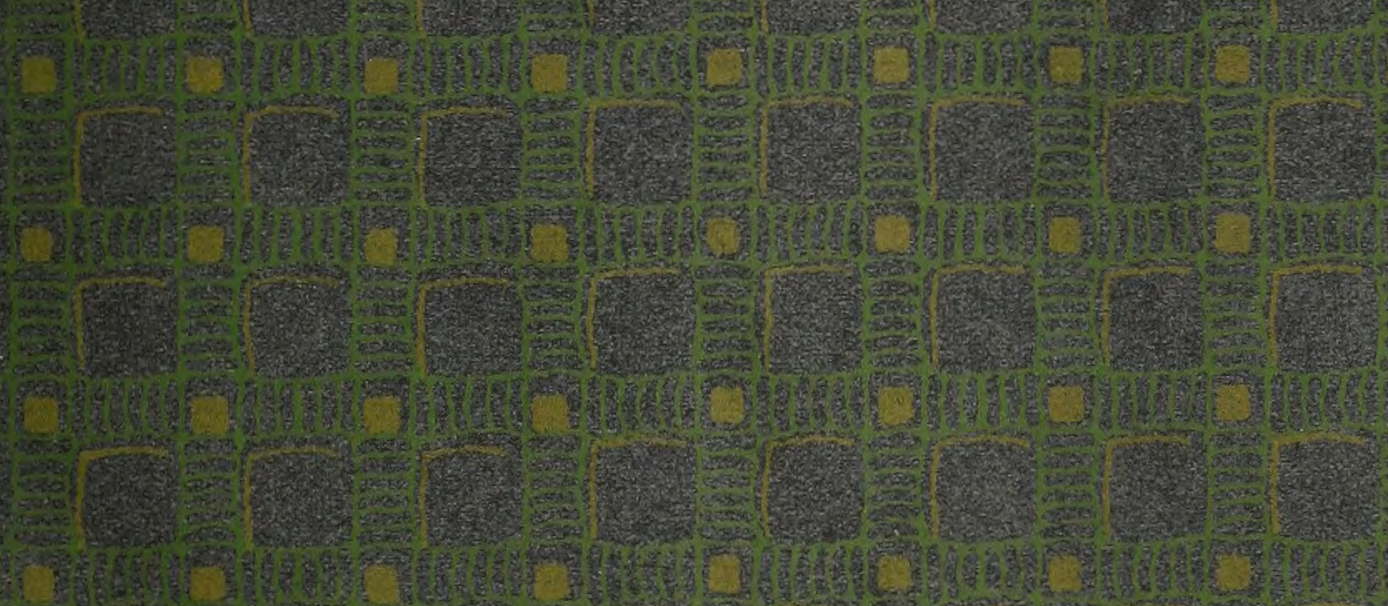

W. 1.
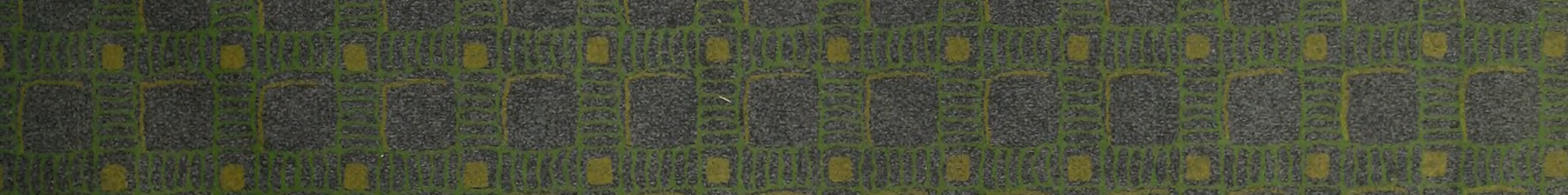

(3)
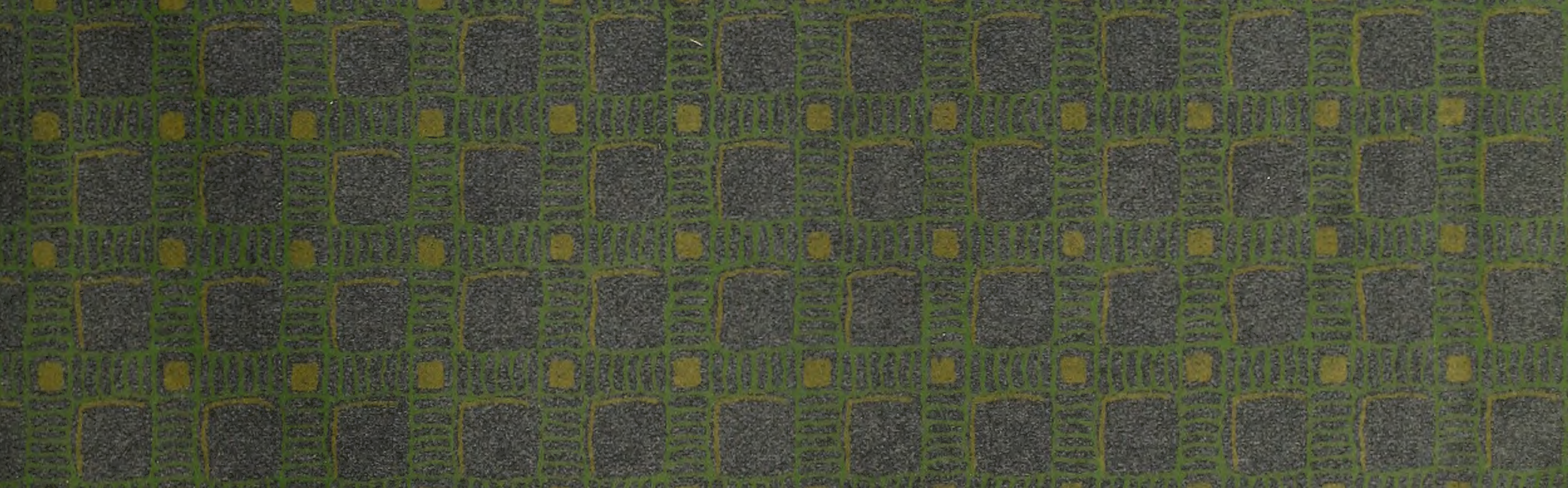

We

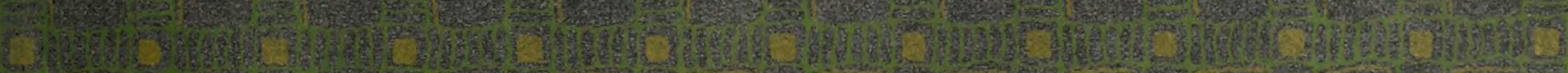

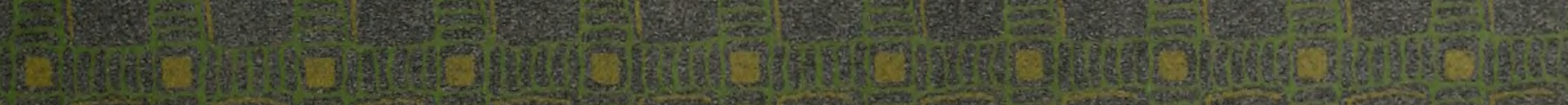


. 


\title{
Die
}

\section{Schmetterlinge Europas}

\begin{abstract}
Mit uiber 3500 Figuren auf 95 Tafeln
$=$ und 505 Abbildungen im Text $=$
\end{abstract}

Dritte Auflage von Prof. E. HOFMANN'S Werk: Die Groß-Schmetterlinge Europas

Unter Mitarbeit von

Dr. med. E. FISCHER (Zürich), weiland Pfarrer A. FUCHS (Bornich), Gymnasiallehrer J. GRIEBEL (Neustadt i. d. Pfalz), Prof. Dr. J. v. KENNEL

(Dorpat), Stadtrat A. MEESS (Karlsruhe i. B.) und Prof. Dr. H. REBEL (Wien)

bearbeitet von

\section{Dr. med. et philos. ARNOLD SPULER}

ao. Universitäts-Professor in Erlangen

\section{B AN D}

Tafelband

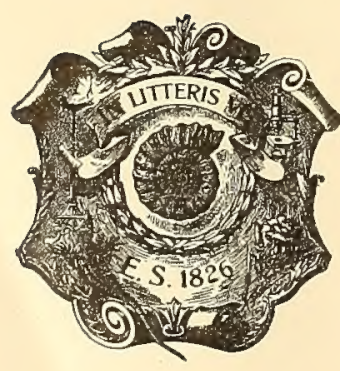

STUTTGART 1910

E. SCHWEIZERBART'SCHE VERLAGSBUCHHANDLUNG

NÄGELE \& DR. SPROESSER. 


\title{
Die
}

\section{Schmetterlinge Europas}

\author{
III. BAND
}

Tafelband

95 Tafeln zum I. und II. Band

mit über 3500 Abbildungen

\section{ARNOLD SPULER}
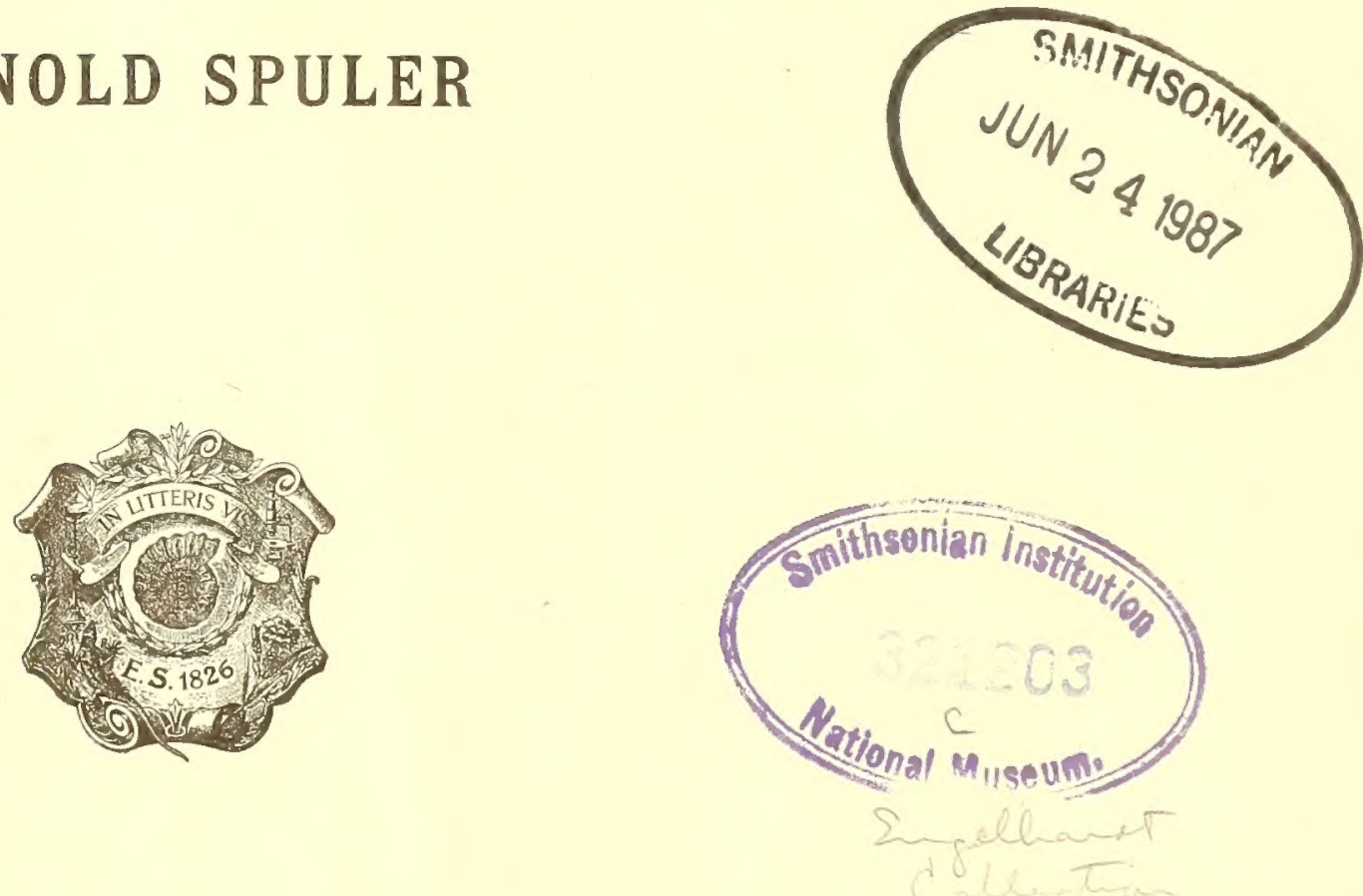

STUTTGART 1910

E. SCHWEIZERBART'SCHE VERLAGSBUCHHANDLUNG NÄGELE \& DR. SPROESSER. 
Alle Rechte vorbehalten.

$$
\begin{gathered}
595.78 \\
5 \% 928
\end{gathered}
$$

Druck der Stuttgarter Vereins-Buchdruckerei. 


\section{Druckfehler-Verzeichnis und Berichtigungen zu den Tafelerklärungen.}

Tafel 1 Fig. 4. P. machaon L.: Ei Taf. 50, 1.

" 8. Die linke Seite für apoltimus zu grau und zu gross, rechte Seite $v$. bellargus Stgr.

"9. P. apollo L.: Ei Taf. 50, 2.

"2 2 6. P. crataegi L.: Ei Taf. 50, 3.

$" 14$ "14. Euchl. belia Cr.: Ei Taf, 50, 4.

$" 3$ "11. C. hyale L.: Ei Taf. $50,5$.

" 4 "14. aetherie: für Hb. zu setzen H.-G.

$" 5$ "3a. Ar. levana L.: Ei Taf. 50, 8.

" 8. Fürsibylla L.zusetzen: cammilla I. (sibylla auct.).

" 9. Für camilla Schiff. zu setzen: rivanláris Scop. (camilla Schiff.).

" 11 "5. S. hermione L.: Für $q$ zu setzen: Unterseite.

" $17 \mathrm{~b}, 15$. Für y m phy ta Ld.zu setzen: tiphon Rott. v. rhodopénsis Elw.

23. Ist bavius Ev., 24. panope Ev.

"22. cyane Ev.: Wie sie ausgefallen ist, gleicht die Abbildung mehr pyláon F. d. W.

$" 20$ 2. Sm. ocellata L.: Ei Taf. 50, Fig. 19.

$" 6$ "6. M.stellatarum L.: Ei, Taf. 50, Fig. 20.

" 23 " 9. P. curtula L.: Ei, Taf. 50, Flg. 54.

"13. Ph. bucephala L.: Ei, Taf. 50, Fig. 53.

" 26 "9. L. eversmanni Ev: Für 119 1. zu lesen $119 \mathbf{r}$.

"10. Er. rimicola Hb.: Für ơ zu setzen q.

"27 "12. Einzufügen: Gast 10 pacha.

"13. Einzufügen: Epicmáprema.

" 32 "26. triangulum Hufn.: Beim Ei für $\mathbf{3 9}$ zu setzen $\mathbf{5 9}$

" 35 8. distinguenda Ld.: Nachzutragen bei S. des I. Bds.: $161 \mathrm{l}$.

" 39 "20. L. virens L.: Nachzutragen bei S. des I. Bds.: 221 r., bei Raupen-Abb.: Ntr.-Taf. V, 7.

$" 41$ "27. Br.meticulosa L.: Ei Taf. 50, Fig. 65.

"48 "7. C. verbasci L.: Beim Ei für 19 zu setzen: 69 .

" 56 "18. N. viridata L.: Ei Taf. 50, 74.

" 57 7. Für corrivallaria zu lesen: corrivalaria,

"30. Für dilectaria zu lesen: dilutaria.
Tafel 58 Fig. 24. God. punctaria L.: Ei Taf. 50, 75. "32. A. grossulariata L.: Ei Taf. 50, 76. 59 für 10 zu setzen: 10 a; hinter L.: ふ7, b ab. musauária Hb. .

Fig. 14. Ennomos quercinaria Hufn.: Ei Taf. $50,77$.

"20 20. Sel. bilunaria Esp.; anzufügen: $\mathrm{a} \sigma^{7}, \mathrm{~b}$ ㅇ.

"21. Sel. Iunaria Schiff.: Ei Fig. 50, 79.

"26. H. pennaria L.; anzufügen: $a \sigma^{7}, \mathrm{~b}$ ㅇ․

60 "4. Eur. dolabraria L.: Ei Taf. 50, 80.

hinter 5. einzufügen $\mathrm{a}$; das a vor $q$ und $\mathrm{b} \sigma^{7}$ zu streichen, anzufügen: 5. smb. sordiáta Fuessl.

Fig. 20. Für Epirranthis zu lesen: EpirFlh ant $h$ is.

61 "5. Phig. pedaria F.: Ei Taf. 50, 81.

"13. B. hirtarius Cl.: Ei Taf. 50, 82.

Bei 15. zu setzen: Amplnidasys betnlanius L.; nachzutragen: Ei Taf. 50, 83 .

" 63 "31. Für Eurranthis zu setzen: Eurrhanthis.

$64 "$ 11. Th. wauaria L.: Ei Taf. 50, 84.

65 "13. C. margarita Hb.: Ei Taf. 50, 85.

67 "6. L. populata L.: Für a zu lesen: b, für $\mathrm{b}: \mathrm{a}$.

8. L. dotata L.: Bei Ei für $26 \mathrm{zu}$ setzen: $\mathbf{8 6}$.

70 "13. L. comitata L.: Ei Taf. 50, 88.

$71 \mathrm{a}, 14$ ist Ac. faillata Fuchs, Fig. 15 nitidata HS.

$71 \mathrm{~b}, 24$. Mal. regelaria Tngstr.: Für 26 l. zu lesen: $\mathbf{B} 61$.

77 "25. A.rhadamantus Esp.: Ei Taf. 50, 24.

80 "4. Hep. humuli L.: Das Zitat des Eis ist zu streichen.

$" 90$ "44. B. thoracella Thnbg: Für 419 l. zu lesen 418/19.

"49. Oen. v. flavum Hw.: Für 421 l. zu lesen $420 \mathrm{r}$.

977. B. antispilella Chrét. i. l. : Für 4201 1. zu lesen 420 l. 



\section{TAFEL 1.}

\author{
Nr. \\ Name \\ 1. Papílio alexánor Esp. 우 \\ 2. " podalírius L. $\sigma^{7}$ \\ 3. " hóspiton Génè ? \\ 4. " macháon L. 우 \\ 5. Thais rumína L., v, eantenéri Stgr. $q$ \\ 6. $"$ cerisyi God. $\sigma^{7}$ \\ 7. " polyxena Sehiff. + \\ 8. Dorítis apollinus Hbst., v. bellárgus \\ 9. Parnássius apóllo L. $\sigma^{7}$ \\ 10. \\ "délius Esp. $\sigma^{7}$
}

Katalog I

2

1

3

4

11 a

9

10

13 u. $13 \mathrm{~b}$

14

20
Seite des I. Bandes

21.

21.

$2 \mathrm{r}$.

21.

$3 \mathrm{r}$.

31.

31.

$3 \mathrm{r}$.

41.

$4 \mathrm{r}$.
Raupen- (Puppen-)

Abbildung

Taf. 6,1

Taf. 1,1 u. 48,1

Taf. 6, 2

$\because 1,2$

" 6,3

Nachtr.-Taf. I, 1

Taf. 1, 3

Nachtr.-Taf. I, 2

Taf. 1, 4

"48, 2 

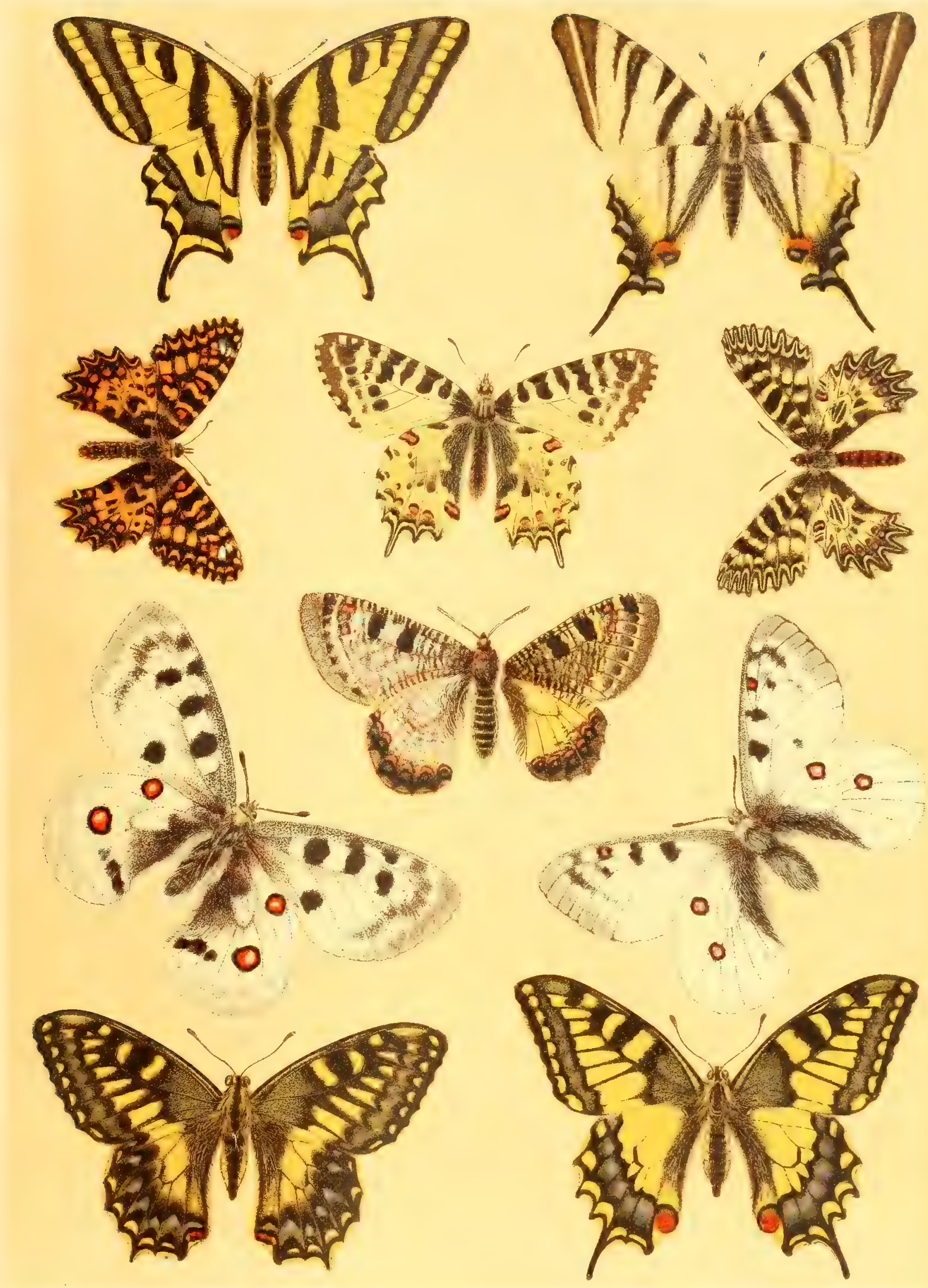



\section{TAFEL 2.}

\begin{tabular}{|c|c|c|c|c|}
\hline Nr. & Name & Katalog I & Seite des I. Bandes & $\begin{array}{l}\text { Raupen- (Puppen-) } \\
\text { Abbildung }\end{array}$ \\
\hline & Píeris nápi L., a $\sigma^{\top}, \mathbf{b}$ v. bryóniae 0 . $q$ & 52 u. $52 \mathrm{c}$ & $6 \mathrm{r}$. & - Taf. 1,9 \\
\hline 2. & " callídice Esp., a $\sigma^{7}, \mathrm{~b}$ 우 & 56 & 71. & $" 1,10$ \\
\hline 3. & Eúchloë tágis $\mathrm{Hb}$. + & 63 & 81. & $" 6,7$ \\
\hline 4. & $\begin{array}{l}\text { grunéri HS. } \sigma^{7} \text { (Exemplar mit relaltiv } \\
\text { breiter innerer Begrenzung der orangen }\end{array}$ & & & \\
\hline & Flügelspitze) & 70 & $8 \mathrm{r}$. & \\
\hline 5. & $" \quad$ belémia Esp. 우 & 60 & $7 \mathrm{r}$. & \\
\hline 6. & Apória crataégi L. $\sigma^{7}$ & 38 & 51. & Taf. 1,6 u. 6,4 \\
\hline 7. & Eủchloë euphenoídes Stgr., a $\sigma^{7}, \mathrm{~b}$ 우 & 73 & $8 \mathrm{r}$. & Taf. 6,8 \\
\hline 8. & $"$ damóne Feisth. $\sigma^{7}$ & 71 & $8 \mathrm{r}$. & \\
\hline 9. & $"$ eardamínes L., a $\sigma^{7}, \mathrm{~b} q$ & 69 & 81. & $" 1,12$ \\
\hline 10. & Píeris brássicae L., rechts $\sigma^{\pi}$, links $q$ & 45 & 61. & $" 1,7$ \\
\hline 11. & "daplídice L., rechts $\sigma^{7}$, links +9 & 57 & 71. & $" 1,11$ \\
\hline 12. & $"$ érgane H.-G. $q$ & 49 & $6 \mathrm{r}$. & Nachtr.-Taf. I, 3 \\
\hline 13. & " rápae L. 우 & 48 & 61. & Taf. 1,8 \\
\hline 14. & Eúchloë bélia Cr., gen. aest. ausónia $\mathrm{Hb}$. 우 & $62 \mathrm{~b}$ & $7 \mathrm{r}$. & $" \quad 6,6$ \\
\hline & Parnássius mnemósyne L. $\sigma^{\lambda}$ & 36 & $4 \mathrm{r}$. & $" 1,5$ \\
\hline 16. & Píris ehlorídice $\mathrm{Hb}$. 우 & 59 & $7 \mathrm{r}$. & \\
\hline
\end{tabular}



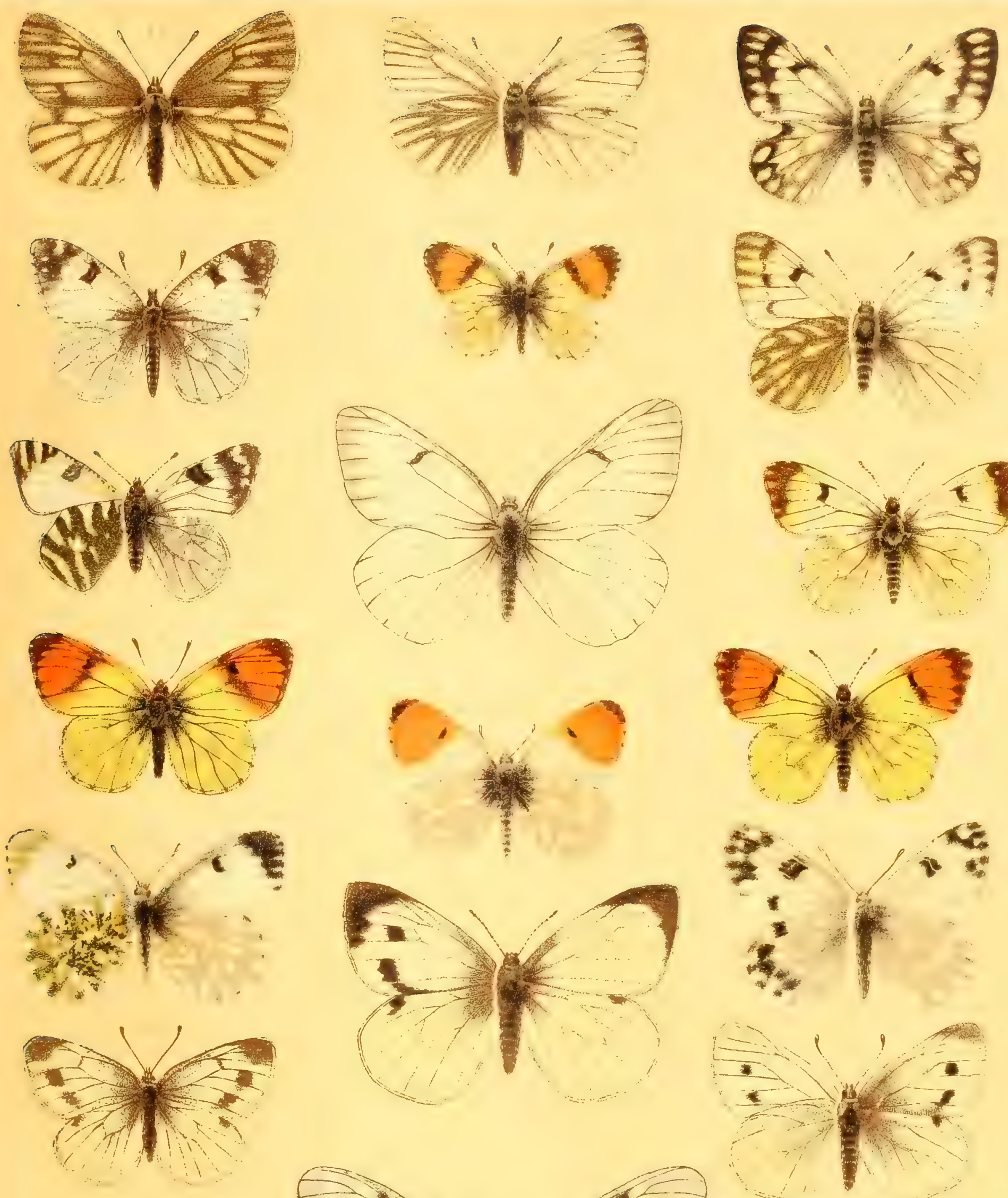

Pa, $2=$ hed
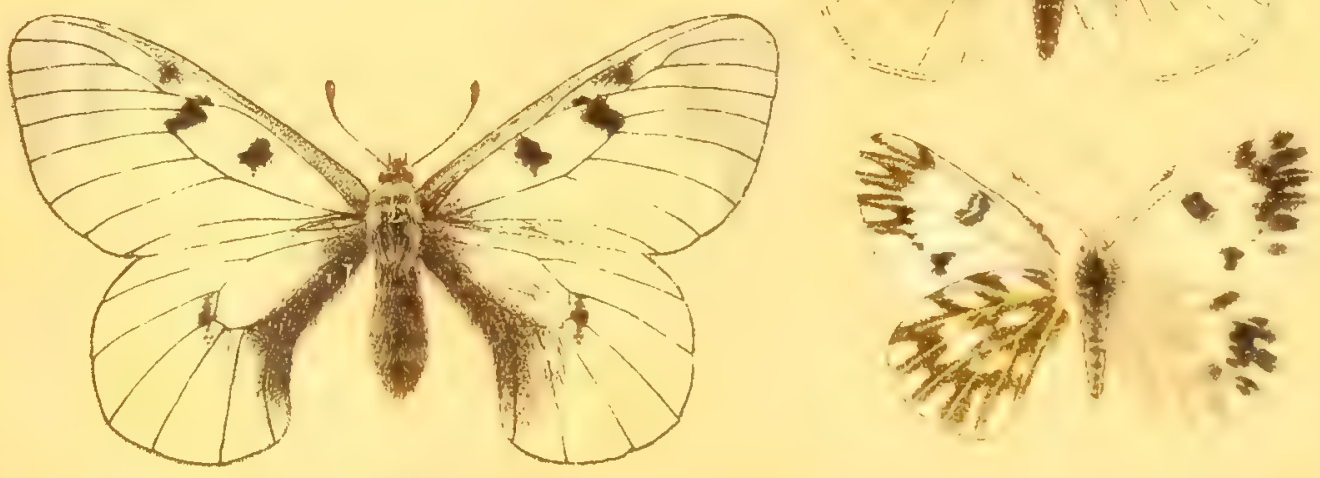



\section{TAFEL 3.}

Nr.

1. Cólias phicomóne Esp., a 우 $\mathrm{b} \sigma^{7}$

2. $"$ myrmídone Esp., a $\sigma^{7}, \mathrm{~b} q$

3. " palaéno L., v. europóme Esp., a $\sigma^{7}, \mathrm{~b} q$

4. Leptídia duponchéli Stgr. $\sigma^{7}$

5. $"$ sinapis L., rechts $\sigma^{7}$, links ㅇ

6. Gonópteryx eleópatra L. $\sigma^{7}$

7. $" \quad$ phámni L., rechts $\widehat{\emptyset}$, links 우

8. Zegris euphéme Esp. $\sigma^{7}$

9. Cólias ehrysótheme Esp., a $\sigma^{7}$,

b kleine Form des $q 111$

10. " edúsa F. (eróceus Fourer.), a $\sigma^{7}$, b $q 113$

11. " hyale L., a $\sigma^{7}, \mathrm{~b}$ ?

12
98

Katalog I

91

114

$86 \mathrm{c}$

83

81

125

124

74

98

99
Seite des I. Bandes

$9 \mathrm{r}$.

$10 \mathrm{r}$.

$9 \mathrm{r}$.

121.

$11 \mathrm{r}$.

111.

111.

91.

101.

$10 \mathrm{r}$.

9/10

101.
Raupen- (Puppen-) Abbildung

Taf. 1,15

Nachtr.-Taf. I, 6

Taf. 1, 14

$$
\begin{aligned}
& \Rightarrow \quad 1,13 \\
& " \quad 2,2 \\
& " \quad 2,1
\end{aligned}
$$

Nachtr.-Taf. I, 5 Taf. 1, 17

Taf. 1, 16 u. 6, 9 


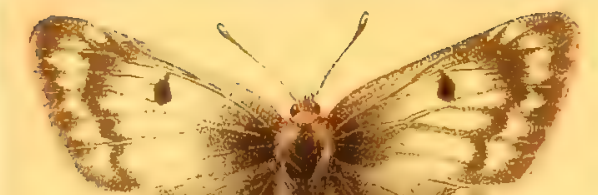

(i)
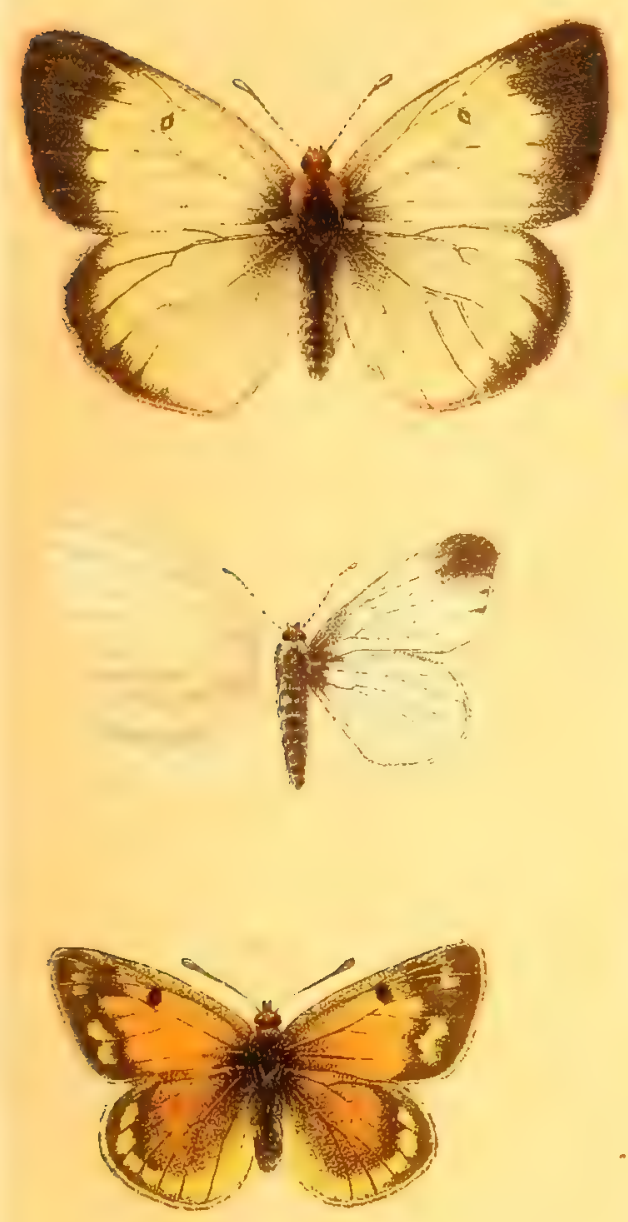
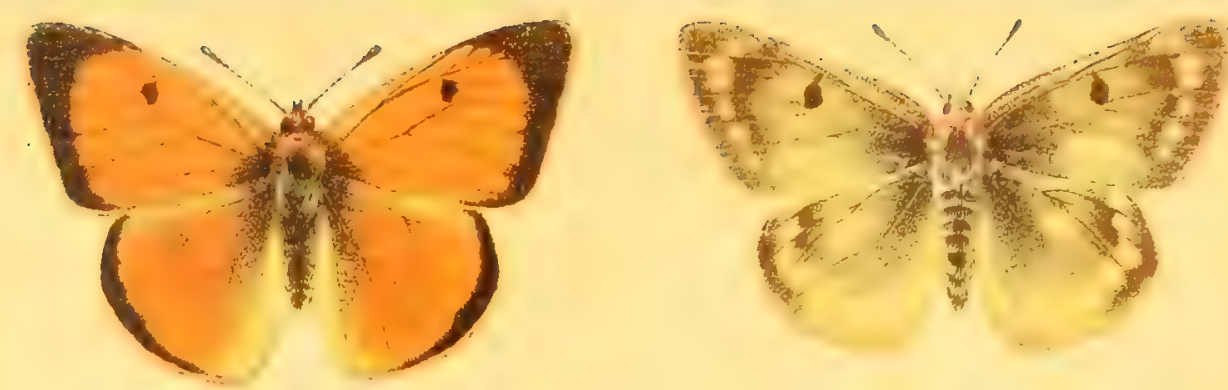

*

iv
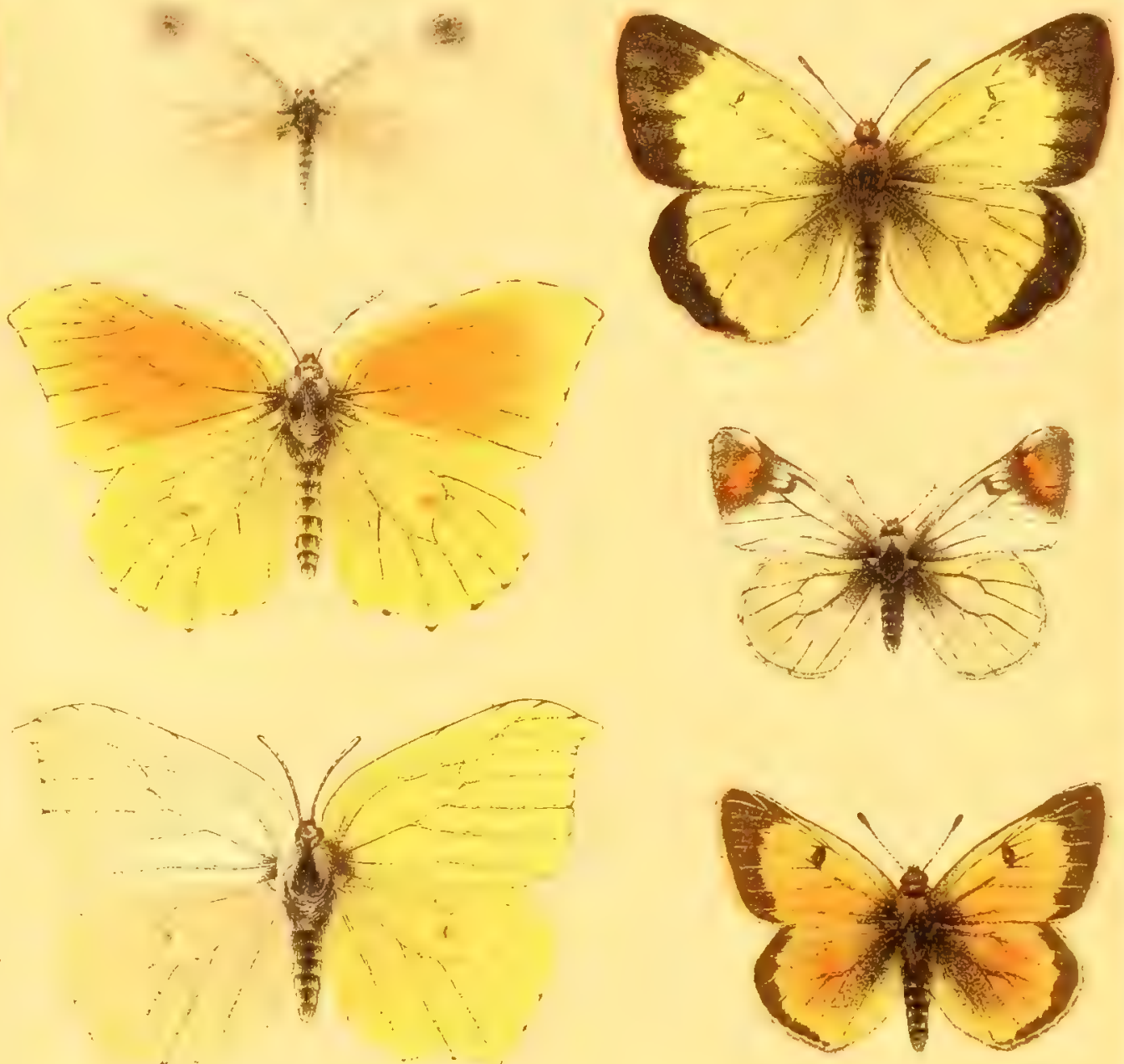
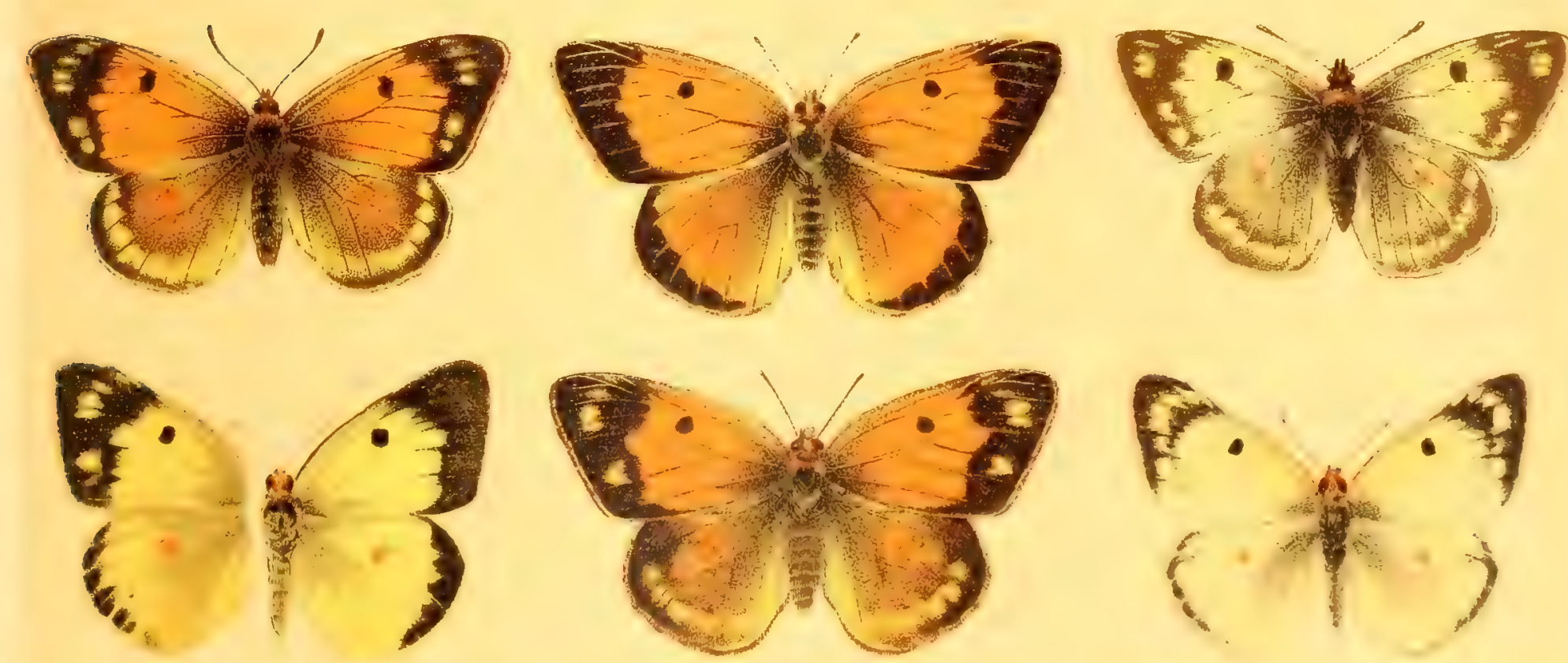




\section{TAFEL 4.}

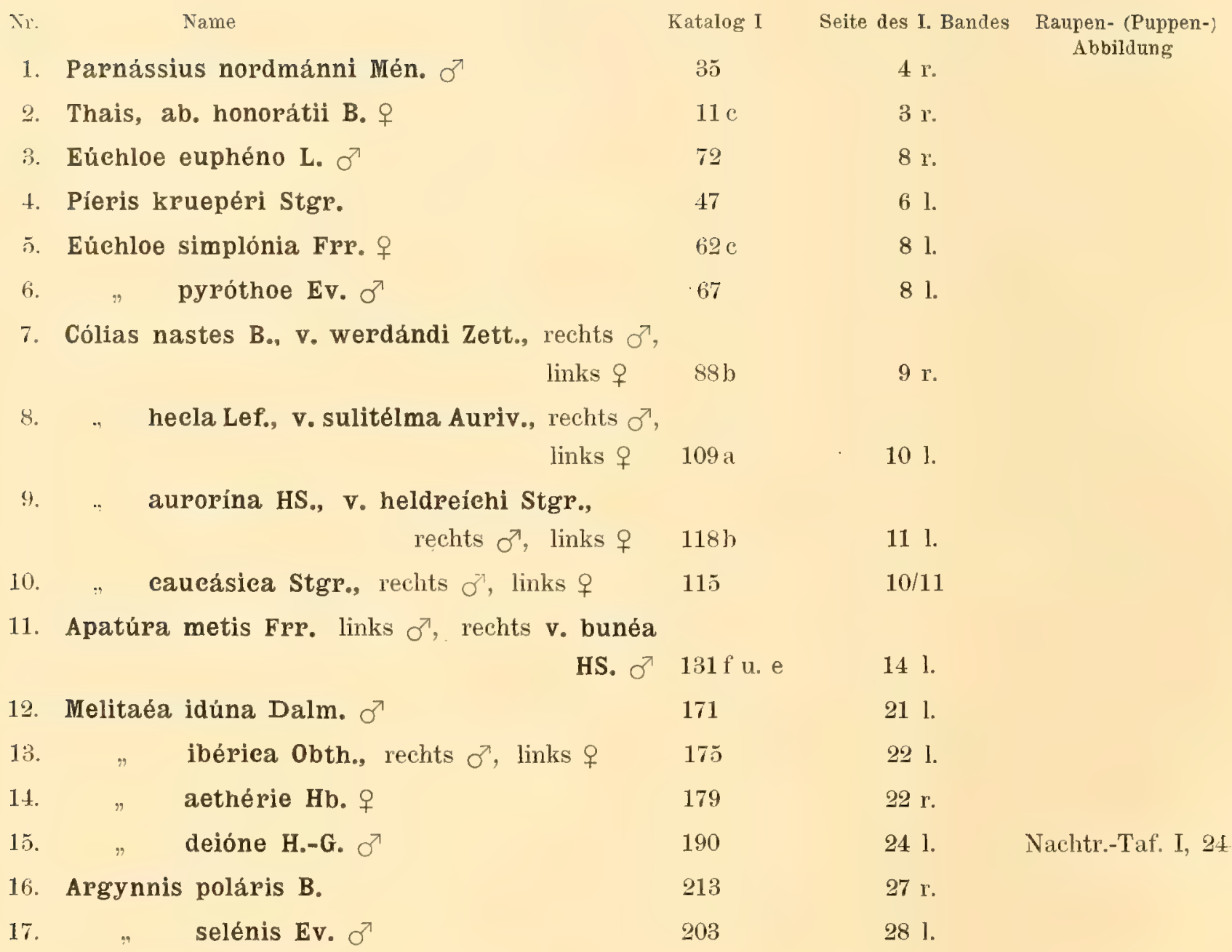




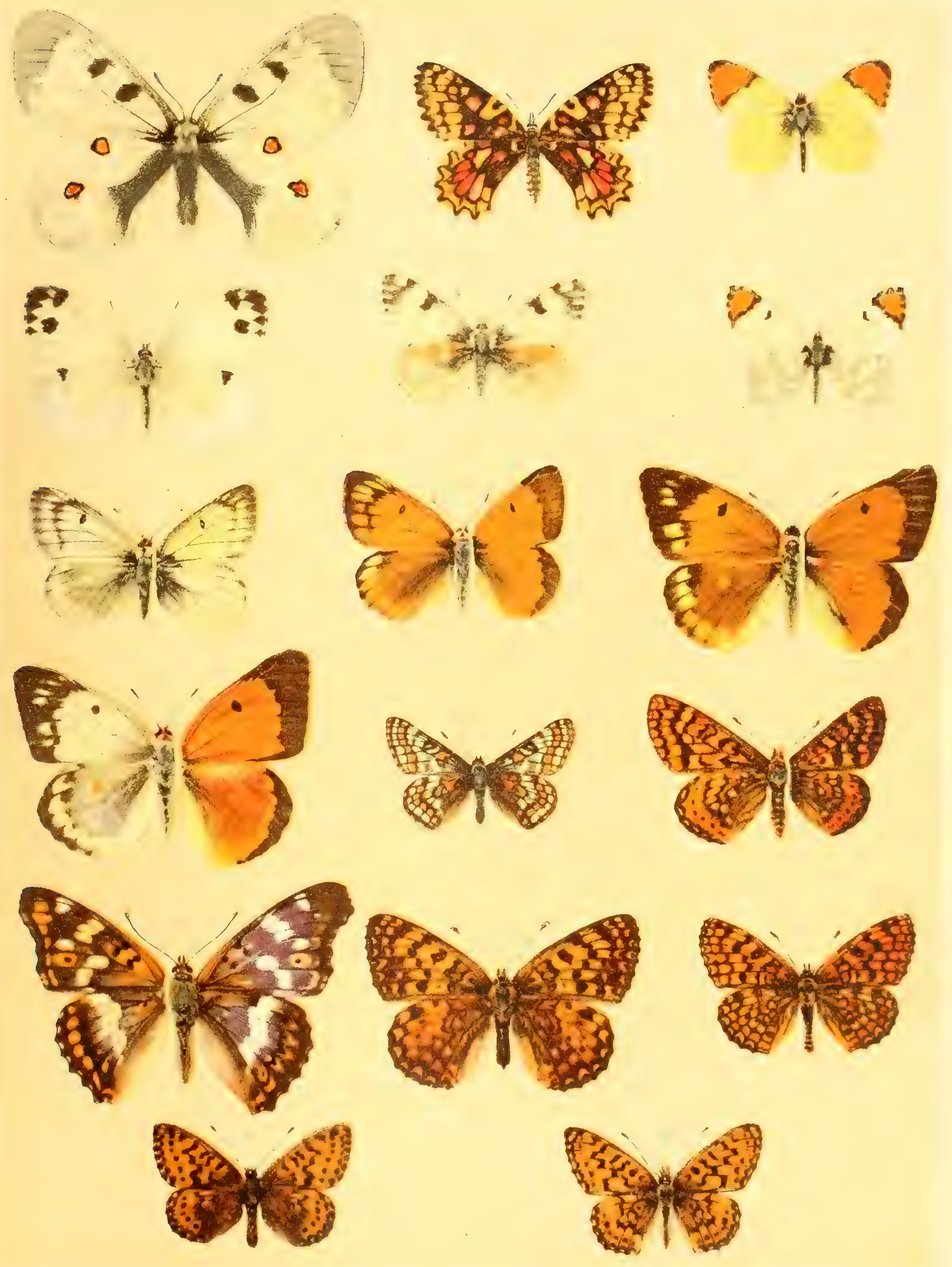






\section{TAFEL 5.}

\begin{tabular}{|c|c|c|c|c|}
\hline Nr. & Name & atalog I & $\begin{array}{l}\text { Seite } \\
\text { des I. Bandes }\end{array}$ & Raupen- (Puppen-) Abbildung \\
\hline $1 \mathrm{a}$. & Apatúra iris L. $\sigma^{7}$ & 131 & $13 \mathrm{r}$ & Taf. 2,36 \\
\hline $1 \mathrm{~b}$. & $" \quad \Rightarrow$ ab. ióle Sehiff. $\sigma^{7}$ & $131 \mathrm{a}$ & $13 \mathrm{r}$ & \\
\hline $2 \mathrm{a}$. & ilia Sehiff. $\sigma^{7}$ & 132 & 141. & $" 2,37 \mathrm{a}, \mathrm{b}$ \\
\hline 21. & $" \quad \quad \quad$ ab. elytie Sehiff. $\sigma^{\pi}$ & $132 \mathrm{~b}$ & 141. & \\
\hline $3 \mathrm{a}$. & Aráehnia levána L. $\sigma^{7}$ & 169 & $20 \mathrm{r}$. & $" 3,5 \mathrm{a}-\mathrm{c}$ \\
\hline $3 \mathrm{~h}$. & $" \quad \quad \quad$ gen. aest. prórsa L. $\sigma^{7}$ & $169 \mathrm{~b}$ & $20 \mathrm{r}$. & \\
\hline $3 \mathrm{c.}$ & $" \quad \Rightarrow \quad$ ab. pórima 0. 우 & $169 \mathrm{a}$ & $20 \mathrm{r}$ & \\
\hline 4. & Limenítis pópuli L., a $\sigma^{7}, \mathrm{~b}$ 우 & 136 & 151 & Taf. 3,1 u. 6,10 \\
\hline 5. & Polygónia e-album L. 우 & 166 & 191. & $" 3,6$ u. Nacht.-Taf. I, 8 \\
\hline 6. & " egéa $\mathrm{Cr}_{.}$, gen. aest. i-album Esp. & 167 & $19 \mathrm{r}$. & $" 6,11$ a, b u. Ntr.-T. I, 9 \\
\hline 7. & Neptis áceris Lepech. ত & 145 & $16 \mathrm{l}$ & "48, 4 u. Nacht.-Taf. I, 7 \\
\hline 8. & Limenitis sibylla L. $q$ & 138 & $15 \mathrm{r}$. & $\left\{\begin{array}{r}\text { Ei Taf. } 50,8 \\
" 3,3\end{array}\right.$ \\
\hline 9. & " camilla Sehiff. & 135 & 151. & $" 3,2 \mathrm{a}, \mathrm{b}$ \\
\hline 10. & Neptis lueílla F. & 144 & 161. & $\Rightarrow 3,4$ \\
\hline
\end{tabular}




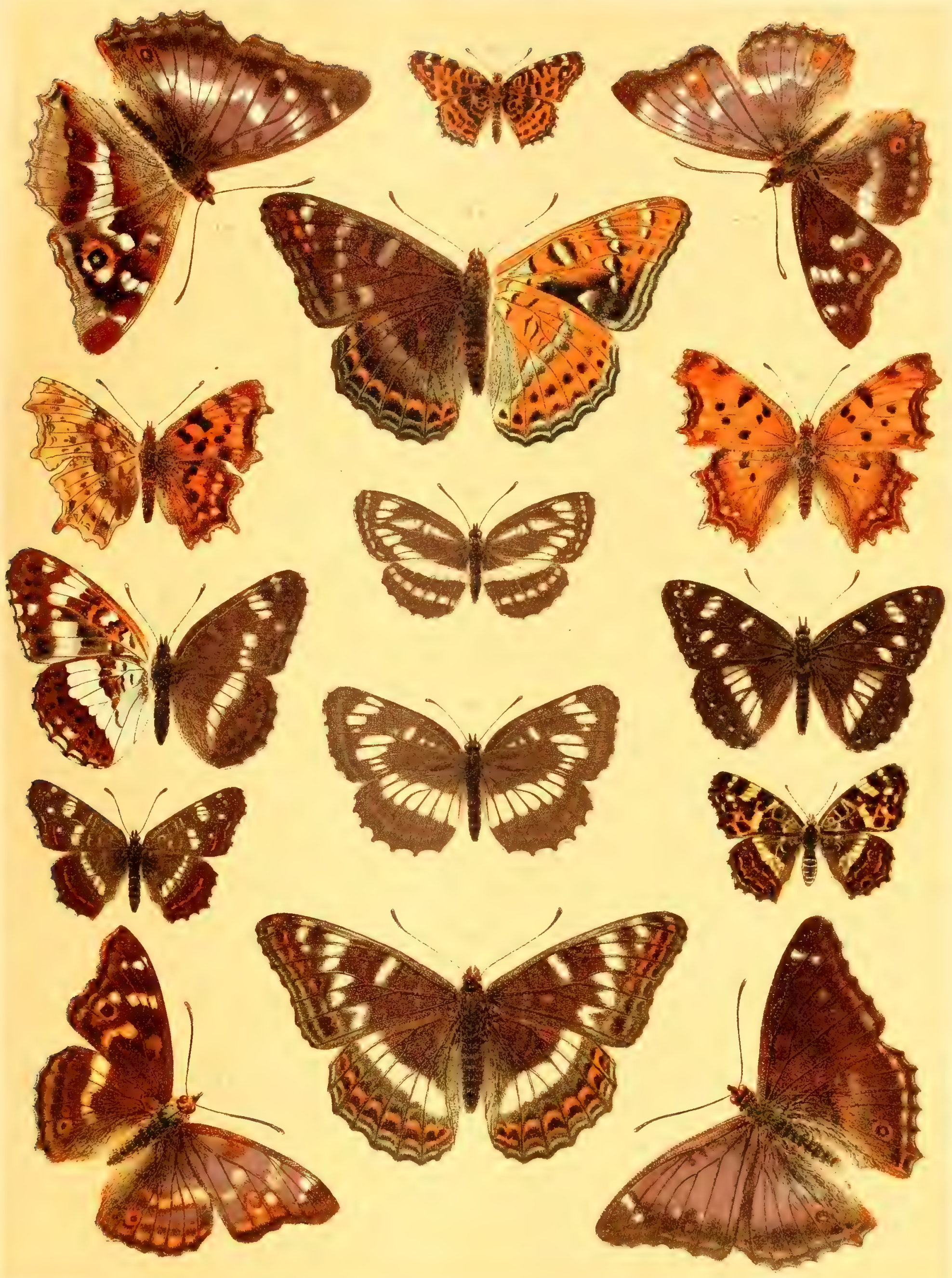






\section{TAFE L 6 .}

\begin{tabular}{|c|c|c|c|c|}
\hline $\mathrm{Nr}$. & Name & Katalog I & Seite des I. Bandes & $\begin{array}{c}\text { Raupen- (Puppen-) } \\
\text { Abbildung }\end{array}$ \\
\hline 1. & Polygónia L-álbum Esp. & 158 & 191. & Taf. 3,8 \\
\hline 2. & Vanéssa polyehlóros L. & 161 & $18 \mathrm{l}$. & $" 3,7$ \\
\hline 5. & atalánta L. & 152 & $19 / 20$ & $" 3,12 \mathrm{a}-\mathrm{d}$ \\
\hline 7. & $" \quad$ urtícae L. & 157 & $17 \mathrm{r}$ & $\Rightarrow 3,9 \mathrm{a}-\mathrm{c}$ \\
\hline $3 \mathrm{a}$. & Melitaéa aurinia Rott. $\sigma^{7}$ & 175 & $21 / 22$ & Taf. 3,16 u. 6,17 \\
\hline $3 \mathrm{~b}$. & " v. mérope Prun. 우 & $175 \mathrm{~b}$ & 221. & \\
\hline 4. & matủrna L. $\sigma^{7}$ & 172 & 21 l. $\{\mathrm{E}$ & $\begin{array}{l}\text { Ei Taf. } 50,10 \\
\qquad 3,15 \text { u. } 6,16\end{array}$ \\
\hline 6. & $"$ eynthia Hb., a $\sigma^{7}, \mathrm{~b}$ ㅇ & 174 & $21 \mathrm{r}$ & Taf. 3,14 \\
\hline 11. & "desfontaínii God., v. baétiea Ramb. 우 & $176 \mathrm{a}$ & $22 \mathrm{r}$ & \\
\hline 8. & Vanéssa antiopa $\mathrm{L}$. & 162 & $17 \mathrm{l}$. & Taf. 3,11 u. 6,14 \\
\hline 9. & Pyramaéis eárdui L. & 153 & 201. & $" 3,13$ u. $6,15 \mathrm{a}, \mathrm{b}$ \\
\hline 10. & Vanéssa io $\mathrm{L}$. & 156 & $17 \mathrm{r}$ & $" 3,10$ u. 6,13 \\
\hline 12. & xanthómelas Esp. & 160 & $18 \mathrm{r}$. & Taf. 6,12 \\
\hline
\end{tabular}




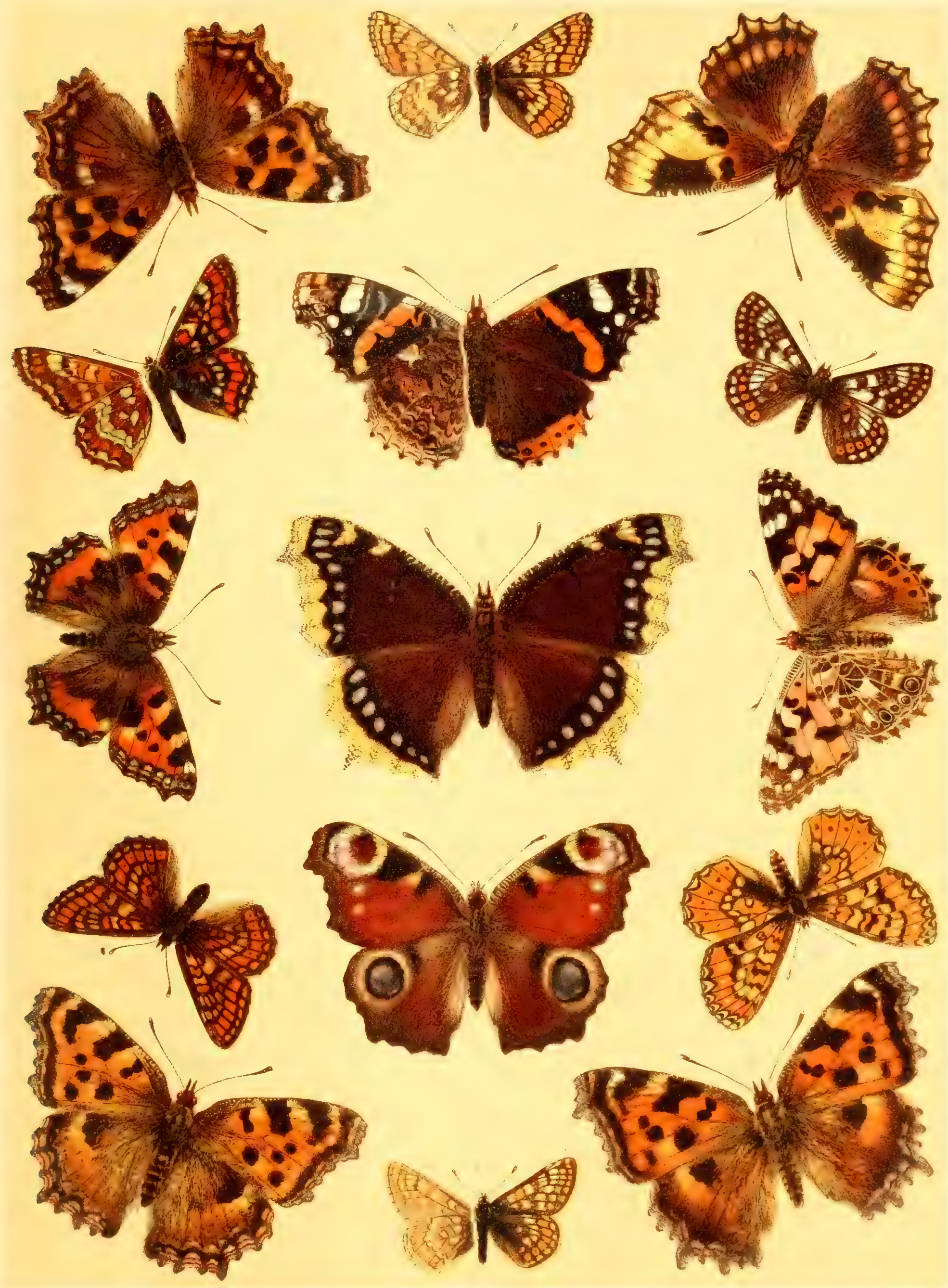






\section{TAFEL $\%$}

\begin{tabular}{|c|c|c|c|c|c|}
\hline $\mathrm{Nr}$ & & Name & Katalog I & Seite des I. Bandes & $\begin{array}{c}\text { Raupen- (Puppen-) } \\
\text { Abbildung }\end{array}$ \\
\hline 1. & Melitaéa & phoébe Knoeh., a $\sigma^{7}, \mathrm{~b}$ 우 & 180 & $22 / 23$ & Taf. 3,18 \\
\hline 2. & $"$ & arduínna Esp. $q$ & 178 & $22 \mathrm{r}$. & \\
\hline 3. & $n$ & trivia Sehiff. $q$ & 186 & $23 \mathrm{r}$ & " 3,19 \\
\hline 4. & $"$ & cínxia L. $q$ & 177 & $22 \mathrm{r}$ & $" 3,17$ \\
\hline 5. & Brenthis & euphrosyne L. Oج & 208 & $26 / 27$ & $\eta \quad 4,3$ \\
\hline 6. & Melitaéa & aurélia Nick. $\sigma^{7}$ & 192 & $24 \mathrm{r}$ & Nachtr.-Taf. I, 11 \\
\hline 7. & Argynnis & aphirápe Hb., a $\sigma^{7}, b q$ & 202 & 261. & Taf. 4,1 \\
\hline 8. & Melitaéa & athália Rott. $q$ & 191 & 241 & $" 3,22$ \\
\hline 9. & $"$ & $\begin{array}{l}\text { parthénie Bkh. 오 zu monoton in } \\
\text { der Färbung }\end{array}$ & 193 & 251. & Nachtr.-Taf. I, 4 \\
\hline 10. & $"$ & dietynna Esp. Оフ & 195 & 251. & Taf. $3,21 \mathrm{a}, \mathrm{b}$ \\
\hline 11. & Brenthis & seléne Sehiff: $\sigma^{7}$ & 204 & $26 \mathrm{r}$ & $" 4,2 \mathrm{a}, \mathrm{b}$ \\
\hline 12. & Melitaéa & astéria Frr. $0^{7}$ & 201 & $25 \mathrm{r}$ & \\
\hline 13. & Brenthis & frigga Thnbg. $\sigma^{7}$ & 216 & 281. & \\
\hline 14. & $"$ & día L. $\sigma^{7}$ & 218 & 281. & $" \quad 4,5$ \\
\hline 15. & $"$ & freía Thnbg. $q$, kleines Exemplar & 212 & $27 \mathrm{r}$ & \\
\hline 16 a. & $"$ & páles Sehiff., ab. ísis $\mathrm{Hb} . q$ & $210 \mathrm{~b}$ & 271 & $" 4,4$ \\
\hline $16 \mathrm{~b}$. & $"$ & $" \quad " \quad$ v. arsiláehe Esp. $\sigma^{\nearrow}$ & $210 \mathrm{e}$ & 271. & \\
\hline 17. & Melitaéa & didyma 0., a $\sigma^{7}, \mathrm{~b}$ 우 & 185 & 231. & $" 3,20$ \\
\hline 18. & Brenthis & $\begin{array}{l}\text { páles Sehiff,, ein aberranter } \sigma^{7} \text { der } \\
\text { ab. napaéa } H b \text {. }\end{array}$ & $210 \mathrm{a}$ & 271 & \\
\hline 19. & Argynnis & dáphne Sehiff. $\sigma^{\nearrow}$ & 223 & $28 / 29$ & $" 4,7$ \\
\hline 20. & Brenthis & thóre Hb. + & 217 & $28 \mathrm{l}$ & \\
\hline 21. & $"$ & amathúsia Esp. 우 & 219 & $28 \mathrm{r}$. & $" 4,6 \mathrm{a}, \mathrm{b}$ \\
\hline
\end{tabular}



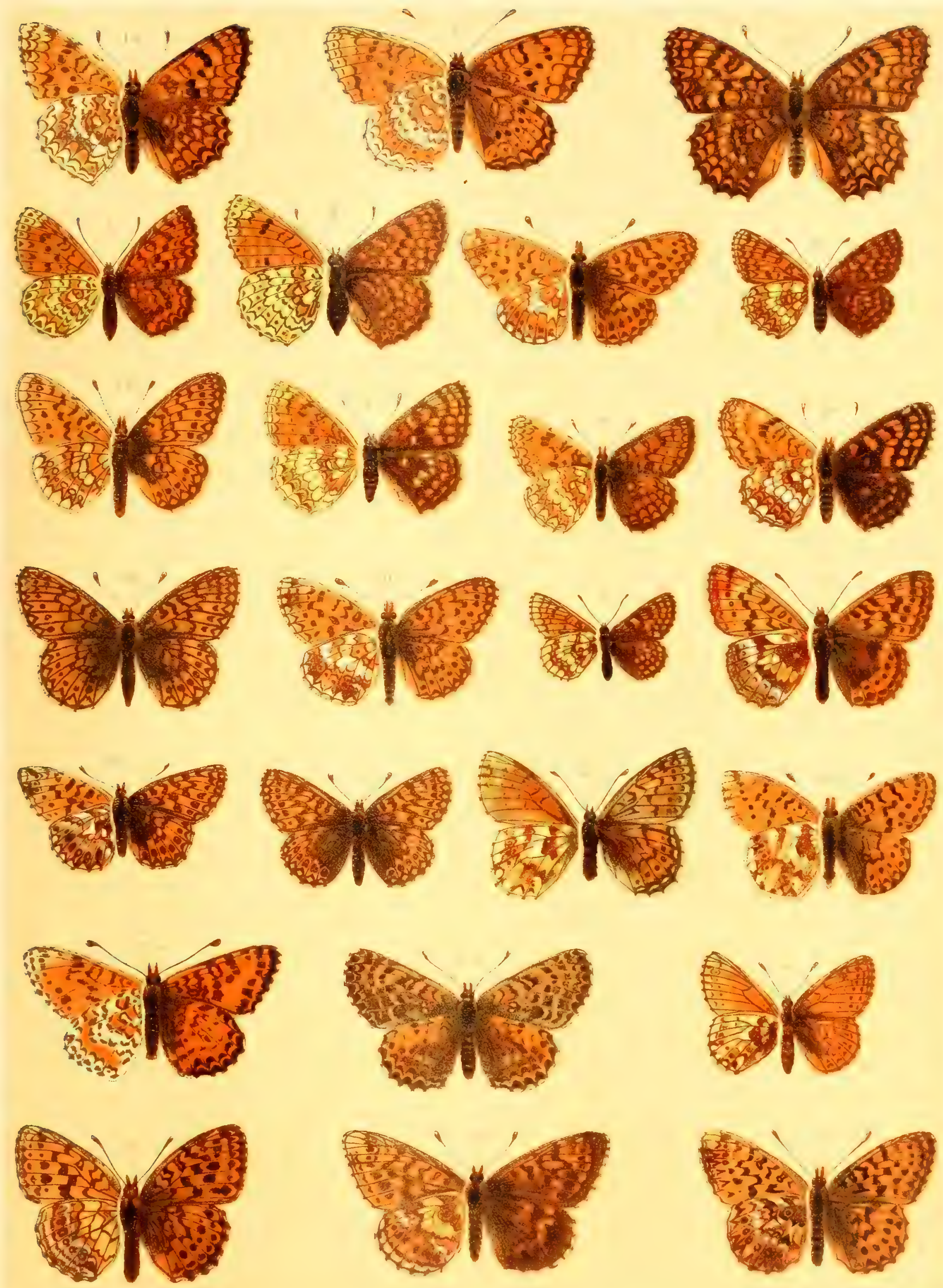




\section{TAFEL 8.}

\begin{tabular}{|c|c|c|c|c|c|}
\hline $\mathrm{Nr}$. & & Name & Katalog I & Seite des I. Bandes & $\begin{array}{l}\text { Raupen-(Puppen-) } \\
\text { Abbildung }\end{array}$ \\
\hline 1. & Argynnis & $\begin{array}{l}\text { níobe L., ab. pelópia Bkh., aus } \\
\text { der 2. Aufl. übernomm. Kopie }\end{array}$ & 231 a & $29 \mathrm{r}$. & \\
\hline 2 a. & $"$ & páphia L. $\sigma^{7}$ & 237 & $30 / 31$ & Taf. $4,13 \mathrm{a}-\mathrm{c}$ \\
\hline $2 \mathrm{~b}$. & $"$ & páphia L., ab. valesína Esp. $q$ & 237 a & 311. & $\because 4,13 \mathrm{a}-\mathrm{c}$ \\
\hline 3. & $"$ & laódice Pall. $\sigma^{7}$ & 234 & $30 \mathrm{r}$ & $\Rightarrow 48,5$ \\
\hline 4. & $n$ & agláia L. O & 230 & $30 \mathrm{r}$. & $" 4,10$ \\
\hline 5. & $"$ & latónia L. $\sigma^{7}$ & 225 & $29 \mathrm{r}$. & $\left\{\begin{array}{c}\text { Ei Taf. } 50,11 \\
, 4,9\end{array}\right.$ \\
\hline $6 \mathrm{a}$. & $"$ & níobe L., v. éris Meig. $\sigma^{7}$ & $231 \mathrm{~b}$ & $29 / 30$ & $" 4,11 \mathrm{a}, \mathrm{b}$ \\
\hline $6 \mathrm{~b}$. & $"$ & níobe $\mathrm{L}$. $q$ & 231 & $29 \mathrm{r}$. & $" 4,11 \mathrm{a}, \mathrm{b}$ \\
\hline 7. & $"$ & íno Rott., kleines $\sigma^{7}$ & 222 & 291. & $" 4,8 \mathrm{a}, \mathrm{b}$ \\
\hline 8. & $"$ & héeate Esp. $0^{7}$ & 221 & 291. & \\
\hline 9. & $"$ & elísa God. $0^{7}$ & 226 & $29 \mathrm{r}$ & Nachtr.-Taf. I, 13 \\
\hline 10. & 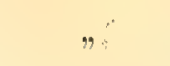 & pandóra Schiff. 우 & 240 & 311. & Taf. 4,14 \\
\hline 11. & $"$ & adíppe L. $\sigma^{7}$ & 232 & 301. & $" 4,12 \mathrm{a}, \mathrm{b}$ \\
\hline 12. & Dánais e & hrysíppus L. ๙ & 241 & $31 \mathrm{r}$ & $" 4,15 \mathrm{a}, \mathrm{b}$ \\
\hline
\end{tabular}



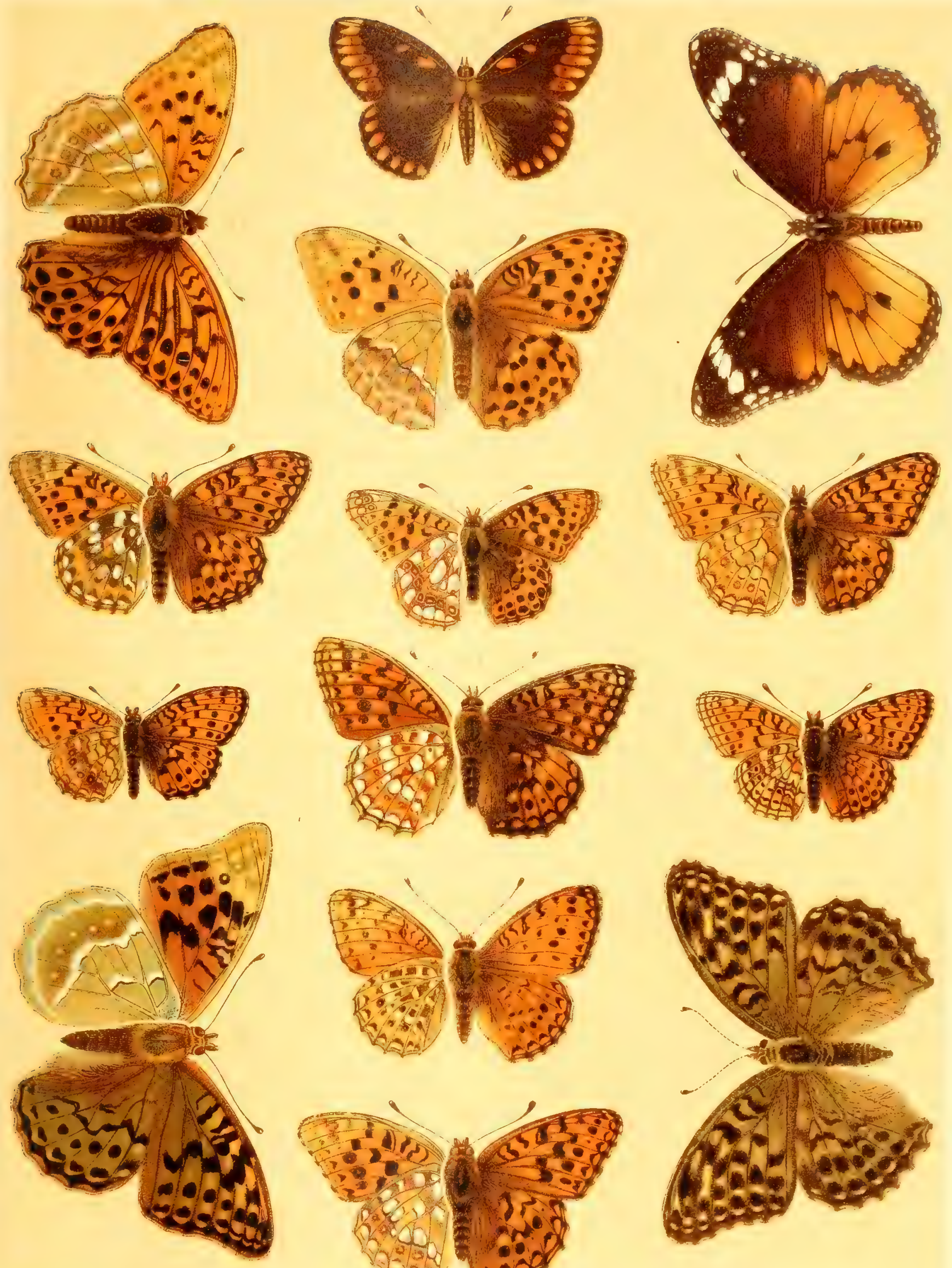



\section{TAFEL 9.}

\begin{tabular}{|c|c|c|c|}
\hline Name & Katalog I & Seite des I. Bandes & $\begin{array}{l}\text { Raupen- (Puppen-) } \\
\text { Abbildung }\end{array}$ \\
\hline 1. Maníola eriphyle Frp $q$ & 265 & 351. & \\
\hline 2. $"$ melámpus Fuessl. $\sigma^{7}$ & 262 & $34 \mathrm{r}$ & \\
\hline 3. Melanảrgia galatéa L. 우 & 246 & $32 / 33$ & Taf. $4,16 \mathrm{a}-\mathrm{c}$ \\
\hline 4. Maníola mnestra $\mathrm{Hb}$, a, $\sigma^{\nwarrow} \mathrm{b} q$ & 267 & 351. & \\
\hline 5. $\quad \quad \quad$ manto Esp. $\sigma^{7}$ & 275 & $35 \mathrm{r}$. & \\
\hline 6. Melanárgia láchesis $\mathrm{Hb}$. 우 & 245 & $32 \mathrm{r}$. & $" 4,17$ \\
\hline 7. Maniola épiphron Kn. O & 261 & 341. & $" 4,19$ \\
\hline 8. Melanárgia pherúsa B. $\sigma^{7}$ & 259 & $33 \mathrm{r}$. & \\
\hline $\begin{array}{l}\text { " laríssa H.-G. }+ \text {, sehr genähert } \\
\text { der } a b \text {. herta } H_{0}-G \text {. }\end{array}$ & 251 & 331. & \\
\hline$" \quad$ ines Hffsgg. $\sigma^{7}$ & 257 & $33 \mathrm{r}$. & \\
\hline 11: Maníola pharte $\mathrm{Hb} \cdot \sigma^{7}$ & 269 & $35 \mathrm{r}$. & \\
\hline 12. Melanárgia iapygia Cyr. $0^{7}$ & 249 & 331. & $9 \quad 4,18$ \\
\hline 13. Maniola aréte $\mathrm{F} . \phi^{*}$ ) & 268 & 351. & \\
\hline oéme Hb. $\sigma^{x}$ & 278 & $36 \mathrm{r}$. & \\
\hline 14 b. " oéme Hb., v. spódia Stgr 우 & $278 \mathrm{~b}$ & $36 \mathrm{r}$. & \\
\hline 15. Melanárgia syllius Hbst. $\sigma^{7}$ & 258 & $33 \mathrm{r.}$ & Nachtr.-Taf. I, 14 \\
\hline 16. Maníola medúsa F. $\sigma^{7}$ & 277 & 361. & Taf. 4,20 \\
\hline eeto $\mathrm{Hb} \cdot \mathrm{O}^{7}$ & 276 & 35,36 & Nachtr.-Taf. I, 14 \\
\hline stygne $0 .$, a $\sigma^{7}, \mathrm{~b}$ 우 & 279 & $36 / 37$ & \\
\hline 19. Melanárgia arge Sulz. $\sigma^{7}$ & 260 & $33 \mathrm{r}$. & \\
\hline
\end{tabular}

*) Die im Probedruck nur im Relief prononciert hervorgehoben erscheinenden Adern der Hfl.Unterseite erscheinen jetzt fälschlich weiß. 

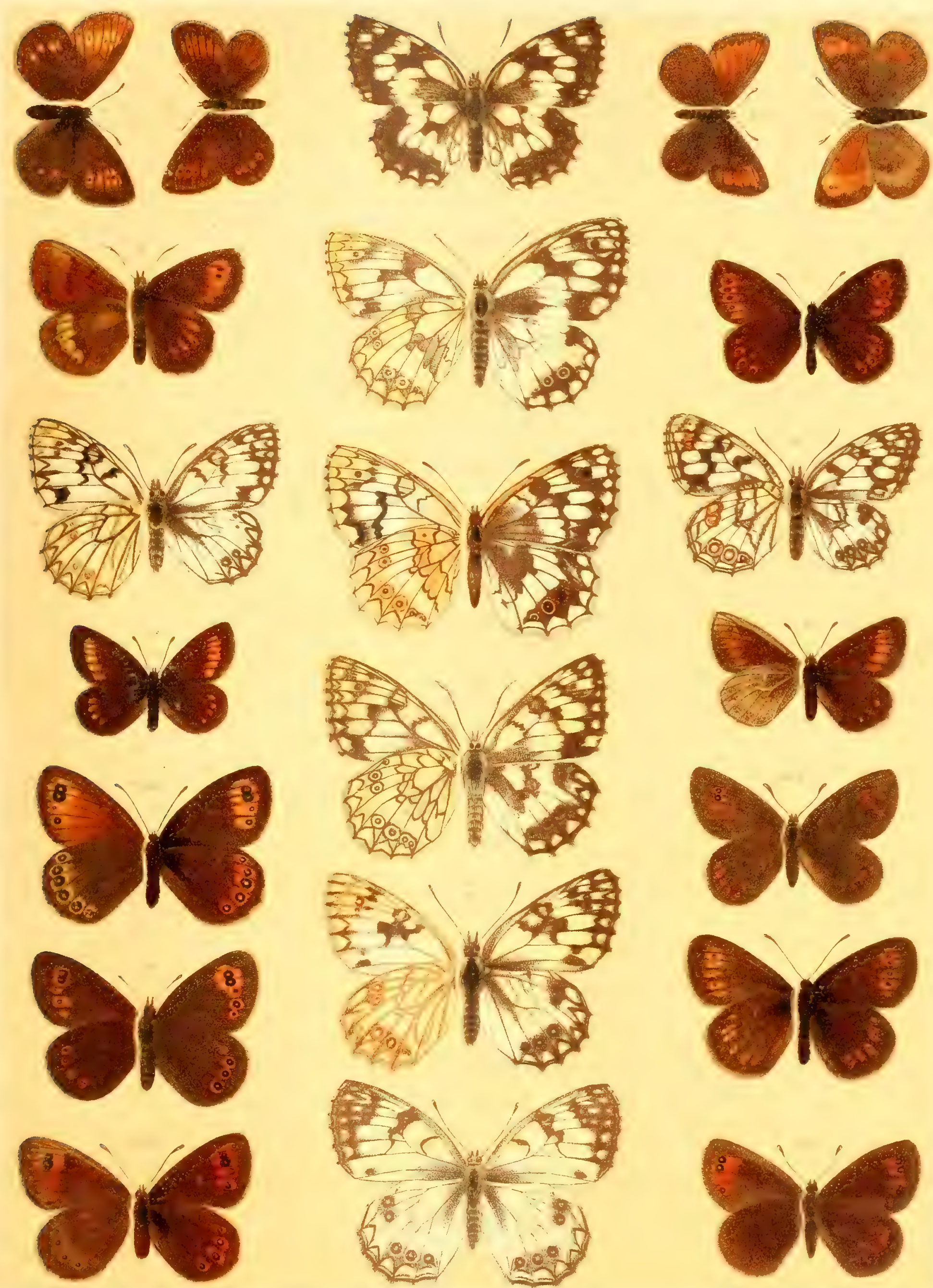



\section{TAFEL 10.}

\begin{tabular}{|c|c|c|c|c|c|}
\hline $\mathrm{Nr}$ & & Name & Katalog I & Seite des I. Bandes & $\begin{array}{c}\text { Raupen-(Puppen-) } \\
\text { Abbildung }\end{array}$ \\
\hline 1 a. & Maníola & neríne Frr ত & 283 & 37 r. & \\
\hline $1 \mathrm{~b}$. & $"$ & neríne Frp, v. reiehlíni HS. 오 & 283 a & $37 \mathrm{r}$ & \\
\hline $2 \mathrm{a}$. & $"$ & évias God. ठ & 280 & 371. & \\
\hline $2 \mathrm{~b}$. & $"$ & évias God., v. hispániea Zapater 0 & $280 \mathrm{~b}$ & 371. & \\
\hline 3. & $"$ & melas Hbst $\sigma^{7}$ & 282 & $37 \mathrm{l}$ & \\
\hline 4. & $"$ & $\begin{array}{c}\text { (hewitsóni Ld. O; eine armenische Art, } \\
\text { gegen des Verf. Absicht abgebildet) }\end{array}$ & 281 & & \\
\hline 5. & $"$ & glaciális Esp. $\sigma^{7}$ & 285 & $37 \mathrm{r.}$ & \\
\hline 6. & $"$ & seipio B. $q$ & 284 & $37 \mathrm{r}$ & \\
\hline 7 & $"$ & epistygne $\mathrm{Hb} . q$ & 289 & 381. & \\
\hline 8. & $"$ & afer Esp. $\sigma^{7}$ & 321 & $40 \mathrm{r}$ & \\
\hline 9. & $"$ & lappóna Esp. $\sigma^{7}$ & 319 & $39 / 40$ & Nachtr.-Taf. I, 15 \\
\hline 10. & " & tyndarus Esp. $\sigma^{7}$ & 320 & 401. & \\
\hline 11. & $"$ & gorge Esp. ठ & 292 & 381. & \\
\hline 12. & $"$ & goánte Esp., a $o^{7}, \mathrm{~b} q$ & 290 & 381. & \\
\hline 13. & $"$ & prónoë Esp. $\sigma^{7}$ & 288 & $37 / 38$ & \\
\hline 14. & $"$ & neópidas B. $\sigma^{7}$ & 293 & $38 \mathrm{r}$ & \\
\hline 15. & $"$ & aéthiops Esp. 우 & 296 & $38 \mathrm{r}$. & Taf. 4,21 \\
\hline 16. & $"$ & zapatéri 0bth. $\sigma^{7}$; kleines Exemplar & 294 & $38 \mathrm{l}$ & \\
\hline 17. & $"$ & ligéa L. $\sigma^{7}$ & 302 & 391. & $" 4,22$ \\
\hline 18. & $"$ & gorge Esp., ab. triópes Stgp 우 & $292 \mathrm{~b}$ & $38 \mathrm{r}$. & \\
\hline
\end{tabular}



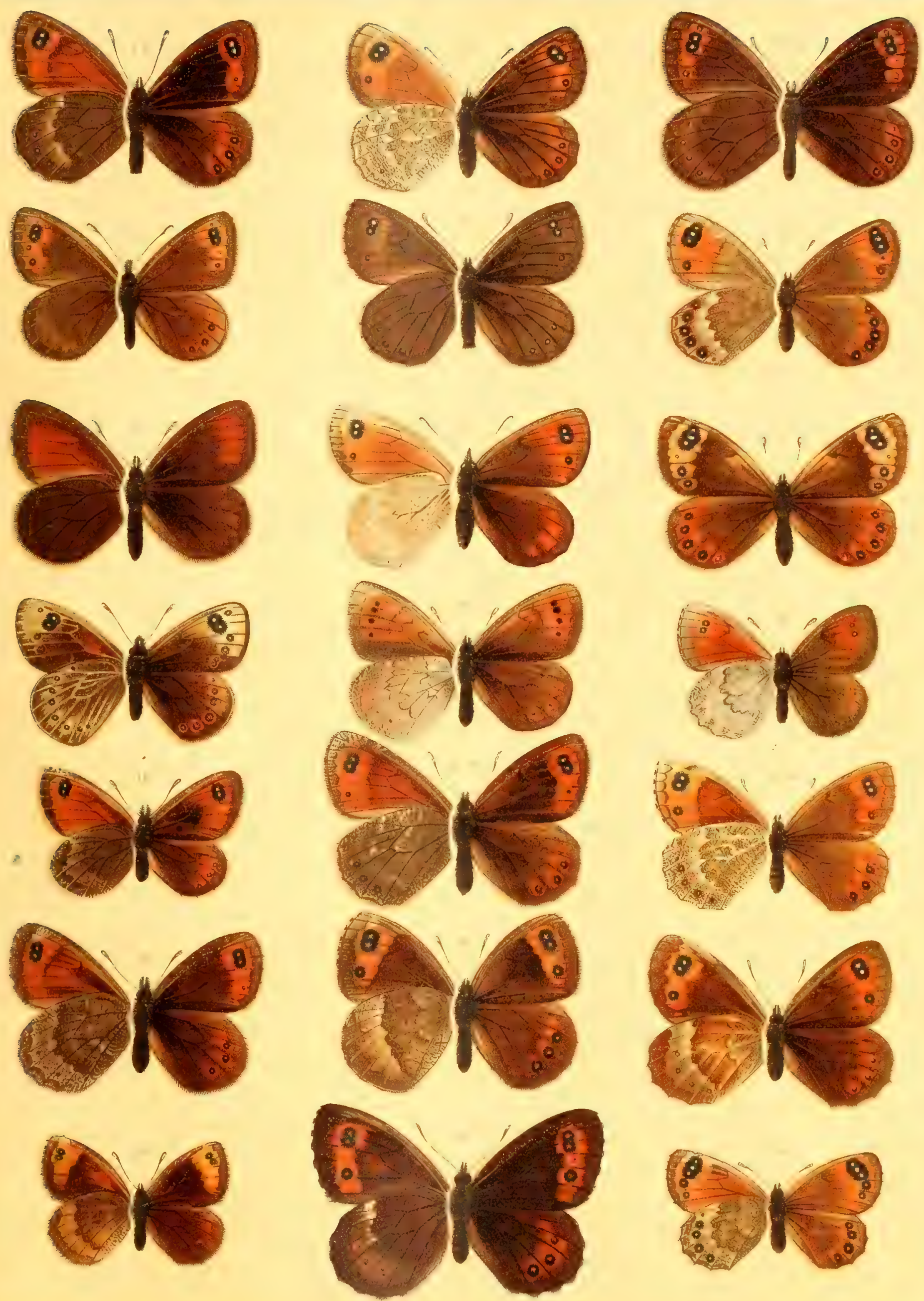




\section{TAFE L 11.}

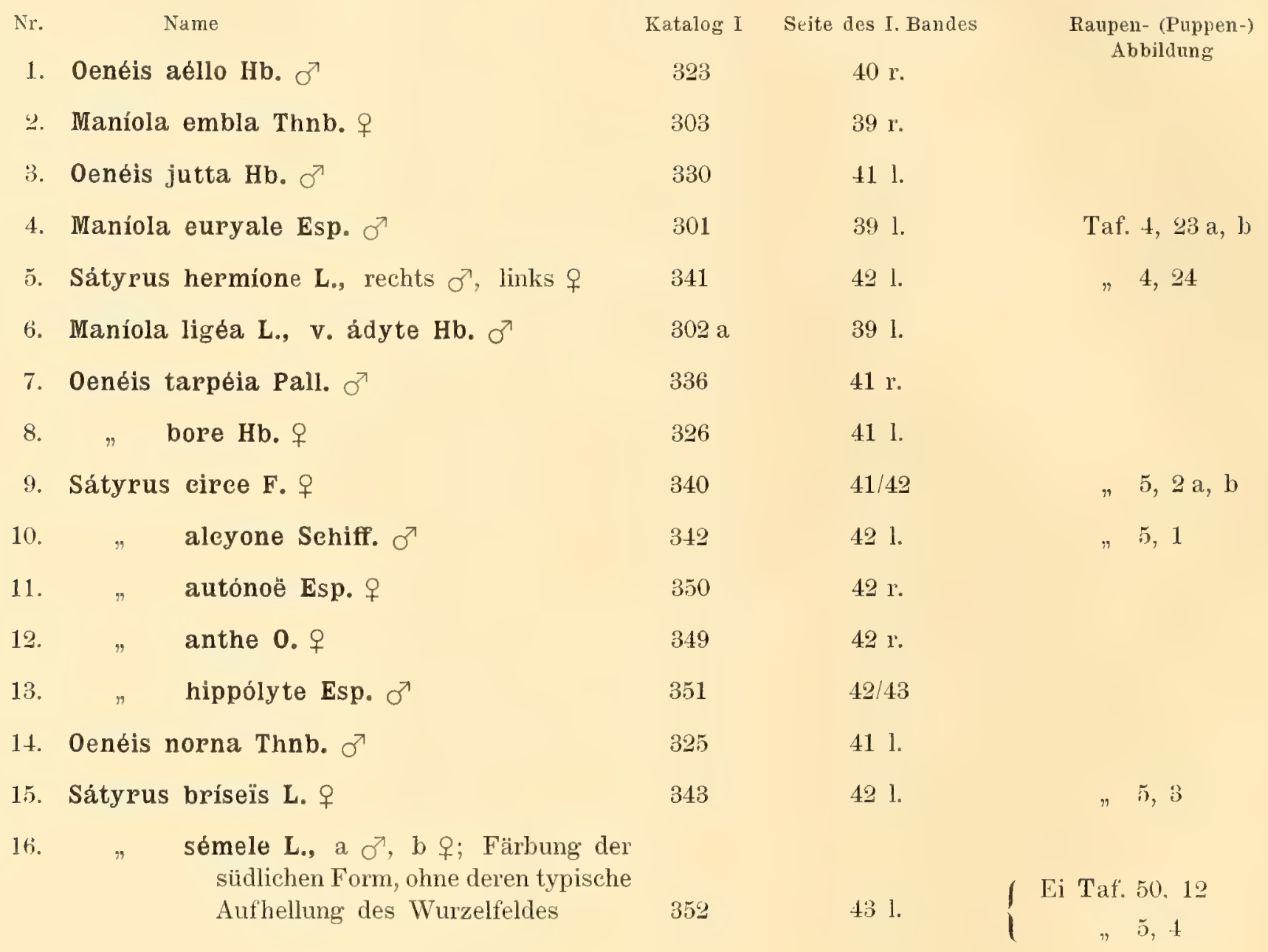



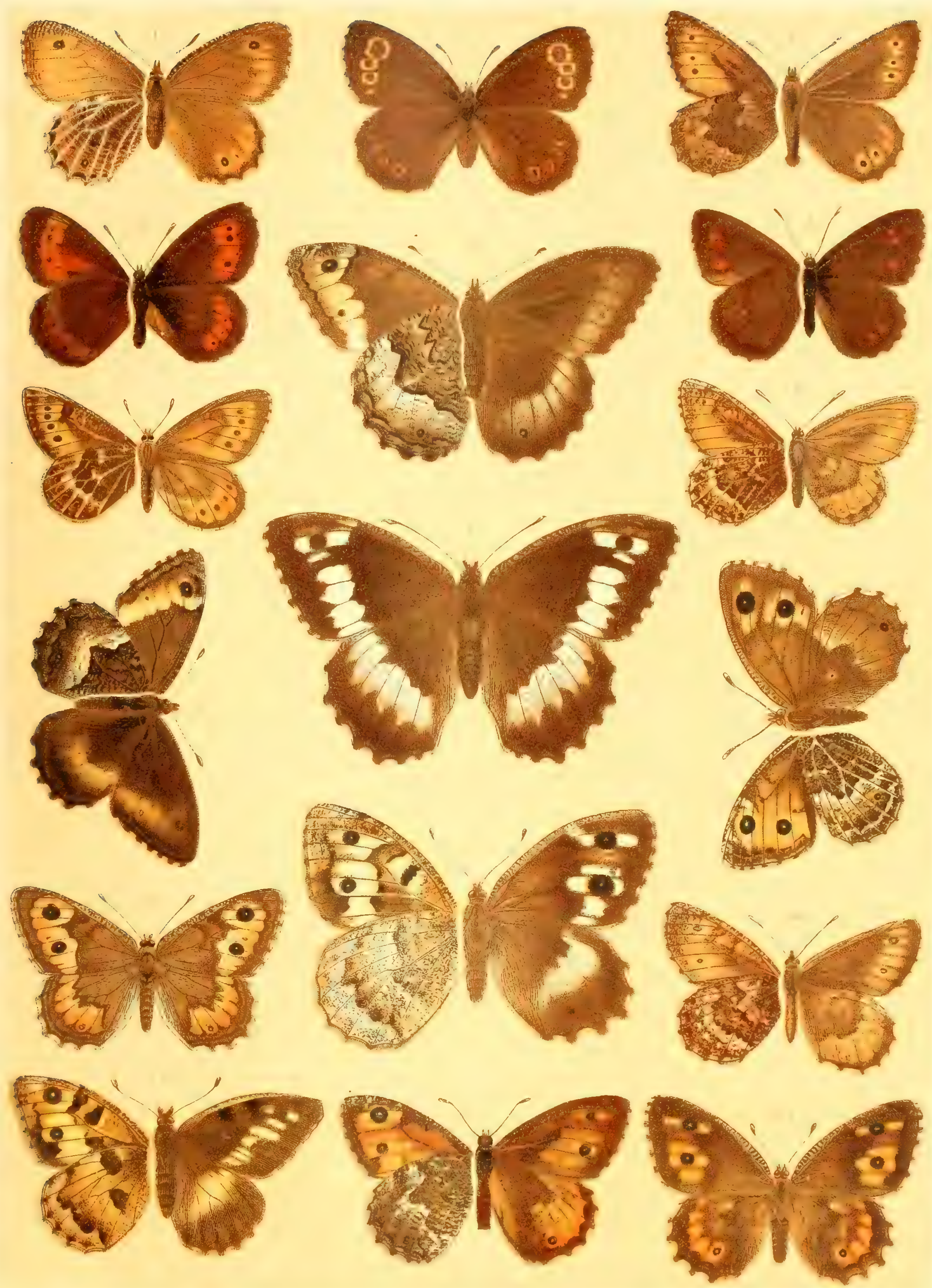




\section{TAFEL 12.}

\begin{tabular}{|c|c|c|c|c|c|}
\hline Nr. & r r & Name & Katalog I & Seite des I. Bandes & $\begin{array}{l}\text { Raupen- (Puppen-) } \\
\text { Abbildung }\end{array}$ \\
\hline 1. & Sátyrus & neoméris God. o & 354 & $43 \mathrm{r}$. & \\
\hline 2. & $"$ & statilinus Hufn. $\sigma^{7}$ & 370 & $43 / 44$ & Nachtr.-Taf. I, 18 \\
\hline 3. & $n$ & arethúsa Esp. ত & 353 & 431. & \\
\hline 4. & Parárge & megaéra L. $\sigma^{\pi}$ & 390 & 501. & $\left\{\begin{array}{c}\text { Ei Taf. } 50,13 \\
n 5,7\end{array}\right.$ \\
\hline $5 \mathrm{a}$. & Sátyrus & aetaéa Esp. O & 378 & 441. & \\
\hline $5 \mathrm{~b}$. & $"$ & aetaéa Esp., v. córdula F. 우 & $378 \mathrm{~b}$ & 441. & Nachtr.-Taf. I, 19 \\
\hline 6. & Parárge & hiera F. $q$ & 391 & 501. & \\
\hline $7 \mathrm{a}$. & $"$ & maéra L. $\sigma^{7}$ & 392 & $50 \mathrm{r}$ & Taf. $5,6 \mathrm{a}, \mathrm{b}$ \\
\hline $7 \mathrm{~b}$. & $"$ & maéra L., v. adrásta Hb. $q$ & $392 \mathrm{~b}$ & $50 \mathrm{r}$ & \\
\hline 8. & Sátyrus & fátua Frr $q$ & 371 & $44 \mathrm{l}$ & \\
\hline 9. & $"$ & fídia L. 우 & 372 & 441. & \\
\hline 10. & $"$ & dryas Se. 우 & 381 & $44 \mathrm{r}$. & $" 5,5$ \\
\hline 11. & Parárge & $\left\{\begin{array}{l}\text { rechts egéria L. } \sigma^{\pi} \\
\text { links v. egerídes Stgr. } \sigma^{\nearrow}\end{array}\right.$ & $\begin{array}{l}385 \\
385 \text { a }\end{array}$ & $\begin{array}{l}49 \mathrm{r} . \\
49 \mathrm{r} .\end{array}$ & $" 5,8$ \\
\hline $12 \mathrm{a}$. & Epinéphe & ele lycáon Rott. + & 405 & $45 / 46$ & $" 5,10 \mathrm{a}-\mathrm{c}$ \\
\hline $12 \mathrm{~b}$. & $"$ & lyeáon Rott., v. lupínus Costa & $\sigma^{7} 405 \mathrm{f}$ & 461. & \\
\hline 13. & Parárge & achíne Seop. $q$ & 394 & $50 / 51$ & $" 5,9$ \\
\hline 14. & $"$ & poxelána $\mathrm{Cr} \cdot \sigma^{7}$ & 389 & 501. & \\
\hline
\end{tabular}



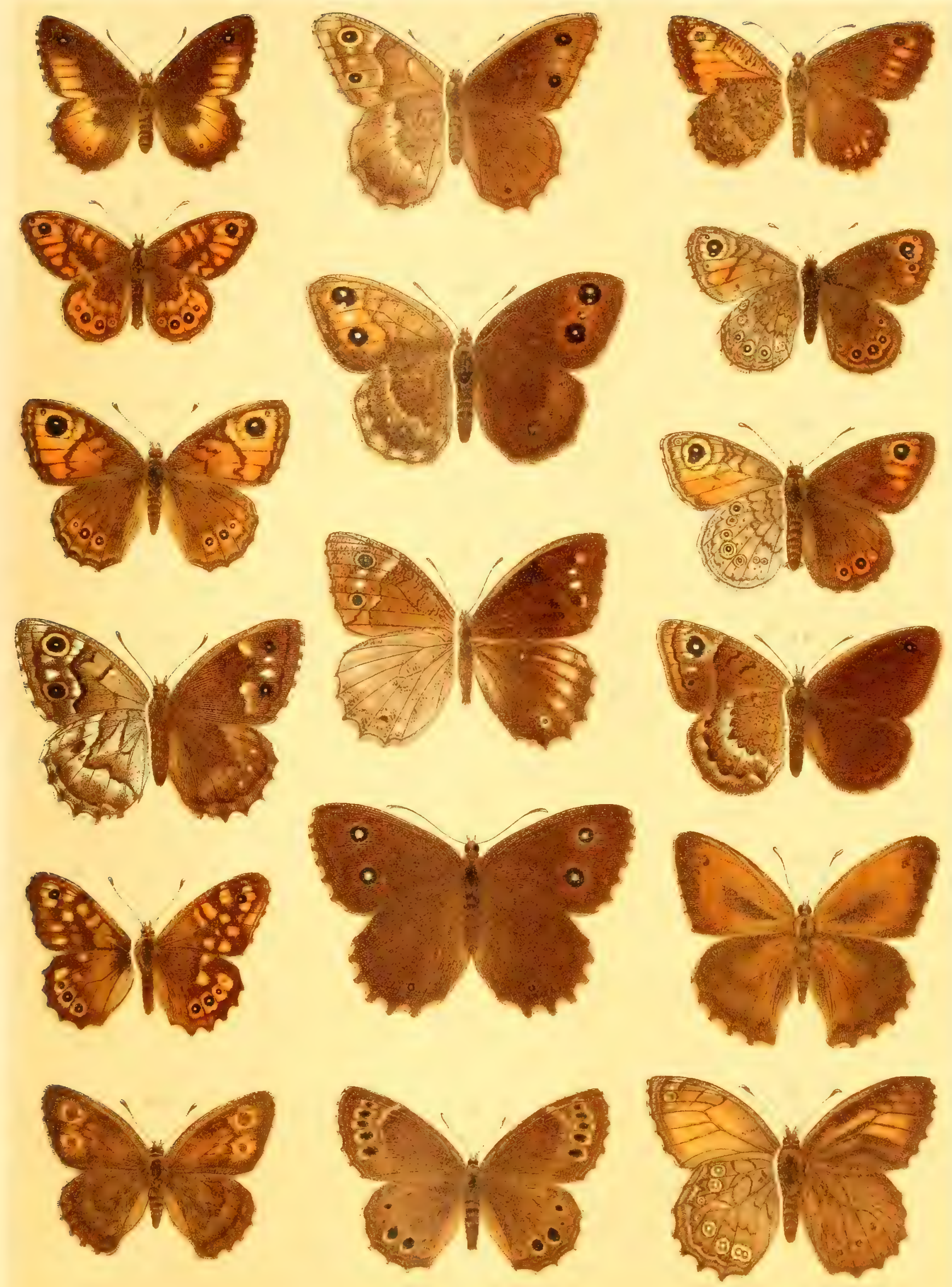



\section{TAFEL 13.}

\begin{tabular}{|c|c|c|c|c|}
\hline Nr. & Name & Katalog I & Seite des I. Bandes & $\begin{array}{l}\text { Raupen- (Puppen-) } \\
\text { Abbildung }\end{array}$ \\
\hline 1. & Epinéphele jurtína L., a $\sigma^{7}, \mathrm{~b}$ 우 & 402 & $45 \mathrm{r}$. & $\left\{\begin{array}{c}\text { Ei Taf. } 50,14 \\
" 5,11\end{array}\right.$ \\
\hline 2. & $" \quad$ ida Esp. $q$ & 423 & $46 \mathrm{r}$. & Taf. 5, 12 \\
\hline 3. & $" \quad$ tithónus L. $q$ & 422 & 461. & "5, $13 \mathrm{a}-\mathrm{c}$ \\
\hline 4. & Coenonympha hero L. $\sigma^{7}$ & 426 & $47 \mathrm{r}$ & Ei Taf. 50,15 \\
\hline 5. & leánder Esp. $\sigma^{7}$ & 428 & $47 \mathrm{r}$. & \\
\hline 6. & Epinéphele pasíphaë Esp. $\sigma^{7}$ & 424 & $46 \mathrm{r}$ & Nachtr.-Taf. I, 20 \\
\hline 7. & Aphántopus hyperánthus L. $q$ & 401 & 451. & Taf. 5,14 \\
\hline 8. & Coenonympha iphis Sehiff., a $\sigma^{7}, \mathrm{~b}$ 우 & 427 & 471. & $" 5,15 \mathrm{a}, \mathrm{b}$ \\
\hline 9. & oédipus F. O & 425 & 471. & \\
\hline $10 \mathrm{a}$. & " areánia L. $q$ & 433 & $47 / 48$ & $\because 5,16$ \\
\hline $10 \mathrm{~J}$. & " arcánia L., v. satyrion Esp. $\sigma^{7}$ & $433 \mathrm{~d}$ & $47 / 48$ & \\
\hline 11. & corínna Hb. $\sigma^{T}$ & 437 & 481 & \\
\hline 12. & $" \quad$ thyrsis Frr $\sigma^{7}$ & 439 & 481. & \\
\hline 13. & $" \quad$ dorus Esp. 우 & 436 & 481. & \\
\hline 14 a. & pámphilus L. $\sigma^{7}$ & 440 & 481. & $95,17 \mathrm{a}, \mathrm{b}$ \\
\hline $1+b$. & $\begin{array}{l}\text { pámphilus L., gen. aest. } \\
\text { lyllus Esp. } \sigma^{7}\end{array}$ & $440 \mathrm{~b}$ & $48 \mathrm{r}$ & \\
\hline 15. & tiphon Rott. $\sigma^{7}$ & 443 & $48 / 49$ & $" 48,6$ \\
\hline 16. & Triphysa phryne Pall., a $\sigma^{\nearrow}, \mathrm{b}$ 우 & 448 & 491. & \\
\hline 17. & Carehárodus Hb. aleéae Esp. ㅇ & 686 & 741 & $" 5,18 \mathrm{a}, \mathrm{b}$ \\
\hline 18. & althaéa Hb. $q$ & 687 & 471 & Nachtr.-Taf. I, 25 \\
\hline 19. & Iavatérae Esp. 우 & 685 & $73 / 74$ & Taf. 48,7 \\
\hline 20. & Hespéria proto Esp. $\sigma^{7}$ & 688 & 751. & \\
\hline 21. & tesséllum Hb. $\sigma^{7}$ & 695 & 751. & \\
\hline 22. & $" \quad$ sidae Esp. $\sigma^{7}$ & 693 & $75 / 76$ & \\
\hline 23. & eárthami Hb. ㅇ & 694 & $76 \mathrm{l}$ & \\
\hline 24. & $" \quad$ álveus $\mathrm{Hb}$. & 703 & 761 & \\
\hline 25. & serrátulae Rbr $\sigma^{7}$ & 701 & $76 \mathrm{r}$ & \\
\hline 26. & " eacáliae Rbr 우 & 704 & $76 \mathrm{r}$. & \\
\hline
\end{tabular}



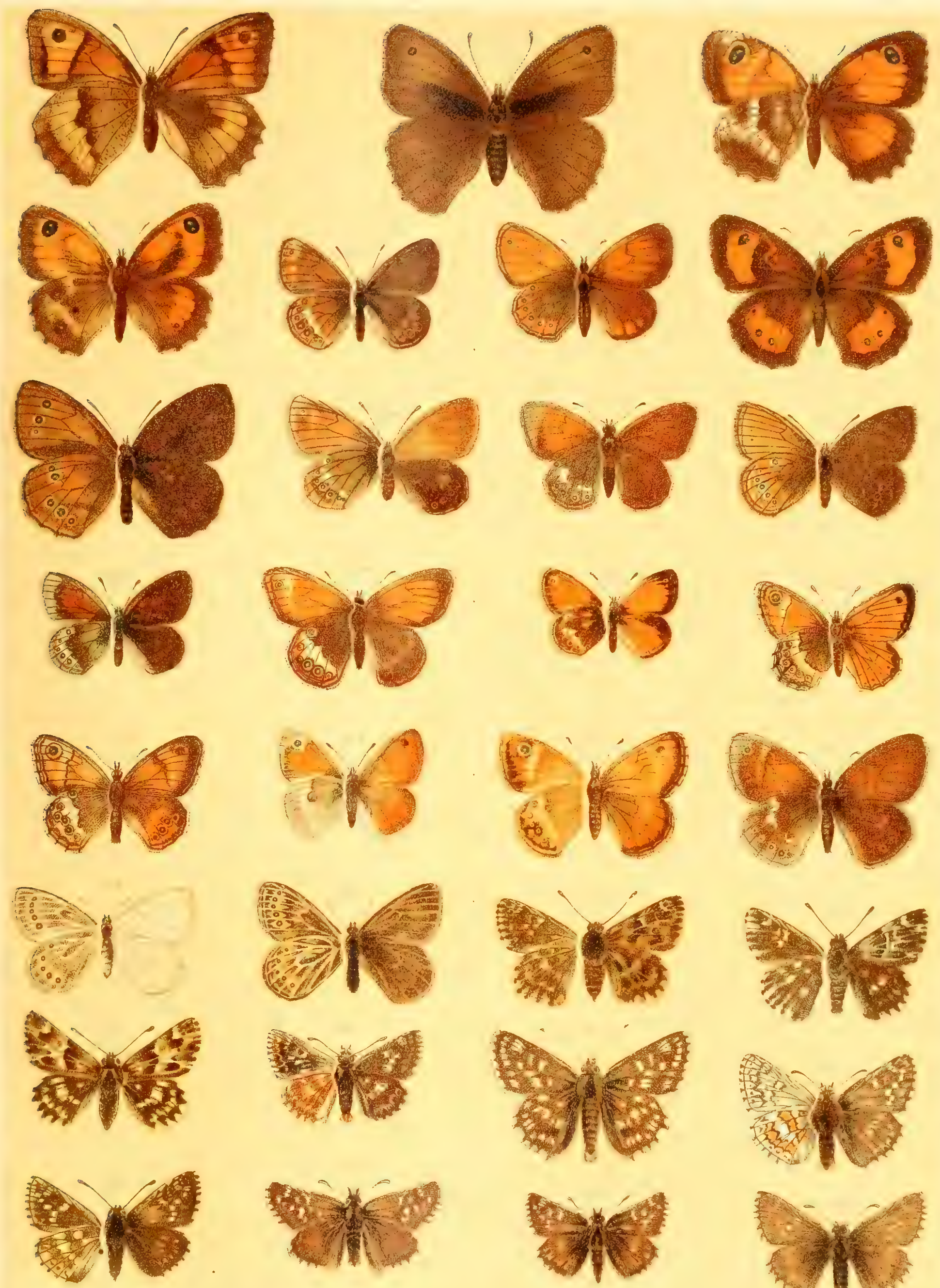
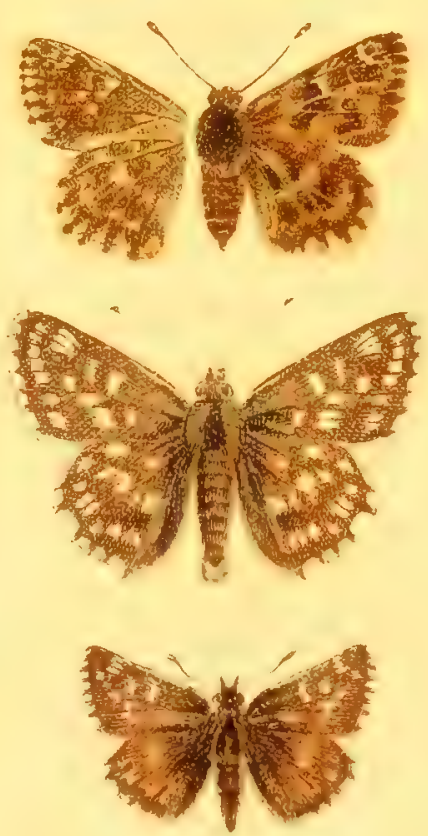
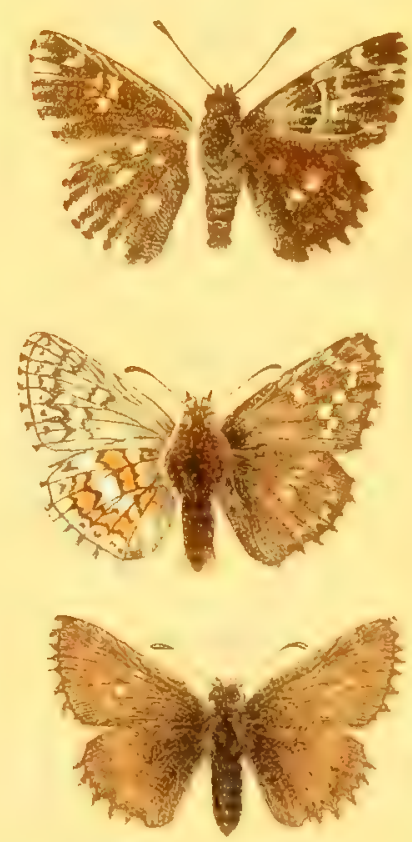



\section{TAFEL 14.}

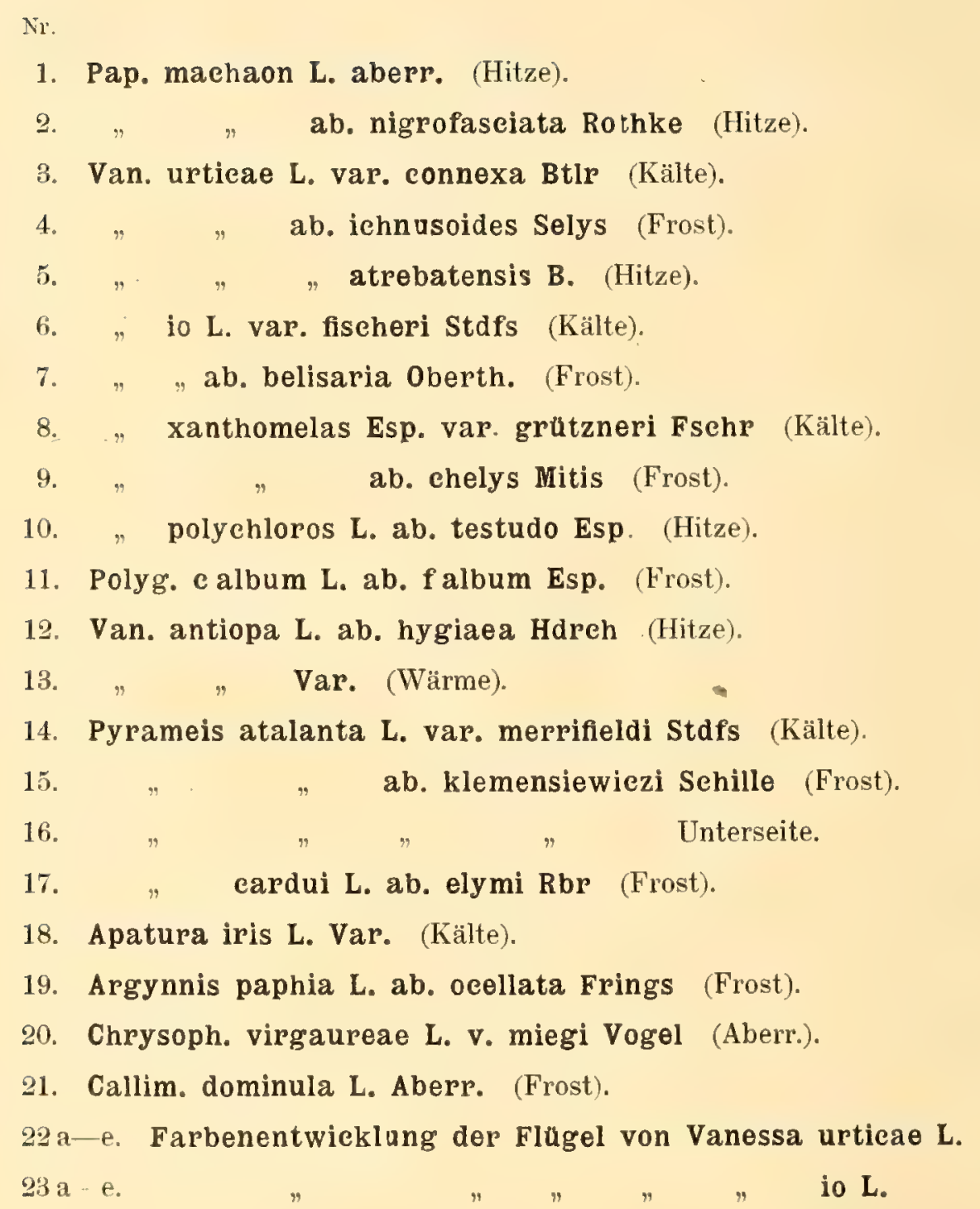

Die Abbildungen 1-21 gehören zu dem Kapitel Temperatur-Experimente, p. XC ff., speziell zu p. CI-CIV; Fig. 22 a-e und 23 a-e zu p. LII. 

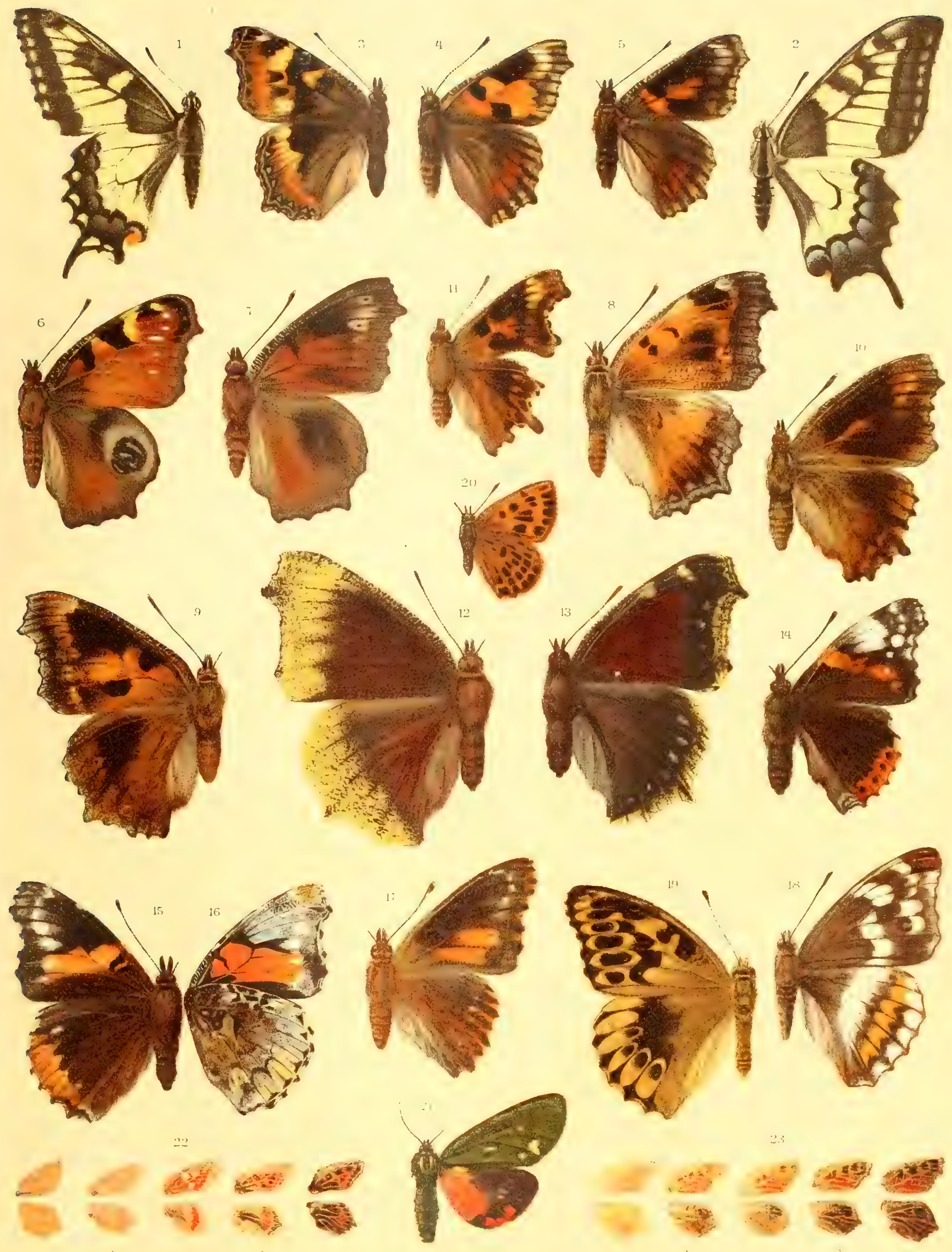



\section{TAFEL 15.}

\begin{tabular}{|c|c|c|c|c|}
\hline Nr. & Name & Katalog I & Seite des I. Bandes & $\begin{array}{c}\text { Raupen- (Puppen-) } \\
\text { Abbildung }\end{array}$ \\
\hline 1. & Zéphypus bétulae L. $q$ & 492 & $54 / 55$ & $\left\{\begin{array}{c}\text { Ei Taf. } 50,6 \\
, 2,3\end{array}\right.$ \\
\hline 2. & Cállophrys rubi L. 우 & 476 & 541. & Taf. 2,9 \\
\hline 3. & Zéphyrus quercus L. 우 & 482 & $54 \mathrm{r}$ & $" 2,8$ \\
\hline 4. & Theela spini Schiff. $\sigma^{7}$ & 460 & $52 / 53$ & $" 2,4 \mathrm{a}, \mathrm{b}$ \\
\hline 5. & $" \quad$ acáciae F. $q$ & 465 & $53 \mathrm{r.}$ & Nachtr.-Taf. I, 22 \\
\hline 6. & $"$ w-album Knoeh. $\sigma^{7}$ & 461 & 531. & Taf. 2,5 \\
\hline 7. & $" \quad$ ilicis Esp. $q$ & 464 & 531. & $\Rightarrow \quad 2,6$ \\
\hline 8. & Thestor ballus F. $q$ & 495 & $55 \mathrm{r}$. & "2, 10 \\
\hline 9. & Laeósopis róboris Esp. ఠ & 457 & 551. & \\
\hline 10. & Chrysophánus virgaúreae L., a $\sigma^{7}, \mathrm{~b}$ 우 & 500 & 561. & $" 2,11$ \\
\hline 11. & Theela pruni L. $q$ & 466 & $53 \mathrm{r}$. & $" 2,7 \mathrm{a}, \mathrm{b}$ \\
\hline 12. & Cpysophánus ottománus Lef. $\sigma^{7}$ & 501 & $56 \mathrm{r}$. & \\
\hline 13. & $" \quad$ thersámon Esp., a $\sigma^{\pi}, \mathrm{b} q$ & 506 & 571. & $" 2,12$ \\
\hline 14. & Thestor nogélli HS. $\sigma^{7}$ & 499 & $55 / 56$ & \\
\hline $15 \mathrm{a}$. & $\begin{array}{c}\text { Chrysophánus phlaéas L., ab. eaeruleopune- } \\
\text { táta Stgr. o der } 1 \text {. Gen. }\end{array}$ & -512 & 581. & $" 2,16$ \\
\hline $15 \mathrm{~b}$. & phlaéas L., v. eléus F. $\sigma^{\gamma}$ & $512 b$ & 581. & \\
\hline 16. & $\begin{array}{l}\text { " dispar Hw., v. rútilus Wernb., } \\
\text { a } \sigma^{7}, \mathrm{~b} \text { 우 }\end{array}$ & $508 \mathrm{a}$ & 57 l. & $" 48,3$ \\
\hline 17. & hippóthoë L., a $\sigma^{7}, \mathrm{~b}$ 우 & 510 & $57 \mathrm{r}$. & $" 2,13 \mathrm{a}, \mathrm{b}$ \\
\hline 18. & dórilis Hufn., a $\sigma^{7}, \mathrm{~b}$ 우 & 513 & $58 \mathrm{r}$. & $" 2,15 \mathrm{a}-\mathrm{c}$ \\
\hline 19. & amphidamas Esp., a $\sigma^{\lambda}, b$ 우 & 514 & $58 / 59$ & $" 2,17 \mathrm{a}, \mathrm{b}$ \\
\hline 20 & áleiphron Rott., a $\sigma^{7}, \mathrm{~b}$ 우 & 511 & $57 / 58$ & $" 2,14$ \\
\hline 21. & Thestor eallímachus Ev. $q$ & 498 & $55 \mathrm{r}$. & Nachtr.-Taf. I, 23 \\
\hline 22. & Lámpides boéticus L., a $\sigma^{7}, \mathrm{~b}$ ㅇ & 529 & $59 \mathrm{r}$ & Taf. $2,18 \mathrm{a}-\mathrm{c}$ \\
\hline 23. & telieánus Lang $\sigma^{\top}$ & 530 & $59 / 60$ & $" 2,19$ \\
\hline 24. & baleánicus Frr ơ & 531 & 601. & \\
\hline
\end{tabular}




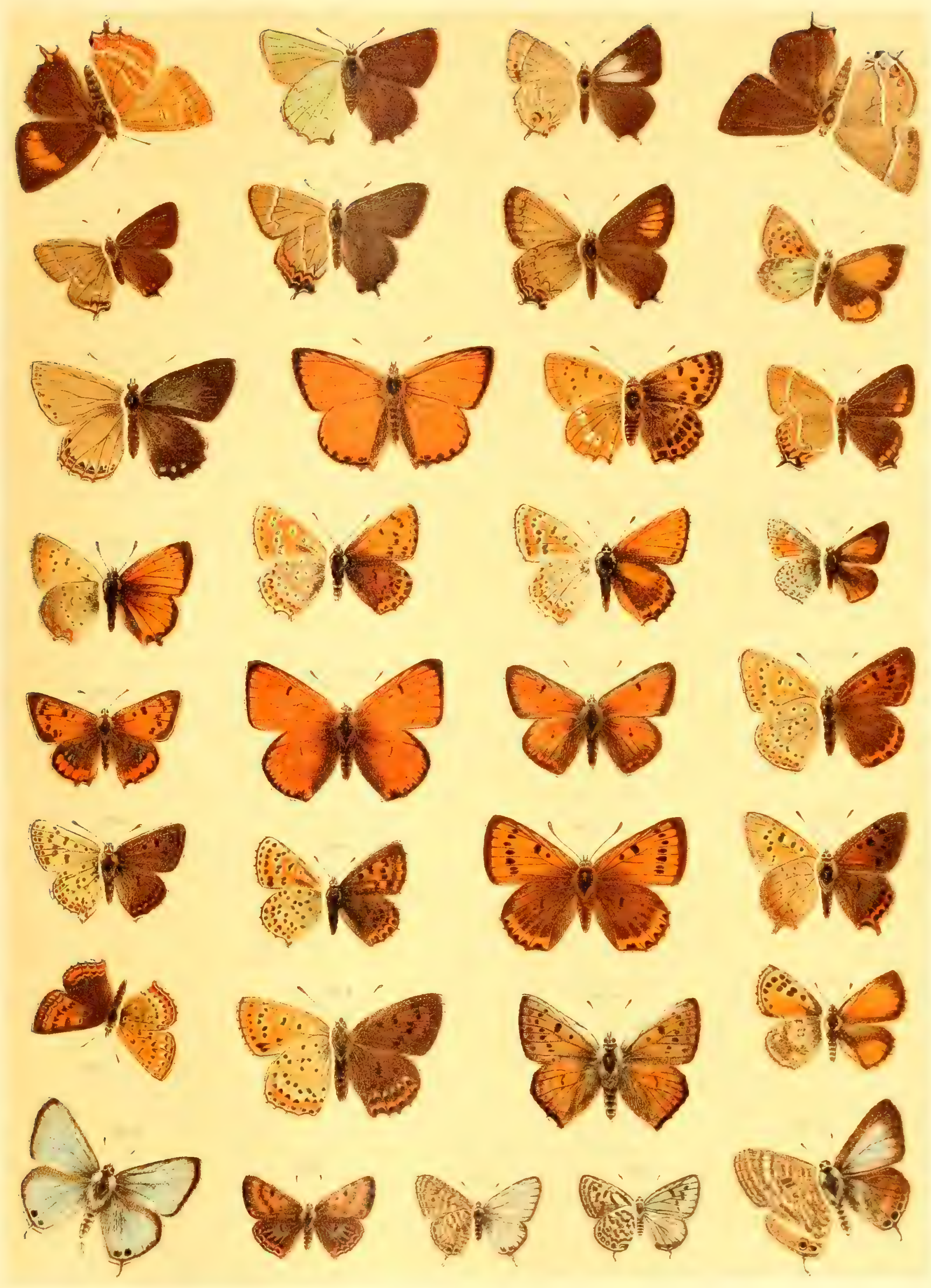





\section{TAFE L 16.}

\begin{tabular}{|c|c|c|c|c|c|}
\hline $\mathrm{Nr}$. & & Name & Ratalog I & Seite des I. Bandes & $\begin{array}{c}\text { Raupen- (Puppen-) } \\
\text { Abbildung }\end{array}$ \\
\hline 1. & Lyeaéna & argus L., a $\sigma^{7}, \mathrm{~b}$ ㅇ & 543 & $60 / 61$ & Taf. $2,20 \mathrm{a}, \mathrm{b}$ \\
\hline 2. & $"$ & argiades Pall., a $\sigma^{7}, \mathrm{~b}$ 오 & 540 & $60 \mathrm{r}$. & Nachtr.-Taf. I, 24 \\
\hline 3. & $"$ & argyrognómon Bergstr., a $\sigma^{7}, \mathrm{~b} q$ & 544 & 611. & Taf. 2,21 \\
\hline 4. & $"$ & zéphypus Friv. ণ & 552 & $61 \mathrm{r}$. & \\
\hline 5. & . & psyllorita Frr. & 588 & $63 \mathrm{r}$ & \\
\hline 6. & , & optiléte Knoch. q & 563 & $61 / 62$ & "2, 22 \\
\hline 7. & $"$ & orion Pall. $\sigma^{7}$ & 574 & $62 \mathrm{r.}$ & Nachtr.-Taf. I, 24b \\
\hline 8. & $"$ & orbitulus Prun., a $\sigma^{7}, b+$ & 581 & $62 / 63$ & \\
\hline 9. & " & pherétes $\mathrm{Hb} . \sigma^{7}$ & 583 & 631. & \\
\hline 10. & $"$ & pyláon F. d.W. Ђ & 551 & $61 \mathrm{r}$. & \\
\hline 11. & Theela $\mathrm{r}$ & hymnus Ev. & 473 & 541. & \\
\hline 12. & Lyeaéna & baton Bergstr. $\sigma^{7}$ & 573 & 621. & $\mathrm{I}, 24 \mathrm{a}$ \\
\hline $13 \mathrm{a}$. & $"$ & astrárche gen. aest. eálida Bell. $\sigma^{7}$ & $589 \mathrm{~b}$ & $63 \mathrm{r}$. & Taf. 2,23 \\
\hline 131. & , & astrárehe Bergstr. 우 & 589 & $63 \mathrm{r}$ & \\
\hline 14. & $"$ & tróchilus Frr. & 539 & 601. & \\
\hline 15. & $"$ & ánteros Frr. $\sigma^{7}$ & 596 & $64 \mathrm{l}$. & \\
\hline 16. & $"$ & icarus Rott., a $\sigma^{7}, \mathrm{~b}$ ㅇ & $604 !$ & $64 \mathrm{r}$ & $" 2,24$ \\
\hline $17 \mathrm{a}$. & $"$ & eros 0. v. eroídes Friv. $\sigma^{7}$ & $597 \mathrm{c} !$ & $64 \mathrm{r}$ & \\
\hline $17 \mathrm{~b}$. & $"$ & eros $0.0^{7}$ & 597 & $64 \mathrm{r}$. & \\
\hline 18. & $"$ & amánda Sehn., a 오, b $\sigma^{7}$ & 607 & 651. & \\
\hline 19. & $"$ & eschéri Hb., a $\sigma^{7}, \mathrm{~b}$ 우 & 612 & $65 \mathrm{r}$. & \\
\hline 20. & $"$ & eúmedon Esp. 우 & 592 & 641. & \\
\hline 21. & $"$ & $\begin{array}{l}\text { bellárgus Rott., a } \sigma^{\top}, \mathrm{b} \text { links 우 } \\
\text { b rechts ab. cerónus Esp. 우 }\end{array}$ & $\begin{array}{l}613 \\
613 \mathrm{~b}\end{array}$ & $\begin{array}{l}65 / 66 \\
65 / 66\end{array}$ & $" 2,25$ \\
\hline 22. & $"$ & lysimon $\mathrm{Hb} \cdot \mathrm{O}^{7}$ & 569 & 621. & \\
\hline 23. & $"$ & hylas Esp., a $\sigma^{7}, \mathrm{~b}$ 우 & 610 & 651. & \\
\hline 24. & $"$ & idas Rbr. $\sigma^{7}$ & 587 & $63 \mathrm{r}$ & \\
\hline 25. & $"$ & eórydon Poda, a $\sigma^{7}, \mathrm{~b}$ 우 & 614 & $66 \mathrm{l}$. & $\Rightarrow \quad 2,26$ \\
\hline
\end{tabular}



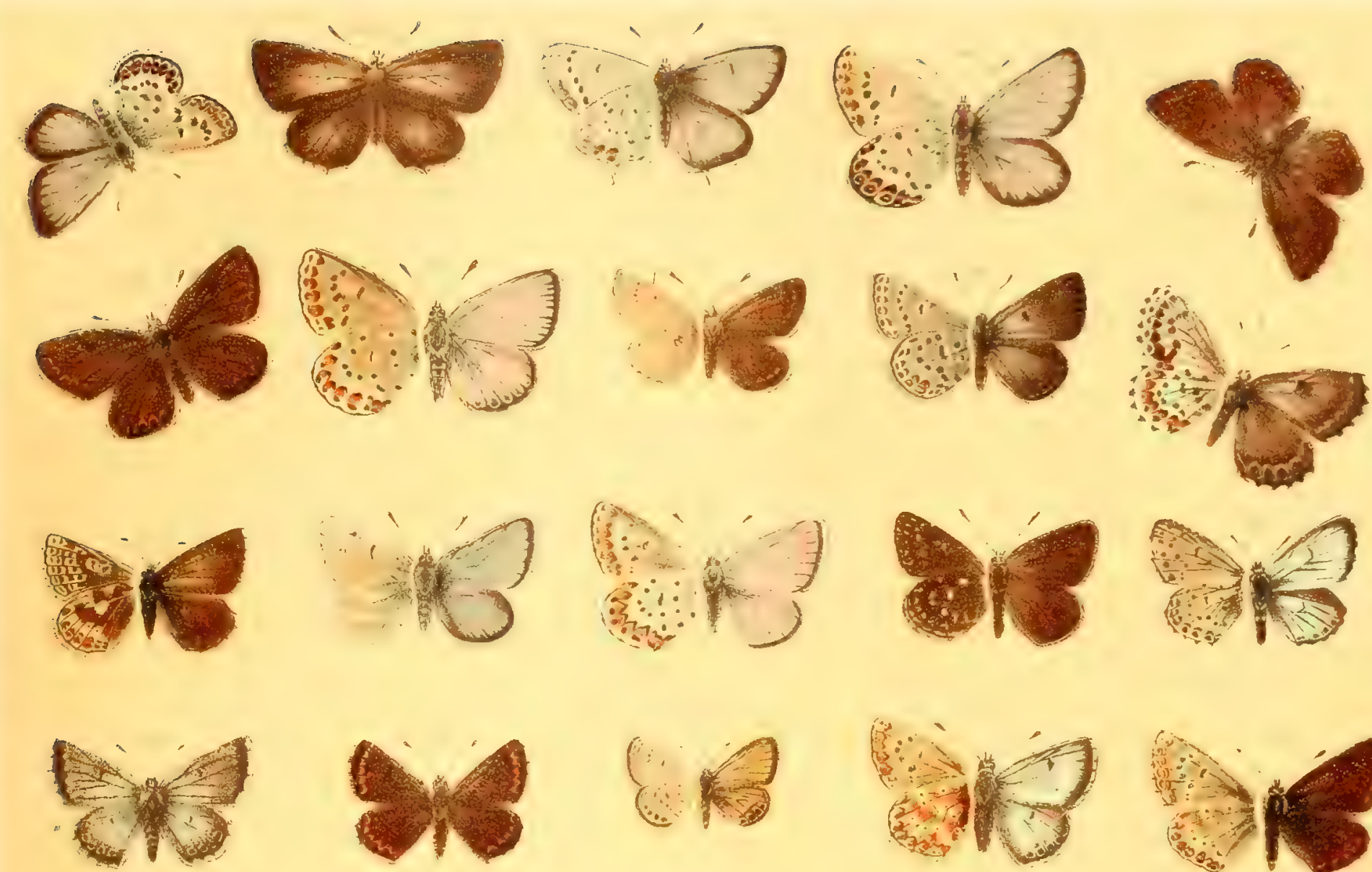

12
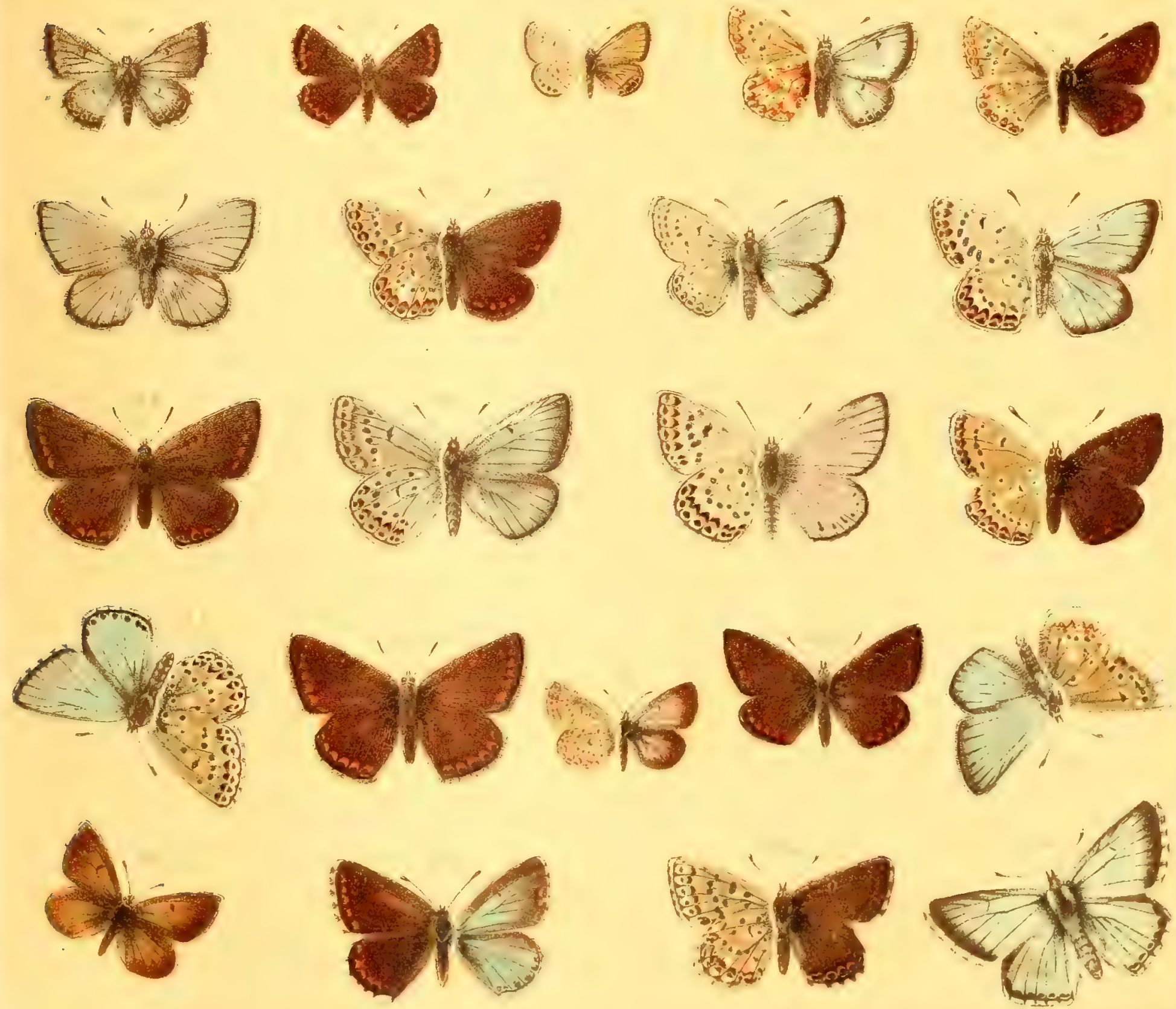




\section{TAFEL 17.}

\begin{tabular}{|c|c|c|c|c|}
\hline $\mathrm{Nr}$. & Name & Katalog I & Seite des I. Bandes & $\begin{array}{c}\text { Raupen-(Puppen-) } \\
\text { Abbildung }\end{array}$ \\
\hline 1. & Lycaéna admétus Esp. & 619 & $66 \mathrm{r}$. & \\
\hline 2. & sebrus B. $\sigma^{7}$ & 633 & $67 \mathrm{l}$ & \\
\hline 3. & Libythea celtis Laicharting & 450 & $51 \mathrm{r}$ & Taf. $2,34 \mathrm{a}-\mathrm{c}$ \\
\hline 4. & donzéli B. $\sigma^{7}$ & 593 & 641. & \\
\hline 5. & Nemeóbius lucína L. 우 & 451 & 521. & $" 2,33 \mathrm{a}, \mathrm{b}$ \\
\hline 6. & Lycaéna meléager Esp., a $\sigma^{7}, b$ 우 & 611 & 651. & \\
\hline 7. & dolus Hb., a $\sigma^{7}, \mathrm{~b}$ 우 & 621 & $66 \mathrm{r}$ & \\
\hline 8. & coelestína Ev. の & 632 & $67 \mathrm{l}$. & \\
\hline 9. & alcon $\mathrm{F} ., \mathrm{a} \sigma^{7}, \mathrm{~b}$ 우 & 644 & 681. & \\
\hline 10. & damon Sehiff. $\sigma^{7}$ & 624 & $66 \mathrm{r}$. & $" 2,27 \mathrm{a}, \mathrm{b}$ \\
\hline 11. & semiárgus Rott., a $\sigma^{7}, \mathrm{~b}$ q & 637 & $67 \mathrm{r}$ & \\
\hline 12. & iólas 0., a $\sigma^{7}, \mathrm{~b} q$ & 630 & 671. & " 2,32 \\
\hline 13. & Charáxes iásius L. & 127 & $13 \mathrm{l}$. & $" 2,35 \mathrm{a}-\mathrm{c}$ \\
\hline 14. & Cyaníris argíolus L., $\mathrm{a}$ ○’, $\mathrm{b}$ 우 & 650 & $69 \mathrm{r}$ & $\left\{\begin{aligned} \text { Ei Taf. } 50,7 \\
, \quad 2,28 \mathrm{a}, \mathrm{b}\end{aligned}\right.$ \\
\hline 15. & Lyeaéna arion L., a $\sigma^{\top}, \mathrm{b}$ 우 & 646 & $69 \mathrm{l}$ & \\
\hline 16. & eyllarus, a $\sigma^{7}, \mathrm{~b}$ 우 & 638 & $68 \mathrm{l}$ & Taf. $2,30 \mathrm{a}, \mathrm{b}$ \\
\hline 17. & mélanops B., a $\sigma^{7}, \mathrm{~b}$ 우 & 639 & 681. & $., \quad 2,31$ \\
\hline 18. & minima Fuessl. 우 & 635 & $67 \mathrm{r}$. & $" 2,29 \mathrm{a}, \mathrm{b}$ \\
\hline 19. & areas Rott., a $\sigma^{7}, \mathrm{~b} q$ & 648 & $69 \mathrm{r}$. & \\
\hline 20. & euphémus Hb., a $\sigma^{7}, \mathrm{~b} q$ & 645 & $68 ; 69$ & \\
\hline
\end{tabular}




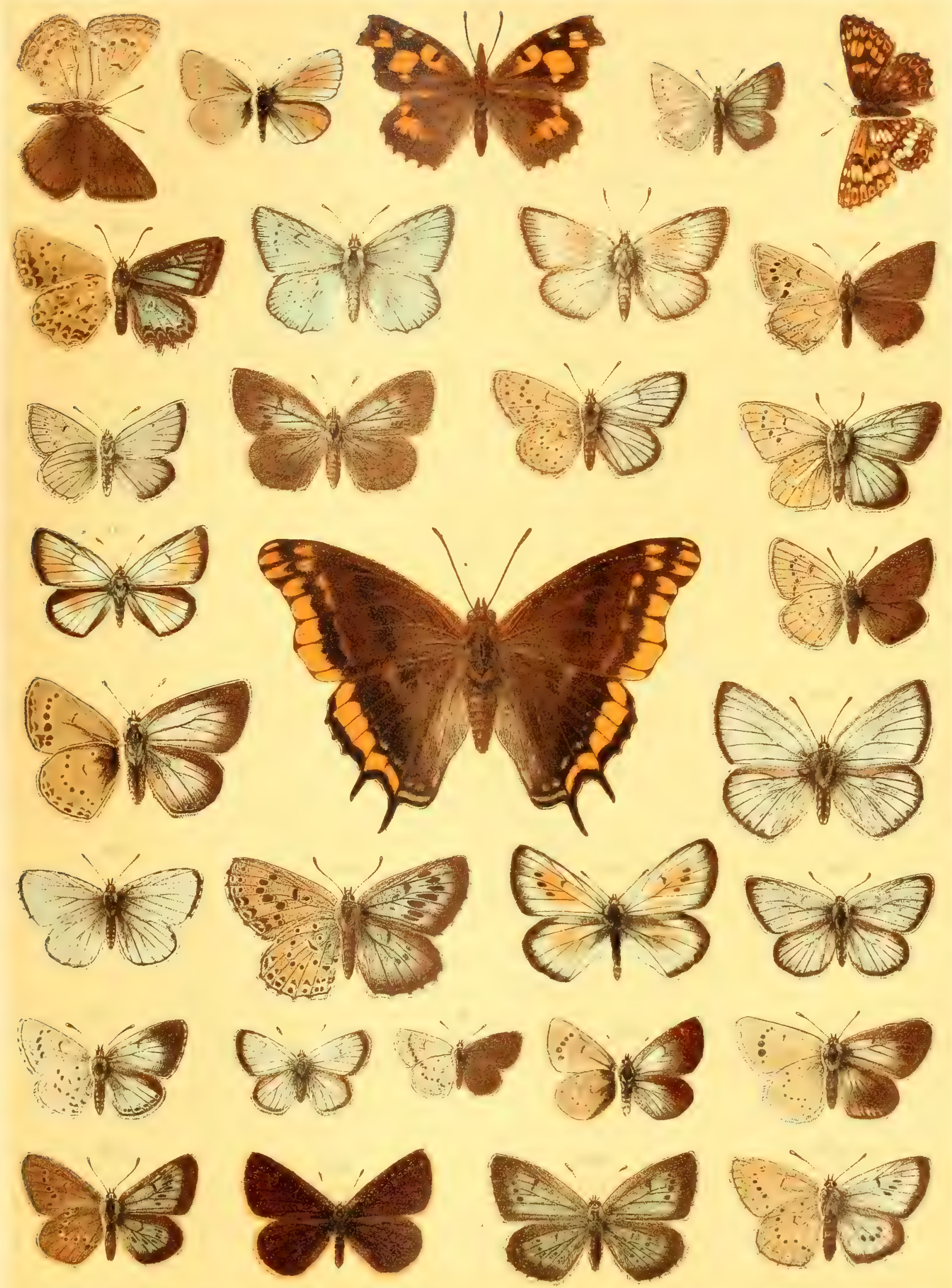





\section{TAFEL $17 \mathrm{~b}$.}

\begin{tabular}{|c|c|c|c|c|c|}
\hline $\mathrm{Nr}$ & & Name & Ratalog I & Seite des I. Bandes & $\begin{array}{l}\text { Raupen-(Puppen-) } \\
\text { Abbildung }\end{array}$ \\
\hline 1. & Maniola & flavofasciáta Heyne $\sigma^{7}$ & 264 & 351. & \\
\hline 2. & $"$ & ehristi Rätzer $q$ & 266 & 351. & \\
\hline 3. & 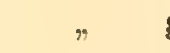 & gorgóne B. 오 & 291 & 381. & \\
\hline 4. & $"$ & eyclópius Ev. $0^{T}$ & 307 & $39 \mathrm{r}$. & \\
\hline $5 \mathrm{a}$. & $"$ & $\begin{array}{l}\text { tyndarus Esp., v. hispánica Butl. ㅇ, } \\
\text { b v. ottomána HS. } \sigma^{7}\end{array}$ & 320 c u. g & 401. & \\
\hline 6. & $"$ & disa Thnbg. & 304 & $39 \mathrm{r}$. & \\
\hline 7. & Satyrus & prieúri Pier. $\sigma^{\nearrow}$ & 348 & $42 \mathrm{r}$ & \\
\hline 8. & $"$ & anthélea Hb., v. amalthéa Friv. 우 & $362 \mathrm{a}$ & 431. & \\
\hline 9. & Epinéphe & ele nurag Ghil. $\sigma^{T}$ & 403 & $45 \mathrm{r}$ & \\
\hline 10. & Sátyrus. & mamúrra HS., v. graéca Stgr. ㅇ & $368 \mathrm{a}$ & 431. & - \\
\hline 11. & Epinéphe & ele nápica $\mathrm{Hb} \cdot \sigma^{7}$ & 410 & 461. & \\
\hline 12. & Coenony & mpha iphioides Stgr. 우 & 429 & $47 \mathrm{r}$. & \\
\hline 13. & $"$ & arcanioídes Pier. $\sigma^{7}$ & 434 & 481. & \\
\hline 14. & $"$ & amaryllis Cram. $q$ & 441 & $48 \mathrm{r}$. & \\
\hline 15. & $"$ & symphyta Ld., v. tiphónides Stgr. & $442 \mathrm{a}$ & $48 \mathrm{r}$ & \\
\hline 16. & Parárge & elymene Esp. $\sigma^{7}$ & 388 & 501. & Nachtr.-Taf. I, 21 \\
\hline 17. & $"$ & deidamía Ev. 우 & 395 & 511. & \\
\hline 18. & Chrysoph & hánus thetis Klug. $\sigma^{7}$ & 502 & $56 / 57$ & \\
\hline 19. & $"$ & óchimus HS. $\sigma^{7}$ & 503 & 571. & \\
\hline 20. & $"$ & dispar Hw. 오 & 508 & 571. & \\
\hline 21. & Lyeaéna & fischéri Ev. $\sigma^{7}$ & 541 & $60 \mathrm{r}$. & \\
\hline 22. & $"$ & eyane Ev. $\sigma^{7}$ & 578 & $61 \mathrm{r}$ & \\
\hline 23. & $"$ & pánope Ev. $\sigma^{\top}$ & 572 & 621. & \\
\hline 24. & $n$ & bávius Ev. $\sigma^{7}$ & 575 & $62 \mathrm{r}$ & \\
\hline 25. & $"$ & (Lámpides) theophrástus F. $\sigma^{T}$ & 532 & 601. & \\
\hline 26. & & & & & \\
\hline 27. & $"$ & damóne Ev. $\sigma^{7}$ & 625 & 671. & \\
\hline 28. & $"$ & lorquíni HS. $\sigma^{7}$ & 634 & $67 \mathrm{l}$. & \\
\hline
\end{tabular}



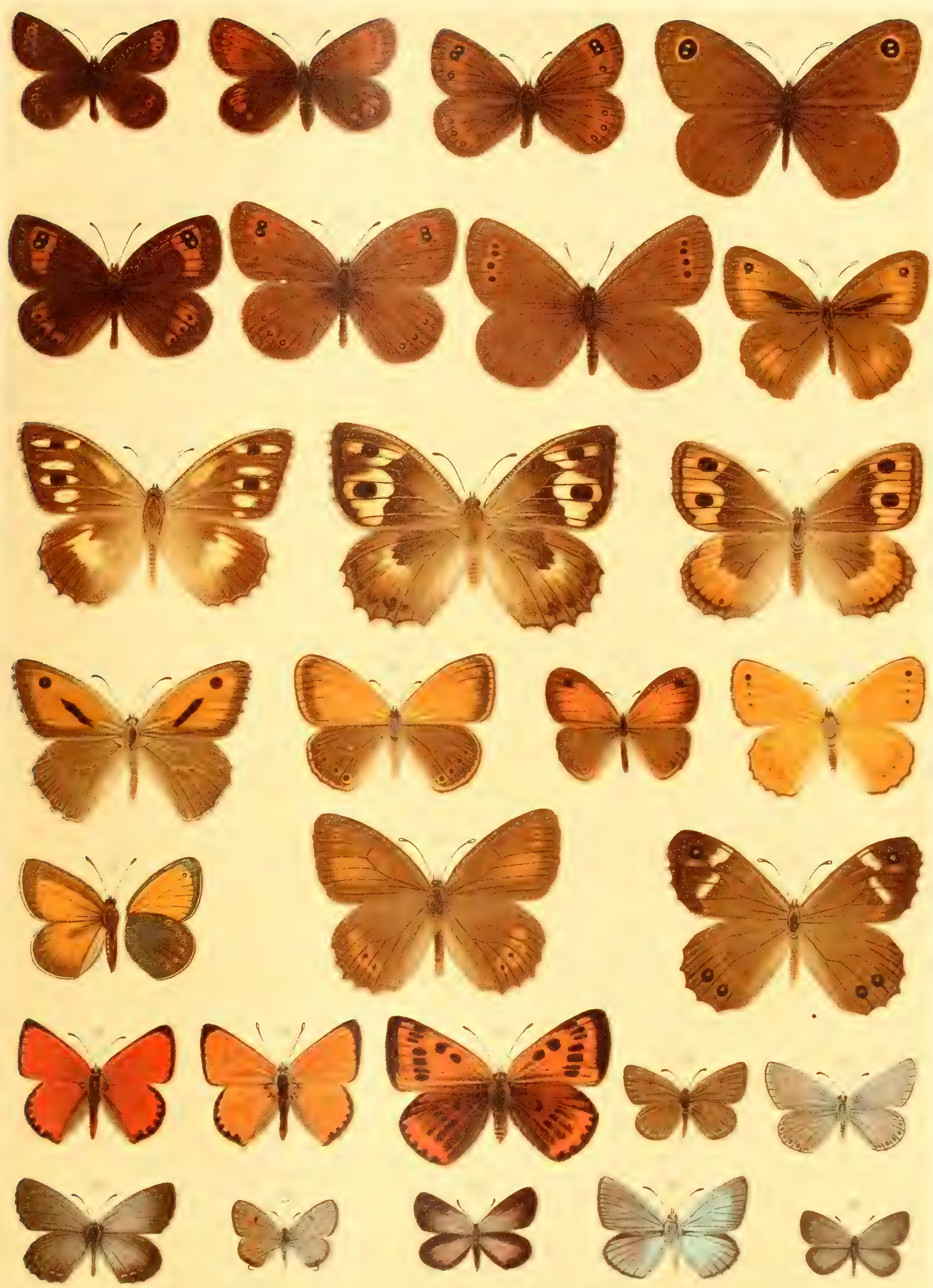



\section{TAFEL $1 \%$.}

\begin{tabular}{|c|c|c|c|c|}
\hline Nr. & Name & Katalog I & Seite des I. Bandes & $\begin{array}{c}\text { Raupen- (Puppen- } \\
\text { Abbildung }\end{array}$ \\
\hline 1. & Heterópterus mórpheus Pall. & 651 & 70 & Taf. 5,26 u. 48,8 \\
\hline 2. & Pámphila palaémon Pall. & 653 & 711. & Taf. 5,27 \\
\hline 3. & sílvius Knoeh., links $\sigma^{\top}$, rechts $\nmid$ & 654 & $71 \mathrm{r}$. & $" \quad 5,28$ \\
\hline 4. & Adopaéa linéola 0., links $0^{7}$, rechts $q$ & 661 & 721. & $" \quad 5,22$ \\
\hline$\overline{5}$ & $\begin{array}{r}\text { flava Brünn. (thaúmas Hufn.) links or, } \\
\text { rechts } \text { 古 }\end{array}$ & 662 & 721 & $" 5,21$ \\
\hline 6. & aetaéon Esp., links $\sigma^{7}$, rechts 우 & 664 & $72 \mathrm{r}$ & $" 5,23$ \\
\hline 7. & Augiades eomma L., links $\sigma^{\nearrow}$, rechts + & 670 & $72 \mathrm{r}$. & $" 5,25$ \\
\hline 8. & sylvánus Esp., links ठ, rechts & 671 & 731. & $" 5,24$ \\
\hline 9. & Párnara nostrodámus F., links $\sigma^{7}$, rechts $q$ & 680 & $73 \mathrm{r}$ & \\
\hline 10. & Carehárodus lavatérae Esp. & 685 & $73 \mathrm{r}$. & $\because 48,7$ \\
\hline 11. & alcéae Esp. & 686 & 741. & $" 5,18$ \\
\hline 12. & althaéae $\mathrm{Hb}$. & 687 & 741. & Nachtr.-Taf. I, 25 \\
\hline 13. & Hespéria proto Esp. & 688 & $75 \mathrm{l}$ & \\
\hline 14. & teséllum $\mathrm{Hb}$. & 695 & 70 & \\
\hline 15. & eribréllum Ev. & 697 & 751. & \\
\hline 16. & phlómidis HS. & 698 & 751. & \\
\hline $1 \overline{7}$. & órbifer $\mathrm{Hb}$. & 699 & $75 \mathrm{r}$. & \\
\hline 18. & sao Hb. & 700 & $75 \mathrm{r}$. & \\
\hline 19. & (v.) therápne Rbr. & $700 \mathrm{c}$ & $75 \mathrm{x}$ & \\
\hline 20. & Seélothrix eynarae Rbr. & 707 & $75 \mathrm{r}$ & \\
\hline 21. & sidae Esp. & 693 & $75 / 76$ & \\
\hline 22. & cápthami Hs. & 694 & 761. & \\
\hline 23. & álveus $\mathrm{Hb}$. & 703 & 761. & \\
\hline 24. & (v.) onopórdi Rbr. & $703 \mathrm{c}$ & $76 \mathrm{l}$. & \\
\hline 25. & seprátulae Rbr. & 701 & $76 \mathrm{r}$. & \\
\hline 26. & eacáliae Rbr. & 704 & $76 \mathrm{x}$ & \\
\hline 27. & andrómedae Wallgr. & 705 & $76 \mathrm{r}$ & \\
\hline 28. & eentaúreae Rbr. & 706 & $76 / 77$ & \\
\hline 29. & malvae L. & 709 & $77 \mathrm{l}$ & Taf. 5,19 \\
\hline 29 a. & malvae L., v. melótis Dup. & $709 \mathrm{e}$ & 771. & \\
\hline 30. & Thánaos tages L. & 713 & $77 \mathrm{r}$. & $" \quad 5,20$ \\
\hline 31. & .. marloyi $\mathrm{B}$. & 715 & $77 \mathrm{r}$ & \\
\hline
\end{tabular}



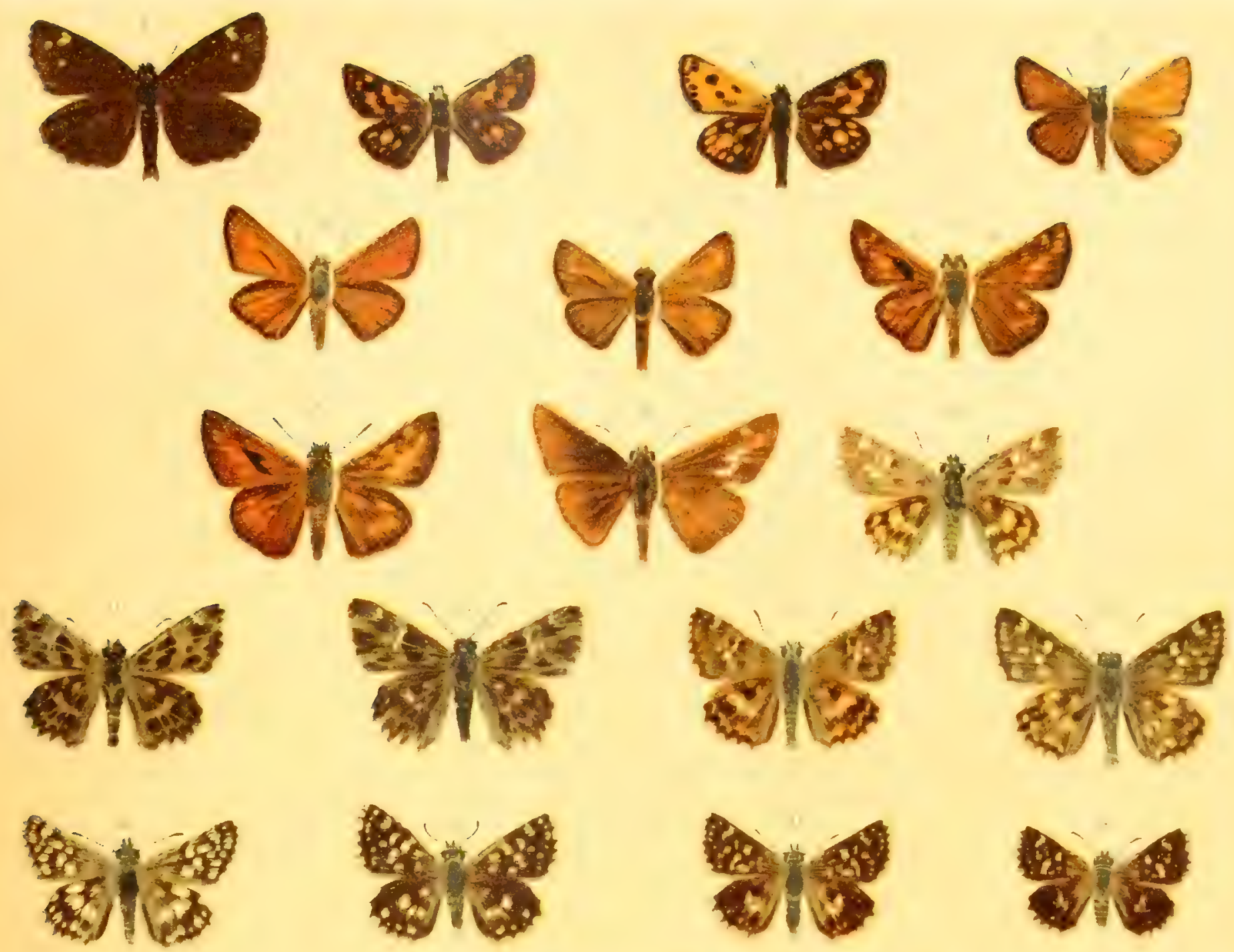

- 15
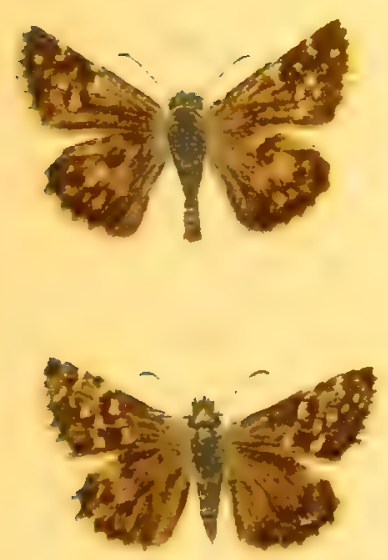
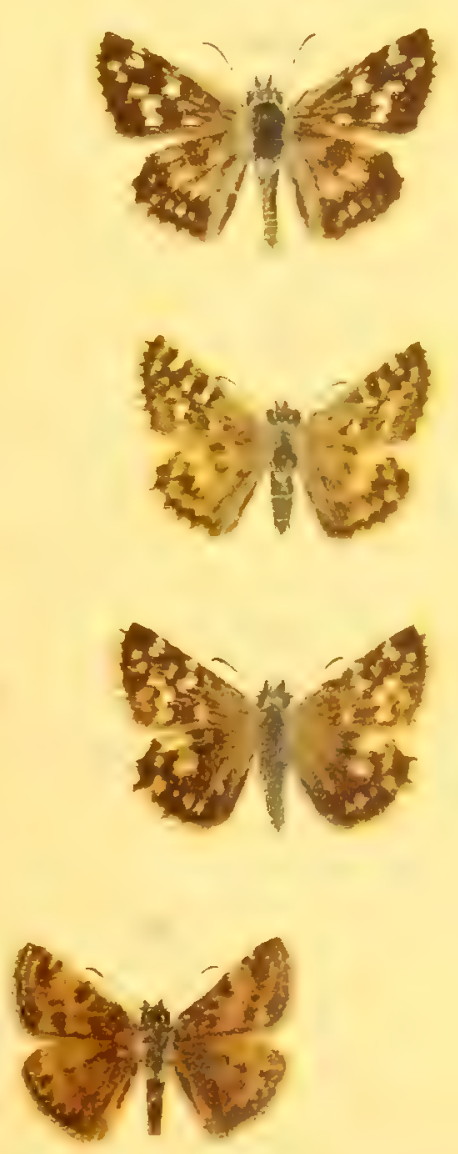
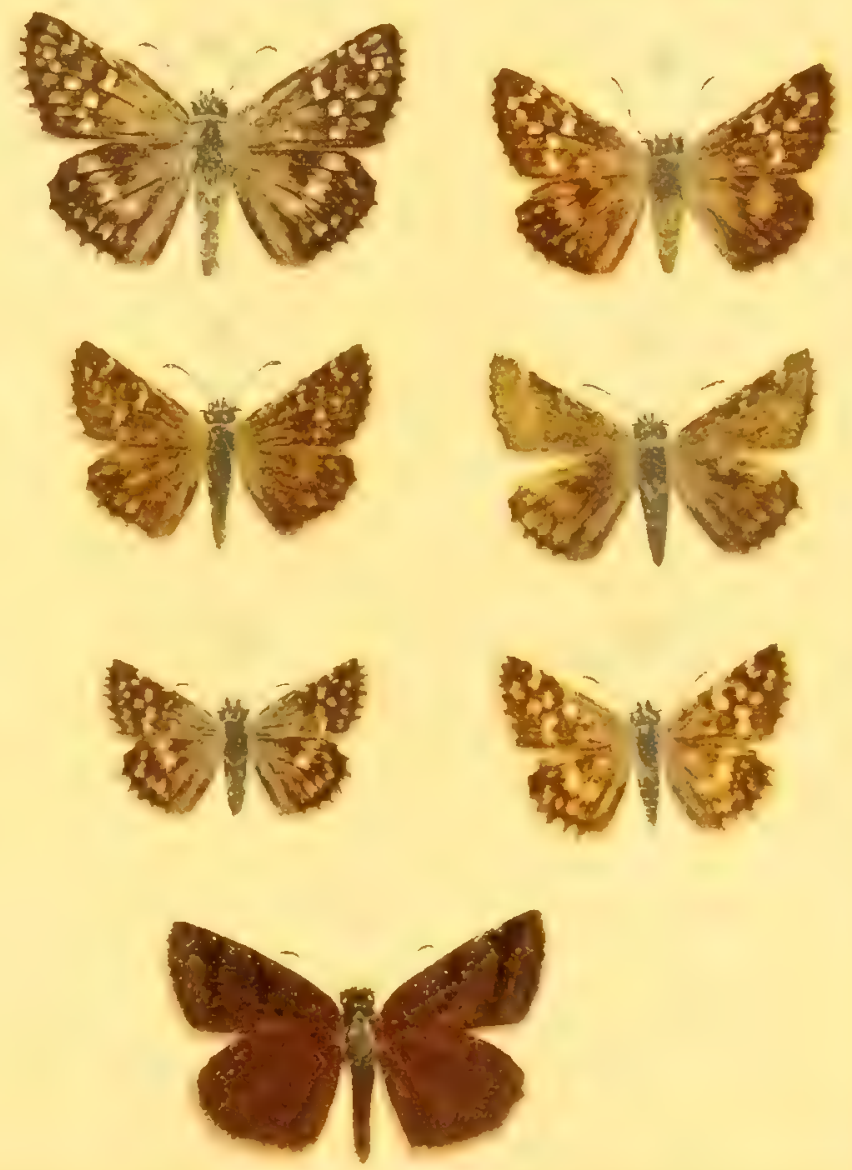




\section{TAF́EL 18.}

\begin{tabular}{|c|c|c|c|c|}
\hline$N x$ & Name & Katalog I & Seite des I. Bandes & $\begin{array}{c}\text { Raupen- (Puppen-) } \\
\text { Abbildung }\end{array}$ \\
\hline 1. & Hespéria andrómedae Wallgr. & 705 & $76 \mathrm{r}$ & \\
\hline 2. & " centaúreae Rbr. & 706 & $76 / 77$ & \\
\hline 3. & malvae L. & 709 & 771 & Taf. $5,19 \mathrm{a}-\mathrm{c}$ \\
\hline 4. & Adopaéa flava Brünn. (thatimas $H u f n$. ), a $\sigma, b q$ & ㅇ 662 & 721. & " 5,21 \\
\hline 5. & Hespéria phlómidis HS. & 698 & $75 \mathrm{l}$ & \\
\hline 6. & Adopaéa linéola $0 .$, a $\sigma^{7}, \mathrm{~b} q$ & 661 & 721. & $" 5,22 \mathrm{a}, \mathrm{b}$ \\
\hline 7. & Thánaos táges L. & 713 & $77 \mathrm{r}$ & $" 5,20 \mathrm{a}, \mathrm{b}$ \\
\hline 8. & Hespéria sao Hb. & 700 & $75 \mathrm{r}$ & \\
\hline 9. & órbifer $\mathrm{Hb}$. & 699 & $75 \mathrm{r.}$ & \\
\hline 10. & Thánaos marloyi B. & 715 & $77 \mathrm{r}$ & \\
\hline 11. & Augíades comma L., a $\sigma^{7}, \mathrm{~b} q$ & 670 & $72 / 73$ & $\Rightarrow \quad 5,25$ \\
\hline 12. & " sylvánus Esp., a $\sigma^{7}, \mathrm{~b} q$ & 671 & 731. & 5,24 \\
\hline 13. & Adopaéa actaéon Rott., a $\sigma$, b 우 & 664 & $72 \mathrm{l}$ & " 5,23 \\
\hline 14. & Pámphila silvius Knoch., a $\sigma^{7}, \mathrm{~b}$ 우 & 654 & $71 \mathrm{r}$ & $" 5,28 \mathrm{a}, \mathrm{b}$ \\
\hline 15. & Heterópterus mórpheus Pall. $\sigma^{7}$ & 651 & Taf. 5 & 5,26 a, b u. Taf. 48,8 \\
\hline 16. & Pámphila aétna $\mathrm{B} .^{*}$ ) & & & \\
\hline 17. & palaémon Pall. & 653 & 711. & Taf. 5,27 \\
\hline 18. & Parnára nostrodámus F., 1. a $\sigma^{7}, \mathrm{r} . \mathrm{b} q$ & 680 & $73 \mathrm{r}$. & \\
\hline 19. & Sphinx pinástri L. 우 & 736 & 871. & $\left\{\begin{array}{c}\text { Ei Taf. } 50,16 \mathrm{a}, \mathrm{b} \\
" 7,2\end{array}\right.$ \\
\hline 20. & $" \quad$ ligústri L. $\sigma^{7}$ & 734 & $86 \mathrm{r}$ & Taf. 7,1 \\
\hline 21. & Aeheróntia átropos L. ㅇ & 717 & $87 / 88$ & $" 6,20 \mathrm{a}, \mathrm{b}$ \\
\hline 22. & Sphinx convólvuli L. $\sigma^{7}$ & 735 & $86 / 87$ & $" 6,21 \mathrm{a}-\mathrm{c}$ \\
\hline
\end{tabular}




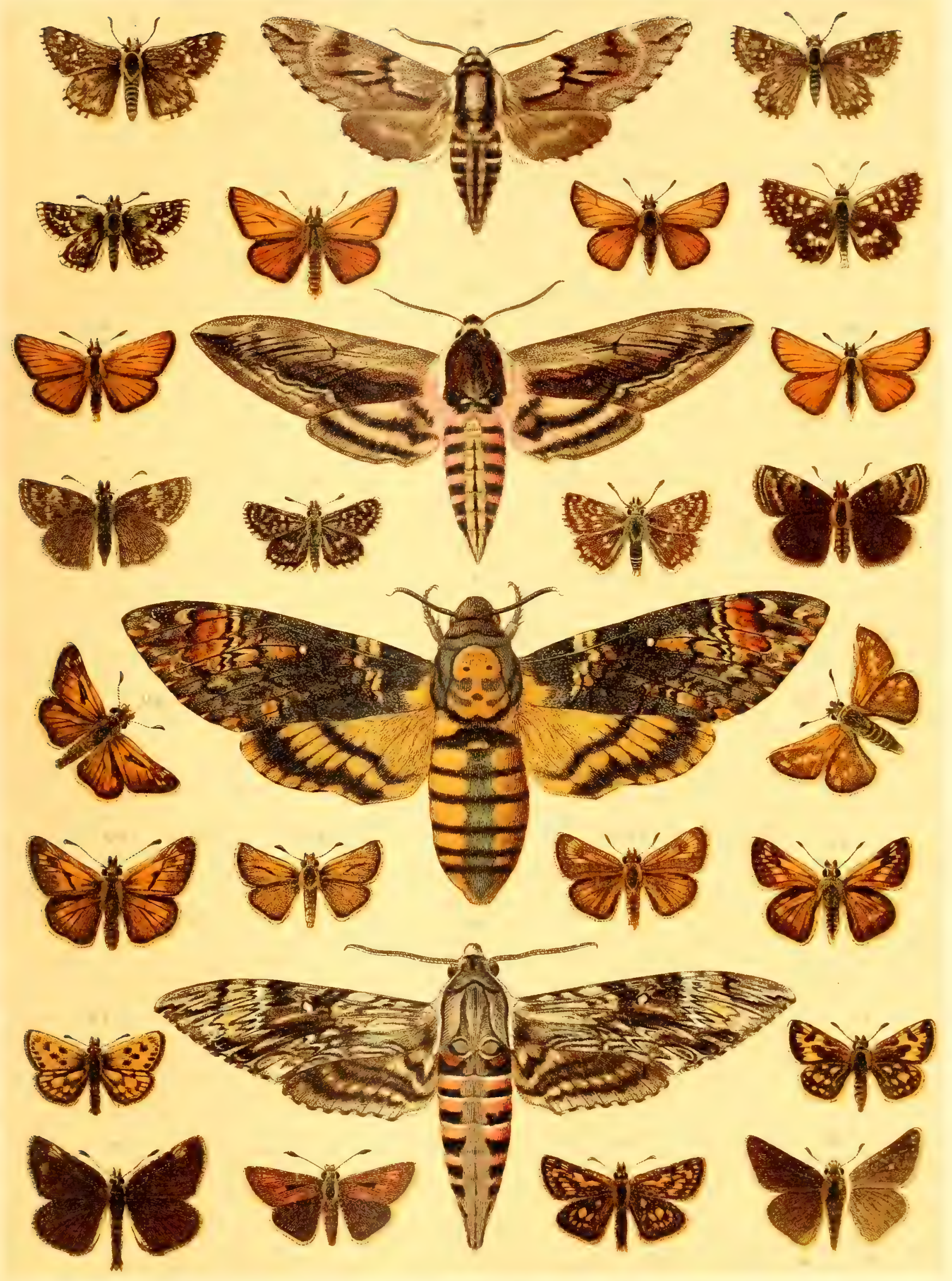






\section{TAFEL 19.}

\begin{tabular}{|c|c|c|c|c|c|}
\hline Nr: & & Name & Katalog I & Seite des I. Bandes & $\begin{array}{l}\text { Raupen-(Puppen-) } \\
\text { Abbildung }\end{array}$ \\
\hline 1. & Deiléphila & vespertílio Esp. $\sigma^{T}$ & 742 & $84 / 85$ & Taf. $7,3 a-c$ \\
\hline 2. & Choerocám & pa elpénor L. & 759 & 811. & $" 8,1 \mathrm{a}, \mathrm{b}$ \\
\hline 3. & Deiléphila & gálii Rott. 우 & 745 & 831. & $" 7,5 \mathrm{a}, \mathrm{b}$ \\
\hline 4. &. & hybr. epilóbỉi B. & $742 \mathrm{~b}$ & 851. & $" 48,11$ \\
\hline 5. & $"$ & lineáta F. v. livórnica Esp. $q$ & $752 \mathrm{a}$ & $81 / 82$ & Taf. 7,8 u. Ntr.-T. I, 27 \\
\hline 6. & $"$ & zygophylli 0. & 744 & $83 / 84$ & Taf. 48,9 \\
\hline 7. & $\because$ & dahli H.-G. & 748 & 831. & $" 6,18$ \\
\hline 8. & .. & nieaéa Prun. + & 750 & 821. & $\eta 7,7 \mathrm{a}, \mathrm{b}$ \\
\hline 9. & $"$ & hippóphaës Esp. & 743 & 841. & $" 7,4$ \\
\hline 10. & \multicolumn{2}{|c|}{ Choerocámpa celério L. $q$} & 753 & $80 / 81$ & $\because 7,9$ \\
\hline 11. & \multicolumn{2}{|c|}{ Deiléphila euphórbiae L. $q$} & 749 & $82 / 83$ & $" 7,6 a-c$ \\
\hline 12. & \multicolumn{2}{|c|}{ Choeroeámpa aléeto L. $\sigma^{7}$} & 757 & 811 & $\Rightarrow 48,10$ \\
\hline
\end{tabular}



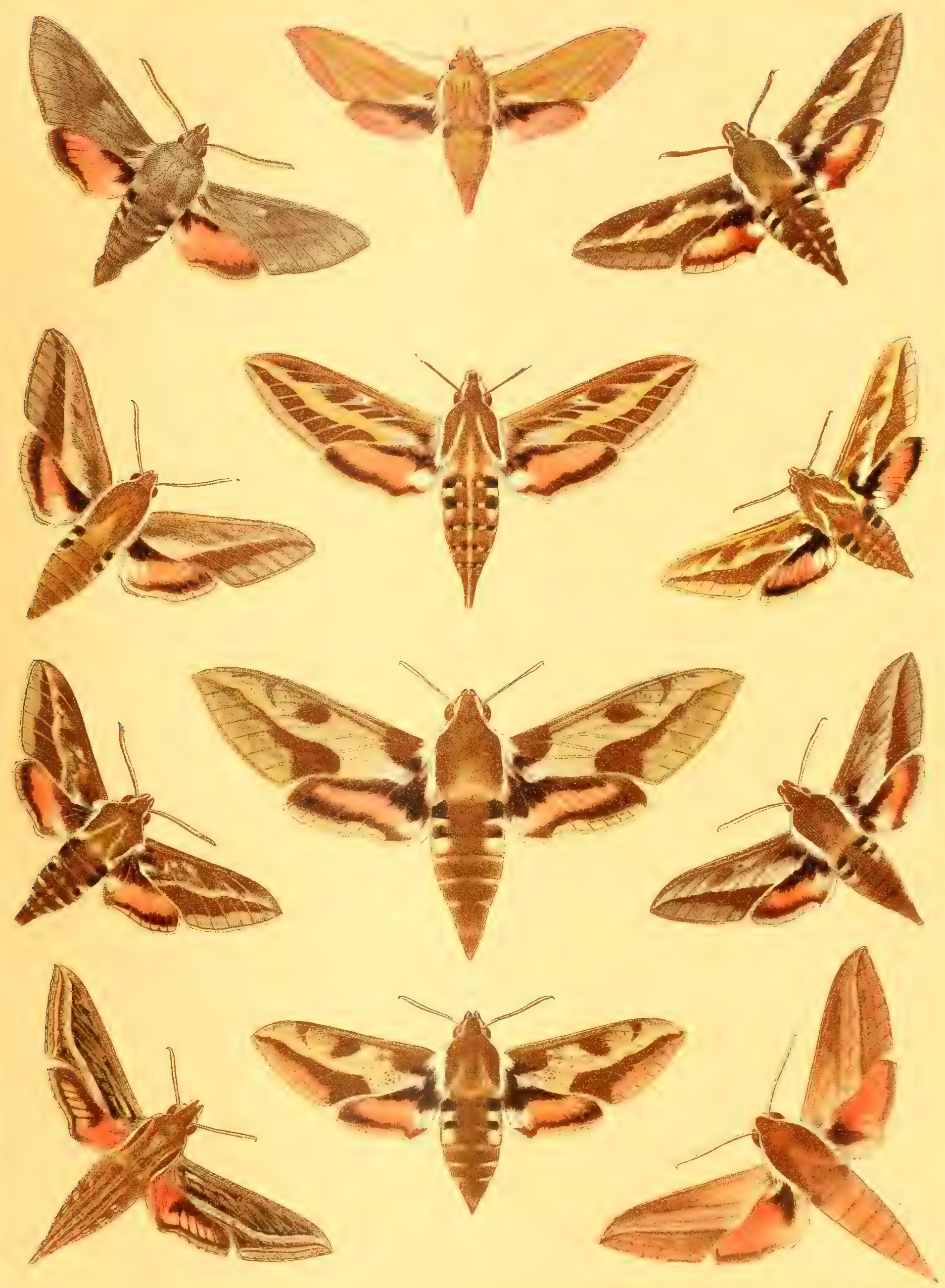



\section{TAFEL 20.}

Nr.

1. Choerocárnpa poreéllus L.

2. Smerínthus ocelláta L. 우

3. Mimas tíliae L. $q$

4. Ptérogon prosérpina Pall.

5. Macroglóssum eroáticum Esp.

6. " stellatárum L.

7. Ptérogon gorgoniades $\mathrm{Hb}$.

8. Daphnis nérii $\mathrm{Hb}$.

9. Macroglóssum fueifórme L.

10. " títyus L.

11. Smerinthus quepeus Sehiff. $\sigma^{7}$

12. " pópuli $\mathrm{L}$.

13. Aegéria melanoeéphala Dalm. ơ

14. Trochílium andrenifórme Lasp.

15. Seiápteron tabanifópme Rott. $\sigma^{7}$

16. Troehílium seolíifórme $\mathrm{Bkh}$.

17. $"$ vespifórme L. ㅇ

18. " eephifórme 0. 오

19. " tipulifórme Cl.

20. " spheeifórme Gerning

21. " myopifórme Bkh.

22. $"$ formieifórme Esp.

23. " stomoxy fórme $\mathrm{Hb}$.

24. Dipsosphécia urocerifórmis Tr.

25. Trochílium conopifórme Esp.

26. Dipsosphécia ichneumonifórmis $\mathrm{F}$.

27. Troehílium eulieifórme L.

28. Chamaesphécia annelláta $\mathrm{Z}$.

29. " masarifórmis $\mathrm{O}$.

30. $"$ empifórmis Esp. $\sigma^{7}$

31. " $\quad$ astatifórmis HS.

32. Aegéria erabronifórmis Lewin $\sigma^{7}$

33. $"$ apifórmis $\mathrm{Cl}$. $\sigma^{7}$

\begin{tabular}{|c|c|c|}
\hline Katalog I & Seite des I. Bandes & $\begin{array}{c}\text { Raupen-(Puppen-) } \\
\text { Abbildung }\end{array}$ \\
\hline 761 & $81 \mathrm{r}$. & $\begin{array}{c}\text { Ei Taf. } 50,18 \\
\text { " } 8,2\end{array}$ \\
\hline 726 & $89 / 90$ & Taf. $8,6 \mathrm{a}, \mathrm{b}$ \\
\hline 730 & 89 r. & $, \quad 8,4$ \\
\hline 765 & 801. & $" 8,8 \mathrm{a}, \mathrm{b}$ \\
\hline 769 & 791. & $" 9,1$ \\
\hline 768 & $79 \mathrm{r}$. & $" 8,9 \mathrm{a}, \mathrm{b}$ \\
\hline 767 & $79 / 80$ & Nachtr.-Taf. I, 26 \\
\hline 733 & 861. & Taf. $8,3 \mathrm{a}, \mathrm{b}$ \\
\hline 774 & $78 / 79$ & $" 9,3$ \\
\hline 771 & 791. & $" 9,2 \mathrm{a}, \mathrm{b}$ \\
\hline 718 & $90 / 91$ & $" 8,5$ \\
\hline 725 & $\begin{array}{c}90 \mathrm{l} . \\
\text { Seite des II. Bandes }\end{array}$ & $" 8,7 \mathrm{a}-\mathrm{c}$ \\
\hline 4536 & 3071. & Nachtr.-Taf. X, 20 \\
\hline 4538 & $307 / 8$ & \\
\hline 4545 & 3091. & Taf. 9, 5 \\
\hline 4555 & $310 \mathrm{r}$. & $" 9,7$ \\
\hline 4551 & 3101. & \\
\hline 4546 & $309 / 10$ & \\
\hline 4557 & $310 \mathrm{r}$. & $" 9,8 \mathrm{a}, \mathrm{b}$ \\
\hline 4566 & $311 \mathrm{l}$. & $" 9,10$ \\
\hline 4564 & 3111. & \\
\hline 4563 & 3111. & $" 9,9$ \\
\hline 4587 & 3141. & \\
\hline 4534 & $307 \mathrm{r}$ & \\
\hline 4532 & 3071. & $\begin{array}{l}\text { Ei Taf. 50, } 21 \\
\qquad \quad 9,4 \text { a, b }\end{array}$ \\
\hline
\end{tabular}

Die nicht fett gedruckten Namen bezeichnen kaum oder nicht kenntliche Abbildungen.

Man vergleiche für die Aegeriiden Taf. 79. 


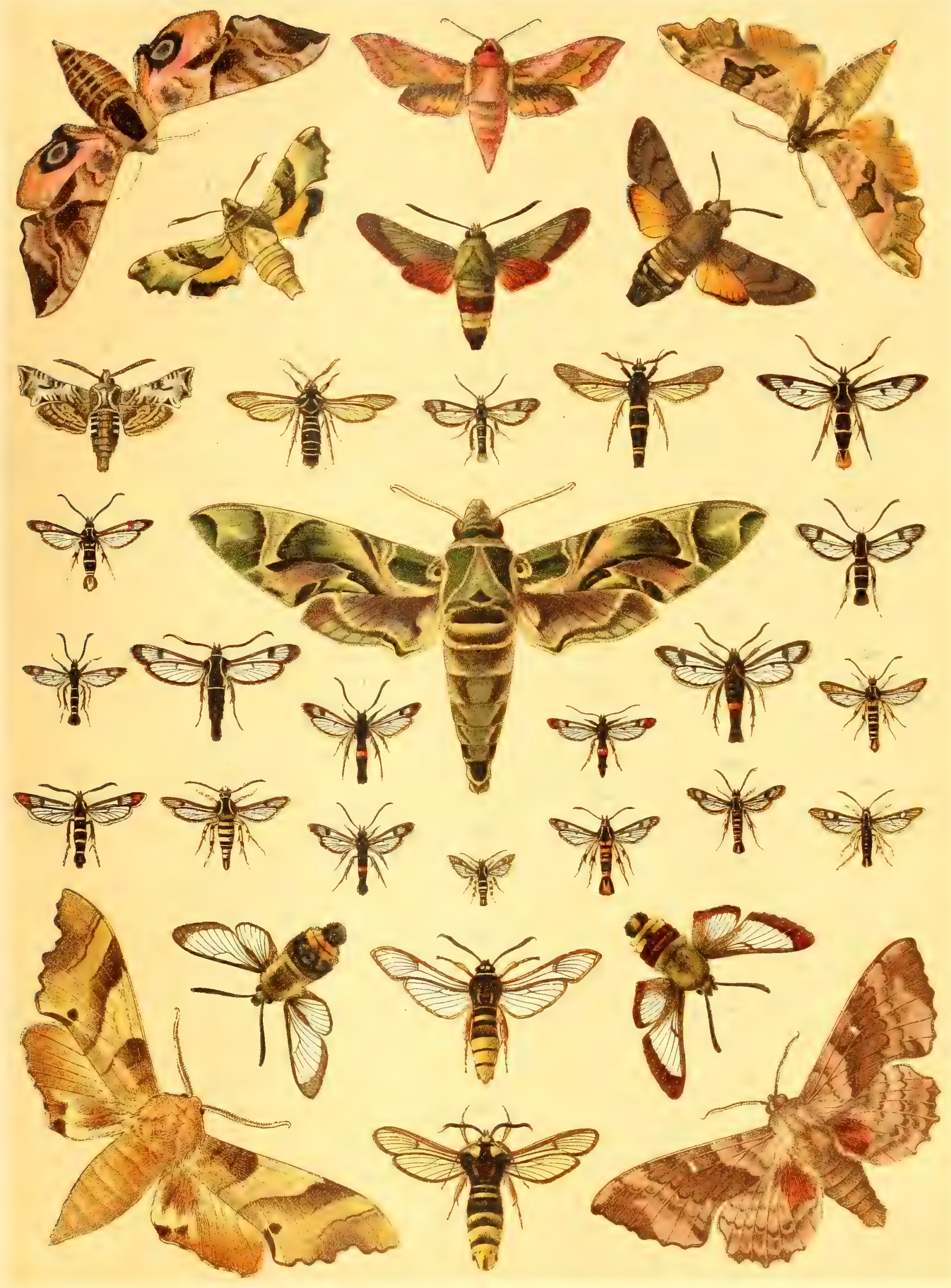





\section{TAFEL 21.}

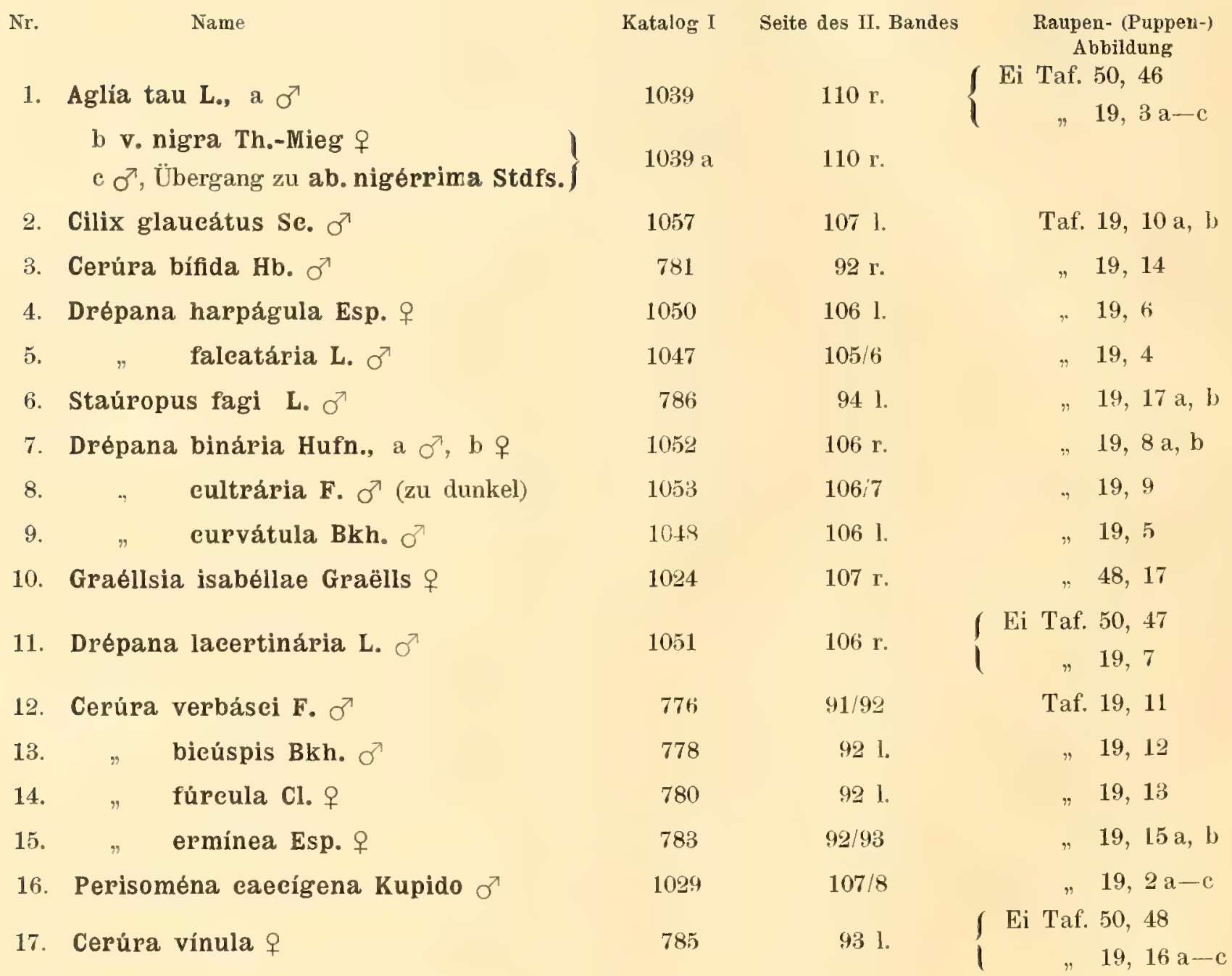



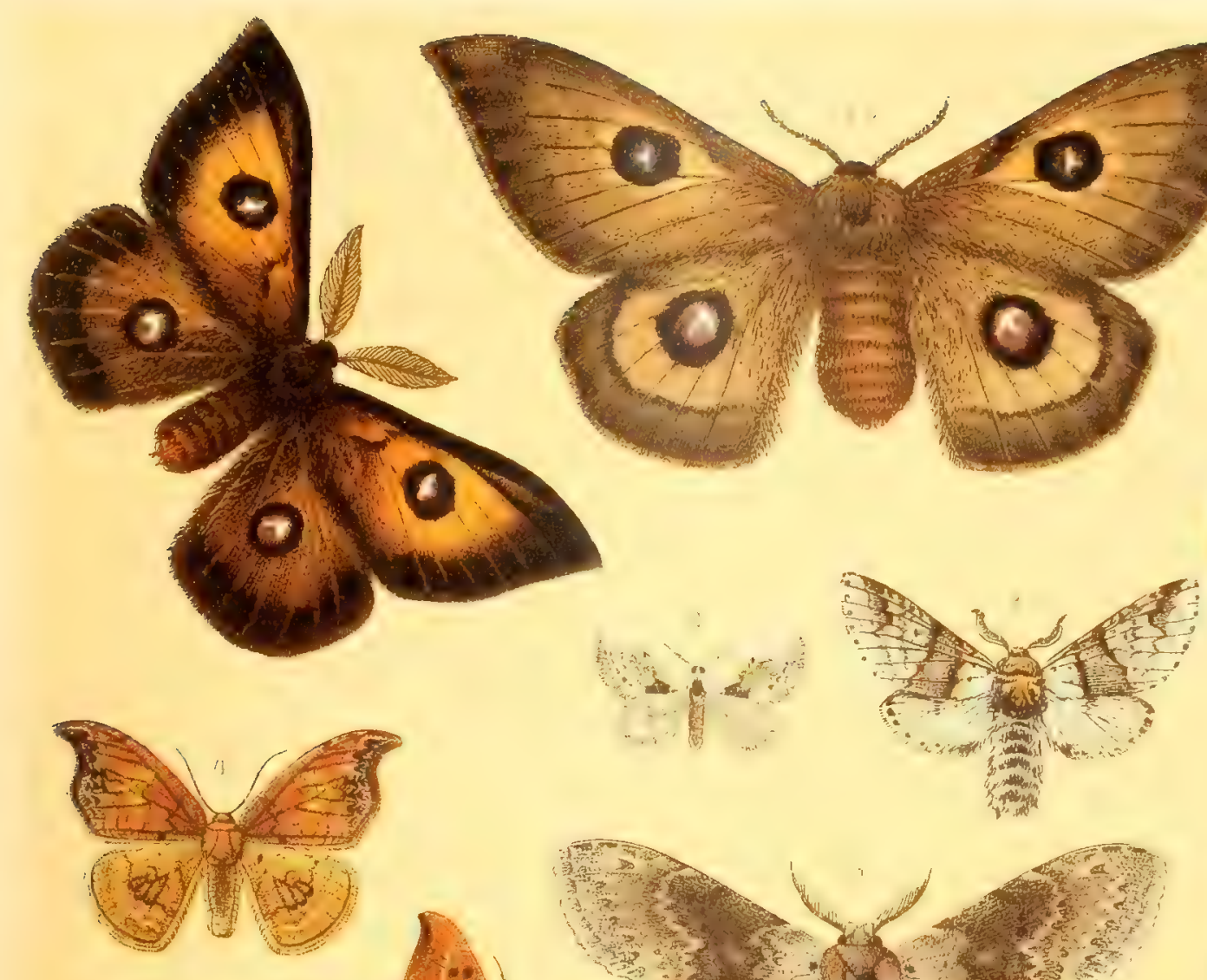

a

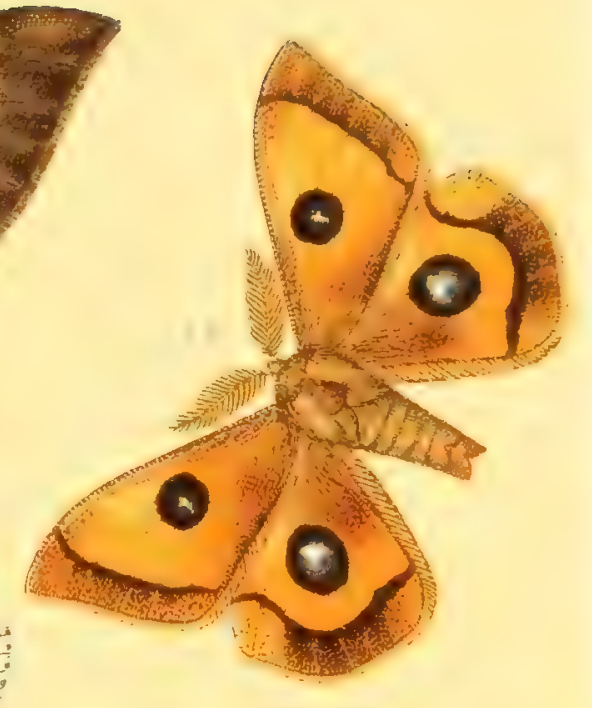

virifor vese (N)
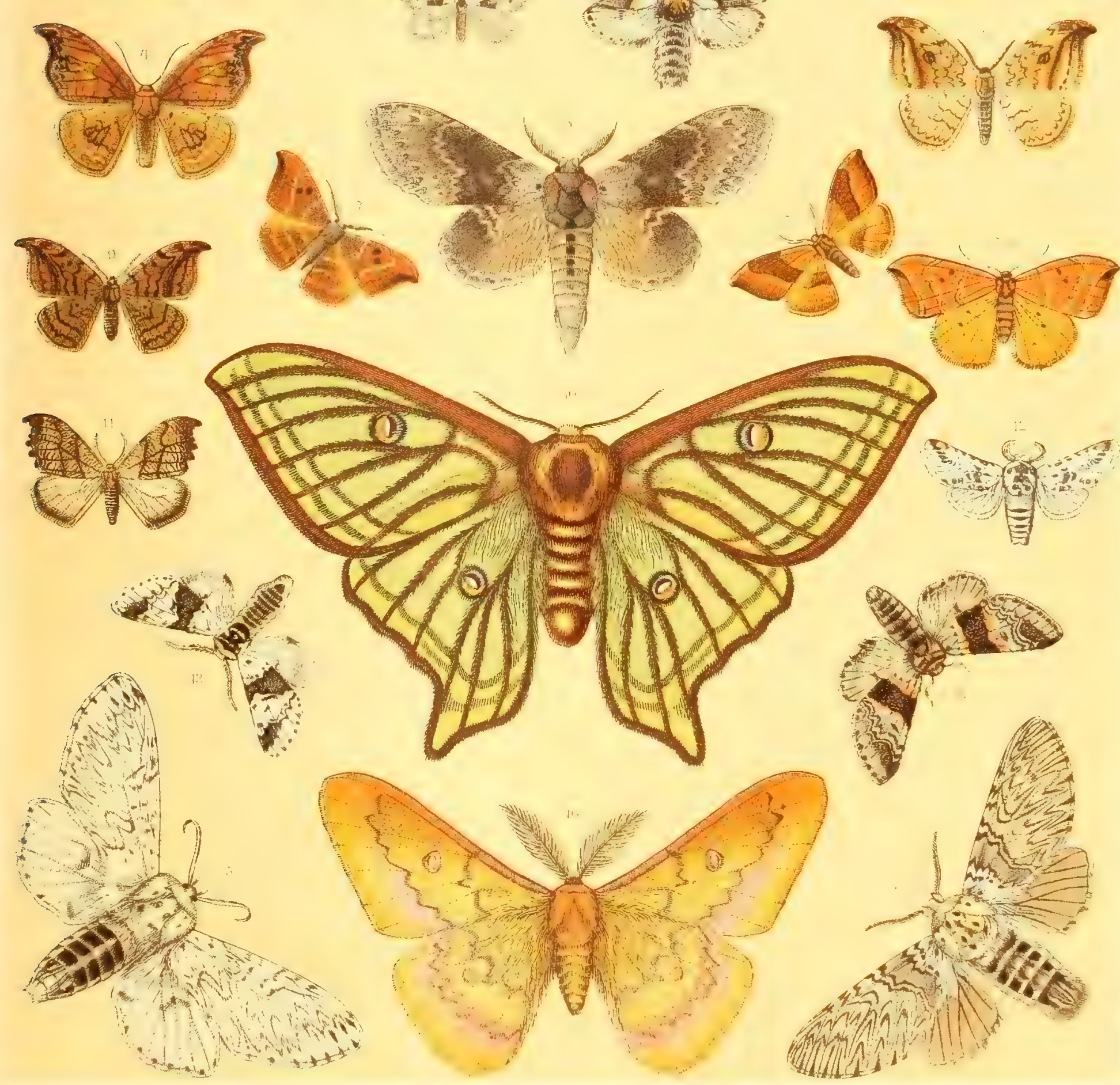




\section{TAFEL 22.}

\begin{tabular}{|c|c|c|c|c|}
\hline $\mathrm{Nr}$. & Name & Katalog I & Seite des I. Bandes & $\begin{array}{l}\text { Raupen- (Puppen-) } \\
\text { Abbildung }\end{array}$ \\
\hline 1. & Exaéreta ulmi Sehiff. $q$ & 789 & $94 \mathrm{r}$. & Taf. 19,18 u. 48,18 \\
\hline 2. & Drymónia ehaónia Hb., a $\sigma^{7}, \mathrm{~b} q$ & 807 & $95 \mathrm{r}$ & Taf. 20,6 \\
\hline 3. & Thaumatopoéa pityoeámpa Sehiff. & 875 & $104 \mathrm{r}$. & $" 20,20 a, b$ \\
\hline 4. & Hoplitis milhauséri $\mathrm{F} \cdot \sigma^{T}$ & 791 & $93 \mathrm{r}$ & $\left\{\begin{aligned} & \text { Ei Taf. } 50,49 \\
& 19,19 \mathrm{a}, \mathrm{b}\end{aligned}\right.$ \\
\hline 5. & Notodónta ziezac L. $q$ & 815 & $96 \mathrm{r}$. & Taf. 20,3 \\
\hline 6. & Oehrostígma velitáris Rott. & 836 & $98 \mathrm{r}$. & $" 20,15 \mathrm{a}, \mathrm{b}$ \\
\hline 7. & Pheósia gnoma F. (dictaeoídes Esp.) $0^{7}$ & 809 & $96 \mathrm{l}$. & $" 20,2 \mathrm{a}, \mathrm{b}$ \\
\hline 8. & Notodónta anceps Goeze (trépida Esp.) ठ & 825 & $97 \mathrm{x}$ & $" 20,4$ \\
\hline 9. & Pheósia trémula $\mathrm{Cl} \cdot \sigma^{7}$ & 808 & 961. & $\left\{\begin{aligned} & \text { Ei Taf. } 50,50 \\
& 20,1 \mathrm{a}-\mathrm{c}\end{aligned}\right.$ \\
\hline 10. & Notodónta phoébe Sieb. 오 & 823 & 971 & Nachtr.-Taf. I, 28 \\
\hline 11. & Drymónia querna F. $\sigma^{7}$ & 804 & 951. & Taf. 20,7 \\
\hline 12. & Notodónta dromedárius L. $q$ & 816 & $96 / 97$ & $" 20, \check{\jmath}$ \\
\hline 13. & Leucodónta bieolóxia Sehiff. OT & 835 & $98 \mathrm{l}$. & $" 20,9$ \\
\hline 14. & Spatália argentína Schiff. $\sigma^{\top}$ & 830 & $97 / 98$ & $" 20,10$ \\
\hline 15. & Drymónia trimácula Esp. $\sigma^{7}$ & 806 & 951. & $" 20,8 \mathrm{a}, \mathrm{b}$ \\
\hline 16. & Odontósia sievérsi Mén. & 839 & 991. & Nachtr.-Taf. I, 29 \\
\hline 17. & Lophópteryx eamelína L. 우 & 841 & $99 \mathrm{r}$. & $\left\{\begin{array}{r}\text { Ei_Taf. } 50,51 \\
, \quad 20,12\end{array}\right.$ \\
\hline 18. & cueúlla Esp. の & 843 & $99 / 100$ & Taf. $20,13 \mathrm{a}-\mathrm{c}$ \\
\hline 19. & Pteróstoma palpínum L. $\sigma^{7}$ & 849 & $100 / 01$ & $" 20,14 \mathrm{a}, \mathrm{b}$ \\
\hline 20. & Oehrostígma melágona Bkh. 우 & 837 & $98 \mathrm{r}$ & $" 20,16$ \\
\hline 21. & Gluphísia erenáta Esp. $q$ & 801 & $94 \mathrm{r}$. & $" 20,17$ \\
\hline 22. & Odontósia earmelíta Esp. 우 & 838 & 991. & $" 20,11 \mathrm{a}, \mathrm{b}$ \\
\hline $\begin{array}{l}23 . \\
24 .\end{array}$ & $\begin{array}{c}\text { Ptilóphora plumígera Esp. } \sigma^{7} \\
", \quad \% \text { (zu grell gefärbt u. gezeichn.) }\end{array}$ & $8 \tilde{a}^{2}$ & 1011. & "20, $18 \mathrm{a}, \mathrm{b}$ \\
\hline 25. & Thaumatopoéa proeessiónea L., a $\sigma^{7}, b$ 우 & $87+$ & 1041. & $\left\{\begin{array}{r}\text { Ei Taf. } 50,52 \\
20,29\end{array}\right.$ \\
\hline 26. & pinívora Tr., a $\sigma^{7}, \mathrm{~b}$ 우 & 876 & 1051. & Nachtr.-Taf. II, 2 \\
\hline 27. & Notodónta tritóphus Esp. ত & 824 & $97 \mathrm{I}$. & \\
\hline 28. & Thaumatopoéa herculeána Rbr, r. $\sigma^{7}, 1$. $q$ & 877 & 1051. & II, 3 \\
\hline
\end{tabular}


propos

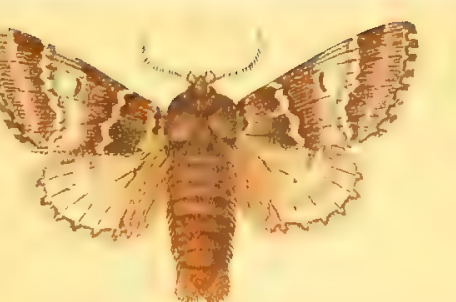

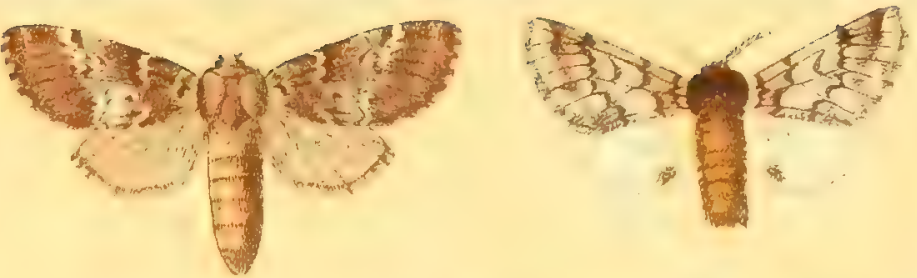

$3=1=3$
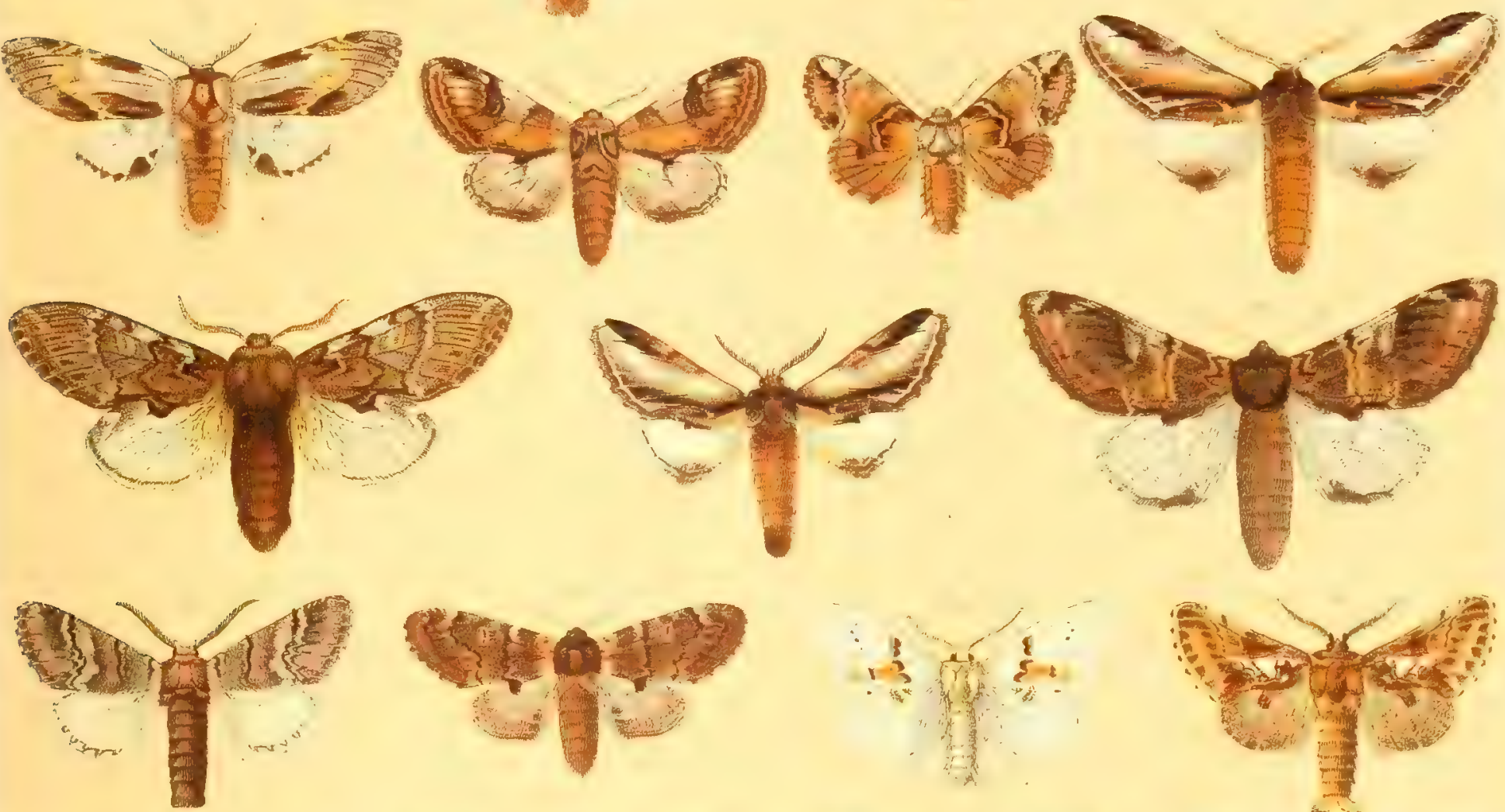

$\because \underbrace{1}_{4} \frac{1}{5-1}$

हो?
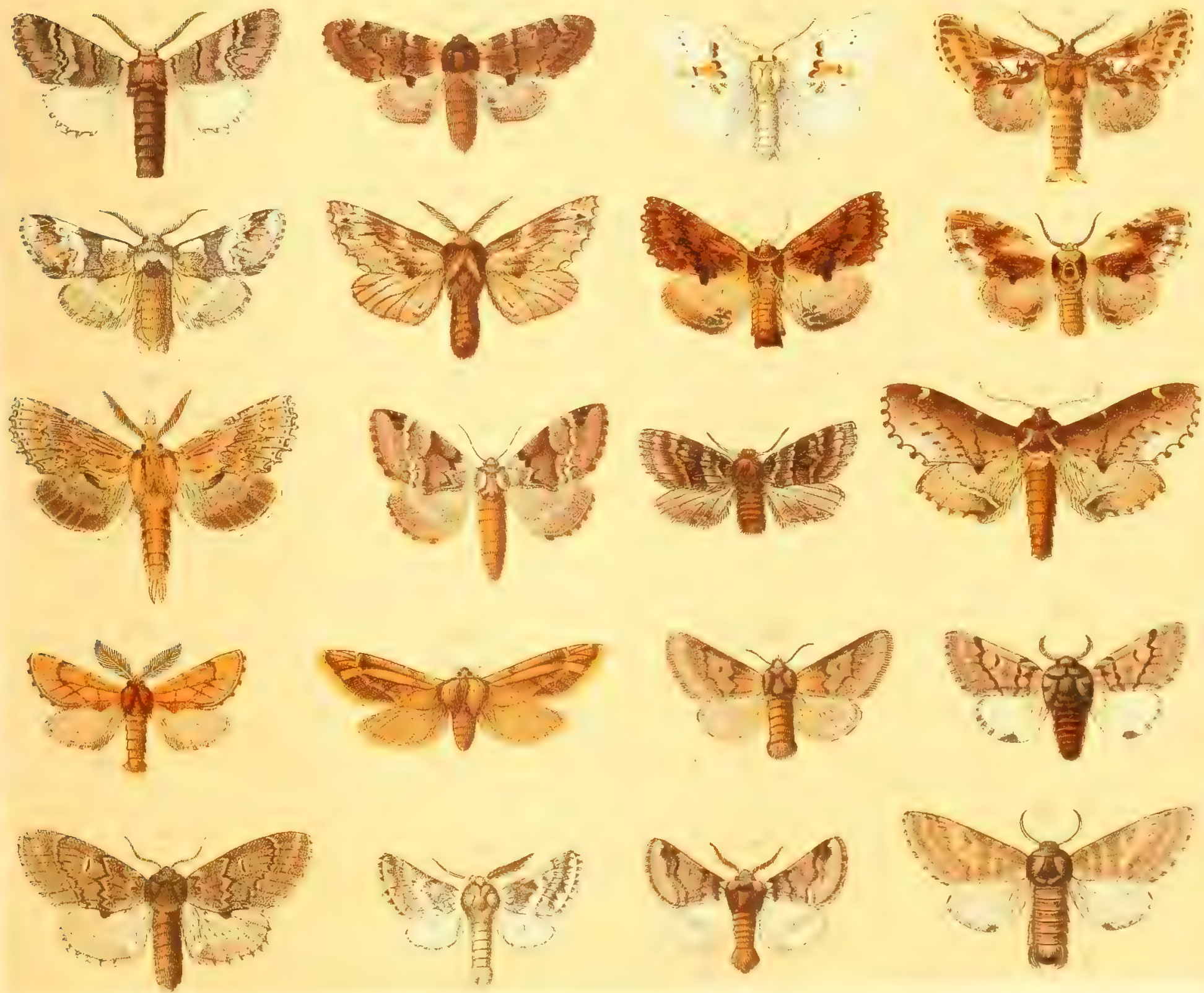


$$
\text { . }
$$




\section{TAFEL 23.}

\begin{tabular}{|c|c|c|c|c|}
\hline Nr. & Name & Katalog I & Seite des I. Bandes & $\begin{array}{l}\text { Raupen- (Puppen-) } \\
\text { A bbildung }\end{array}$ \\
\hline 1. & Cerúra aeruginósa Chr. $\sigma^{7}$ & 777 & 921. & \\
\hline 2. & interrúpta $\mathrm{Chr} . \sigma^{\top}$ & 782 & $92 \mathrm{r}$ & \\
\hline 3. & vínula L., v. phantóma Dalm. ত & 785 & 931. & . \\
\hline 4. & Drymónia vittáta Stgr. & 802 & 951. & \\
\hline 5. & " trimácula Esp., v. et ab. dodonaéa $\mathrm{Hb} . q$ & $q 806 \mathrm{~b}$ & $95 \mathrm{l}$ & Taf. $20,8 \mathrm{a}$ \\
\hline 6. & Rhegmatóphila alpina Bell. $\sigma^{7}$ & 847 & $100 \mathrm{l}$ & Nachtr.-Taf. II, 1b \\
\hline 7. & Pygaéra pigra Hufn. $\sigma^{7}$ & 870 & 1031. & Taf. 21,5 \\
\hline 8. & timon Hb., a $\sigma^{\top}, \mathrm{b} q$ & 861 & $102 \mathrm{r}$. & \\
\hline 9. & eúptula L. $\sigma^{7}$ & 866 & $102 / 3$ & $" 21,3 a, b$ \\
\hline 10. & anachoréta $\mathrm{F} .+9$ & 869 & 1031. & $" 21,4$ \\
\hline 11. & anastomósis L. $\sigma^{7}$ & 865 & $102 \mathrm{r}$. & $" 21,2$ \\
\hline $12 \mathrm{a}$. & hybr. paesehkéi Stdfs. ఠ & & $103 \mathrm{r}$. & \\
\hline $12 \mathrm{~b}$. & hybr. próava Stdfs. + & & $103 \mathrm{r}$. & \\
\hline 13. & Phaléra bucéphala L. $\sigma^{T}$ & 858 & $101 \mathrm{r}$. & Taf. $20,21 \mathrm{a}, \mathrm{b}$ \\
\hline 14. & $" \quad$ bueephaloídes $0 . q$ & 859 & 1021. & $" 21,1$ \\
\hline 15. & Thaumatopoéa solitária Frr., a $\sigma^{7}, \mathrm{~b}$ 오 & 873 & 1041. & \\
\hline 16. & Lemónia ballióni Chr. ఠ & 1017 & $111 \mathrm{r}$ & \\
\hline 17. & philópalus Donz. જ & 1018 & $111 \mathrm{r}$ & \\
\hline 18. & Chondróstega vandalíeia Mill., a $\sigma^{\pi}, \mathrm{b}$ 우 & 951 & $113 \mathrm{r}$. & Nachtr.-Taf. II, 6 \\
\hline 19. & Poecilocámpa pópuli L., v. canénsis Mill. $\sigma^{7}$ & $962 \mathrm{~b}$ & $114 \mathrm{r}$. & \\
\hline 20. & Eriogáster lanéstris L., v. arbủseulae Frr. O & $965 \mathrm{a}$ & 1171. & Taf. 48,15 \\
\hline 21. & henkéi Stgre. $\sigma^{7}$ & 967 & $117 \mathrm{r}$ & \\
\hline 22. & Taragáma repánda $\mathrm{Hb} .$, a $\sigma^{7}, \mathrm{~b}$ 우 & 1007 & 1251. & . $48,16 \mathrm{a}$, \\
\hline
\end{tabular}




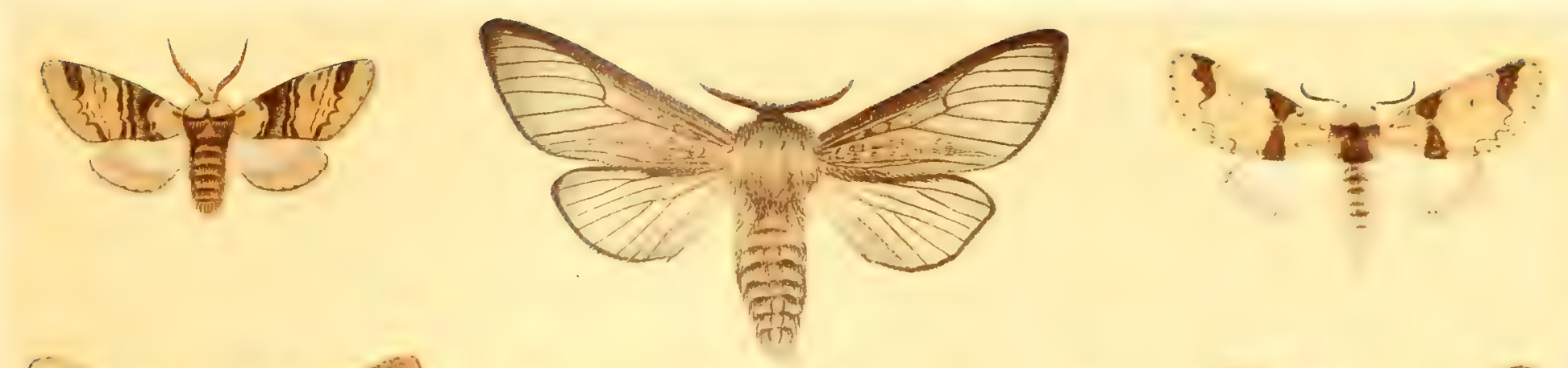

Q0

41

tog.

(a)

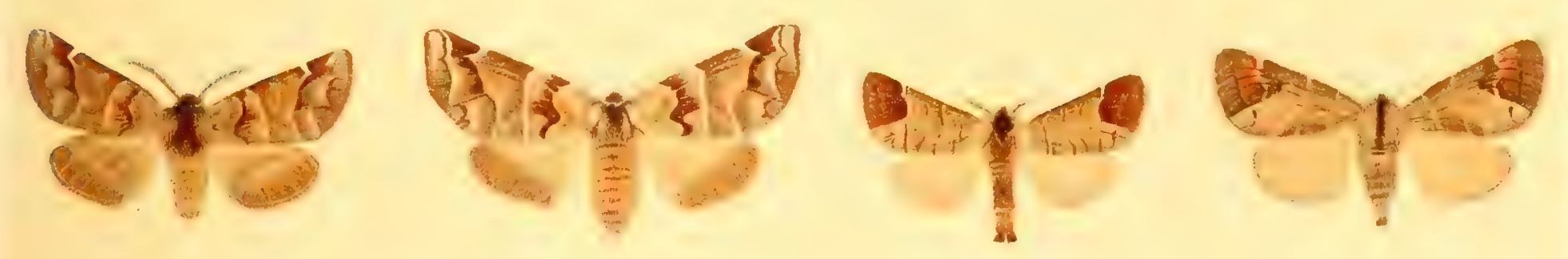

Qrove breng is

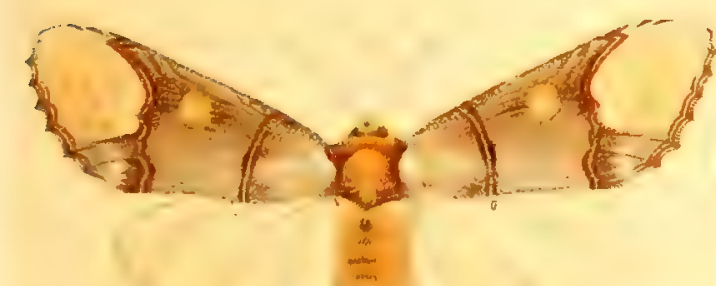

$\rightarrow-1$
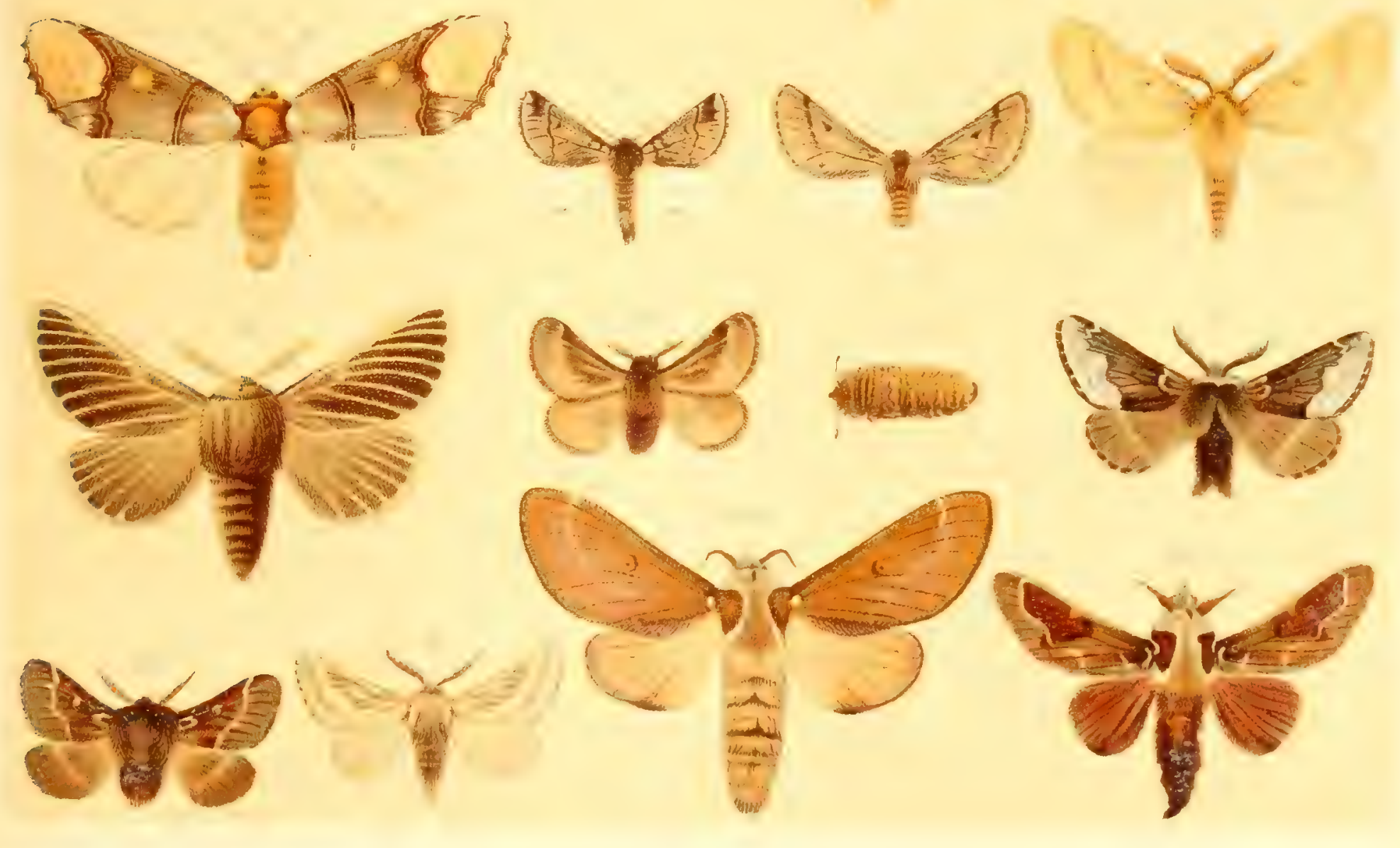



\section{TAFE L 24.}

Nr.

1. Pachypása otus Drury $\sigma^{7}$

2. Éndromis versicolóra L., a $\sigma^{7}, \mathrm{~b}$.

3. Satúrnia pavónia L., a $\sigma^{7}, \mathrm{~b}$ 오

t.,$\quad$ pyri Sehiff. $\sigma^{x}$

5. . . hybr. daúbi Stdfs, a $\sigma^{7}, \quad$ jo 우

b. $\quad$ spini Sehiff. $\sigma^{7}$
Katalog I Seite des I. Bandes

1004

1014

1037

1034

1035

$124 \mathrm{r}$.

1121.

$108 / 9$

1081.

$109 \mathrm{r}$.

$108 \mathrm{r}$.

$$
\text { Seite des I. Bandes }
$$

ง. 18,12
Raupen- (Puppen-) Abbildung

Taf. $48,9 \mathrm{a}, \mathrm{b}$

(Ei Taf. 50,44

( $\quad 18,10$

( Ei Taf. 50, 45

" $19,1 \mathrm{a}-\mathrm{c}$

Taf. 18, $11 \mathrm{a}-\mathrm{c}$ 


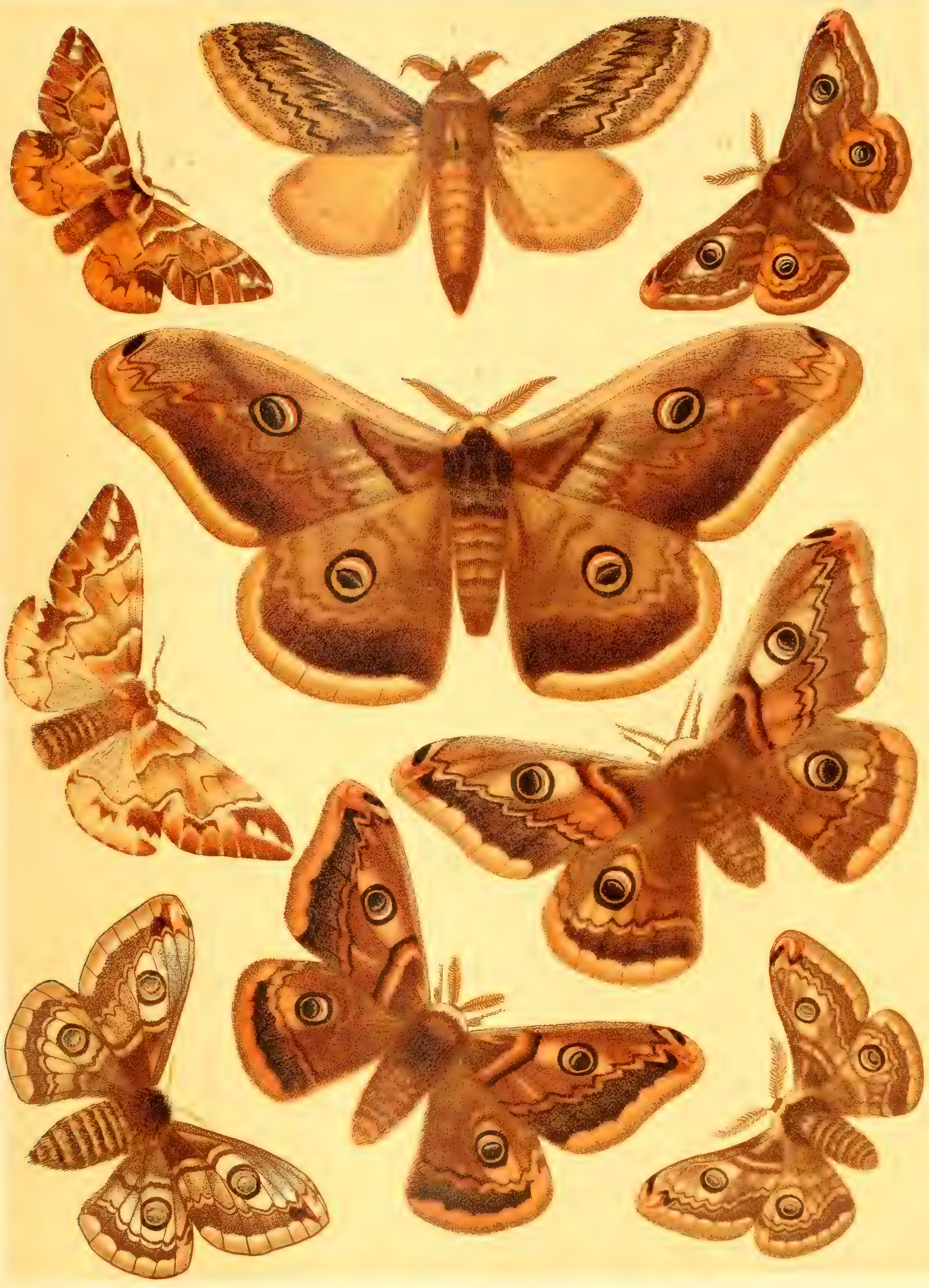




\section{•}




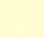




\section{TAFEL 25.}

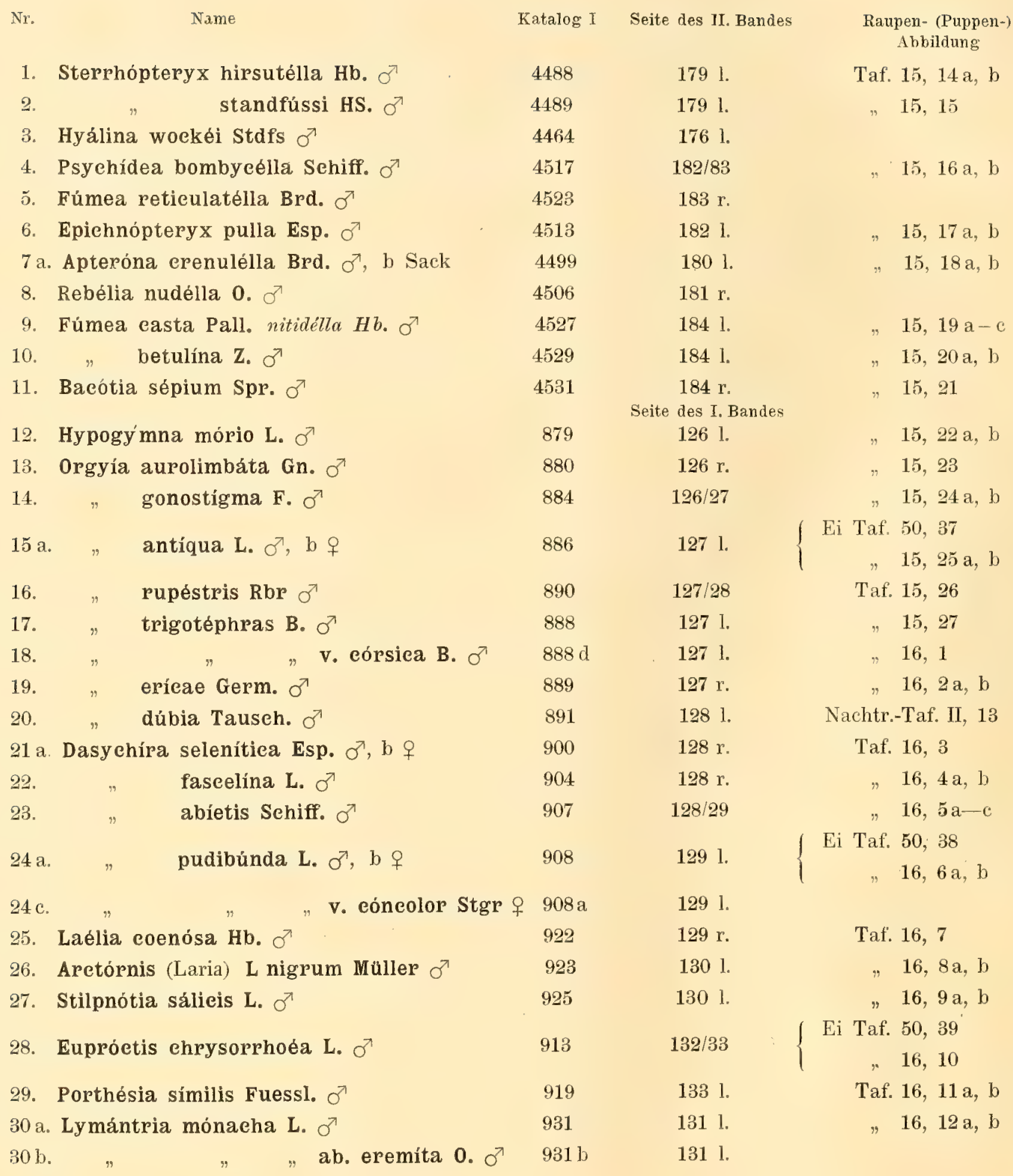




\section{ers}

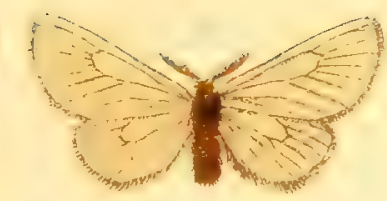

y

1.X.

$+\frac{19}{2}+3$

ore of
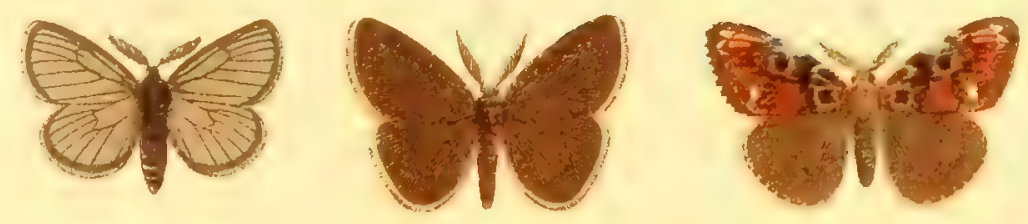

ans (n)
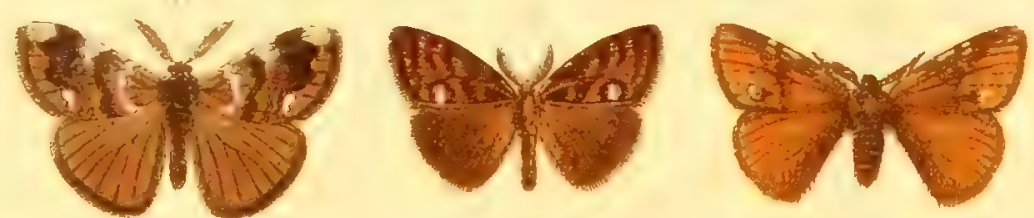

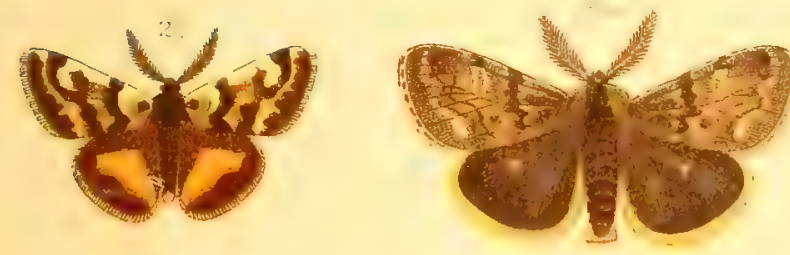
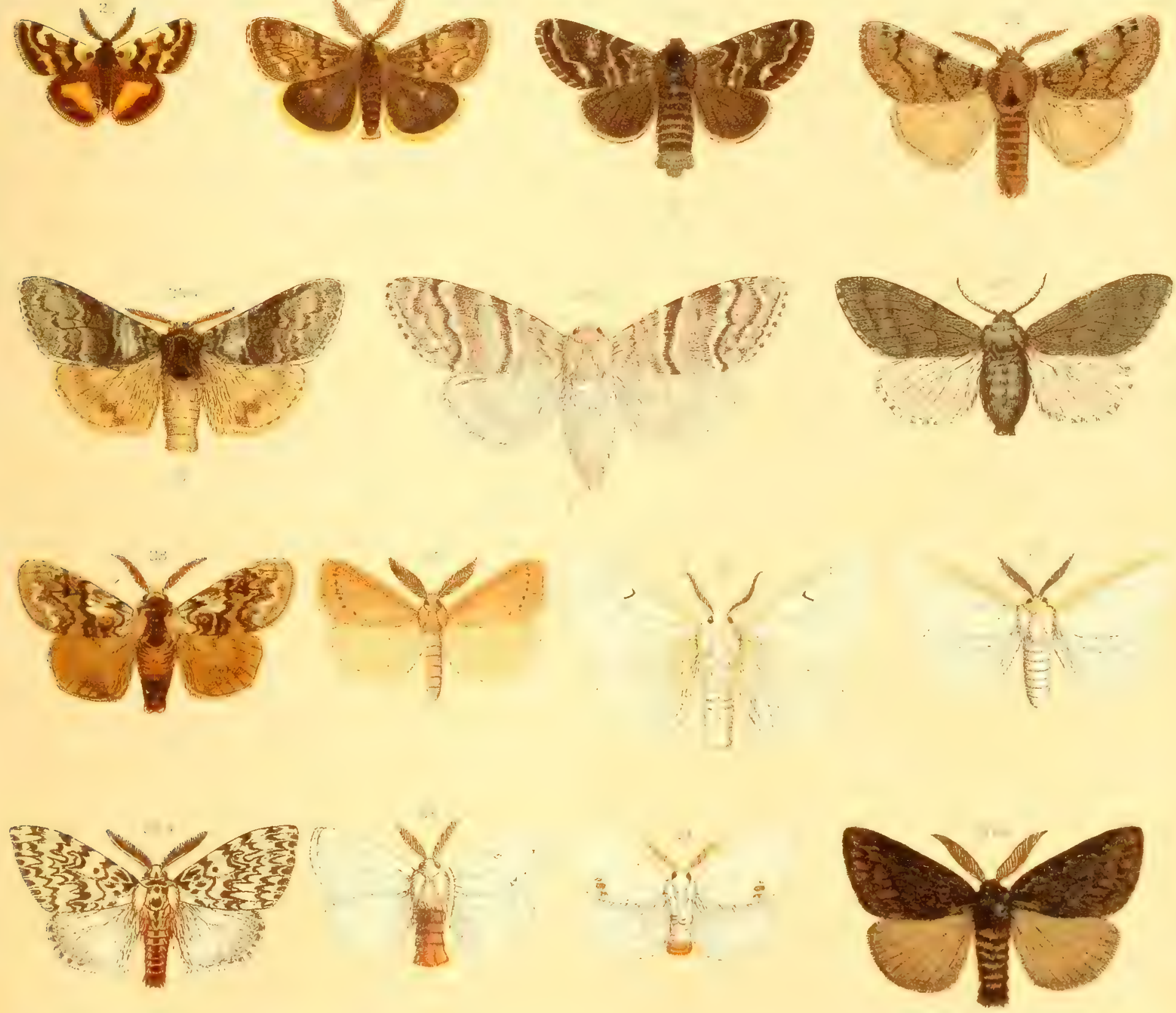




\section{TAFEL 26.}

\begin{tabular}{|c|c|c|c|c|}
\hline & Name & Katalog I & Seite des I. Bandes & $\begin{array}{l}\text { Raupen-(Puppen-) } \\
\text { Abbildung }\end{array}$ \\
\hline & Oenéria terebínthi Frp ơ & 939 & 1321. & Nachtr.-Taf. II, 12 \\
\hline 2. & detrita Esp. $\sigma^{7}$ & 938 & 1321. & Taf. 17,1 \\
\hline & Malacosóma franeónicum Esp. $\sigma^{7}, \mathrm{~b}$ 우 & 958 & 1161. & $" 17,6$ \\
\hline & Triehiủpa ílieis Rbr & 961 & 1141. & $" 17,3$ \\
\hline 5 a. & Lymántria dispar L. $\sigma^{7}, \mathrm{~b}$ q & 929 & 1311. & $\left\{\begin{array}{r}\text { Ei Taf. } 50,40 \mathrm{a} \\
, 16,13 \mathrm{a}, \mathrm{b}\end{array}\right.$ \\
\hline & Trichiủra erataégi L. $\sigma^{7}$ & 960 & 1141. & Taf. $17,4 \mathrm{a}-\mathrm{e}$ \\
\hline & Eriogáster lanéstris L. $\sigma^{7}$ & 965 & $117 \mathrm{l}$. & $" 17,10$ \\
\hline & Lymántria atlántica Rbr. $\sigma^{7}$ & 932 & $131 \mathrm{r}$. & \\
\hline & Lasiocámpa eversmánni Ev. $\sigma^{T}$ & 978 & 1191. & Nachtr.-Taf. II, 8 \\
\hline & Eriogáster rimieola Hb. б & 963 & $116 / 17$ & Taf. $17,12 \mathrm{a}, \mathrm{b}$ \\
\hline $11 \mathrm{a}$. & Lasioeámpa trifólii Esp. $\sigma^{\nearrow}$ b $q$ & 976 & $118 / 19$ & "17,13 \\
\hline 12. & Eriogáster lanéstris L. $q$ & 965 & 1171. & $" 17,10$ \\
\hline 13. & Lasioeámpa quereus L. $\sigma^{7}$ & 970 & 1181. & $\left\{\begin{array}{r}\text { Ei Taf. } 50,41 \\
" 17,14\end{array}\right.$ \\
\hline & Oenéria rúbea $\mathrm{F}$. 우 & 944 & 1321. & Tat'. $17,2 a, b$ \\
\hline & Poeeiloeámpa pópuli L. O & 962 & $114 / 15$ & $" 17,5 \mathrm{a}-\mathrm{c}$ \\
\hline 16. & Malacosóma castrénse L. 우 & 957 & $115 / 16$ & $" 17,8$ \\
\hline $17 \mathrm{a}$. & $" \quad$ neústrium L. $\sigma^{7}, \mathrm{~b}$ \& & 956 & $115 \mathrm{r}$. & $\left\{\begin{array}{c}\text { Gelege Taf. } 50,40 \mathrm{~b} \\
" 17,9\end{array}\right.$ \\
\hline & Oenéria rúbea F. $\sigma^{7}$ & 944 & $132 \mathrm{l}$. & Taf. $17,2 \mathrm{a}, \mathrm{b}$ \\
\hline $19 \mathrm{a}$. & Malacosóma alpicolum Stgr. $\sigma^{7}, \mathrm{~b}$ ㅇ & 959 & 116 & $" 17,7 \mathrm{a}, \mathrm{b}$ \\
\hline 20. & Eriogáster néogena F. d. W. O' & 968 & $117 \mathrm{r}$. & Nachtr.-Taf. II, 7 \\
\hline $21 \mathrm{a}$. & Diplúra loti $0 . \sigma^{7}, \mathrm{~b} q$ & 984 & 1201. & II, 9 \\
\hline & Malacosóma eastrénse L. $\sigma^{T}$ & 957 & $115 / 16$ & Taf. 17,8 \\
\hline 23. & Trichiủra crataégi L. 우 & 960 & 1141. & $\because \quad 17,4 \mathrm{a}-\mathrm{e}$ \\
\hline & Eriogáster lanéstris L. v. apbúseulae Frr. $0^{7}$ & $965 \mathrm{a}$ & 1171. & $" 48,15$ \\
\hline $25 \mathrm{a}$. & $\operatorname{eatax}$ L. $\sigma^{7}, \mathrm{~b}$ ㅇ & 964 & 117 & $" 17,11$ \\
\hline
\end{tabular}



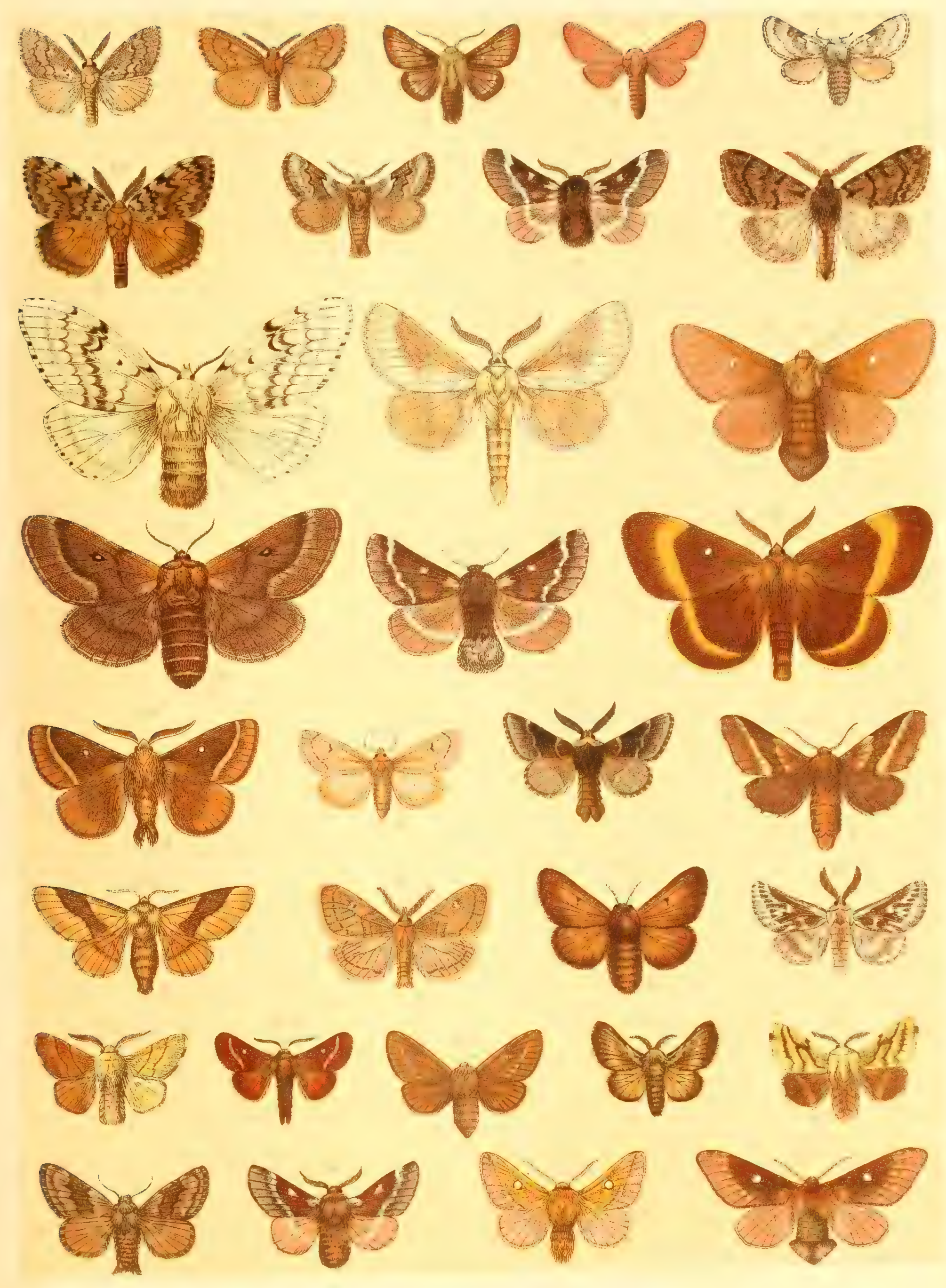



\section{TAFEL $2 \%$}

\begin{tabular}{|c|c|c|c|}
\hline Name & Katalog I & Seite des I. Bandes & $\begin{array}{l}\text { Raupen-(Puppen-) } \\
\text { Abbildung }\end{array}$ \\
\hline $1 \mathrm{a}$. Lemónia baleániea HS. $\sigma^{7}$ & 1019 & $111 \mathrm{r}$ & Nachtr.-Taf. II, 5 \\
\hline 2a. Epienáptera suberifólia Dup. $\sigma^{7}, \mathrm{~b} q$ & 997 & 1221. & II, 10 \\
\hline 3. Lemónia taráxaci Esp. $0^{7}$ & 1015 & $111 \mathrm{r}$. & Taf. 17.16 \\
\hline 4a. Macrothylácia rubi L. $\sigma^{7}$, b $q$ & 982 & $119 / 20$ & $" 17,15 \mathrm{a}, \mathrm{b}$ \\
\hline 5. Lasiocámpa quercus L. $q$ & 970 & 1181. & $\left\{\begin{array}{r}\text { Ei Taf. } 50,41 \\
, 17,14\end{array}\right.$ \\
\hline 6. Lemónia dumi L. $\sigma$ & 1020 & $111 / 12$ & $\left\{\begin{array}{r}\text { Ei Taf. } 50,42 \\
, 17,17 \mathrm{a}, \mathrm{b}\end{array}\right.$ \\
\hline 7. Odonéstis pruni L. $\sigma^{7}$ & 1000 & 1231. & Taf. 18,2 \\
\hline 8. Dendrolimus pini L. + , r. Flügel des $\sigma^{7}$ & 1001 & 1241. & $" 18,8 \mathrm{a}, \mathrm{b}$ \\
\hline 8a. $\quad " \quad$ v. montána Stgr. $\sigma^{7}$ & $1001 \mathrm{a}$ & 1241 & \\
\hline 9 a. Cosmotriehe potatória L. $\sigma^{7}, \mathrm{~b} q$ & 990 & 1211. & Taf. 18, 1 \\
\hline 10. Gastrópacha quereifólia L. $\sigma^{\top}$ & $99 \times$ & $122 \mathrm{r}$ & $\left\{\begin{array}{r}\text { Ei Taf. } 50,43 \\
" 18,3\end{array}\right.$ \\
\hline 11. Epienáptera ilieifólia L. 우 & 994 & $121 \mathrm{r}$. & Taf. 18,6 \\
\hline populifólia Esp. $\sigma^{7}$ & 999 & 1231. & $" 18,4$ \\
\hline 13. $"$ tremulifólia Hb. $q$ & 995 & $121 / 22$ & $" 18,5$ \\
\hline 14a. Selenéphera lunígera Esp. v. lobulína Esp. $q$ & f $993 a$ & $120 \mathrm{r}$. & $. \quad 18,7 \mathrm{~b}$ \\
\hline 141). $\quad " \quad$ lunígera Esp. & 993 & $120 \mathrm{r}$ & $\because \quad 18,7 \mathrm{a}-\mathrm{c}$ \\
\hline 15. Pachypása lineósa Vill. $\sigma^{7}$ & 1005 & $124 / 25$ & Nachtr.-Taf. II, 11 \\
\hline
\end{tabular}



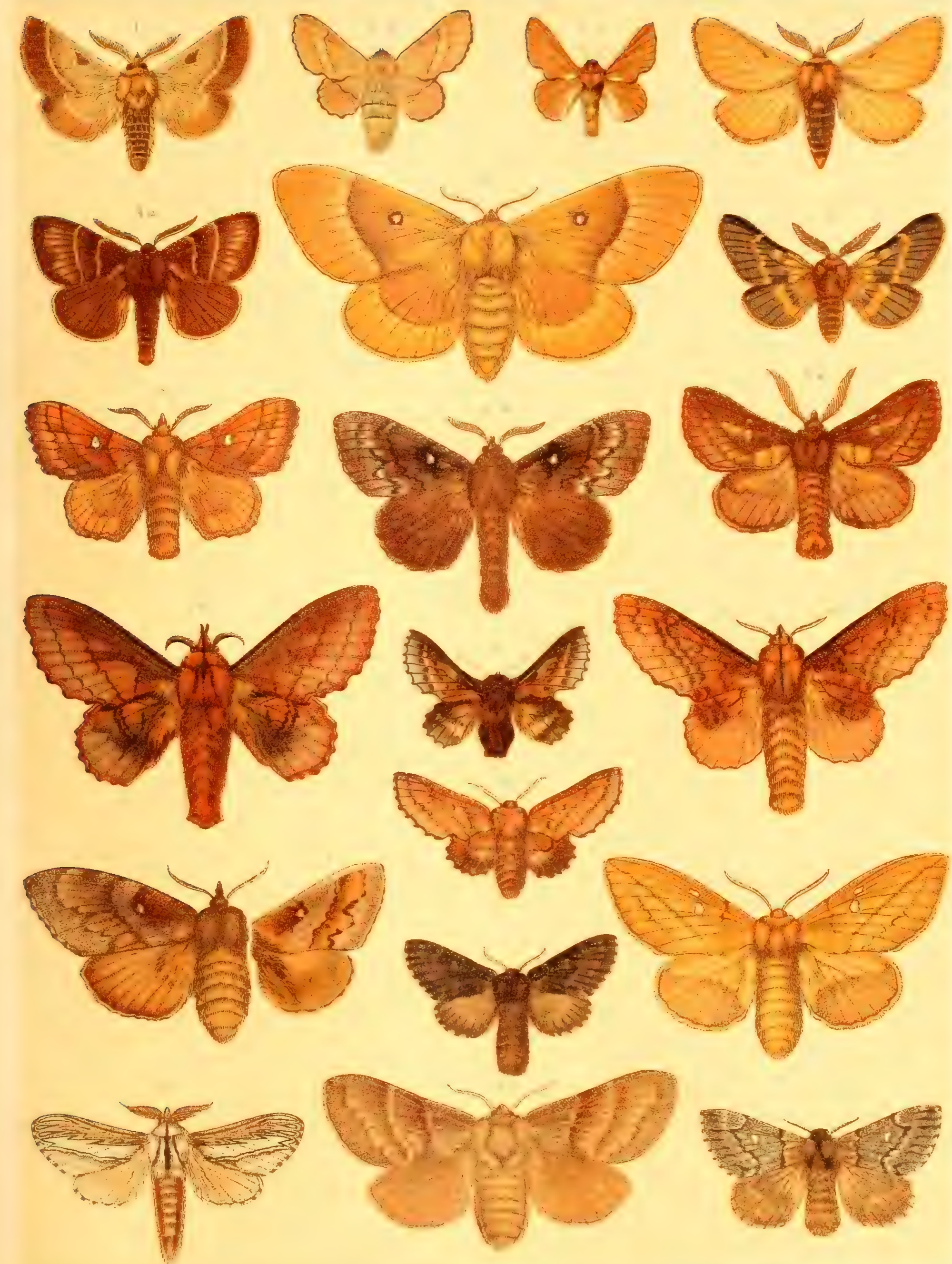



\section{TAFEL 28.}

\begin{tabular}{|c|c|c|c|c|}
\hline$N x$. & Name & Katalog I & Seite des I. Bandes & $\begin{array}{l}\text { Raupen- (Puppen-) } \\
\text { Abbildung }\end{array}$ \\
\hline 1. & Lasiocámpa terréni HS., 1. フ, r. 우 & $976 \mathrm{f}$ & 1191. & \\
\hline 2. & rétamae HS., 1. $0^{7}$, r. + & $976 \mathrm{c}$ & 1191. & \\
\hline 3. & séprula Gn., l. $\sigma^{7}$, r. 우 & 972 & $119 \mathrm{l}$. & \\
\hline 4. & Eógena contaminéi Ev. & 1112 & $140 / 41$ & Nachtr-Taf. II, 16 \\
\hline 5. & Simýra buettnéri Hering 우 & 1117 & $141 \mathrm{r}$. & \\
\hline 6. & Âgrotis sqálida Gn. ㅇ & 1147 & $145 \mathrm{r}$. & \\
\hline 7. & imperita $\mathrm{Hb} . \sigma^{\pi}$ & 1164 & 1471. & \\
\hline 8. & " teeta Hb. $\sigma^{7}$ & 1166 & 1471. & \\
\hline 9. & insignáta Ld. $\sigma^{7}$ & 1180 & 1491. & \\
\hline 10. & chaldáica B. $\sigma^{7}$ & 1182 & 1491. & \\
\hline 11. & kollári Ld. శ & 1189 & $149 \mathrm{r}$. & \\
\hline 12. & deplanáta Ev. O & 1225 & 1521. & \\
\hline 13. & luperinoides Gn. $\sigma^{7}$ & 1234 & $152 \mathrm{r}$. & \\
\hline 14. & anaehoréta HS., 1. $\sigma^{7}$, r. 우 & 1235 & $152 \mathrm{r}$. & \\
\hline 15. & wiskótti Stdfs $\sigma^{7}$ & 1297 & 1551. & \\
\hline 16. & $"$ hydrilloídes Alph. caradrinoides Stgr $\sigma^{7}$ & 1306 & $155 / 56$ & \\
\hline 17. & quadrángula Zett. 우 & 1310 & $156 \mathrm{l}$. & \\
\hline 18. & squalórum Ev. $q$ & 1317 & 1561. & \\
\hline 19. & vallesiaea B. ఠ & 1318 & $156 \mathrm{r}$. & \\
\hline 20. & multicúspis Ev. $q$ & 1334 & 1571. & \\
\hline 21. & endogaéa B. $\sigma^{7}$ & 1337 & $157 \mathrm{l}$. & \\
\hline 22. & flavína HS. $q$ & 1352 & $158 \mathrm{r}$. & \\
\hline 23. & " serraticórnis Stgr $\sigma^{7}$ & $1352 \mathrm{a}$ & 1591. & \\
\hline 24. & adumbráta Ev. $q$ & 1371 & 1601. & \\
\hline 25. & islándiea Stgr v. róssiea Stgr q & $1373 \mathrm{a}$ & 1601. & \\
\hline 26. & ehristóphi Stgre $\sigma^{7}$ & 1380 & $161 \mathrm{l}$. & \\
\hline 27. & basigrámma Stgr $\sigma^{7}$ & 1386 & $161 \mathrm{r}$. & \\
\hline 28. & multífida Ld. 우 & 1389 & 1621. & \\
\hline 29. & trifúpea Ev. ㅇ & 1412 & $164 \mathrm{l}$. & \\
\hline 30. & $" \quad$ robústa Ev. & 1414 & 1641. & \\
\hline 31. & Phlebóis rognéda Stgr $\sigma^{7}$ & 1429 & $166 \mathrm{r}$ & \\
\hline 32. & Maméstra spalax Alph. $\sigma^{7}$ & 1445 & $168 \mathrm{r}$. & \\
\hline
\end{tabular}

Diese Tafel enthält Nachträge zu den Tafeln 26 und 31-36. 

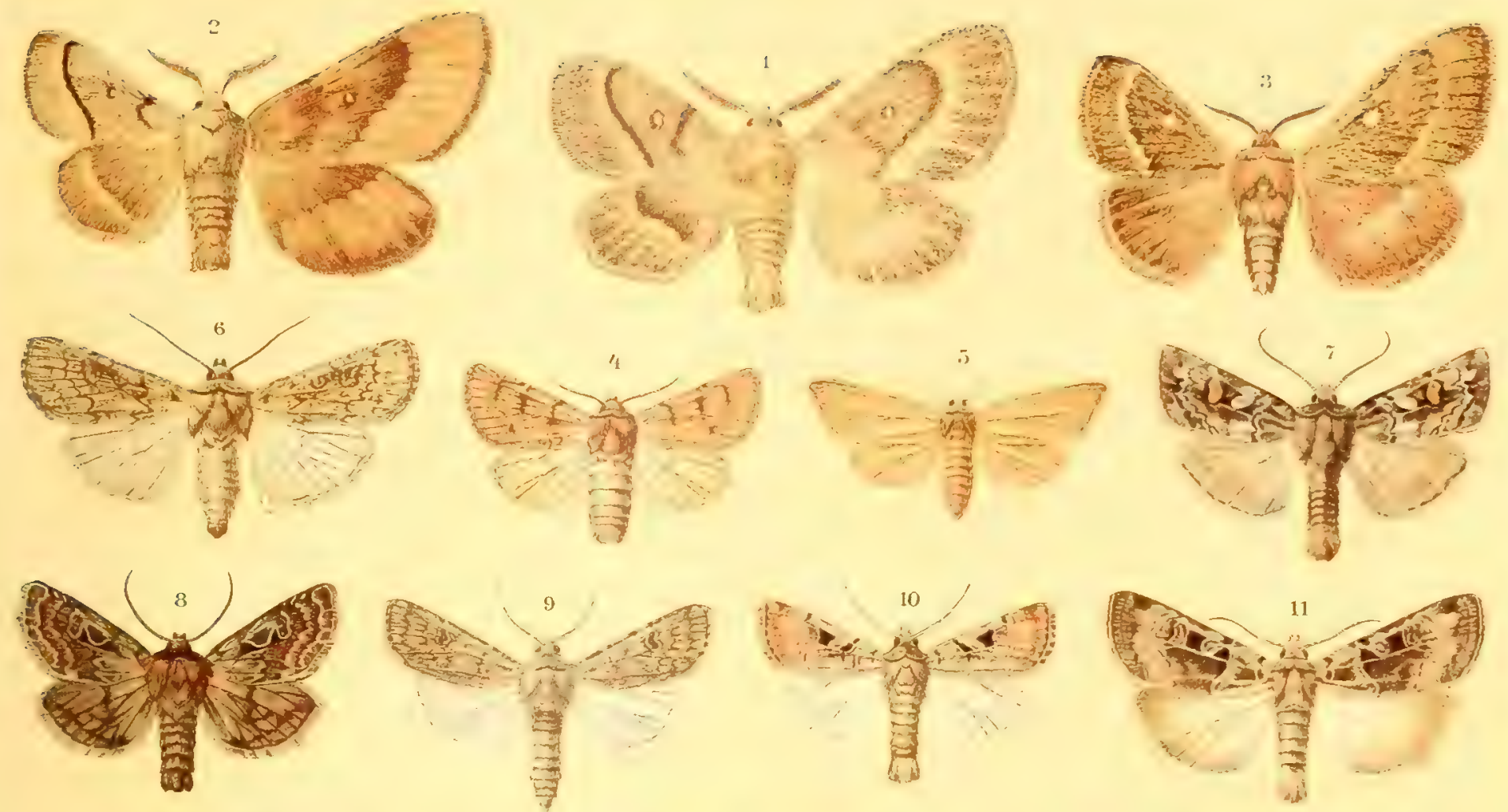

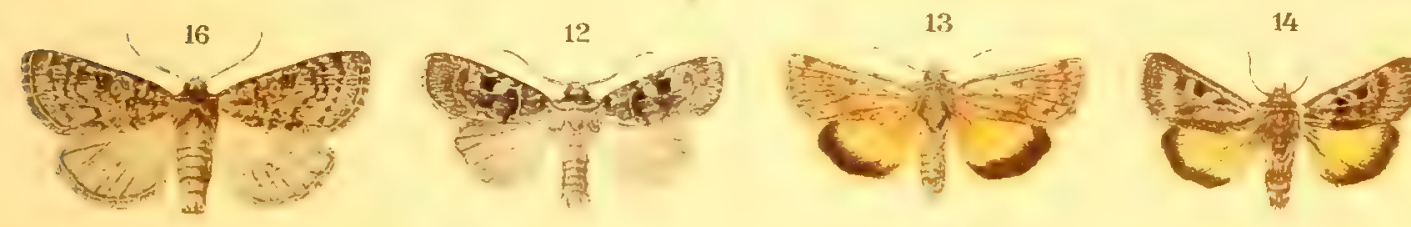
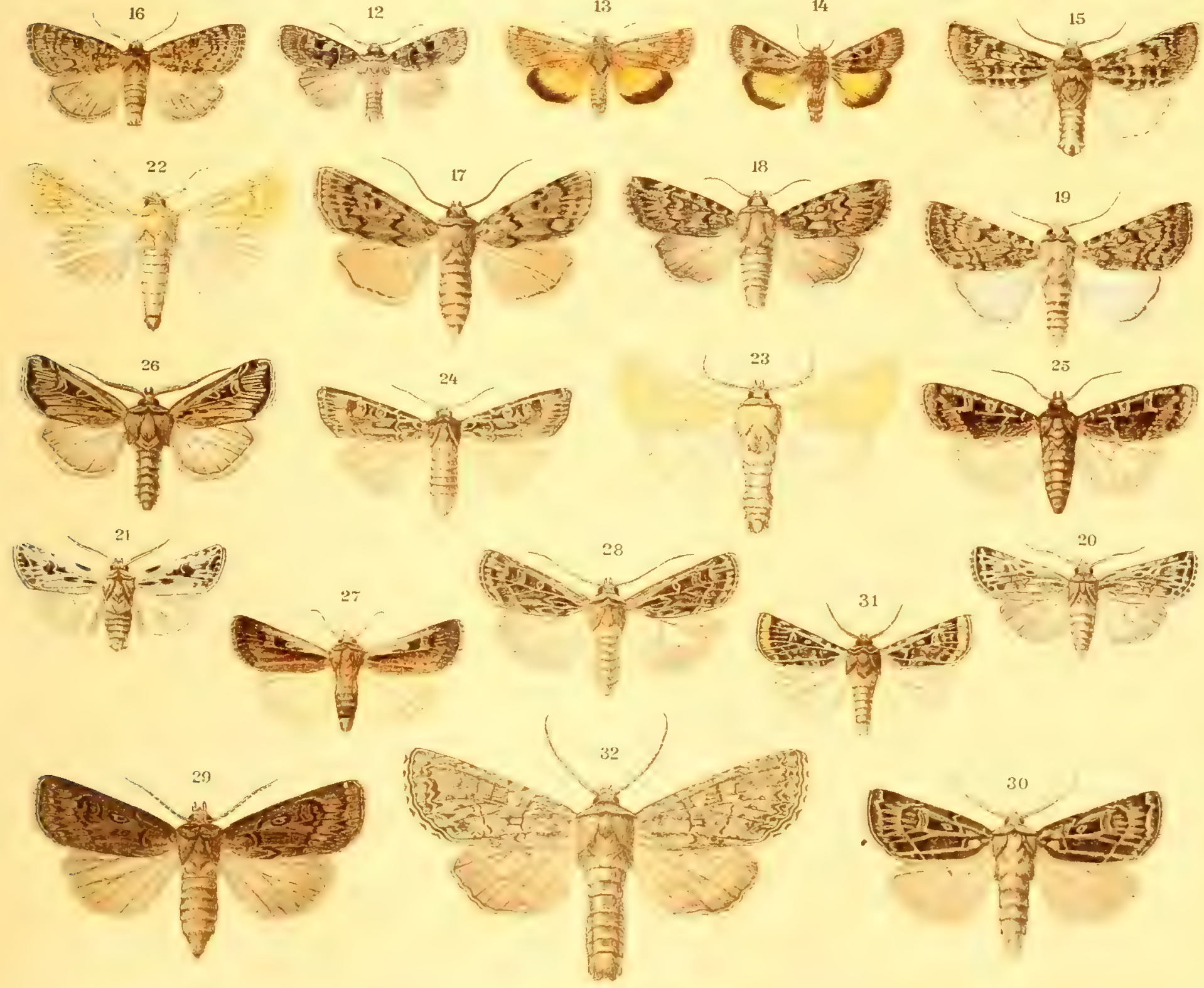




\section{TAFEL 29.}

\begin{tabular}{|c|c|c|c|c|}
\hline Nr. & Name & Katalog I & Seite des I. Bandes & $\begin{array}{l}\text { Raupen- (Puppen-) } \\
\text { Abbildung }\end{array}$ \\
\hline 1. & Maméstra impléxa Hb.*) & 1473 & $172 / 73$ & \\
\hline 2. & stigmósa Chr. ठ & 1479 & $173 \mathrm{l}$. & \\
\hline 3. & skraelingia HS. 우 & 1485 & $173 \mathrm{r}$. & \\
\hline 4. & ealberlái Stgr $q$ & 1491 & 1741. & \\
\hline 5. & furca Ev. $\sigma^{x}$ & 1507 & 1751. & \\
\hline 6. & córsica Ramb. $q$ & $1514 \mathrm{c}$ & $176 \mathrm{l}$. & \\
\hline $6 \mathrm{a}$ & - Saragóssa siceanórum Stgr $q$ & 1517 & 1761. & \\
\hline 7. & Dianthoécia dovrénsis Weke $\sigma^{7}$ & 1535 & $177 l^{1}$ & \\
\hline 8. & earpóphaga Bkh. $q$ & 1553 & $179 \mathrm{r}$. & Taf. 2519 , \\
\hline 9. & ehristóphi Moesehl. 우 & 1555 & 1801. & \\
\hline 10. & Miána mieroglóssa Ramb. & 1572 & 1821. & \\
\hline 11. & Bryóphila galatéa Mill. $q$ & 1590 & $183 / 84$ & \\
\hline 12. & Thalpóphila vittálba Frp & 1622 & $187 \mathrm{l}$. & Nachtr.-Taf. III, 19 \\
\hline 13. & Psoudohadéna immúnda Ev. $q$ & 1647 & $188 \mathrm{r}$. & \\
\hline 14. & " hálimi Mill. $\sigma^{7}$ & $1647 \mathrm{a}$ & $188 \mathrm{r}$. & III, 21 \\
\hline 15. & Hadéna ehristóphi Alph. $\left.{ }^{* *}\right)$ & 1657 & $189 \mathrm{l}$. & \\
\hline 16. & leuconóta HS. $\sigma^{\nearrow}$ & 1659 & $189 \mathrm{r}$. & \\
\hline 17. & zeta Tr. + & 1674 & 1911. & \\
\hline 18. & áretica Frr $q$ & 1688 & $192 \mathrm{r}$. & \\
\hline 19. & Oneoenémis nigrieula Ev. $\sigma^{\top}$ & 1730 & $196 \mathrm{r}$. & \\
\hline 20. & Episéma ledéri Chr. $\sigma^{\top}$ & 1738 & $197 \mathrm{l}$. & \\
\hline 21. & Helióphobus fallax Stgr $q$ & 1746 & $198 \mathrm{l}$. & \\
\hline 22. & Apor. lutulénta Bkh. v. lúneburgénsis Frr $q$ & q $1761 \mathrm{a}$ & $198 / 99$ & Taf'. $26,5 \mathrm{a}, \mathrm{b}$ \\
\hline 23. & Pólia aeúta Frr o & 1798 & 2031. & \\
\hline 24. & Nonágria brevilínea Fenn $\sigma^{7}$ & 1899 & $217 / 18$ & \\
\hline 25. & Coenóbia stigmática Ev. $\sigma^{\pi}$ & 1903 & 2181. & \\
\hline 26. & Epipsámmia desertícola Stgr $q$ & 1924 & 2211. & \\
\hline 27. & Leueánia lineáta Ev. ㅇ & 1953 & $225 \mathrm{r}$. & \\
\hline 28. & unipuneta $\mathrm{Hw}$. ㅇ & 1962 & 2271. & \\
\hline 29. & Mithýmna impar Stgr ठ7 & 1979 & $228 \mathrm{r}$. & \\
\hline 30. & Caradrina vicína Stgr $\sigma^{7}$ & 1995 & 2301. & \\
\hline 31. & $" \quad$ albína Ev. $\left.\sigma^{7 * * *}\right)$ & 2001 & $230 \mathrm{r}$. & \\
\hline 32. & $\begin{array}{r}\text { ménétrièsi Kretsehm. v. pouge- } \\
\text { monti (Püng.) }\end{array}$ & & $231 \mathrm{r}$. & \\
\hline 33. & grisea Ev. $\sigma^{\pi}$ & 2008 & $231 \mathrm{r}$ & \\
\hline 34. & albisignáta $0 \mathrm{bth} \cdot \sigma^{7}$ & 2021 & $233 / 34$ & \\
\hline 35. & Hydrilla lepígone Moesehl. $q$ & 2029 & 2351. & \\
\hline 36. & Netrocerócara quadriplága Bort. $\sigma^{7}$ & & 2361. & \\
\hline
\end{tabular}

*) Kopie nach Mém. Rom. V, t. 12, fig. 1.

**) Kopie nach Mém. Rom. V, t. 12, fig. 2.

***) Die Hfl. sind wei $B$, stark blauviolett irisierend.

Diese Tafel enthält Nachträge zu den Tafeln 37-44. 

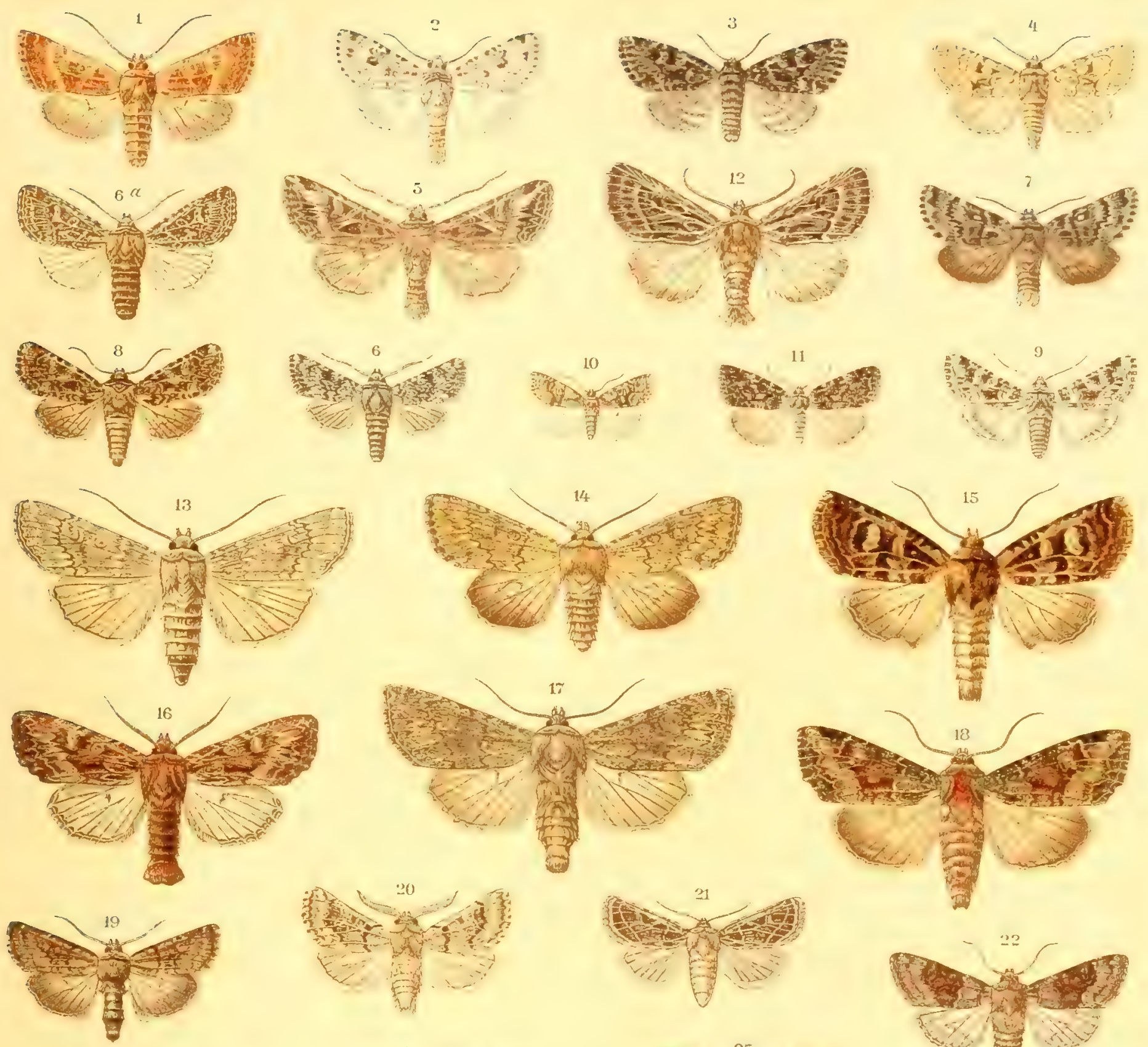

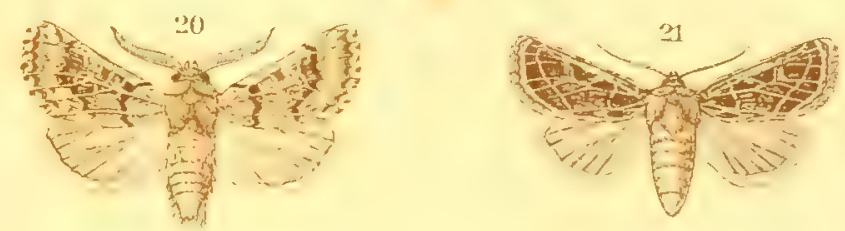
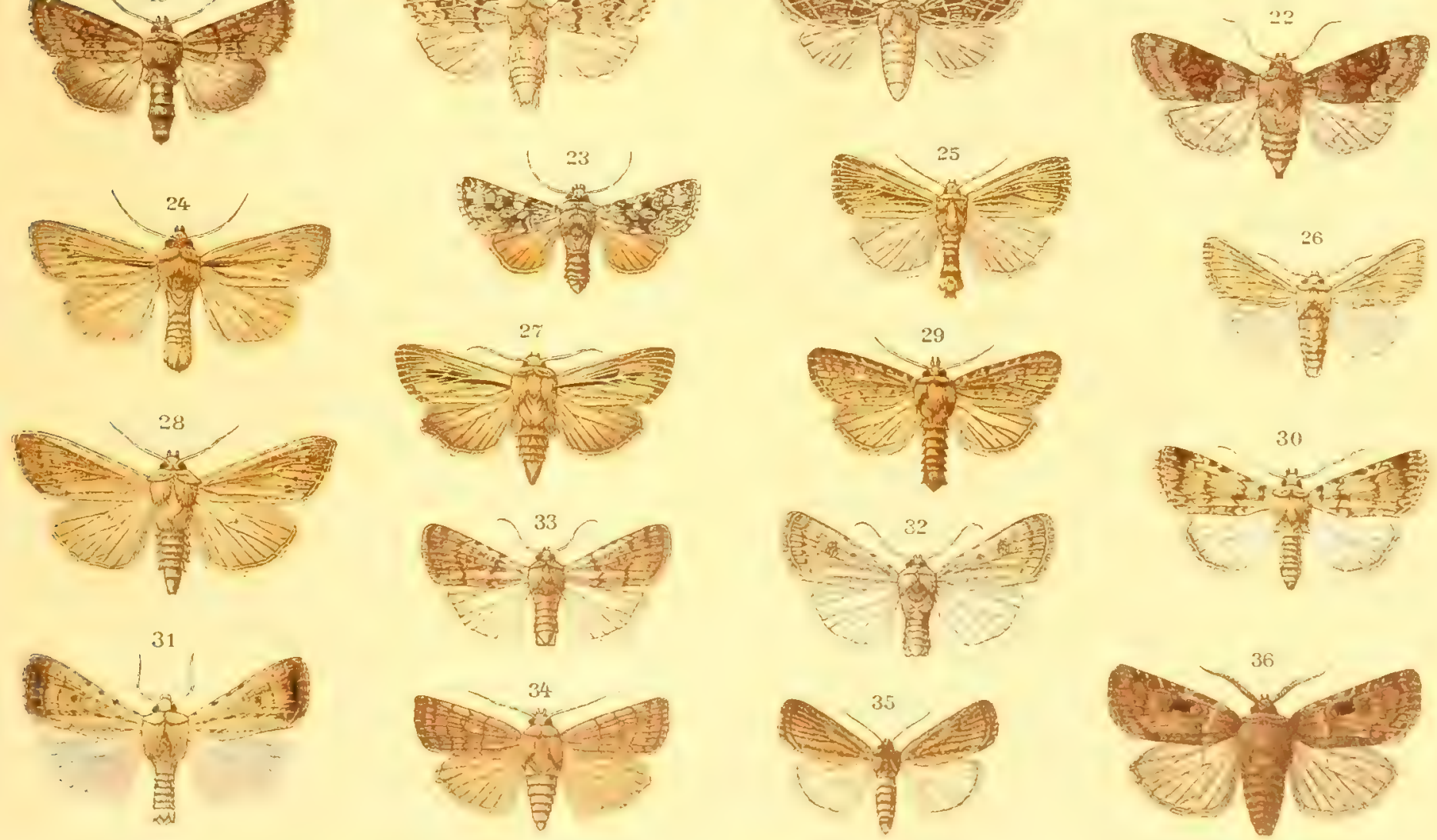


. 


\section{TAFEL 30.}

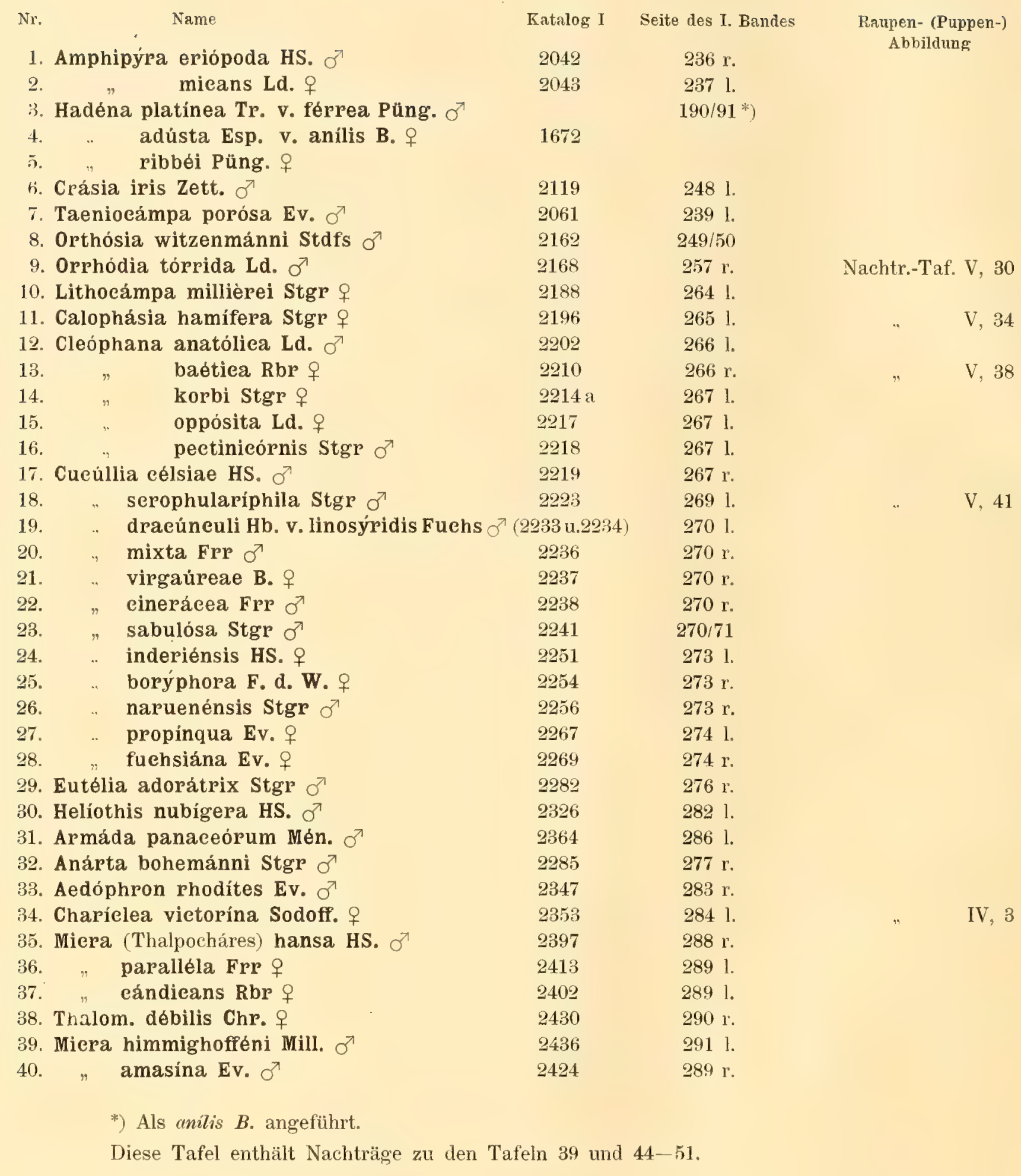



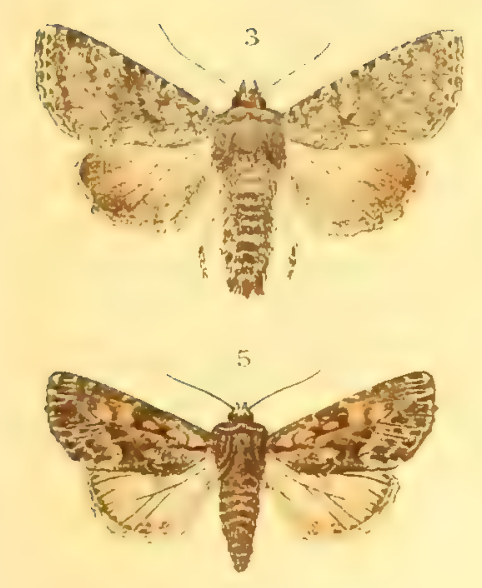

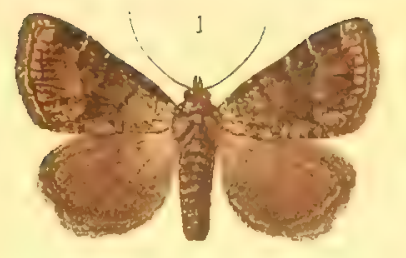

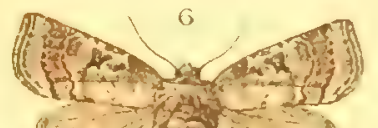

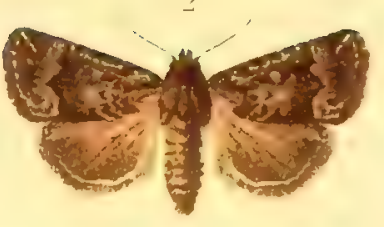

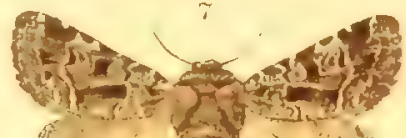
तु
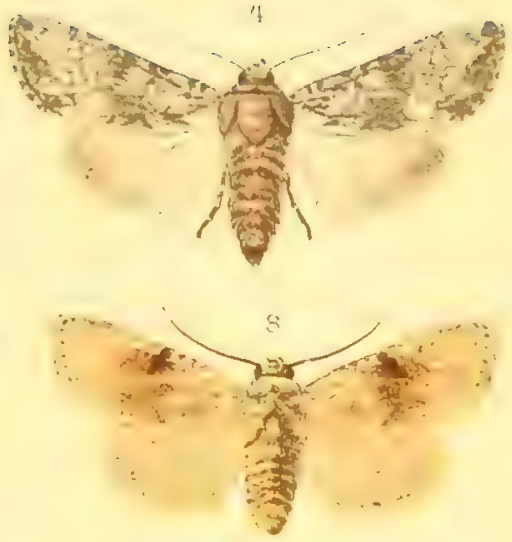

\section{(2)}
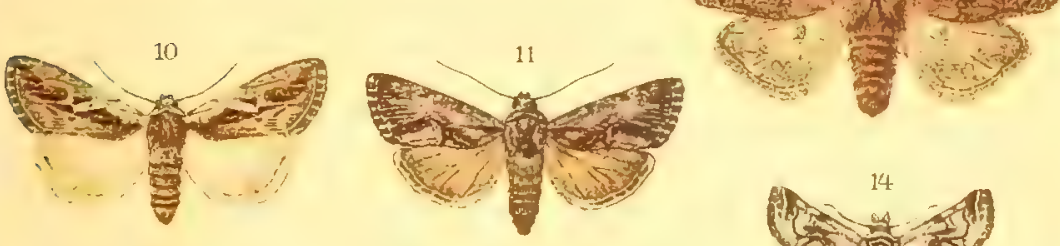

(14

(3)

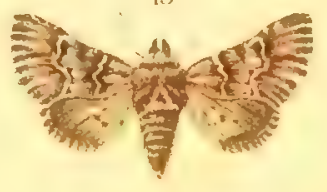

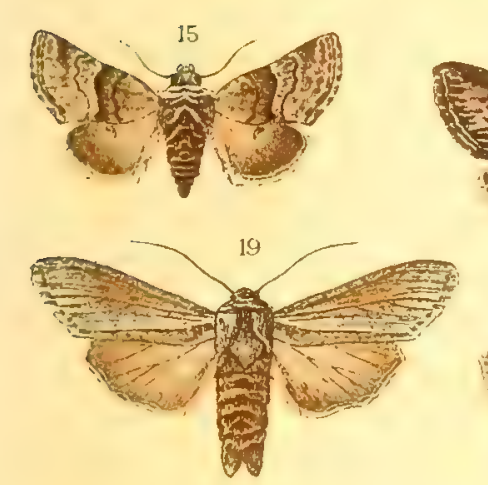

$\because \frac{1}{2}$

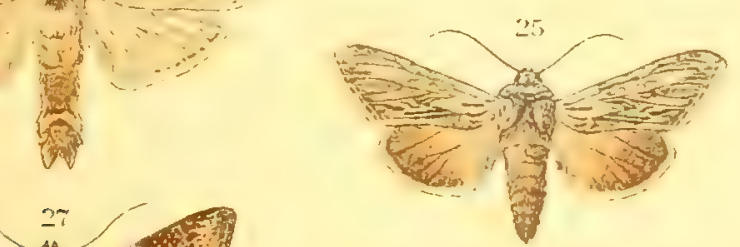

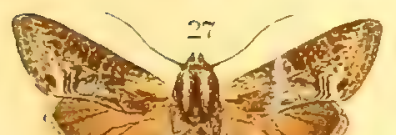
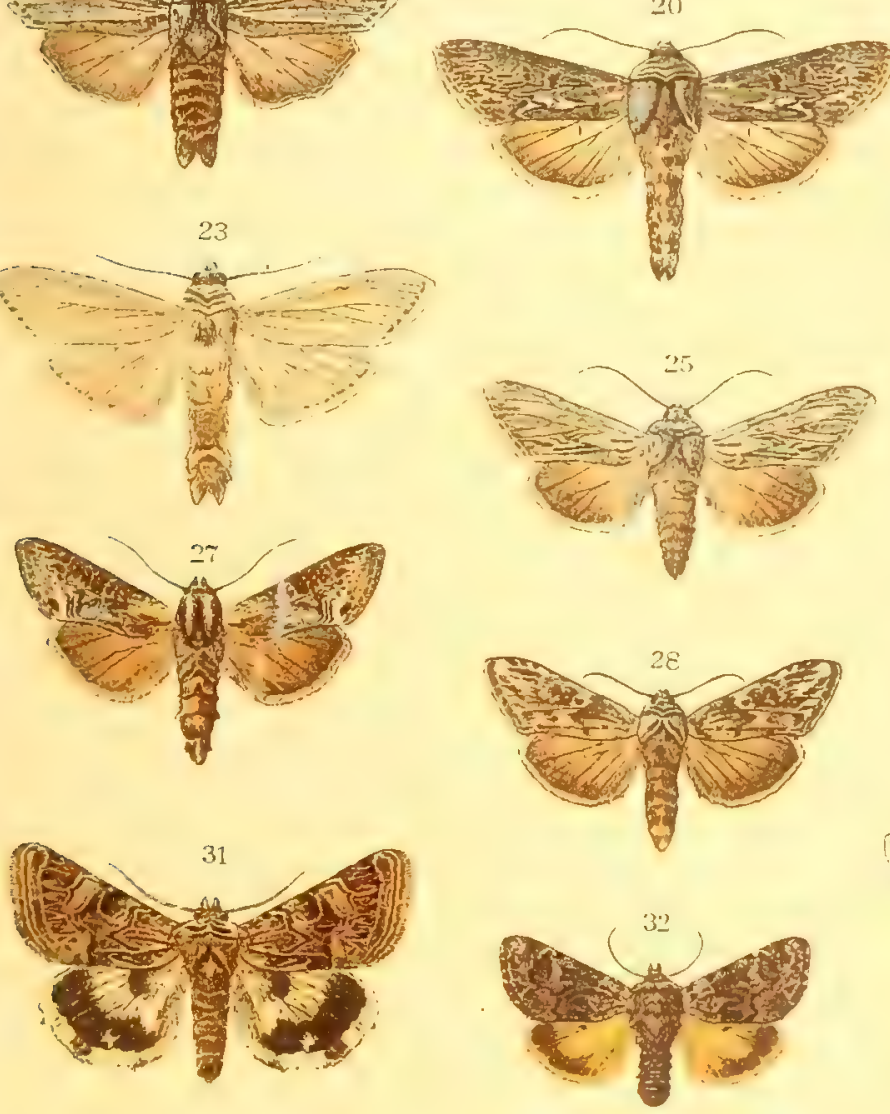
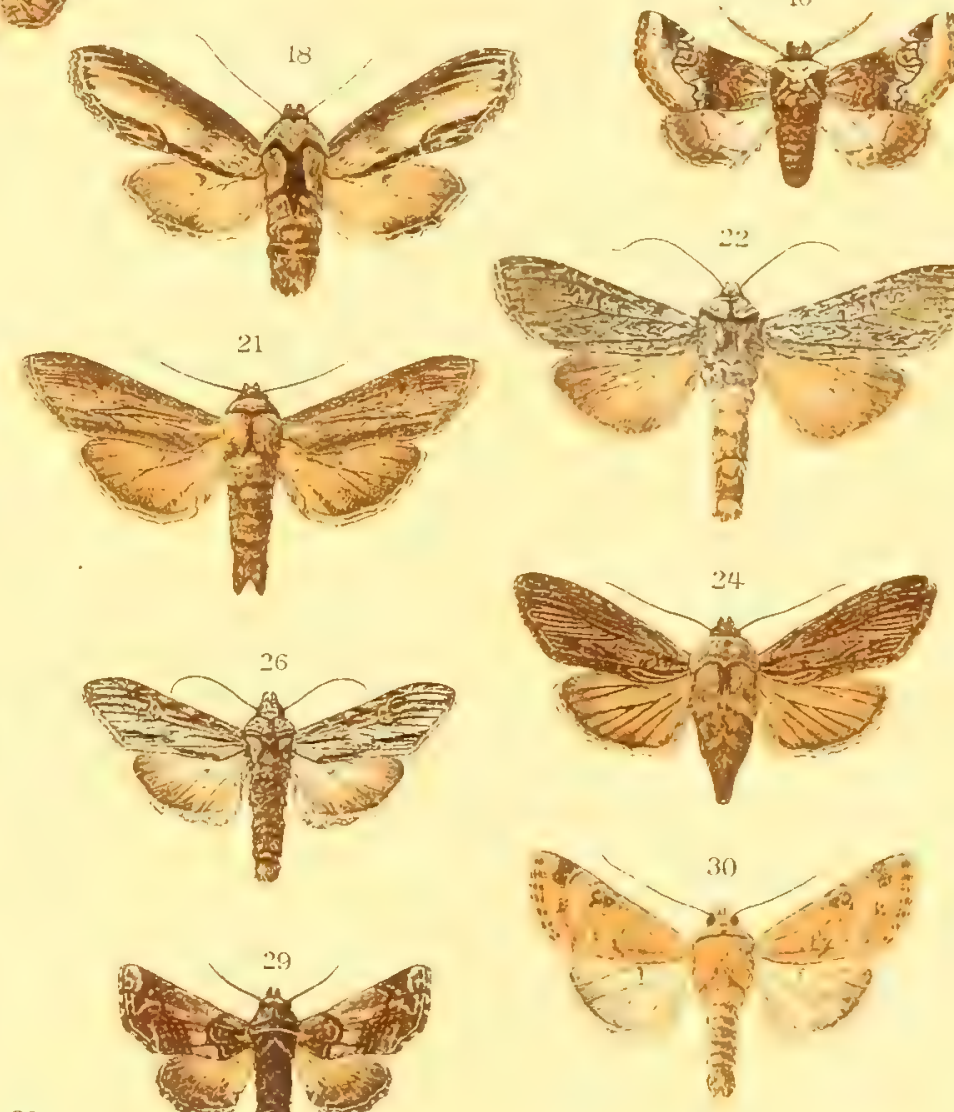

$\frac{38}{\frac{3 x}{20}}$
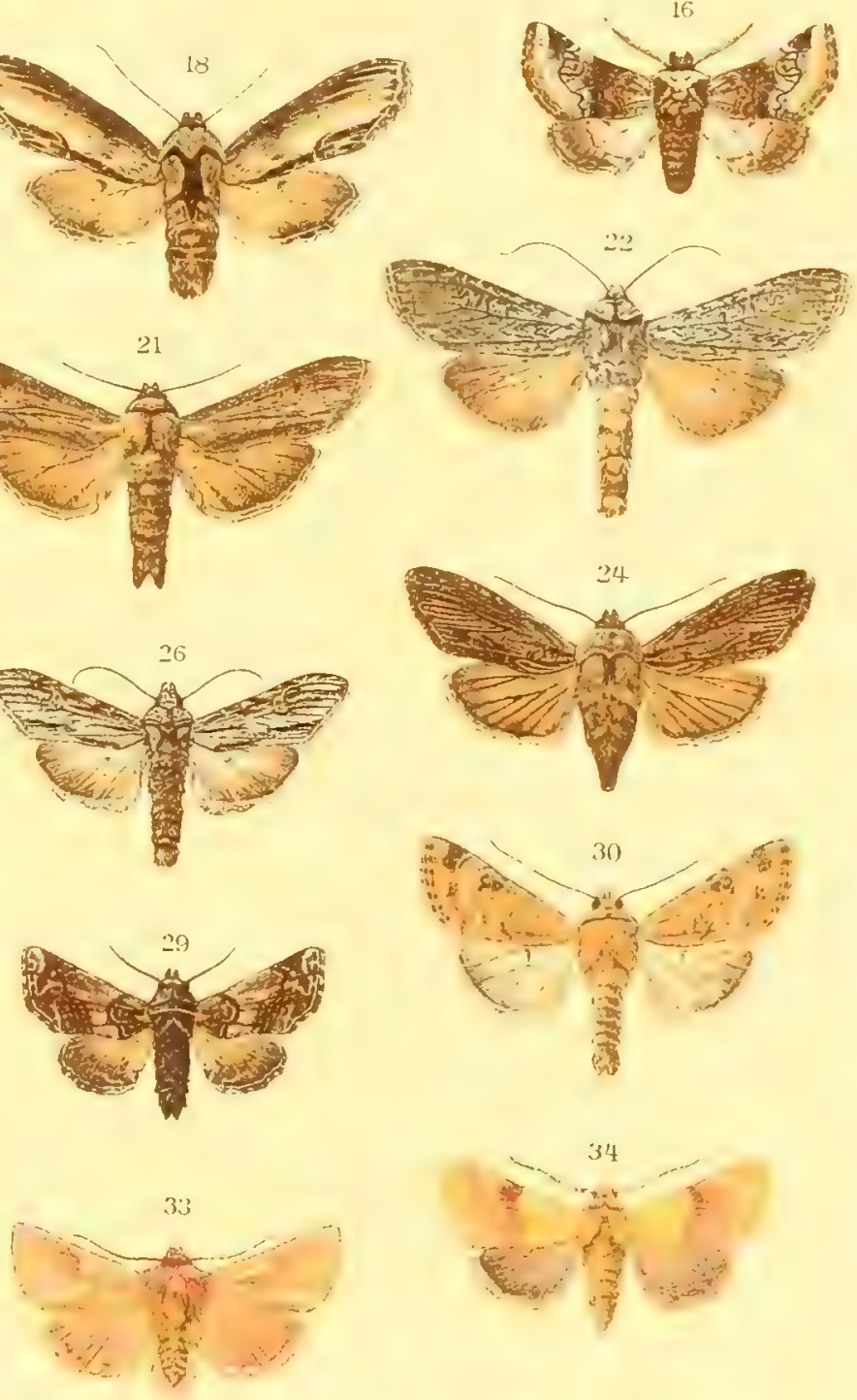

$$
\text { (f) dis } 15
$$
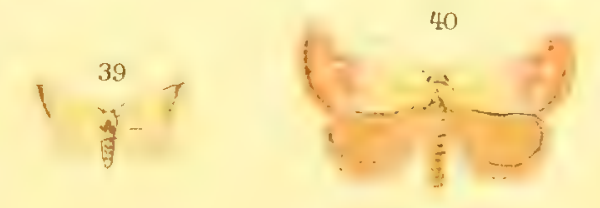



\section{TAFEL 31 .}

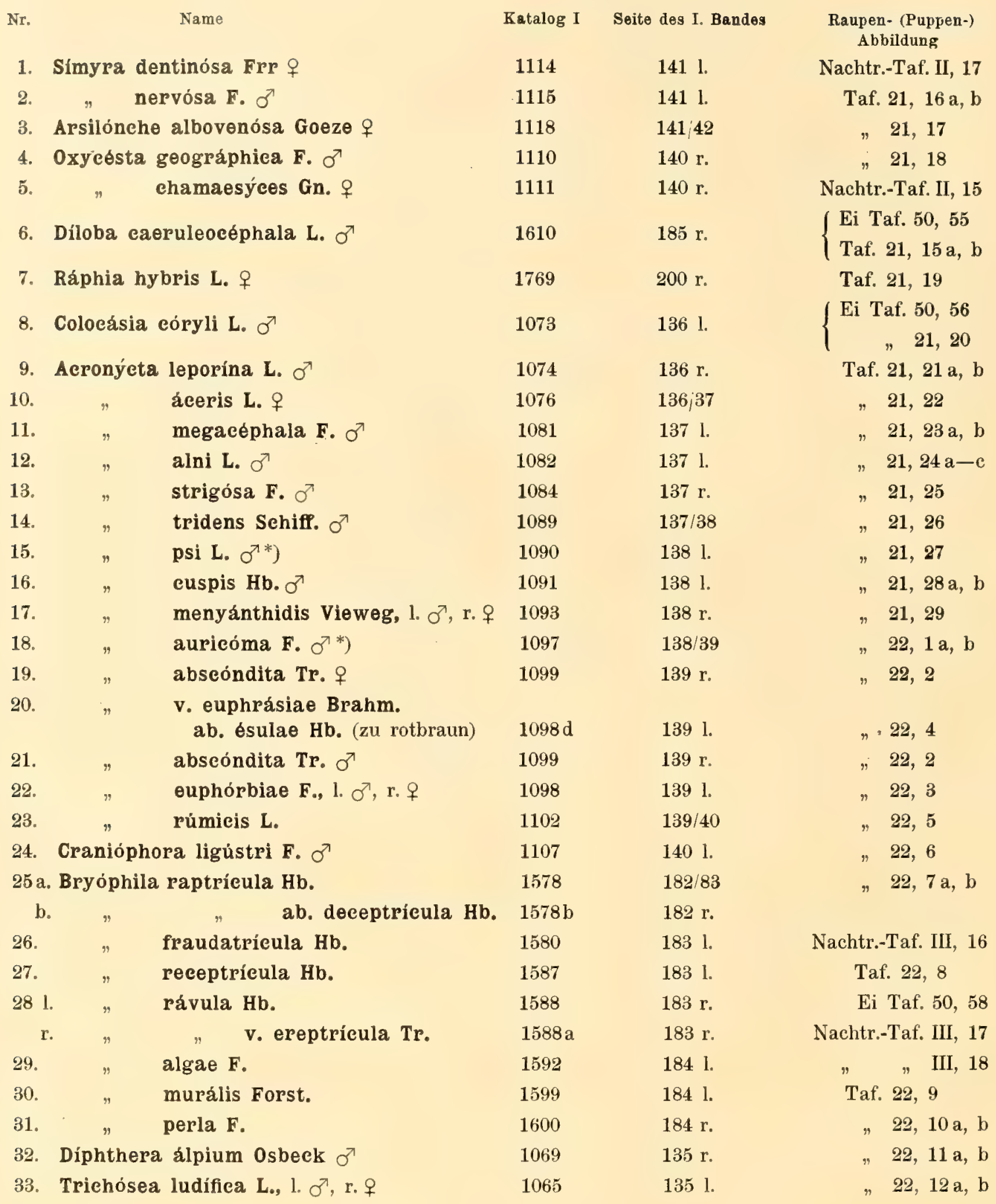

*) Zu bräunlich. 

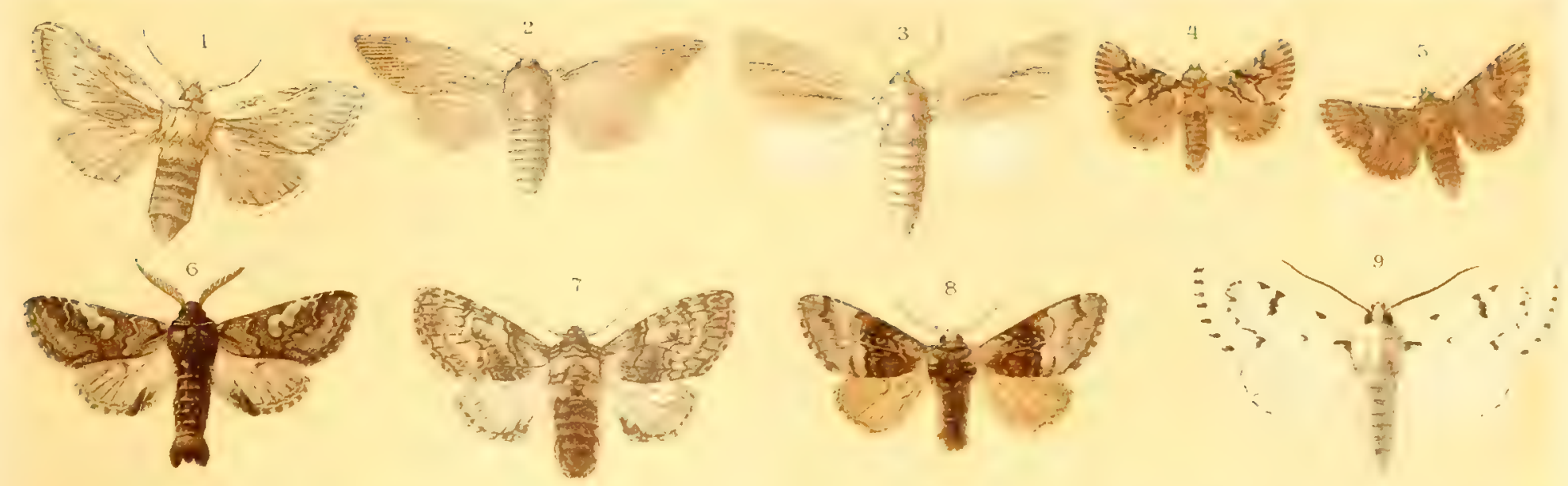

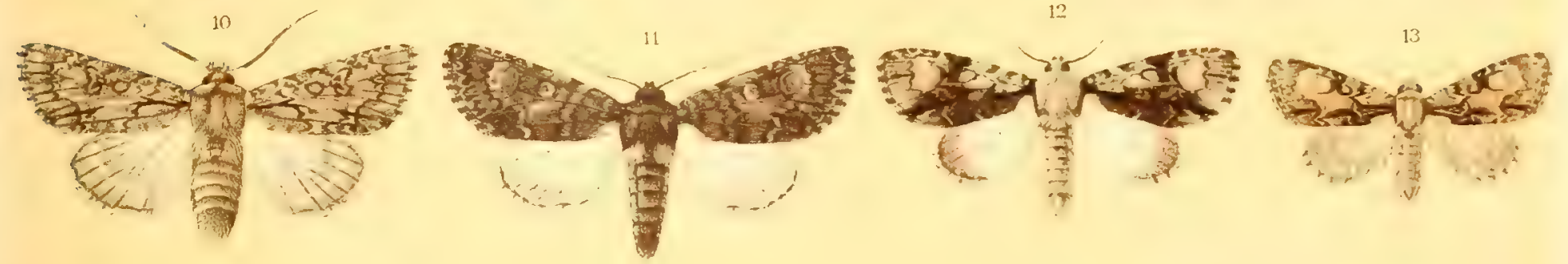

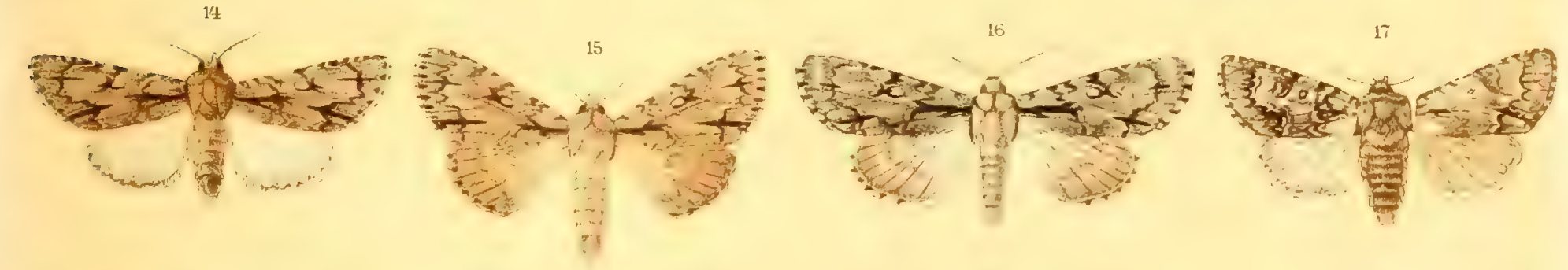
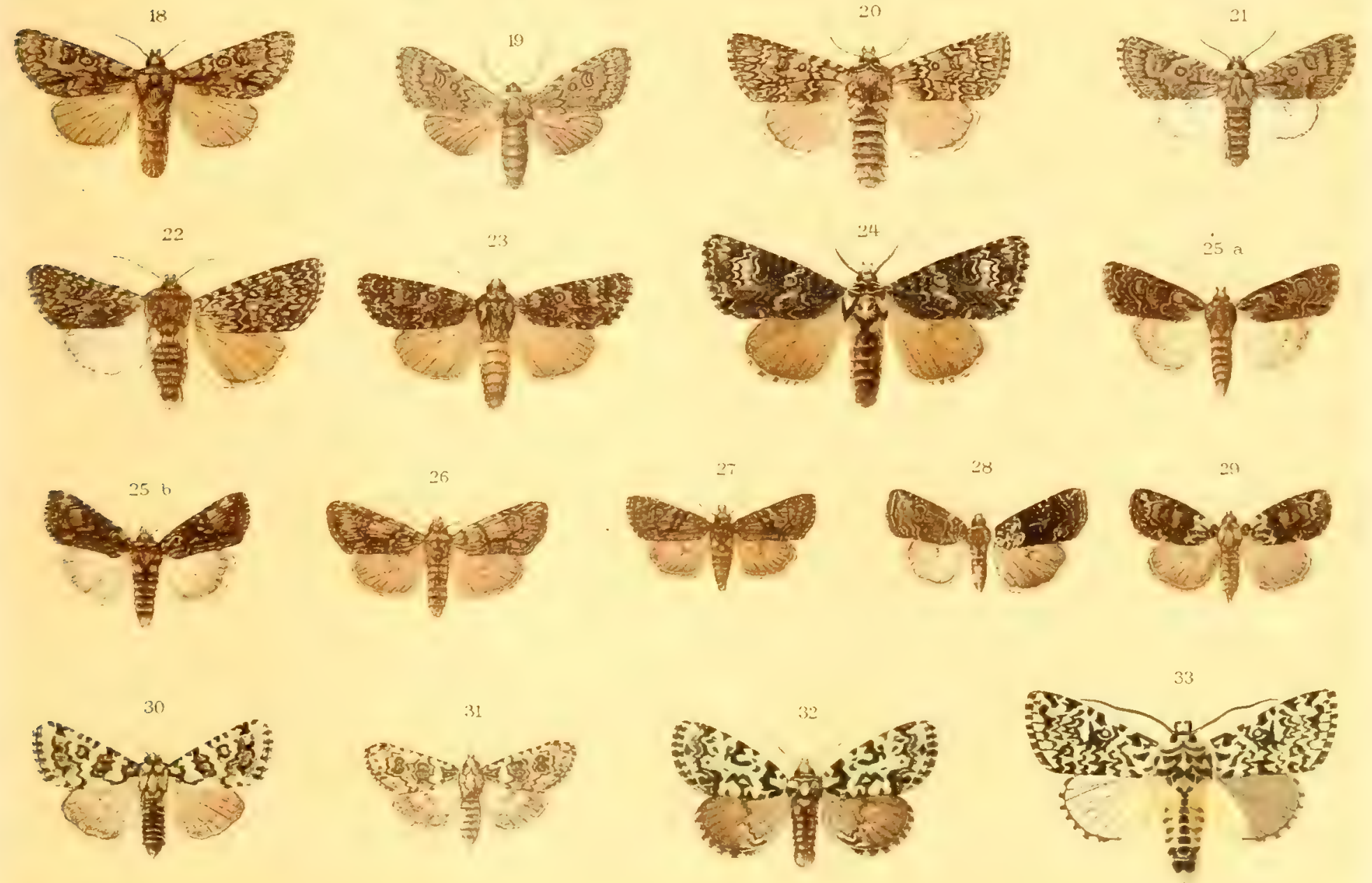




\section{TAFEL 32.}

Nr.

1. Pánthea coenobíta Esp. $\sigma^{7}$

2. Agrótis strigula Thnb. ㅇ

3. " molothína Esp. $\sigma^{7}$

4. " polýgona F. + .

5. $"$ signum F. 우

6. " subrósea Steph. $\sigma^{7}$

7. $"$ ianthína Esp. $\sigma^{7}$

8. " linogrísea Sehiff. 우

9. $"$ interiécta $\mathrm{Hb}$. $\mathrm{O}^{7}$

10. $"$ ehardínyi B. $\sigma^{7}$

11. $"$ sobrina Gn, $\sigma^{7}$

12. " punicea Hb. $\sigma^{7}$

13. $"$ aủgur F. $\sigma^{7}$

14. " fímbria L. ㅇ

15. " senna HG. +

16a. " prónuba L. $\sigma^{7}$

b. "ab. innuba Tr.

17. " erythrína Ramb. $\sigma^{7}$

18. " obseúra Brahm. $q$

19. $"$ sủbsequa $\mathrm{Hb} . \sigma^{7}$

20. " orbóna Hufn. 우

21a. " castánea Esp. 우

b. " $"$ v. neglécta $\mathrm{Hb} \cdot \sigma^{\nearrow}$

22. " oblóngula Stgr $\sigma^{7}$

23. " hyperbórea Zett. 우

24. " collína B. $\sigma^{7}$

25. " agathína Dup. $\sigma^{7}$

26. " triángulum Hufn. ㅇ

27. "baía F. $\sigma^{7}$
Katalog I Seite des I. Bandes

134/35

1064

1119

1120

1121

1122

1123

1125

1126

1128

1129

1131

1132

1136

1127

1139

1152

$1152 \mathrm{a}$

1141

1143

1153

1154

1156

$1156 \mathrm{a}$

1157

1165

1167

1168

1169

1172
$142 \mathrm{r}$.

$142 / 43$

$143 \mathrm{l}$.

1431.

$143 \mathrm{l}$.

$143 \mathrm{r}$.

14344

1441.

$144 \mathrm{r}$.

$144 \mathrm{r}$.

$144 \mathrm{r}$.

$144 / 45$

1441.

1451.

$145 \mathrm{r}$.

145 r.

$145 \mathrm{l}$.

$145 \mathrm{l}$.

$146 \mathrm{l}$.

$146 \mathrm{l}$.

$146 \mathrm{r}$.

$146 \mathrm{r}$.

$146 \mathrm{r}$.

$147 \mathrm{l}$.

$147 \mathrm{r}$.

$147 \mathrm{r}$.

$147 \mathrm{r}$.

$148 \mathrm{l}$.
Raupen- (Puppen-) Abbildung

Taf. 22, $13 a, b$ und Nachtr.-Taf.II, 14 a,b Taf. $22,14 \mathrm{a}, \mathrm{b}$

Nachtr.-Taf. II, 18

Taf. 22, 15

, 22,16

Taf. 22, 17

" 22,18

Nacht.-Taf. II, 19

Taf. 22, $20 \mathrm{a}, \mathrm{b}$

" 22,21

"22, 19

" 48, 21

"22, $22 \mathrm{a}, \mathrm{b}$

Nachtr.-Taf. II, 20

Taf. 22, 23

"22, 24

" 22,25

Nachtr.-Taf. II, 21

Taf. 23, 1

Ei Taf. 50, 39 ,23, $2 \mathrm{a}-\mathrm{c}$

Taf. 23, 3 

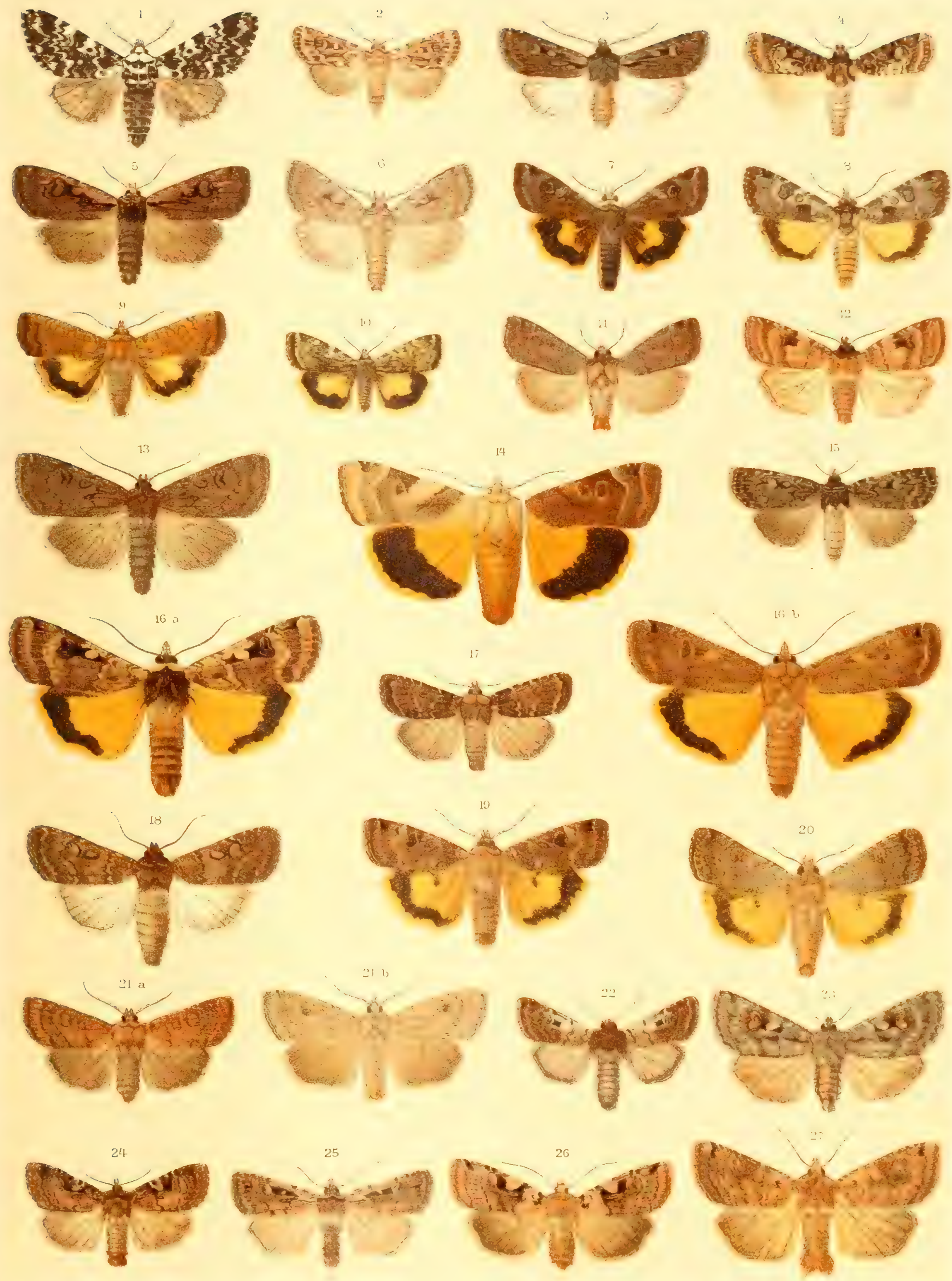



\section{TAFEL 33.}

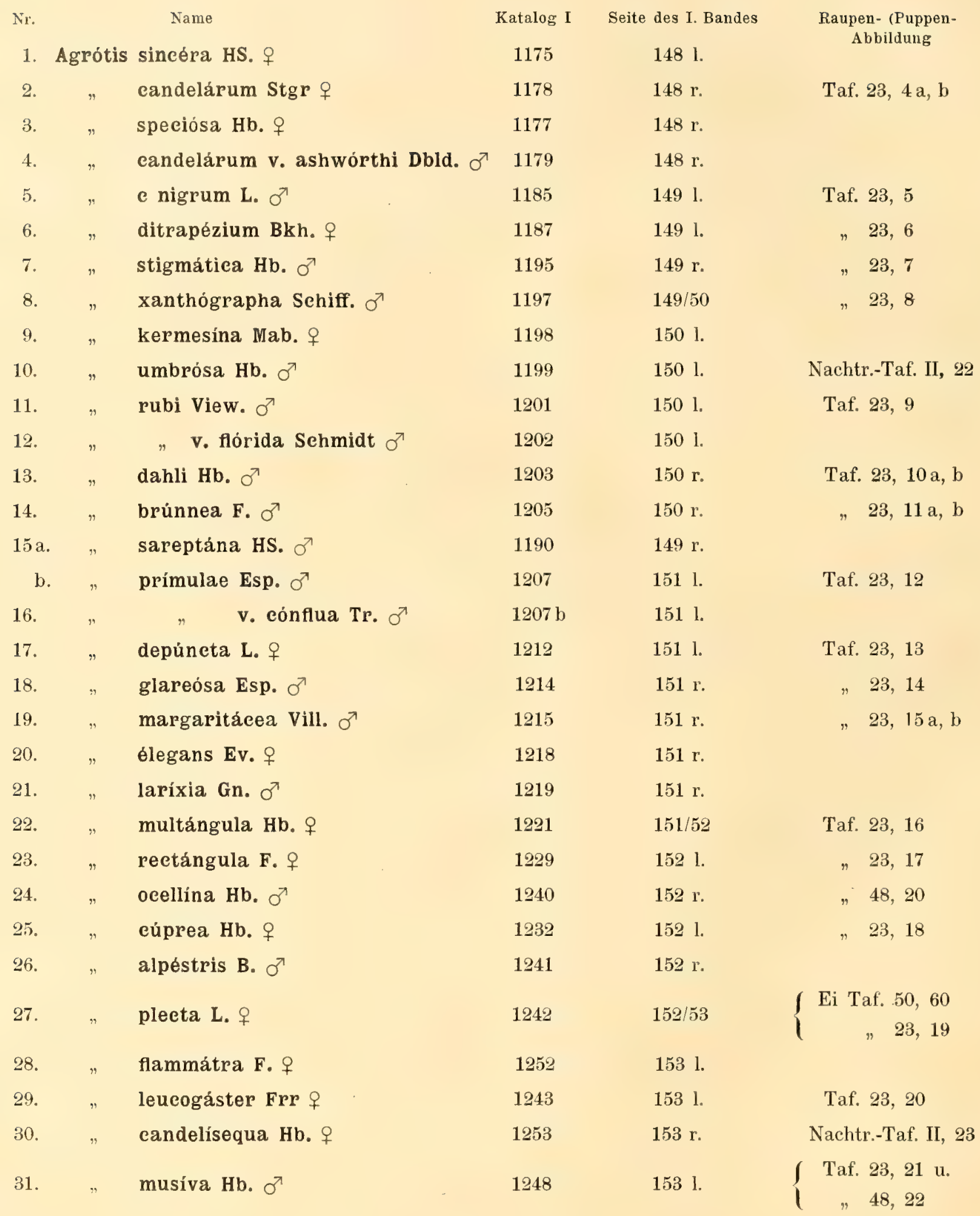


(4)

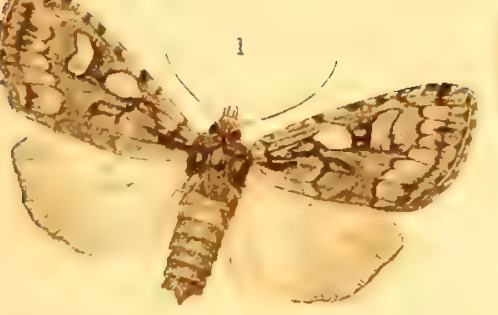

$(n+2)$

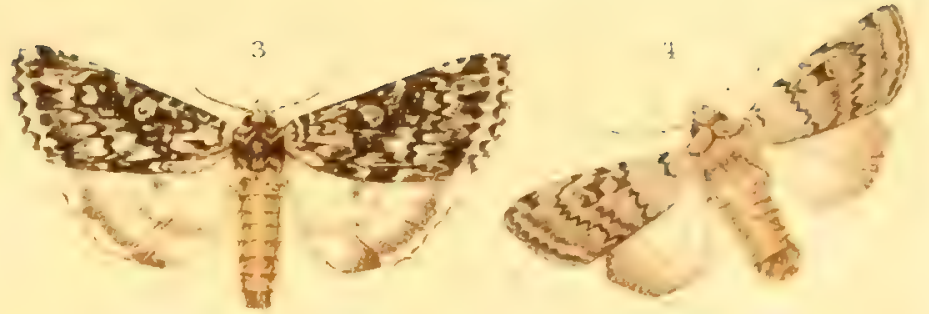

(not)
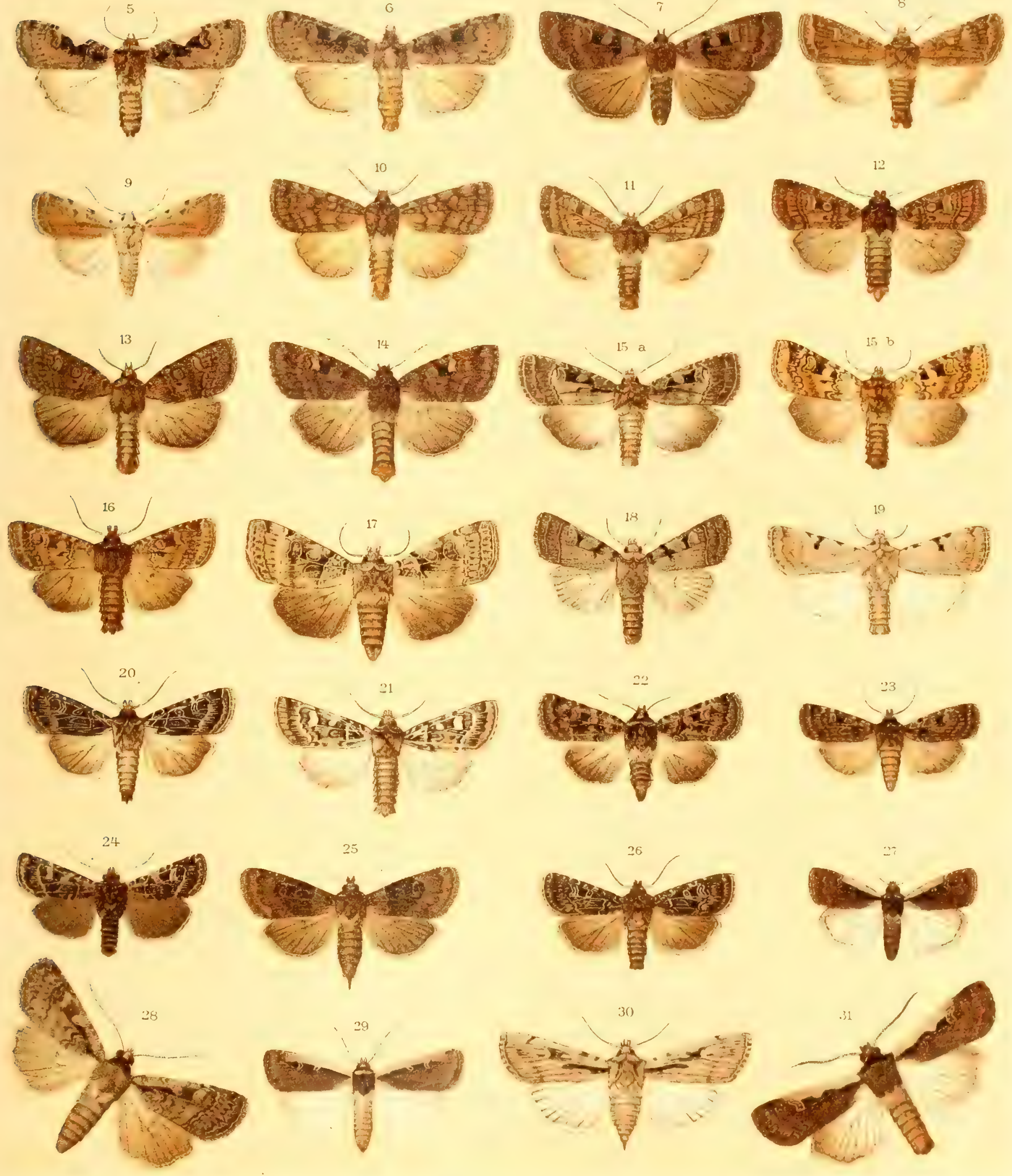



\section{TAFEL 34.}

\begin{tabular}{|c|c|c|c|c|c|}
\hline Nr. & & Name & Katalog I & Seite des I. Bandes & $\begin{array}{l}\text { Raupen- (Puppen-) } \\
\text { Abbildung }\end{array}$ \\
\hline 1. & Agrótis & fénnica Tausch. 우 & 1255 & $153 \mathrm{r}$. & \\
\hline 2. & $"$ & lucérnea L. $\sigma^{7}$ & 1259 & 1541. & Nachtr.-Taf. II, 24 \\
\hline 3. & $"$ & nychthémera B. $q$ & 1260 & 1541. & \\
\hline 4. & $"$ & eulminicola Stgr & 1296 & 1551. & \\
\hline 5. & $"$ & constánti Mill. శ & 1293 & 1551. & \\
\hline 6. & $"$ & helvetína B. $\sigma^{7}$ & 1273 & $154 \mathrm{r}$. & \\
\hline 7. & $"$ & signifera F. 우 & 1330 & $157 \mathrm{l}$. & Taf. 23,24 \\
\hline 8. & $"$ & putris L. $\sigma^{7}$ & 1346 & 1581. & " $23,23 a, b$ \\
\hline 9. & $"$ & lueípeta $\mathrm{F}$. 우 & 1270 & $154 \mathrm{r}$ & Nachtr.-Taf. II, 25 \\
\hline 10. & $"$ & fugax Tr. 우 & 1267 & $154 \mathrm{r}$ & Taf. 23,22 \\
\hline 11. & $"$ & símulans Hufn. $\sigma^{7}$ & 1256 & 1541. & \\
\hline 12. & $"$ & celsícola Bell. 우 & 1333 & $157 \mathrm{l}$. & \\
\hline 13. & $"$ & forcípula $\mathrm{Hb} \cdot \sigma^{7}$ & 1323 & $156 /$ ŏ & $\left\{\begin{array}{c}\text { Taf. } 23,25 \mathrm{a}, \mathrm{b} u . \\
" 49,1\end{array}\right.$ \\
\hline 14. & $"$ & fimbriola Esp. $\sigma^{\nearrow}$ & 1322 & $156 \mathrm{r}$. & Taf. 48,19 \\
\hline 1.$)$. & $"$ & latens $\mathrm{Hb}$. 우 & 1308 & 1561. & $" \quad 23,26$ \\
\hline 16. & $"$ & griséseens Tr. $\sigma^{7}$ & 1303 & $155 \mathrm{r}$. & \\
\hline 17. & $"$ & birivia $\mathrm{Hb} \cdot \sigma^{\pi}$ & 1276 & $154 \mathrm{r}$. & \\
\hline 18. & $"$ & decóra Hb. 우 & 1281 & $154 / 55$ & Taf. 49,2 \\
\hline 19. & $"$ & puta $\mathrm{Hb}$. ab. renítens $\mathrm{Hb} . \sigma^{\pi}$ & 1345 & $157 / 58$ & \\
\hline 20. & $"$ & renigera $\mathrm{Hb} . q$ & 1299 & $155 \mathrm{r}$. & \\
\hline 21. & $"$ & $\cos \mathrm{Hb} \cdot \sigma^{7}$ & 1313 & 1561. & Nachtr.-Taf. II, 27 \\
\hline 22. & $"$ & cinérea Hb. $\sigma^{7}$ & 1347 & 1581. & \\
\hline 23. & $"$ & simplónia $\mathrm{H}-\mathrm{G}$. $\sigma^{7}$ & 1302 & $155 \mathrm{r}$. & Nachtr.-Taf. II, 26 \\
\hline 21. & $"$ & exelamatiónis L. 우 & 1349 & $158 \mathrm{r}$. & Taf. $23,27 \mathrm{a}, \mathrm{b}$ \\
\hline 25. & $"$ & spinífera Hb. 우 & 1344 & $157 \mathrm{r}$. & $\Rightarrow \quad 24,1$ \\
\hline $26 \mathrm{a}$. & $"$ & ripae $\mathrm{Hb} . \sigma^{7}$ & 1355 & 1591. & Nachtr.-Taf. II, 28 \\
\hline b. & $"$ & $" \quad " \quad$ v. weissenbórni Frr & $1355 \mathrm{~b}$ & 1591. & \\
\hline 27. & $"$ & $" \quad " \quad$ v. desertórum B. $q$ & $1355 \mathrm{c}$ & 1591. & \\
\hline
\end{tabular}



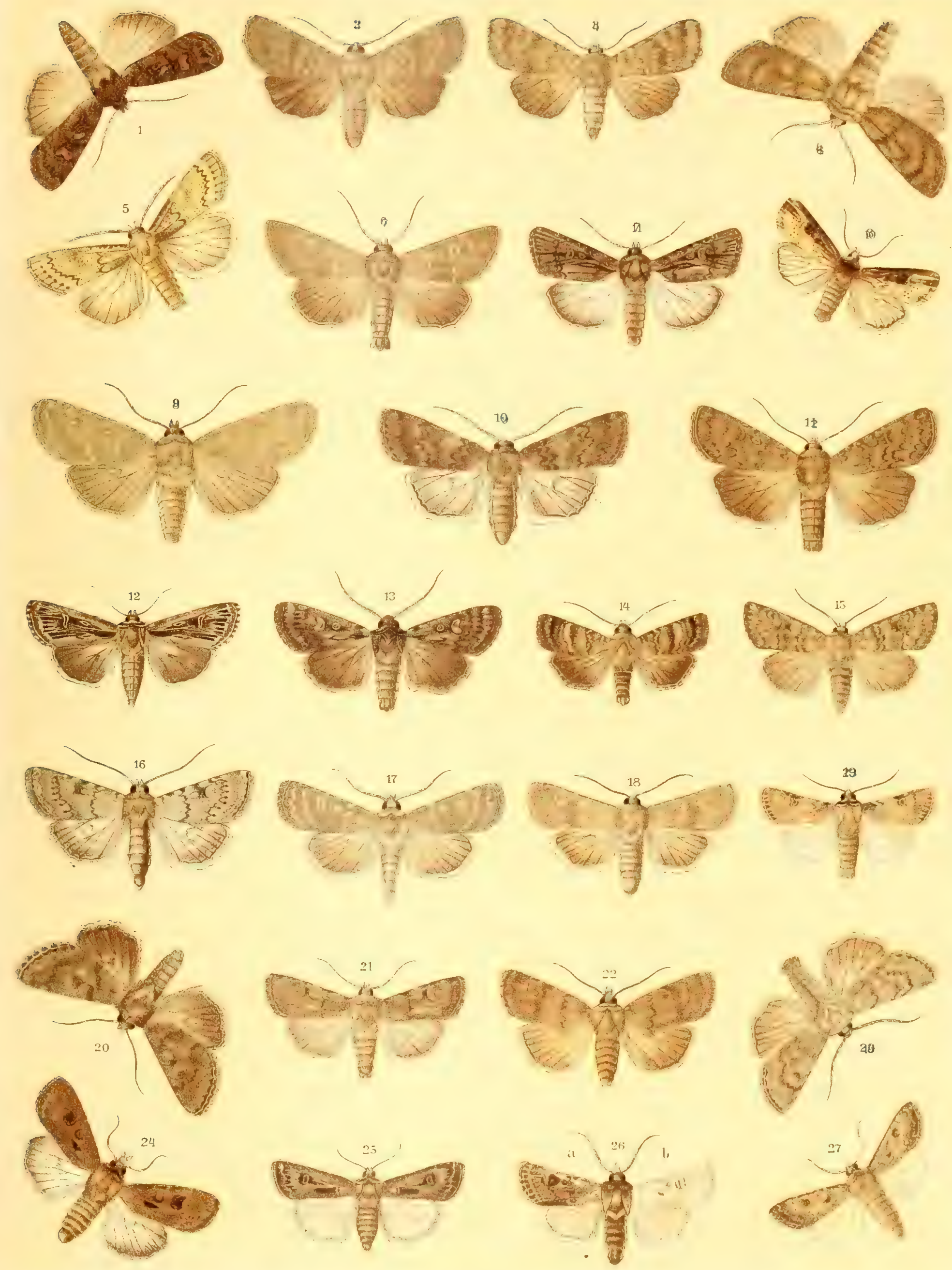




\section{TAFE L 35.}

\begin{tabular}{|c|c|c|c|c|c|}
\hline Nir. & & Name & Katalog I & Seite des I. Bandes & $\begin{array}{c}\text { Raupen- (Puppen-) } \\
\text { Abbildung }\end{array}$ \\
\hline 1. & Agrótis & eursória Hufn. $\sigma^{7}$ & 1358 & 1591. & Taf. 24,2 \\
\hline 2. & $"$ & reeússa $\mathrm{Hb}$. 우 & 1369 & $159 \mathrm{r}$. & Nachtr.-Taf. II, 29 \\
\hline 3. & $"$ & transsylvánica HS. $\sigma^{7}$ & 1369 & $159 \mathrm{r}$. & \\
\hline 4. & $"$ & nígrieans L. + & 1370 & 1601. & \\
\hline 5. & $"$ & lídia $\mathrm{Cr}$. & 1372 & 1601. & \\
\hline 6ia. & 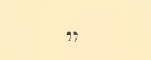 & trítici L. $\sigma^{7}$ & 1375 & $160 / 61$ & Nachtr.-Taf. II, 30 \\
\hline b. & $"$ & .. " v. éputa Hb. ㅇ & $1375 \mathrm{a}$ & $160 \mathrm{r}$. & \\
\hline c. & $"$ & $" \quad "$ v. aquilína $\mathrm{Hb} \cdot \sigma^{7}$ & $1475 \mathrm{c}$ & $160 \mathrm{r}$ & \\
\hline 7. & $"$ & vitta $\mathrm{Hb} .+$ & 1379 & 1611. & \\
\hline 8. & $"$ & distinguénda Ld. $\sigma^{\top}$ & 1378 & & \\
\hline 9 a. & $"$ & obelísea Hb. $\sigma^{T}$ & 1387 & $161 \mathrm{r}$ & \\
\hline b. & $"$ & " v. puris Hb. $\sigma^{7}$ & $1387 \mathrm{a}$ & $161 \mathrm{r}$. & \\
\hline 10. & $"$ & hastifera Donz. $\sigma^{7}$ & 1388 & $161 / 62$ & \\
\hline $11 \mathrm{a}$. & $"$ & conspieua $\mathrm{Hb} \cdot \sigma^{7}$ & 1408 & 1631. & \\
\hline b. & $"$ & $" \quad$ v. lyeárum HS. & 1403 & $163 \mathrm{r}$. & \\
\hline 12. & $"$ & saúeia $\mathrm{Hb} \cdot \sigma^{7}$ & 1402 & 1631. & Taf. 24,3 \\
\hline 13. & $"$ & ypsilon Rott. $\sigma^{7}$ & 1399 & 1621. & Nachtr.-Taf. II, 31 \\
\hline $14 \mathrm{a}$. & $"$ & trux $\mathrm{Hb} . \sigma^{7}, \mathrm{~b}$ ㅇ & 1401 & $162 / 63$ & " $\quad$ II, 32 \\
\hline $14 c$ & $"$ & $" \quad$ " v. alpína Spul. ơ, & $1401 \mathrm{a}$ & $162 / 63$ & \\
\hline $14 \mathrm{~d}$. & $"$ & $\begin{array}{l}" \quad \text { v. lunigera Steph. } \\
\text { ab. suffúsa Tutt. } q\end{array}$ & $1401 \mathrm{a}$ & $162 / 36$ & \\
\hline 15. & $"$ & ségetum Sehiff. or & 1400 & $162 \mathrm{r}$. & Taf. $24,4 \mathrm{a}, \mathrm{b}$ \\
\hline 16. & $"$ & cortícea $\mathrm{Hb} \cdot \mathrm{O}^{7}$ & 1396 & 1621. & $" 24,5$ \\
\hline 17. & $"$ & vestigiális Rott. $\sigma^{\lambda}$ & 1411 & 1641. & $" 24,7$ \\
\hline 18. & $"$ & obésa B. $\sigma^{7}$ & 1408 & $163 / 64$ & $" \quad 24,6$ \\
\hline 19. & $"$ & graslíni B. $\sigma^{7}$ & 1410 & $164 \mathrm{l}$. & \\
\hline 20. & $"$ & erassa $\mathrm{Hb} .{ }^{\circ}+$ & 1405 & $163 \mathrm{r}$ & Nachtr.-Taf. II, 33 \\
\hline 21. & $"$ & fatidica $\mathrm{Hb} ., 1 .+$, r. $\sigma^{7}$ & 1416 & $164 \mathrm{r}$. & Taf. 24,8 \\
\hline 22. & $"$ & praécox L. O & 1418 & $164 \mathrm{r}$ & $" \quad 24,9$ \\
\hline
\end{tabular}


(2x)
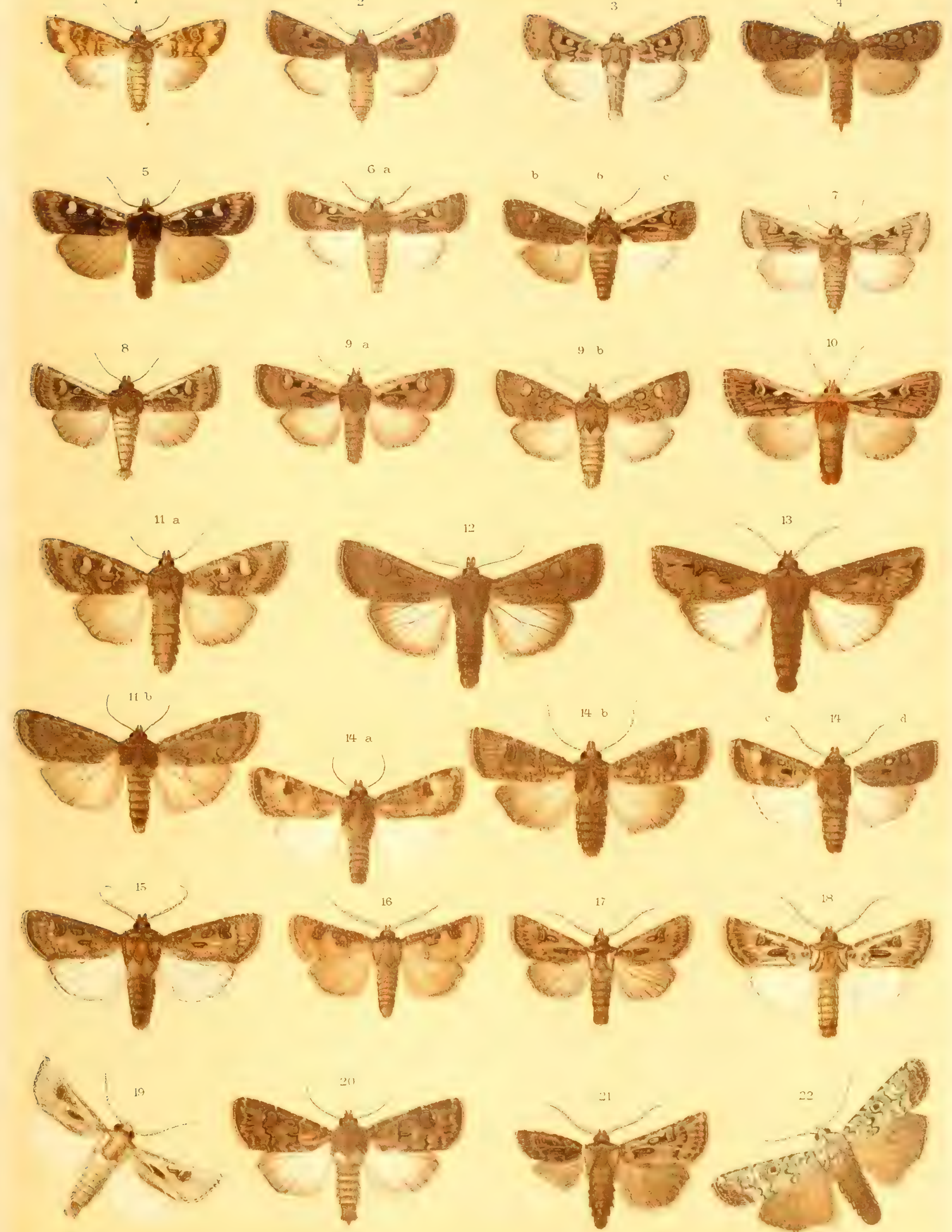

. 


\section{TAFE L 36.}

\begin{tabular}{|c|c|c|c|c|}
\hline Nr. & Name & Katalog I & Seite des I. Bandes & $\begin{array}{c}\text { Raupen-(Puppen-) } \\
\text { Abbildung }\end{array}$ \\
\hline 1. & Agrótis prásina F. $q$ & 1420 & $164 / 65$ & Taf. 24,9 \\
\hline 2. & oecúlta L. + & 1422 & 1651. & $" 24,10 \mathrm{a}-\mathrm{c}$ \\
\hline 3. & Glóttula encaústa $\mathrm{Hb}$. $q$ & 1436 & $166 \mathrm{l}$. & \\
\hline 4. & panerátii Cyr. $q$ & 1435 & 1661. & Nachtr.-Taf. III, 3 \\
\hline 5. & Charaéas gráminis L. $\sigma^{*}$ ) & 1438 & 1671. & $\left\{\begin{array}{r}\text { Ei Taf. } \\
, \quad \mathbf{2 4}, 12\end{array}\right.$ \\
\hline 6. & Epineurónia populáris F. $q$ & 1439 & $167 \mathrm{r}$. & Taf. 24,13 \\
\hline 7. & céspitis F. O & 1440 & $167 \mathrm{r}$. & $" 24,14 \mathrm{a}-\mathrm{d}$ \\
\hline 8. & Maméstra leucophaéa View. & 1441 & $168 \mathrm{l}$. & $" 24,15 \mathrm{a}, \mathrm{b}$ \\
\hline 9. & serratilinea Tr. $\sigma^{7}$ & 1444 & $168 \mathrm{r}$. & Taf. 24,16 u. 49,3 \\
\hline 10. & ádvena F. $q$ & 1446 & $168 / 69$ & Taf. $24,17 \mathrm{a}-\mathrm{c}$ \\
\hline 11. & tineta Brahm. 우 & 1449 & 1691. & $\eta 24,18$ \\
\hline 12. & dissímilis Knoeh 아 & 1467 & 1711. & Nachtr.-Taf. III, 6 \\
\hline 13. & nebulósa Hufn. $q$ & 1452 & 1691. & Taf. $24,19 \mathrm{a}, \mathrm{b}$ \\
\hline 14. & eontígua Vill. $\sigma^{7}$ & 1469 & 1721. & $" 24,20 \mathrm{a}-\mathrm{e}$ \\
\hline 15. & Hadéna alpígena B. $\left.q^{* *}\right)$ & 1492 & $190 \mathrm{r}$. & \\
\hline 16. & Maméstra thalassína Rott. $q$ & 1468 & $171 / 72$ & Taf. $24,21 \mathrm{a}, \mathrm{b}$ \\
\hline 17. & pisi L. $\sigma^{7}$ & 1471 & 1721. & $\eta 25,1 \mathrm{a}, \mathrm{b}$ \\
\hline 18. & brássicae L. $q$ & 1454 & $169 / 70$ & $n 25,2 a-d$ \\
\hline 19. & leinéri Frp $q$ & 1472 & $172 \mathrm{r}$. & Nachtr.-Taf. III, 7 \\
\hline 20. & persicáriae L. 우 & 1456 & 1701. & $\left\{\begin{array}{r}\text { Ei Taf. } 50,62 \\
, 25,3 a-c\end{array}\right.$ \\
\hline 21. & albícolon $\mathrm{Hb} . \sigma^{\top}$ & 1457 & 1701. & Nachtr.-Taf. III, 4 \\
\hline 22. & aliéna $\mathrm{Hb}$. 우 & 1465 & $171 \mathrm{l}$. & $" \quad$ III, 5 \\
\hline 23. & splendens Hb. $\sigma^{\top}$ & 1463 & $170 \mathrm{r}$. & Taf. $25,4 \mathrm{a}, \mathrm{b}$ \\
\hline 24. & olerácea L. $q$ & 1464 & $170 / 71$ & $" 25,5 \mathrm{a}-\mathrm{c}$ \\
\hline 25. & genistae Bkh. の & 1466 & $171 \mathrm{l}$ & $" 25,6$ \\
\hline
\end{tabular}



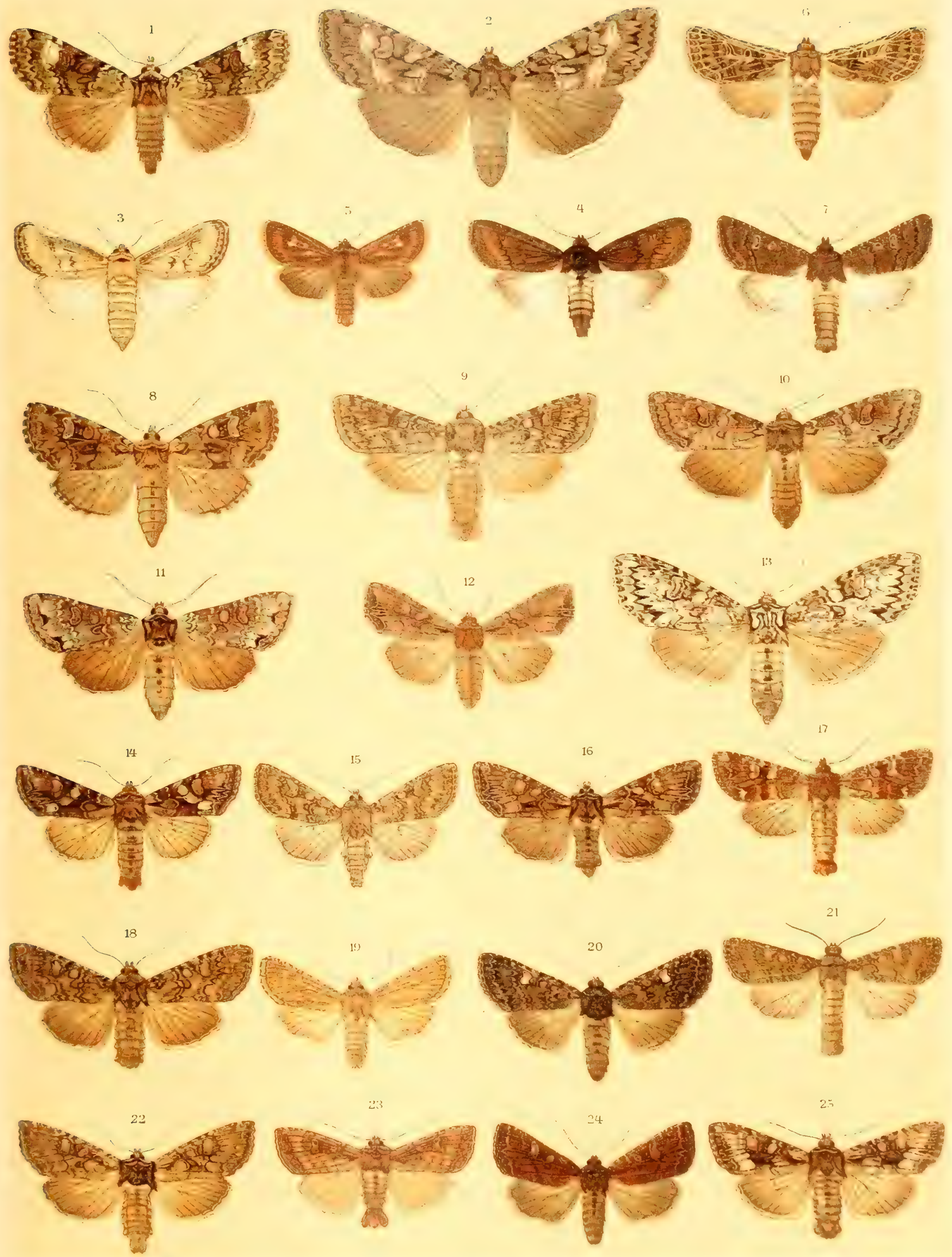




\section{TAFEL $3 \%$}

\begin{tabular}{|c|c|c|c|c|c|}
\hline Nr. & & ame & Katalog I & Seite des I. Bandes & $\begin{array}{l}\text { Raupen- (Puppen-) } \\
\text { Abbildung }\end{array}$ \\
\hline 1. & Maméstra $\mathrm{g}$ & glaúca $\mathrm{Hb}$. 우 & 1484 & $173 \mathrm{r}$. & Taf. 25,7 \\
\hline 2. & $"$ & nana Hufn. dentina Esp. & 1487 & 1741. & $" 25,8 \mathrm{a}, \mathrm{b}$ \\
\hline 3. & , & peregrína Tr. ㅇ & 1493 & $174 \mathrm{r}$. & Nachtr.-Taf. III, 9 \\
\hline 4. & " & diánthi Tausch. $\sigma^{7}$ & 1506 & 1751. & \\
\hline 5. & $"$ & praédita $\mathrm{Hb} \cdot \sigma^{7}$ & 1504 & $175 \mathrm{l}$ & \\
\hline 6. & $"$ & marmorósa Bkh. $\sigma^{7}$ & 1496 & $174 \mathrm{r}$. & Nachtr.-Taf. III, 10 \\
\hline 7. & $"$ & treitsehkéi B. $\sigma^{\top}$ & 1495 & $174 \mathrm{r}$. & \\
\hline 8. & " & trifólii Rott. $\sigma^{T}$ & 1477 & $173 \mathrm{l}$. & Taf. $25,9 \mathrm{a}-\mathrm{c}$ \\
\hline 9 & $"$ & sociábilis Grasl. $\sigma^{\pi}$ & 1481 & $173 \mathrm{l}$. & \\
\hline 10. & $"$ & sodae $\mathrm{Rbr} \sigma^{7}$ & 1478 & 1731. & \\
\hline 11. & , & eavernósa Ev. $\sigma^{7}$ & 1502 & 1751. & \\
\hline 12. & $"$ & reticuláta Vill. の & 1499 & $174 / 75$ & Taf. 25,10 \\
\hline 13. & $"$ & ehrysozóna Bkh. $\sigma^{7}$ & 1513 & $175 \mathrm{r}$. & $" 25,11 \mathrm{a}, \mathrm{b}$ \\
\hline 14. & $"$ & seréna $\mathbf{F} \cdot \sigma^{\top}$ & 1514 & $175 / 76$ & \# 25,12 \\
\hline 15. & $"$ & eappa $\mathrm{Hb} \cdot \sigma^{7}$ & 1515 & 1761. & $\Rightarrow \quad 25,13 \mathrm{a}-\mathrm{c}$ \\
\hline 16. & Dianthoécia & a luteágo $\mathrm{Hb} \cdot \sigma^{7}$ & 1527 & $176 \mathrm{r}$. & Nachtr.-Taf. III, 11 \\
\hline 17. & Maméstra $p$ & próxima Hb. $q$ & 1530 & $177 \mathrm{l}$ & \\
\hline 18. & Dianthoécia & eaésia Bkh. ㅇ & 1539 & 1771. & Taf. 49,4 \\
\hline 19. & $"$ & filigrámma Esp. ㅇ & 1542 & $177 \mathrm{r}$. & $" 49,5$ \\
\hline 20. & $"$ & tephroleúea B. 우 & 1543 & 1781. & \\
\hline 21. & $"$ & magnóli B. $\sigma^{\top}$ & 1544 & $178 \mathrm{l}$. & Nachtr.-Taf. III, 12 \\
\hline 22. & $"$ & eonspureáta Fre $\sigma^{7}$ & 1545 & 1781. & \\
\hline 23. & $"$ & conspérsa Esp. (nana Rott.d. Kat.) $q$ & 1547 & 1781. & Taf. $25,14 \mathrm{a}, \mathrm{b}$ \\
\hline 24. & $"$ & albimáeula Bkh. $\sigma^{7}$ & 1546 & 1781. & $" \quad 25,15$ \\
\hline 25. & $"$ & compta F. $q$ & 1548 & $178 \mathrm{r}$. & $" 25,16$ \\
\hline 26. & " & capsíneola $\mathrm{Hb} \cdot \sigma^{\top}$ & 1550 & $179 \mathrm{l}$. & $" 25,17 \mathrm{a}, \mathrm{b}$ \\
\hline 27. & $"$ & rivósa Ström. cucúbali Fuessl. $\sigma^{7}$ & 1552 & 1791. & $\Rightarrow \quad 25,18$ \\
\hline 28. & $"$ & earpóphaga Bkh.*) & 1553 & $179 \mathrm{r}$. & $" \quad 25,19$ \\
\hline 29. & $"$ & v. eapsóphila Dup. & $1553 a$ & $179 \mathrm{r}$. & \\
\hline 30. & $"$ & silénes $\mathrm{Hb}$. 우 & 1556 & 1801. & Nachtr.-Taf. III, 13 \\
\hline 31. & $"$ & irreguláris Hufn. 우 & 1559 & 1801. & Taf. 26,1 \\
\hline 32. & Metopócera & s cantenéri Dup. & 1724 & $196 \mathrm{l}$. & \\
\hline
\end{tabular}

*) Siehe die'bessere Abbildung Taf. 29, Fig. 8. 


\section{Dosonsol}
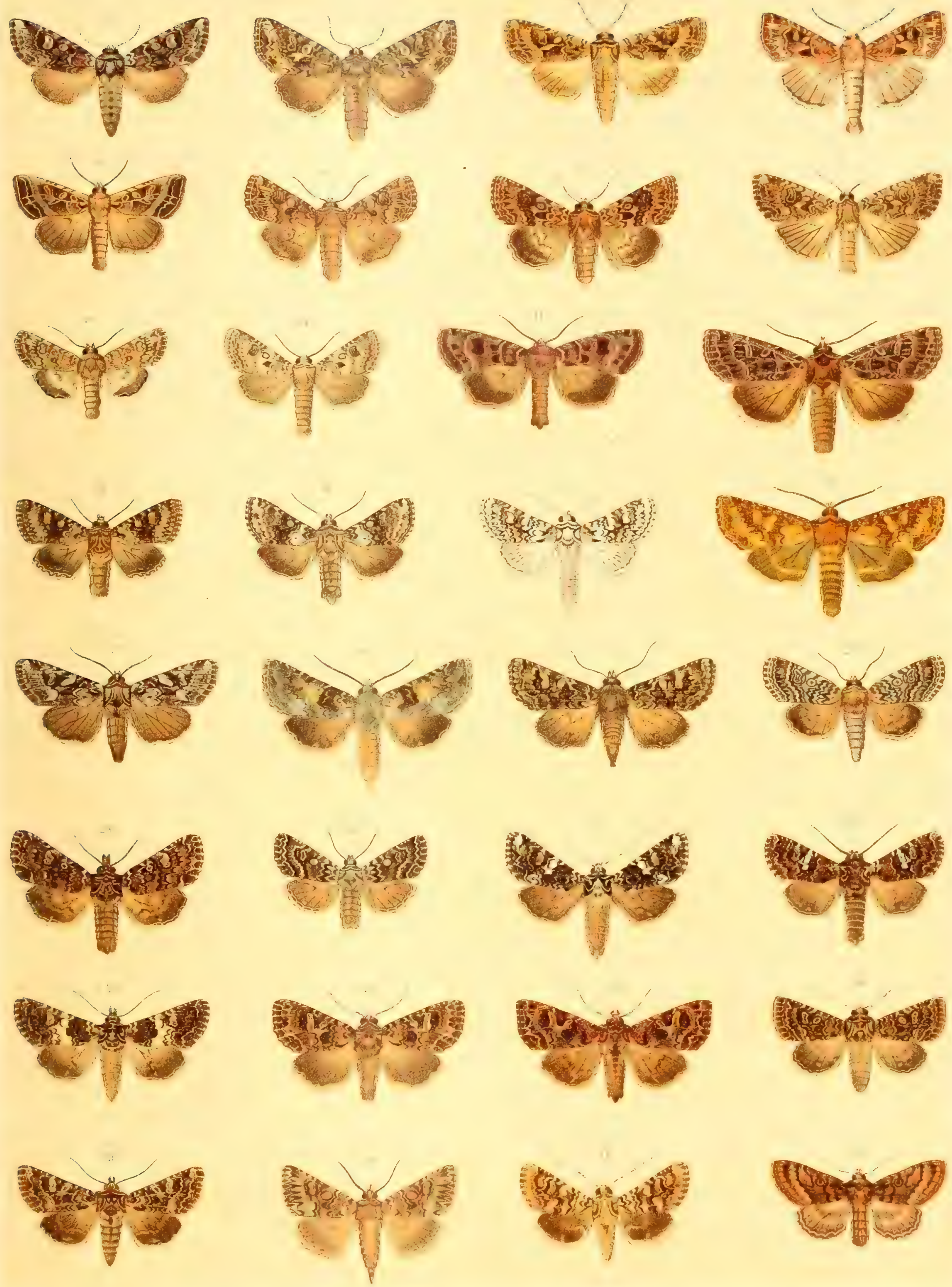



\section{TAFE L 38.}

\begin{tabular}{|c|c|c|c|c|}
\hline Nr. & Name & Katalog I & Seite des I. Bandes & $\begin{array}{c}\text { Raupen- (Puppen-) } \\
\text { Abbildung }\end{array}$ \\
\hline & Metopóceras felicína Donz. & 1725 & $196 \mathrm{r}$. & \\
\hline & Oneoenémis confúsa Frp & 1729 & $196 \mathrm{r}$. & \\
\hline 3. & Cladócera optábilis B. $\sigma^{7}$ & 1734 & 1971. & Taf. 26,2 \\
\hline 4. & baétiea $\mathrm{B}$. & 1735 & $197 \mathrm{l}$. & \\
\hline $5 \mathrm{a}$. & Episéma glaueína Esp. ఠ & 1737 & 1971. & Nachtr.-Taf. III, 26 \\
\hline $5 \mathrm{~b}$. & $"$ v. dentimácula & $1737 \mathrm{~b}$ & $197 \mathrm{r}$. & \\
\hline $6 \mathrm{a}$. & scoriácea Esp. の゙, b. 우 & 1741 & $197 / 98$ & Nachtr.-Taf. III, 27 \\
\hline & Helióphobus híspidus H.-G. $\sigma^{7}$ & 1742 & 1981. & Taf. $26,3 \mathrm{a}, \mathrm{b}$ \\
\hline & Ulochlaéna hirta Hb. $\sigma^{7}$ & 1759 & $198 \mathrm{r}$. & $" 26,4$ \\
\hline 9. & Aporophyla lutulénta Bkh. & 1761 & $198 / 99$ & $" 26,5 \mathrm{a}, \mathrm{b}$ \\
\hline 10. & $" \quad$ mioleúea Tr. $\sigma^{7}$ & 1762 & 1991. & \\
\hline 11. & nigra $\mathrm{Hw} \cdot \mathrm{O}^{7}$ & 1765 & $199 \mathrm{r}$. & Taf. 26,6 \\
\hline 12. & austrális B. $\sigma^{7}$ & 1763 & $199 \mathrm{l}$. & $" 26,7$ \\
\hline & Ammocónia caecimácula $\mathrm{F}$. + & 1767 & 2001. & $" 26,8 \mathrm{a}, \mathrm{b}$ \\
\hline & senex H.-G. vétula Dup. б & 1768 & 2001. & $" 26,9 \mathrm{a}, \mathrm{b}$ \\
\hline & Epúnda lichénea $\mathrm{Hb} \cdot \mathrm{O}^{7}$ & 1771 & $200 / 01$ & \\
\hline 16. 1 & Pólia serpentína Tr. $\sigma^{7}$ & 1774 & 2011. & Taf. 26,10 \\
\hline & $"$ polymita L. & 1775 & 2011. & $" 26,11$ \\
\hline 18. & $" \quad$ flavieíneta $F$. & 1777 & $201 \mathrm{r}$ & $" 26,12 \mathrm{a}, \mathrm{b}$ \\
\hline & " pufocíneta H.-G. & 1778 & $201 \mathrm{r}$. & $" 26,13 \mathrm{a}, \mathrm{b}$ \\
\hline 20. & " dúbia Dup. & 1782 & 2021. & $" 26,14$ \\
\hline & " xanthomista $\mathrm{Hb}$. & 1786 & 2021. & $" 26,15$ \\
\hline & $" \quad$ venústa $B \cdot \sigma^{T}$ & 1785 & 2021. & $" 26,16$ \\
\hline 23. & $"$ eanéscens Dup. $\sigma^{\pi}$ & 1787 & 202 r. & Nachtr.-Taf. III, 28 \\
\hline & $"$ suda H.-G. $\sigma^{7}$ & 1789 & 202 r. & III, 29 \\
\hline 25. & $" \quad$ chi L. + & 1797 & $202 / 3$ & Taf. 26,17 \\
\hline & Thecóphora fóvea Tr. OT & 1819 & 2061. & $" 26,18 \mathrm{a}, \mathrm{b}$ \\
\hline & Dryóbota furva Esp. $\sigma^{7}$ & 1820 & 2061. & " 26,19 \\
\hline 28. & róboris $B$. & 1821 & $206 \mathrm{r}$. & $" 26,20$ \\
\hline
\end{tabular}



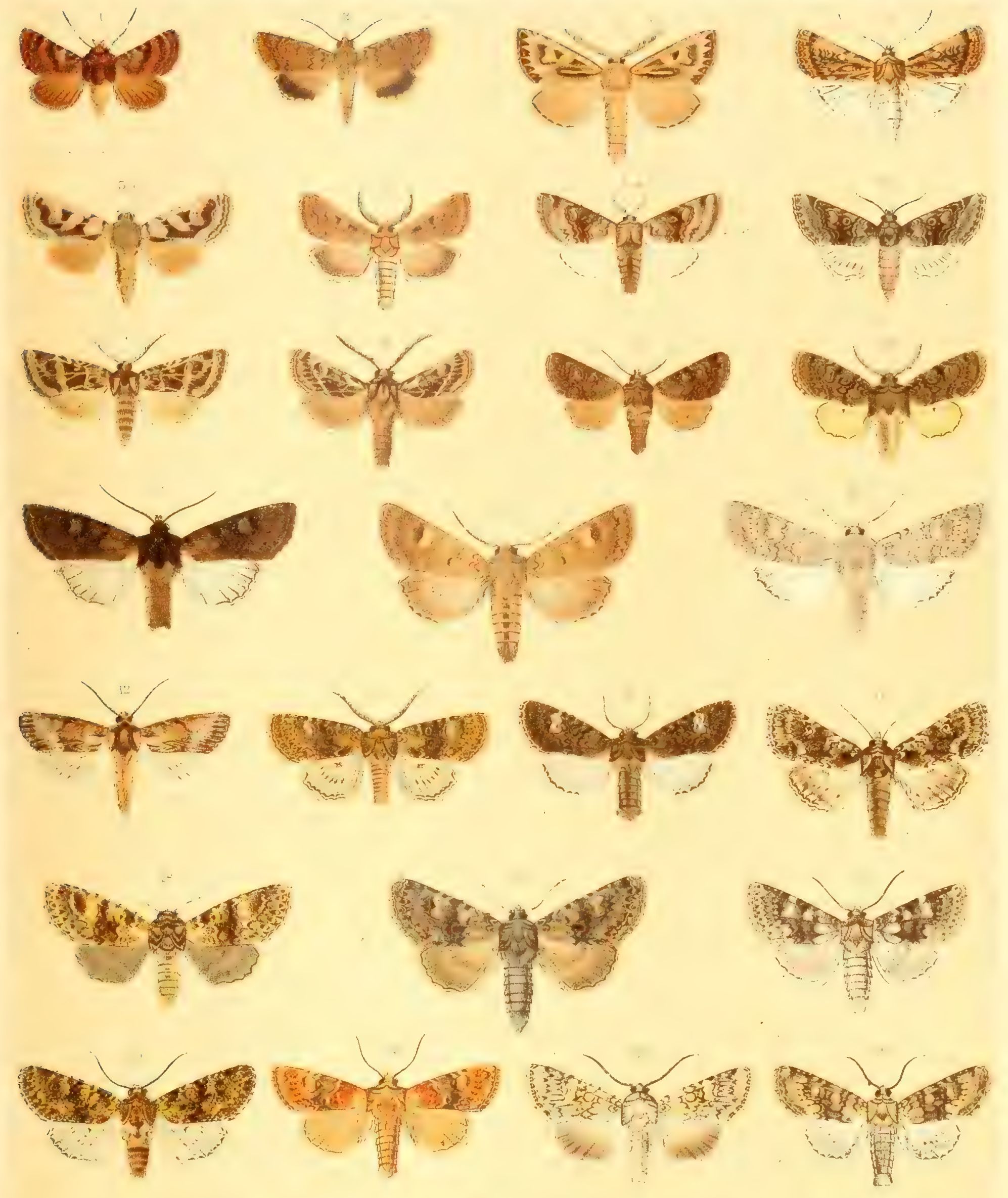

140.03
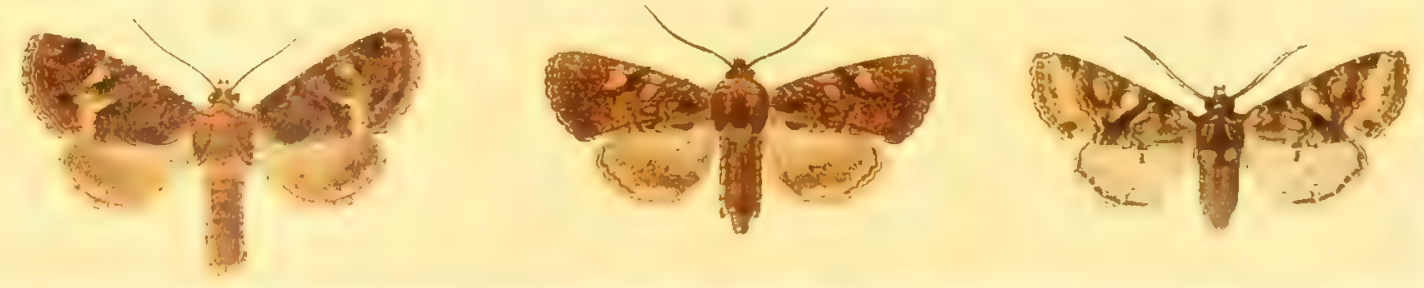



\section{TAFE L 39.}

\begin{tabular}{|c|c|c|c|c|}
\hline Nr. & Name & Katalog I & Seite des I. Bandes & $\begin{array}{c}\text { Raupen- (Puppeu-) } \\
\text { Abbildung }\end{array}$ \\
\hline 1. & Dryóbota sapórtae Dup. $\sigma^{7}$ & 1823 & $206 \mathrm{r}$. & \\
\hline 2. & monochróma Esp. $q$ & 1824 & $206 \mathrm{r}$. & Nachtr.-Taf. III, 30 \\
\hline 3. & prótea Bkh. $\sigma^{7}$ & 1825 & $206 / 7$ & Taf. $26,21 \mathrm{a}-\mathrm{c}$ \\
\hline 4. & Diehónia convérgens F. & 1818 & $205 \mathrm{r}$. & " 26,22 \\
\hline 5 & aerugínea $\mathrm{Hb}$. & 1817 & 2051. & $" 26,23$ \\
\hline 6. & $" \quad$ aprilina L. $q$ & 1816 & 2051. & $\left\{\begin{aligned} \text { Ei Taf. } 50,63 \\
, 26,24 \mathrm{a}, \mathrm{b}\end{aligned}\right.$ \\
\hline 7. & Chariptera vipidána Waleh. & 1815 & $204 / 5$ & Taf. $27,1 \mathrm{a}, \mathrm{b}$ \\
\hline 8. & Misélia bímaculósa L. $\sigma^{7}$ & 1810 & 2041. & $" 27,2$ \\
\hline 9. & " oyacánthae L. $q$ & 1813 & $204 \mathrm{r}$. & $\left\{\begin{array}{c}\text { Ei Taf. } 50,64 \\
" 27,3\end{array}\right.$ \\
\hline 10. & Valéria iaspidea Vill. $\sigma^{7}$ & 1611 & $185 \mathrm{r}$. & Taf. $27,4 \mathrm{a}, \mathrm{b}$ \\
\hline 11. & $" \quad$ oleagina F. $\sigma^{7}$ & 1614 & $185 \mathrm{r}$ & $\because 27, \tilde{a}$ \\
\hline 12. & Oxytrypia orbiculósa Esp. $\sigma^{7}$ & 1615 & 1861. & \\
\hline 13. & Apaméa testácea Hb. $\sigma^{7}$ & 1618 & $186 \mathrm{l}$. & Taf. 27,6 \\
\hline 14. & $" \quad$ niekérli Frp. $q$ & 1619 & $186 \mathrm{r}$. & \\
\hline 15. & $" \quad$ dumeríli Dup. $q$ & 1620 & $186 \mathrm{r}$. & \\
\hline 16. & Celaéna hawórthi Curt. $\sigma^{\top}$ & 1621 & $186 \mathrm{r}$. & Taf. 49,6 \\
\hline 17. & Thalpóphila amathúsia Rbr 우 & 1622 (bis) & $.187 \mathrm{l}$ & \\
\hline 18. & matúra Hufn. ㅇ & 1623 & $187 \mathrm{l}$. & Taf. $27,7 \mathrm{a}, \mathrm{b}$ \\
\hline 19. & Luperína rubélla Dup. $\sigma^{7}$ & 1624 & $187 \mathrm{r}$. & \\
\hline 20. & Lucéria virens L. $\sigma^{7}$ & 1927 & & \\
\hline 21. & Hadéna ferrágo Ev. $\sigma^{T}$ & 1695 & 1931. & \\
\hline 22. & Luperina zollikoféri Frr & 1625 & $187 / 88$ & \\
\hline 23. & dumetórum H.-G. & 1626 & 1881. & \\
\hline 24. & Pseudohadéna chenopodíphaga Rbr $q$ & 1649 & $188 / 89$ & Taf. 27,8 \\
\hline 25. & Hadéna amíca Tr. 우 & 1655 & 1891. & \\
\hline 26. & porphyrea Esp. $\sigma^{\pi}$ & 1661 & $189 \mathrm{r}$. & Taf. 27,9 \\
\hline 27. & " funérea Hein. $\sigma^{7}$ & 1663 & $189 / 90$ & \\
\hline
\end{tabular}



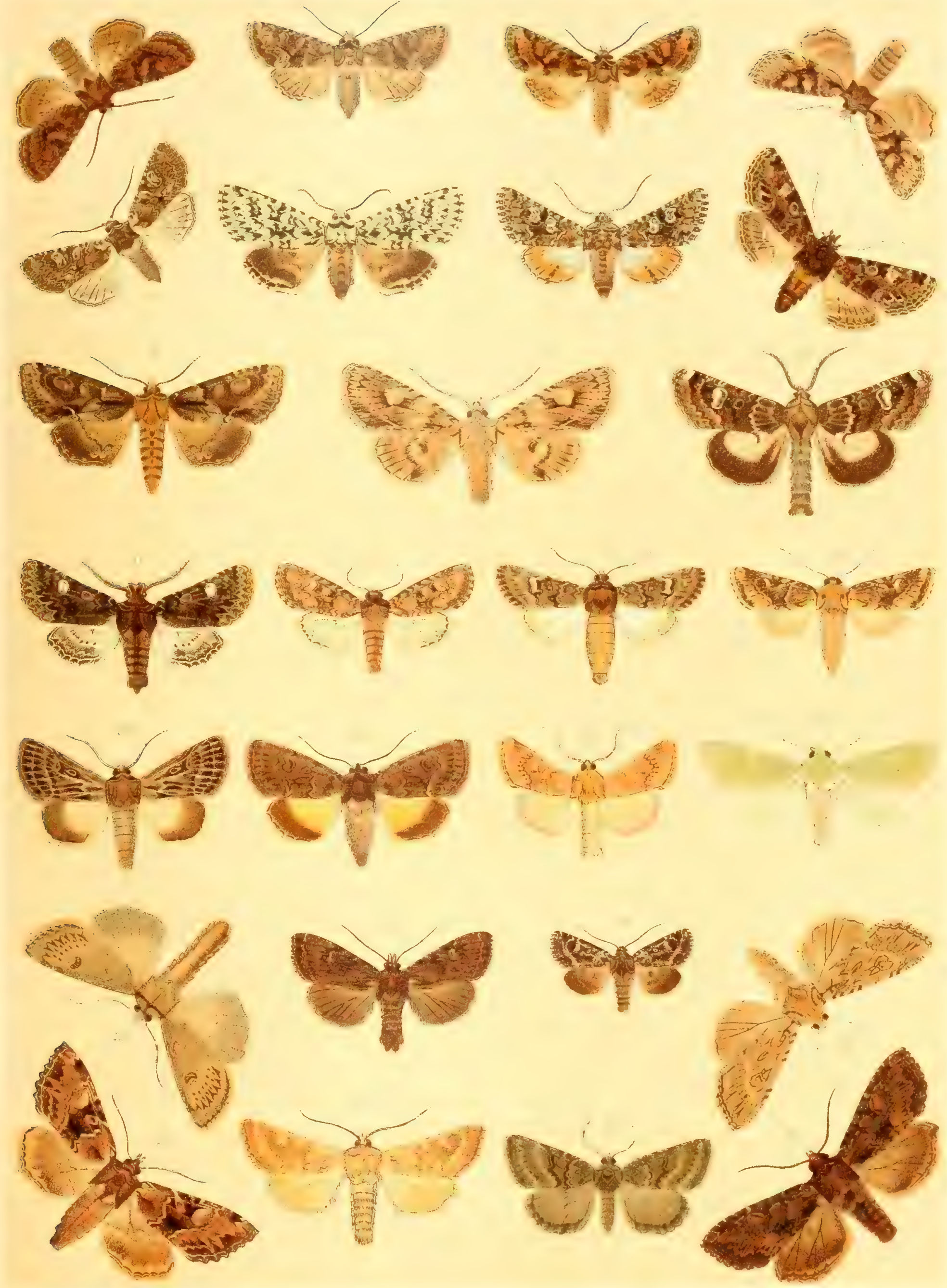



\section{TAFEL 40.}

\begin{tabular}{|c|c|c|c|c|c|}
\hline Nr. & & Name & Katalog I & Seite des I. Bandes & $\begin{array}{c}\text { Raupen-(Puppen-) } \\
\text { Abbildung }\end{array}$ \\
\hline 1. & Hadéna & adústa Esp. $\sigma^{7}$ & 1665 & $190 \mathrm{l}$. & Taf. 27,10 \\
\hline 2. & $"$ & somméri Lef. $q$ & 1666 & $190 \mathrm{r}$. & Nachtr.-Taf. III, 22 \\
\hline 3. & $"$ & soliéri B. O & 1664 & 1901. & Taf. 27,11 \\
\hline 4. & $"$ & ochroleúca Esp. + ? & 1670 & $190 \mathrm{r}$. & $" 27,12$ \\
\hline 5. & $"$ & platínea Tr. & 1673 & 1911. & \\
\hline 6. & $"$ & zeta $\left.\operatorname{Tr} \cdot 0^{7 *}\right)$ & 1674 & $191 \mathrm{l}$ & \\
\hline 7. & , & maillárdi H.-G. $\sigma^{\top}$ & 1677 & 1911. & \\
\hline 8. & $"$ & dífflua $\mathrm{Hb} \cdot \mathrm{O}^{7}$ & $1677 \mathrm{~b}$ & $191 r$ & \\
\hline 9. & $"$ & adústa v. sylvática Bell. $\sigma^{7}$ & $1665 \mathrm{c}$ & 1901. & \\
\hline 10. & $"$ & gémmea Tr. જ & 1682 & 1921. & Nachtr.-Taf. III, 23 \\
\hline 11. & $"$ & pubripéna Tr. $\sigma^{7}$ & 1686 & $192 \mathrm{r}$. & \\
\hline 12. & $"$ & furva $\mathrm{Hb} . \mathrm{O}^{7}$ & 1678 & $191 / 92$ & Taf. 27,13 \\
\hline 13. & $"$ & abiécta Hb. ㅇ & 1693 & 1931. & \\
\hline 14. & $"$ & laterítia Hufn. $\sigma^{7}$ & 1694 & 1931. & Taf. 27, 14 \\
\hline 15. & $"$ & monóglypha Hufn. $\sigma^{7}$ & 1690 & $192 / 93$ & $" 27,15$ \\
\hline 16. & $"$ & lithoxylea F. $\sigma^{7}$ & 1700 & $193 \mathrm{r}$. & Nachtr.-Taf. III, 24 \\
\hline 17. & $"$ & sublústris Esp. & 1701 & $193 \mathrm{r}$. & \\
\hline 18. & $"$ & sórdida Bkh. $\sigma^{7}$ & 1679 & 1921. & \\
\hline 19. & $"$ & leúcodon Ev. ఠ & 1680 & 1921. & \\
\hline 20. & $"$ & basilínea $\mathbf{F}$. & 1710 & $194 \mathrm{r}$. & Taf. 27, 16 \\
\hline $21 \mathrm{a}$. & $"$ & rúrea $\mathbf{F} \cdot \sigma^{7}$ & 1706 & $193 / 94$ & $" 27,17$ \\
\hline $21 b$. & $"$ & $" \quad "$ ab. alopecúrus Esp. $\sigma^{7}$ & $1706 a$ & 1941. & \\
\hline 22. & $"$ & seolopácina Esp. $0^{7}$ & 1709 & $194 \mathrm{r}$. & $" 27,18$ \\
\hline 23. & $"$ & hepática $\mathrm{Hb} \cdot \sigma^{\pi}$ & 1707 & 1941. & $" 27,19$ \\
\hline 24. & $"$ & gémina $\mathrm{Hb}$. ab. remíssa Tr. & $1712 \mathrm{a}$ & $194 / 95$ & $" 27,20$ \\
\hline
\end{tabular}

*) Siehe die bessere Abbildung Taf. 29, Fig. 17. 

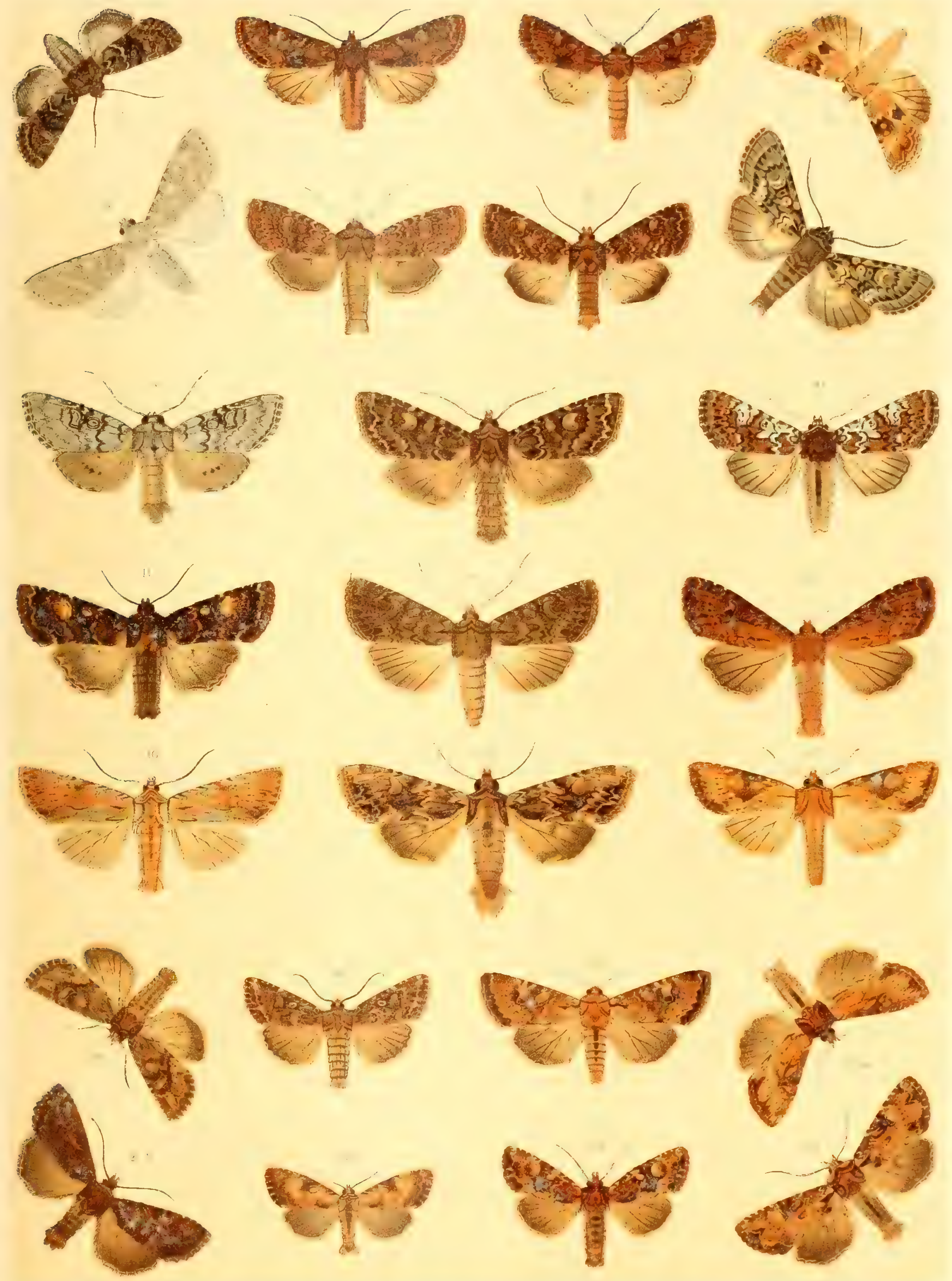




\section{TAFE L 41.}

\begin{tabular}{|c|c|c|c|}
\hline Name & Katalog I & Seite des I. Bandes & $\begin{array}{l}\text { Raupen-(Puppen-) } \\
\text { Abbildung }\end{array}$ \\
\hline 1. Hadéna unánimis Tr. + & 1713 & $195 \mathrm{l}$. & Taf. 27, 21 \\
\hline illýpiea Frr & 1714 & $195 \mathrm{r}$ & \\
\hline $\begin{array}{r}\text { seeális L. (dídyma Esp.) 우 U Über- } \\
\text { gang zu ab. secalina Hb. }\end{array}$ & 1715 & $195 / 96$ & . $27,22 \mathrm{a}, \mathrm{b}$ \\
\hline$" \quad a b$. leueostigma Esp. $\sigma^{7}$ & $1715 b$ & $195 \mathrm{r}$. & \\
\hline pabulatríeula Brahm. & 1717 & 1961. & $\Rightarrow 27,23$ \\
\hline 5. Miána ophiográmma Esp. 우 & 1561 & $180 \mathrm{r}$. & Nachtr.-Taf. III, 15 \\
\hline literósa $\mathrm{Hw}$. & 1566 & $180 / 81$ & Taf. $49,9 \mathrm{a}, \mathrm{b}$ \\
\hline strigilis $\mathrm{Cl}$. ㅇ & 1567 & 1811. & $\because 49,8 \mathrm{a}, \mathrm{b}$ \\
\hline$" \quad$ "ab. aéthiops Hw. $\sigma^{\pi}$ & $1567 \mathrm{~b}$ & 1811. & \\
\hline faseiúneula Hw. $\sigma^{\pi}$ & 1568 & $181 \mathrm{r}$. & $" 49,11$ \\
\hline " bicolória Vill. & 1569 & $181 / 82$ & $" 49,10$ \\
\hline 10. Thalerástria bipartíta HS. & 2452 & 2921. & \\
\hline 11. Dipterýgia seabriủseula L. $\sigma^{7}$ & 1827 & 2071. & $" 27,24$ \\
\hline 12. Hyppa rectilínea Esp. $\sigma^{7}$ & 1828 & $207 \mathrm{r}$. & $" 28,1 \mathrm{a}-\mathrm{c}$ \\
\hline 13. Rhizográmma detérsa Esp. О & 1829 & 2081. & $" 49,12$ \\
\hline 14. Chloántha hypérici F. 우 & 1843 & $208 \mathrm{r}$. & $" 28,2 \mathrm{a}, \mathrm{b}$ \\
\hline$" \quad$ polyodon $\mathrm{Cl} \cdot \sigma^{7}$ & 1840 & $208 \mathrm{r}$. & $" 28,3$ \\
\hline radiósa Esp. 우 & 1839 & 2081. & $" 28,4$ \\
\hline 17. Callopístria purpureofaseiáta Piller $q$ & 1846 & 2091. & $" 28,5 \mathrm{a}, \mathrm{b}$ \\
\hline latreillei Dup. $q$ & 1848 & $209 \mathrm{l}$. & $" 28,6 \mathrm{a}, \mathrm{b}$ \\
\hline 19. Polyphaénis sericáta Esp. $\sigma^{7}$ & 1850 & $209 \mathrm{r}$. & $" 28,7 a-c$ \\
\hline xanthoehlópis B. 우 & 1852 & $209 / 10$ & \\
\hline 21. Traehéa atríplieis L. $\sigma^{7}$ & 1854 & $210 \mathrm{l}$. & $" 28,8 \mathrm{a}, \mathrm{b}$ \\
\hline 22. Prodénia littorális $B$. & 1857 & $210 \mathrm{r}$. & \\
\hline 23. Trigonóphora flámmea Esp. & 1858 & $210 \mathrm{r}$. & \\
\hline iódea Gn. OT & 1859 & 2111. & \\
\hline 25. Eupléxia lucípara L. $\sigma^{7}$ & 1861 & 2111. & $" 28,9 \mathrm{a}-\mathrm{c}$ \\
\hline 26. Phlogóphora seita $\mathrm{Hb}$. & 1865 & $211 \mathrm{r}$. & $" 28,10$ \\
\hline 27. Brotolómia metieulósa L. $\sigma^{7}$ & 1867 & $211 / 12$ & $\Rightarrow 28,11 \mathrm{a}-\mathrm{c}$ \\
\hline 28. Mánia maúra L. & 1870 & 2121. & "28, $12 \mathrm{a}, \mathrm{b}$ \\
\hline 29. Naénia & 1871 & $212 / 13$ & $\begin{array}{r}\text { Ei Taf. } 50,66 \\
28,13\end{array}$ \\
\hline
\end{tabular}




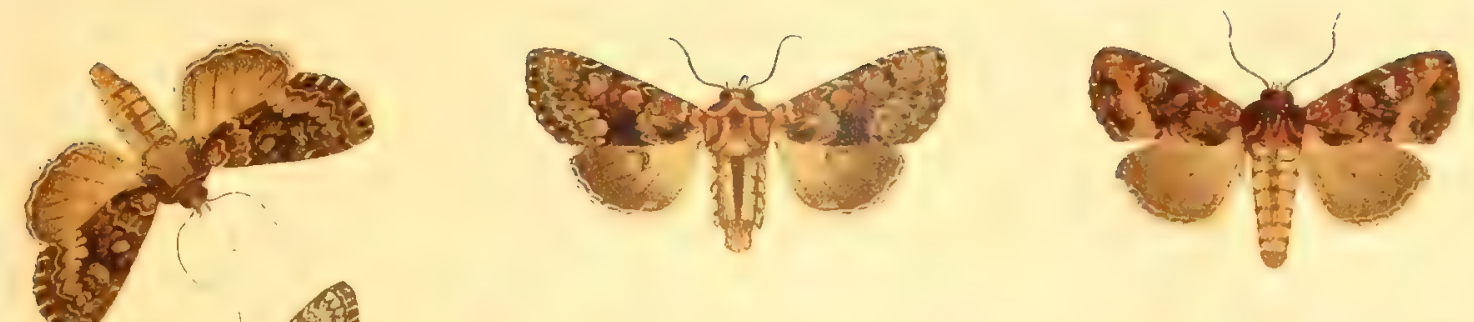

20

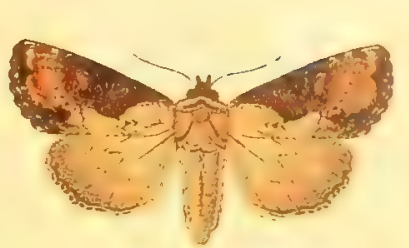

nors

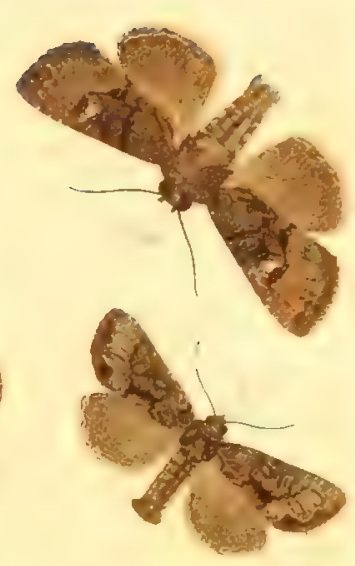

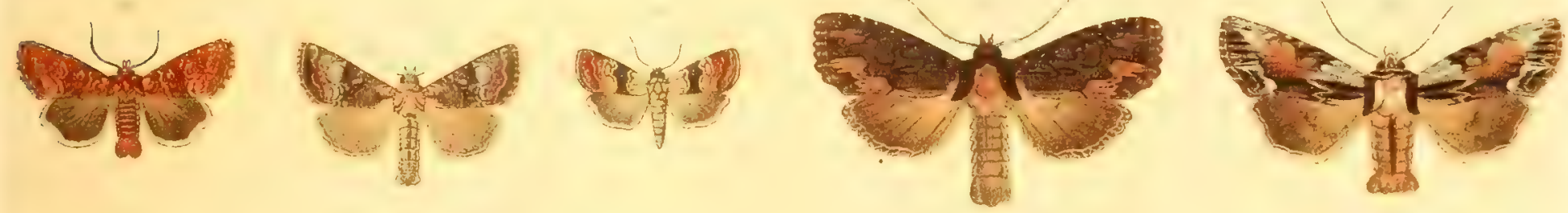
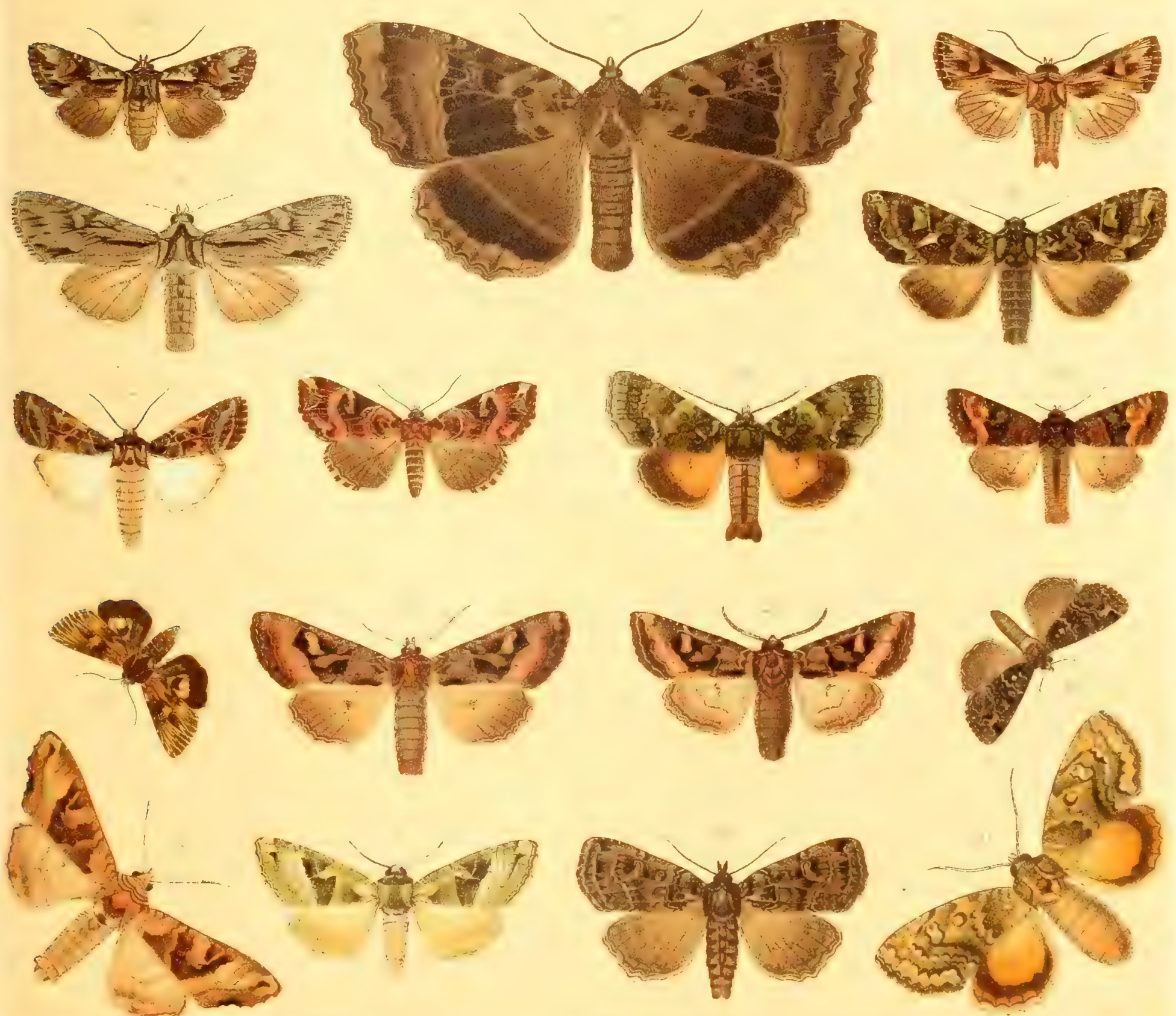



\section{TAFE L 42.}

\begin{tabular}{|c|c|c|c|c|}
\hline Nr. & Name & Katalog I & Seite des I. Bandes & $\begin{array}{l}\text { Raupen- (Puppen-) } \\
\text { Abbildung }\end{array}$ \\
\hline 1. & Iaspidea eélsia L. $\sigma^{\top}$ & 1874 & 2131. & Taf. 28,14 \\
\hline 2. & Helótropha leucostigma Ld. $\sigma^{7}$ & 1876 & $213 \mathrm{r}$ & " 49, 13 \\
\hline $3 a$. & Hydroéeia lueens Frp $q$ & $1877 b$ & 2141. & \\
\hline $3 \mathrm{~b}$. & níctitans $\mathrm{Bkh}$. 우 & 1877 & 214 1. Taf. 49 &, $14 a-c$ u. N.-T. III, 31 \\
\hline 4. & micácea Esp. $q$ & 1879 & $214 \mathrm{r}$. & Taf. 28,15 \\
\hline 5. & petásitis Dbld. & 1880 & 2151. & $" 28,16 \mathrm{a}, \mathrm{b}$ \\
\hline 6. & Gortýna oehrácea Hb. v. xanthénes Germ. & 1881 & $215 \mathrm{r}$. & Nachtr.-Taf. III, 34 \\
\hline 7. & Hydroécia moesiaca HS. $q$ & 1882 & 2151. & \\
\hline 8. & leueógrapha Bkh. 운 & 1883 & 2151. & III, 33 \\
\hline 9. & Gortýna oehrácea Hb. & 1887 & $215 \mathrm{r}$ & $\left\{\begin{array}{r}\text { Ei Taf. 50, } 67 \\
, 28,17\end{array}\right.$ \\
\hline 10. & Nonágria nexa $\mathrm{Hb}$. & 1891 & 2161. & Nachtr.-Taf. V, 4 \\
\hline 11. & cannae $0.0^{7}$ & 1892 & $216 \mathrm{l}$. & $\mathrm{V}, 2$ \\
\hline 12. & spargánii Esp. $\sigma^{7}$ & 1893 & $216 \mathrm{r}$. & Taf. 28,18 \\
\hline 13. & typhae Thunb. $\sigma^{T}$ & 1894 & $216 / 17$ & $" 28,19 \mathrm{a}, \mathrm{b}$ \\
\hline 14. & geminipúneta Hateh. $\sigma^{7}$ & 1895 & 2171. & $" 29,1$ \\
\hline 15. & neúrica $\mathrm{Hb}$. & 1896 & 2171. & Nachtr.-Taf. V, 3 \\
\hline 16. & dissolủta Tr. $\sigma^{7}$ & 1897 & $217 \mathrm{r}$. & \\
\hline 17. & Coenóbia rufa Hw. $\sigma^{\nearrow}$ & 1902 & 2181. & \\
\hline 18. & Senta maritima Tauseh. $\sigma^{7}$ & 1906 & $218 \mathrm{r}$. & Taf. 29,2 \\
\hline 19. & Myctéroplus puniceágo B. & 2332 & 2831. & $, \quad 29,3$ \\
\hline 20. & Tapinóstola museulósa $\mathrm{Hb} \cdot \sigma^{7}$ & 1913 & $219 / 20$ & \\
\hline 21. & fulva $\mathrm{Hb}$ & 1923 & $220 \mathrm{r}$ & $. .92,4$ \\
\hline 22. & hellmánni Ev. Oフ & 1922 & $220 \mathrm{r}$ & Nachtr.-Taf. V, 1 \\
\hline 23. & extréma Hb. & 1921 & 2201. & \\
\hline 24. & bóndi Knaggs. の & 1920 & 2201. & \\
\hline 25. & élymi Tr. $\sigma^{7}$ & 1915 & 2201. & Taf. 29,5 \\
\hline 26. & Sesámia nonagrioídes Lef. $\sigma^{7}$ & 1925 & 2211. & Nachtr.-Taf. V, 5 \\
\hline 27. & $" \quad$ erética Ld. 우 & 1926 & $221 \mathrm{r}$. & $\mathrm{V}, 6$ \\
\hline 28. & Calámia lutósa $\mathrm{Hb} \cdot \mathrm{O}^{7}$ & 1928 & 2221. & $\mathrm{~V}, 8$ \\
\hline 29. & phragmitidis $\mathrm{Hb}$. & 1929 & 2221. & Taf. 49,15 \\
\hline 30. & Argyrospíla succinea Esp. & 1910 & $219 \mathrm{r}$. & \\
\hline 31. & Meliána flámmea Curt. $\sigma^{7}$ & 1909 & 2191. & $" 49,16 \mathrm{a}, \mathrm{b}$ \\
\hline & Leueánia ímpudens Hb. $\sigma^{7}$ & 1932 & $222 / 23$ & $. \quad 29,6 \mathrm{a}-\mathrm{c}$ \\
\hline
\end{tabular}



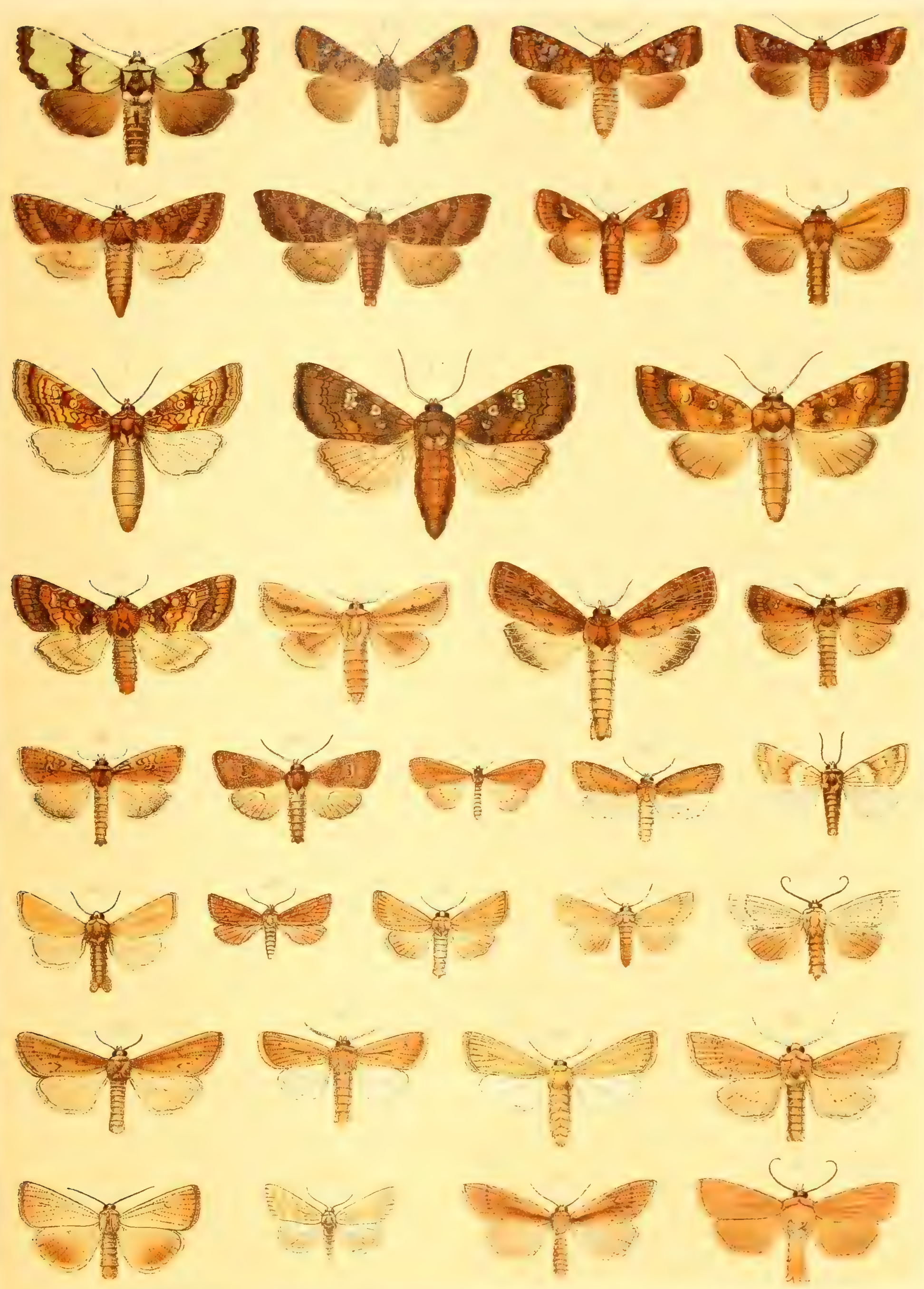


\section{TAFEL 43.}

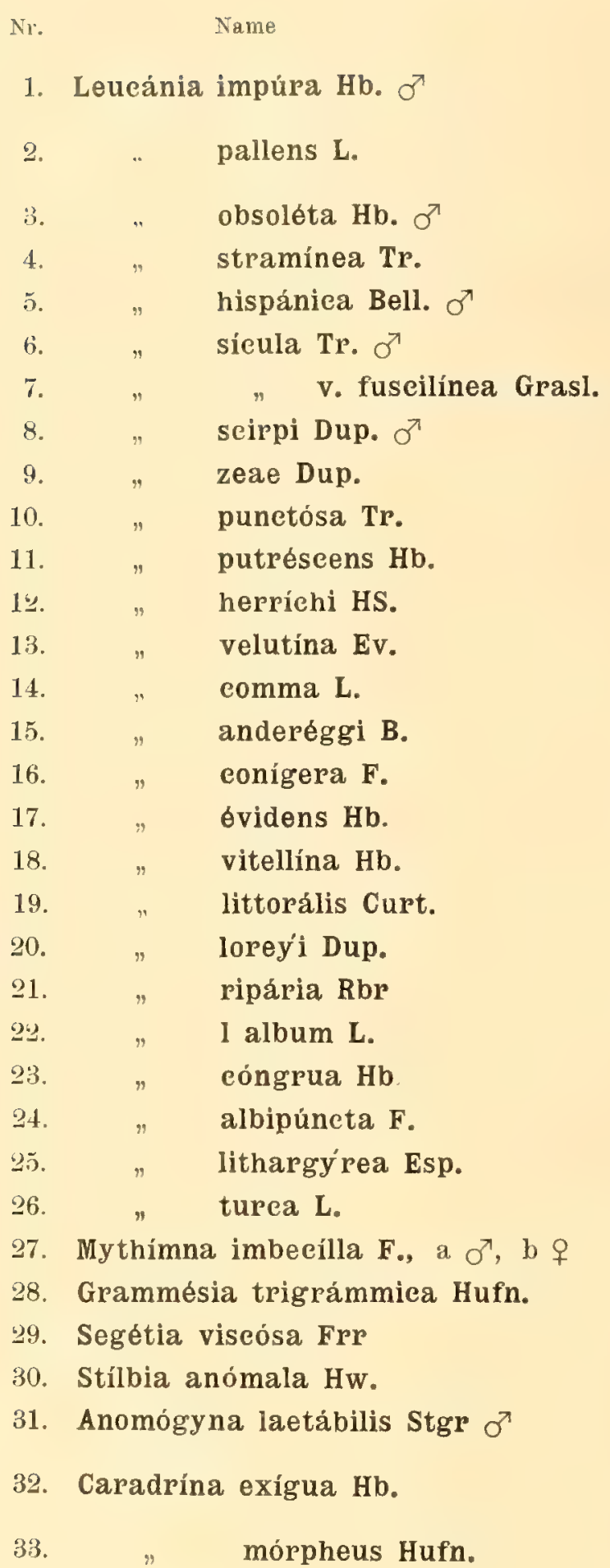

\begin{tabular}{|c|c|c|}
\hline Katalog I & Seite des I. Bandes & $\begin{array}{c}\text { Raupen- (Puppen-) } \\
\text { Abbildung }\end{array}$ \\
\hline 1933 & 2231. & Taf. 29,7 \\
\hline 1935 & 2231. & $\left\{\begin{array}{c}\text { Ei Taf. } 50,68 \text { a, b } \\
n 29,8\end{array}\right.$ \\
\hline 1936 & 2241. & Taf. $29,9 \mathrm{a} \mathrm{b}$ \\
\hline 1938 & 2241. & $" 29,10$ \\
\hline 1939 & 2241. & \\
\hline 1940 & $224 \mathrm{r}$. & \\
\hline $1940 \mathrm{a}$ & 224 r. & \\
\hline 1942 & $224 r$. & \\
\hline 1945 & $224 / 25$ & Nachtr.-Taf. V, 9 \\
\hline 1946 & $225 \mathrm{l}$. & Taf. 29,11 \\
\hline 1947 & 2251. & $" \quad 29,12$ \\
\hline 1949 & $255 \mathrm{r}$. & \\
\hline 1950 & $225 \mathrm{r}$ & \\
\hline 1951 & $225 \mathrm{r}$. & $\Rightarrow \quad 29,13$ \\
\hline 1952 & $225 \mathrm{r}$. & \\
\hline 1964 & $227 \mathrm{l}$. & $" 29,14 \mathrm{a}, \mathrm{b}$ \\
\hline 1963 & 2271. & Nachtr.-Taf. V, 13 \\
\hline 1961 & $226 \mathrm{r}$. & Taf. 29,16 \\
\hline 1958 & 226 r. & Nachtr.-Taf. V, 12 \\
\hline 1957 & 2261. & $\mathrm{~V}, 11$ \\
\hline 1955 & 2261. & \\
\hline 1954 & 2261. & $\mathrm{~V}, 10$ \\
\hline 1956 & 2261. & Taf. 29,16 \\
\hline 1966 & $227 \mathrm{l}$. & $" 29,17$ \\
\hline 1967 & 227 r. & $" \quad 29,18$ \\
\hline 1969 & $227 / 28$ & $" \quad 29,19$ \\
\hline 1977 & 2281. & $\Rightarrow \quad 29,20$ \\
\hline 1986 & $229 \mathrm{r}$. & $" \quad 29,21 \mathrm{a}, \mathrm{b}$ \\
\hline 1634 & $188 \mathrm{l}$. & Nachtr.-Taf. III, 20 \\
\hline 1981 & $228 / 29$ & Taf. $29,22 \mathrm{a}, \mathrm{b}$ \\
\hline 1985 & 2291. & \\
\hline 1990 & 2801. & $\left\{\begin{array}{c}\text { Ei Taf. } 50,68 \mathrm{c} \\
, 29,23\end{array}\right.$ \\
\hline 2016 & $232 / 33$ & Taf. 29,24 \\
\hline
\end{tabular}



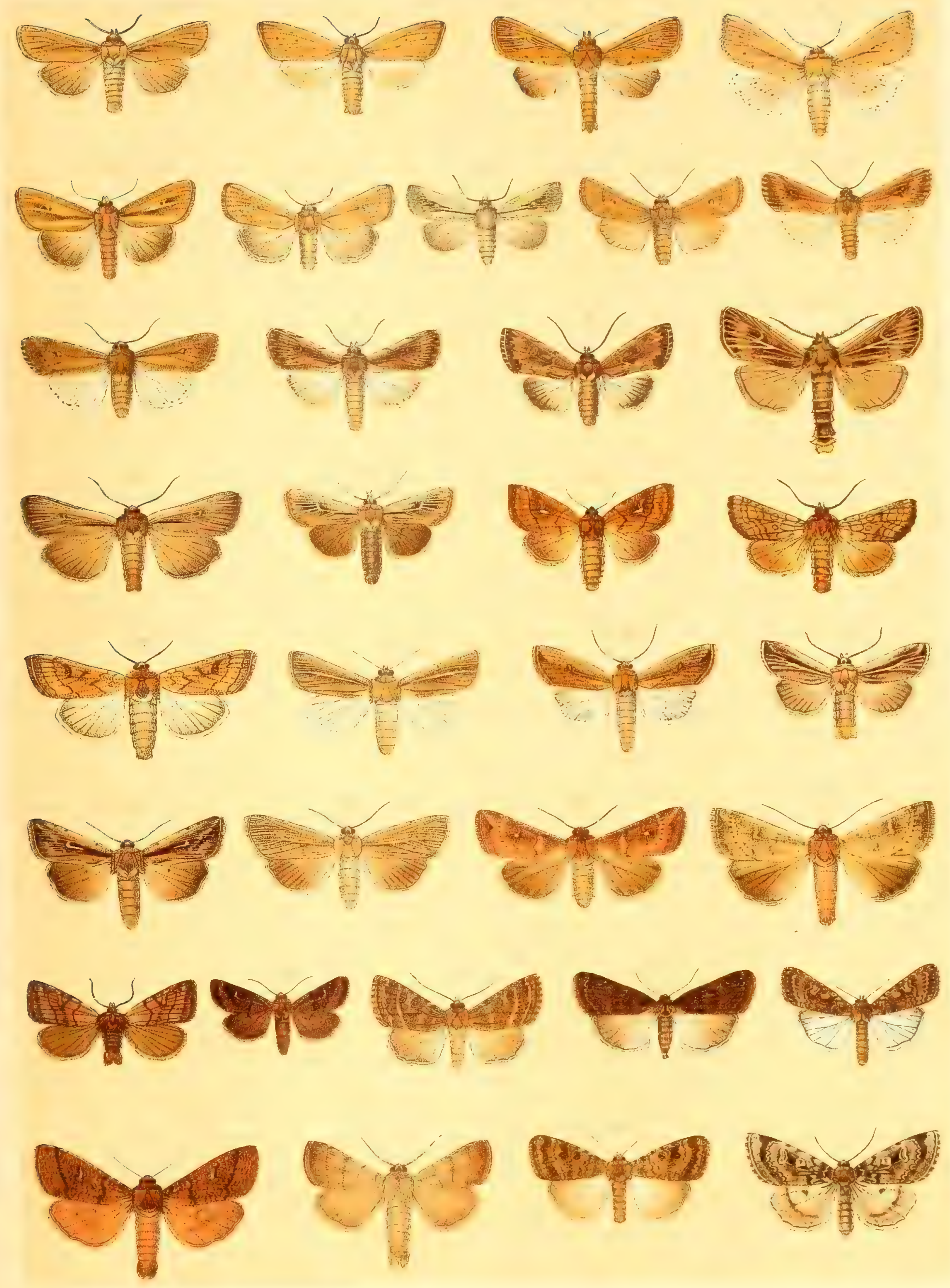



\section{TAFE L 44.}

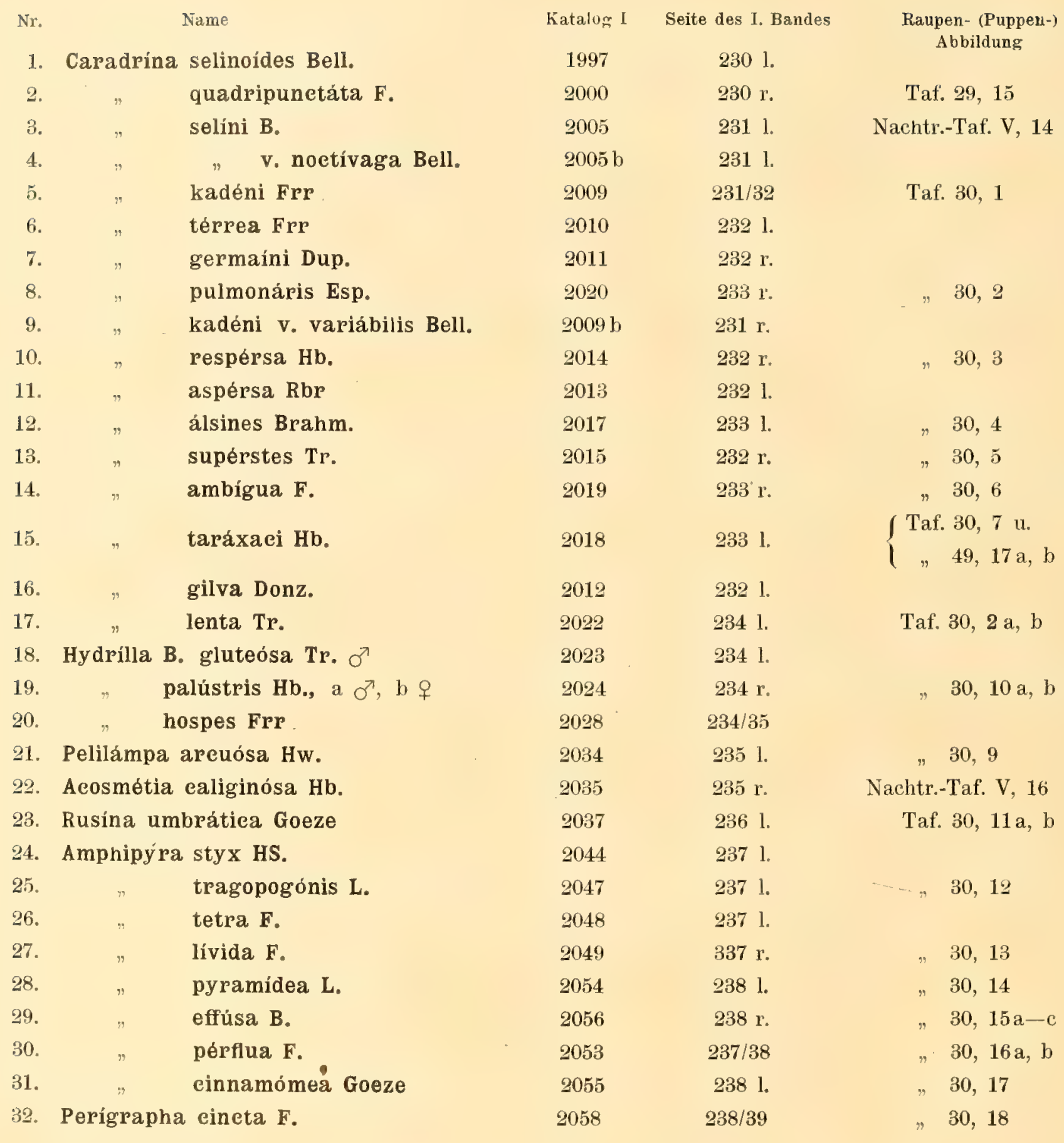



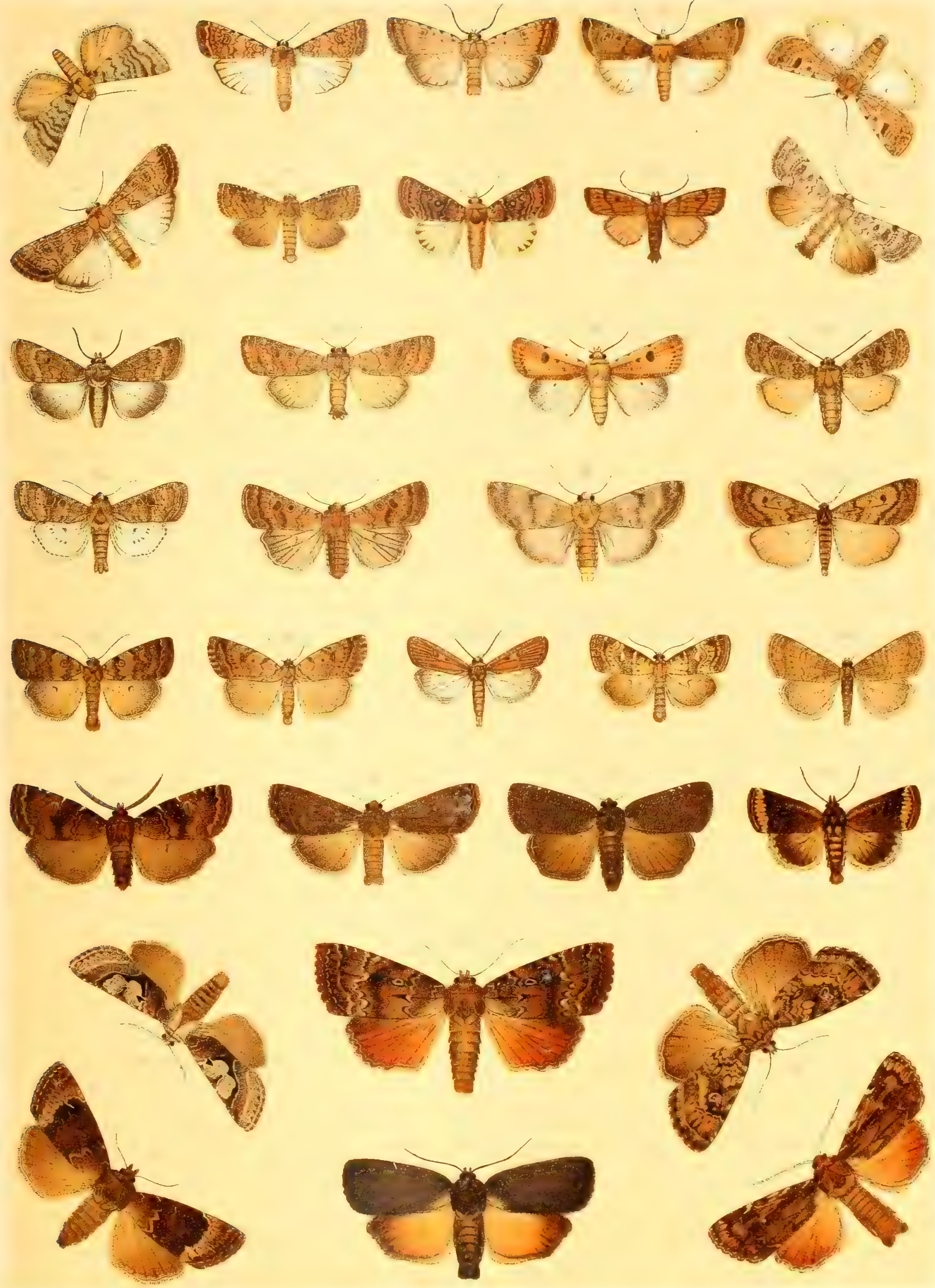



\section{TAFEL 45.}

\begin{tabular}{|c|c|c|c|c|}
\hline $\mathrm{Nr}$. & Name & Katalog I & Seite des I. Bandes & $\begin{array}{c}\text { Raupen-(Puppen-) } \\
\text { Abbildung }\end{array}$ \\
\hline 1. & Taeniocámpa góthica L. & 2062 & $239 \mathrm{l}$. & Taf. 30,19 \\
\hline 2. & rórida HS. శ & 2069 & $240 \mathrm{r}$ & \\
\hline 3. & miniósa $\mathrm{F}$. & 2065 & $239 / 40$ & $\because \quad 30,20$ \\
\hline 4. & pulverulénta Esp. & 2066 & 2401. & $\Rightarrow 30,21$ \\
\hline 5. & pópuli Stroem. populéti $T r$. & 2067 & 2401. & Nachtr.-Taf. V, 17 \\
\hline 6. & stábilis View. & 2068 & $240 \mathrm{r}$ & Taf. 30,22 \\
\hline 7. & gráeilis F. & 2072 & $241 \mathrm{r}$. & $" 30,23$ \\
\hline $8 \mathrm{a}$. & incérta Hufn. ab. fuscáta $\mathrm{Hw}$. & 2070 & 2411. & \\
\hline 81 . & incérta Hufn. & 2070 & $240 / 41$ & $" 30,24$ \\
\hline 9. & opíma Hb. & 2071 & 2411. & Nachtr.-Taf. V, 18 \\
\hline 10. & munda Esp. & 2073 & $241 / 42$ & Taf. 30,25 \\
\hline 11. & Pánolis griseovariegáta Goeze $q$ & 2074 & 2421. & $" 31,1 \mathrm{a}, \mathrm{b}$ \\
\hline 12. & Sora leucógrapha $\mathrm{Hb}$. & 1424 & $165 / 66$ & Nachtr.-Taf. III, 2 \\
\hline 13. & " facéta Tr. & 1425 & 1661. & \\
\hline 14. & $"$ rubrieósa F. & 1423 & $165 \mathrm{r}$. & Taf. 31,2 a b \\
\hline 15. & Ágotis tecta $\mathrm{Hb}$. & 1166 & 1471. & \\
\hline 16. & Mesogóna oxalína Hb. $\sigma^{7}$ & 2077 & $242 \mathrm{r}$ & $" 31,3 \mathrm{a}, \mathrm{b}$ \\
\hline 17. & acetoséllae F. $\sigma^{7}$ & 2078 & 2431. & $\Rightarrow 31,4$ \\
\hline 18. & Hiptélia ochreágo Hb. & 2079 & 2431. & Nachtr.-Taf. V, 19 \\
\hline 19. & lorézi Stgr. & 2081 & 2431. & \\
\hline 20. & miniágo Frr & 2082 & 2431. & \\
\hline 21. & Díeyla $00 \mathrm{~L}$. & 2085 & $243 r$ & Taf. 31,5 \\
\hline 22. & Calýmnia pyralína View. & 2087 & 2441. & $\Rightarrow 31,6 \mathrm{a}, \mathrm{b}$ \\
\hline 23. & diffinis $L$. & 2089 & 2441. & $" 31,7 \mathrm{a}, \mathrm{b}$ \\
\hline 24. & affínis L. & 2088 & 2441. & $" 31,8$ \\
\hline 25. & trapezina L. & 2098 & $244 \mathrm{r}$ & $\Rightarrow \quad 31,9$ \\
\hline 26. & Cósmia paleácea Esp. & 2099 & 2451. & Nachtr.-Taf. V, 21 \\
\hline 27. & ablúta Hb. & 2101 & 2451. & $V, 20$ \\
\hline 28. & contúsa Frr & 2108 & $245 \mathrm{r}^{*}$ & Taf. $31,10 a, b$ \\
\hline 29. & Dysehorísta suspéeta Hb. $q$ & 2109 & $245 / 46$ & Nachtr.-Taf. V, 22 \\
\hline 30. & fissipúneta Hw. $\sigma^{7}$ & 2111 & 2461. & Taf. 31,11 \\
\hline 31. & Plâstenis retúsa L. $\sigma^{7}$ & 2114 & $246 \mathrm{r}$ & $" \quad 31,12$ \\
\hline
\end{tabular}



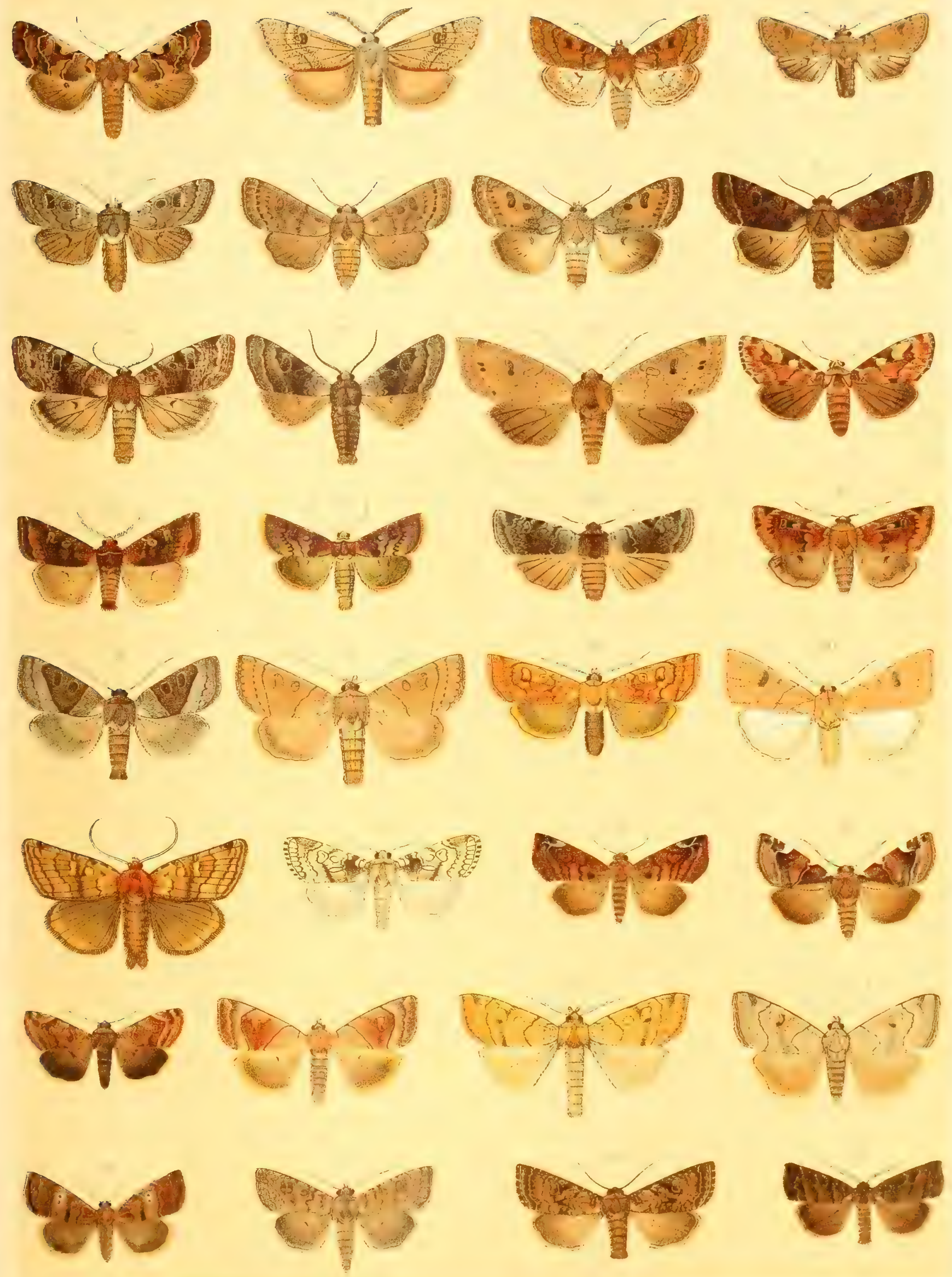




\section{TAFEL 46.}

\begin{tabular}{|c|c|c|c|c|c|}
\hline Nr. & & Name & Katalog I & Seite des I. Bandes & $\begin{array}{l}\text { Raupen-(Fuppen-) } \\
\text { Abbildung }\end{array}$ \\
\hline 1. PI & lástenis & is subtúsa $\mathbf{F} \cdot \sigma^{x}$ & 2115 & $246 r$ & Taf. 31,13 \\
\hline 2. $\mathrm{Ci}$ & iprhoéd & dia ambústa $F$, 우 & 2116 & $246 / 47$ & $" 31,14$ \\
\hline 3. & $"$ & xerampélina $\mathrm{Hb}$. & 2117 & 2471. & 1731,15 \\
\hline 4. & $"$ & úlieis Stgr or & 2103 & 2471. & \\
\hline 5. $\mathrm{Bo}$ & ombýcis & ia viminális $\mathrm{F} \cdot \mathrm{O}^{7}$ & 1560 & $180 \mathrm{r}$. & Nachtr.-Taf. III, 14 \\
\hline 6. $\mathrm{Ar}$ & nehoeél & olis lunósa Hw. & 2118 & $247 \mathrm{r}$. & V, 23 \\
\hline 7. $0 \mathrm{r}$ & rthósia & a rutieílla Esp. & 2120 & $248 \mathrm{r}$. & \\
\hline 8. & " & lota Cl. ab. pufa Tutt & 2122 & $248 / 49$ & Taf. 31,16 \\
\hline 9. & $"$ & macilénta $\mathrm{Hb}$. & 2123 & 2491. & $" 31,17 \mathrm{a}$ \\
\hline 10. & $"$ & eircelláris Hufn. & 2124 & 2491. & $" 31,18$ \\
\hline 11. & $"$ & hélvola L. & 2125 & 249 r. & $\Rightarrow 31,19 \mathrm{a}-\mathrm{c}$ \\
\hline $12 \mathrm{a}$. & $"$ & pistácina $\mathrm{F}$. ab. lýchnidis $\mathrm{Hb}$. & 2127 & 2501. & $" 31,20$ \\
\hline $12 \mathrm{~b}$. & $"$ & $" \quad \quad$ ab. canária Esp. & $2127 \mathrm{a}$ & 2501. & \\
\hline 13. & $n$ & haematídea Dup. & 2129 & 2501. & \\
\hline 14. & $"$ & nítida $\mathrm{F}$. & 2130 & $250 \mathrm{r}$. & $" 31,21$ \\
\hline 15. & $"$ & húmilis $\mathrm{F}$. & 2132 & $250 \mathrm{r}$. & $" \quad 31,22$ \\
\hline 16. & $"$ & laévis $\mathrm{Hb}$. & 2133 & $250 / 51$ & \\
\hline 17. & $"$ & kindermánni F. R. & 2136 & 2511. & \\
\hline 18. & $"$ & litúra L. & 2138 & 2511. & $" 32,1 \mathrm{a}-\mathrm{c}$ \\
\hline 19. $\mathrm{X}$ & ánthia & citrágo L. & 2143 & $251 / 52$ & $" 32,2$ \\
\hline 20. & $"$ & sulfurágo $\mathrm{F}_{\text {. }}$ & 2144 & 2521. & $" 32,3$ \\
\hline 21. link & ks $n$ & aurágo F. & 2145 & 2521. & Nachtr.-Taf. V, 24 \\
\hline 21. $r$. & $"$ & ". Übergang zu ab. fucáta Esp. & 2145 a & 2521. & \\
\hline 22. & $"$ & lútea Ström. & 2146 & $252 \mathrm{r}$. & Taf. 49,18 \\
\hline $23 \mathrm{a}$. & $"$ & fulvágo L. & 2148 & $252 / 53$ & $" 32,4$ \\
\hline $23 \mathrm{~b}$. & $"$ & $" \quad$ "ab. flavéscens Esp. & $2148 \mathrm{a}$ & $252 \mathrm{r}$. & \\
\hline 24. & $"$ & gilvágo Esp. & 2151 & 2531. & Nachtr.-Taf. V, 25 \\
\hline 25. & $"$ & ocelláris Bkh. & 2152 & 2531. & $\mathrm{~V}, 26$ \\
\hline 26. $\mathrm{Op}$ & porína & eroceágo F. & 2155 & $253 / 54$ & Taf. 32,5 \\
\hline 27. $0 \mathrm{r}$ & rrhódia & a fragáriae Esp. & 2156 & 2541. & $" 32,6 \mathrm{a}, \mathrm{b}$ \\
\hline $28 a$. & $"$ & erythrocéphala $\mathrm{F}$. & 2157 & $254 \mathrm{r}$. & $" 32,7$ \\
\hline $28 \mathrm{~b}$. & $"$ & $" \quad$ ab. glabra $\mathrm{Hb}$ & $2157 \mathrm{a}$ & $254 \mathrm{r}$. & \\
\hline 29. & $"$ & veronícae $\mathrm{Hb}$. & 2158 & $254: 55$ & Nachtr.-Taf. V, 27 \\
\hline 30. & $"$ & vau punetátum Esp. & 2159 & 2551. & $\mathrm{~V}, 28$ \\
\hline 31. & $"$ & daúbei Dup. & 2161 & 2551. & $\mathrm{~V}, 29$ \\
\hline $32 \mathrm{a}$. & $"$ & vaceínii L. & 2164 & $255 / 56$ & Taf. 32,9 \\
\hline $32 \mathrm{~b}$. & $"$ & $" \quad$ " v. spadícea $\mathrm{Hb}$. & $2164 \mathrm{a}$ & 2561. & \\
\hline 33. & $n$ & lígula Esp. & 2165 & $256 / 57$ & $" \quad 32,10$ \\
\hline
\end{tabular}




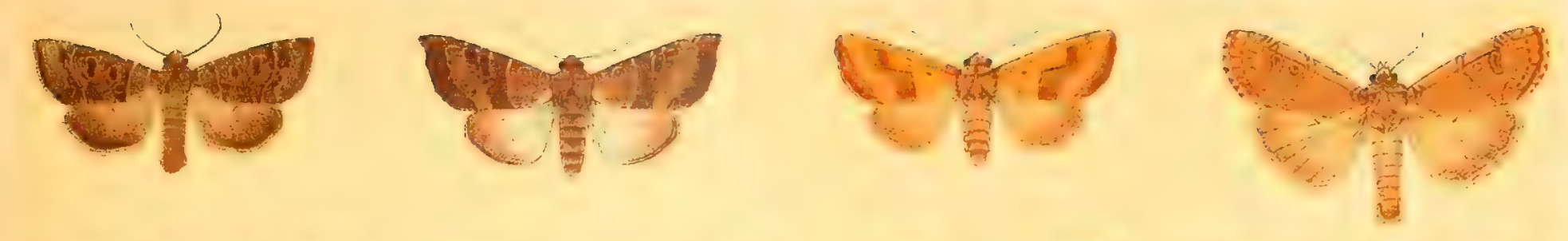

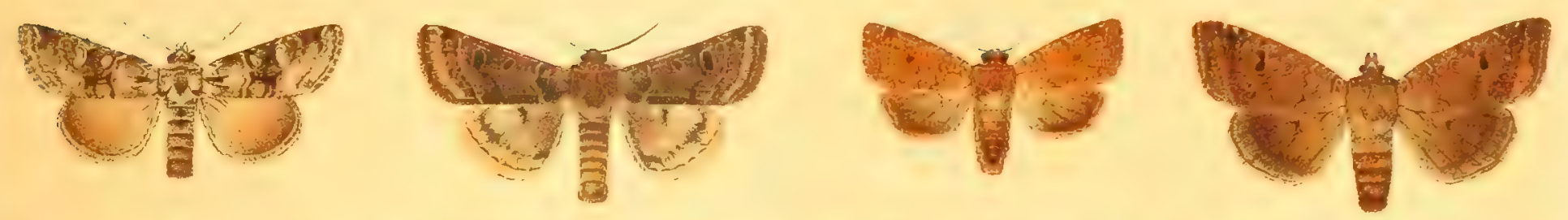
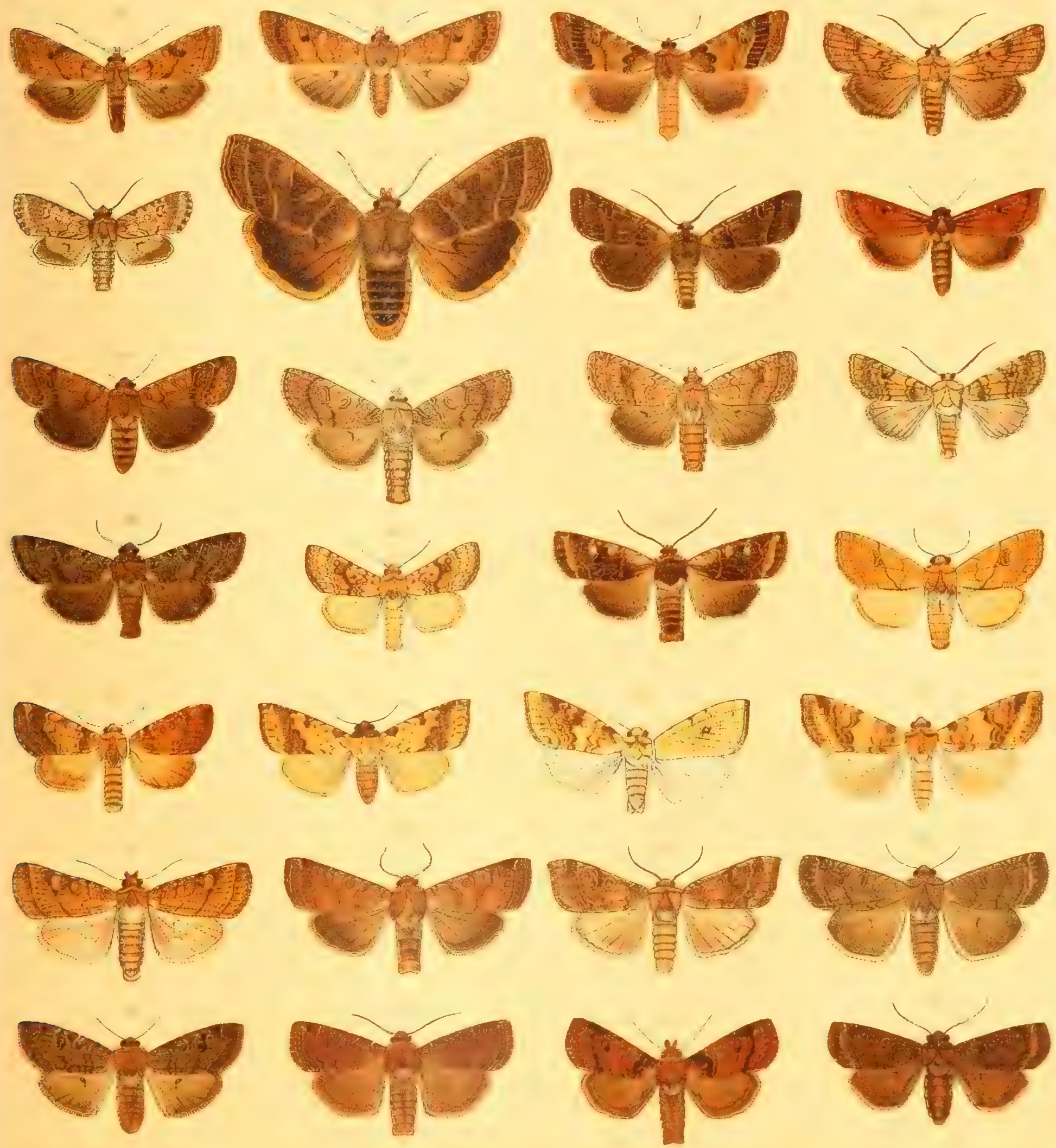



\section{TAFEL $4 \%$.}

\begin{tabular}{|c|c|c|c|c|c|}
\hline $\mathrm{N}_{\mathrm{r}}$ & Name & & Katalog I & Seite des I. Bandes & $\begin{array}{c}\text { Raupen- (Puppen-) } \\
\text { Abbildung }\end{array}$ \\
\hline 1. & Orrhódia rubigínea F. & & 2167 & 2571. & Taf. $32,11 \mathrm{a}-\mathrm{c}$ \\
\hline 2. & lígula Esp. ab. staudingéri & Grasl. & $2165 \mathrm{c}$ & 2571 & \\
\hline 3. & Scopelosóma satellítium L. & & 2169 & 2581. & $" 32,12$ \\
\hline 4. & Scoliópteryx libátrix L. & $\cdot$ & 2502 & 297 r. & $. \quad 32,13$ \\
\hline 5. & Xýlina semmibrúnnea Hw. & & 2170 & $258 \mathrm{r}$ & $" 32,14 \mathrm{a}, \mathrm{b}$ \\
\hline 6. & sóeia Rott. & & 2172 & $258 / 59$ & Nachtr.-Taf. V, 31 \\
\hline 7. & .. furcífera Hufn. & & 2173 & 2591. & Taf. $32,15 \mathrm{a}, \mathrm{b}$ \\
\hline 8. & , ingriea HS. & & 2174 & $259 \mathrm{r}$. & \\
\hline 9 a. & " lámbda $\mathrm{F}$. & & 2176 & $260 \mathrm{l}$. & $" 32,16$ \\
\hline 97$).$ & .. $\quad$ v. zinckéni Tr. & & $2176 \mathrm{~b}$ & $260 \mathrm{l}$. & \\
\hline 10. & .. orníthopus Rott. & & 2177 & 2601. & , 32,17 \\
\hline 11. & .. lapídea $\mathrm{Hb}$. & & 2178 & $260 \mathrm{r}$. & $. \quad 32,18$ \\
\hline 12. & " méreki Rbr & & 2179 & 2611 & " 32,19 \\
\hline 13. & Calocámpa vetústa $\mathrm{Hb}$. & & 2180 & 2611. & $" 32,20$ \\
\hline 14. & exoléta L. & & 2181 & $261 / 62$ & $" \quad 32,21 \mathrm{a}-\mathrm{c}$ \\
\hline 15. & solidáginis $\mathrm{Hb}$. & & 2182 & 2621. & $" \quad 32,22 a, b$ \\
\hline 16. & Xyl. conspieilláris L. ab. intermédia & Tutt & 2183 & 262 r. & Nachtr.-Taf. V, 32 \\
\hline 17. & Seotochrósta pulla $\mathrm{Hb}$. ㅇ & & 2184 & 2621. & $\mathrm{~V}, 33$ \\
\hline 18. & Brachyónyeha nubeculósa Fsp. $\sigma^{7}$ & & 1808 & 203 r. & Taf. 32,23 \\
\hline 19. & sphinx Hufn. $\sigma^{7}$ & & 1809 & $203 / 4$ & . $\quad 32,24$ \\
\hline 20. & Dasypólia templi Thunb. & & 1802 & 2031. & $" 33,1$ \\
\hline 21. & Xylocámpa aréola Esp. 우 & & 2186 & $263 \mathrm{r}$ & $. .33,2$ \\
\hline 22. & Lithoeámpa ramósa Esp. О & & 2187 & $263 / 64$ & $. \quad 33,3 a-c$ \\
\hline 23. & Epiméeia ủstula Frr $\sigma^{7}$ & & 2191 & 2641. & $" \quad 33,4 \mathrm{a}, \mathrm{b}$ \\
\hline 24. & Calophásia easta Bkh. & & 2194 & $264 / 65$ & $" 33,5 \mathrm{a}, \mathrm{b}$ \\
\hline 25. & platýptera Esp. & & 2195 & 2651. & Nachtr.-Taf. V, 34 \\
\hline 26. & almoravída Grasl. & & 2197 & 2651. & \\
\hline 27. & freyéri Friv. & & 2200 & $265 \mathrm{r}$. & \\
\hline 28. & lúnula Hufn. & & 2199 & $265 \mathrm{r}$ & Taf. $33,6 \mathrm{a}, \mathrm{b}$ \\
\hline 29. & Cleóphana antirrhíni $\mathrm{Hb}$. & & 2201 & 2661. & $" 33,7$ \\
\hline
\end{tabular}



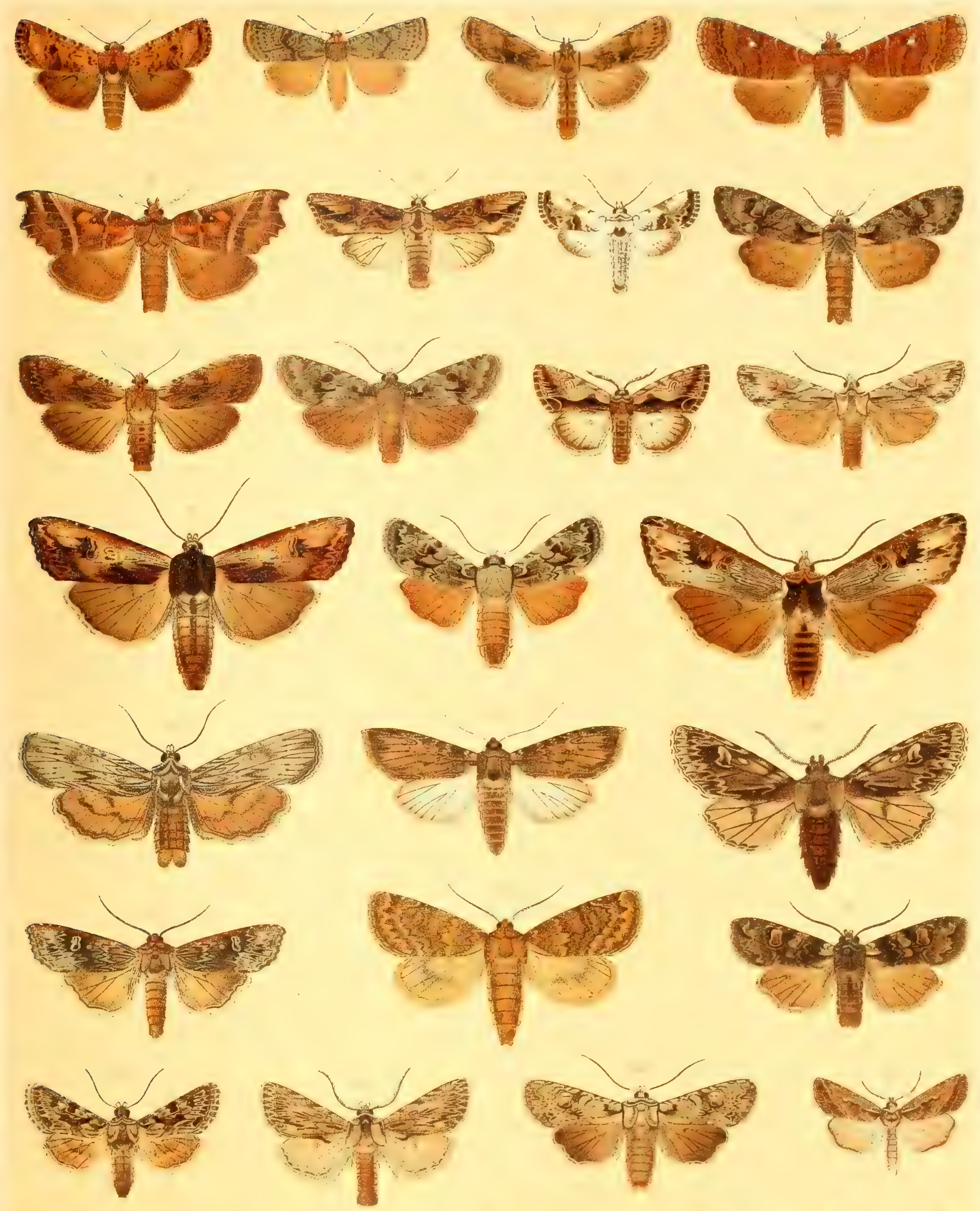

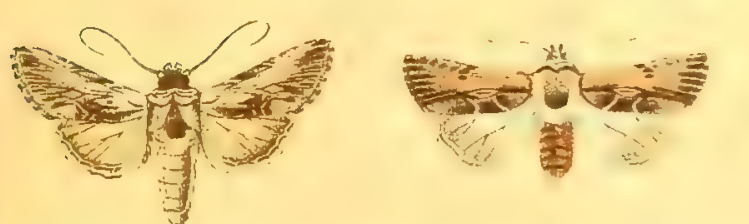
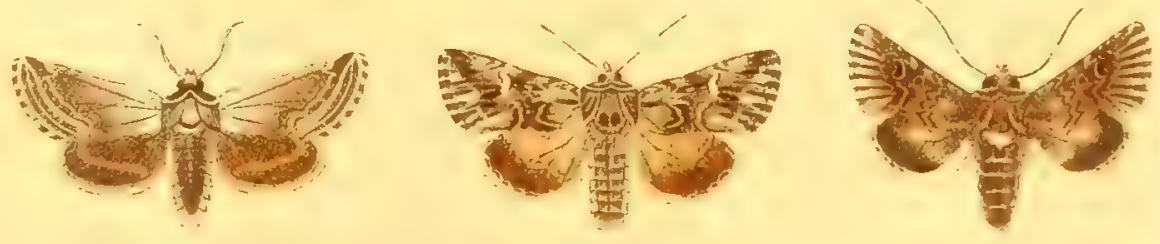




\section{TAFEL 48.}

\begin{tabular}{|c|c|c|}
\hline ir. & & Name \\
\hline 1. & Cleóphana & na serráta Tr. \\
\hline 2. &. & olivina HS. \\
\hline 3. & $"$ & anarrhíni Dup. \\
\hline 4. &. & déjeáni Dup. \\
\hline 5. & $\because$ & yváni Dup. \\
\hline 6. & Cueúllia $\mathrm{r}$ & prenánthis B. \\
\hline 7. & . & verbásci L. \\
\hline 8. & $"$ & serophulápiae Capieux \\
\hline 9. &. & lyehnitidis $\mathrm{Rbr}$ \\
\hline 10. & $"$ & thapsíphaga Tr. \\
\hline 11. & $" n$ & serophulariphaga Rbr \\
\hline 12. & $"$ & blattáriae Esp. 우 \\
\hline 13. & .. & ásteris Sehiff. \\
\hline 14. & $"$ & dracúnculi $\mathrm{Hb}$. \\
\hline 15. & 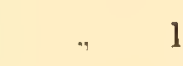 & láctea $\mathrm{F}$. \\
\hline 16. & $"$ & biornáta F. d. W. \\
\hline 17. & $"$ & balsamitae $B$. \\
\hline 18. & $"$ & umbrática L. \\
\hline 19. & $"$ & lactúcae Esp. \\
\hline 20. & $\because$ & lueífuga $\mathrm{Hb}$. \\
\hline 21. & .. & eampánulae Frr \\
\hline 22. & $"$ & santolínae Rbr \\
\hline 23. & $"$ & ehamomíllae Schiff. \\
\hline 24. & , & tanacéti Schiff. \\
\hline 25. & $"$ & santóniei $\mathrm{Hb}$. \\
\hline 26. &. & praecána Ev. \\
\hline 27. &, & xeránthemi B. \\
\hline
\end{tabular}

\begin{tabular}{|c|c|c|}
\hline Katalog I & Seite des I. Bandes & $\begin{array}{c}\text { Raupen-(Puppen-) } \\
\text { Abbildung }\end{array}$ \\
\hline 2204 & 2661. & Nachtr.-Taf. V, 36 \\
\hline 2206 & 2661. & $\mathrm{~V}, 37$ \\
\hline 2208 & $266 \mathrm{r}$. & \\
\hline 2211 & $266 / 67$ & $\mathrm{~V}, 39$ \\
\hline 2214 & 2671. & $V, 40$ \\
\hline 2220 & $267 / 68$ & Taf. 33,8 \\
\hline 2221 & 2681. & $\begin{array}{l}\text { Ei Taf. } 50,19 \\
n 33,9 \mathrm{a}, \mathrm{b}\end{array}$ \\
\hline 2222 & 2681. & Taf. 33,10 \\
\hline 2224 & $268 \mathrm{r}$. & "33, 11 \\
\hline 2225 & $268 / 69$ & $\Rightarrow \quad 33,12 \mathrm{a}, \mathrm{b}$ \\
\hline 2226 & 2691. & \\
\hline 2227 . & $269 \mathrm{r}$. & $" 33,13$ \\
\hline 2229 & $269 \mathrm{r}$. & . $33,14 \mathrm{a}, \mathrm{b}$ \\
\hline 2333 & 2701. & $\begin{array}{l}\text { 34, } 4 \\
\text { (v. anthémidis) }\end{array}$ \\
\hline 2240 & $270 \mathrm{r}$ & \\
\hline 2242 & $271 r$ & \\
\hline 2243 & 2711. & Taf. 33,15 \\
\hline 2245 & $271 / 72$ & $" 33,16 \mathrm{a}-\mathrm{c}$ \\
\hline 2248 & $272 \mathrm{r}$. & " 33,17 \\
\hline 2247 & 2721. & $" 33,18 \mathrm{a}, \mathrm{b}$ \\
\hline 2246 & 2721. & $" 34,1$ \\
\hline 2249 & $272 / 73$ & $" 34,2$ \\
\hline 2250 & 2731. & $" 34,3$ \\
\hline 2244 & $271 \mathrm{l}$ & $" 34,5$ \\
\hline 2258 & $273 r$ & $" 34,6$ \\
\hline 2235 & 2701. & Nachtr.-Taf. V, 43 \\
\hline 2265 & 2741. & Taf. 34,7 \\
\hline
\end{tabular}



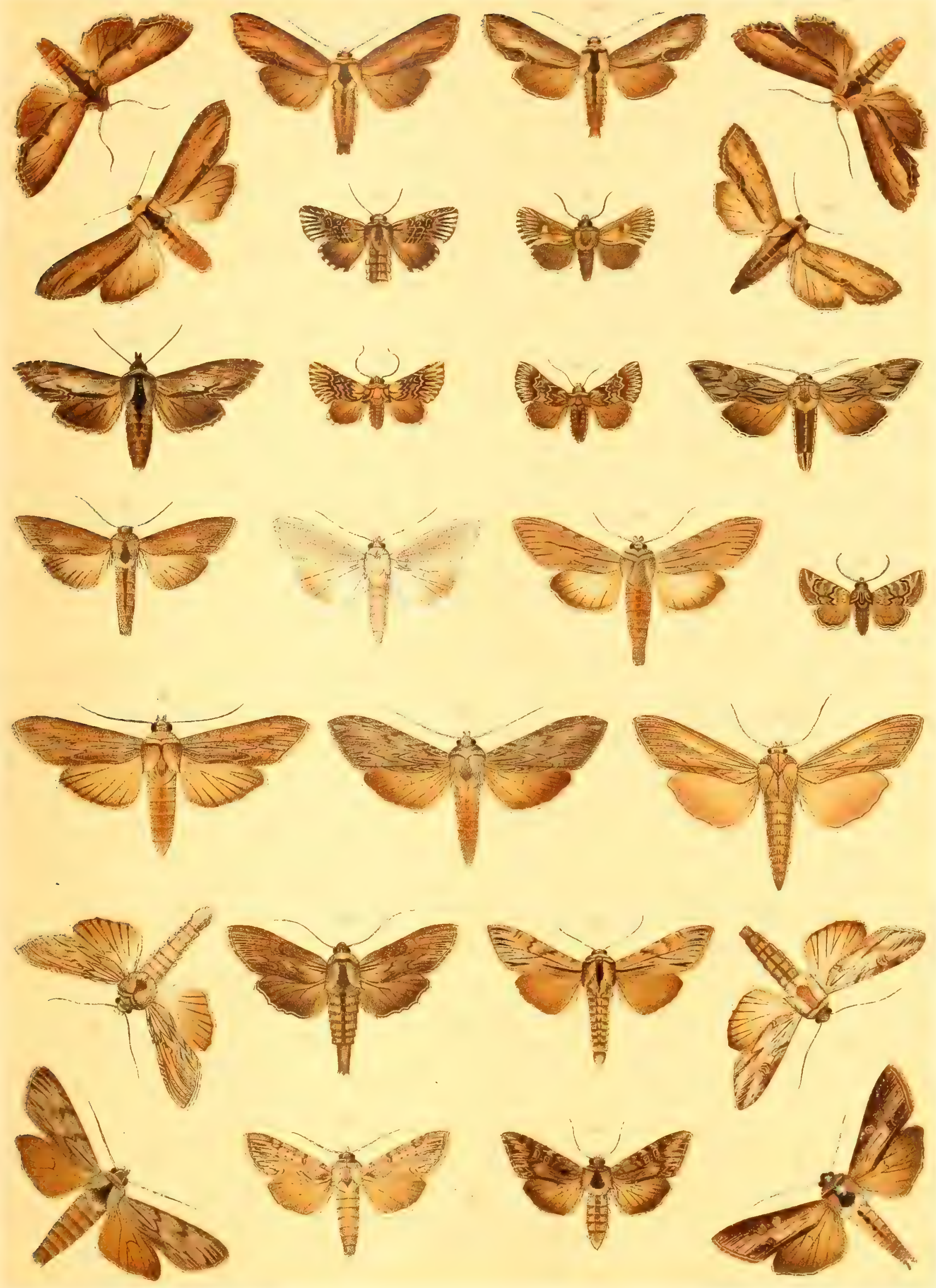




\section{TAFEL 49.}

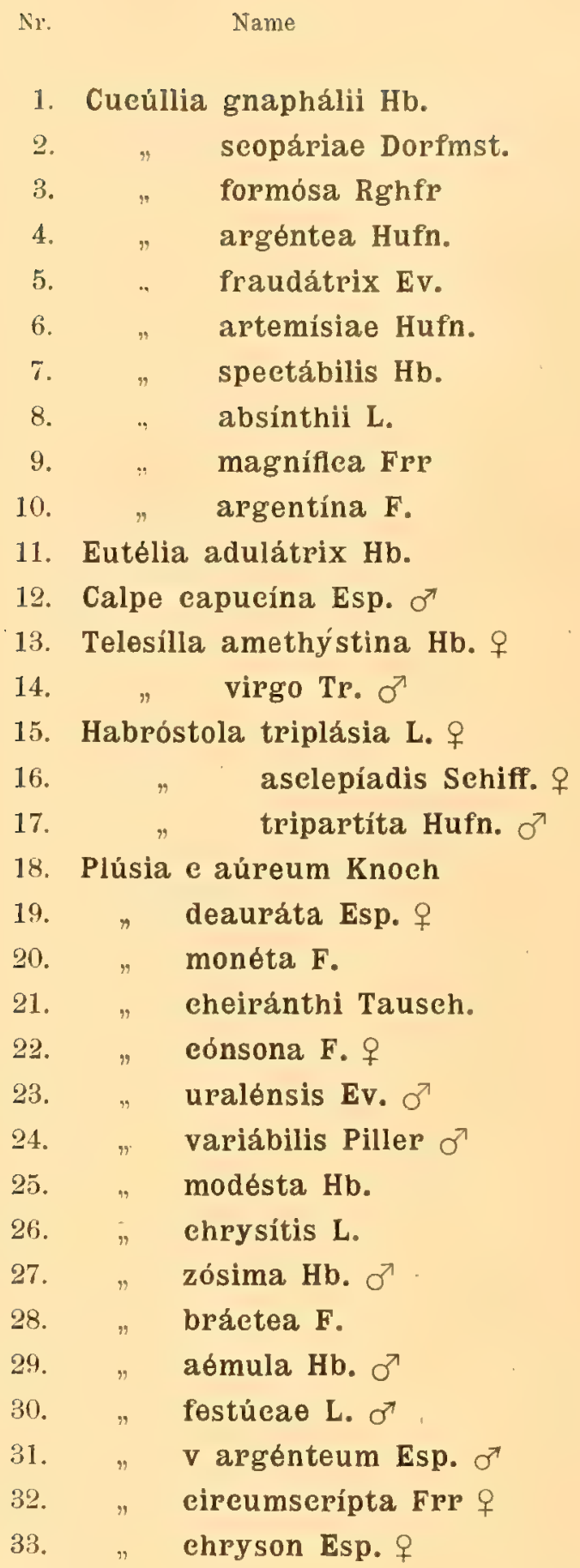

\begin{tabular}{|c|c|c|}
\hline Katalog I & Seite des I. Bandes & $\begin{array}{c}\text { Raupen- (Puppen-) } \\
\text { Abbildung }\end{array}$ \\
\hline 2264 & $273 / 74$ & Tafi: 34,8 \\
\hline 2272 & $274 / 75$ & $" 34,9$ \\
\hline 2275 & $275 \mathrm{x}$ & $" 34,12$ \\
\hline 2278 & $275 / 76$ & $" \quad 34,13$ \\
\hline 2271 & $274 \mathrm{r}$. & Nachtr.-Taf. V, 43 \\
\hline 2273 & 2751. & Taf. $34,10 \mathrm{a}, \mathrm{b}$ \\
\hline 2268 & $274 \mathrm{r}$ & Nachtr.-Taf. V, 44 \\
\hline 2274 & $275 \mathrm{l}$. & Taf. $34,11 \mathrm{a}, \mathrm{b}$ \\
\hline 2277 & $275 \mathrm{r}$. & \\
\hline 2279 & 2761. & $\Rightarrow \quad 34,14$ \\
\hline 2281 & $276 \mathrm{r}$ & $" 34,15 \mathrm{a}-\mathrm{d}$ \\
\hline 2507 & 2981. & $" 34,16$ \\
\hline 2512 & 2981. & $\Rightarrow 34,17$ \\
\hline 2513 & 298 r. & \\
\hline 2515 & $298 \mathrm{r}$. & $" 34,18 \mathrm{a}-\mathrm{c}$ \\
\hline 2516 & 2991. & $" 34,19$ \\
\hline 2517 & 2991. & $" 35,1$ \\
\hline 2518 & $299 / 300$ & " $3 \tilde{5}, 2 \mathrm{a}, \mathrm{b}$ \\
\hline 2519 & 3001. & \\
\hline 2520 & 3001. & $" 35,3 \mathrm{a}-\mathrm{c}$ \\
\hline 2524 & $300 \mathrm{r}$. & $" 35,4$ \\
\hline 2532 & 301 l. & $" 35,5$ \\
\hline 2529 & $300 \mathrm{r}$. & \\
\hline 2530 & $300 / 01$ & $" 35,6$ \\
\hline 2531 & 3011. & $" 35,7$ \\
\hline 2539 & $301 / 2$ & $" 35,8 \mathrm{a}, \mathrm{J}$ \\
\hline 2540 & 3021. & \\
\hline 2543 & 3021. & $, \quad 49,19$ \\
\hline 2545 & $302 \mathrm{r}$. & \\
\hline 2546 & $302 / 3$ & $" 35,10$ \\
\hline 2549 & 3031. & Nachtr--Taf. IV, 21 \\
\hline 2552 & 3031. & \\
\hline 2542 & 3021. & Taf. 35,9 \\
\hline
\end{tabular}




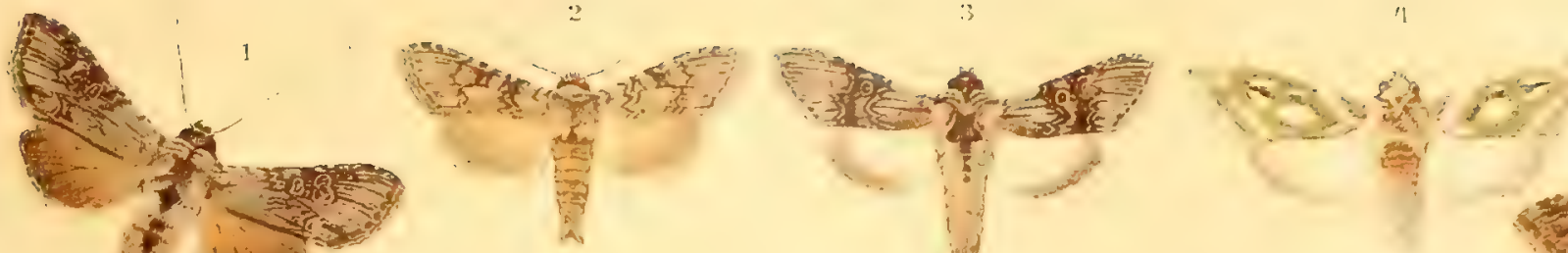

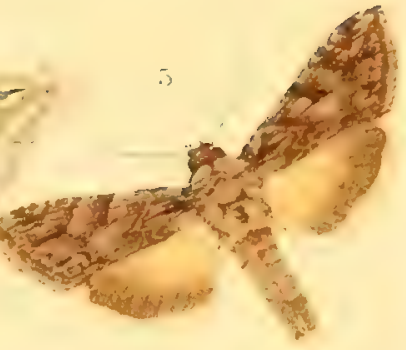

(t)

Und
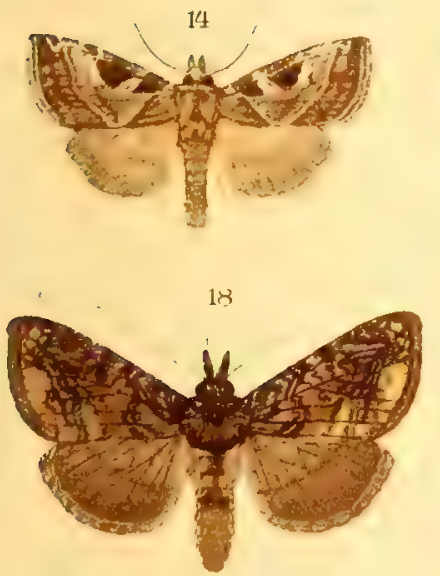

phente
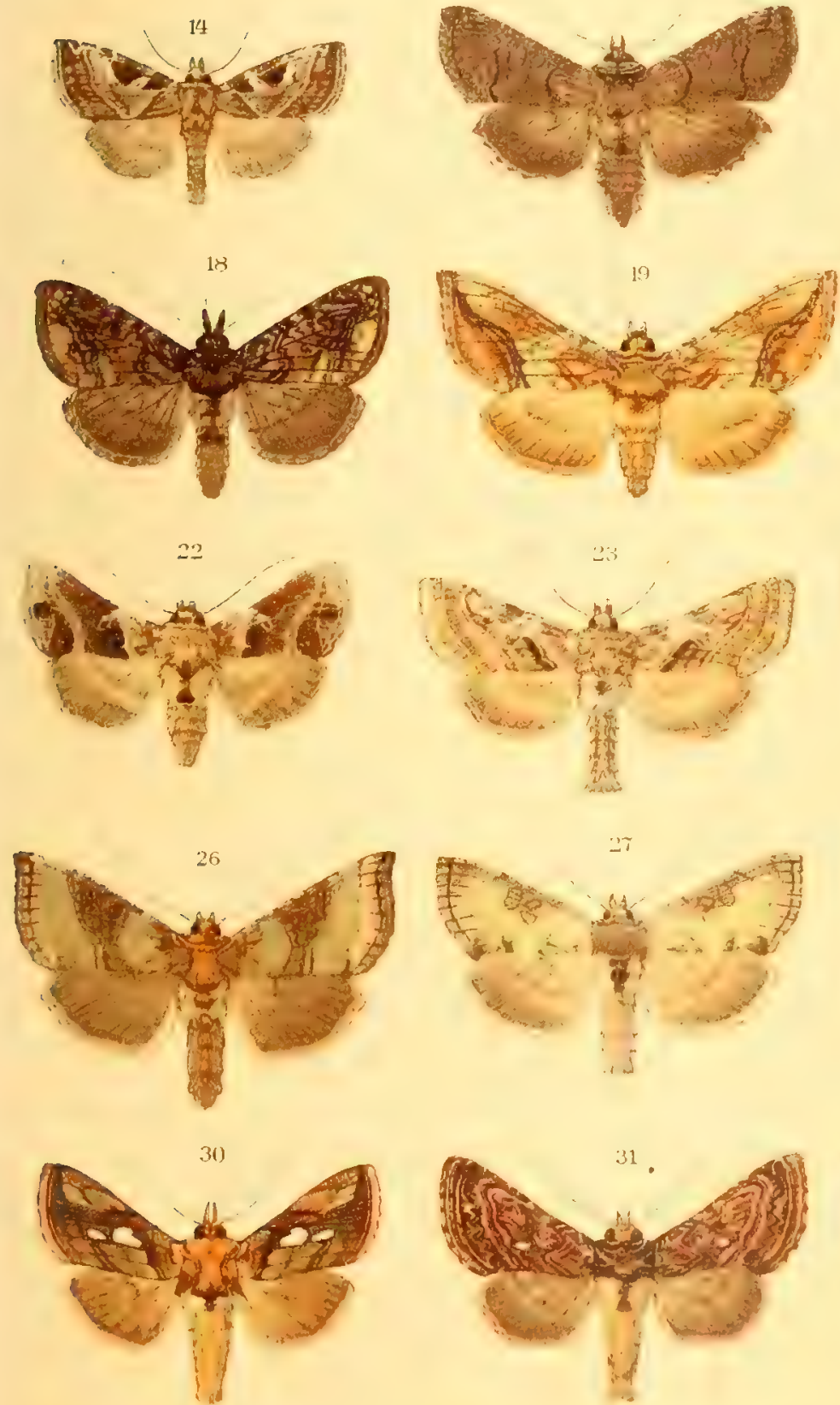

霹
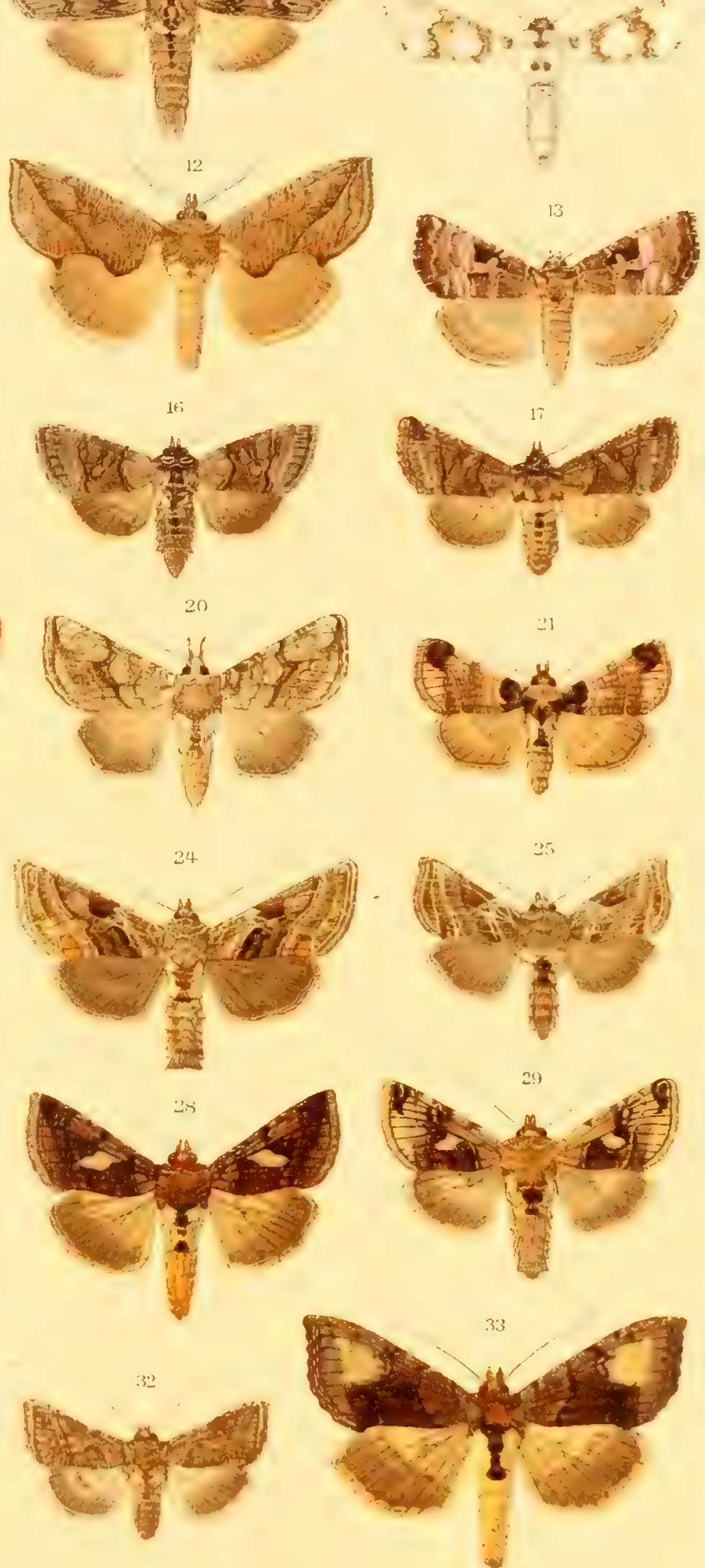



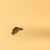




\section{TAFEL 50.}

$\mathrm{Nr}$.

- Plúsia accentífera Lef. $\sigma^{7}$ gutta Gn.

chaleítes Esp. $0^{7}$

ióta L.

pulehrína $\mathrm{Hw}$.

maerogámma Ev. ơ

7. "gamma L. $\sigma^{7}$

8. " daubéi B. $0^{7}$

9. " eireumfléxa L. $\sigma^{7}$

10. " ni Hb.

11. " interrogatiónis L. $\sigma^{*}$

12. .. ain Hochenw. of

13.

14.

21.

22.

23.

24.

25.

26.

27.

28.

29.

30

31

párilis Hb. 오

migrogámma $\mathrm{Hb}$. ㅇ

devérgens $\mathrm{Hb}$. o $^{7}$

Anóphia leueómelas L.

Aédia funésta Esp.

Anárta myrtíli L.

eordigera Thnbg

" melaleủea Thnbg

" melanópa Thnbg 우

nigríta $\mathrm{Hb}$.

funébris $\mathrm{Hb}$.

" richardsóni Curt.

" leueocýcla Stgr

" lappónica Thnbg 우

" zetterstédti Stgr

"quiéta $\mathrm{Hb}$. o $^{7}$

Panheméria tenebráta Se.

Heliódes pupícola Hb. $\sigma^{7}$

Omia eymbaláriae Hb.

" eyclópea Grasl. o

Heliothis cognáta Frp 우

eárdui Hb.

córa Ev.

onónidis F. $\sigma^{7}$

dipsácea L.

seutósa Sehiff.

peltígera Sehiff. hochenwápthi Hoehenw, o7

ab. variegáta Tutt ơ 2284

$$
\text { v. staudingéri Auriv. } q
$$

Ianthínea frivaldsky'i Friv. $\sigma^{7}$

purpuráseens Tausch.

v.? imperiális Stgr or

2579

2576

2577

2578

2660

2661

2283

2286

2293

2292

2289

2290

\author{
Ka
}

$\begin{array}{ccc}\text { Katalog I } & \text { Seite des I. Bandes } & \begin{array}{c}\text { Raupen- (Puppen-) } \\ \text { Abbildung }\end{array} \\ 2566 & 304 \mathrm{r} . & \text { Nachtr.Taf. IV, 23 } \\ 2551 & 303 \mathrm{l} . & \text { Taf. 35, 11 } \\ 2557 & 303 \mathrm{r} . & 35,12 \\ 2560 & 304 \mathrm{l} . & 35,13 \\ 2559 & 303 \mathrm{r} . & \text { Nachtr.-Taf. IV, 22 } \\ 2561 & 304 \mathrm{l} . & \text { ( Ei Taf. 50, 70 } \\ 2562 & 304 \mathrm{l} . & \text { ( } " 35,14 \\ 2567 & 304 / 5 & \text { Taf. 32, } 8\end{array}$

Nachtr.-Taf. IV, 24

2573

2574

$304 \mathrm{r}$.

3051.

3051 .

$305 \mathrm{r}$.

3061.

$305 / 6$

3061.

$306 \mathrm{l}$.

312,13

3131.

2771.

2771.

277 r.

$277 / 78$

$278 \mathrm{r}$.

$278 \mathrm{r}$.

278 !.

2781.

$278 / 79$

$278 \mathrm{r}$.

2791.

$279 \mathrm{l}$.

$279 \mathrm{r}$.

$280 \mathrm{l}$.

2801.

2801.

$280 \mathrm{r}$.

$280 \mathrm{r}$.

280/81

$281 \mathrm{l}$.

281 i.

2811.

$280 / 81$

281/82

2821.
IV, 25

$$
\begin{array}{ll}
" & I V, 26 \\
" & I V, 27 \\
" & \text { IV, 28 }
\end{array}
$$

Taf'. $35,15 \mathrm{a}, \mathrm{b}$

, 35,16

" 35,17

1) 35,18

$" \quad 49,20$

" 35,19

" 35,20

Nachtr.-Taf. IV, 1

Taf. 35, $21 \mathrm{a}-\mathrm{c}$

" $35,22 \mathrm{a}, \mathrm{b}$

" 35,23

" 35,24 


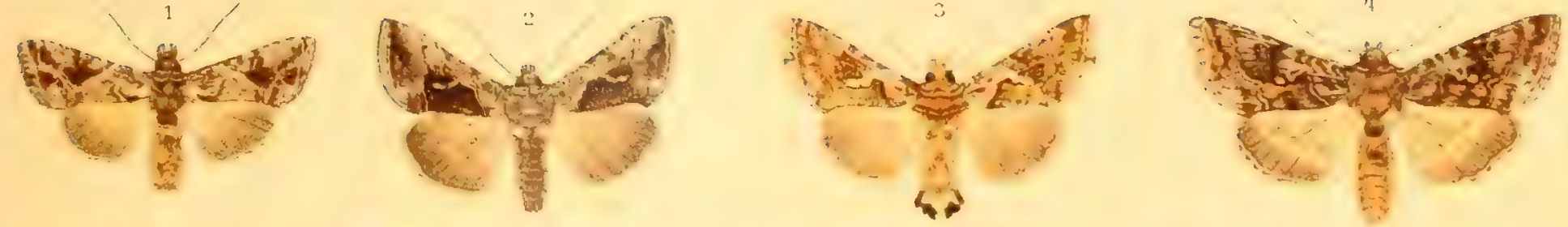

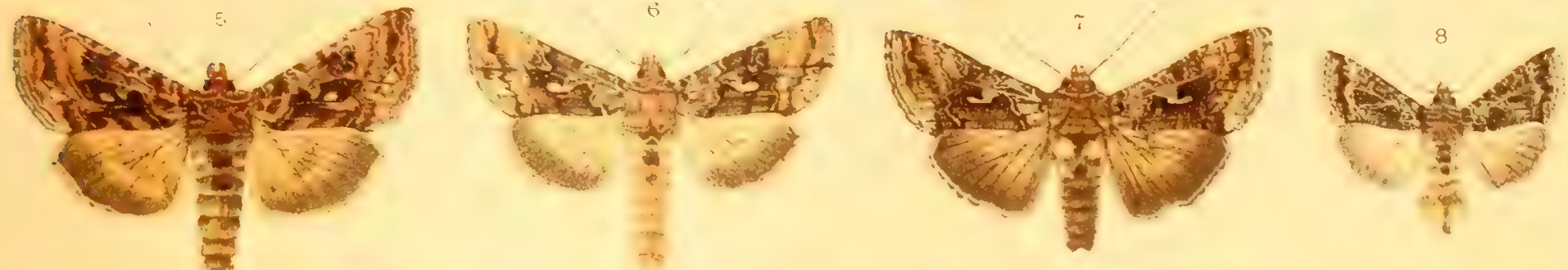

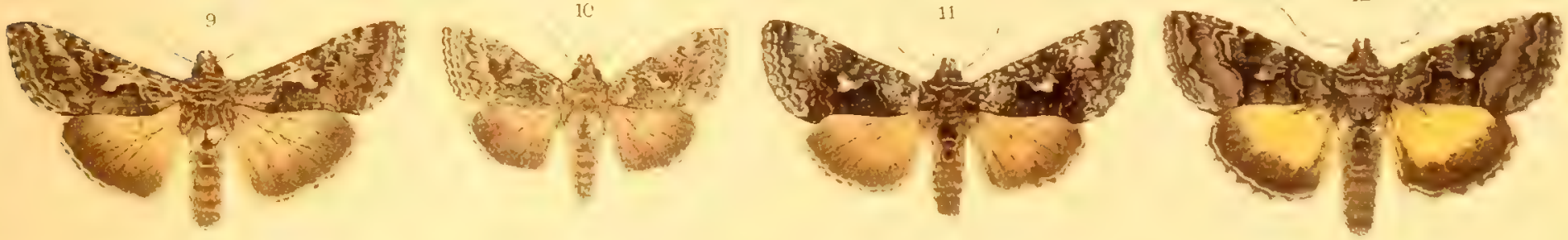

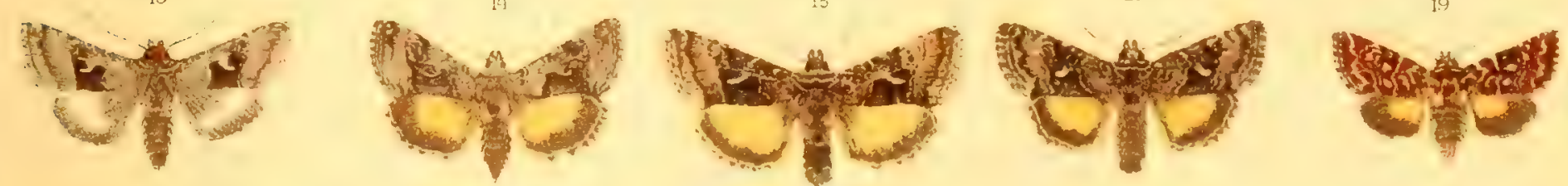

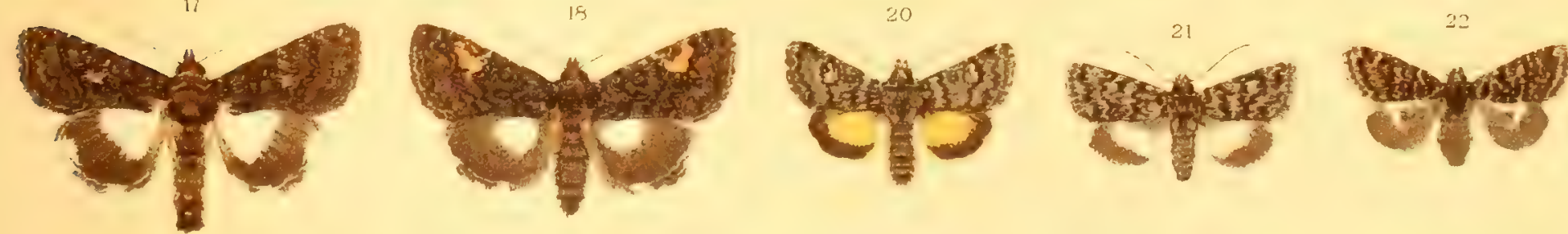

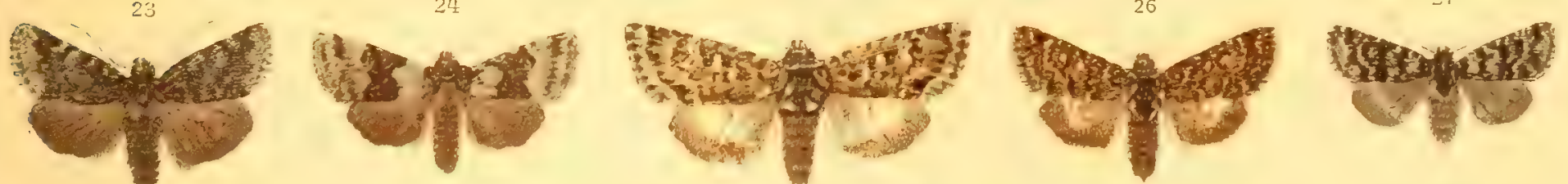
10010.

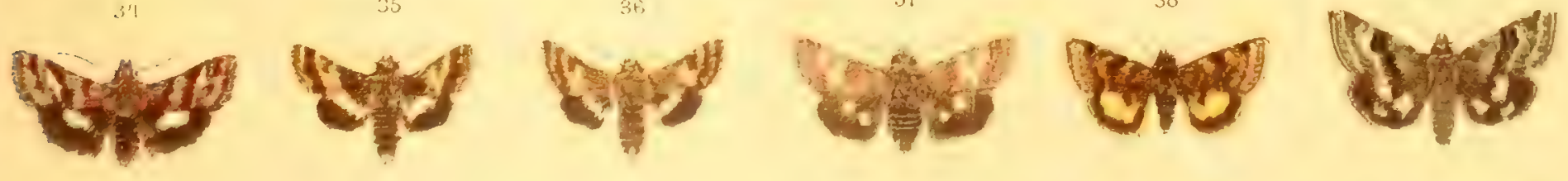
Dyes toide
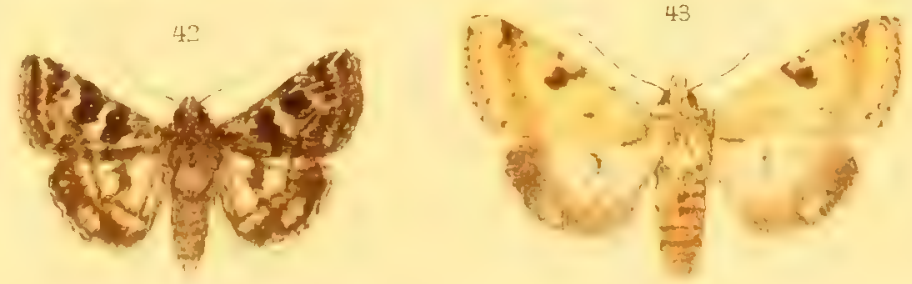



\begin{tabular}{|c|c|c|c|c|}
\hline $\mathrm{Nr}$. & Name & Katalog I & Seite des I. Bandes & $\begin{array}{c}\text { Raupen-(Puppen-) } \\
\Delta \text { bbildung }\end{array}$ \\
\hline 1. & Heliothis armígera $\mathrm{Hb}$. & 2326 & $282 \mathrm{r}$ & Taf. 36,1 \\
\hline 2. & " incarnáta Frp. & 2329 & $282 / 83$ & Nachtr.-Taf. IV, 2 \\
\hline 3. & Charíclea delphínii L. $q$ & 2352 & 2841. & Taf. $36,2 \mathrm{a}, \mathrm{b}$ \\
\hline 4. & " treitsehkéi Friv. & 2354 & $284 \mathrm{l}$. & Nachtr.-Taf. IV, 4 \\
\hline 5. & Pýrrhia purpurites Tr. & 2357 & $284 \mathrm{r}$. & Taf. $36,3 \mathrm{a}, \mathrm{b}$ \\
\hline 6. & $"$ umbra Hufn. 우 & 2358 & $284 / 85$ & $" 36,4 \mathrm{a}, \mathrm{b}$ \\
\hline 7. & Micra lacernácia $\mathrm{Hb}$. & 2396 & $288 \mathrm{r}$. & Nachtr.-Taf. IV, 9 \\
\hline 8. & Xanthódes graéllsi Feist. & 2362 & $285 \mathrm{r}$. & IV, 6 \\
\hline 9. & $"$ malvae Esp. 1. $\sigma^{7}$, r. 우 & 2361 & 285 l. Taf. 36,5 & $\mathrm{a}, \mathrm{b}$ u.N.-T.IV, $5 \mathrm{a}, \mathrm{b}$ \\
\hline 10. & Eutérpia loudéti B. $\sigma^{7}$ & 2363 & $285 / 86$ & Taf. 36,6 \\
\hline 11. & Acóntia uránia Friv. o & 2376 & 2861. & Nachtr.-Taf. IV, 7 \\
\hline 12. & titánia Esp. & 2377 & 2861. & IV, 8 \\
\hline 13. & lúeida Hufn. & 2378 & $286 r$. & Taf. 36,7 \\
\hline 14. & luetuósa Esp. & 2380 & $286 / 87$ & $" 36,8 \mathrm{a}, \mathrm{b}$ \\
\hline 15. & Acontiola moldavieola HS. & 2384 & 2871. & \\
\hline 16. & Eublémma areuínna $\mathrm{Hb} . \sigma^{\top}$ & 2389 & 287 r. & \\
\hline 17. & $" \quad$ suávis $\mathrm{Hb} \cdot \mathrm{O}^{7}$ & 2391 & 287 r. & \\
\hline 18. & iucúnda $\mathrm{Hb}$. & 2392 & $287 \mathrm{r}$. & \\
\hline 19. & Miera velox Hb. $\sigma^{7}$ & 2394 & 2881. & \\
\hline 20. & " dardouíni $\mathrm{B}$. & 2395 & 2881. & $\eta \quad 36,9$ \\
\hline 21. & " respérsa $\mathrm{Hb}$. & 2403 & 2891. & $. \quad 36,10$ \\
\hline 22. & " eoneínnula B. & 2414 & 2891. & \\
\hline 23. & " ragusána Frr & 2415 & 2891. & \\
\hline 24. & " polygrámma Dup. & 2417 & 2891. & \\
\hline 25. & Oratocélis communimácula $\mathrm{Hb}$. & 2422 & $291 \mathrm{r}$. & Nachtr.-Taf. IV, 10 \\
\hline 26. & Miera pannónica Fre & 2423 & 289 r. & Taf. 36,11 \\
\hline 27. & " purpurína $\mathrm{Hb}$. & 2426 & 2901. & Nachtr.-Taf. IV, 12 \\
\hline 28. & $"$ rósea Hb. & 2425 & $289 / 90$ & $, \quad I V, 11$ \\
\hline 29. & " ostrina $\mathrm{Hb}$. & 2428 & 2901. & IV, 13 \\
\hline 30. & $" \quad$ papva $\mathrm{Hb}$ & 2429 & $290 \mathrm{r}$. & \\
\hline 31. & $"$ paula $\mathrm{Hb}$. & 2431 & $290 \mathrm{r}$. & IV, 14 \\
\hline 32. & " eandidána v. heliehrýsi Rbr & 2435 & 2911. & \\
\hline 33. & $" \quad$ v. virídula Gn. & 2434 & 2911. & \\
\hline 34. & $" \quad$ eandidána $\mathrm{F}$. & 2433 & 2911. & IV, 15 \\
\hline 35. & $" \quad$ pura $\mathrm{Hb}$ & 2437 & $291 \mathrm{r}$. & \\
\hline 36. & Erástria argéntula $\mathrm{Hb}$. & 2453 & $292 / 93$ & Taf. 36,12 \\
\hline 37. & "úneula $\mathrm{Cl}$. & 2454 & 2931. & $" 49,21$ \\
\hline 38. & obliteráta Rbr & 2455 & 2931. & Nachtr.-Taf. IV, 16 \\
\hline 39. & pusilla View. & 2460 & $293 r$. & Taf. $36,13 \mathrm{a}, \mathrm{b}$ \\
\hline 40. & venústula $\mathrm{Hb}$. & 2458 & $293 \mathrm{r}$. & Nachtr.-Taf. IV, 17 \\
\hline 41. & Coceidíphaga scitula Rbr & 2449 & 2921. & \\
\hline 42. & Erástria numérica $B$. & 2468 & 2941. & \\
\hline 43. & deceptória Se. & 2462 & $293 / 94$ & Taf. 36,14 \\
\hline 44. & fasciána $L$. & 2464 & 2941. & $\% 36,15$ \\
\hline 45. & Miána eaptiúneula Tr. & 1571 & 1821. & \\
\hline 46. & Prothymia viridária $\mathrm{Cl}$. & 2482 & $295 \mathrm{r}$. & Nachtr.-Taf. IV, 18 \\
\hline 47. & sanetifloréntis B. 1. $\sigma^{\pi}$, r. q & 2483 & $295 \mathrm{r}$. & \\
\hline 48. & Mesotrósta signális Tr. & 2488 & $295 / 96$ & \\
\hline 49. & Emmélia trabeális Se. & 2490 & 2961. & Taf. $36,16 \mathrm{a}-\mathrm{c}$ \\
\hline 50. & Haemerósia renális $\mathrm{Hb} . \sigma^{T}$ & 2492 & $296 \mathrm{r}$. & $\Rightarrow \quad 36,17$ \\
\hline 51. & Metopónia koekeritziána Hb. & 2493 & $296 / 97$ & $" 36,18 \mathrm{a}, \mathrm{b}$ \\
\hline
\end{tabular}




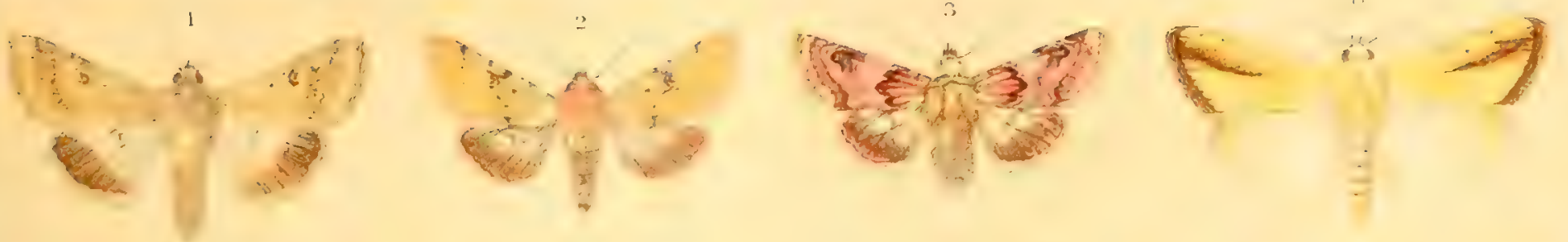

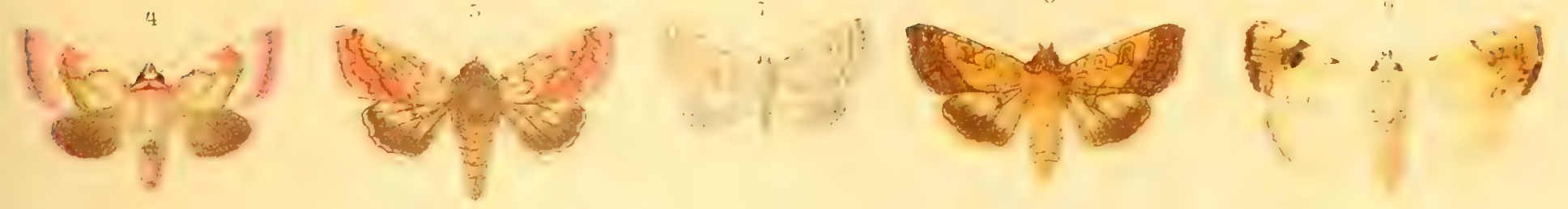

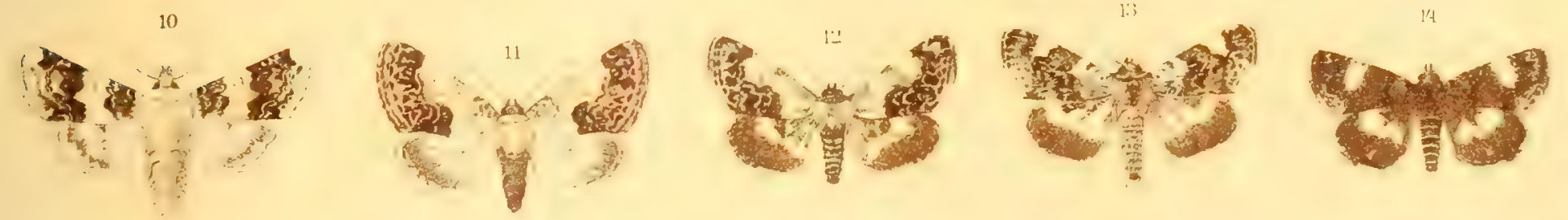

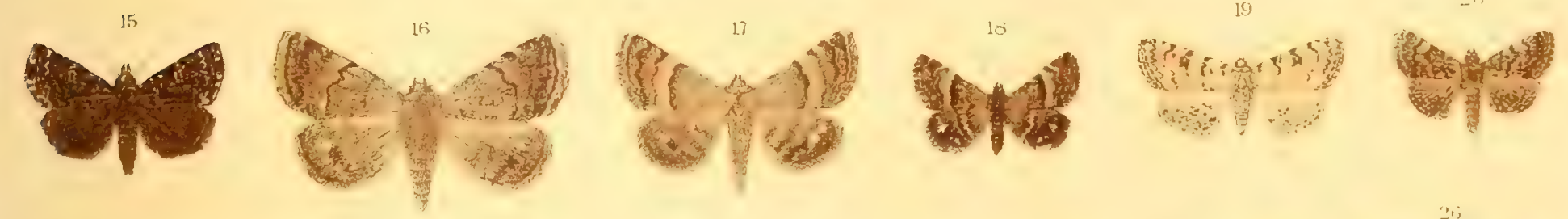

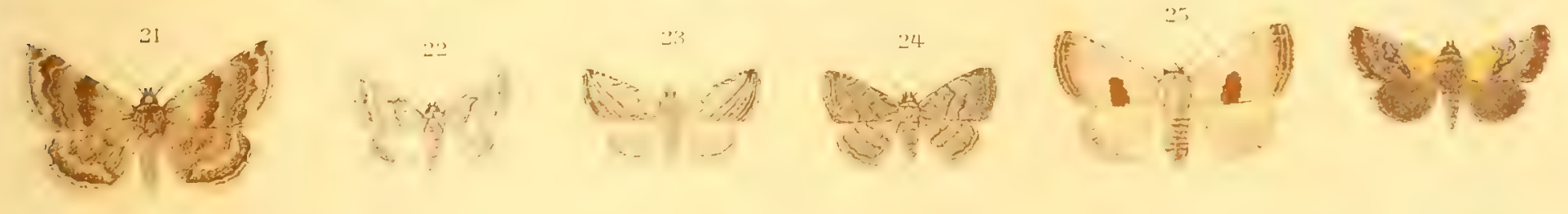

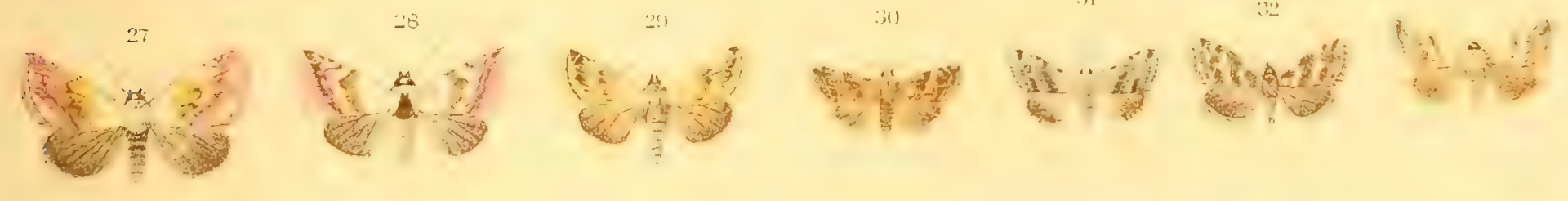

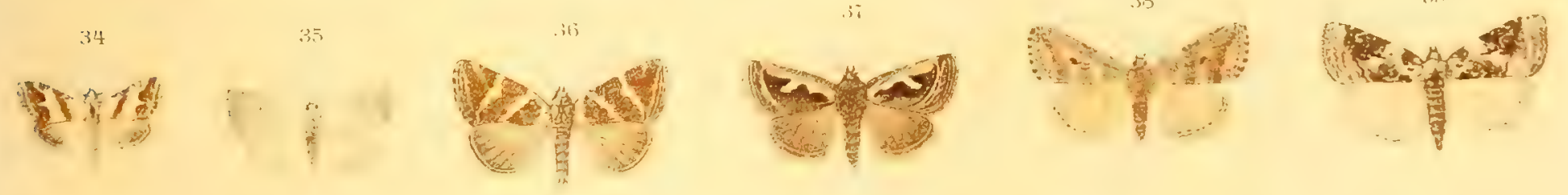

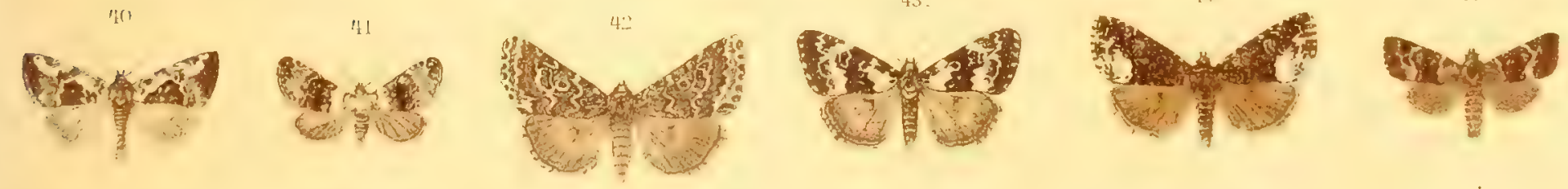

no 




\section{TAFEL 52.}

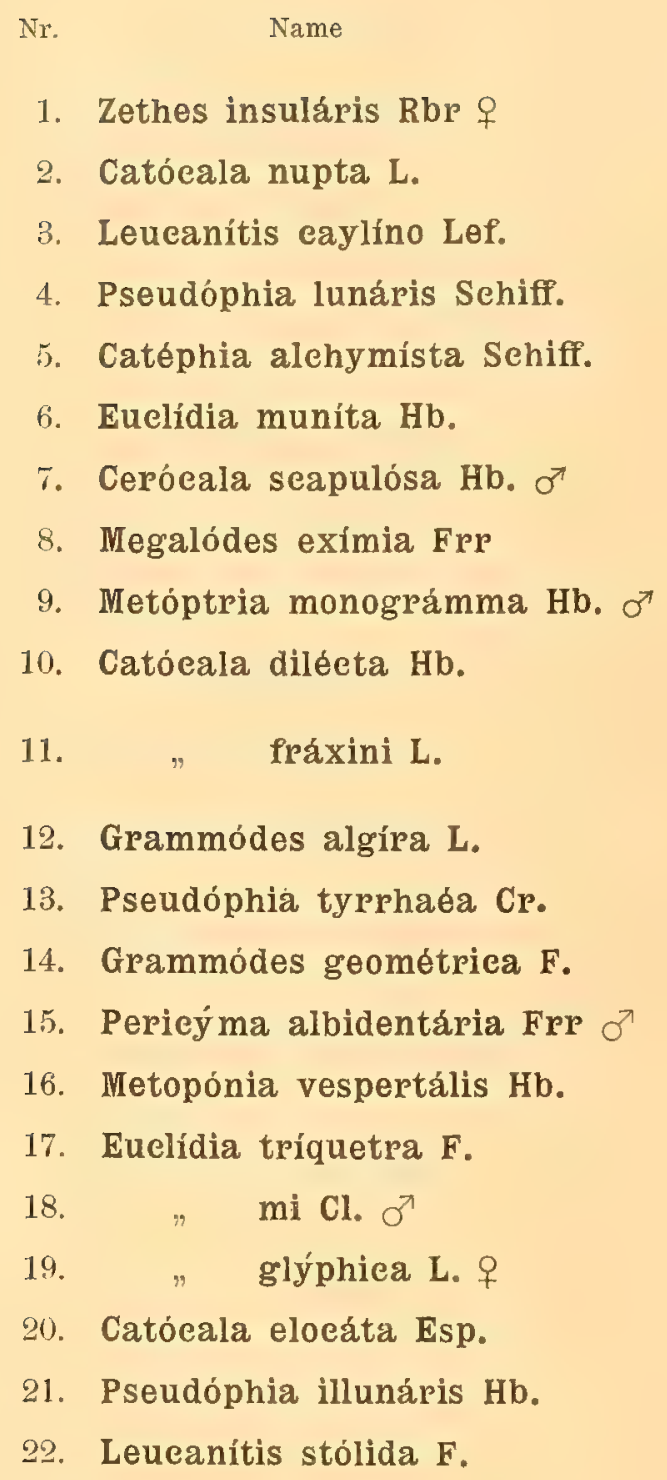

\begin{tabular}{|c|c|c|}
\hline Katalog I & Seite des I. Bandes & $\begin{array}{l}\text { Raupen-(Puppen-) } \\
\text { Abbildung }\end{array}$ \\
\hline 2598 & 3091. & Nachtr.-Taf. IV, 32 \\
\hline 2678 & $315 / 16$ & Taf. $37,6 \mathrm{a}-\mathrm{c}$ \\
\hline 2627 & 3101. & $" 36,23$ \\
\hline 2655 & 3121. & $" 37,1 \mathrm{a}-\mathrm{c}$ \\
\hline 2662 & $313 \mathrm{r}$. & $" 37,3$ \\
\hline 2590 & $307 / 08$ & \\
\hline 2594 & $308 \mathrm{r}$. & Nachtr.-Taf. IV, 30 \\
\hline 2580 & $306 \mathrm{r}$. & $" \quad$ IV, 29 \\
\hline 2583 & $306 \mathrm{r}$. & Taf. 36,19 \\
\hline 2681 & $316 \mathrm{r}$. & $" 37,7$ \\
\hline 2667 & 3141. & $\begin{aligned} \text { Ei Taf. } 50,72 \\
\\
" 37,4 \mathrm{a}, \mathrm{b}\end{aligned}$ \\
\hline 2644 & 3111. & Taf. 36,25 \\
\hline 2657 & $312 \mathrm{r}$ & $" 37,2 \mathrm{a}, \mathrm{b}$ \\
\hline 2646 & 3111. & $" 36,24 \mathrm{a}, \mathrm{b}$ \\
\hline 2595 & $308 / 09$ & \\
\hline 2496 & 2971. & Nachtr-Taf. IV, 19 \\
\hline 2591 & 3081. & Taf. 36,22 \\
\hline 2586 & 3071. & $" 36,20 \mathrm{a}, \mathrm{b}$ \\
\hline 2589 & $307 \mathrm{r}$. & $\eta \quad 36,21 \mathrm{a}, \mathrm{b}$ \\
\hline 2670 & $314 / 15$ & $\because \quad 37,5$ \\
\hline 2651 & $311 / 12$ & Nachtr-Taf. IV, 34 \\
\hline 2642 & $310 \mathrm{r}$. & IV, 33 \\
\hline
\end{tabular}




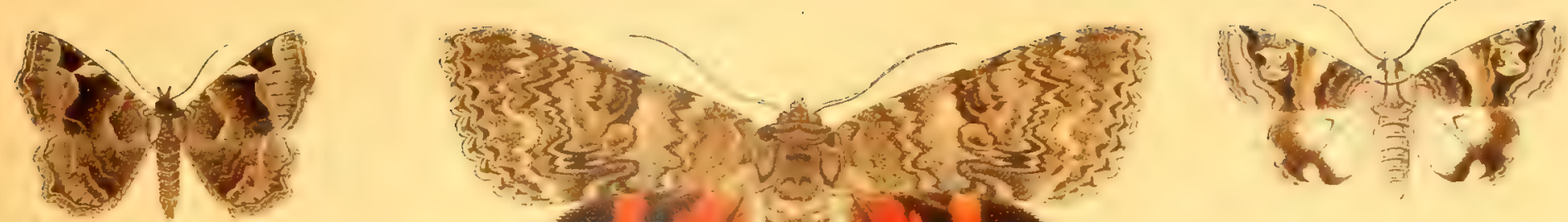

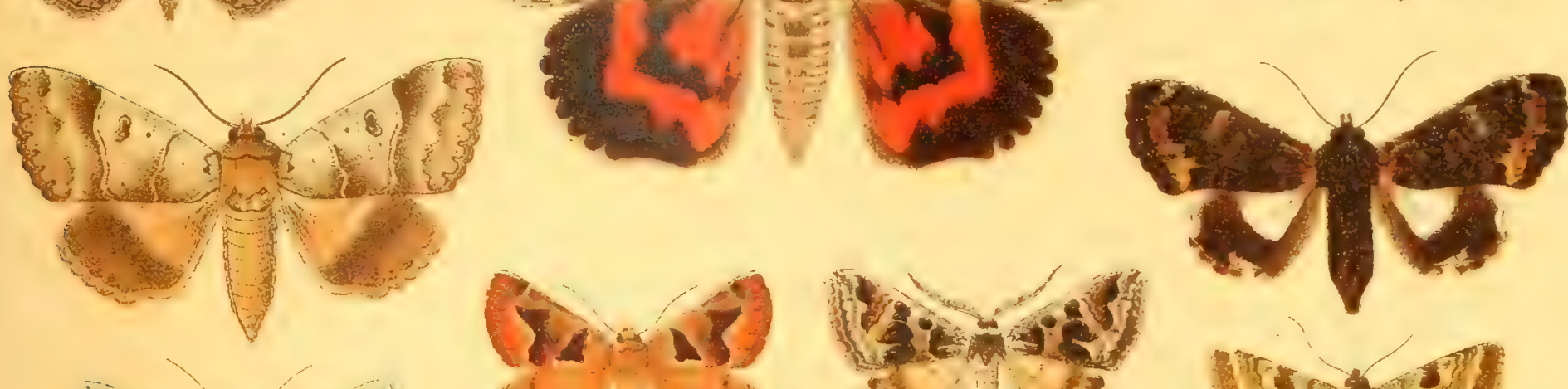

90.
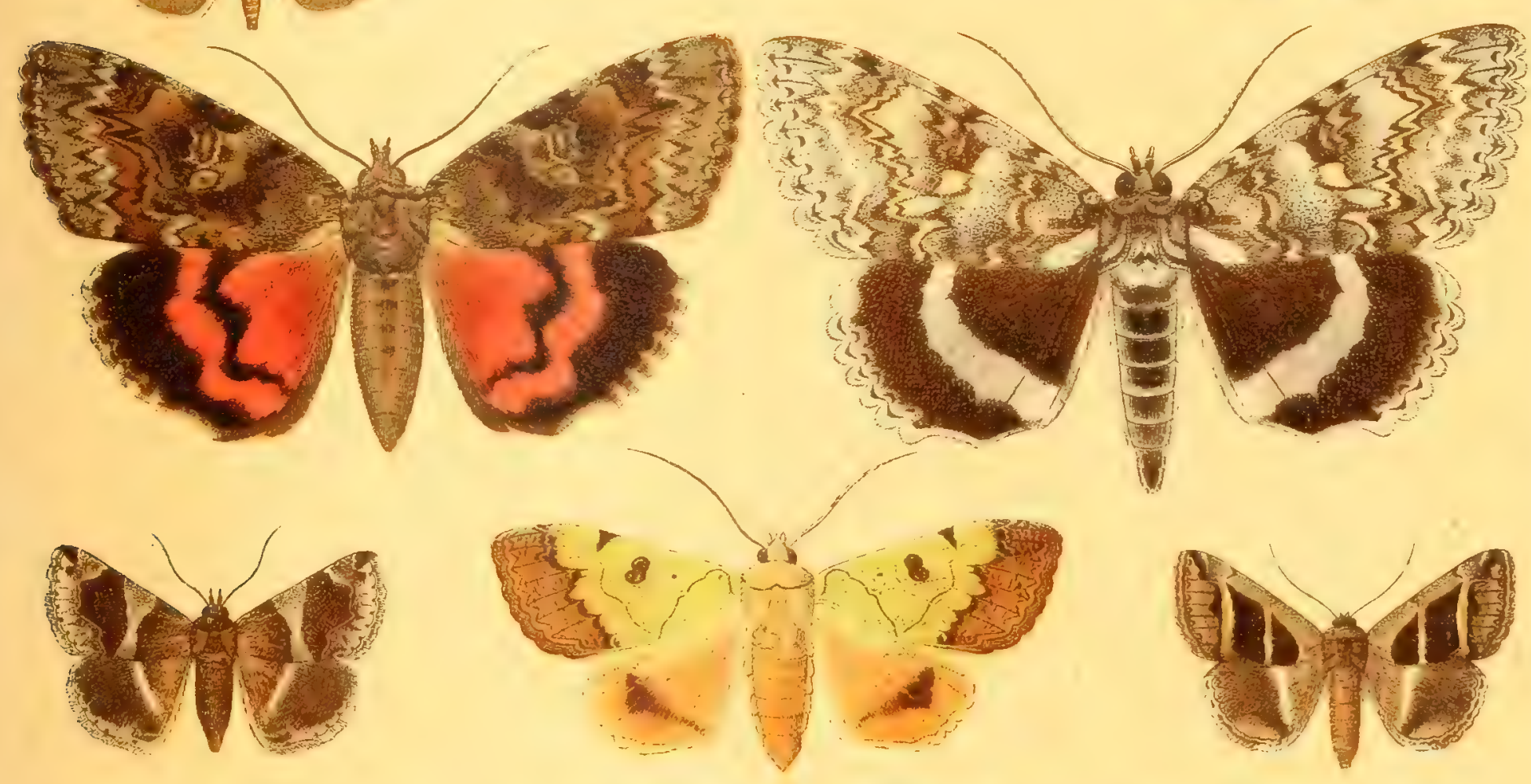

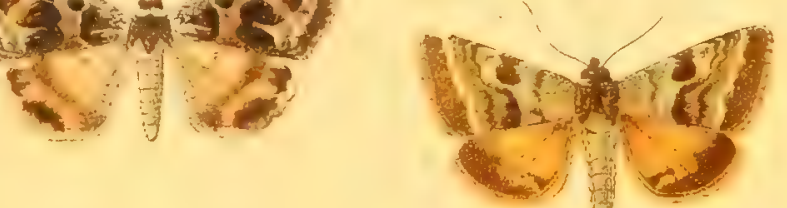






\section{TAFEL 53.}

\begin{tabular}{|c|c|c|}
\hline $\mathrm{Nr}$. & & Name \\
\hline 1. & Catóeala & neoný mpha Esp. \\
\hline 2. & $"$ & paeta L. \\
\hline 3. & $"$ & lupína HS. \\
\hline 4. & $"$ & fulmínea Seop. \\
\hline 5. & $"$ & sponsa L. \\
\hline 6. & $"$ & eléeta Bkh. \\
\hline 7. & $"$ & convérsa Esp. $q$ \\
\hline 8. & $"$ & puérpera Giorna \\
\hline 9. & $"$ & optáta God. \\
\hline 10 . & $"$ & nymphaéa Esp. \\
\hline 11. & $"$ & promissa Esp. \\
\hline 12. & $"$ & hymenaéa Sehiff \\
\hline 13. & $"$ & coniúncta Esp. \\
\hline
\end{tabular}

\begin{tabular}{|c|c|c|}
\hline Katalog I & Seite des I. Bandes & $\begin{array}{c}\text { Raupen- (Puppen-) } \\
\text { Abbildung }\end{array}$ \\
\hline 2698 & 3181. & Nachtr.-Taf. IV, 36 \\
\hline 2692 & $317 \mathrm{r}$ & Taf. 37,10 \\
\hline 2689 & 3171. & \\
\hline 2696 & $317 / 18$ & $" 37,13 \mathrm{a}, \mathrm{b}$ \\
\hline 2682 & 3161. & $" 37,8 \mathrm{a}, \mathrm{b}$ \\
\hline 2669 & $314 \mathrm{r}$. & $" \quad 37,11$ \\
\hline 2713 & $318 \mathrm{r}$. & $" 37,15 \mathrm{a}, \mathrm{b}$ \\
\hline 2673 & 3151. & $" \quad 37,12$ \\
\hline 2690 & 3171. & Nachtr.-Taf. IV, 35 \\
\hline 2697 & 3181. & Taf. 37,16 \\
\hline 2684 & $316 \mathrm{r}$. & $" 37,9$ \\
\hline 2694 & $317 \mathrm{r}$. & " 37,14 \\
\hline 2685 & $316 / 17$ & \\
\hline
\end{tabular}



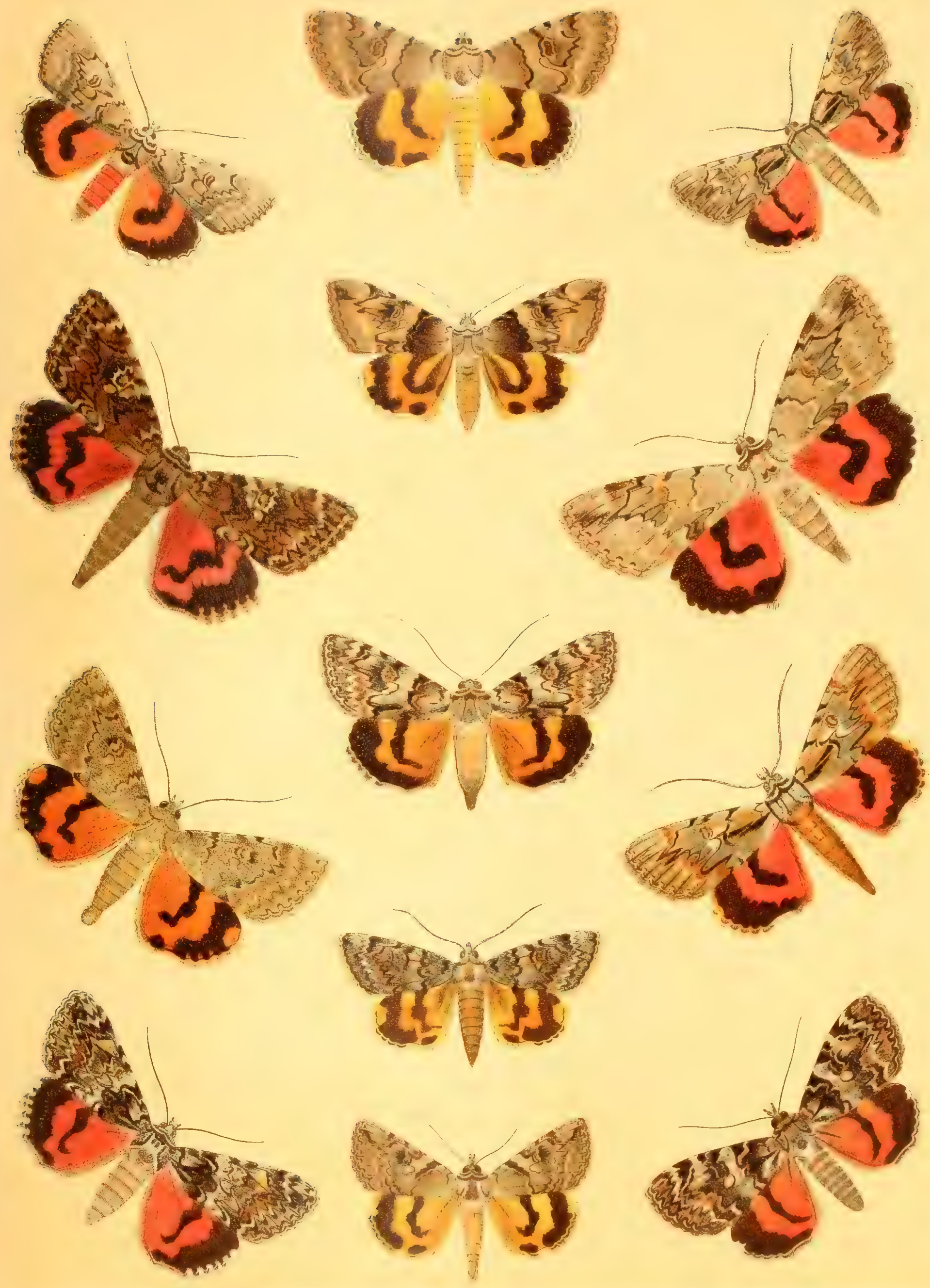




\section{TAFEL 54 .}

\begin{tabular}{|c|c|c|c|c|}
\hline Nr. & Name & Katalog I & Seite des I. Bandes & $\begin{array}{c}\text { Raupen- (Puppen-) } \\
\text { Abbildung }\end{array}$ \\
\hline 1. & Catócala eutýchea Tr. & 2714 & $318 \mathrm{r}$. & \\
\hline 2. & divérsa H.-G. & 2718 & 3191. & \\
\hline 3. & disiuneta $\mathrm{H}_{0}-\mathrm{G}$. & 2717 & 3191. & \\
\hline 4. & ny mphagóga Esp. & 2715 & $318 / 19$ & Taf. 38,1 \\
\hline$\check{5}$ & Parascótia fuliginária L. $\sigma^{7}$ & 2752 & $323 / 24$ & Taf. 38,10 u. 49,25 \\
\hline 6. & Laspeýria fléxula Schiff. & $27 \pm 7$ & $323 \mathrm{r}$ & $\Rightarrow 38,9$ u. 49,24 \\
\hline 7. & Zanelógnatha tarsierinális Knoeh. & 2767 & 3261. & Taf. 38,13 \\
\hline 8. & Apopéstes eatáphanes $\mathrm{Hb} . q$ & 2721 & $319 / 20$ & $" 38,3$ \\
\hline 9. & dilúcida $\mathrm{Hb}$. & 2723 & 3201. & Nachtr.-Taf. IV, 37 \\
\hline 10. & Exophýla rectanguláris $\mathrm{H}_{\text {-G. }}$. & 2733 & 32021 & Taf. 38,4 \\
\hline 11. & Zanel. griseális Hb. gen. aestivális Spul. & 2768 & 3201. & Nachtr.-Taf. IV, 39 \\
\hline 12. & Éecrita lúdiera Hb. & 2734 & 3211. & Taf. 38,5 \\
\hline 13. & Toxocámpa Iusória L. & 2735 & $321 \mathrm{r}$. & $.38,6$ \\
\hline 14. & Apopéstes spectrum Esp. $q$ & 2720 & $319 \mathrm{r}$. & . 38,2 \\
\hline 15. & Toxocámpa pástinum Tr. & 2741 & 3221. & $. \quad 38,7 \mathrm{a}, \mathrm{b}$ \\
\hline 16. & " víciae $\mathrm{Hb} \cdot \sigma^{7}$ & 2742 & $322 \mathrm{r}$ & $" 49,22$ \\
\hline 17. & Simplícia rectális Ev. & 2762 & $32+r$ & Nachtr.-Taf. IV, 38 \\
\hline 18. & Nodária nodosális HS. O & 2763 & 3251. & \\
\hline 19. & Toxocámpa eráceae F. $\sigma^{7}$ & 2743 & 3221. & Taf. 49,23 \\
\hline 20. & limósa Tr. & 2746 & 3231. & " 38,8 \\
\hline 21. & Graeilipálpus ephiáltes Hb. & 2039 & $236 \mathrm{r}$ & \\
\hline 22. & Epizeúxis ealvária F. $q$ & 2756 & 3241. & $\because 38,11 \mathrm{a}, \mathrm{b}$ \\
\hline 23. & Zanelógnatha tarsiplumális $\mathrm{Hb}$. $q$ & 2765 & $325 \mathrm{r}$. & $" 38,12$ \\
\hline 24. & tarsieristális HS. & 2771 & $326 \mathrm{r}$ & Nachtr.-Taf. IV, 40 \\
\hline 25. & tarsipennális $\mathrm{Tr} . \sigma^{7}$ & 2766 & $325 / 26$ & \\
\hline 26. & $"$ " ab. bidentális Hein. & $2766 \mathrm{a}$ & 3261. & \\
\hline 27. & Standfússia emortuális Sehiff. & 2781 & 3271. & Taf. $38,14 \mathrm{a}, \mathrm{b}$ \\
\hline
\end{tabular}


Wa 6 4)
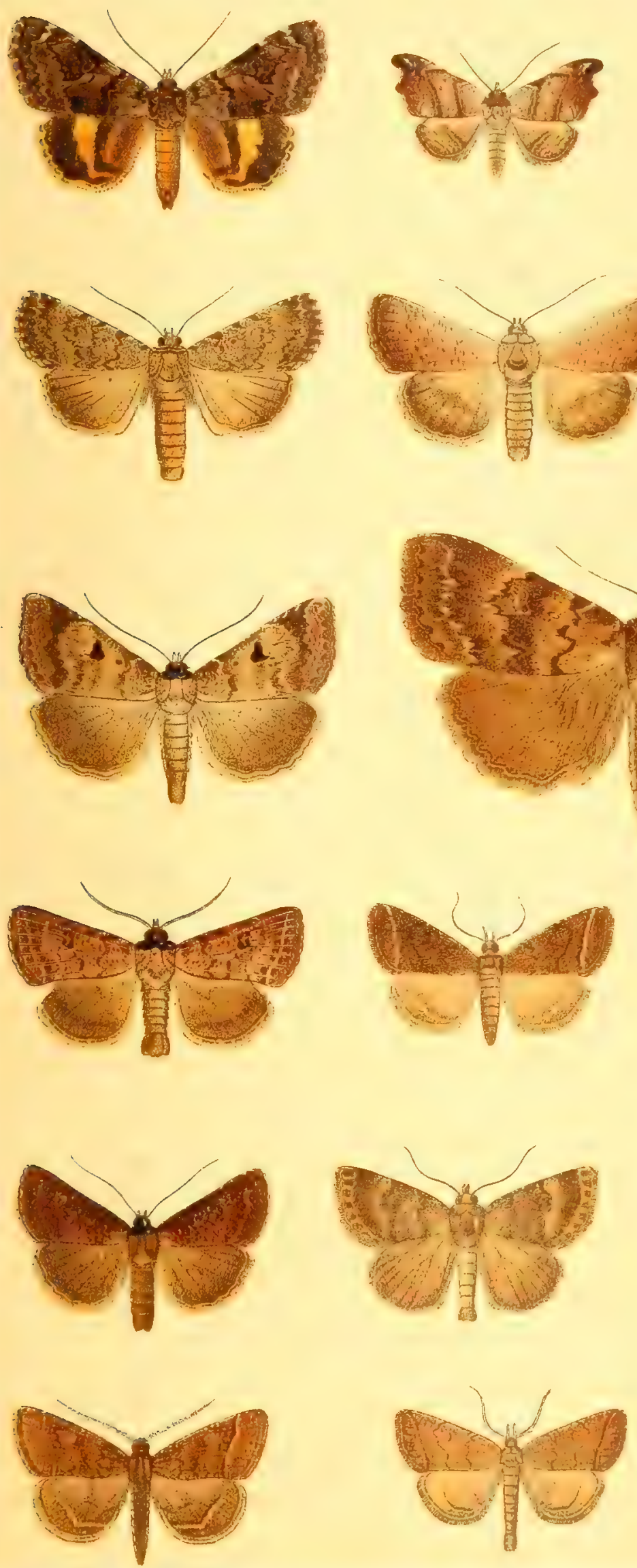
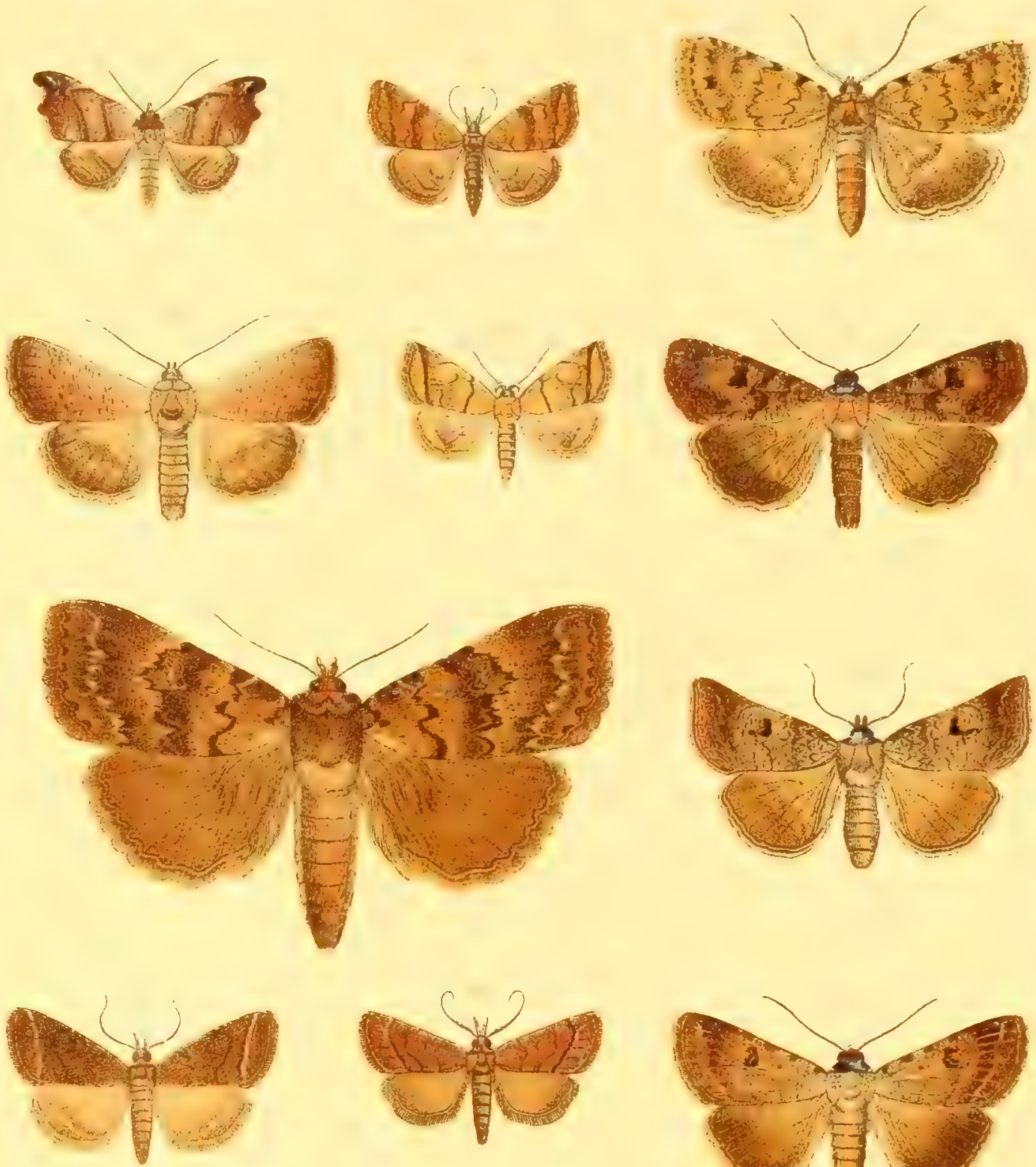
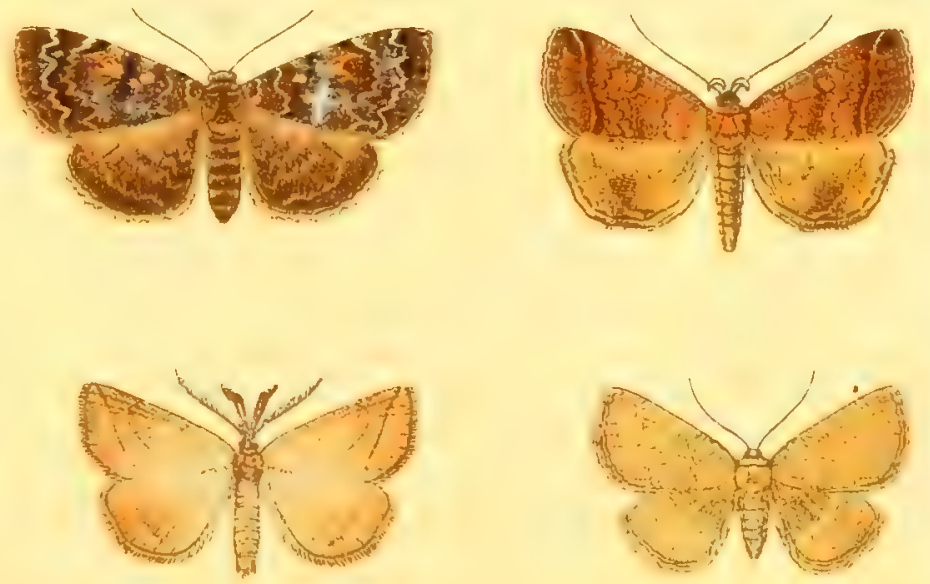




\section{TAFEL 55.}

\begin{tabular}{|c|c|c|c|}
\hline Nr. Name & Katalog I & Seite des I. Bandes & $\begin{array}{l}\text { Raupen- (Puppen-) } \\
\text { Abbildung }\end{array}$ \\
\hline 1. Hermínia eribrális Hb. $\sigma^{7}$ & 2795 & $327 / 28$ & \\
\hline$"$ gryphális HS. न & 2799 & 3281. & \\
\hline$" \quad$ erinális Tr. $\sigma^{7}$ & 2797 & 3281. & Taf. 38,16 \\
\hline tentaculápia L. & 2801 & 3281. & \\
\hline derivális $\mathrm{Hb}$. & 2800 & $328 \mathrm{r}$. & Nachtr.-Taf. IV, 41 \\
\hline 7. Pechypógon barbális $\mathrm{Cl}$. & 2803 & 3291. & Taf. 38,17 \\
\hline 8. Bomólocha fontis Thnbg & 2804 & $329 \mathrm{r}$. & $" 38,18 \mathrm{a}, \mathrm{b}$ \\
\hline 9. Hypéna antiquális $\mathrm{Hb}$. & 2825 & $330 \mathrm{l}$. & \\
\hline$" \quad$ ravális HS. & 2824 & 3311. & \\
\hline lividális $\mathrm{Hb}$. & 2820 & 3311. & \\
\hline rostrális $\mathrm{L}$. & 2819 & $330 / 31$ & $" 38,19$ \\
\hline proboscidális L. & 2814 & 3301. & $" 38,20$ \\
\hline$" \quad$ munitális $\mathrm{Mn}$ & 2811 & $329 / 30$ & \\
\hline " palpális $\mathrm{Hb}$. & 2815 & 3301. & \\
\hline " obesális Tr. & 2816 & 3301. & Nachtr.-Taf. IV, 42 \\
\hline 17 a. " obsitális Hb. $\sigma^{7}$, b. $q$ & 2818 & $330 \mathrm{r}$. & \\
\hline 18. Hypenódes costaestrigális Stph. & 2828 & 3321. & \\
\hline$n \quad$ taeniális $\mathrm{Hb}$. & 2827 & $331 / 32$ & \\
\hline 20. Tholómiges turfosális Wek. & 2831 & $332 \mathrm{r}$. & \\
\hline 21. Oréctis proboseidáta HS. & 2826 & $331 \mathrm{r}$. & \\
\hline 22. Rívula sericeảlis Se. & 2475 & $294 / 95$ & \\
\hline 23. Brephos parthénias L. & 2854 & $336 / 37$ & Taf. $38,22 \mathrm{a}, \mathrm{b}$ \\
\hline notha $\mathrm{Hb}$. & 2856 & 3371. & $" 38,23 \mathrm{a}, \mathrm{b}$ \\
\hline$" \quad$ puélla Esp. & 2858 & $337 \mathrm{r}$. & $" \quad 38,24$ \\
\hline 26. Catóeala adúltera Mén.*) & 2679 & 3161. & \\
\hline 27. Toxocámpa lúbrica Frr & 2740 & 3221. & \\
\hline glyeyrrhízae Rbr 우 & 2736 & 3221. & \\
\hline 29. Apopéstes hirsúta Stgr & 2726 & $320 \mathrm{r}$. & \\
\hline exsiceáta Ld. & 2732 & $320 \mathrm{r}$ & \\
\hline 31. Leueanitis pieta Chr. & 2624 & $310 \mathrm{l}$. & \\
\hline 32. Zanclógnatha tenuiális $\mathrm{Rbl} \sigma^{7}$ & 2775 & $326 / 27$ & \\
\hline 33. Hypenódes kalehbérgi Stgr & 2830 & 3321. & \\
\hline 34. Hypéna ravulális Stgr & 2822 & 3311. & \\
\hline 35. Madópa salicális Schiff. & 2790 & $327 \mathrm{r}$. & $\eta \quad 38,15 a-c$ \\
\hline
\end{tabular}

*) Siehe Taf. 78, Fig. 18. 


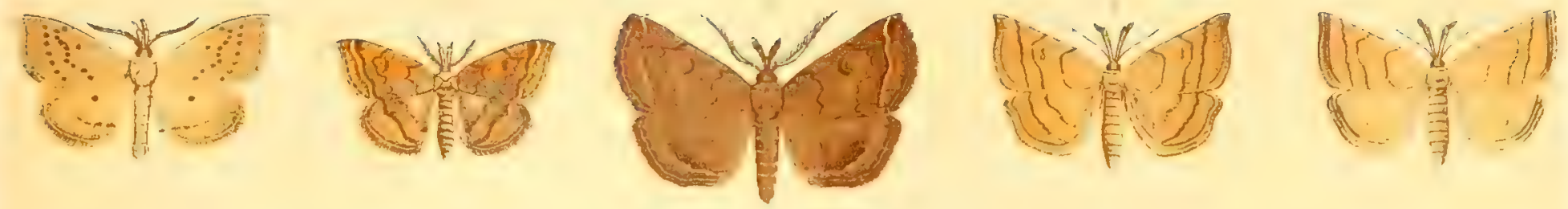

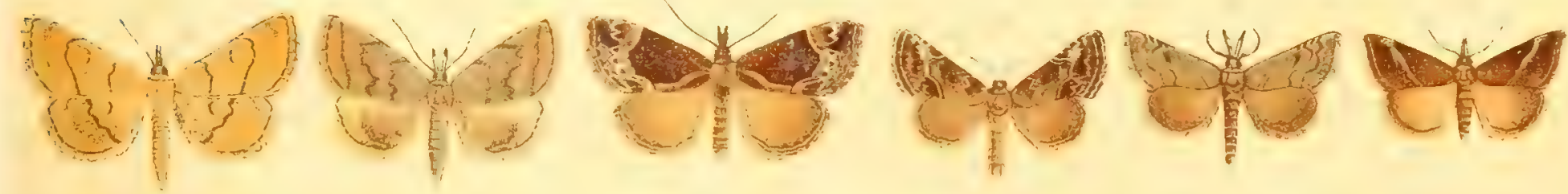
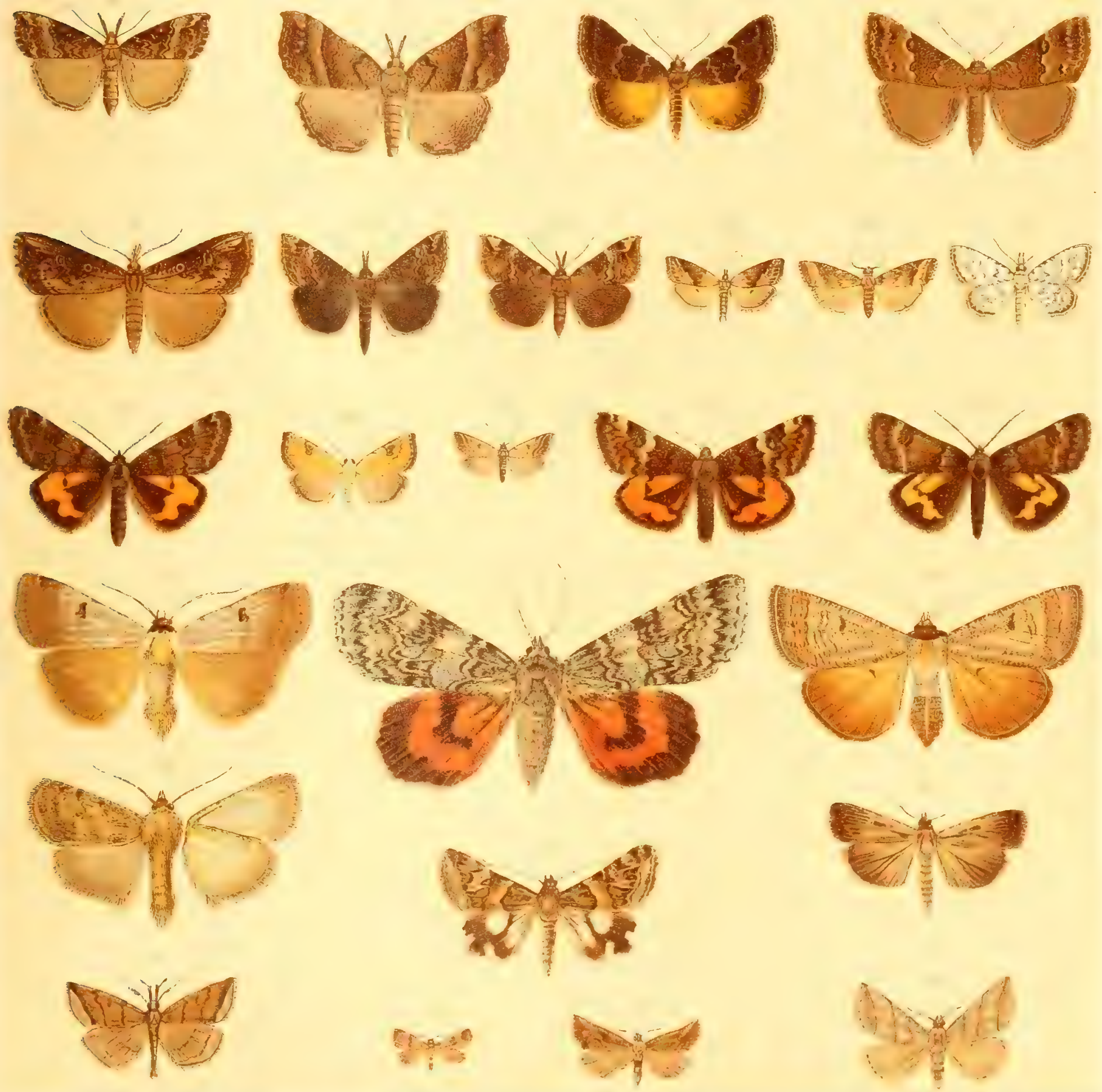




\section{TAFEL 56.}

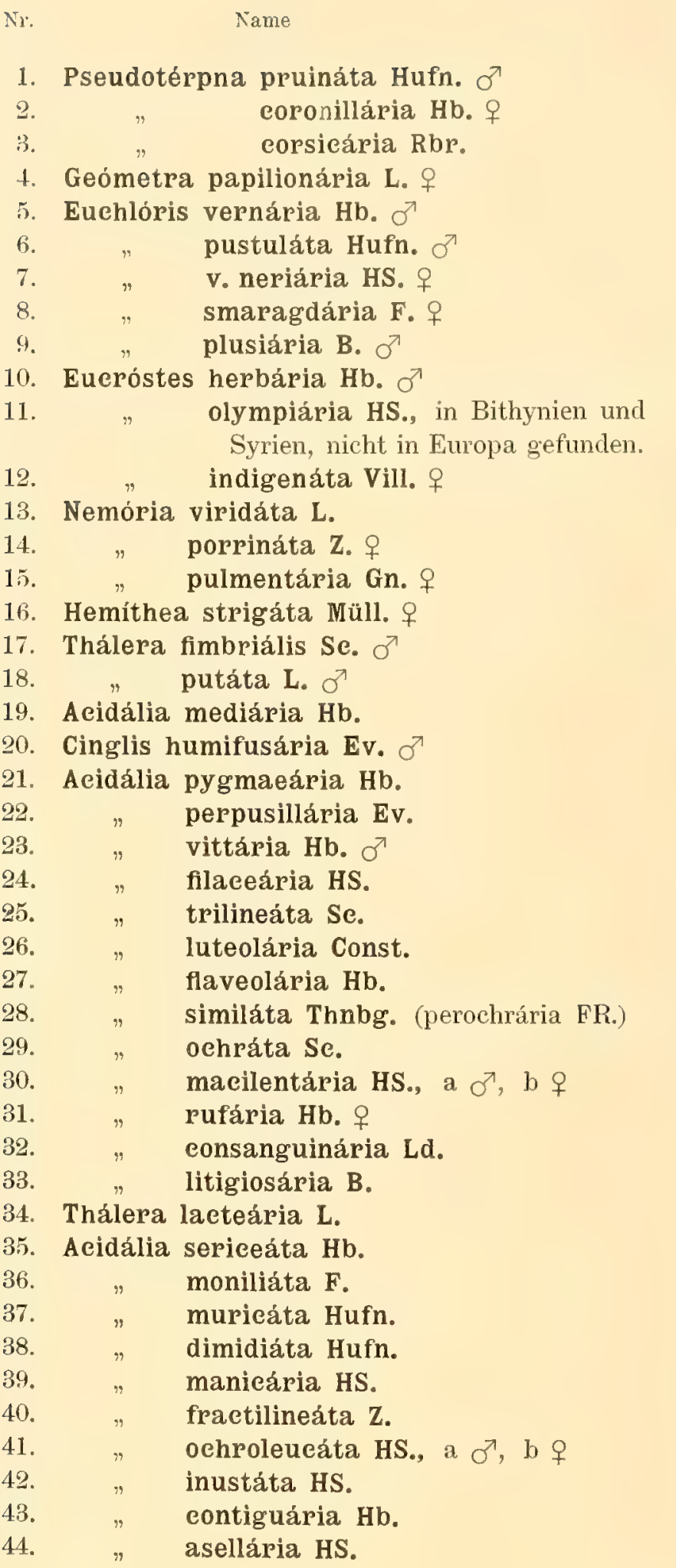

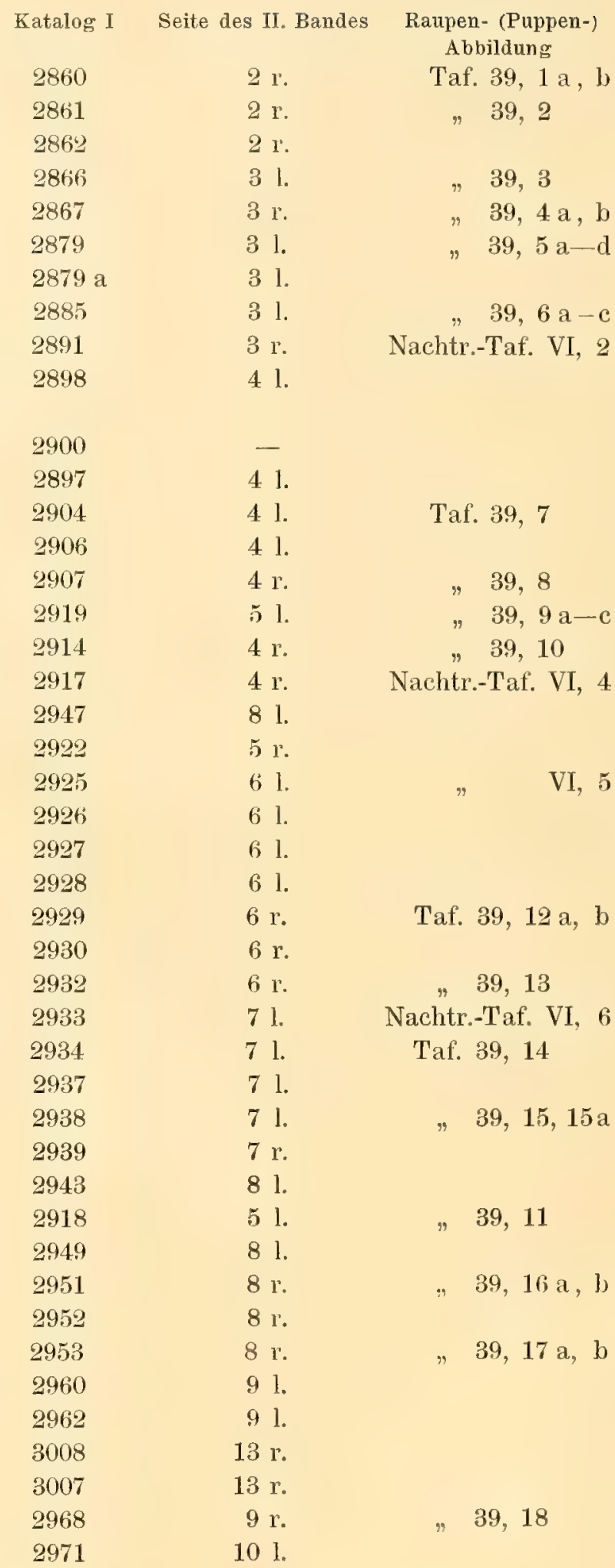

Siehe ferner: Euchlór. imparária Gn. Taf. 71 a, Fig. 1; Euer. beryllária Mn. ib., Fig. 2; Nem. faustináta Mill. ib., Fig. 3; Acidália aceretáta Fuehs ib. Fig. 4; Ae. Pubelláta Rbr. ib., Fig. 5; Ac. geministrigáta Fuehs ib. Fig. 6; Ac. lambessáta 0bthr. ib., Fig. 7 ; Ae. exilária Gn. ib., Fig. 8; Ae. fatimáta Stgr. ib., Fig. 9; Ac. consolidáta Ld. ib., Fig. 11 a; Ac. cervantária Mill. Taf. 71 b, Fig. 26 und Ae. isabellária Mill. Taf. 71 b, Fig. 27. 

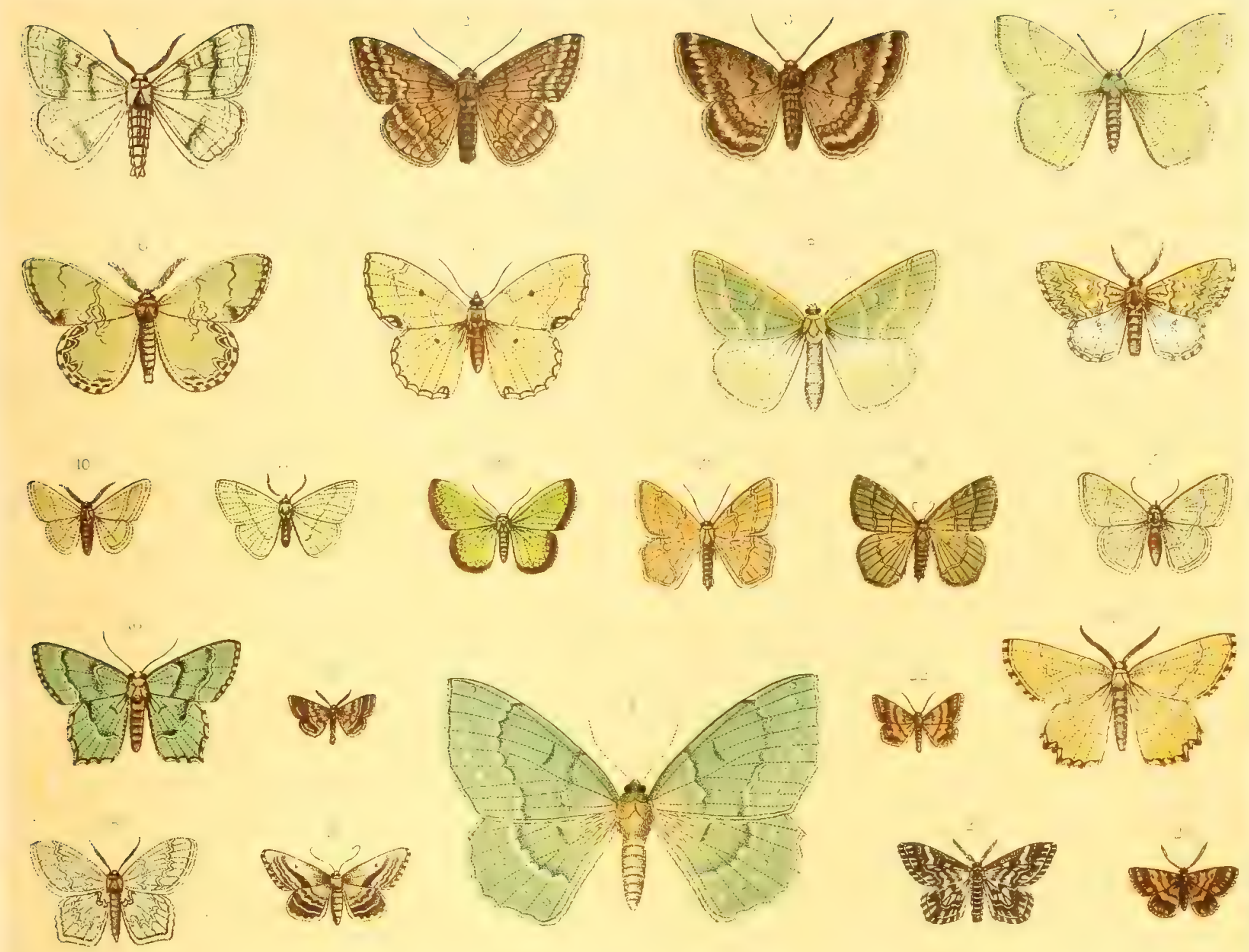

U D M
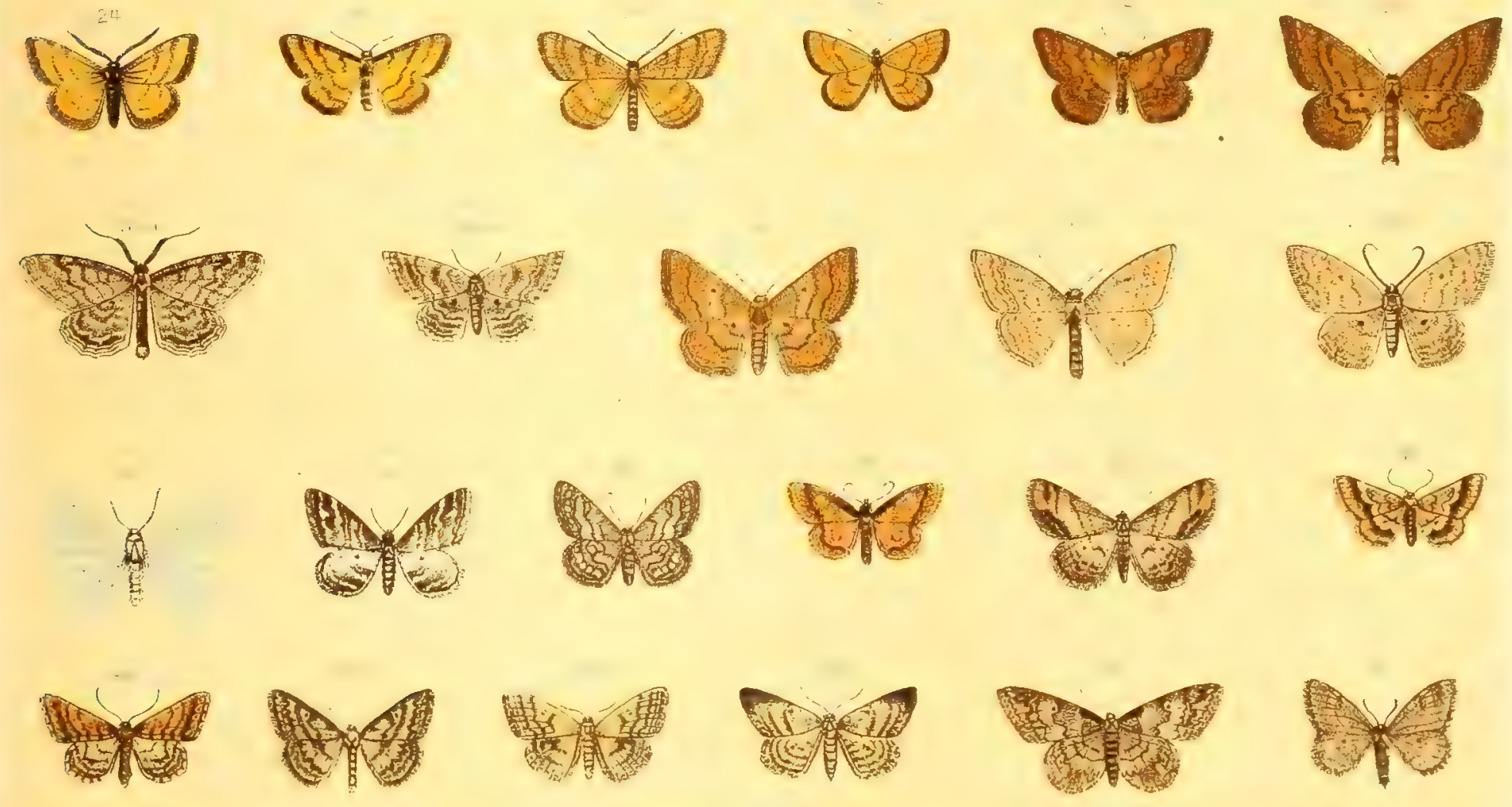




\section{TAFEL $5 \%$.}

\begin{tabular}{|c|c|c|c|c|c|}
\hline Nis. & & Name & Katalog I & Seite des II. Bandes & $\begin{array}{c}\text { Raupen- (Puppen-) } \\
\text { Abbildung }\end{array}$ \\
\hline 1. Ae & dális & a eampária HS. & 2980 & 111. & \\
\hline 2. & . & sodaliária HS. & 2981 & $11 \mathrm{r}$. & \\
\hline 3. & $"$ & nexáta $\mathrm{Hb}$. & 2923 & 61. & \\
\hline $4 \mathrm{a}, \mathrm{J}$. & $\eta$ & virgulária Hb. & 2983 & $11 / 12$ & Taf. 39,20 \\
\hline 5. & ", & stramináta Tr. & 2997 & $12 / 13$ & \\
\hline 6. & ", & pallidáta Bkh. & 2990 & $12 \mathrm{l}$. & Nachtr.-Taf. VI, 7 \\
\hline 7. & $"$ & subsericeáta $\mathrm{Hw}$. & 2995 & $12 \mathrm{r}$. & Taf. $39,21 \mathrm{a}, \mathrm{b}$ \\
\hline 8. & $"$ & laevigáta Se. & 3002 & 131. & $" \quad 39,19$ \\
\hline 9. & $"$ & extarsária HS. & 3003 & $13 \mathrm{r}$. & \\
\hline 10. & " & subsaturáta Gn.*) & 2954 & 91. & \\
\hline 11. & $"$ & extarsária HS. v. eriopodáta Grasl. & $3003 \mathrm{a}$ & $13 \mathrm{r}$. & \\
\hline 12. & $"$ & attenuária $\mathrm{Rbr}$ & 3005 & $13 \mathrm{r}$. & \\
\hline 13. & $"$ & infirmária $\mathrm{Rbr}$ & 3006 & $13 \mathrm{r}$. & \\
\hline 14. & $"$ & helianthemáta Mill.**) & 3014 & $14 \mathrm{r}$ & \\
\hline 15. & $"$ & infirmária Rbr v. aquitanárla Const. & $3006 \mathrm{a}$ & $13 \mathrm{r}$. & \\
\hline 16. & $n$ & obsoletária Rbr & 3010 & 141. & \\
\hline 17. & 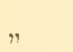 & inearnária HS. & 3011 & 141. & Nachtr.-Taf. VI, 8 \\
\hline 18. & " & ostrinária $\mathrm{Hb}$. & 3016 & 151. & \\
\hline 19. & ." & cireuitária Hb. & 3017 & $15 \mathrm{l}$. & \\
\hline 20. & $"$ & hepbariáta F. & 3020 & $15 \mathrm{r}$. & VI, 9 \\
\hline 21. & , & eallunetária Stgr & 3022 & $15 / 16$ & \\
\hline 22. & $"$ & elongária Rbr & 3023 & 161. & \\
\hline 23. & 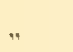 & bisetáta Hufn. & 3025 & 161. & \\
\hline 24. & $"$ & trigemináta $\mathrm{Hw}$. & 3026 & $16 \mathrm{r}$. & \\
\hline 25. & $"$ & politáta $\mathrm{Hb}$. & 3031 & $16 / 17$ & \\
\hline 26. & , & filieáta Hb. & 3032 & 171. & VI, 10 \\
\hline 27. & , & rusticáta F. & 3034 & $17 \mathrm{r}$. & Taf: 39,22 \\
\hline 28. & $"$ & humiliáta Hufn. & 3040 & $18 \mathrm{r}$. & $" \quad 39,23$ \\
\hline 29. & $"$ & interiectária B. & 3039 & 181. & \\
\hline 30. & $"$ & dileetária $\mathrm{Hb}$. & 3038 & $18 \mathrm{l}$. & $" 39,24 \mathrm{a}, \mathrm{b}$ \\
\hline 31. &. & diffluáta HS. & $3047 \mathrm{a}$ & $20 \mathrm{r}$. & \\
\hline 32. &. & degenerária Hb. & 3043 & 191. & $" 39,25 \mathrm{a}, \mathrm{b}$ \\
\hline 33. & . & inornáta $\mathrm{Hw}$. & $3046^{\circ}$ & 201 & \\
\hline $34 a$. & , & aversáta L. & 3048 & $20 / 21$ & $\eta \quad 39,26 \mathrm{a}, \mathrm{b}$ \\
\hline $34 \mathrm{~b}$. & $"$ & " $\quad$ v. spoliáta Stgr & 3048 a & $20 \mathrm{r}$ & \\
\hline 35. &. & emargináta L. & - 3050 & 211. & Nachtr.-Taf. VI, 11 \\
\hline 36. & . & immoráta L. & 3051 & 211. & Taf. $39,27 \mathrm{a}, \mathrm{b}$ \\
\hline 37. & $"$ & tessellária B. & $3051 \mathrm{a}$ & $21 x$ & \\
\hline 38. & $"$ & rubigináta Hufn. & 3053 & $21 \mathrm{r}$. & $" 39,28$ \\
\hline 39. & $"$ & turbidária HS. & 3054 & $21 \mathrm{r}$ & \\
\hline 40. & $"$ & albieerária HS. & 3059 & $21 / 22$ & \\
\hline 41. & $"$ & marginepunetáta Goeze & 3064 & 221. & $" 39,29 \mathrm{a}, \mathrm{b}$ \\
\hline 42. & $"$ & luridáta $\mathrm{Z}$ & 3066 & $22 / 23$ & \\
\hline 43. & $"$ & " $\quad$ v. rufomixtáta Rbr & $3066 \mathrm{~d}$ & $22 \mathrm{r}$ & \\
\hline 44. & $"$ & submutáta Tr. & 3068 & $22 \mathrm{r}$ & $" \quad 40,1$ \\
\hline 45. & $"$ & incanáta L. & 3069 & 221. & \\
\hline
\end{tabular}

Siehe ferner: Ac. fathmária 0bth. Taf. 71 a, Fig. 11; longária HS. ib., Fig. 10 ; eugeniáta Mill. ib., Fig. $11 \mathrm{~b}$; belemiáta Mill. Taf. $71 \mathrm{~b}$, Fig. 25; pobigináta Stgr Taf. $71 \mathrm{a}$, Fig. 12 ; lutulentária Stgr ib., Fig. 13; nitidáta HS. ib., Fig. 14; failláta Fuchs ib., Fig. 15; rubrária Stgr. ib., Fig. 16; v. bilineária Fuehs ib., Fig. 16 a deversária HS. ib., Fig. 17; beckerária Ld. ib., Fig. 18 und eoenosária Ld. ib., Fig. 19.

*) Vfl.-Spitze zu stumpf.

**) Zu_rot, Hfl. zu rund, Querlinien der Saumfelder recht mangelhaft. 
1

4 a

$4 b$

5

6

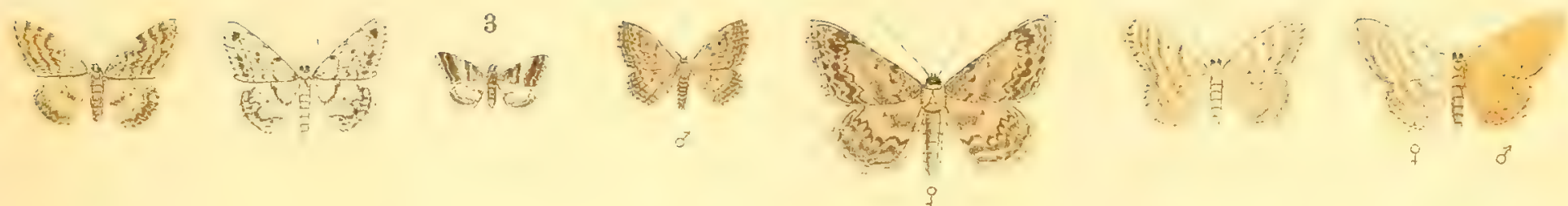

7

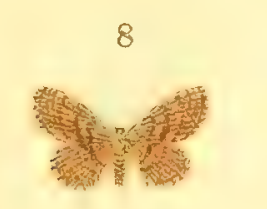

10

11

12

13

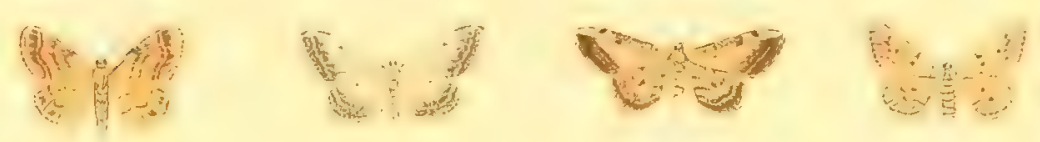

17

(Giveng)

$-17$

18

19

(1)

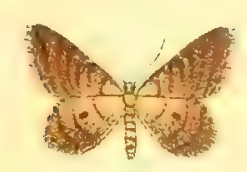

21

22

23

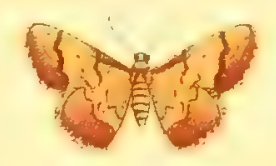

$(10)$

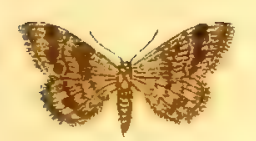

Non

Nution

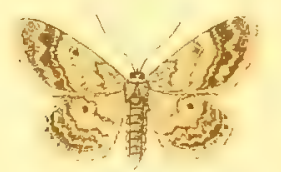

24

25

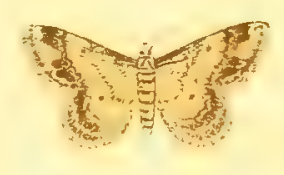

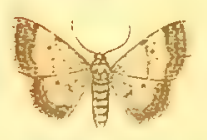

26

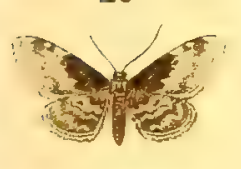

27

28

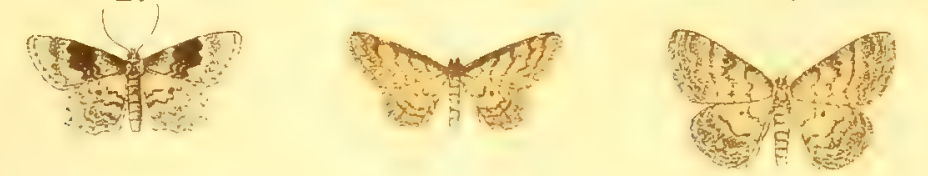

29

30

31

(1)
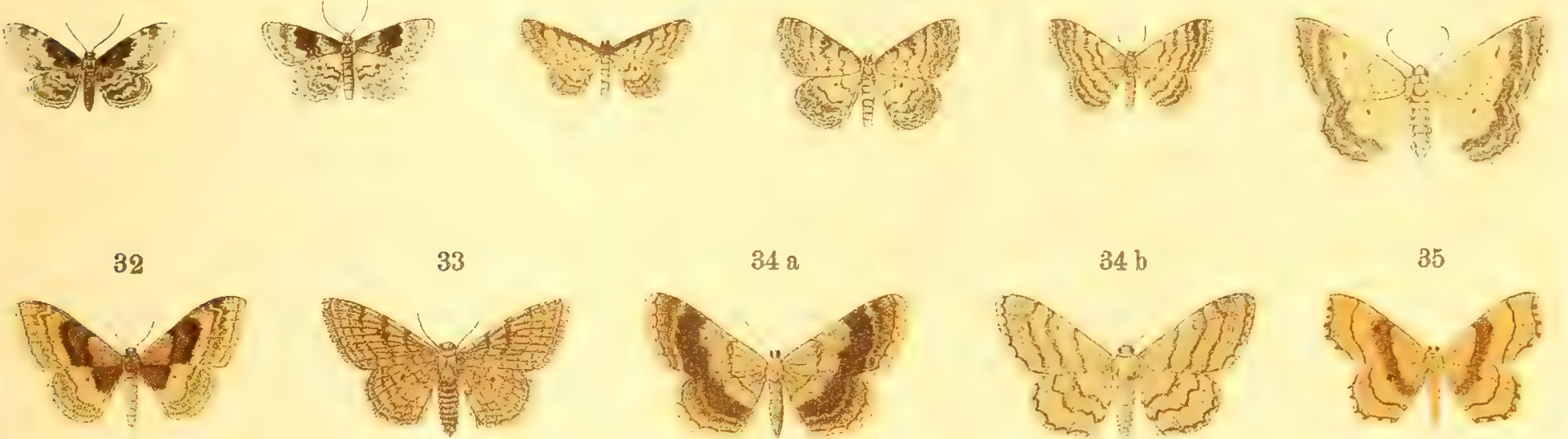

33
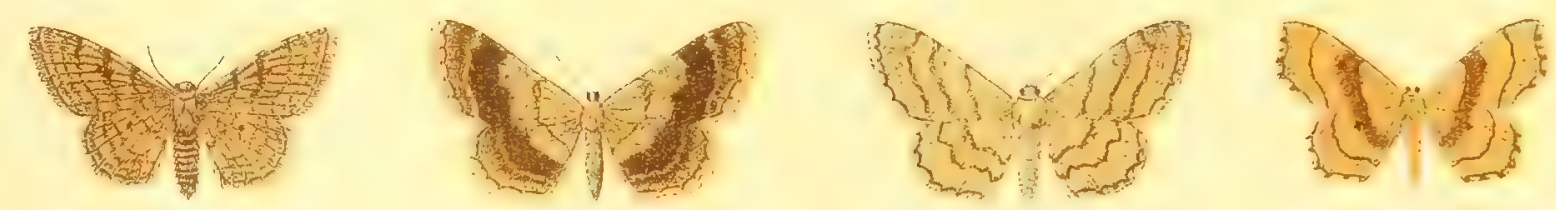

36

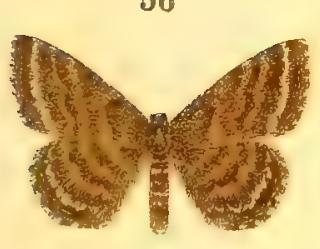

37

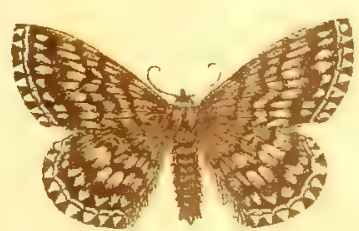

38
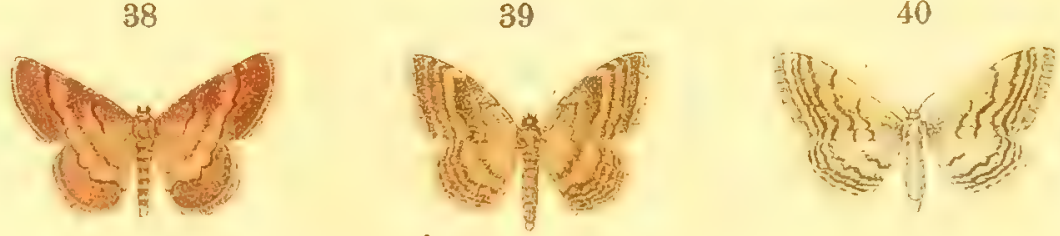

$\Delta 1$

$4: 2$

43
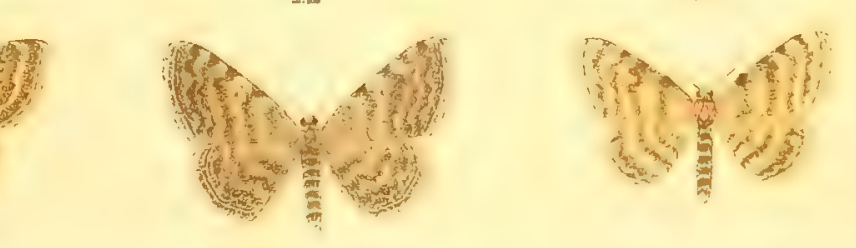

44

45
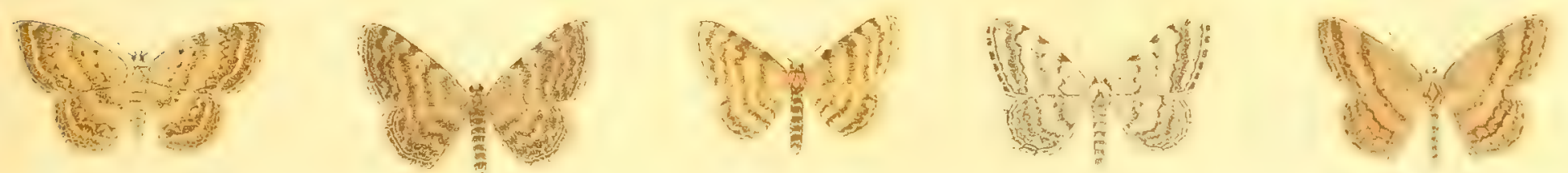




\section{TAFEL 58.}

\begin{tabular}{|c|c|c|c|c|c|}
\hline $\mathrm{Nr}$. & & Name & Katalog I & Seite des II. Bandes & $\begin{array}{c}\text { Raupen-(Puppen-) } \\
\text { Abbildung }\end{array}$ \\
\hline 1. & Aeidália & fumáta Stph. & 3072 & 231. & \\
\hline 2. & ." & remutária $\mathrm{Hb}$. & 3074 & $23 \mathrm{r}$ & Taf. $40,2 \mathrm{a}, \mathrm{b}$ \\
\hline 3. & $"$ & nemorária Hb. & 3077 & $23 r$. & \\
\hline 4. & $"$ & punetáta Se. & 3078 & 23 r. & \\
\hline 5. & $"$ & carieária Reutti & 3079 & 241. & $" 40,3$ \\
\hline 6. & $"$ & immutáta L. & 3081 & 241. & $" 40,4$ \\
\hline 7. & $"$ & corrivallária Kretsehmar & 3082 & $24 \mathrm{r}$ & \\
\hline 8. & $"$ & strigária Hb. & 3083 & $24 \mathrm{r}$. & Taf. $40,5 \mathrm{a}, \mathrm{b}$ \\
\hline 9. & $"$ & umbellápia $\mathrm{Hb}$. & 3085 & 251. & $\Rightarrow 40,6 \mathrm{a}, \mathrm{b}$ \\
\hline 10. & $"$ & strigillária Hb. & 3086 & 251. & $\because 40,7$ \\
\hline 11. & $"$ & emutária Hb. & 3090 & $25 \mathrm{r}$. & $\Rightarrow \quad 40,8$ \\
\hline 12. & $"$ & flaceidária Z. & 3091 & $25 \mathrm{r}$. & \\
\hline 13. & $"$ & imitária Hb. & 3093 & $25 \mathrm{r}$ & \\
\hline 14. & $"$ & coneinnária Dup. & 3094 & 261. & \\
\hline 15. & $"$ & ornáta Se. & 3095 & 261. & $" 40,9 \mathrm{a}, \mathrm{b}$ \\
\hline 16. & $"$ & eongruáta $\mathrm{Z}$. & 3096 & 261. & \\
\hline 17. & $"$ & v. decoráta Bkh. & $3097 \mathrm{a}$ & 261. & $" 40,10 \mathrm{a}, \mathrm{b}$ \\
\hline 18. & Codónia & pendulária $\mathrm{Cl}$. & 3108 & 271. & $" 40,11$ \\
\hline 19. & $"$ & orbiculária Hb. & 3109 & 271. & Nachtr.-Taf. VI, 12 \\
\hline 20. & $"$ & annuláta Sehulze & 3111 & $27 \mathrm{r}$. & VI, 14 \\
\hline 21. & $"$ & albiocellária Hb. & 3110 & $27 \mathrm{r}$ & VI, 13 \\
\hline 22. & $"$ & pupillária Hb. & 3112 & $27 \mathrm{r}$. & \\
\hline 23. & $"$ & popáta F. & 3113 & 27 r. & VI, 15 \\
\hline 24. & $"$ & punetária L: & 3115 & 281. & Taf. 40,12 \\
\hline 25. & $"$ & lineária Hb. & 3117 & $28 \mathrm{r}$. & \\
\hline 26. & Timándr & ra amáta L. $\sigma^{7}$ & 3139 & 291. & $" 40,13$ \\
\hline 27. & Oehodón & atia adustária F. d. W. & 3142 & $29 \mathrm{r}$. & Nachtr.-Taf. VI, 16 \\
\hline 28. & Rhodostr & róphia vibieária $\mathrm{Cl}$. & 3122 & $28 \mathrm{r}$ & Taf. 40,14 \\
\hline 29. & $"$ & sieanária $\mathrm{Z}$. & 3123 & 291. & \\
\hline 30. & $"$ & ealabrária $\mathrm{Z}$. & 3124 & 291. & $" 40,15$ \\
\hline 31. & Ariehánn & Ina melanária L. $\sigma^{\top}$ & 3691 & 87 r. & $" 40,16$ \\
\hline 32. & Abráxas & s grossulariáta L. & 3697 & 881. & $n \quad 40,17$ \\
\hline 33. & $"$ & pantária L. & 3699 & $88 \mathrm{r}$. & $" 40,18$ \\
\hline 34. & $"$ & sylváta Se. & 3698 & 881. & $" 40,19$ \\
\hline 35. & $n$ & adustáta Sehiff. & 3701 & $88 \mathrm{r}$. & $" 40,20$ \\
\hline 36. & $"$ & margináta $\mathrm{L}$. & 3700 & $88 \mathrm{r}$. & $\Rightarrow 40,21$ \\
\hline 37. & Opthostí & íxis eribrária $\mathrm{Hb}$. & 3683 & 861. & \\
\hline 38. & Bapta pi & oietária Curt. & 3702 & 891. & Nachtr.-Taf. VIII, 21 \\
\hline
\end{tabular}

Siehe ferner: Acidália frigidária vo sehoyéni Sp.-Sehneid. Taf. 71 a, Fig. 20; Ac, coprivallária Kretsehm. auch Taf. 71 a, Fig. 21; Ae. subtiláta Chr. Taf. 71 b, Fig. 28, Cod. v. lennigiária Fuchs Taf. 71 a, Fig. 24 c; Cod. quereimontária Bastelb. ibid., Fig. 23; Cod. rufieiliária HS. ib., Fig. 24 a, bo und Eusárea interpunetária HS. Taf. 71 b, Fig. 1. 

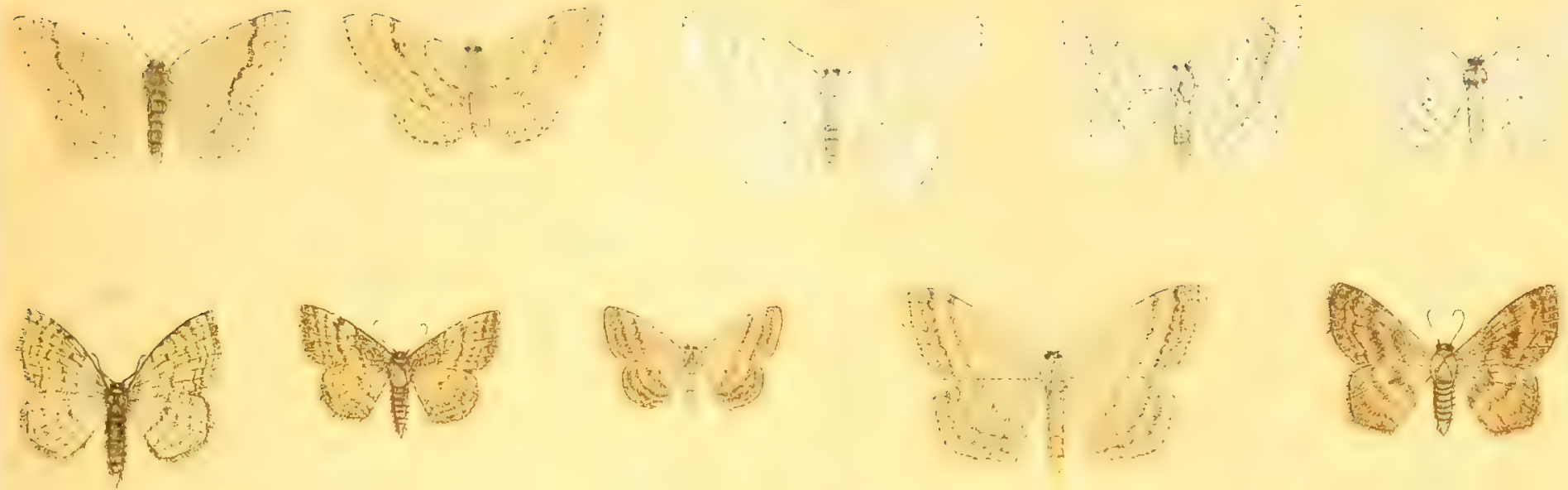

De lo dis and

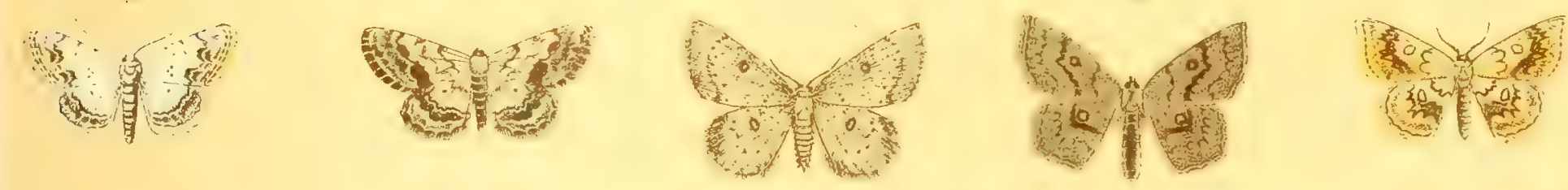
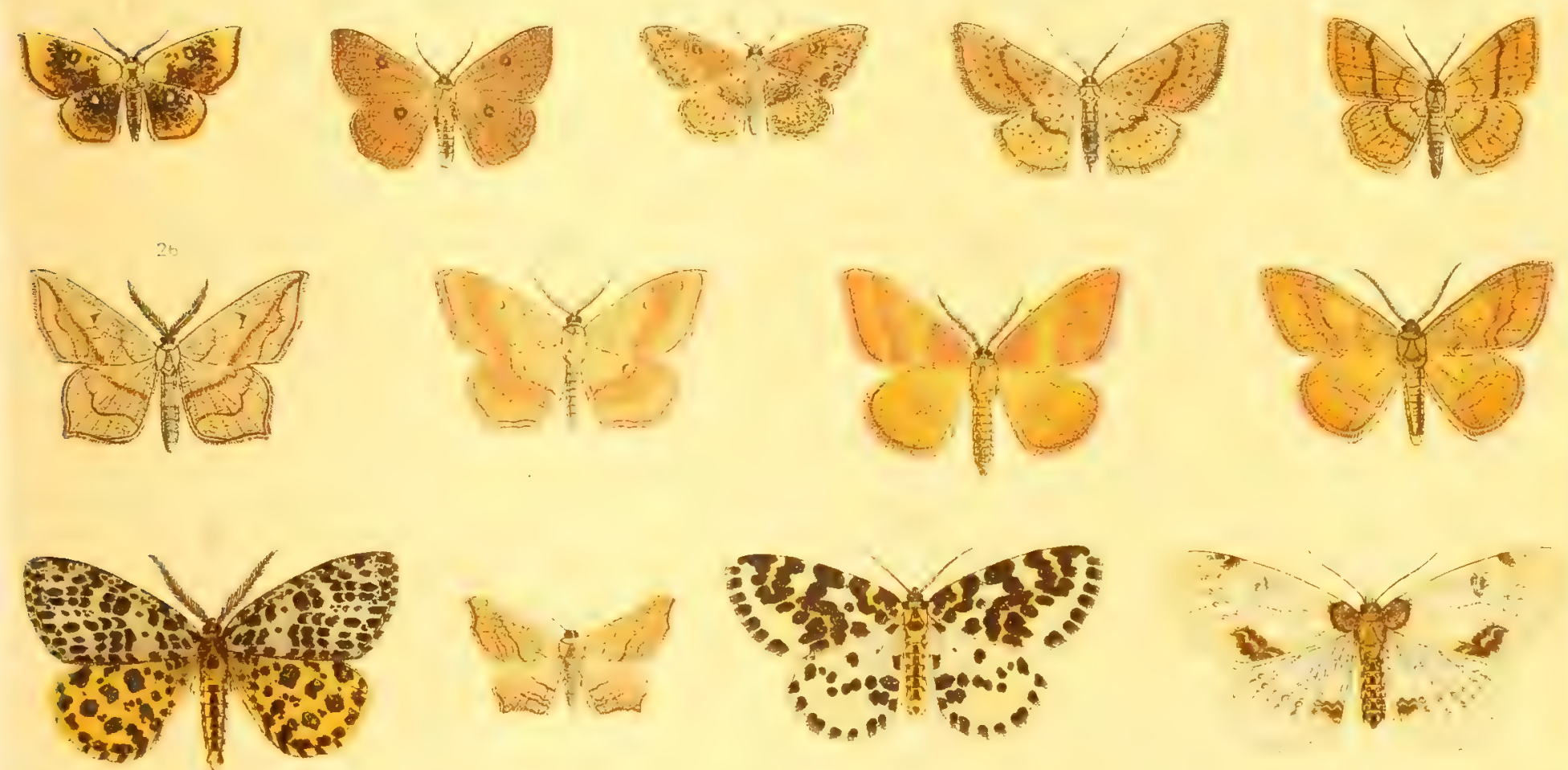

(a)
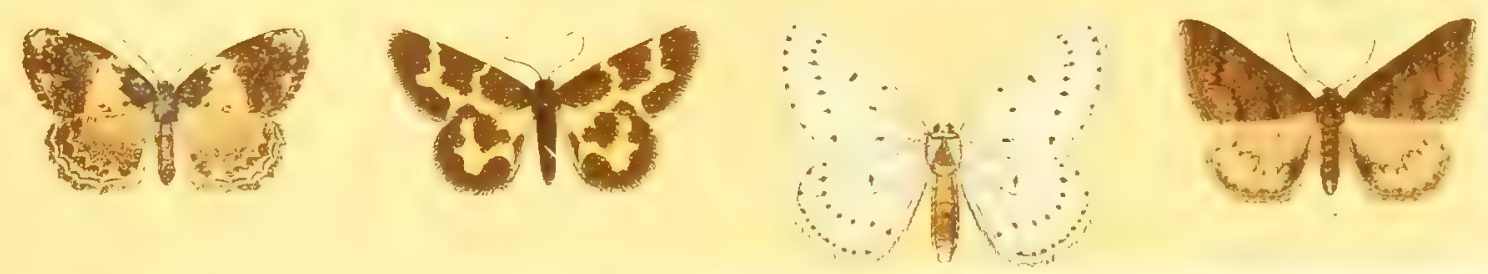




\section{TAFE L 59.}

\begin{tabular}{|c|c|c|c|c|}
\hline $\mathrm{Nr}$ & Name & Katalog I & Seite des II. Bandes & $\begin{array}{c}\text { Raupen-(Puppen-) } \\
\text { Abbildung }\end{array}$ \\
\hline 1. & Bapta bimaeuláta F. & 3703 & 891. & \\
\hline 2. & " temeráta $\mathrm{Hb}$. & 3704 & 891. & 'Taf. 40,22 \\
\hline 3. & Stegánia trimaeuláta Vill. & 3707 & 891. & $" \quad 40,23$ \\
\hline 4. & " dilectária $\mathrm{Hb}$. & 3708 & $89 r$ & Nachtr.-Taf. VI, 31 \\
\hline 5. & " earária $\mathrm{Hb}$. & 3709 & $89 \mathrm{r}$. & \\
\hline 6. & Deilínia pusária L. & 3713 & $89 \mathrm{r}$. & Taf. 40,24 \\
\hline 7. & " exanthemáta Se. & 3714 & 901. & Nachtr.-Taf. VI, 32 \\
\hline 8. & Numéria pulverária L. & 3715 & 901 & Taf. 40,25 \\
\hline 9. & " capreolária F. & 3716 & 901. & \\
\hline 10. & Ellópia prosapiária L. & 3720 & $90 \mathrm{r.}$ & $\eta \quad 41,1$ \\
\hline 11. & Gnophos benesignáta Bell. & 3958 & $109 \mathrm{r}$. & \\
\hline 12. & Metrocámpa margaritária L. & 3723 & $90 \mathrm{r}$ & $" 41,2$ \\
\hline 13. & " honorária Sehiff. & 3724 & 911. & $" 41,3$ \\
\hline 14. & Énnomos quereinária Hufn. & 3726 & $91 \mathrm{r.}$ & $n \quad 41,4$ \\
\hline 15. & " autumnária Wernb. & 3725 & 911. & $" 41,5$ \\
\hline 16. & " alniária L. & 3727 & $91 \mathrm{r}$ & \\
\hline 17. & " fuscántária Frr. & 3728 & $91 \mathrm{r}$ & Nachtr.-Taf. VI, 33 \\
\hline 18. & " erosária $\mathrm{Hb}$. & 3730 & 921. & Taf. 41,6 \\
\hline 19. & " quercária $\mathrm{Hb}$. & 3731 & 921. & Nachtr.-Taf. VI, 34 \\
\hline 20. & Selénia bilunária Esp. & 3733 & $92 \mathrm{r}$. & Taf. 41,7 \\
\hline 21. & " Iunápia Sehiff. & 3734 & $92 r$ & $\because 41,8$ \\
\hline 22. & $" \quad$ tetralunária Hufn. & 3735 & $92 r$ & $" \quad 41,9$ \\
\hline 23. & Hygrochróa syringária L. & 3736 & 931. & $" 41,10$ \\
\hline 24. & Therápis evonymária Sehiff. & 3738 & 931. & $" 41,11$ \\
\hline 25. & Gonodóntis bidentáta $\mathrm{Cl}$. & 3743 & $93 \mathrm{r}$. & $" \quad 41,12$ \\
\hline 26. & Hímera pennária L. & 3746 & $93 \mathrm{r}$. & $" 41,13$ \\
\hline
\end{tabular}

Siehe ferner: Stegan. dalmatária Gn. Taf. 71b, Fig. 2; Eum. regína Stgr. ib., Fig. 2 a und Dasyeeph. modésta Stgr. ib., Fig. 1 a. 


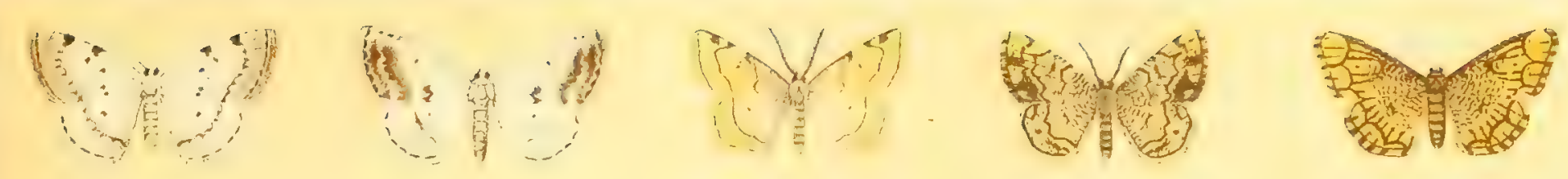
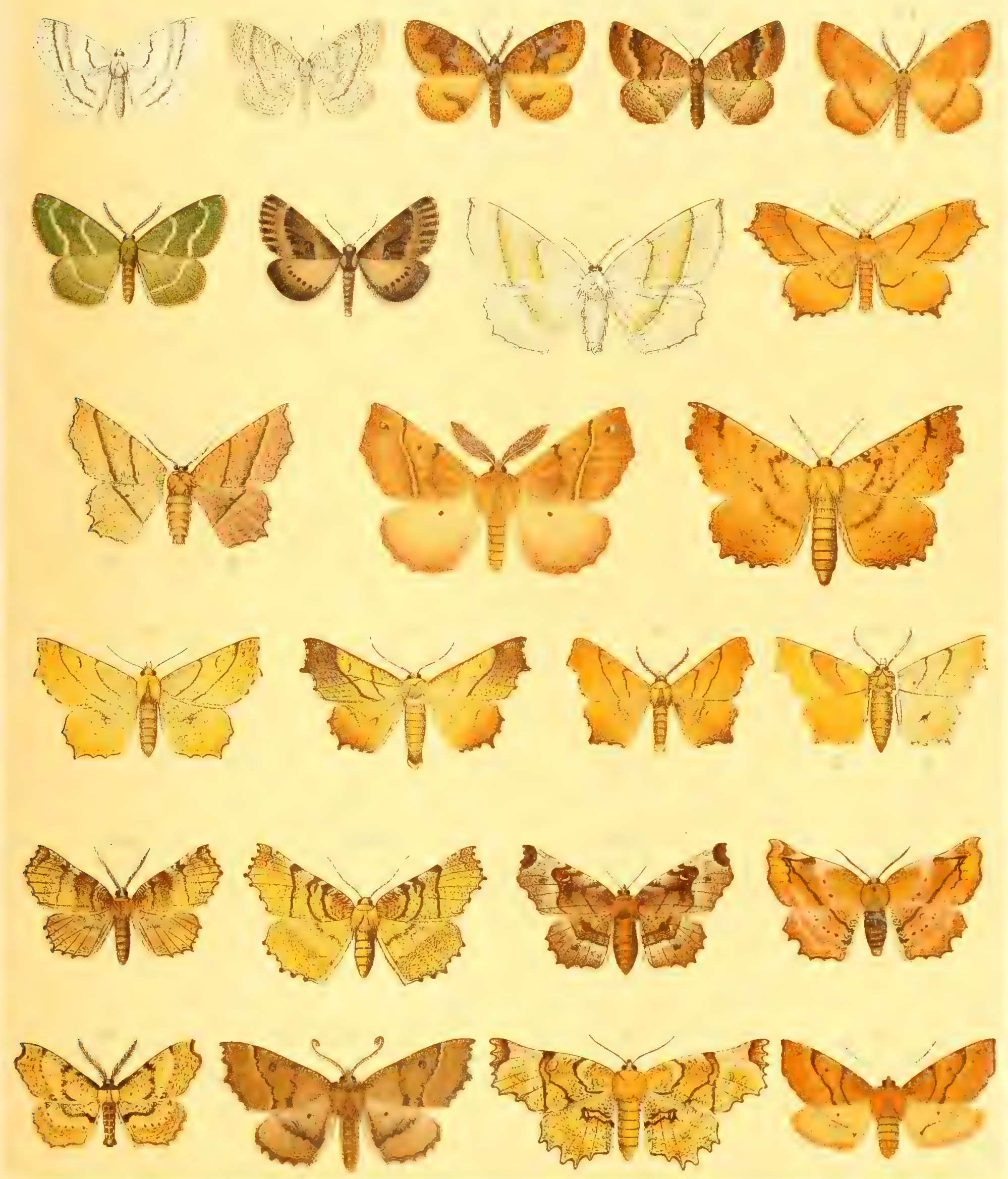




\section{TAFEL 60.}

$\mathrm{Nr}$.

1. Crocállis tusciária Bkh. $\sigma^{7}$

2. " elinguária L. ㅇ

3. "dordoinária Donz.

4. Euryméne dolabrária L. 우

5. Angeróna prunária L., a $q, \mathrm{~b} \sigma^{7}$

6. Urápteryx sambueária L. +

7. Opisthográptis luteoláta L.

8. Epíone apieiária Schiff.

9. " parallelária Sehiff.

10. $"$ advenária $\mathrm{Hb}$.

11. Hypopléetis adspersária $\mathrm{Hb}$.

12. Caustolóma flavieária $\mathrm{Hb}$.

13. Venília maculária L.

14. Eilierínia cordiária $\mathrm{Hb}$.

15. " trinotáta Metzner

16. Semiothísa notáta $\mathrm{L}$.

17. $"$ alternária $\mathrm{Hb}$.

18. " signária $\mathrm{Hb}$.

$19 . \quad$ aestimária $\mathrm{Hb}$.

20. " lituráta $\mathrm{Cl}$.

21. Epirránthis diversáta Gerning (pulveráta Thnbg.)

22. Chemerína ealigineária Rbr.

23. Lignyóptera fumidária $\mathrm{Hb}$.

24. Hibérnia rupieaprária $\mathrm{Hb}$.

25. " baiária Sehiff.

26. " Ieucophaeária Sehiff.

27. " aurantiária Esp.

28. " marginária $\mathrm{Bkh}$.
Katalog

3748

3749

3751

3760

3754

3757

3761

3763

3764

3765

3769

3771

3773

3777

3780

3782

3783

3785

3792

3790

3689

3690

3793

3794

3796

3997

3798

3799
Seite des II. Bandes

941.

$94 \mathrm{l}$.

$94 \mathrm{r}$.

$95 \mathrm{r}$.

$94 \mathrm{r}$.

951.

$95 \mathrm{r}$.

$95 \mathrm{r}$.

961.

$96 \mathrm{l}$.

961.

$96 \mathrm{r}$.

$96 \mathrm{r}$.

971

$97 \mathrm{l}$.

$97 \mathrm{r}$.

$97 \mathrm{r}$.

$97 \mathrm{r}$.

$97 \mathrm{r}$.

$97 \mathrm{r}$

$86 \mathrm{r}$.

87 l.

981.

$98 \mathrm{r}$.

$98 \mathrm{x}$.

$98 \mathrm{r}$.

$98 \mathrm{r}$.

991 .
, 42, 5

Nachtr.-Taf. VI, 40

Taf. 42,6

Nachtr.-Taf. VI, 41

Taf. 42,7

Raupen- (Puppen-)

Abbildung

Nachtr.-Taf. VI, 35

Taf. 41,14

Nachtr.-Taf. VI, 36

Taf. 41, 15

"41, 16

$" 41,17$

" 42, 1

Nachtr.-Taf. VI, 37

Taf. 42,2

Nachtr.-Taf. VI, 38

Taf. 42,3

Nachtr.-Taf. VI, 39

Taf. 42,4

Nachtr.-Taf. VI, 30 $\because \quad$ VIII, 20

"VI, 42

Taf. 42,8

"42, 9

Nachtr.-Taf. VI, 43

Taf. 42,10

" 42,11

Siehe ferner: Crocall. aubérti Obthr. Taf. 71 b, Fig. 1 b; Eilier. eauteriáta Stgr.ib., Fig. 1 c; Eil. subcordária HS. ib., Fig. 3 und Lignyopț. thaumastária Rbl. ib., Fig. 4. 

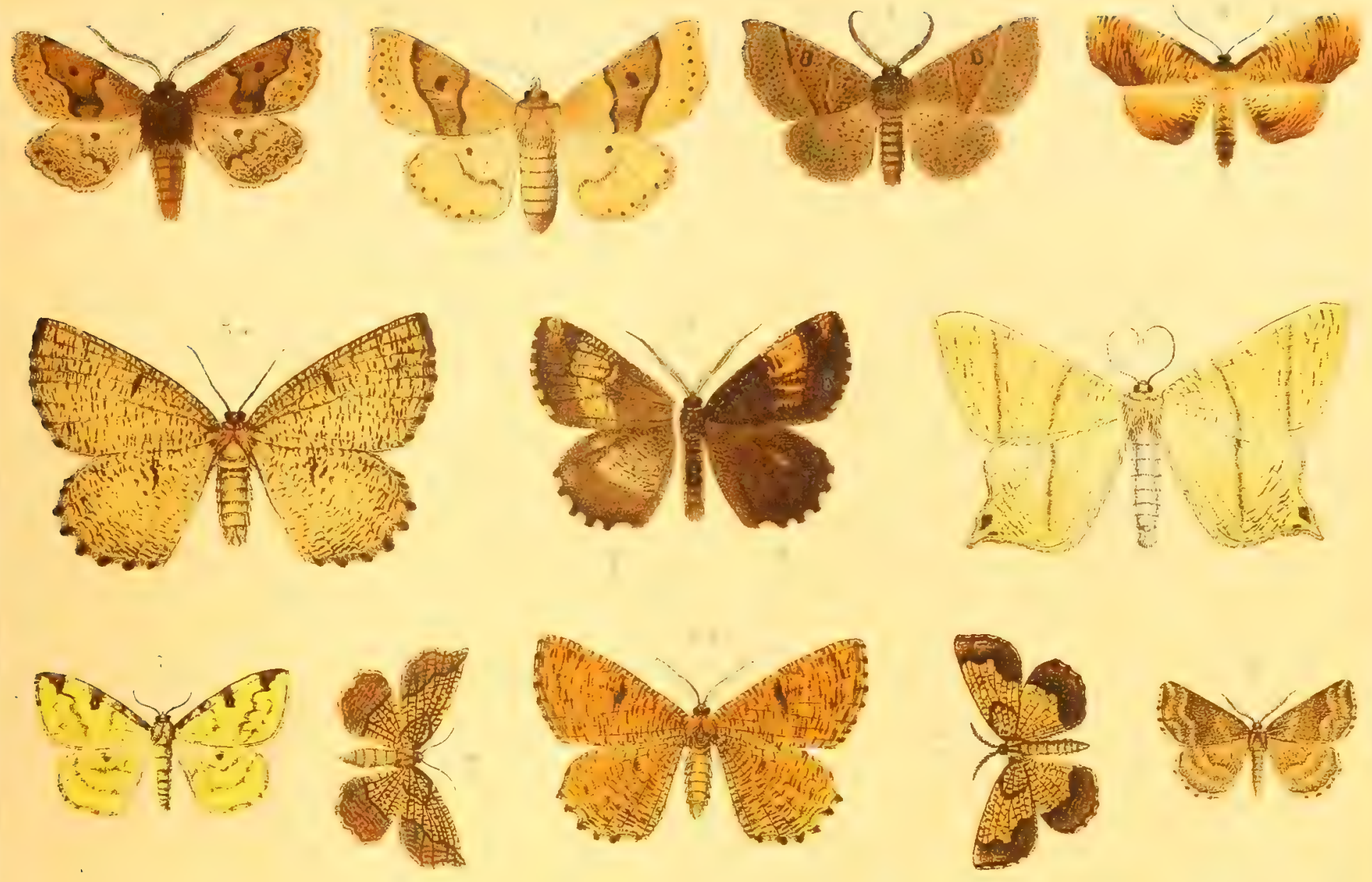

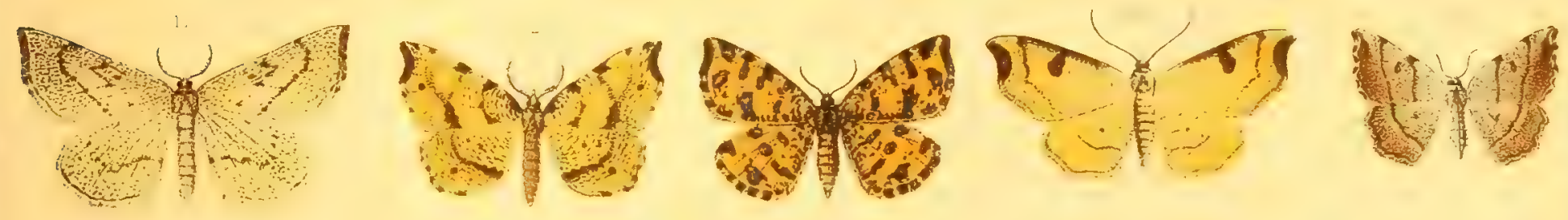
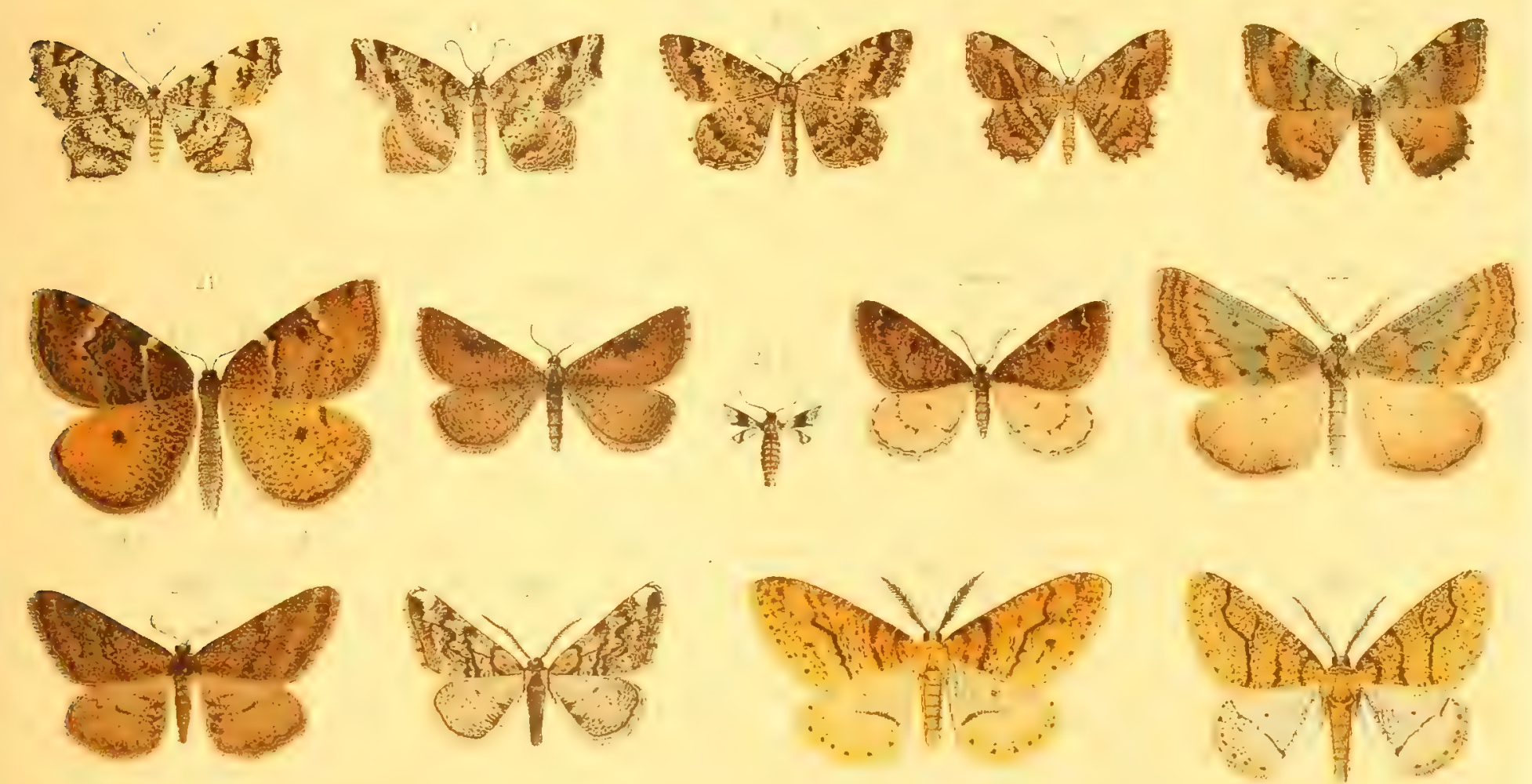




\section{TAFEL 61 .}

\begin{tabular}{|c|c|c|c|c|}
\hline $\mathrm{Nr}$ & Name & Katalog I & Seite des II. Bandes & $\begin{array}{c}\text { Raupen- (Puppen-) } \\
\text { Abbildung }\end{array}$ \\
\hline 1. & Hibérnia ankerária Stgr. & 3800 & 991. & \\
\hline 2. & defoliária $\mathrm{Cl}$. & 3802 & 991. & Taf. 42,12 \\
\hline 3. & Anisópteryx acerária Sehiff. & 3807 & $99 \mathrm{r}$ & " 42, 13 \\
\hline 4. & aesculária Schiff. & 3809 & $99 \mathrm{r}$ & Nachtr.-Taf. VI, 44 \\
\hline 5. & Phigália pedápia F. & 3812 & $100 \mathrm{l}$. & Taf. 42,16 \\
\hline 6. & Chondrosóma fidueiárium Anker & 3813 & 1001. & \\
\hline 7. & Biston hispidápius $\mathrm{F}$. & 3814 & $100 \mathrm{r}$. & $" 42,15$ \\
\hline 8. & " pomonáeius $\mathrm{Hb}$. & 3816 & $100 \mathrm{r}$. & $" 42,14$ \\
\hline 9. & " lapponárius $\mathrm{B}$. & 3817 & $100 \mathrm{r}$ & $" 42,17$ \\
\hline 10. & " zonárius Sehiff. & 3819 & $101 \mathrm{l}$ & $" 42,18$ \\
\hline 11. & " alpínus Sulz. & 3821 & $101 \mathrm{l}$ & $" 42,19$ \\
\hline 12. & " graecárius Stgr. & 3822 & $101 \mathrm{l}$. & Nachtr.-Taf. VI, 46 \\
\hline 13. & " hiptárius $\mathrm{Cl}$. & 3825 & $101 \mathrm{r}$ & Taf. 42,20 \\
\hline 14. & " stratárius Hufn. & 3826 & $101 \mathrm{r}$. & $" \quad 42,21$ \\
\hline 15. & Amphidásis betulápia L. & 3832 & 1021. & $" 43,1$ \\
\hline 16. & Zamáera flabellária Heeger & 3830 & $101 \mathrm{r}$. & Nachtr.-Taf. VI, 47 \\
\hline 17. & Hemeróphila abruptária Thnog. & 3845 & $102 \mathrm{r}$ & Taf. 43,2 \\
\hline 18. & ny ehthemerária $H_{0}-$ G. & 3847 & $102 \mathrm{r}$. & $" 43,3$ \\
\hline 19. & " v. bareinonária Bell. & $3843 \mathrm{a}$ & $102 \mathrm{r}$. & , \\
\hline 20. & Nyehiódes lividária $\mathrm{Hb}$. & 3839 & 1021. & Nachtr.-Taf. VI, 48 \\
\hline 21. & amygdalária HS. & 3840 & $102 \mathrm{r}$. & \\
\hline 22. & Synópsia soeiária Hb. & 3853 & 1031. & Taf. 43,4 \\
\hline 23. & Boármia solierária Rbr. & 3858 & $103 \mathrm{r}$. & \\
\hline 24. & " atlanticária Stgr. & 3859 & $103 \mathrm{r}$. & Nachtr.-Taf. VI, 49 \\
\hline 25. & " oceitanária Dup. & 3861 & $103 \mathrm{r}$. & VI, 50 \\
\hline 26. & " bastelieária Bell. & 3866 & 1041. & \\
\hline
\end{tabular}

Siehe ferner: Synópsia serpulária Ev. Taf. 71 b, Fig. 5. 

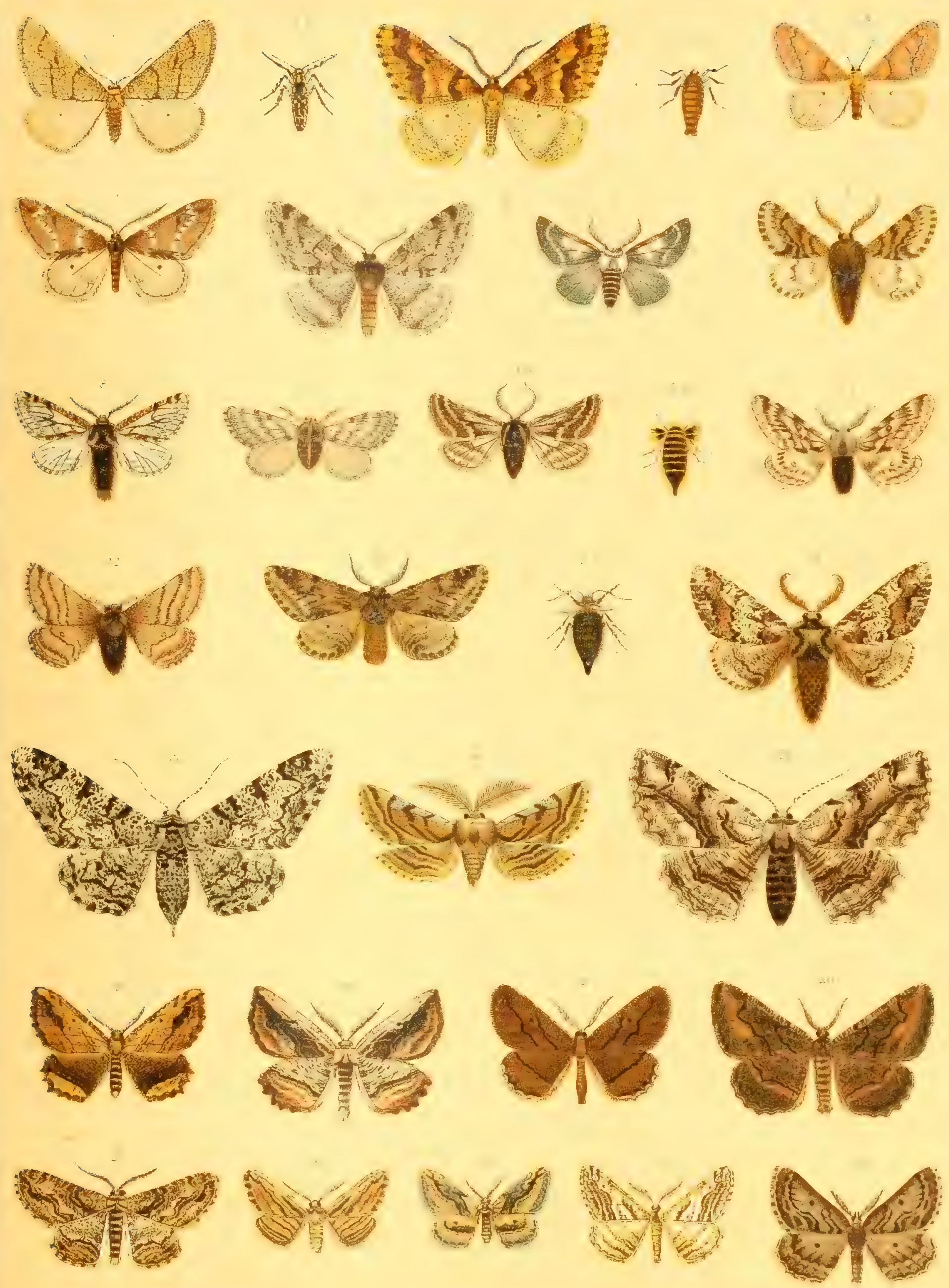




\section{TAFEL 62.}

\begin{tabular}{|c|c|c|c|c|c|}
\hline $\mathrm{Nr}$ & & Name & Katalog I & Seite des II. Bandes & $\begin{array}{c}\text { Raupen- (Puppen-) } \\
\text { Abbildung }\end{array}$ \\
\hline 1. & Boármia & perversária B. & 3864 & $103 \mathrm{r}$. & \\
\hline 2. & $"$ & einetária Schiff. & 3874 & 1041. & Taf. 43,5 \\
\hline 3. & $"$ & gemmária Brahm & 3876 & 1041. & $" 43,6$ \\
\hline 4. &. & ilicária H.-G. & 3879 & $104 \mathrm{r}$ & Nachtr.-Taf. VI, 51 \\
\hline 5. &. & secundária Esp. & 3882 & $104 \mathrm{r}$ & Taf. 43,7 \\
\hline 6. & .. & ribeáta Cl. (abietária Hb.) & 3883 & $104 \mathrm{r}$. & $" 43,8$ \\
\hline 7. & $"$ & umbrária Hb. & 3886 & $104 \mathrm{r}$. & Nachtr.-Taf. VI, 53 \\
\hline 8. & 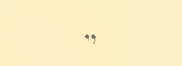 & pepandáta L. & 3891 & $104 \mathrm{r}$ & Taf. 43,9 \\
\hline 9. & $"$ & roborária Sehiff. & 3894 & 1051. & $" \quad 43,10 a, b$ \\
\hline 10. & ", & consortária F. & 3895 & 1051. & $" 43,11 \mathrm{a}, \mathrm{b}$ \\
\hline 11. & " & angulápia Thnbg. & 3896 & $105 \mathrm{r}$. & \\
\hline 12. & 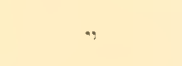 & liehenária Hufn. & 3897 & $105 \mathrm{r}$. & $. \quad 43,12 \mathrm{a}-\mathrm{c}$ \\
\hline 13. & $"$ & jubáta Thnbg. (glabrária Hb.) & 3900 & $105 \mathrm{r}$ & \\
\hline 14. & 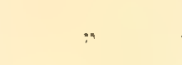 & selenária $\mathrm{Hb}$. & 3901 & $105 \mathrm{r}$. & $" \quad 43,13$ \\
\hline 15. & $"$ & erepuseulária ab. defessária Frr. & 3903 a & 1061 & \\
\hline 16. & $"$ & erepuseulária Hb. & 3903 & 1061. & $" \quad 43,14$ \\
\hline 17. & $"$ & consonápia Hb. & 3906 & 1061. & Nachtr.-Taf. VI, 52 \\
\hline 18. & $"$ & luridáta Bkh. & 3908 & 1061. & T'af. 43,15 \\
\hline 19. & $"$ & punetulária Hb. & 3910 & 1061. & $" \quad 43,16 \mathrm{a}, \mathrm{b}$ \\
\hline 20. & Tephrónia & la sepiária Hufn. & 3911 & $106 \mathrm{r}$. & $" \quad 43,17$ \\
\hline 21. & 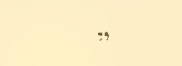 & eremiária Frr. & 3912 & $106 \mathrm{r}$ & $" 43,18 \mathrm{a}, \mathrm{b}$ \\
\hline 22. & Pachyené & émia hippoeastanápia Hb. & 3917 & 1071. & $" \quad 43,19 \mathrm{a}-\mathrm{c}$ \\
\hline 23. & Gnophos: & stevenária $B$. & 3922 & $107 \mathrm{r}$. & \\
\hline 24. & $"$ & dumetáta Tr. & 3923 & $107 \mathrm{r}$. & \\
\hline 25. & $"$ & furváta $F$. & 3925 & $107 \mathrm{r}$ & $" 43,20 \mathrm{a}, \mathrm{b}$ \\
\hline 26. & . & respersária Hb. & 3927 & $107 \mathrm{r}$ & Nachtr.-Taf. VIII, 22 \\
\hline 27. & $"$ & sartáta Tr. & 3929 & 1081 & \\
\hline 28. & $"$ & obseurária Hb. & 3931 & $108 \mathrm{l}$. & Taf. 43,21 \\
\hline 29. & $"$ & ambiguáta Dup. & 3933 & 1081. & $" 43,22$ \\
\hline
\end{tabular}

Siehe ferner: Boármia viértli Bhtseh. Taf. 71b, Fig. 6; B. maeotieária Alph. ib., Fig. 7 ; B. bistortáta Goeze ib., Fig. 8; Tephr. oppositária Mn. ib., Fig. 9 und Gn. onustária HS. ib., Fig. 10. 

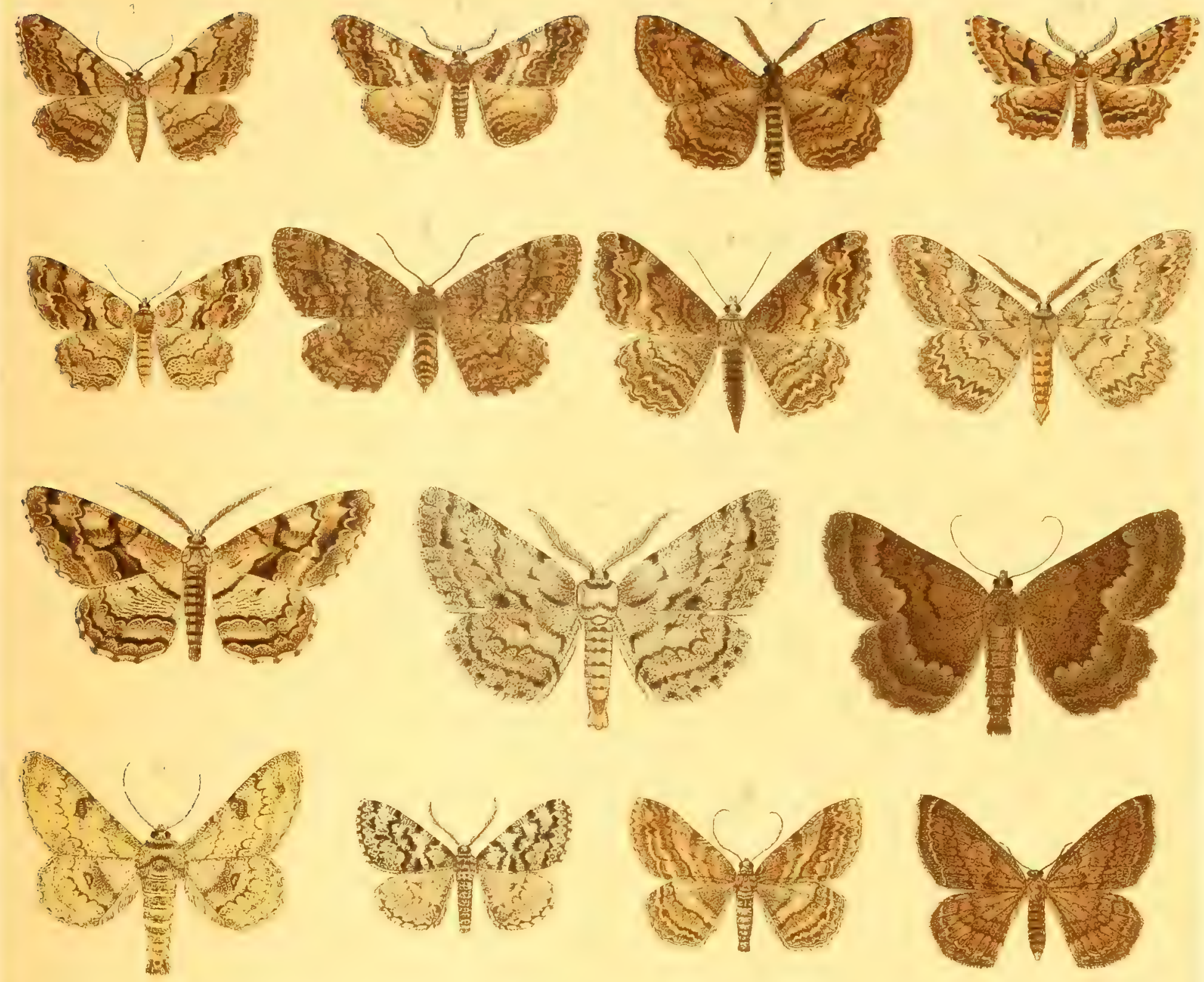

(2)
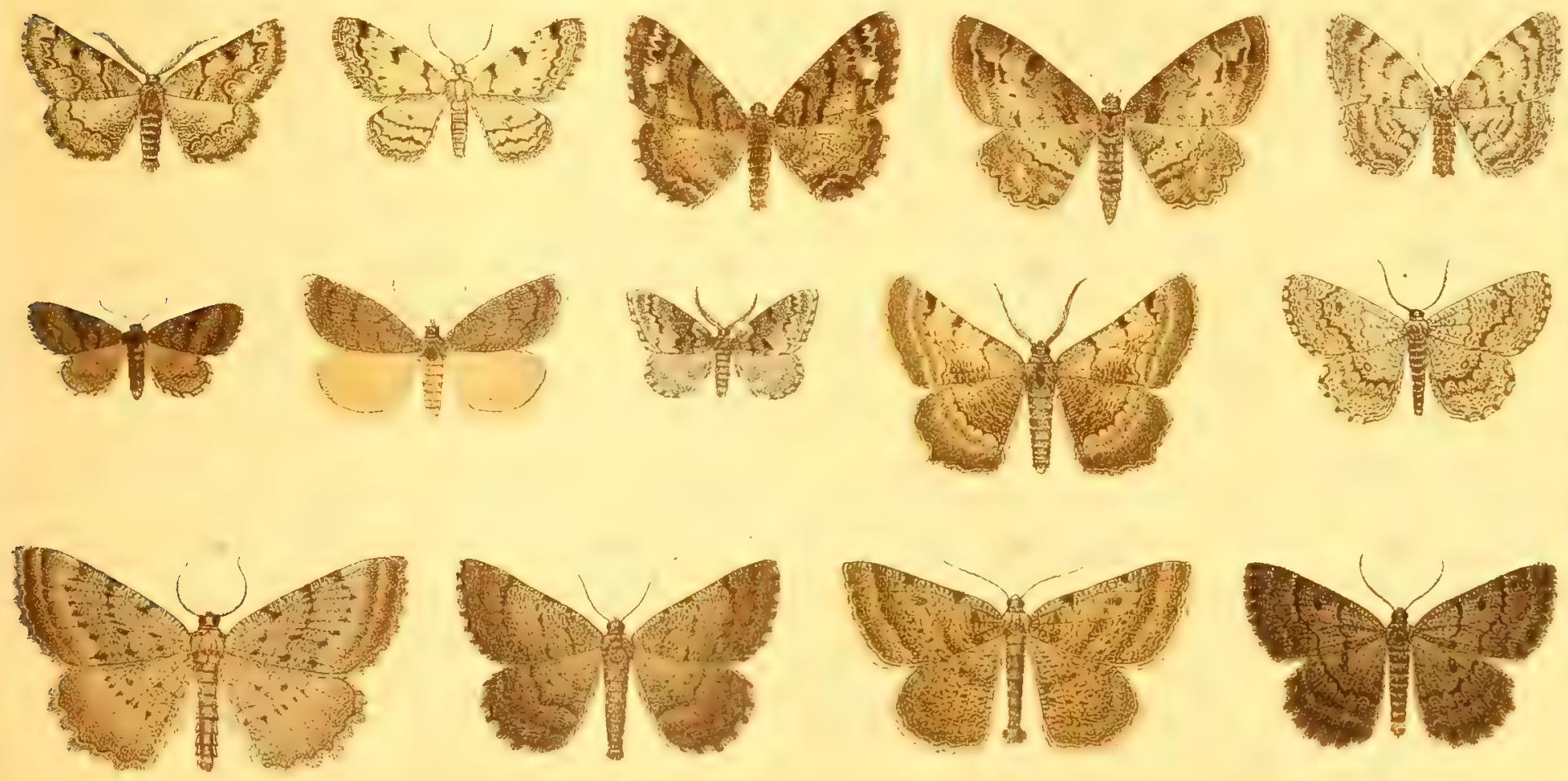



\section{TAFEL 63.}

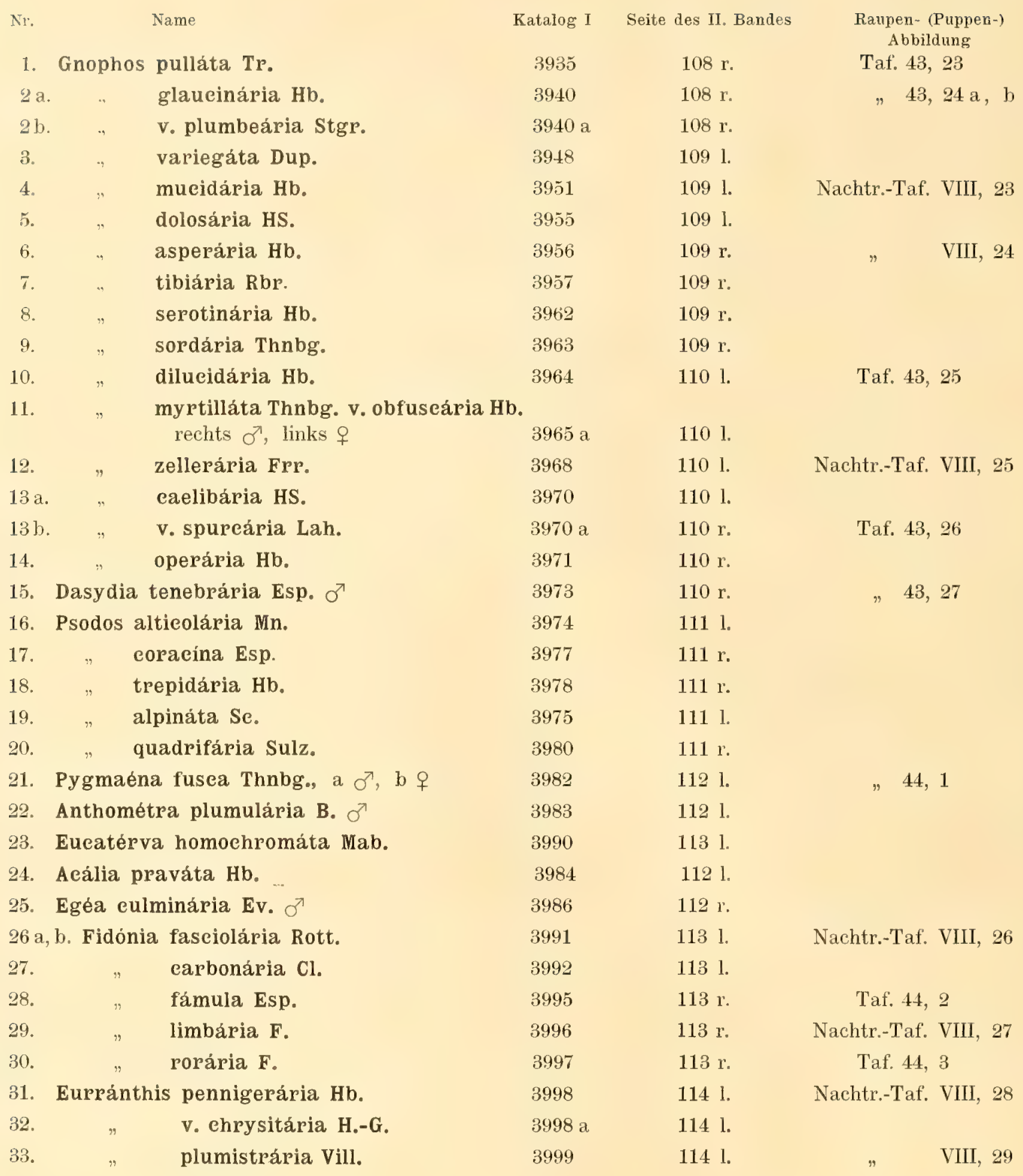

Siehe ferner: Gn. benesignáta Bell. Taf. 59, Fig. 11; Gn. grunerária Stgr. Taf. 71b, Fig. 11; Gn. andereggária Lah. ib., Fig. 12; Psodos noricána Wagner ib., Fig. 13; Eg. eacuminária Rbr. ib., Fig. 14 und Fid. pratána F. ib., Fig. 15. 

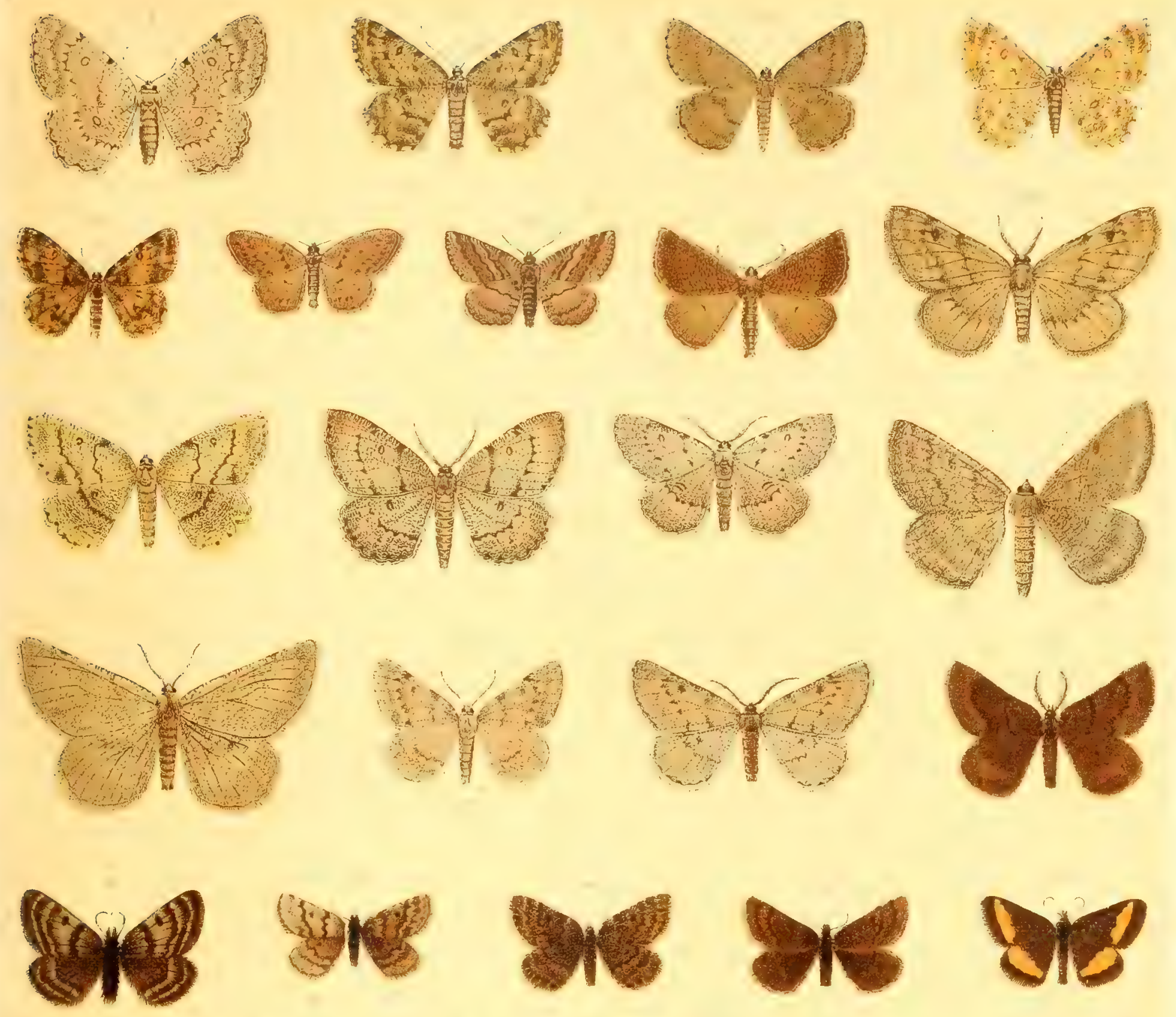

\section{or to ve 83 sye th}
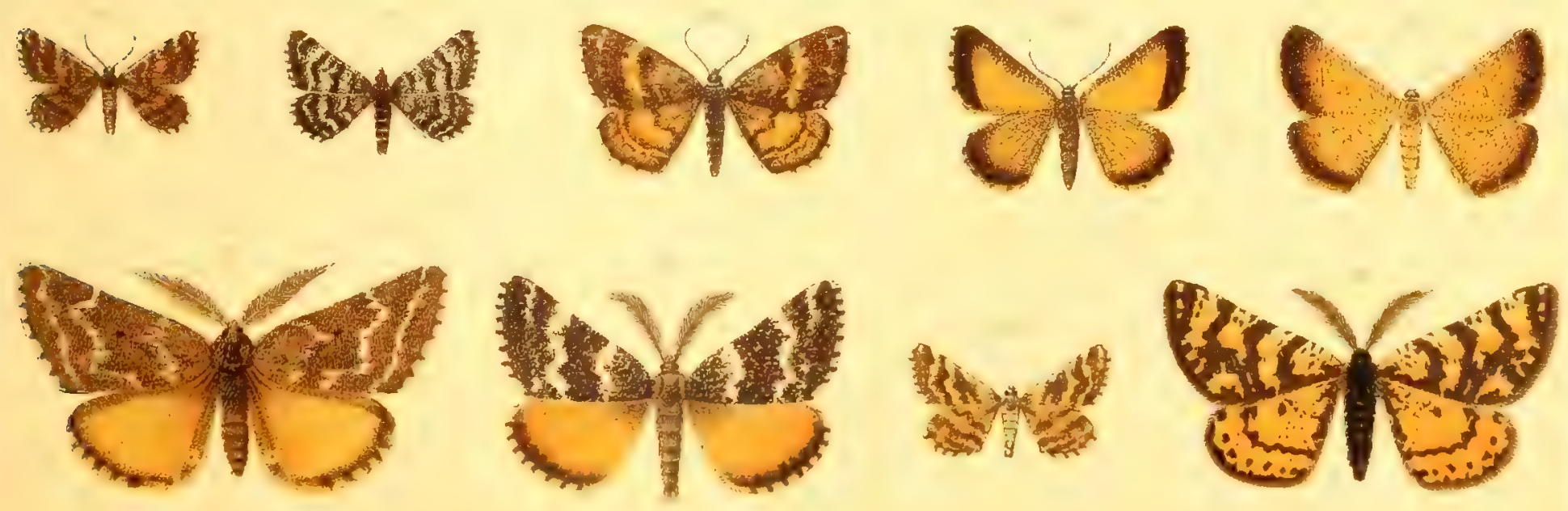




\section{TAFEL 64.}

\begin{tabular}{|c|c|c|c|c|}
\hline Nr. & Name & Katalog I & Seite des II. Bandes & $\begin{array}{c}\text { Raupen- (Puppen-) } \\
\text { Abbildung }\end{array}$ \\
\hline 1. & Hematúrga atomária L., a $\sigma^{7}, \mathrm{~b}$ ㅇ & 4000 & $114 \mathrm{r}$. & Taf. 44,4 \\
\hline 2. & Búpalus piniárius L., a $\sigma^{7}, \mathrm{~b}$ 우 & 4001 & $114 \mathrm{r}$. & Nachtr.-Taf. VIII, 30 \\
\hline 3. & Selidoséma ericetária Vill., 'a $\sigma^{7}$, b ㅇ & 4003 & 1151. & Taf. 44,5 \\
\hline 4. & taeniolária $\mathrm{Hb}$. $q$ & 4005 & 1151. & Nachtr.-Taf. VIII, 31 \\
\hline 5. & ambustária $H_{0}-$ G. $0^{\top}$ & 4007 & $115 \mathrm{l}$. & \\
\hline 6. & Thamnonóma vineulária $\mathrm{Hb}$. + & 4008 & $115 \mathrm{r}$. & VIII, 32 \\
\hline 7. & semicanária Frr. $0^{7}$ & 4009 & $115 \mathrm{r}$. & \\
\hline 8. & gestieulária Hb. $\sigma^{7}$ & 4016 & 1161. & \\
\hline 9. & contaminária $\mathrm{Hb} \cdot \sigma^{7}$ & 4017 & 1161. & VIII, 33 \\
\hline 10. & loricária Ev. ఠ & 4012 & $115 \mathrm{r}$. & \\
\hline 11. & wauária L. $\sigma^{7}$ & 4013 & $115 / 16$ & Taf. 44,6 \\
\hline 12. & brunneáta Thnbg. $\sigma^{7}$ & 4018 & 1161. & $\because 44,7$ \\
\hline 13. & Diastictis artesiária F. $q$ & 4019 & $116 \mathrm{l}$. & $" 44,8$ \\
\hline 14. & Phasíane petrária $\mathrm{Hb}$. 우 & 4023 & $116 \mathrm{r}$. & Nachtr.-Taf. VIII, 34 \\
\hline 15. & partitária $\mathrm{Hb} .+$ & 4028 & 1171. & VIII, 36 \\
\hline 16. & seutulária Dup. $0^{7}$ & 4026 & $116 / 17$ & VIII, 35 \\
\hline 17. & rippertária Dup. + & 4024 & $116 \mathrm{r}$. & \\
\hline 18. & glareária Brahm o & 4033 & 1171. & \\
\hline 19. & $" \quad$ elathráta L. $\sigma^{7}$ & 4032 & 1171. & Taf. 44,9 \\
\hline 20. & Eubólia arenaceária $\mathrm{Hb} . \sigma^{7}$ & 4037 & $117 \mathrm{r}$. & Nachtr.-Taf. VIII, 37 \\
\hline 21. & $"$ murinápia F., a $\sigma^{7}, \mathrm{~b}$ 우 & 4038 & $117 \mathrm{r}$. & Taf. 44,10 \\
\hline 22. & $" \quad$ pumicária Ld. + & 4044 & 1181. & \\
\hline 23. & $" \quad$ assimilária Rbr. $\sigma^{\top}$ & 4041 & $117 \mathrm{r}$. & \\
\hline 24. & Enconista miniosária Dup. $\sigma^{7}$ & 4050 & 1181. & Nachtr-Taf. VIII, 39 \\
\hline 25. & agarithária Dardoin $\sigma^{7}$ & 4053 & 1181. & VIII, 40 \\
\hline 26. & Seodióna emueidária Dup. $\sigma^{7}$ & 4056 & $118 \mathrm{r}$. & \\
\hline 27. & fagária Thnbg. $\sigma^{7}$ & 4058 & $118 / 19$ & VIII, 41 \\
\hline 28. & pennulatária $\mathrm{Hb} \cdot \sigma^{\top}$ & 4057 & $118 \mathrm{r}$. & VIII, 42 \\
\hline 29. & $" \quad$ conspersária F. $\sigma^{7}$ & 4059 & 1191. & Taf. 44,11 \\
\hline 30. & " lentiseária Donz. o" & 4061 & 1191. & \\
\hline 31. & Cleógene luteária F. $\sigma^{\top}$ & 4064 & 1191. & $" 44,12$ \\
\hline
\end{tabular}

Siehe ferner: Thamn. buffonária Mill. Taf. 71 b, Fig. 16; Thamn. sparsária Hb. ib., Fig. 17; Phas. binaeváta Mab. ib., Fig. 18; Eubol. griseolária Ev. ib., Fig. 19; Eub. catalaunária Gn. ib., Fig. 20 und Ene. unieolorária Rbr. ib., Fig. 21. 

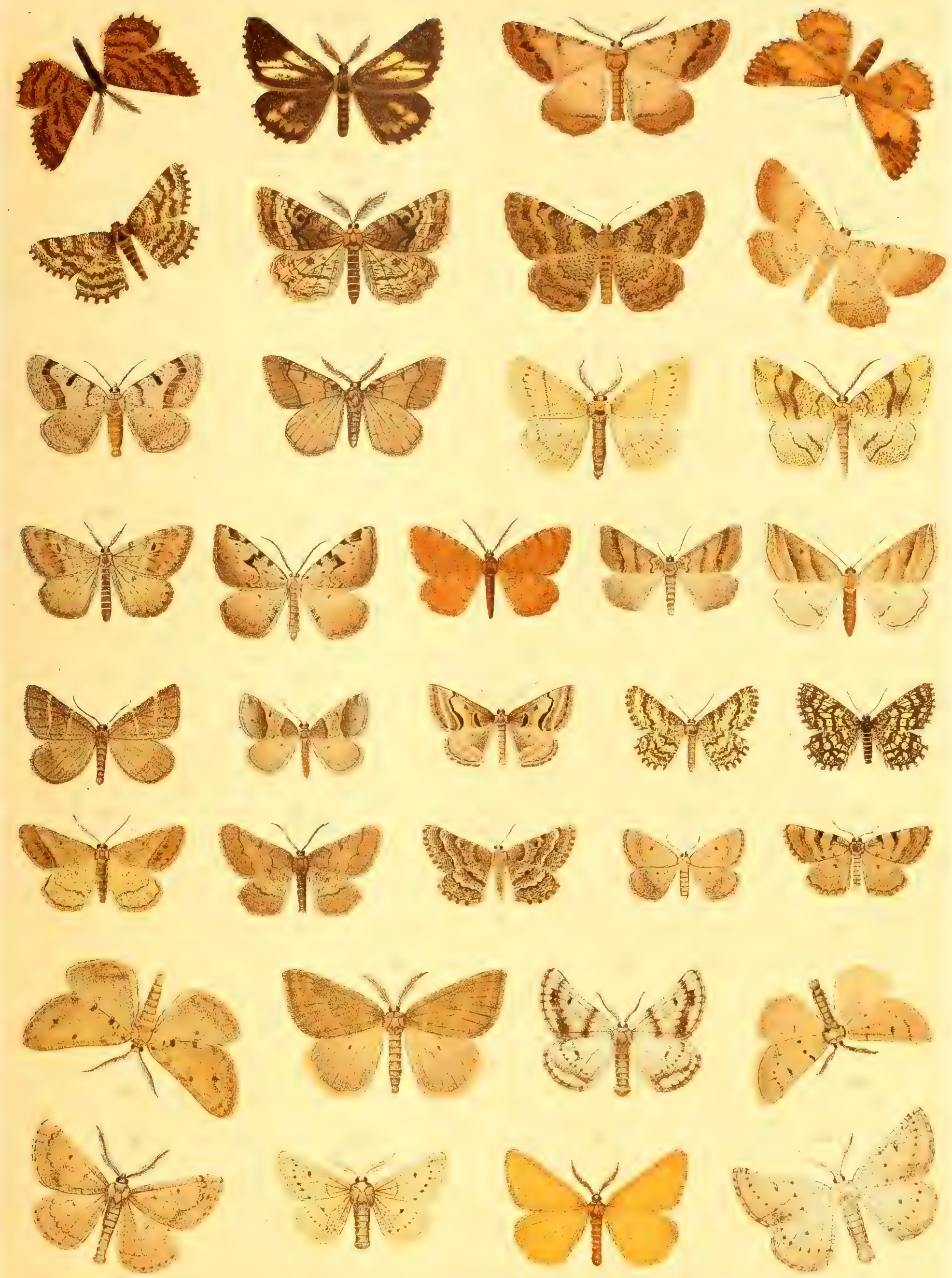




\section{TAFEL 65.}

\begin{tabular}{|c|c|c|c|c|}
\hline Nr. & Name & Katalog I & Seite des II. Bandes & $\begin{array}{l}\text { Raupen- (Puppen-) } \\
\text { Abbildung }\end{array}$ \\
\hline 1. & Cleógene niveáta Scop. $\sigma^{7}$ & 4065 & $119 \mathrm{r}$. & \\
\hline 2. & peletierária Dup. $\sigma^{7}$ & 4066 & $119 \mathrm{r}$. & \\
\hline 3. & Seória lineáta Seop. $q$ & 4067 & $119 \mathrm{r}$. & Taf. $44,13 \mathrm{a}, \mathrm{b}$ \\
\hline 4. & Aspilátes mundatária $\mathrm{Cr} \cdot \sigma^{7}$ & 4070 & 1201. & \\
\hline 5. & formosária Ev. O & 4071 & 1201. & \\
\hline 6. & gilvária F. $\sigma^{7}$ & 4075 & 1201. & $" 44,14$ \\
\hline 7. & ochreária Rossi $\sigma^{7}$ & 4077 & $120 \mathrm{l}$. & $" 44,15 \mathrm{a}, \mathrm{b}$ \\
\hline 8. & Pereónia strigillária Hb. তٓ & 4079 & $120 \mathrm{r}$. & $" 44,16$ \\
\hline 9. & Rhodostróphia badiária Frp. O & 3133 & 291. & \\
\hline 10. & iaculária Hb. О7 & 3137 & 291. & Nachtr.-Taf. VII, 1 \\
\hline 11. & Prosopólopha opacária Hb., 1. $\sigma^{7}$, r. $q$ & 4082 & $121 \mathrm{l}$. & VIII, 43 \\
\hline 12. & jourdanária Vill. $\sigma^{\top}$ & 4084 & 1211. & VIII, 44 \\
\hline 13. & Cimélia margarita $\mathrm{Hb} . \sigma^{7}$ & 4088 & $121 \mathrm{r}$. & \\
\hline 14. & Helióthea discoidária B. $\sigma^{\top}$ & 4086 & $121 \mathrm{r}$. & VIII, 45 \\
\hline 15. & Aplásta ononária Fuesl. & 2859 & 21. & VI, 1 \\
\hline 16. & Gypsochróa renitidáta Hb. & 3686 & 861. & \\
\hline 17. & Rhodométra (Sterrha) saerária L. O & 3143 & $29 \mathrm{r}$. & Taf. $44,17 \mathrm{a}, \mathrm{b}$ \\
\hline 18. & " anthophilária $\mathrm{Hb}$. $\sigma^{7}$ & 3144 & 301. & \\
\hline 19. & Lythria plumulária Frr. $\sigma^{7}$ & 3145 & 301. & \\
\hline 20. & purpurária L. đ & 3147 & $30 \mathrm{r}$. & $" 44,18$ \\
\hline 21. & sanguinária Dup. $\sigma^{7}$ & 3148 & $30 \mathrm{r}$ & Nachtr.-Taf. VII, 2 \\
\hline 22. & Ortholítha coaretáta F. & 3150 & 311. & VII, 3 \\
\hline 23. & plumbária F. 우 & 3151 & 311 & Taf. 44,19 \\
\hline 24. & eepvináta Sehiff. $\sigma^{7}$ & 3152 & 311. & $\Rightarrow 44,20$ \\
\hline 25. & limitáta Se. ত & 3155 & 311. & Nachtr.-Taf. VI, 17 \\
\hline 26. & moeniáta Se. + & 3156 & 311. & VI, 18 \\
\hline 27. & coelinária Grasl. $\sigma^{7}$ & 3160 & $31 \mathrm{r}$ & \\
\hline 28. & periboláta Hb. ఠ & 3159 & $31 \mathrm{r}$. & \\
\hline 29. & obvallária Mab. ㅇ & 3157 & $31 \mathrm{r}$. & \\
\hline 30. & burgária Ev. & 3170 & $31 \mathrm{r}$. & \\
\hline 31. & vieinária Dup. $\sigma^{7}$ & 3169 & $31 \mathrm{r}$. & \\
\hline 32. & bipunetária Sehiff. $\sigma^{\top}$ & 3174 & $31 \mathrm{r}$. & Nachtr.-Taf. VI, 19 \\
\hline 33. & Mesotype virgáta Rott. & 3180 & 321. & Taf. 44,21 \\
\hline 34. & Minóa murináta Se. & 3183 & 321. & $" 44,22 \mathrm{a}-\mathrm{c}$ \\
\hline 35. & Odézia atráta L. & 3191 & $32 \mathrm{r}$. & Nachtr.-Taf. VI, 20 \\
\hline
\end{tabular}

Siehe ferner: Perc. baeticária Rbr. Taf. 71 b, Fig. 22 ; Prosop argentária HS. ib., Fig. 23 ; Eus. interpunetária HS. ib., Fig. 1 und Orthol. proximária Rbr. Taf. 71 b, Fig. 25. 

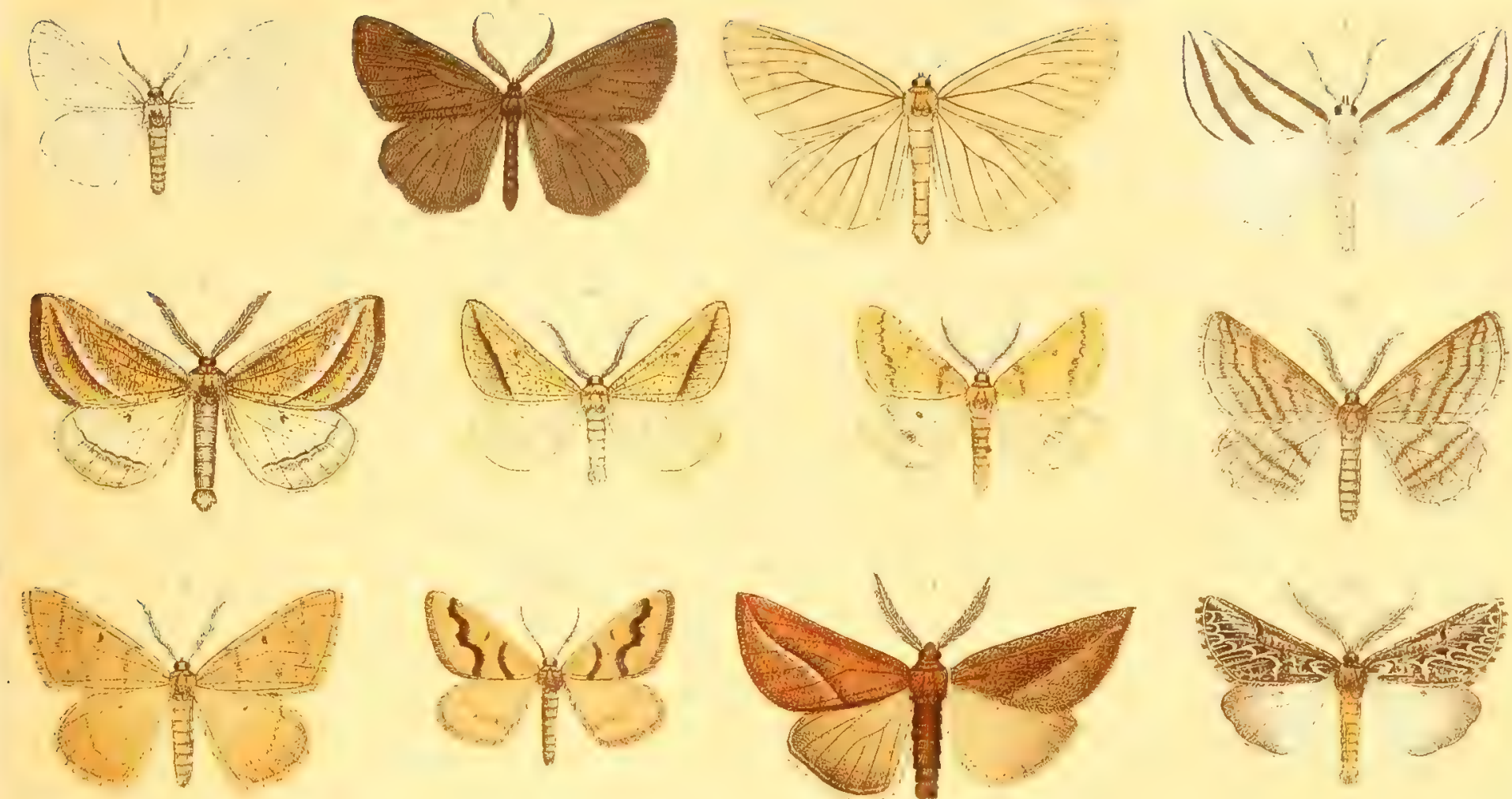

3,18
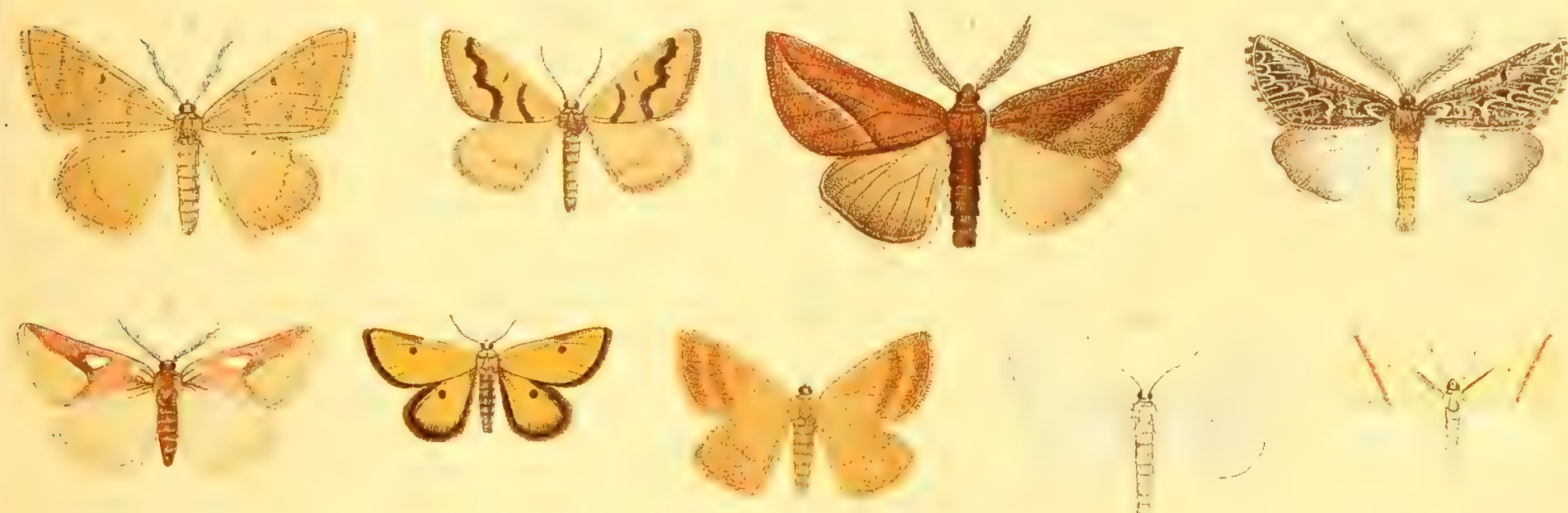

dits $19 x$
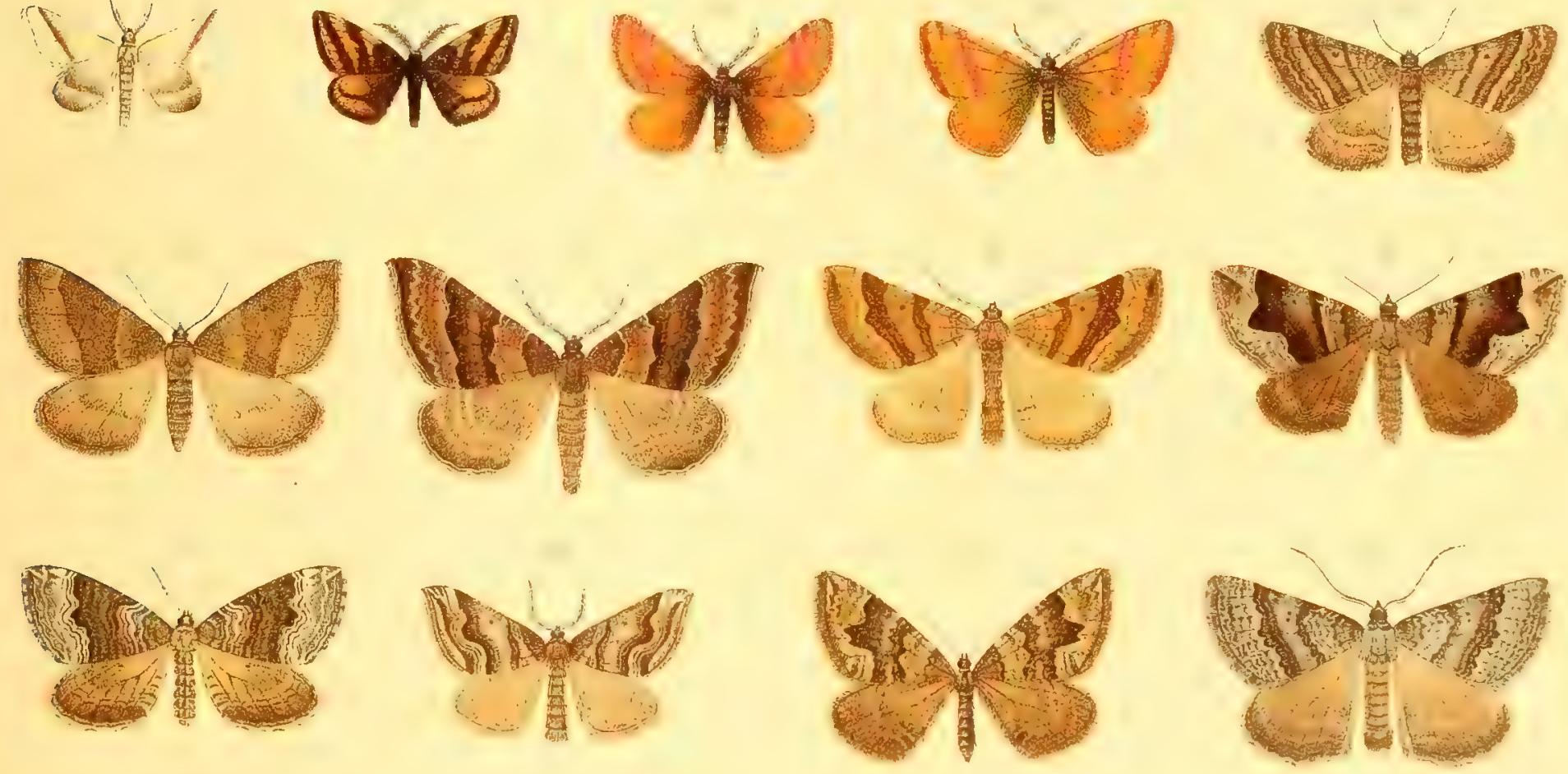

nian thents
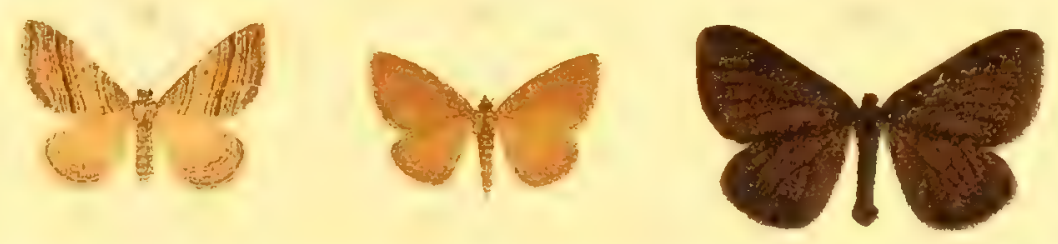




\section{TAFEL 66 .}

\begin{tabular}{|c|c|c|c|c|}
\hline $\mathrm{Nr}$ & Name & Katalog I & Seite des $\Pi$. Bandes & $\begin{array}{l}\text { Raupen- (Puppen-) } \\
\text { Abbildung }\end{array}$ \\
\hline 1. & Odézia tibiále Esp. & 3192 & $32 \mathrm{r}$. & Nachtr.-Taf. VI, 21 \\
\hline 2. & Siona decussáta Bkh. + & 3193 & $32 \mathrm{r}$. & \\
\hline 3. & Lithóstege griseáta Schiff. & 3197 & 331. & VI, 22 \\
\hline 4. & duplicáta Hb. 우 & 3199 & 33 r. & \\
\hline 5. & farináta Hufn. & 3195 & 331. & \\
\hline 6. & Anaitis lithoxyláta $\mathrm{Hb}$. 우 & 3212 & $33 \mathrm{r}$. & \\
\hline 7. & " praeformáta $\mathrm{Hb}$. 우 & 3218 & $33 \mathrm{r}$ & Taf. $44,23 \mathrm{a}, \mathrm{b}$ \\
\hline 8. & " plagiáta L. $q$ & 3220 & 341. & $" \quad 44,24$ \\
\hline 9. & " simpliciáta Tr. $\sigma^{7}$ & 3222 & 341. & \\
\hline 10. & " paludáta Thnbg. 우 & 3228 & 341. & \\
\hline 11. & Chésias spartiáta Herbst 우 & 3229 & $34 \mathrm{r}$ & $" 44,25 \mathrm{a}-\mathrm{d}$ \\
\hline 12. & " pufáta F. + & 3230 & $34 \mathrm{r}$. & Nachtr.-Taf. VI, 23 \\
\hline 13. & Lobóphora externáta HS. & 3233 & 34 r. & \\
\hline 14. & polyeommáta $\mathrm{Hb}$. & 3236 & 351. & Taf. 44,26 \\
\hline 15. & sabináta H.-G. & 3235 & 351. & Nachtr.-Taf. VII, 4 \\
\hline 16. & sertáta $\mathrm{Hb}$. & 3240 & 351. & 'Taf. 44,27 \\
\hline 17. & earpináta $\mathrm{Bkh}$. & 3241 & 351. & $" 44,28$ \\
\hline 18. & halteráta Hufn. $\sigma^{7}$ & 3243 & 351. & Nachtr.-Taf. VI, 24 \\
\hline 19. & sexaláta $\mathrm{Hb} \cdot \sigma^{7}$ & 3244 & 351. & Taf. $44,29 \mathrm{a}, \mathrm{b}$ \\
\hline 20. & appensáta Ev. & 3245 & $35 \mathrm{r}$. & $\Rightarrow \quad 44,30$ \\
\hline 21. & viretáta $\mathrm{Hb}$. & 3246 & $35 \mathrm{r}$. & $" 45,1 \mathrm{a}-\mathrm{c}$ \\
\hline 22. & Sparta paradoxária Stgr. & 3248 & $35 \mathrm{r}$. & \\
\hline 23. & $\begin{array}{r}\text { Operóphthera (Cheimatóbia) brumáta L., } \\
\qquad a \sigma^{7}, \mathrm{~b} \text { 오 }\end{array}$ & 3256 & $36 \mathrm{r}$ & $" 45,2 \mathrm{a}, \mathrm{b}$ \\
\hline 24. & $" \quad$ boreáta Hb. $\sigma^{7}$ & 3255 & 361. & $" 45,3 \mathrm{a}, \mathrm{b}$ \\
\hline 25. & Triphósa sabaudiáta Dup. & 3258 & $36 \mathrm{r}$ & Nachtr.-Taf. VI, 25 \\
\hline 26. & $" \quad$ dubitáta $\mathrm{L}$. & 3259 & $36 \mathrm{r}$ & Taf. $45,4 \mathrm{a}, \mathrm{b}$ \\
\hline 27. & Eucósmia certáta $\mathrm{Hb}$. & 3264 & $37 \mathrm{l}$. & $" 45,5$ \\
\hline 28. & $" \quad$ montivagáta Dup. $\sigma^{7}$ & 3267 & 371. & Nachtr.-Taf. VI, 26 \\
\hline 29. & unduláta L. & 3270 & 371. & Taf. 45,6 \\
\hline 30. & Seotósia vetuláta Sehiff. & 3278 & $37 \mathrm{r}$. & $" 45,7 \mathrm{a}, \mathrm{b}$ \\
\hline 31. & rhamnáta Sehiff. $0^{7}$ & 3281 & $37 \mathrm{r}$. & $" 45,8 \mathrm{a}, \mathrm{b}$ \\
\hline
\end{tabular}

Siehe ferner: Síona nubilária Hb. Taf. 71 a, Fig. 26; Lithost. flavieornáta Z. ib., Fig. 27 ; Lith. castiliária Stgr. ib., Fig. 28 und Malae. regelária Tngstr. Taf. 71 b, Fig. 24. 

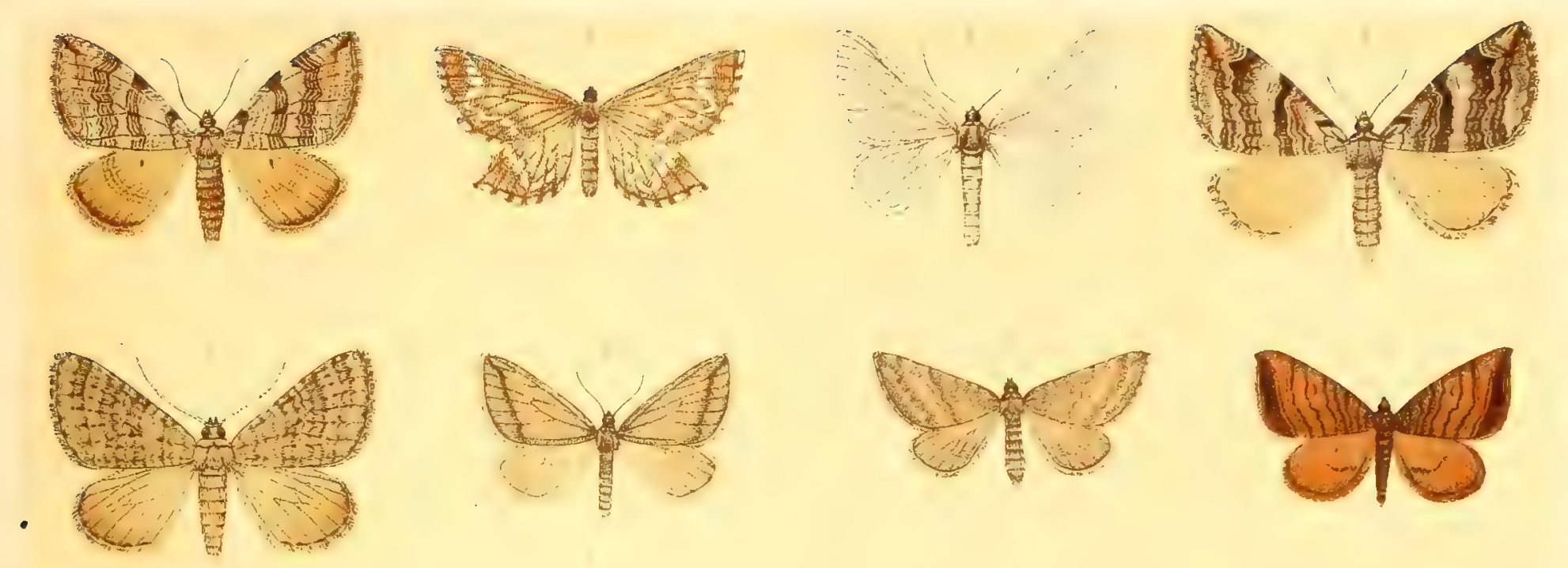

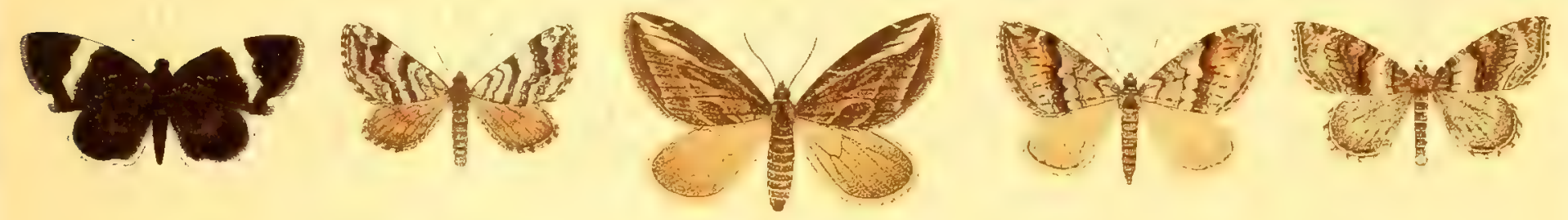

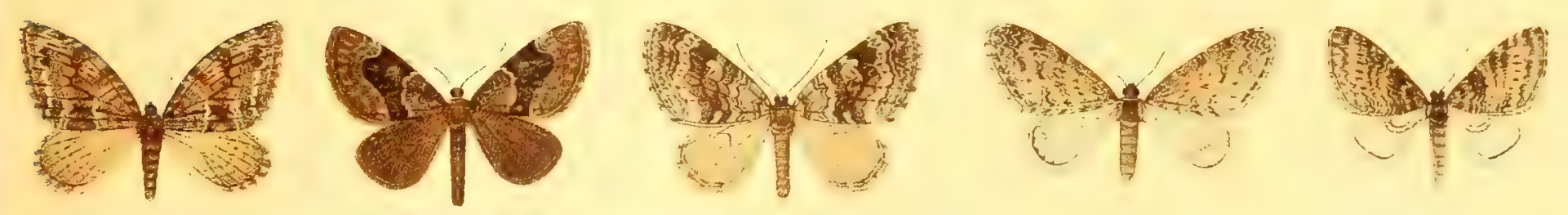

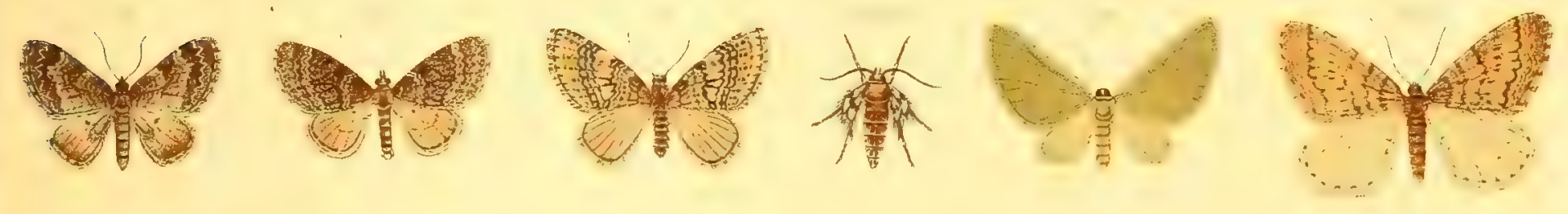
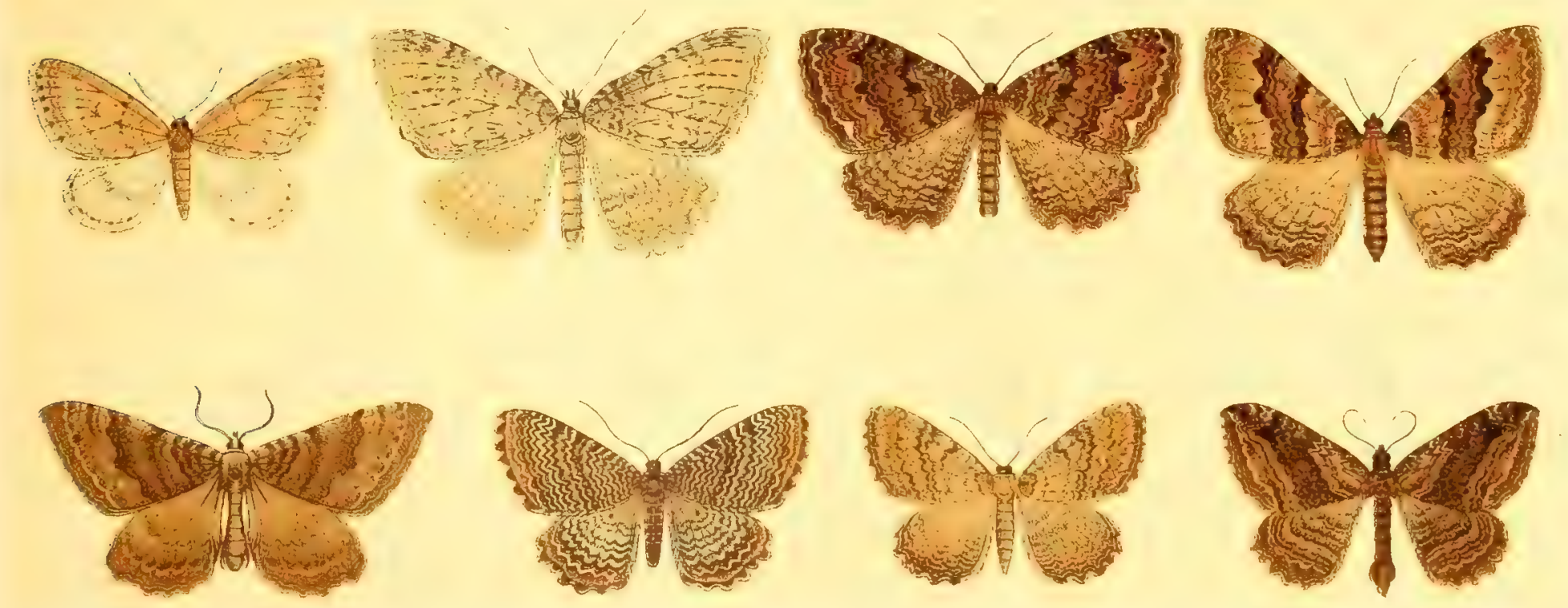




\section{TAFEL $6 \%$.}

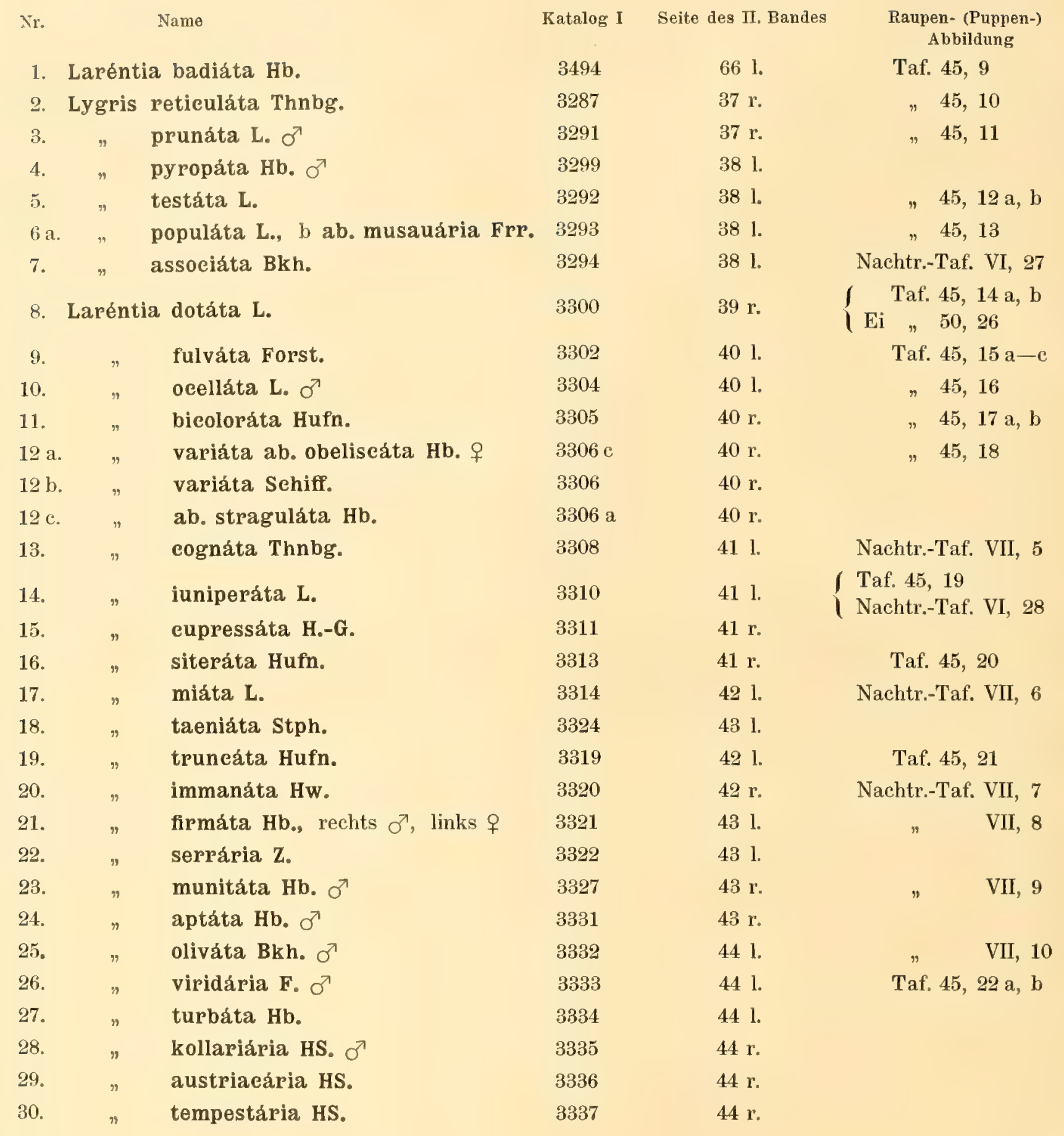

Siehe ferner: Lar. püngeléri Stertz Taf. 71 a, Fig. 29. 

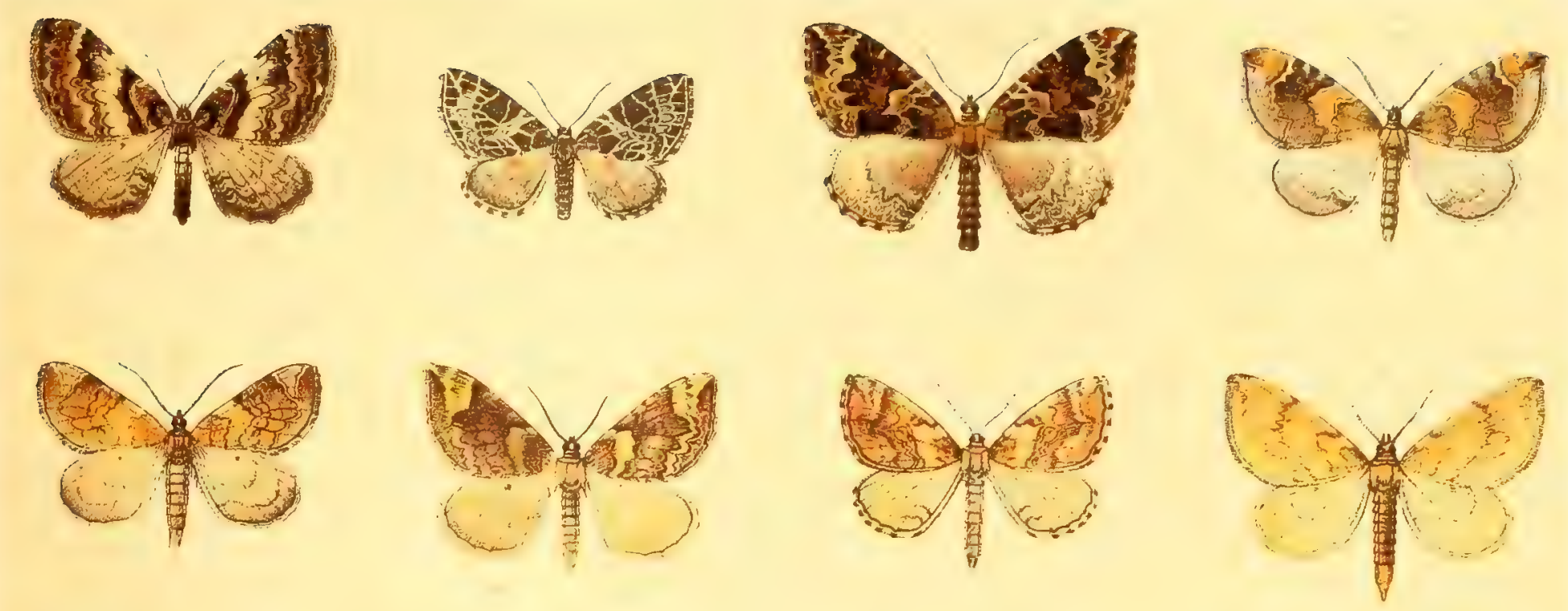

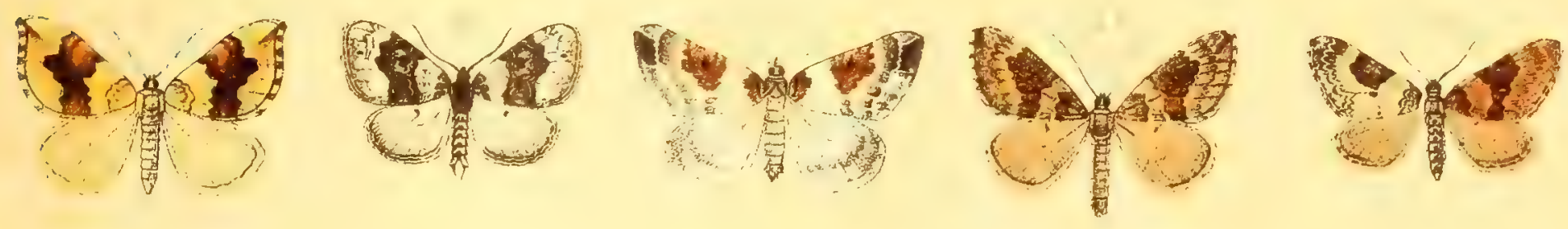
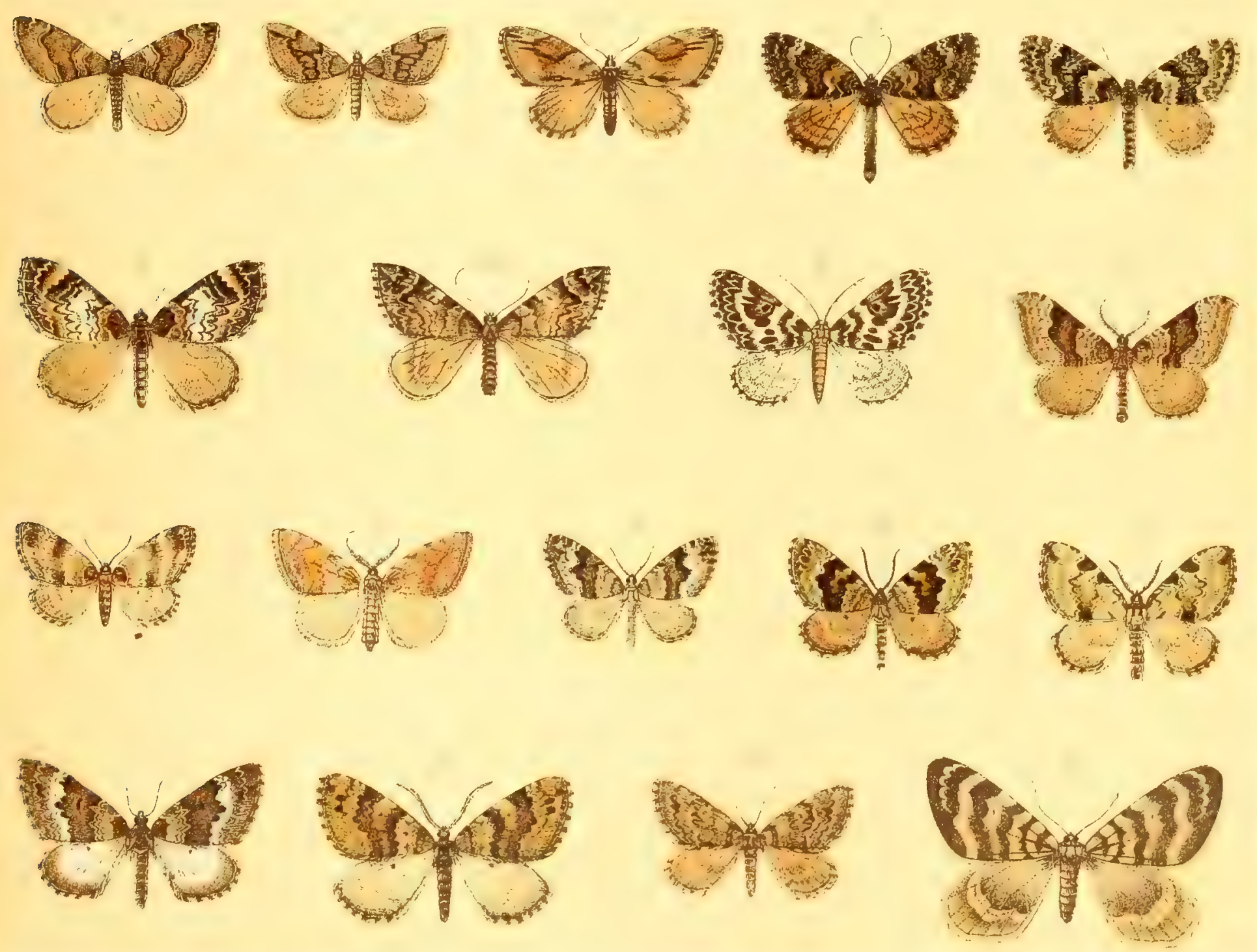




\section{TAFEL 68.}

\begin{tabular}{|c|c|c|c|c|c|}
\hline $\mathrm{Nr}$ & & Name & Katalog I & Seite des II. Bandes & $\begin{array}{c}\text { Raupen- (Puppen-) } \\
\text { Abbildung }\end{array}$ \\
\hline 1. 1 & Laréntia & aqueáta $\mathrm{Hb}$. & 3338 & $44 \mathrm{r}$ & \\
\hline 2. & $"$ & salicáta Hb. $0^{x}$ & 3340 & 451. & Nachtr.-Taf. VII, 11 \\
\hline 3. & $"$ & multistrigária Hw. & 3356 & 461. & VII, 12 \\
\hline 4. & $"$ & didymáta L., a $\sigma^{\pi}, \mathrm{b}$ 우 & 3358 & $46 \mathrm{r}$. & \\
\hline 5. & $"$ & cámbrica Curt. ơ & 3359 & $46 \mathrm{r}$ & VII, 13 \\
\hline 6. & $"$ & $\begin{array}{l}\text { parallelolineáta Retz. (versper- } \\
\text { tária Bkh.) }\end{array}$ & 3360 & 471. & VII, 14 \\
\hline 7. & $n$ & incursáta Hb. $\sigma^{7}$ & 3361 & 471. & VII, 15 \\
\hline 8. & $"$ & fluetuáta L. $\sigma^{7}$ & 3344 & $45 \mathrm{r}$. & Taf. 45,23 \\
\hline 9. & $"$ & montanáta Sehiff. $\sigma^{7}$ & 3363 & $47 \mathrm{r}$ & $" 45,24 \mathrm{a}, \mathrm{b}$ \\
\hline 10. & $"$ & quadrifasciária $\mathrm{Cl} .9$ & 3368 & 481. & $" 45,25 \mathrm{a}, \mathrm{b}$ \\
\hline 11. & $"$ & ferrugáta $\mathrm{Cl} \cdot \sigma^{7}$ & 3369 & $48 \mathrm{r}$ & $" 45,26 \mathrm{a}, \mathrm{b}$ \\
\hline 12. & " & unidentária $\mathrm{Hw}$. \& & 3370 & 491. & \\
\hline 13. & $"$ & suffumáta $\mathrm{Hb} \cdot \sigma^{\top}$ & 3367 & 481. & Nachtr.-Taf. VII, 17 \\
\hline 14. & $"$ & pomoeriária Ev. $\sigma^{7}$ & 3373 & $49 \mathrm{I}$. & Taf. $45,27 \mathrm{a}, \mathrm{b}$ \\
\hline 15. & $"$ & designáta Rott. $\sigma^{7}$ & 3374 & $49 \mathrm{r}$ & Nachtr.-Taf. VII, 16 \\
\hline 16. & $"$ & abrasária HS. & 3377 & $49 \mathrm{r}$ & \\
\hline 17. & $"$ & fluviáta $\mathrm{Hb} \cdot \mathrm{O}^{7}$ & 3378 & 501. & Taf. $46,1 \mathrm{a}-\mathrm{c}$ \\
\hline 18. & $"$ & vittáta $\mathrm{Bkh}$. & 3379 & 501. & \\
\hline $19 \mathrm{a}$. & $"$ & dilutáta ab. obscuráta Stgr. & $3380 \mathrm{a}$ & $50 \mathrm{r}$. & $" 45,28 \mathrm{a}, \mathrm{b}$ \\
\hline $19 \mathrm{~b}$. & $"$ & autumnáta $\mathrm{Bkh}$. & 3381 & $50 \mathrm{r}$. & \\
\hline 20. & $n$ & v. filigrammária HS. & 3381 a & $50 \mathrm{r}$. & \\
\hline 21. & $"$ & poláta Dup. & 3382 & $50 \mathrm{r}$. & \\
\hline 22. & $n$ & eaesiáta Lang & 3385 & 511. & Nachtr.-Taf. VII, 18 \\
\hline 23. & $"$ & flavieinetáta $\mathrm{Hb}$. & 3387 & 511. & \\
\hline 24. & $"$ & infidária Lah. & 3388 & $51 \mathrm{r}$. & Taf. 46,2 \\
\hline 25. & $"$ & eyanáta $\mathrm{Hb}$. & 3390 & 521. & Nachtr.-Taf. VII, 19 \\
\hline 26. & $"$ & tophaceáta $\mathrm{Hb}$. & 3393 & 521. & Taf. 46,3 \\
\hline 27. & $n$ & nobiliária HS. $\sigma^{7}$ & 3394 & $52 \mathrm{r}$. & Nachtr.-Taf. VII, 20 \\
\hline 28. & $"$ & aehromária Lah. & 3403 & 531. & VII, 21 \\
\hline 29. & $"$ & ineultária HS. & 3404 & $53 \mathrm{r}$ & Taf. 46,4 \\
\hline 30. & $"$ & nebuláta Tr. & 3399 & $53 \mathrm{l}$. & \\
\hline 31. & $"$ & verberáta Se. & 3398 & $52 \mathrm{r}$ & \\
\hline 32. & $"$ & senectária HS. & 3408 & 541. & \\
\hline 33. & $"$ & sandosária HS. & 3411 & $54 \mathrm{r}$. & \\
\hline 34. & $n$ & frustáta Tr. & 3410 & 541. & \\
\hline
\end{tabular}

Siehe ferner: Lar. flavolineáta Stgr. Taf. 71 a, Fig. 30; L. disiunetária Lah. ib., Fig. 31; L. frigidária Gn. ib., Fig. 32; L. alfacária Stgr. ib., Fig. 33; L. byssáta Auriv. ib., Fig. 34 und L. alfacariáta Rbr., ibericáta Stgr. ib., Fig. 35. 


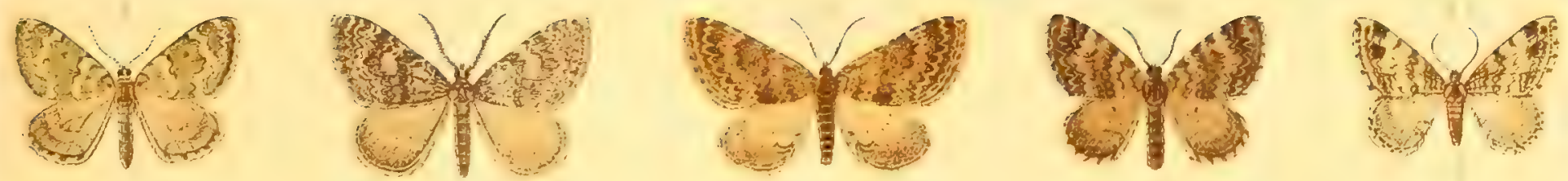
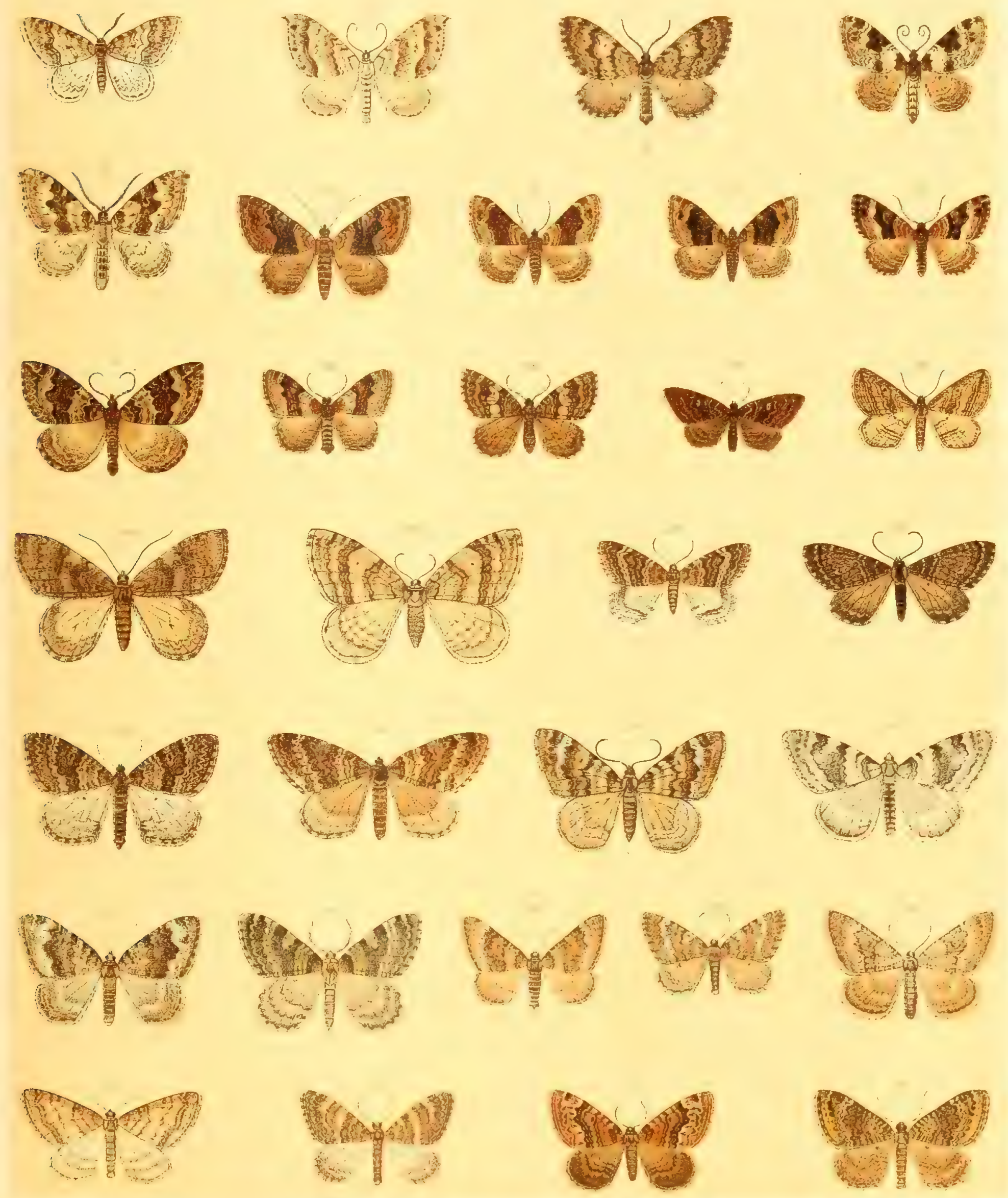




\section{TAFEL 69.}

\begin{tabular}{|c|c|c|c|c|c|}
\hline Nr. & & Name & Katalog I & Seite des Ir. Bandes & $\begin{array}{l}\text { Raupen-(Puppen-) } \\
\text { Abbildung }\end{array}$ \\
\hline 1. & Laréntia & cupreáta HS. & 3419 & 551. & \\
\hline 2. & $"$ & dissimuláta Rbr. & 3412 & $54 \mathrm{r}$. & \\
\hline 3. & $"$ & seripturáta Hb. & 3415 & 551. & \\
\hline 4. & $"$ & piguáta $\mathrm{Hb}$. & 3417 & 551. & Taf. 46,5 \\
\hline 5. & $"$ & adumbrária HS. & 3397 & $52 \mathrm{r}$ & \\
\hline 6. & $"$ & alpicolária HS. & 3426 & 561. & $\Rightarrow \quad 46,6$ \\
\hline 7. & $"$ & pieáta Hb. & 3439 & $57 \mathrm{r}$. & Nachtr.-Taf. VII, 23 \\
\hline 8. & $"$ & malváta Rbr. & 3424 & $55 \mathrm{r}$. & \\
\hline 9. & $"$ & unicáta Gn. & 3431 & $56 \mathrm{r}$ & \\
\hline 10. & $\eta$ & permixtária HS. & 3430 & 561. & \\
\hline 11. & $n$ & eueulláta Hufn. & 3432 & $56 \mathrm{r}$. & Taf. $46,7 \mathrm{a}, \mathrm{b}$ \\
\hline 12. & $"$ & galiáta Hb. & 3434 & $56 \mathrm{r}$. & $" 46,8 \mathrm{a}, \mathrm{b}$ \\
\hline 13. & $"$ & riváta $\mathrm{Hb}$. & 3436 & $57 \mathrm{l}$. & $" 46,9$ \\
\hline 14. & 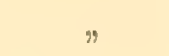 & soeiáta Bkh. & 3437 & $57 \mathrm{l}$. & $" 46,10$ \\
\hline 15. & 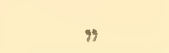 & unanguláta $\mathrm{Hw}$. & 3438 & $57 \mathrm{r}$. & Nachtr.-Taf. VII, 22 \\
\hline 16. & $"$ & alaudária Frr. & 3440 & 581. & \\
\hline 17. & $"$ & albieilláta L. & 3442 & 581. & Taf. 46,11 \\
\hline 18. & $n$ & procelláta $\mathrm{F}$. & 3443 & $58 \mathrm{r}$. & Nachtr.-Taf. VII, 24 \\
\hline 19. & $"$ & transversáta Thnbg. (lugubráta & 3444 & $58 \mathrm{r}$. & Taf. 46, $12 \mathrm{a}-\mathrm{c}$ \\
\hline 20. & ” & thuleária HS. & 3448 & $59 \mathrm{r}$. & Nachtr.-Taf. VII, 25 \\
\hline 21. & $n$ & hastáta L. & 3447 & - 591. & Taf. $46,13 \mathrm{a}, \mathrm{b}$ \\
\hline 22. & $"$ & tristáta L. $\mathrm{a}, \mathrm{b}$ & 3449 & $60 \mathrm{l}$. & $\eta \quad 46,14$ \\
\hline 23. & $\eta$ & luetuáta Hb. & 3450 & 601. & $" 46,15$ \\
\hline 24. & $"$ & pupilláta Thnbg. & 3451 & $60 \mathrm{r}$. & \\
\hline 25. & $"$ & mollugináta $\mathrm{Hb}$. & 3450 & $60 \mathrm{r}$ & $" 46,16 \mathrm{a}, \mathrm{b}$ \\
\hline 26. & $"$ & affinitáta Stph. & 3455 & 611. & \\
\hline 27. & $n$ & alehemilláta $\mathrm{L}$. & 3456 & $61 \mathrm{l}$. & $" 46,17 \mathrm{a}, \mathrm{b}$ \\
\hline 28. & $"$ & hydráta Tr. & 3457 & $61 \mathrm{r}$ & $" 46,18$ \\
\hline 29. & $"$ & lugdunária HS. & 3458 & $61 \mathrm{r}$. & \\
\hline 30. & $"$ & unifasciáta $\mathrm{Hw}$. & 3459 & $61 \mathrm{r}$. & $\because \quad 46,19$ \\
\hline 31. & $"$ & minoráta Tr. & 3463 & 621. & Nachtr.-Taf. VII, 26 \\
\hline 32. & $"$ & adaequáta $\mathrm{Bkh}$. & 3464 & $62 \mathrm{l}$. & Taf. 46,20 \\
\hline 33. & $"$ & albuláta Sehiff. & 3465 & $62 \mathrm{r}$. & $\eta 46,21 \mathrm{a}, \mathrm{b}$ \\
\hline 34. & Ásthena & eandidáta Sehiff. & 3505 & 681. & Nachtr.-Taf. VII, 30 \\
\hline 35. & Laréntia & testaceáta Don. & 3471 & $62 \mathrm{r}$ & Taf. 46,22 \\
\hline 36. & $"$ & bloméri Curt. & 3472 & $62 \mathrm{r}$. & Nachtr.-Taf. VII, 27 \\
\hline 37. & $"$ & flavofasciáta Thnbg. & 3476 & $63 \mathrm{r}$ & VII, 28 \\
\hline 38. & $"$ & luteảta Sehiff. & 3475 & $63 \mathrm{r}$. & \\
\hline 39. & $"$ & obliteráta Hufn. & 3474 & 631. & Taf. 46,23 \\
\hline
\end{tabular}

Siehe ferner: Lar. basochesiáta Dup. Taf. 71 a, Fig. 36, L. easeária Const. ib., Fig. 37 ; L. timozzária Const. ib., Fig. 38; L. putridária HS. Taf. 71 b, Fig. 30 ; L. oxybiáta Mill. Taf. 71 b, Fig. 31 ; L. corydalária Graes. Taf. 71 b, Fig. 29 ; Asth. anserária HS. Taf. 71 a, Fig. 40 und Asth. nymphuláta Gn. Taf. 71 b, Fig. 32. 


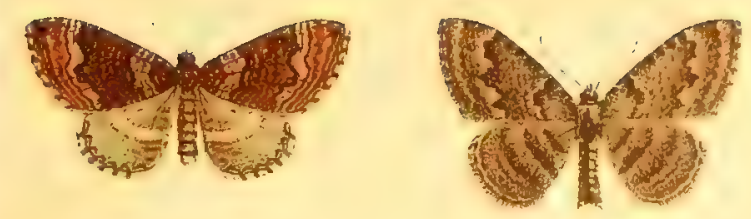
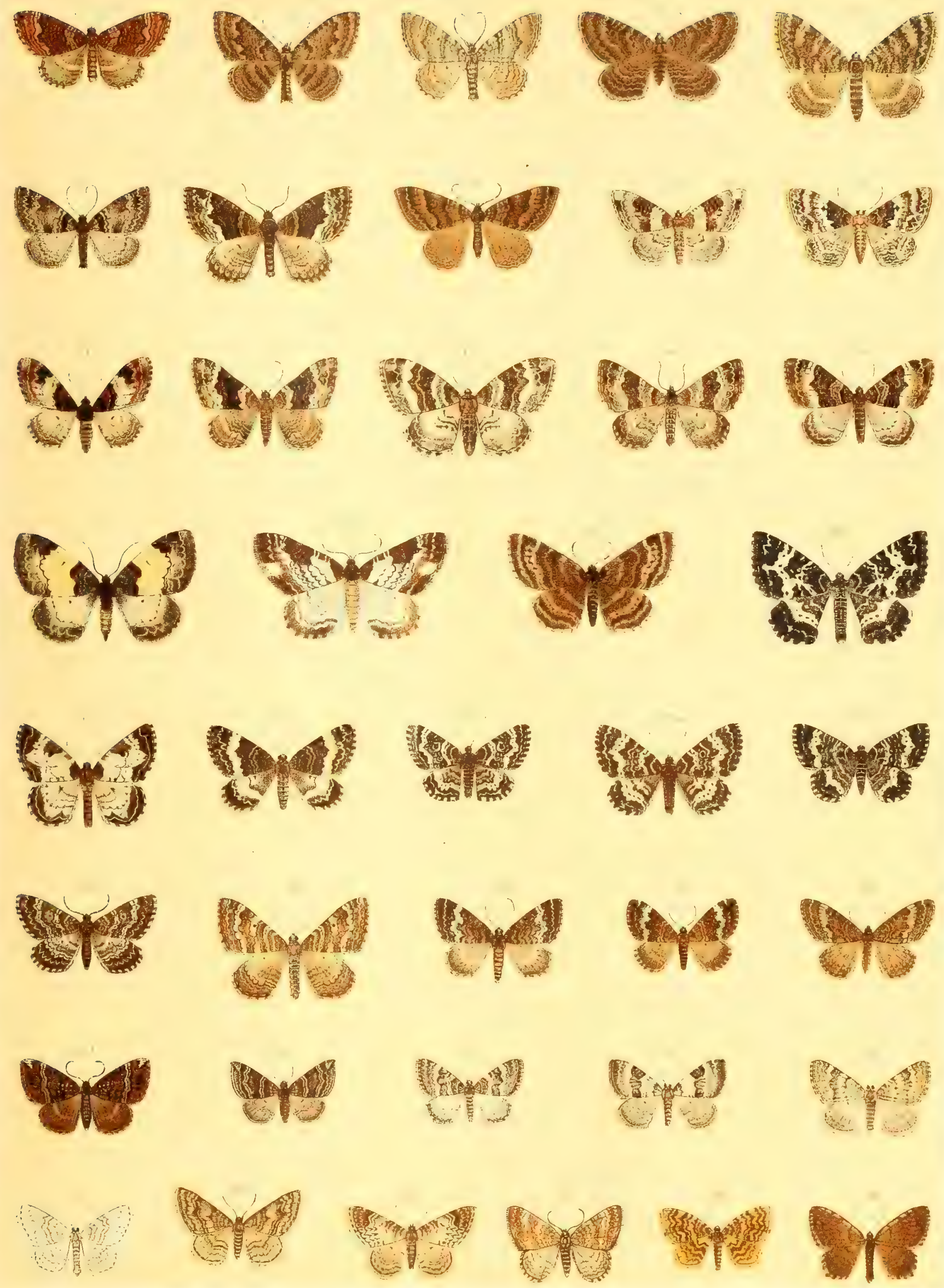




\section{TAFEL $\% 0$.}

\begin{tabular}{|c|c|c|c|c|c|}
\hline $\mathrm{Nr}$. & & Name & Katalog I & Seite des II. Bandes & $\begin{array}{l}\text { Raupen-(Puppeu-) } \\
\text { Abbildung }\end{array}$ \\
\hline 1. & Laréntia & bistrigáta Tr. & 3483 & 641. & \\
\hline 2. & $"$ & bilineáta L. $\sigma^{7}$ & 3481 & $63 \mathrm{r}$. & Taf. 46,24 \\
\hline 3 a. & $"$ & sordidáta $\mathrm{F} \cdot \mathrm{O}^{\lambda}$ & 3485 & 641. & $" 46,25 \mathrm{a}-\mathrm{c}$ \\
\hline $3 \mathrm{~b}$. & $"$ & ab. fusco-undáta Don. $\sigma^{7}$ & 3485 a & 641. & \\
\hline 4. & $"$ & autumnális Ström. & 3486 & $64 \mathrm{r}$ & $\Rightarrow 46,26$ \\
\hline 5. & $n$ & puberáta Frr. & 3487 & $64 \mathrm{r}$ & , \\
\hline 6. & $"$ & capitáta HS. & 3488 & 651. & $\Rightarrow \quad 46,27 \mathrm{a}-\mathrm{c}$ \\
\hline 7. & $"$ & silaceáta $\mathrm{Hb} . \sigma^{7}$ & 3489 & 651. & $" 46,28 \mathrm{a}, \mathrm{b}$ \\
\hline 8. & $"$ & coryláta Thnbg. $q$ & 3491 & $65 \mathrm{r}$ & $\left\{\begin{array}{l}\text { Taf. } 46,29 \mathrm{a}-\mathrm{c} \\
\mathrm{Ei} \quad 50,87\end{array}\right.$ \\
\hline 9. & $n$ & berberáta Sehiff. & 3498 & $66 \mathrm{l}$. & Taf. $46,30 \mathrm{a}$ \\
\hline 10. & $"$ & nigrofasciária Goeze & 3500 & $66 \mathrm{r}$. & $\because 46,31$ \\
\hline 11. & $"$ & rubidáta $\mathrm{F}$. & 3502 & $66 \mathrm{r}$. & " 46,32 \\
\hline 12. & $"$ & sagittáta $\mathrm{F}$. & 3504 & $67 \mathrm{r}$ & Nachtr.-Taf, VII, 29 \\
\hline 13. & $"$ & comitáta L. & 3503 & 671. & Taf. 46,33 \\
\hline 14. & Phibalápte & eryx lapidáta $\mathrm{Hb}$. & 3669 & 851. & \\
\hline 15. & $n$ & polygrammáta Bkh. & 3666 & $84 \mathrm{r}$. & $" 46,34$ \\
\hline 16. & $"$ & aquáta $\mathrm{Hb}$. & 3670 & 851. & Nachtr-Taf. VIII, 16 \\
\hline 17. & $"$ & vitalbáta $\mathrm{Hb}$. & 3671 & 851. & Taf. $49,7 \mathrm{a}, \mathrm{b}$ \\
\hline 18. & $"$ & corticáta Tr. & 3673 & $85 \mathrm{r}$. & Nachtr.-Taf. VIII, 17 \\
\hline 19. & $"$ & tersáta Hb. $\sigma^{7}$ & 3674 & $85 \mathrm{r}$. & $" \quad$ VIII, 18 \\
\hline 20. & $"$ & aemuláta $\mathrm{Hb} . q$ & 3679 & $85 \mathrm{r}$. & Taf. 46,35 \\
\hline 21. & $"$ & ealligrapháta HS. $q$ & 3681 & $85 \mathrm{r}$ & Nachtr.-Taf. VIII, 19 \\
\hline 22. & Collix spar & psáta Tr. & 3665 & $84 \mathrm{r}$ & $" \quad$ VIII, 15 \\
\hline 23. & Tephroelys & stia gratiosáta HS. & 3510 & 691. & VII, 31 \\
\hline 24. & $"$ & oblongáta Thnbg. & 3511 & $69 \mathrm{r}$ & Taf. $47,1 \mathrm{a}, \mathrm{b}$ \\
\hline 25. & $"$ & breviculáta Donz. & 3512 & $69 \mathrm{r}$. & Nachtr.-Taf. VII, 32 \\
\hline 26. & $"$ & extremáta F. & 3513 & $69 \mathrm{r}$ & \\
\hline 27. & $"$ & ipriguáta Hb. & 3531 & 711 & \\
\hline 28. & $"$ & insigniáta $\mathrm{Hb}$. & 3541 & $71 \mathrm{r}$. & \\
\hline 29. & $"$ & venosáta $\mathrm{F}$. & 3543 & 721. & Taf. $47,2 \mathrm{a}, \mathrm{b}$ \\
\hline 30. & $"$ & silenieoláta Mab. & 3542 & 721. & $" \quad 47,3$ \\
\hline 31. & $\eta$ & alliápia Stgr. & 3549 & $72 \mathrm{r}$ & $" 47,4$ \\
\hline 32. & $"$ & subnotáta Hb. & 3580 & $75 \mathrm{r}$ & $" 47,5$ \\
\hline 33. & $"$ & puleheliáta Stph. & 3522 & 701. & Nachtr.-Taf. VII, 34 \\
\hline 34. & $"$ & linariáta $L$. & 3520 & 701 & Taf. $47,6 \mathrm{a}-\mathrm{c}$ \\
\hline 35. & $"$ & laqueária HS. & 3523 & $70 \mathrm{r}$ & \\
\hline 36. & $"$ & pusilláta $\mathrm{F}$. & 3535 & 711. & Nachtr.-Taf. VII, 36 \\
\hline 37. & $"$ & strobiláta $\mathrm{Hb}$ & 3538 & $71 \mathrm{r}$ & VII, 38 \\
\hline 38. & $"$ & abietápia Goeze (togáta $\mathrm{Hb}$.) & 3539 & $71 \mathrm{r}$ & \\
\hline 39. & Chloroelyst & tis debiliáta $\mathrm{Hb}$. & 3661 & 841. & Taf. 47,7 \\
\hline 40. & $n$ & eoponáta Hb. & 3659 & 841. & $" 47,8 \mathrm{a}-\mathrm{c}$ \\
\hline 41. & $"$ & reetanguláta L. & 3660 & 841. & $" 47,9$ \\
\hline 42. & $\eta$ & chloeráta Mab. & 3662 & $84 \mathrm{r}$ & $" 47,10$ \\
\hline
\end{tabular}

Siehe ferner: Lar. alhambráta Stgr. Taf. 71 a, Fig. 39; Tephroel. guenéeáta Mill. Taf. 71 a, Fig. 42; T. pyreneáta Mab. Taf. 71 b, Fig. 33; T. liguriáta Mill. Taf. 71 b, Fig. 34; T. tedaldiáta Fuehs Taf. 71 a, Fig. 41 ; T. sehieferéri Bhtseh. Taf. 71 b, Fig. 35 ; T. earpophagáta Rbr. Taf. 71 b, Fig. 37 und T. egenária HS. Taf. 71 a, Fig. 43. 


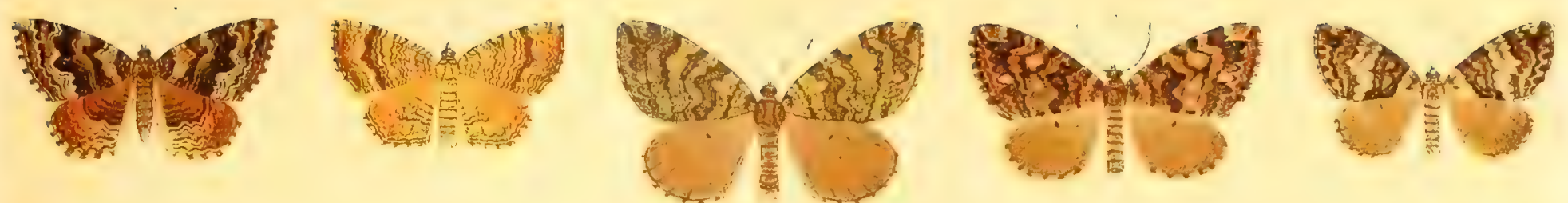

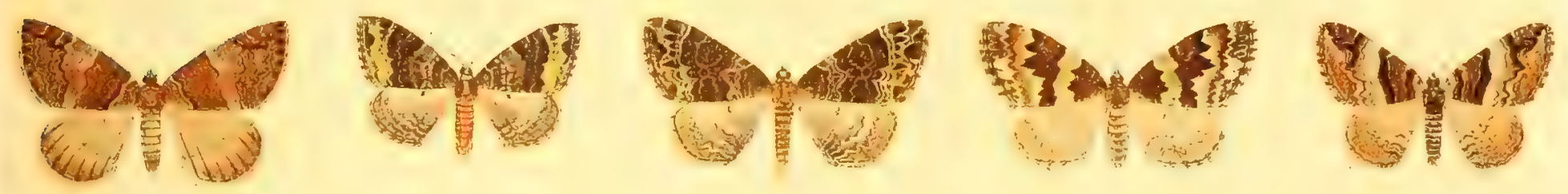

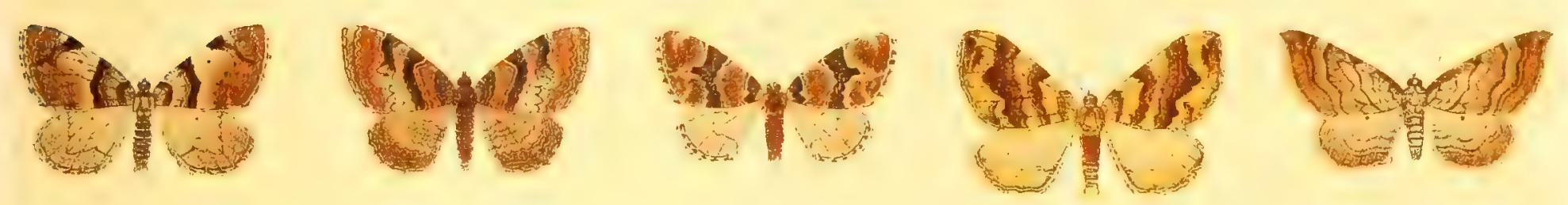
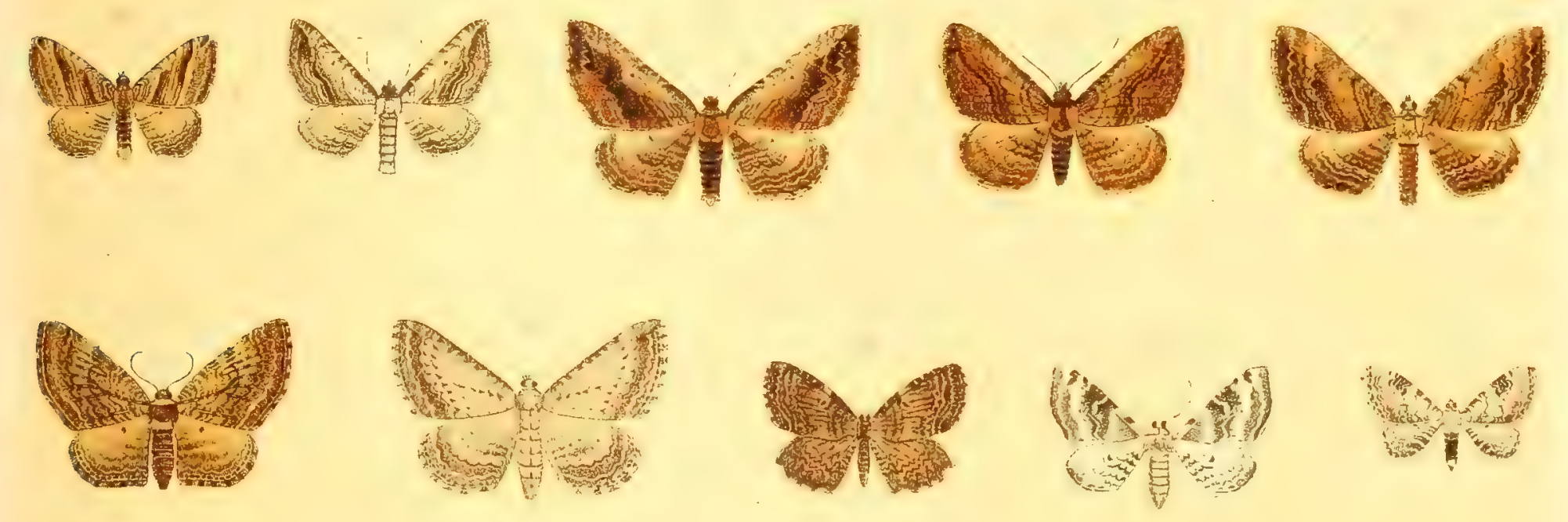

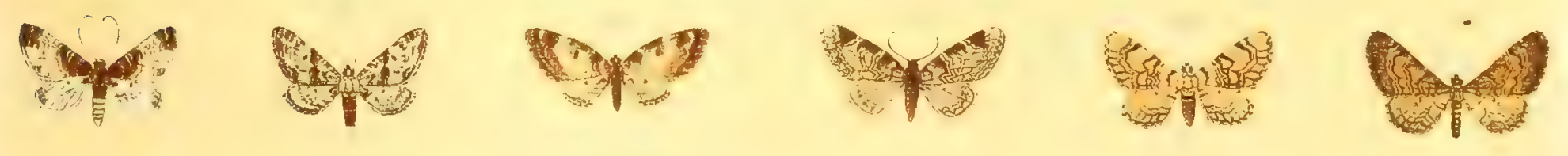

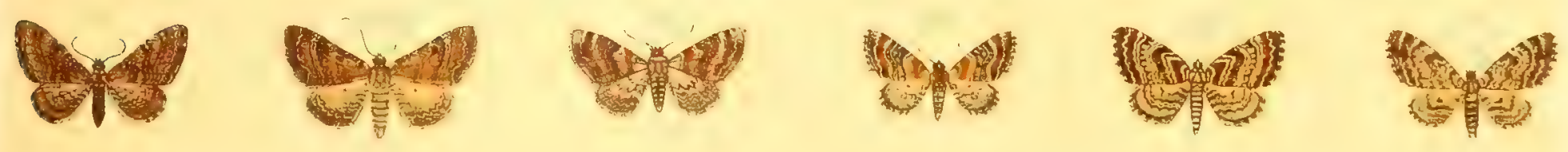

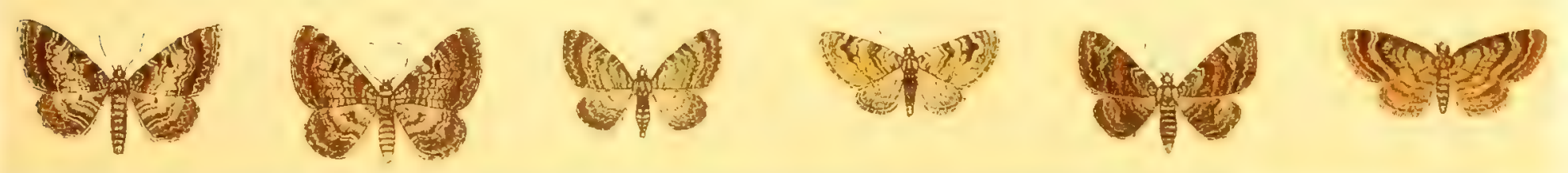






\section{TAFEL, ri1.}

\begin{tabular}{|c|c|c|c|c|c|}
\hline $\mathrm{Nr}$. & & Tame & Katalog I & Seite des II. Bandes & $\begin{array}{l}\text { Raupen-(Puppen-) } \\
\text { Abbildung }\end{array}$ \\
\hline 1. & Tephroelystia & scabiosáta Bkh. & 3604 & 781. & Taf. 47,11 \\
\hline פ. &. & denticuláta Tr. & 3606 & $78 \mathrm{r}$. & \\
\hline 3. & .. & millefoliáta Rössl. & 3603 & 781 & $" 47,12 \mathrm{a}-\mathrm{c}$ \\
\hline 4. & ." & succenturiáta L. & 3600 & $77 \mathrm{r}$. & $" 47,13$ \\
\hline$\therefore$. & " & v. subfulváta $\mathrm{Hw}$. & $3600 \mathrm{c}$ & $77 \mathrm{r}$. & $\because 47,14 \mathrm{a}, \mathrm{b}$ \\
\hline 6. & ., & seopariáta Rbr & 3597 & 771. & 2. $47,15 \mathrm{a}, \mathrm{b}$ \\
\hline 7. & $"$ & nanáta $\mathrm{Hb}$. & 3634 & 811. & $\because \quad 47,16$ \\
\hline 8. & " & innotáta Hufn. & $3636^{\circ}$ & $81 \mathrm{x}$ & $4 \quad 47,17$ \\
\hline 9. & $"$ & v. tamariseiáta Frr & 3636 a & $81 r$ & $" 47,18$ \\
\hline 10. &. & v. fraxináta Crewe & $3636 \mathrm{~b}$ & $811^{1}$ & \\
\hline 11. & ." & extensária Frp & 3585 & 761. & \\
\hline 12. &. & impupáta Hb. & 3607 & $78 \mathrm{r}$ & Nachtr.-Taf. VII, 53 \\
\hline 13. & $"$ & semigrapháta Brd & 3608 & $78 \mathrm{r}$. & Taf. 47,19 \\
\hline 14. & $"$ & unitária HS. & 3612 & 791. & \\
\hline 15. & $"$ & grapháta Tr. & 3614 & $79 \mathrm{l}$. & \\
\hline 16. & $"$ & scriptária HS. & 3616 & $79 \mathrm{r}$. & Nachtr.-Taf. VIII, 2 \\
\hline 17. & $"$ & mayéri Mn. & 3615 & 791. & VIII, 1 \\
\hline 18. & :" & ripária HS. & 3618 & $79 \mathrm{r}$. & \\
\hline 19. &. & spissilineáta Metzner & 3620 & $79 \mathrm{r}$. & \\
\hline 20. & $\because$ & pygmaeáta $\mathrm{Hb}$. & 3627 & $80 \mathrm{r}$. & VIII, 5 \\
\hline 21. & . & ultimária B. & 3628 & $80 \mathrm{r}$. & \\
\hline 2. & $"$ & isogrammária HS. & 3625 & 801. & VI, 29 \\
\hline 23. & $"$ & tenuiáta $\mathrm{Hb}$. & 3630 & $80 \mathrm{r}$. & Taf. 47,20 \\
\hline 24. &. & inturbáta $\mathrm{Hb}$. & 3631 & 811. & Nachtr.-Taf. VIII, 6 \\
\hline 25. &. & plumbeoláta HW. & 3623 & $79 \mathrm{r}$ & VIII, 3 \\
\hline 26. & $\because$ & valerianáta Hb. & 3626 & $80 \mathrm{l}$. & Taf. $47,21 \mathrm{a}, \mathrm{b}$ \\
\hline 27 & $\because$ & immundáta Z. & 3624 & 801. & Nachtr.-Taf. VIII, 4 \\
\hline 28. &. & eauehyáta Dup. & 3594 & $77 \mathrm{l}$ & VII, 52 \\
\hline 29. & $"$ & satyráta Hb. & 3595 & 771 & Taf. 47,22 \\
\hline 30. & $"$ & vepatrária HS. & 3591 & $76 \mathrm{r}$. & Nachtr.-Taf. VII, 51 \\
\hline 31. & :, & helveticária B. & 3592 & $76 \mathrm{r}$. & Taf. 47, 23 \\
\hline 32. & $"$ & eastigáta Hb. & 3575 & $75 \mathrm{r}$ & $n 47,24 a-c$ \\
\hline 33. &. & trisignária HS. & 3573 & 751. & $\because 47,25$ \\
\hline 34. & $"$ & virgaureáta Dbld. & 3571 & 751. & Nachtr.-Taf. VII, 49 \\
\hline 35. & $"$ & vulgáta Hw. & 3569 & 751. & VII, 47 \\
\hline 36. & $"$ & denotáta $\mathrm{Hb}$. & 3563 & 741 & VII, 46 \\
\hline 37 & $"$ & albipunetáta Hw. & 3567 & $74 \mathrm{r}$. & Taf. 47,26 \\
\hline 38. & $"$ & aetaeáta Walderdorff & 3565 & $7+\mathrm{r}$. & $\Rightarrow \quad 47,27$ \\
\hline 39. & " & assimiláta Gn. & 3559 & $73 \mathrm{r}$. & Nachtr.-Taf. VII, 45 \\
\hline 40. & $"$ & eallúnae $\mathrm{Spr}$ & 3561 & $7+1$ & Taf. $47,28 \mathrm{a}-\mathrm{c}$ \\
\hline 41. & $"$ & absinthiáta $\mathrm{Cl}$. & 3560 & $7 \pm 1$. & $" 47,29$ \\
\hline 42. & $"$ & expallidáta Gn. & 3558 & $73 \mathrm{r}$. & Nachtr.-Taf. VII, 44 \\
\hline 43. & $"$ & pimpinelláta $\mathrm{Hb}$. & 3553 & 731. & Taf. 47,30 \\
\hline 44. & ., & euphrasiáta HS. & 3552 & 731. & Nachtr.-Taf. VII, 40 \\
\hline 45. & $"$ & gemelláta HS. & 3554 & 731. & VII, +1 \\
\hline 46. & $"$ & $\begin{array}{l}\text { Kopie der albifronsáta Grasl., } \\
\text { Synonym der folgenden }\end{array}$ & & & \\
\hline 47. & $"$ & distinetápia HS. & $3556^{\circ}$ & $73 \mathrm{r}$. & VII, +2 \\
\hline 48. & $"$ & contermináta $\mathrm{Z}$. & 3536 & 711. & \\
\hline 49. & $"$ & indigáta Hb. & 3537 & $711 \%$ & VII, 37 \\
\hline 50. & $"$ & lapieiáta Frp & 3574 & $75 \mathrm{r}$ & VII, 50 \\
\hline 51. & $"$ & silenáta Stdfs sen. & 3596 & 771. & Taf. 47,32 \\
\hline 52. & $"$ & abbreviáta Stph. & 3646 & $82 \mathrm{l}$ & Nachtr.-Taf. VIII, 11 \\
\hline 53. & $"$ & dodonaeáta Gn. & 3648 & $82 \mathrm{r}$ & VIII, 12 \\
\hline 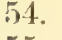 & $n$ & exiguáta $\mathrm{Hb}$. & 3650 & $82 \mathrm{r}$. & Taf. 47,31 \\
\hline 55. & $"$ & lanceáta $\mathrm{Hb}$ & 3653 & 831. & Nachtr.-Taf. VIII, 13 \\
\hline 56. & $"$ & phoeniceáta Rbp & 3641 & 821. & VIII, 9 \\
\hline 37. & $"$ & oxycedráta Rbp & 3643 & 821. & VIII, 10 \\
\hline 58. & $"$ & sobrináta Hb. & 3656 & 831. & Taf. $47,33 \mathrm{a}-\mathrm{e}$ \\
\hline 59 & . & pumiláta $\mathrm{Hb}$. & 36.58 & $83 \mathrm{r}$. & Nachtr.-Taf. VIII, 14 \\
\hline
\end{tabular}

Siehe ferner: Tephroel. extraversária HS. Taf. 71 a, Fig. 44; T. fenestráta Mill. ib., Fig. 45 ; T. druentiáta Dietze ib., Fig. 46; T. santolináta Mab. ib., Fig. 47; T. massiliáta Mill. ib., Fig. 48; T. unedonáta Mab. ib., Fig. 49; T. sinuosápia Ev. ib., Fig. 50; T. selináta HS. Taf. 71 b, Fig. 36 ; T. aggregáta Gn. ib., Fig. 38; T. pantellária Mill. ib., Fig. 39; T. hyperporeáta Stgr ib., Fig. 40; T. rosmarináta Mill. ib., Fig. 41; T. semitinetária Mab. ib., Fig. 42; T. lentiseáta Mab. ib., Fig. 43; T. ericeáta Rbr ib., Fig. 44. 


\section{(ben?
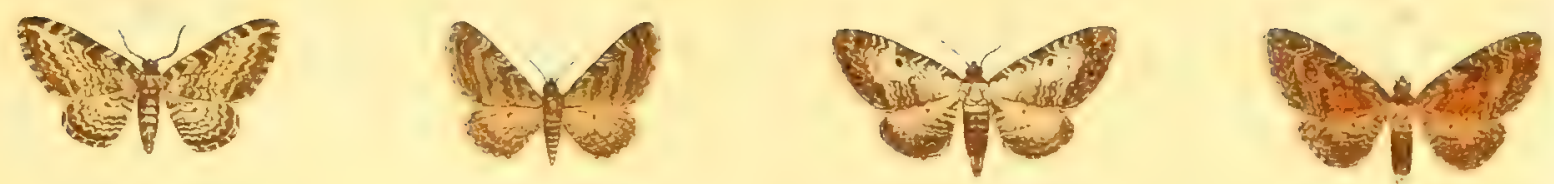

Q1e
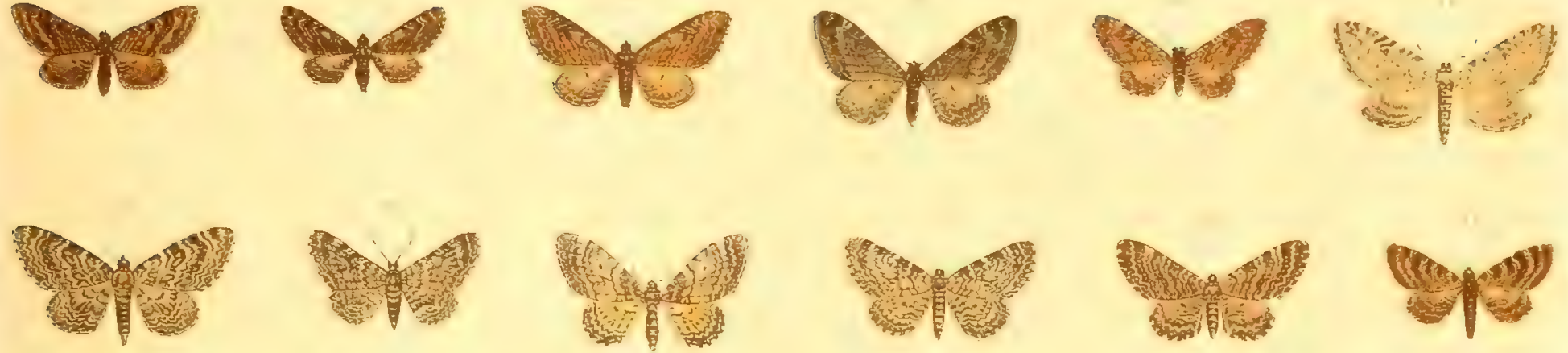

(ave)

Mafle

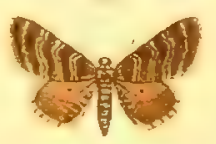

19u
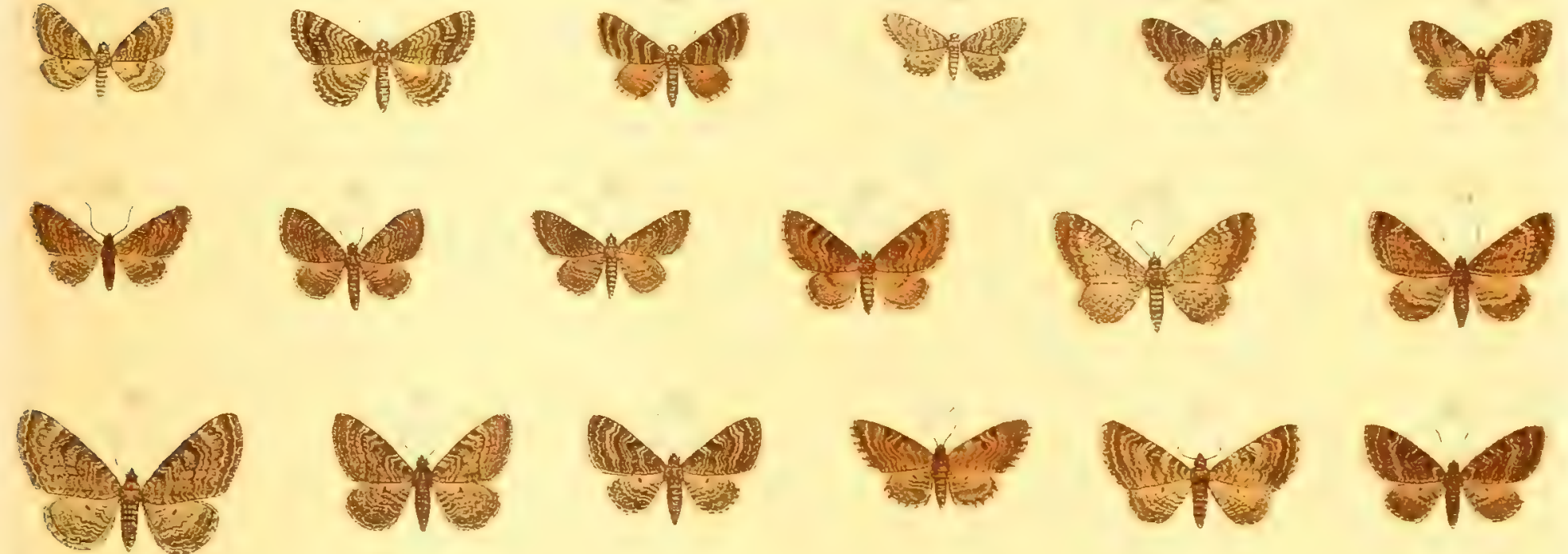

\section{1.}
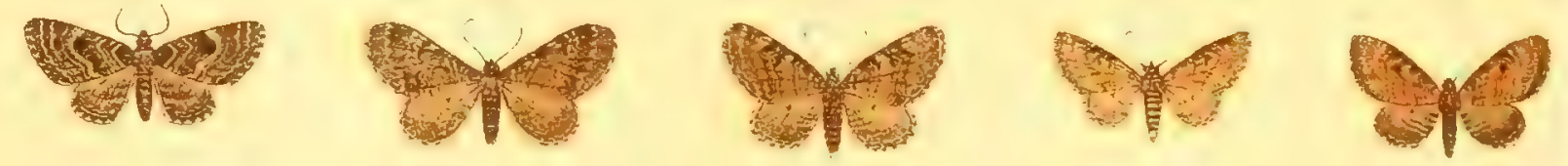

ences
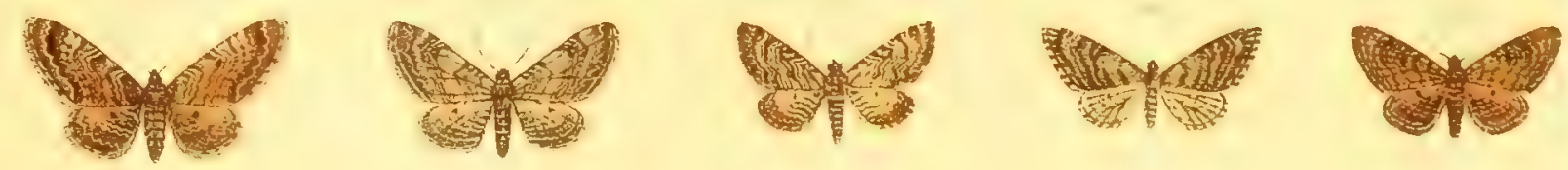

(and
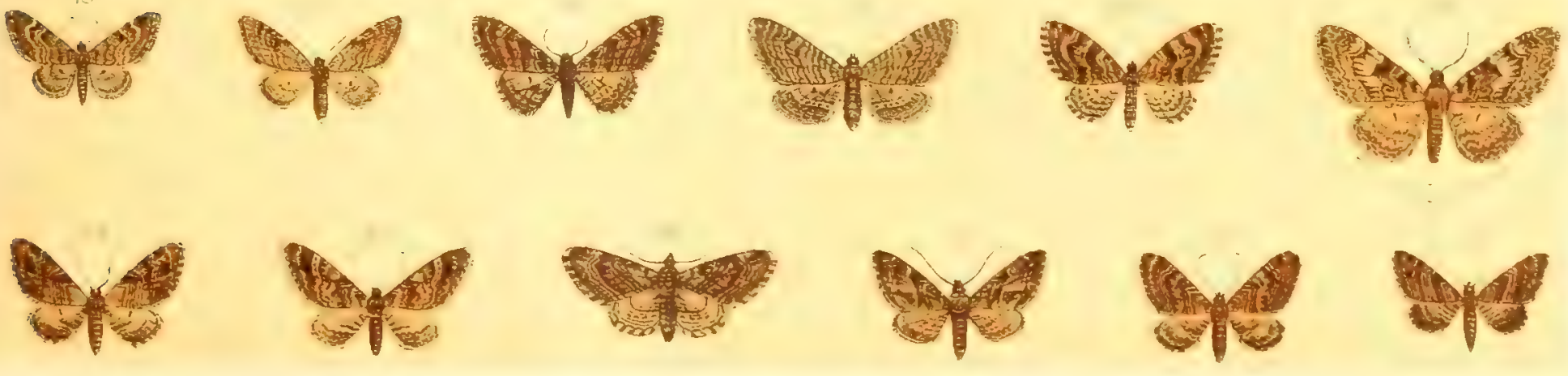




\section{TAFE L 71a.}

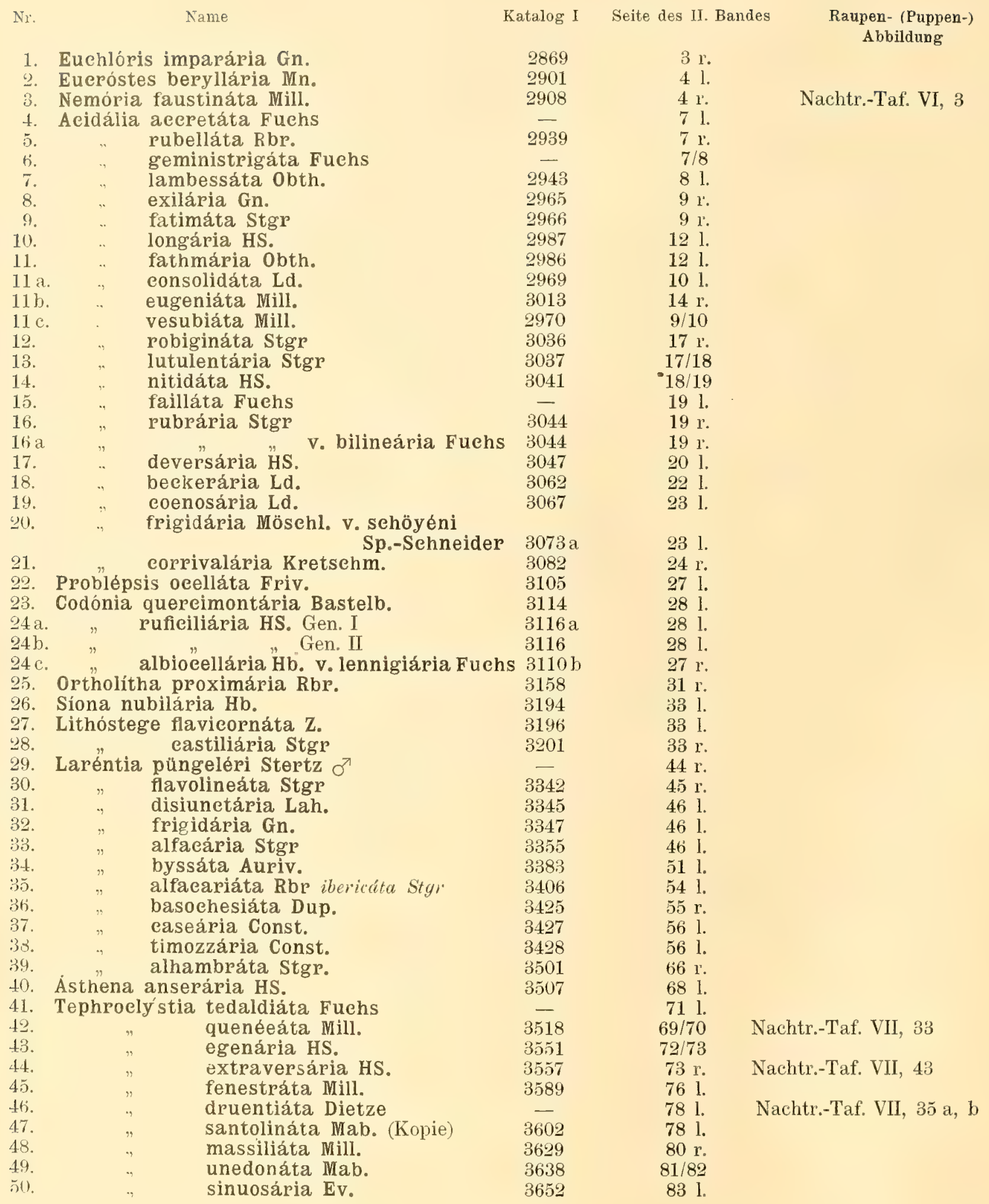




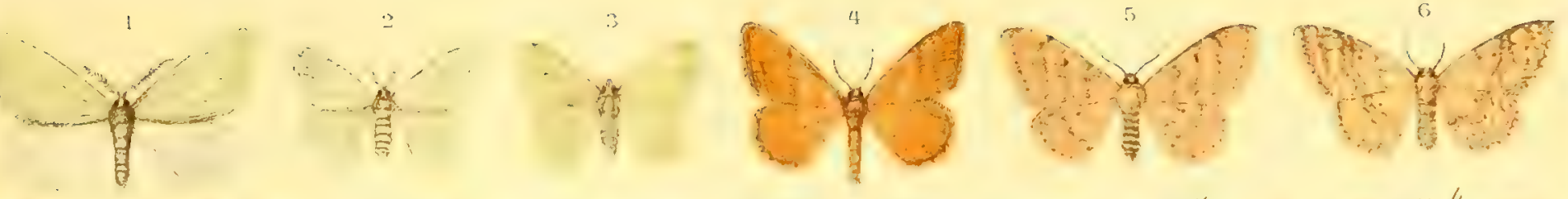

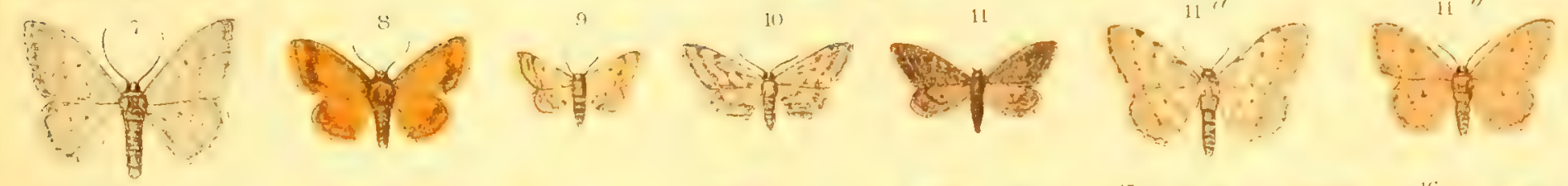
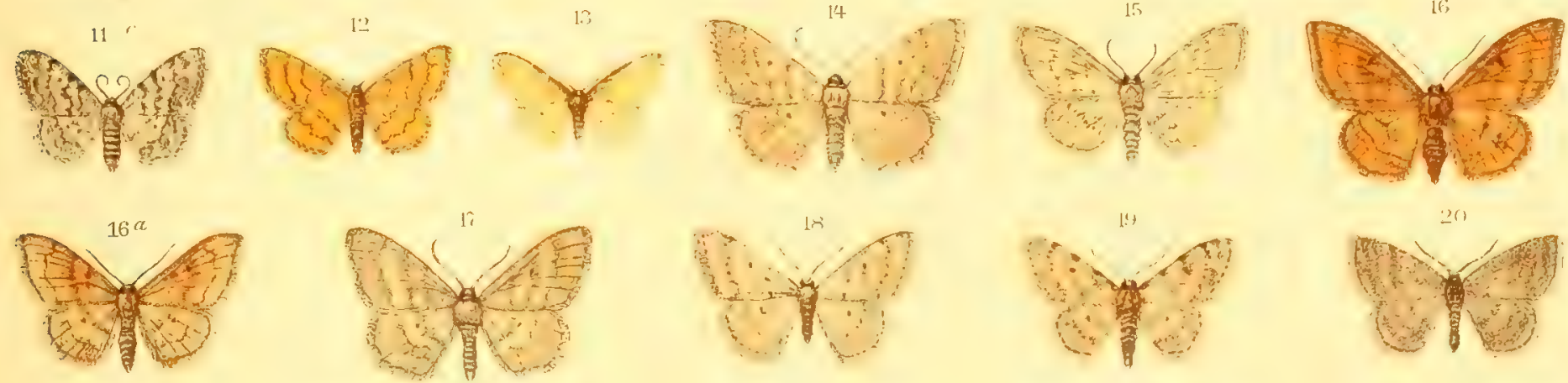

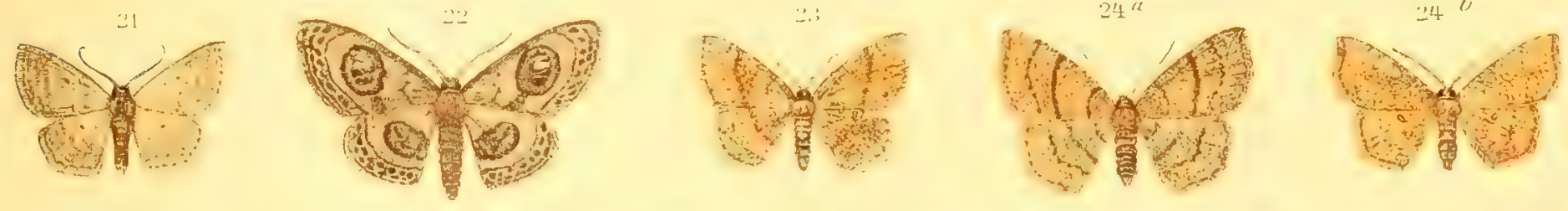
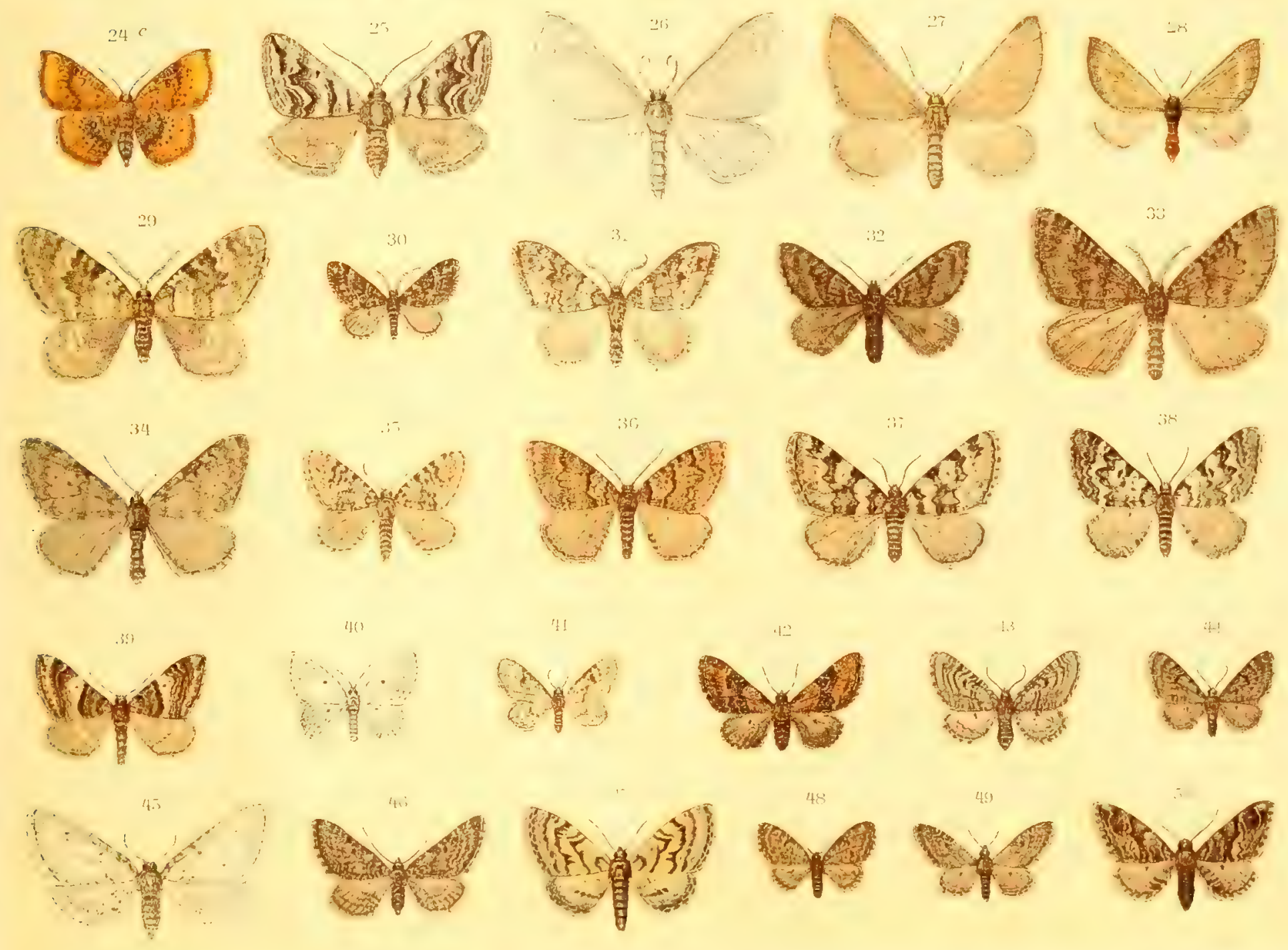




\section{TAFEL \%1b.}

\begin{tabular}{|c|c|c|c|c|c|}
\hline $\mathrm{Nr}$. & Name & Katalog I & Seite des II. Bandes & \multirow{2}{*}{\multicolumn{2}{|c|}{$\begin{array}{c}\text { Rrupen- (Puppen-) } \\
\text { Abbildung }\end{array}$}} \\
\hline 1. & Eusárea interpunctária HS. (Kopie) & 3687 & $86 \mathrm{r}$. & & \\
\hline & Dasyeéphala modésta Stgr જフ & 3747 & 941. & & \\
\hline 11 & Croeállis aubérti 0bth. (Kopie) & 3750 & $94 \mathrm{r}$. & & \\
\hline $1 \mathrm{c}$ & Eilierínia eauteriáta Stgr (Kopie) & 3776 & 971. & & \\
\hline 2. & Stegánia dalmatária Gn. $\sigma^{7}$ & 3710 & $89 \mathrm{r}$. & & \\
\hline 3. & Eúmera regína Stgr or & 3732 & 921. & & \\
\hline & Lignyóptera thaumastária $\mathrm{Rbl}$ & & $98 \mathrm{l}$. & & \\
\hline & Ilierínia subeordária HS. $q$ & 3778 & 971. & & \\
\hline & Synópsia serrulária Ev. $\sigma^{7}$ & 3856 & 1031. & & \\
\hline & Boármia viértli Bhtseh ठ & 3875 & 1041. & & \\
\hline 7. & " maeoticária Alph. (Kopie) & 3899 & $105 \mathrm{r}$. & & \\
\hline 8. & bistortáta Goeze $q$ & 3904 & 1061. & & \\
\hline 9. & Tephrỏnia oppositária Mn. & 3913 & $106 \mathrm{r}$. & & \\
\hline & Gnophos onustária H.S. v. serrária Gn. $q$ & $3932 \mathrm{a}$ & 1081. & & \\
\hline 11. & " grunerária Stgr (Kopie) & 3961 & $109 \mathrm{r}$. & & \\
\hline 12. & andereggária Lah. $\sigma^{7}$ & 3969 & 1101. & & \\
\hline & Psodos noricána Wagner & 3976 & 1111. & & \\
\hline & Egéa eacuminária Rbr ơ & 3989 & $112 \mathrm{r}$. & & \\
\hline & Fidónia pratána F. ㅇ (Kopie) & 3994 & 1131. & & \\
\hline & Thamnonóma buffonária Mill. 우 (Kopie) & 4011 & $115 \mathrm{r}$. & & \\
\hline 17. & " sparsária Hb. व & 4015 & 1161. & & \\
\hline & Phasiane binaeváta Mab. (Kopie) & 4029 & 1171. & & \\
\hline & Eubólia griseolária Ev. (Kopie) & 4039 & $117 \mathrm{r}$. & & \\
\hline 20. & $" \quad$ eatalaunária Gn. $\sigma^{7}$ & 4043 & $117 / 18$ & Nachtr.-Taf. & VIII, 38 \\
\hline & Enconista unieolária Rbr ơ (Kopie) & 4051 & $118 \mathrm{l}$. & & \\
\hline & Percónia baetieária Rbr $\sigma^{7}$ (Kopie) & 4080 & $120 \mathrm{r}$ & & \\
\hline & Prosopólopha argentária HS. Оフ (Kopie) & 4083 & 1211. & & \\
\hline & Malacódea regelária Tngstr. & 3254 & 261. & & \\
\hline & Acidália belemiáta Mill. (Kopie) & 3030 & $16 \mathrm{r}$ & & \\
\hline 26. & eepvantária Mill. (Kopie) & 2978 & $10 / 11$ & & \\
\hline 27. & isabellária Mill. (Kopie) & 2979 & $11 \mathrm{i}$. & & \\
\hline 28. & subtiláta Chr. & 3098 & $26 \mathrm{r}$ & & \\
\hline & Laréntia corydalária Graes. & 3453 & $60 \mathrm{r}$. & & \\
\hline :30. & . $\quad$ putridária HS. & 3429 & 561. & & \\
\hline 31. & oxybiáta Mill. (Kopie) & 3435 & 571. & & \\
\hline & Ásthena nymphuláta Gn. (Kopie) & 3509 & $68 \mathrm{r}$. & & \\
\hline 33. & Tephroelýstia pyreneáta Mab. & 3521 & 701. & & \\
\hline 34. & liguriáta Mill. roederária Stdfs & 3525 & $70 \mathrm{r}$ & & \\
\hline 35. & sehieferéri Bhtseh & $3543 b$ & 721. & & \\
\hline 36. & selináta HS. & 3572 & 751. & , & VII, 48 \\
\hline 37. & carpophagáta Rbr (Ko],ie) & $3546 ; 47$ & 721. & , & VII, 39 \\
\hline 38. & aggregáta Gn. (Kopie) & 3593 & $76 / 77$ & & \\
\hline 39. & pantellária Mill. (Kopie) & 3613 & $79 \mathrm{l}$ & & \\
\hline 41). & hyperboreáta Stgr & 3635 & 811. &.. & VIII, 7 \\
\hline 41. & rosmarináta Mill. & 3640 & 821 & $"$ & VIII, 8 \\
\hline 42. & semitinetápia Mab. (Kopie) & 3647 & $82 \mathrm{r}$. & & \\
\hline 43. & lentiseáta Mab. (Kopie) & 3655 & 831. & & \\
\hline 44. & erieeáta Rbr & 3657 & $83 \mathrm{r}$. & & \\
\hline 45. & Phibalápteryx exoletáta HS. (Kopie) & 3676 & $85 \mathrm{r}$. & & \\
\hline
\end{tabular}


$(4) \frac{-19}{13}$
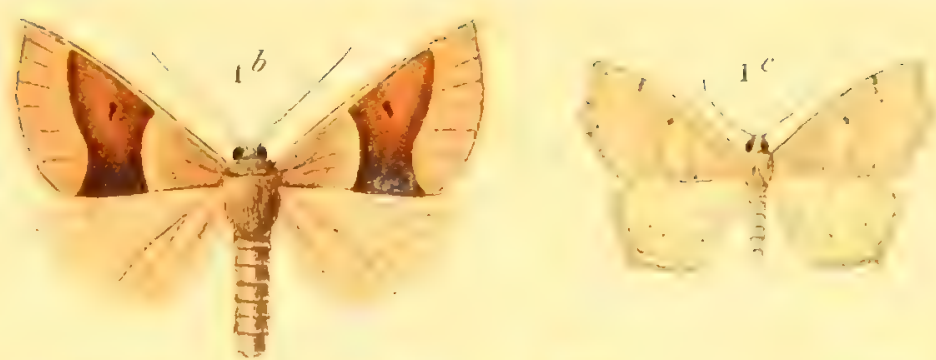

int digt?

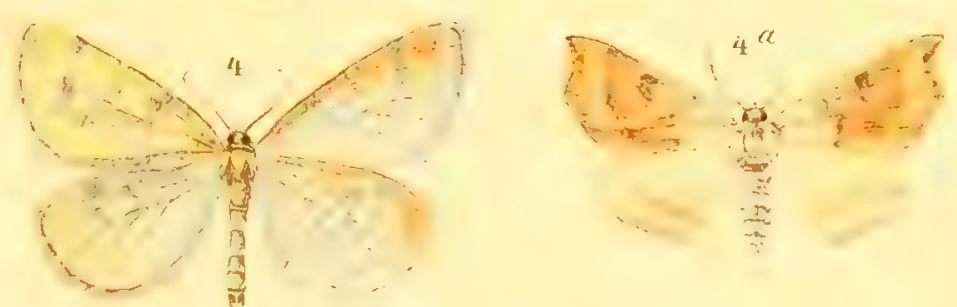

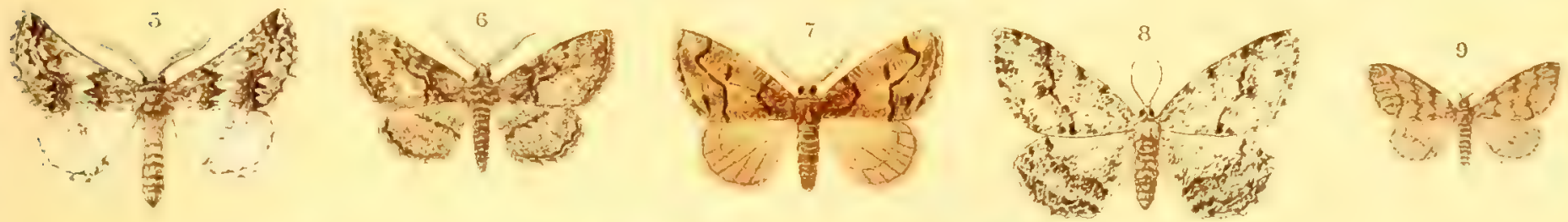
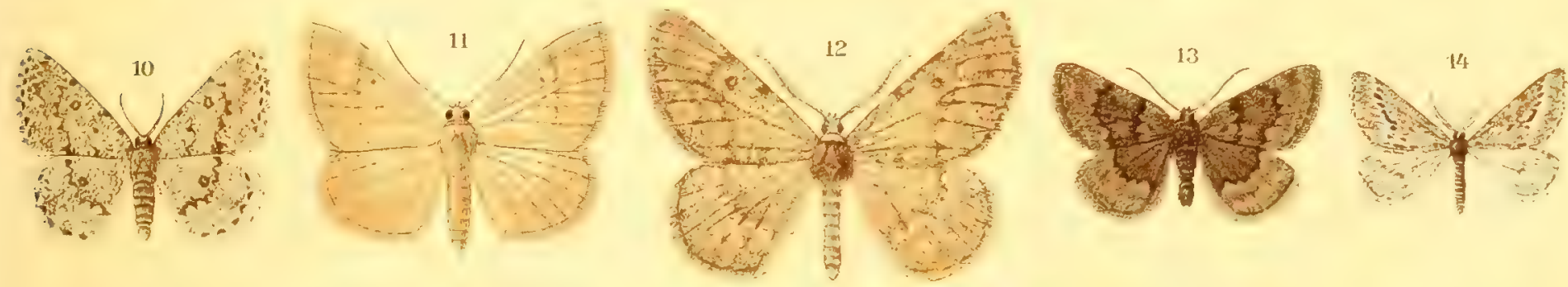

(150)
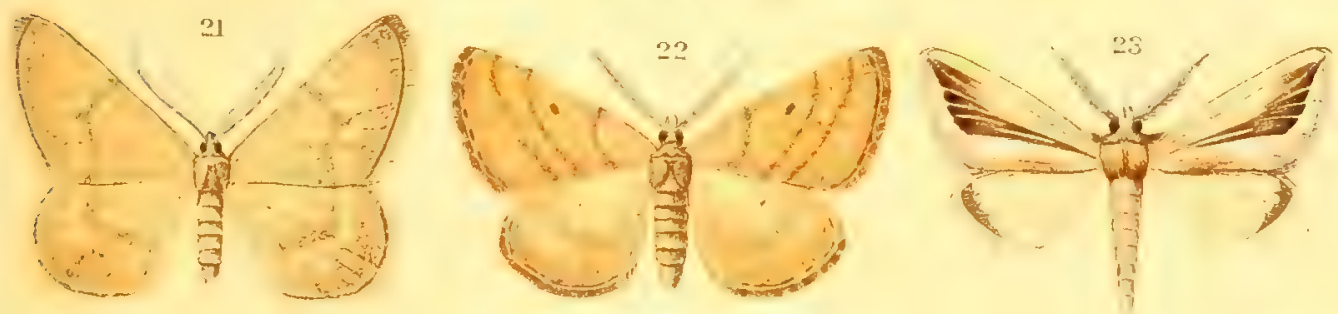

ment 8

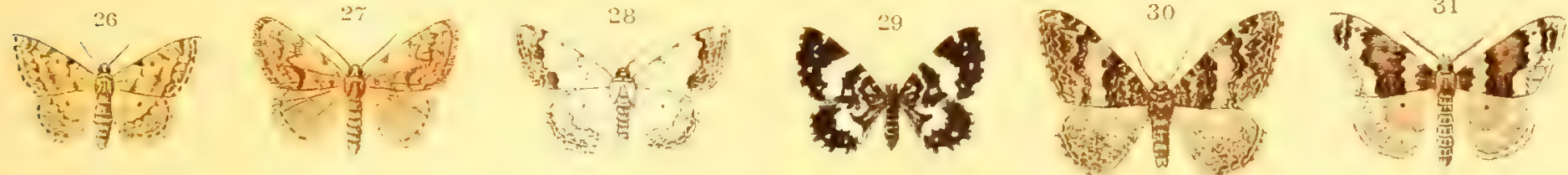

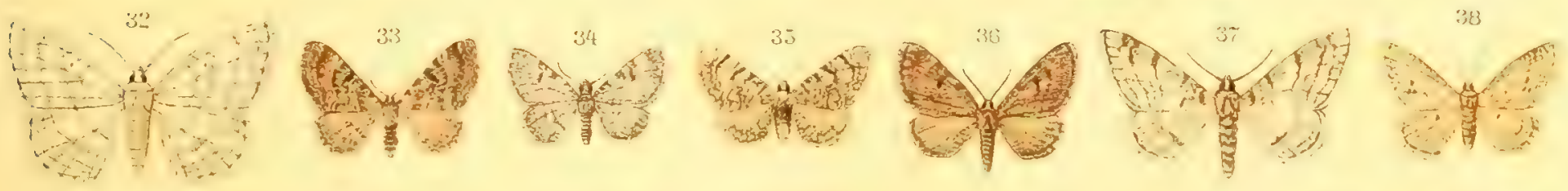

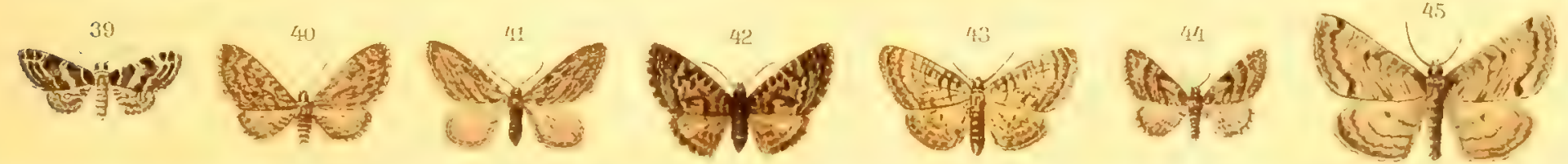






\section{TAFEL 72}

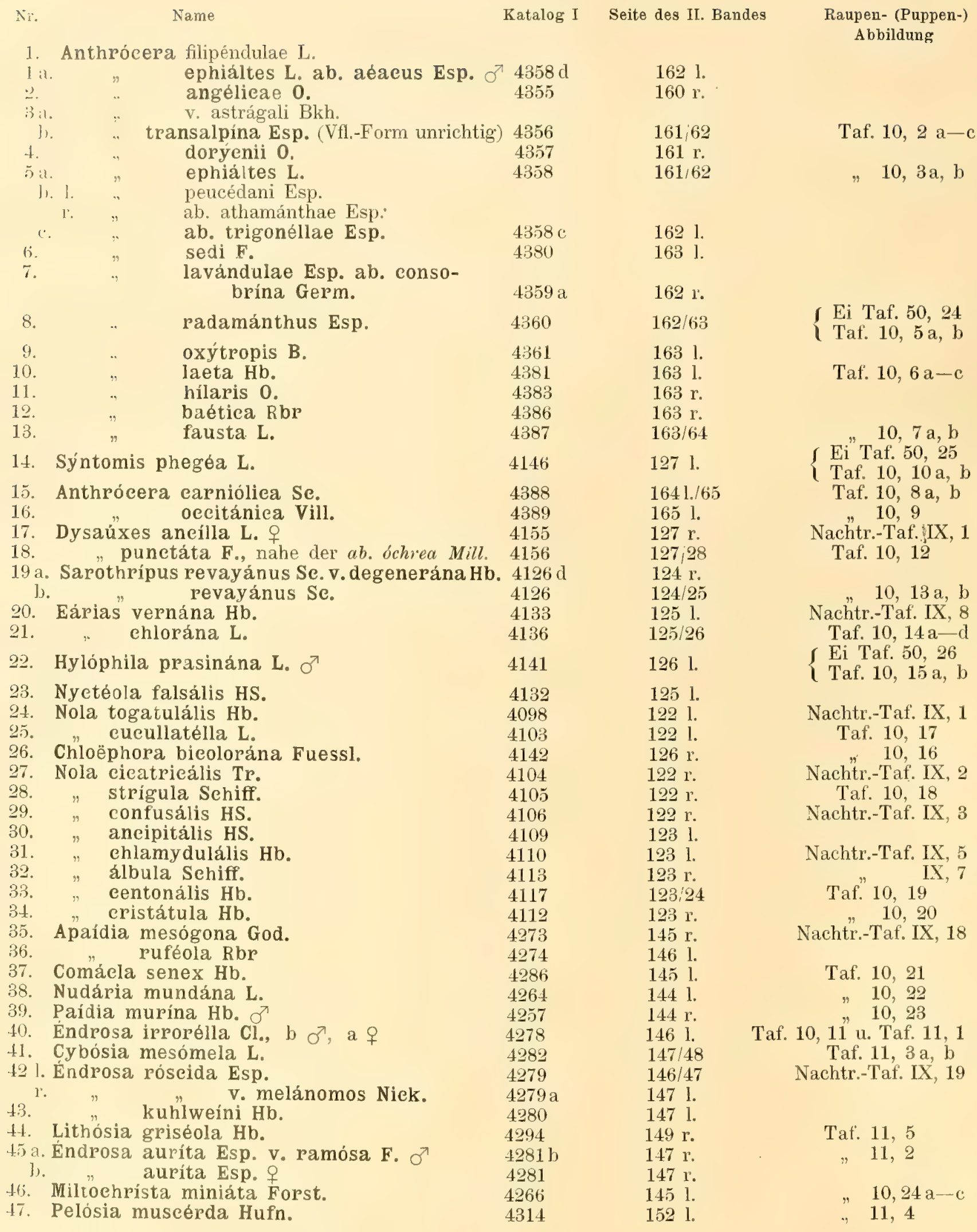

Siche auch Taf. 76 und 77.

Die Figuren 1, 3a und $5 \mathrm{~b}$ unkenntlich. 


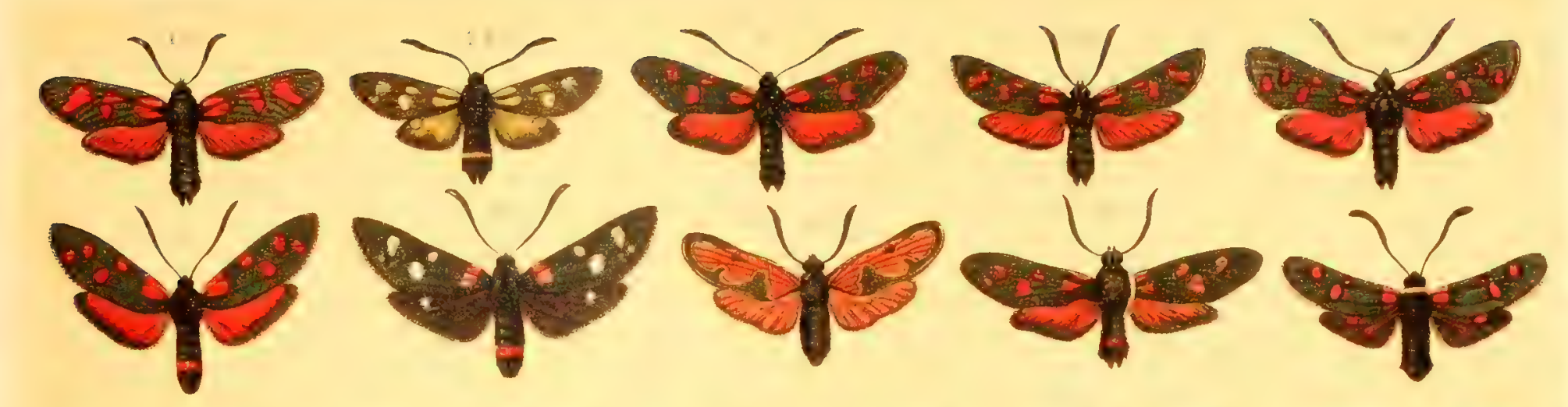

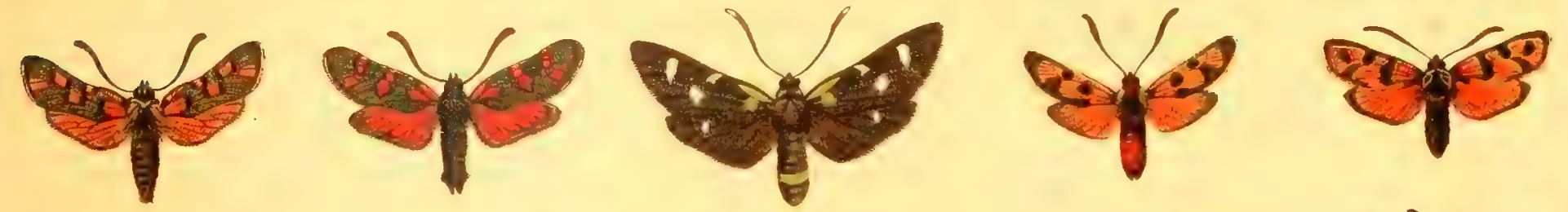

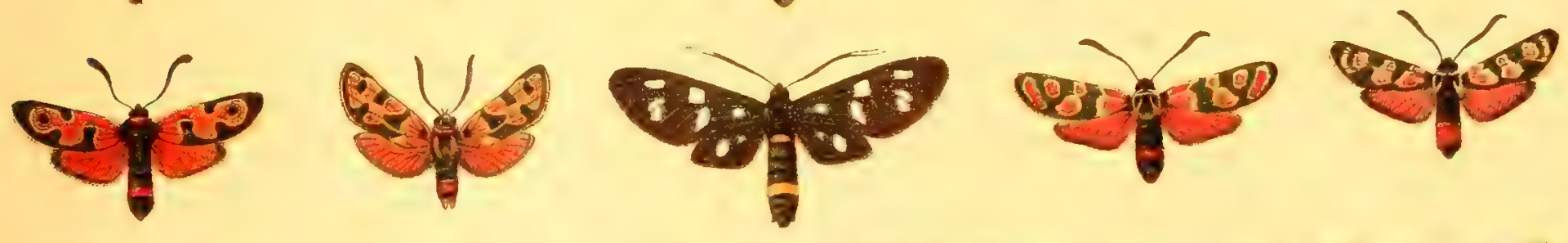

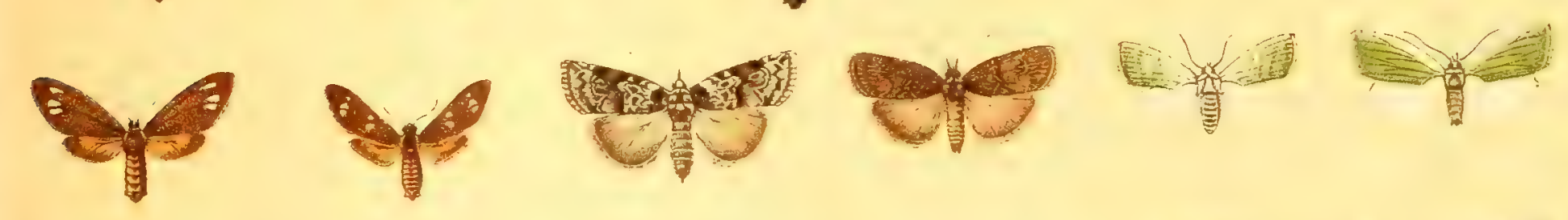

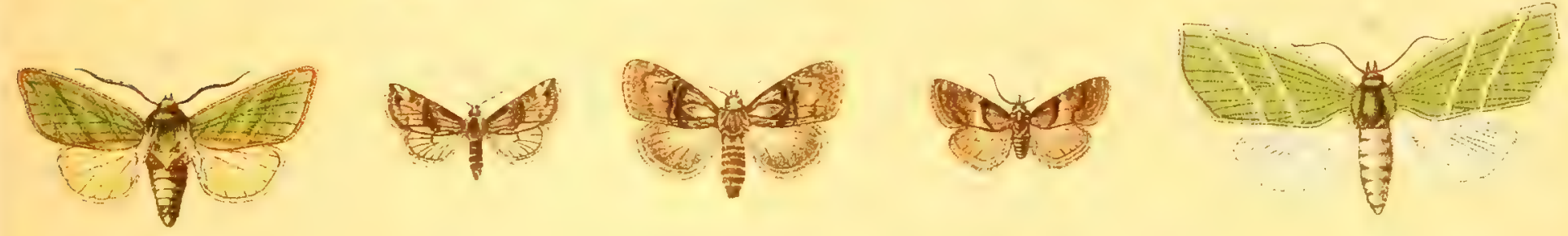

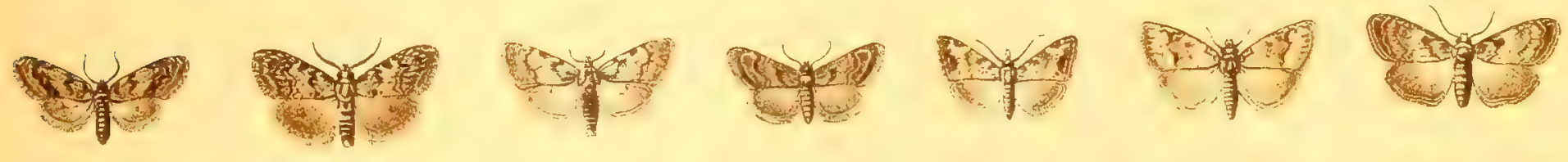

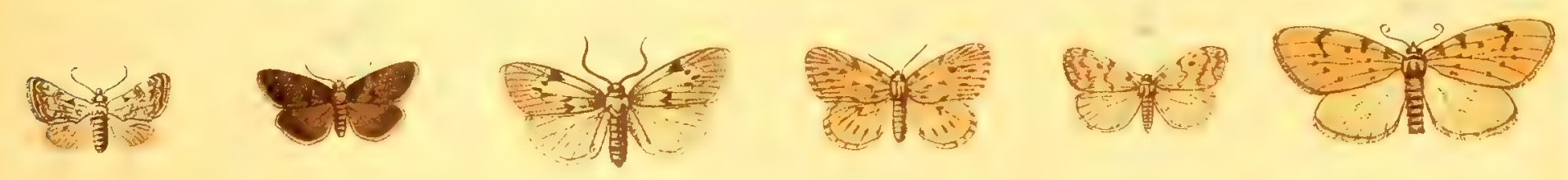
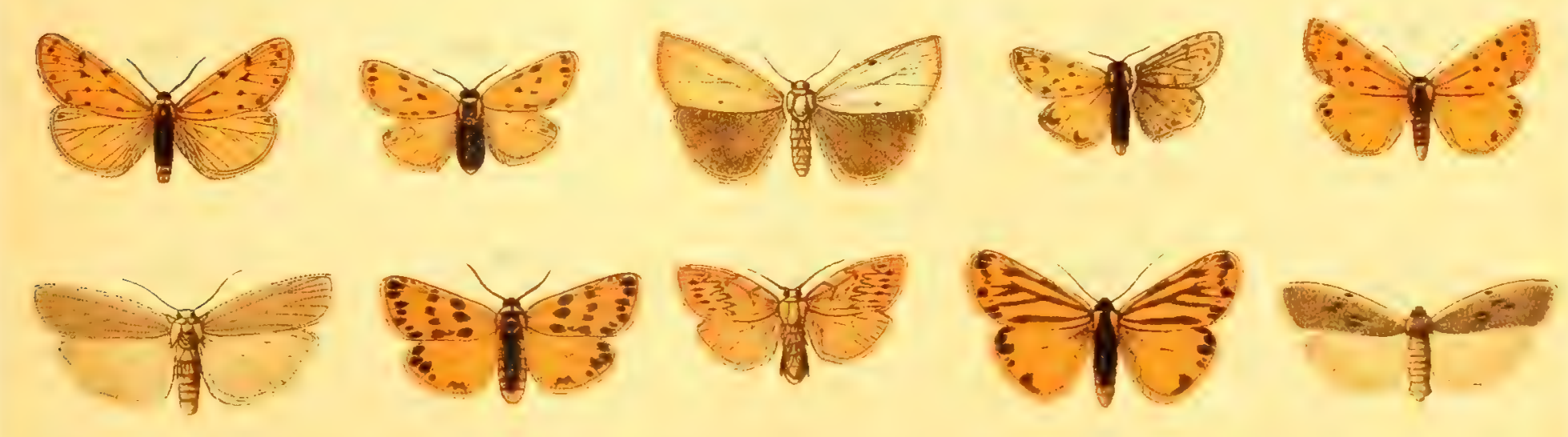




\section{TAFEL 73.}

\begin{tabular}{|c|c|c|c|}
\hline Name & Katalog I & Seite des II. Bandes & $\begin{array}{c}\text { Raupen-(Puppen-) } \\
\text { Abbildung }\end{array}$ \\
\hline 1a. Ảretia maculósa Gerning $\sigma^{7}$ & 4217 & $137 / 38$ & Taf. 13,4 \\
\hline b. $\quad " \quad$ simplóniea B. $\sigma^{7}$ & $4217 \mathrm{~b}$ & $137 \mathrm{r}$. & \\
\hline 2. Aretinia caesárea Goeze & \pm 187 & 1331. & $\Rightarrow 13,13 \mathrm{a}, \mathrm{b}$ \\
\hline 3. Trichosóma parasíta $\mathrm{Hb}$, , a $\sigma^{7}, \mathrm{~b} q$ & 4194 & 1341. & $" 13,11$ \\
\hline 1. Ảretia latréillei God. 우 & 4220 & $138 \mathrm{r}$. & Nachtr.-Taf. IX, 17 \\
\hline 5. $"$ easta Esp. $\sigma^{7}$ & 4218 & 1381. & Taf. $13,5 \mathrm{a}-\mathrm{c}$ \\
\hline 6. Spilosóma luetuósum H.-G. $\sigma^{7}$ & 4171 & $128 \mathrm{l}$ & Nachtr.-Taf. IX, 11 \\
\hline 7. Triehosóma hemigenum Grasl. $\sigma^{7}$ & 4195 & 1341. & IX, 15 \\
\hline " baéticum Rbr $\sigma^{\pi}$ & 4189 & $133 / 34$ & IX, 14 \\
\hline 9. Rhypária purpuráta L. ㅇ & $\$ 179$ & $131 \mathrm{r}$. & Taf. $12,7 \mathrm{a}-\mathrm{c}$ \\
\hline 10. Áretia hébe L. ㅇ & 4215 & 1371. & n $13,1 \mathrm{a}, \mathrm{b}$ \\
\hline " festíva Bkh. + & 4210 & $136 / 37$ & \\
\hline " faseiáta Esp. & 4204 & 1361. & $" 12,8$ \\
\hline " eaía L., a $\sigma^{7}, \mathrm{~b}$ extreme dunkle Aberr. & 4201 & $134 / 35$ & $\left\{\begin{array}{r}\text { Ei Taf. } 50,30 \\
, 12,4 \mathrm{a}-\mathrm{c}\end{array}\right.$ \\
\hline 14. Euprépia púdica Esp. $\sigma^{\nearrow}$ & 4238 & 1391. & Taf. 13,9 \\
\hline 15. Âretia flávia Fuessl. $q$ & 4202 & 1351. & $" 12,5$ \\
\hline " aúlica L. $\sigma^{7}$ & 4207 & 1361. & $" 13,2$ \\
\hline$" \quad$ villiea L. $\sigma^{7}$ & 4203 & $135 / 36$ & $" 12,6$ \\
\hline$" \quad$ dejeáni God. $\sigma^{7}$ & 4209 & $136 r$ & Nachtr.-Taf. IX, 16 \\
\hline$"$ testudinária Foure. $\sigma^{7}$ & 4208 & $136 \mathrm{r}$ & Taf. 13,3 \\
\hline$" \quad$ spectábilis Tauseh. $\sigma^{7}$ & 4235 & 1331. & $" 13,8$ \\
\hline 21. Spilosóma mendieum $\mathrm{Cl} ., \mathrm{a}^{7}, \mathrm{~b}$ 우 & 4158 & 1291. & $" 14,1 \mathrm{a}-\mathrm{c}$ \\
\hline 22. Triehosóma córsicum Rbr ơ & 4188 & $133 \mathrm{r}$. & $" 13,10$ \\
\hline zoraída Grasl. $\sigma^{7}$ & 4196 & 1341. & " 48,13 \\
\hline 24. Ảretia eepvíni Fallou 우 & 4231 & $138 ; 39$ & "13,6 \\
\hline 25. Spilosóma sórdidum Hb., a $\sigma^{7}, \mathrm{~b} q$ & 4170 & $128 / 29$ & $" 13,14 \mathrm{a}-\mathrm{c}$ \\
\hline 26. Phragmatóbia fuliginósa L. 우 & 4168 & 1301. & $\begin{array}{l}\text { Ei Taf. } 50,31 \\
\quad " 13,12 \mathrm{a}, \mathrm{b}\end{array}$ \\
\hline 27. Áretia quenséli Payk. の ] & 4232 & 1391. & Taf. 13,7 \\
\hline
\end{tabular}

Siehe funer Taf, 76. 

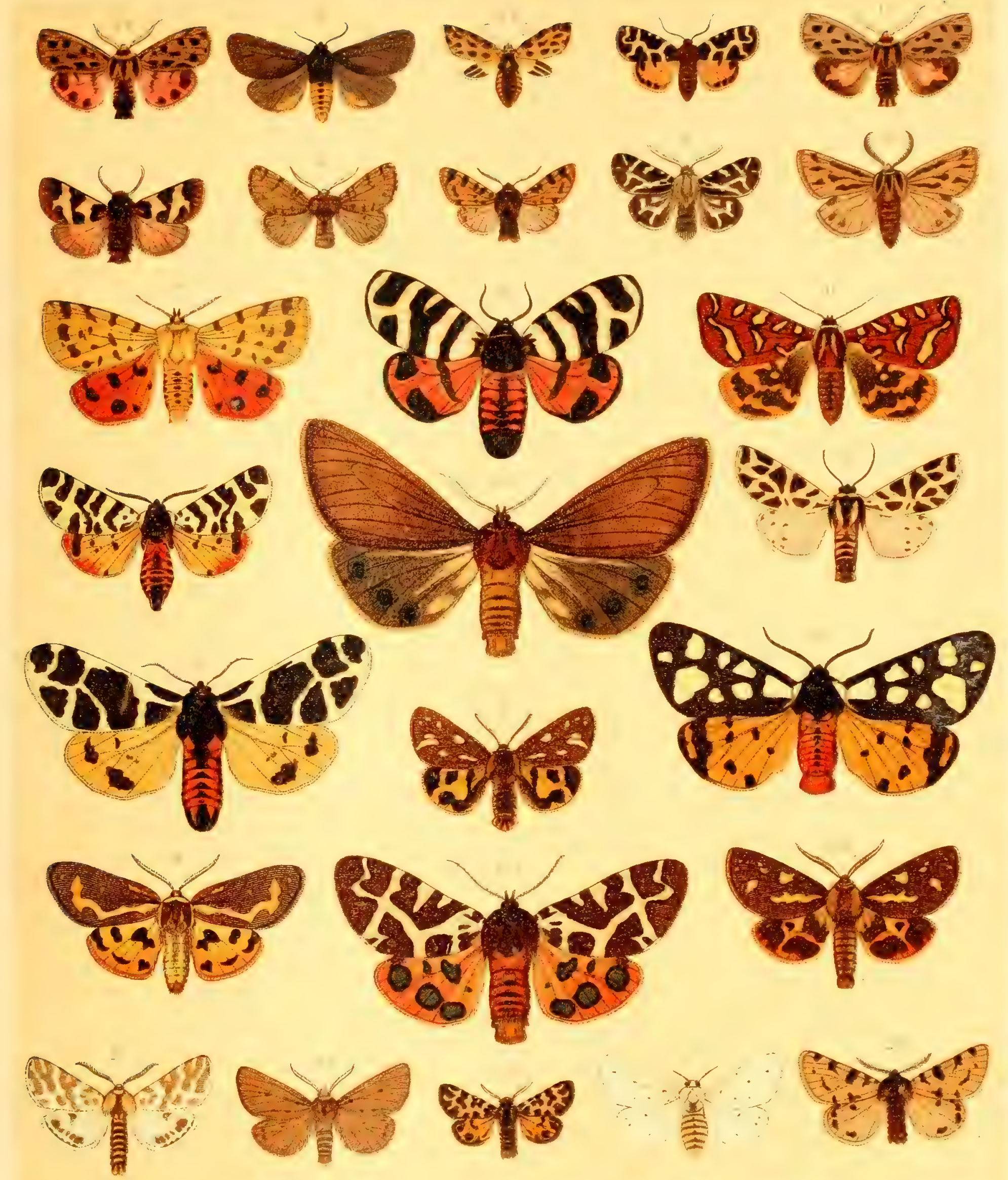

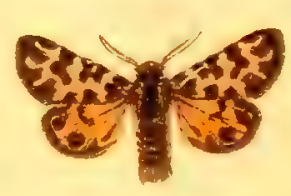

1.
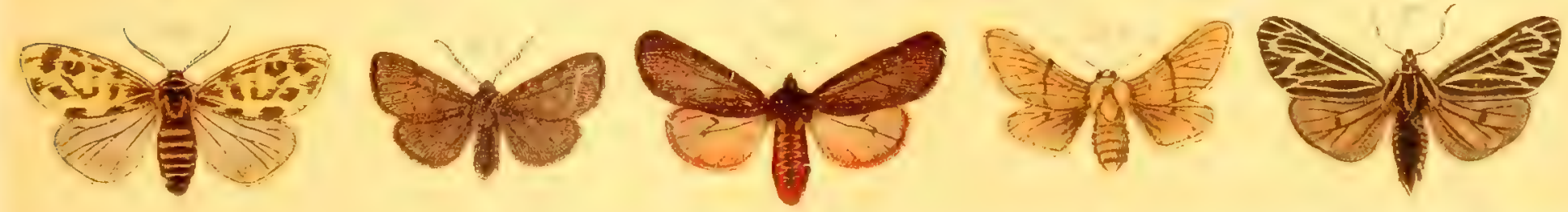




\section{TAFE L 74.}

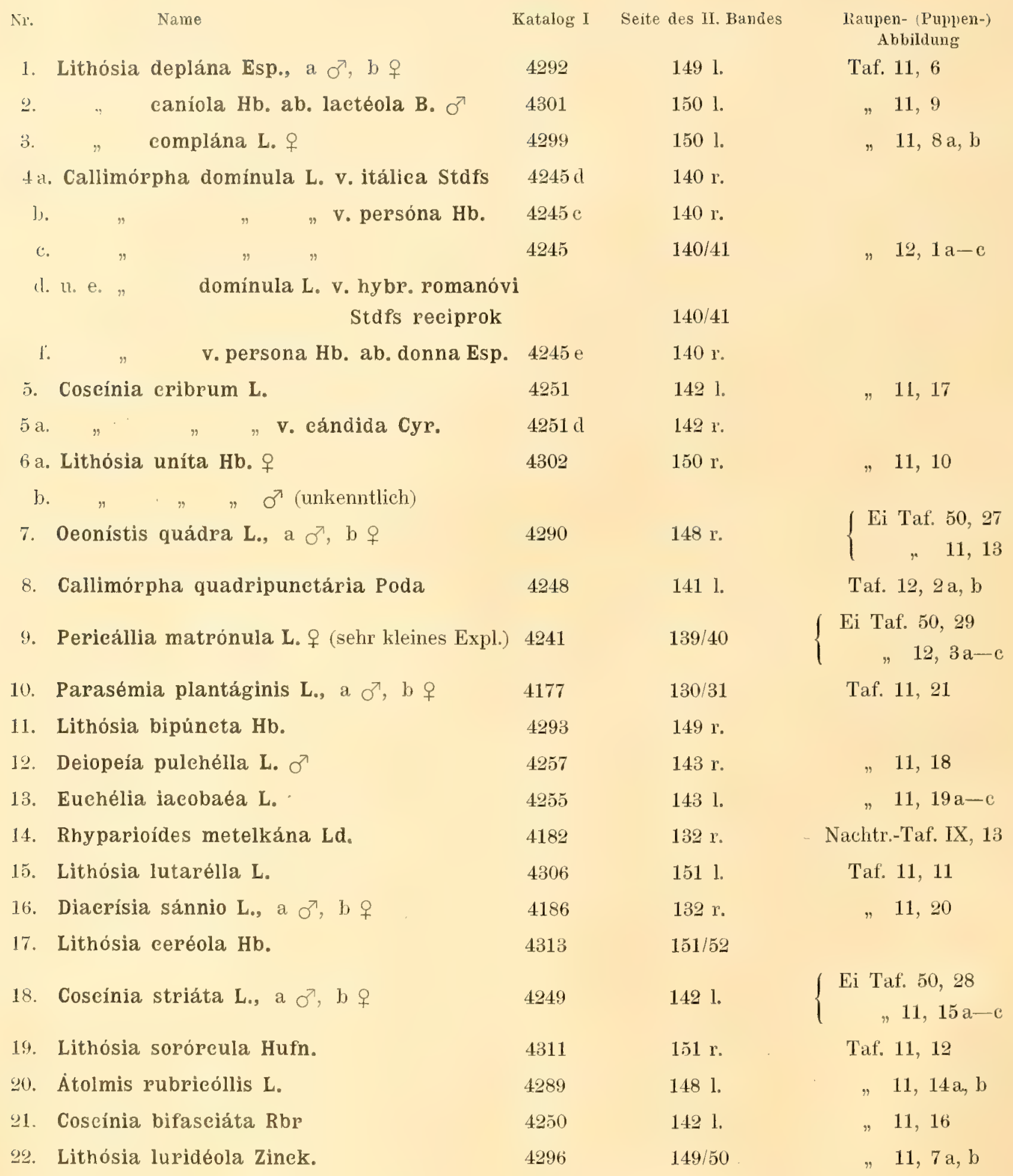

Siehe auch Titf. 76. 

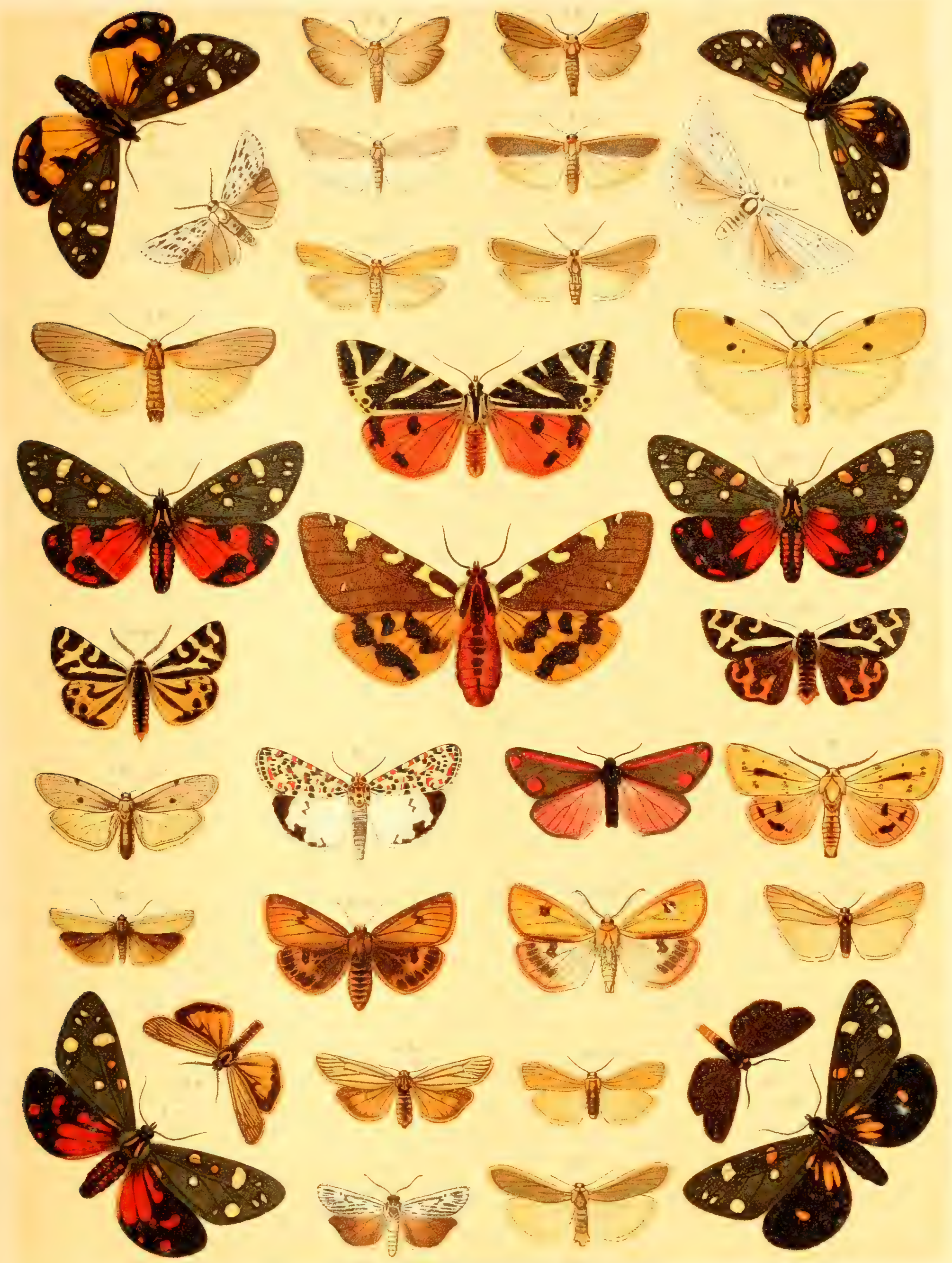




\section{TAFEL 75.}

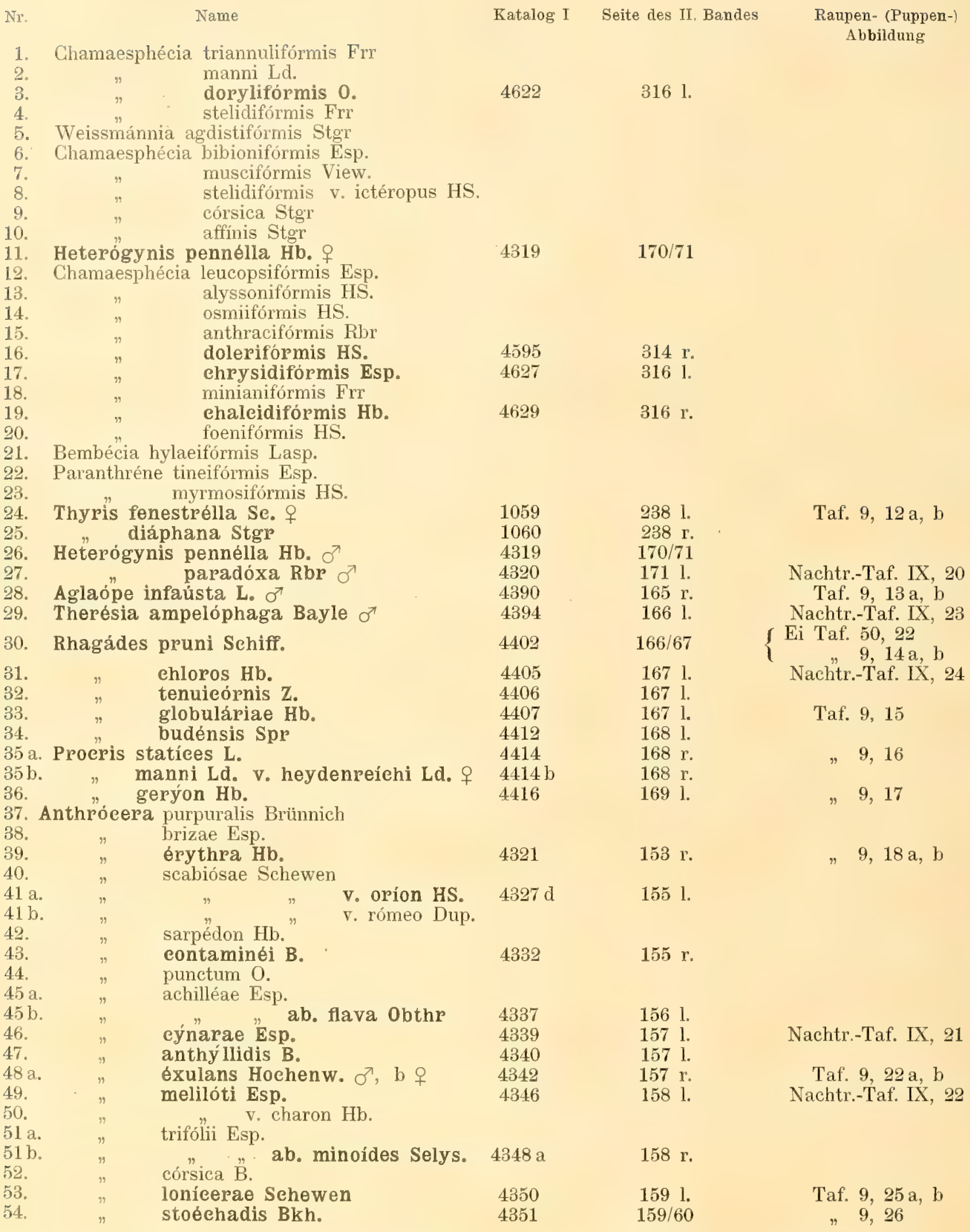

Die nicht fett gedruckten Namen bezeichnen kaum oder nicht kenntliche Abbildungen.

Siehe auch für die Anthroeeriden Taf. 77 u. für die Aegeriiden Taf. 79. 


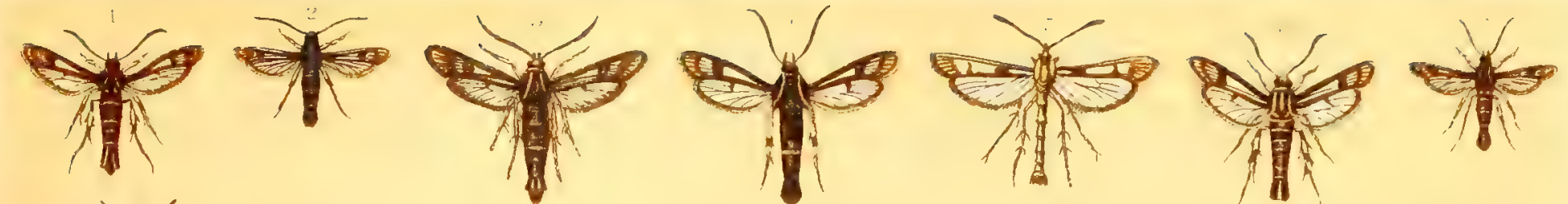

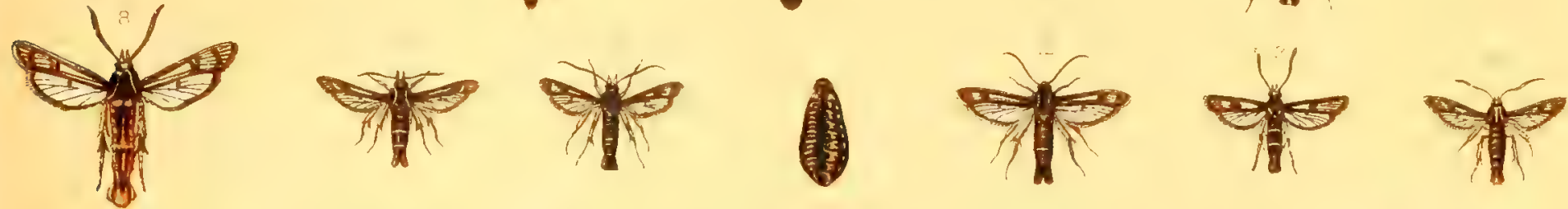

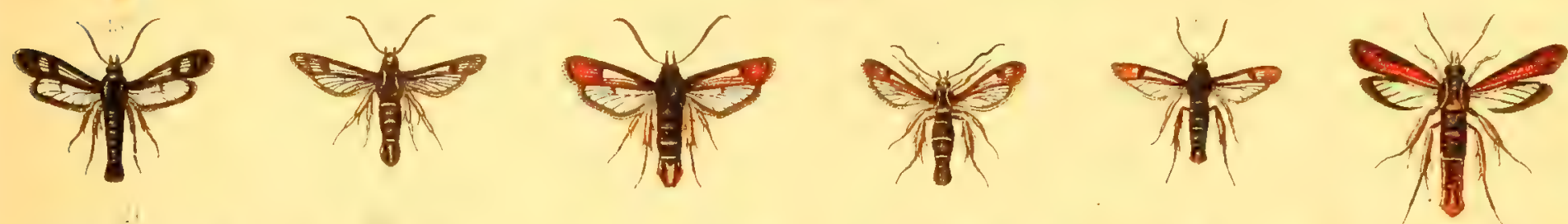

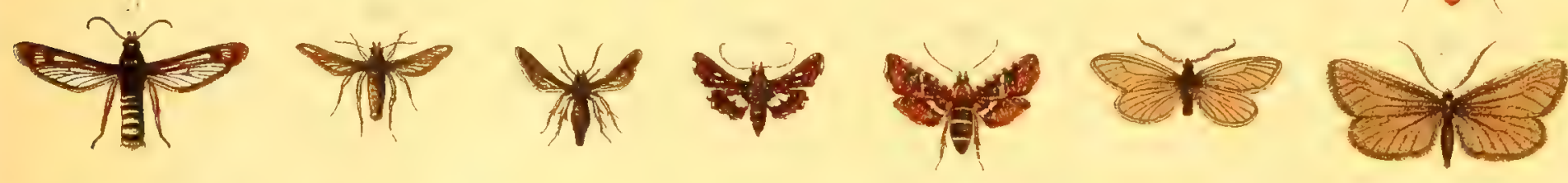

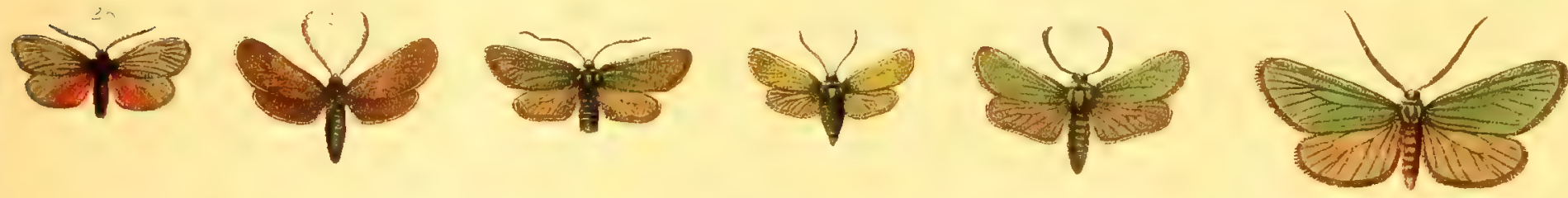

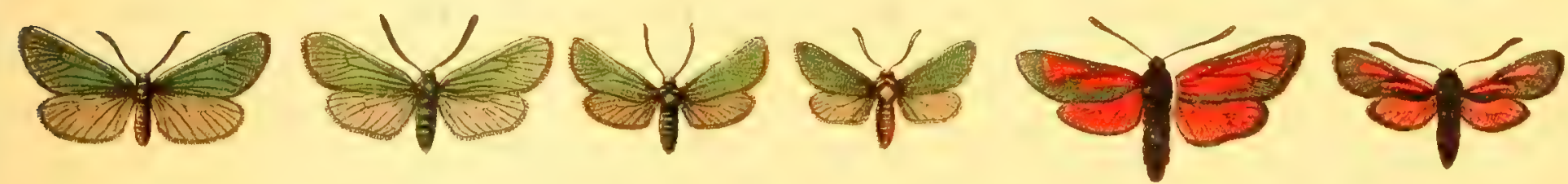

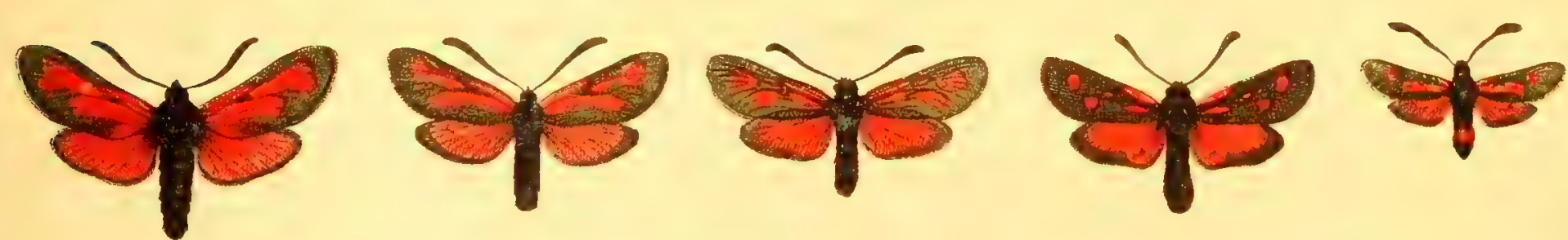

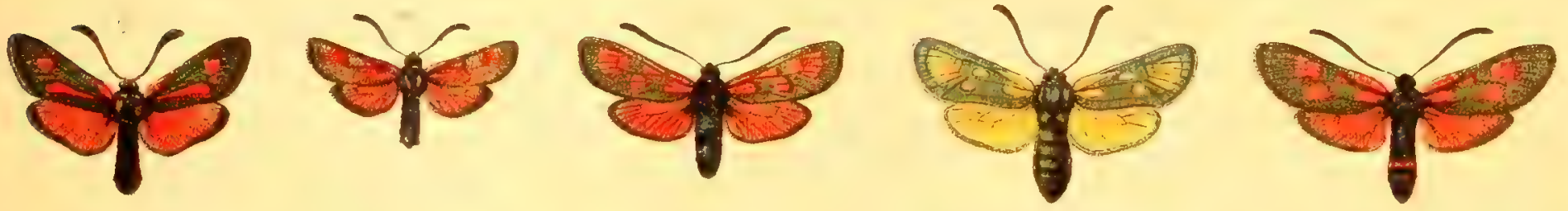

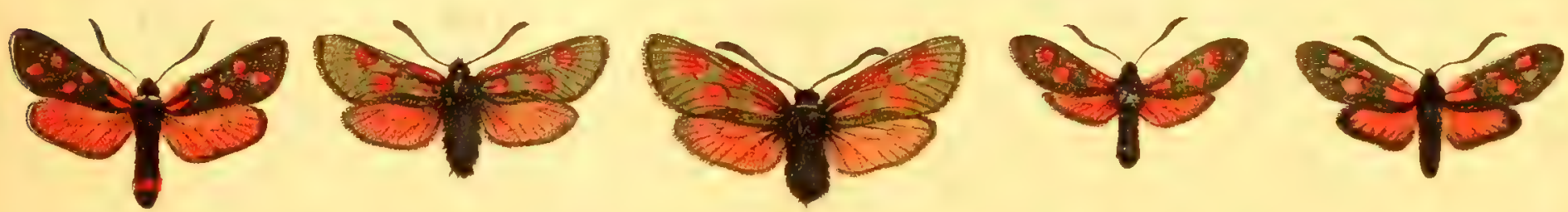

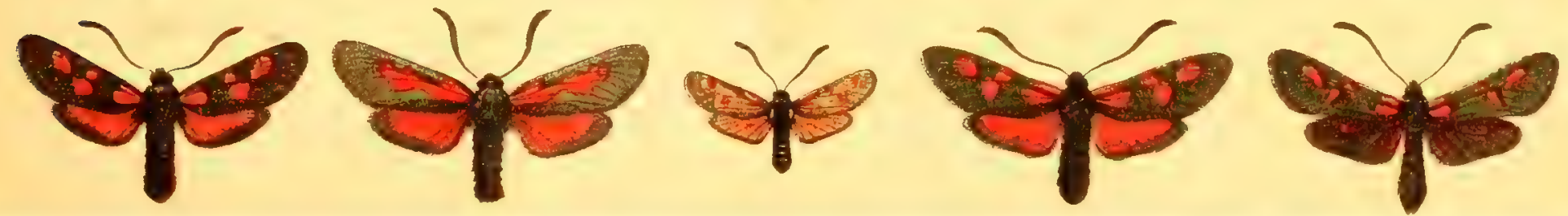






\section{TAFEL 76.}

\begin{tabular}{|c|c|c|c|c|}
\hline Nr. & Name & Katalog I & Seite des II. Bandes & $\begin{array}{c}\text { Raupen- (Puppen-) } \\
\text { Abbildung }\end{array}$ \\
\hline 1. & Nola thýmula Mill. 우 & 4108 & $122 / 23$ & Nachtr.-Taf. IX, 4 \\
\hline $1 \mathrm{a}$. & . togatulális $\mathrm{Hb}$. & 4098 & 1221. & IX, 1 \\
\hline 1). & " eicatricális $\mathrm{Tr}$. & 4104 & $122 \mathrm{r}$ & IX, 2 \\
\hline $1 c$. & .. strigula Sehiff. $q$ & 4105 & $122 \mathrm{r}$. & Taf. 10,18 \\
\hline $1 \mathrm{~d}$. & . ancipitális Tr. $\sigma^{7}$ & 4109 & 1231. & \\
\hline 2. & " subchlamydula Stgr $q$ & 4111 & 1231. & Nachtr.-Taf. IX, 6 \\
\hline 2 a. & . álbula Sehiff. $\sigma^{7}$ & 4113 & $123 \mathrm{l}$. & IX, 7 \\
\hline $2 \mathrm{~b}$. & " centonális $\mathrm{Hb}$. & 4117 & $123 / 24$ & Taf. 10,19 \\
\hline 3. & " squálida Stgr o7 & 4123 & 1241. & \\
\hline 4. & Eảrias insulána B. 우 & 4134 & $125 \mathrm{r}$. & \\
\hline 5. & Sýntomis eáspiea Stgr $q$ & 4151 & 1271. & \\
\hline 6. & Nyetéola falsális HS. $q$ & 4132 & 1251. & \\
\hline $7 \mathrm{a}$. & Trichosóma pudens Lue. $\sigma, b$ 우 & 4174 & 1301. & \\
\hline 8. & Phragmatóbia pláeida Friv. 우 & 4169 & $130 \mathrm{r}$. & Nachtr.-Taf. IX, 10 \\
\hline 9. & Parasémia caucásica Mén. ab. flava Spul. o7 & $4177 \mathrm{e}$ & $131 \mathrm{l}$. & \\
\hline 10. & Aretia festíva $\mathrm{Bkh} \cdot \mathrm{O}^{7}$ & 4210 & $136 / 37$ & \\
\hline $10 \mathrm{a}$. & $" \quad$ testudinária Fourer. $q$ & 4208 & $136 \mathrm{r}$. & Taf. 13,3 \\
\hline $10 \mathrm{~b}$. & $"$ déjeáni God. $\sigma^{7}$ & 4209 & $136 \mathrm{r}$. & Nachtr.-Taf. IX, 16 \\
\hline 11. & " mannerheími Dup. o & $4217 \mathrm{c}$ & $137 \mathrm{r}$. & \\
\hline 12. & " kindermánni Stgr $\sigma^{7}$ & 4219 & $138 \mathrm{r}$. & \\
\hline 13. & Lithósia seríeea Gregs. 우 & 4300 & 1501. & \\
\hline 14. & " máreida $\mathrm{Mn} \sigma^{7}$ & 4305 & $150 / 51$ & \\
\hline 15. & pállifrons Z. o & 4307 & 1511. & \\
\hline 16. & Proeris (Rhag.) turátii Bart. $\sigma^{7}$ & & $167 \mathrm{r}$. & \\
\hline 17. & $" \quad$ notáta Z. & 4408 & 167,68 & \\
\hline 18. & cognáta HS. $\sigma^{\pi}$ & 4409 & 1681. & \\
\hline 19. & v? subsolána Stgr or & $4409 \mathrm{a}$ & 1681. & \\
\hline 20. & Aeanthopsýehe zelléri Mn $\sigma^{7}$ & 4447 & 1741. & Taf. 15,7 \\
\hline 21. & Amicta lútea Stgr $\sigma^{7}$ & 4456 & $175 \mathrm{l}$. & \\
\hline 22. & Hyálina álbida Esp. v. lorquiniélla Brd $\sigma^{7}$ & 4463 a & $175 / 76$ & \\
\hline 23. & $" \quad$ malvinélla Mill. $\sigma^{7}$ & 446 כั & $176 \mathrm{l}$. & \\
\hline 24. & Oreopsyche kahri Ld. $\sigma^{7}$ & 4469 & $176 \mathrm{r}$. & \\
\hline 25. & "leschenaúlti Stgr v. nigricans Stgr $\sigma^{7}$ & 4470 & 1761 & $" 15,8 \mathrm{a}, \mathrm{b}$ \\
\hline 26. & Psyche viadrina Stgr $\sigma^{7}$ & 4484 & $178 \mathrm{r}$. & \\
\hline 27. & $"$ eonstancélla Brd $\sigma^{7}$ & 4485 & $178 \mathrm{r}$ & Nachtr.-Taf. X, 65 \\
\hline 28. & turátii Stgr $\sigma^{7}$ & 4486 & $178 \mathrm{r}$. & $\mathrm{X}, 66$ \\
\hline 29. & Rebélia sappho Mill. $\sigma^{7}$ & 4501 & $181 \mathrm{l}$. & \\
\hline 30. & Psychídea peetinélla F. $\sigma^{7}$ & 4518 & 1831. & \\
\hline 31. & Lúffia lapidélla Goeze Љ & $\begin{array}{c}\text { Katalog II } \\
4435\end{array}$ & 1851. & \\
\hline 32. & Dissóctena granigerélla Stgr $\sigma^{7}$ & 4420 & $185 \mathrm{r}$. & \\
\hline 33. & Thalaepópia tubulósa Retz. $\sigma^{\pi}$ & 4423 & 1861. & \\
\hline 34. & Bankésia alpestrélla Hein. $\sigma^{7}$ & 4430 & $187 \mathrm{l}$. & \\
\hline 35. & Solenóbia pinéti $\mathrm{Z} \cdot \sigma^{7}$ & $\begin{array}{c}4441 \\
\text { Katalog I }\end{array}$ & 1881. & \\
\hline 36. & Dyspéssa marmoráta Rbr $\sigma^{7}$ & 4689 a & $303 \mathrm{r}$. & \\
\hline 37. & Holcócerus volgénsis Chr. $\sigma^{\pi}$ & 4676 & $302 \mathrm{r}$. & \\
\hline 38. & $" \quad$ arenieola Stgr $\sigma^{T}$ & 4678 & 3031. & \\
\hline 39. & Dyspéssa salicícola Ev. & 4692 & $303 / 04$ & \\
\hline 40. & Stýgia eólchiea HS. 우 & 4707 & 3041. & \\
\hline 41. & Phassus sehamýli Chr. $\sigma^{7}$ & 4724 & $484 \mathrm{r}$. & \\
\hline 42. & Hepiolus amasínus HS. $0^{7}$ & 4728 & $485 \mathrm{l}$. & \\
\hline 43. & pyrenáieus Donz. $\sigma^{7}$ & 4737 & $485 \mathrm{r}$. & \\
\hline $44 \mathrm{a}$. & altícola 0bthr $\sigma^{7}, \mathrm{~b}$ 우 & 4737 a & $485 \mathrm{r}$. & \\
\hline
\end{tabular}




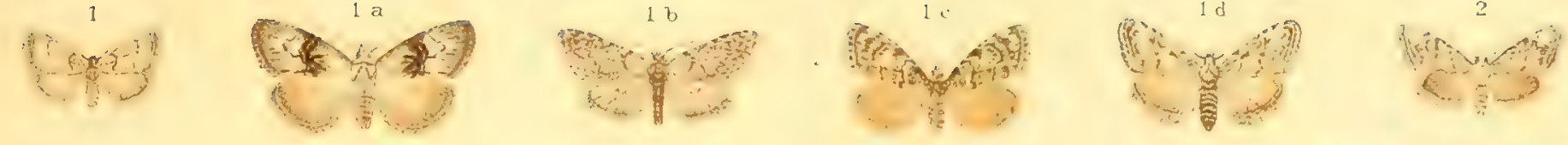

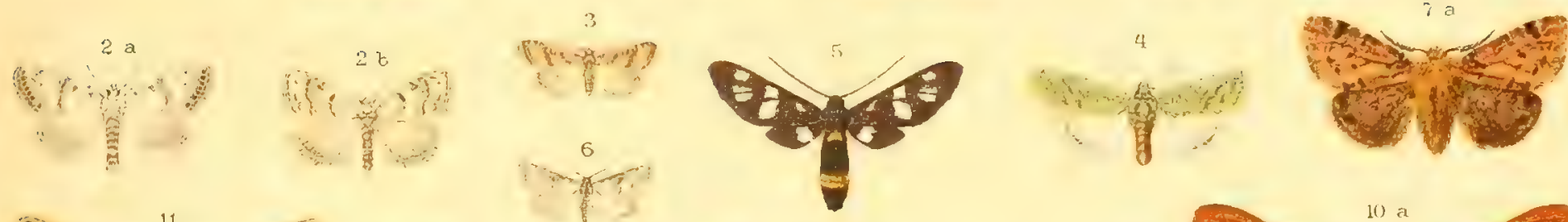
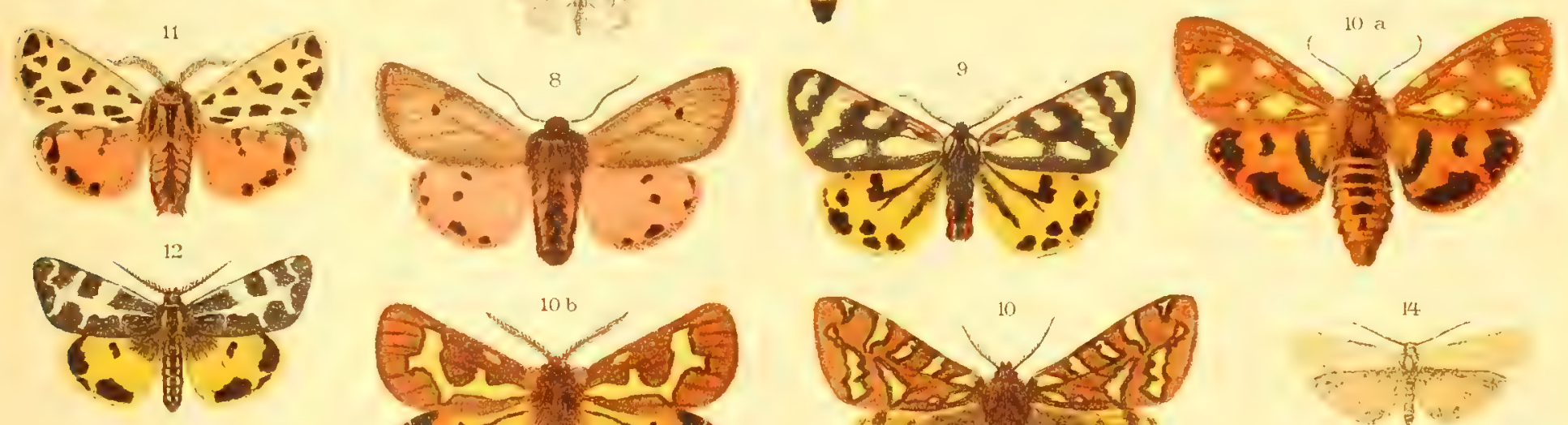

${ }_{\cdots}+\ldots$
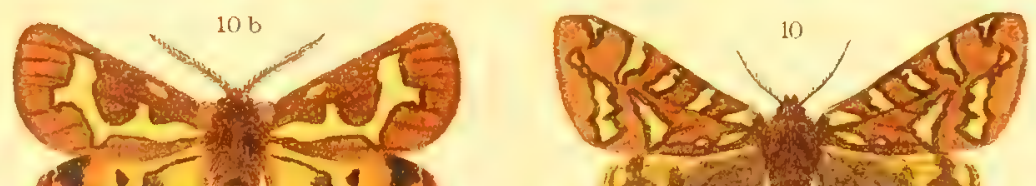
(4)
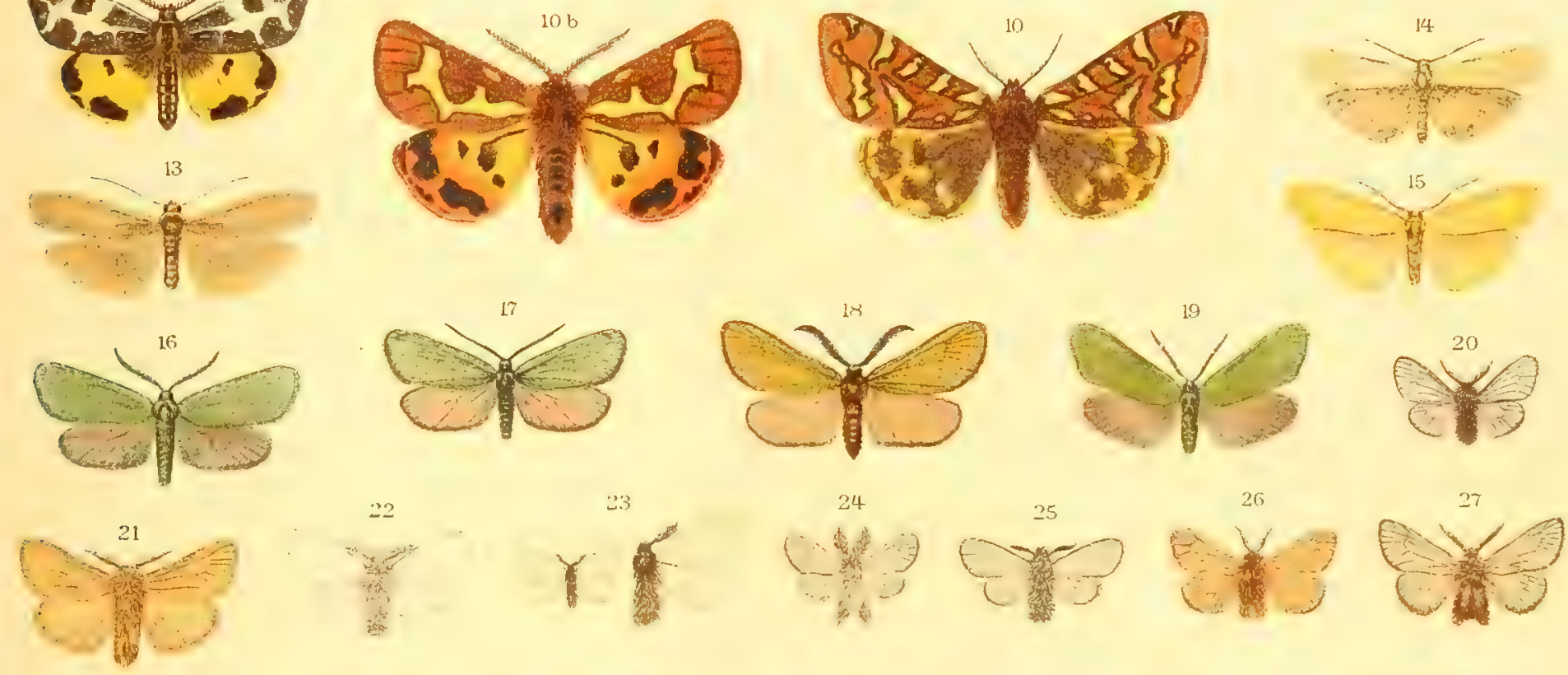

yor a de
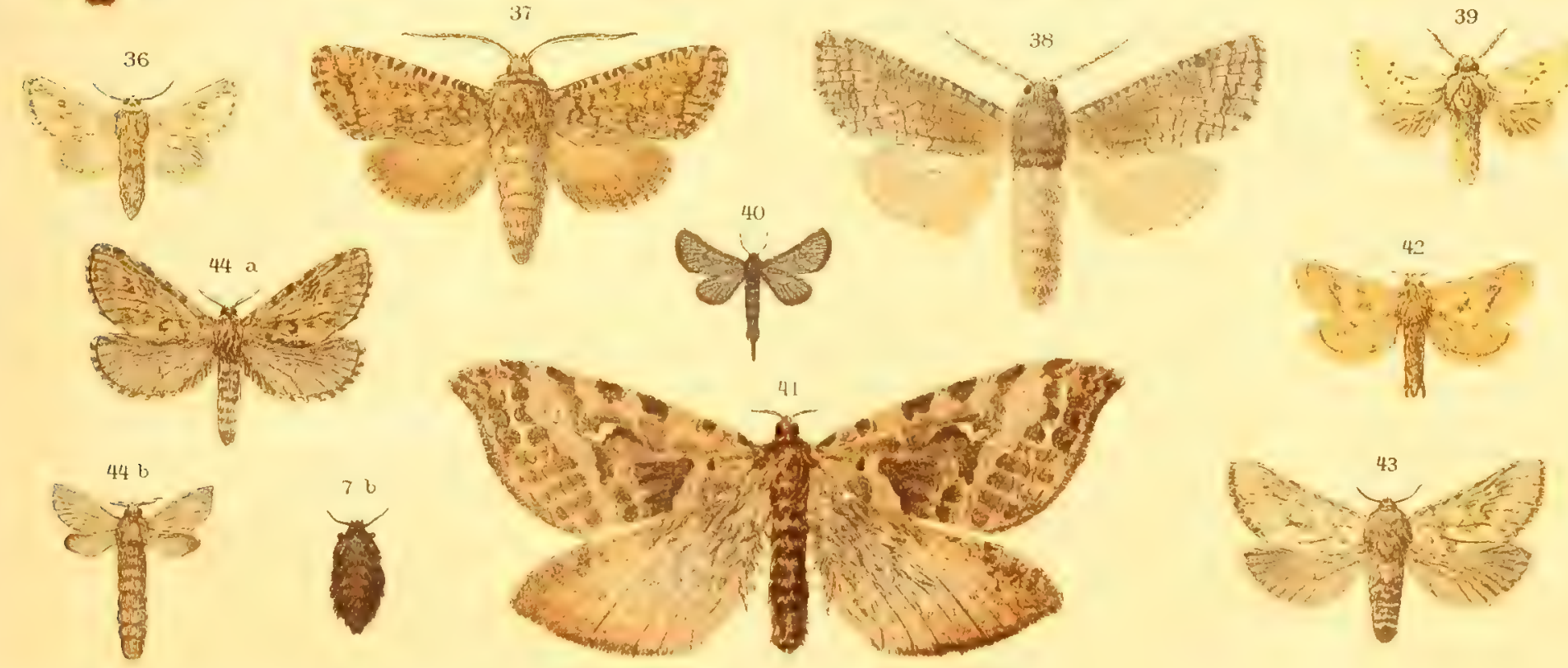




\section{TAFEL $1 \%$}

\begin{tabular}{|c|c|c|c|c|c|}
\hline$\therefore i:$ & & ame & Katalog I & Seite des II. Bandes & $\begin{array}{l}\text { Raupell- (Puppen-) } \\
\text { Abbildung }\end{array}$ \\
\hline 1. & Anthrócera & érythra Hb. & 4321 & $153 \mathrm{r}$. & Taf. $9,18 \mathrm{a}, \mathrm{b}$ \\
\hline$\therefore$. & $"$ & rubicúnda $\mathrm{Hb}$. & 4322 & $153 \mathrm{r}$. & \\
\hline 3. & $"$ & purpurális Brünnich & 4323 & $153 / 54$ & $" 9,19 \mathrm{a}, \mathrm{b} ?$ \\
\hline bit. &. & .. V. pluto 0 & $4323 \mathrm{~b}$ & 1541. & \\
\hline ]). & “. & v. nubígena Ld. & $4323 \mathrm{f}$ & 1541. & \\
\hline $\begin{array}{l} \pm . \\
5 .\end{array}$ & ". scabi & $\begin{array}{l}\text { brizae Esp. } \\
\text { ósae Seheven, fast ab. divísa Stgr }\end{array}$ & 4324 & $154 \mathrm{l}$. & \\
\hline $5 \mathrm{ad}$. & . & $\begin{array}{l}\text { lósae Seheven, fast ab. divísa Stgr } \\
\quad \text { v. oríon HS. }\end{array}$ & $\begin{array}{l}4327 \\
4327 \mathrm{~d}\end{array}$ & $\begin{array}{l}154 / 55 \\
155 \mathrm{l} .\end{array}$ & \\
\hline ). & $n$ & " v. neapolitána Calb. & $4327 \mathrm{~g}$ & $155 \mathrm{l}$. & \\
\hline (¿. &. & $\mathrm{V}$. nevadensis $\mathrm{RbP}$ & $4327 \mathrm{~h}$ & 1551. & \\
\hline 6. & " & sarpédon $\mathrm{Hb}$ & 4328 & $155 \mathrm{r}$. & $" 9,20$ \\
\hline 7. & $"$ & contaminéi $B$. & 4332 & $155 \mathrm{r}$. & \\
\hline 8. & $\cdot$ & punetum 0 & 4333 & $155 / 56$ & $. \quad 48,12$ \\
\hline 8a. &. & v. dystrepta F. d. W. & $4333 \mathrm{c}$ & $156 \mathrm{i}$. & \\
\hline 4. & .. & achilléae Esp. & 4337 & 1561. & $., 9,21$ \\
\hline 10. & " & wagnéri Milí. & 4338 & $156 / 57$ & \\
\hline $\begin{array}{l}10 \mathrm{a} . \\
\mathrm{h} .\end{array}$ & $\because$ & " ab. achilleoídes Wagn. & & $156 \mathrm{r}$. & \\
\hline 1. & ". & $\begin{array}{l}\text { eýnarae Esp. giesekíngi Wagn. } \\
\text { eỹ }\end{array}$ & 4339 & $157 \mathrm{l}$. & Nachtr.-Taf. IX, 21 \\
\hline $1 \mathrm{al}$ & $"$ & " v. genistae HS. & $4339]$ & 1571. & \\
\hline 12. &. & centaúreae F. d. W. & $4339 c$ & $157 \mathrm{l}$. & \\
\hline $\begin{array}{l}12 . \\
13 .\end{array}$ &.. & anthýlidis $B$. & 4340 & $157 \mathrm{l}$. & \\
\hline $\begin{array}{l}13 . \\
13 \mathrm{a} .\end{array}$ &.. & éxulans' Hoehenw. & 4342 & $157 \mathrm{r}$. & Taf. $9,22 \mathrm{a}, \mathrm{b}$ \\
\hline $\begin{array}{l}3 \mathrm{a} . \\
4 .\end{array}$ &. & v. vánadis Dalm. & 4342 a & $157 \mathrm{r}$ & \\
\hline $\begin{array}{l}14 . \\
15 .\end{array}$ & "* & córsiea B. & 4345 & $157 / 58$ & $9,23 \mathrm{a}, \mathrm{b}$ \\
\hline $\begin{array}{l}15 . \\
15 a .\end{array}$ & $"$ & melilóti Esp. & 4346 & 1581. & Nachtr.-Taf. IX, 22 \\
\hline $\begin{array}{l}15 \mathrm{a} . \\
\mathrm{j} .\end{array}$ & 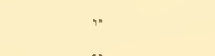 & " v. eharon $\mathrm{Hb}$. & $4346 \mathrm{~d}$ & 1581. & \\
\hline 16. & .. & $\begin{array}{l}\text { V. stentzi Frr } \\
\text { trifólii Esp. }\end{array}$ & $4346 \mathrm{a}$ & $158 \mathrm{l}$. & \\
\hline $6 \mathrm{a}$. & .. & $\begin{array}{l}\text { trifólii Esp. } \\
\quad " \quad a b . \text { minoídes Selys }\end{array}$ & 4348 & $\begin{array}{l}158 / 59 \\
158\end{array}$ & Taf. $9,24 a, b$ \\
\hline ]). &. & " v. sypacúsiae Z. & $\begin{array}{l}4548 \mathrm{a} \\
4348 \mathrm{c}\end{array}$ & 1591. & \\
\hline $\begin{array}{l}17 . \\
18 .\end{array}$ &. & lonícerae Seheven & 4350 & 1591. & $\Rightarrow 9,25 \mathrm{a}, \mathrm{b}$ \\
\hline $\begin{array}{l}18 . \\
18 \mathrm{a} .\end{array}$ &. & stoéchadis Bkh. & 4351 & $159 / 60$ & 9,26 \\
\hline $\begin{array}{l}18 \mathrm{a} . \\
19 .\end{array}$ & 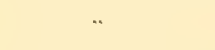 & $" \quad$ v. dúbia Stgr & $4351 \mathrm{~b}$ & $159 x$ & \\
\hline $\begin{array}{l}19 . \\
19 \mathrm{A.}\end{array}$ &. & filipéndulae L. & 4352 & $160 \mathrm{l}$. & $\left\{\begin{array}{l}\text { Ei Taf. } 50,23 \\
10,1\end{array}\right.$ \\
\hline 19 д. & $\begin{array}{l}. . \\
. .\end{array}$ & $\begin{array}{l}\text { ab. eýtisi } \mathrm{Hb} \text {. } \\
\mathrm{v} \text { ochsenheiméri } \mathrm{Z} \text {. }\end{array}$ & $\begin{array}{l}4352 \mathrm{a} \\
4352 \mathrm{e}\end{array}$ & $\begin{array}{l}160 \mathrm{l} \\
160 \mathrm{l}\end{array}$ & \\
\hline 20 & .. & angélicae 0. & 4355 & $160 \mathrm{l}$. & \\
\hline $\begin{array}{ll}21 . \\
21 \mathrm{il} .\end{array}$ & .. & transalpína Esp. v. férulae Ld. & 4356 & $160 / 61$ & Taf. $10,2 \mathrm{a}-\mathrm{c}$ \\
\hline $\begin{array}{l}21 \mathrm{i} . \\
1 \text {. }\end{array}$ &. & v. astrágali Bkh. & $4356 \mathrm{~d}$ & $161 \mathrm{l}$. & \\
\hline $\begin{array}{l}\text { ]). } \\
\text { c. }\end{array}$ & .. & v. calábriea Calb. & $4356 \mathrm{~b}$ & $161 \mathrm{l}$. & \\
\hline 29. &. & v. sorrentína Calb. & $.4356 \mathrm{a}$ & $161 \mathrm{l}$. & \\
\hline 23. & .. & $\begin{array}{l}\text { doryenii } 0 . \\
\text { ephiáltes L. }\end{array}$ & $\begin{array}{l}4357 \\
4358\end{array}$ & $\begin{array}{l}161 \mathrm{r} \\
161 / 62\end{array}$ & \\
\hline $23 a$. & 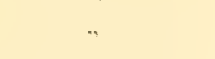 & " v. coroníllae Esp. & $4358 \mathrm{~b}$ & 1621. & \\
\hline $\begin{array}{l}\text { ]). } \\
\text { c. }\end{array}$ &. & ab. trigonéllae Esp. & $4358 \mathrm{c}$ & $162 \mathrm{l}$. & \\
\hline c. &.. & v. peucédani Esp. & $4358 \mathrm{e}$ & 1621. & $\Rightarrow \quad 10,3 \mathrm{a}, \mathrm{b}$ \\
\hline 24. & ". & $\begin{array}{l}\text { ab. athamánthae Esp. } \\
\text { lavăndulae Esp. }\end{array}$ & . $4358 \mathrm{f}$ & $162 \mathrm{l}$. & \\
\hline 25. &. & $\begin{array}{l}\text { lavándulae Esp. } \\
\text { rhadamánthus Esp. }\end{array}$ & 4359 & $162 \mathrm{r}$ & $" 10,4 \mathrm{a}, \mathrm{b}$ \\
\hline 26. & .. & $\begin{array}{l}\text { Phadamanthus Esp. } \\
\text { oxýtropis B. }\end{array}$ & $\begin{array}{l}4360 \\
4361\end{array}$ & $\begin{array}{l}162 / 63 \\
1631 .\end{array}$ & $" 10,5 \mathrm{a}, \mathrm{b}$ \\
\hline 27. & . & sedi $F$. & 4380 & 1631. & \\
\hline 28. & .. & laeta $\mathrm{Hb}$. & 4381 & $163 \mathrm{l}$. & $" 10,6 \mathrm{a}-\mathrm{c}$ \\
\hline 29. & " & ignifera Korb & 4382 & 1631. & \\
\hline 30. & $"$ & hilaris 0 . & 4383 & $163 r$. & \\
\hline $\begin{array}{l}31 . \\
32 .\end{array}$ &. & baétiea RbP & 4386 & $163 \mathrm{r}$ & \\
\hline $\begin{array}{l}32 . \\
32 \mathrm{a} .\end{array}$ & .. & fausta L. & 4387 & $163 / 64$ & $" 10,7 \mathrm{a}, \mathrm{b}$ \\
\hline $\begin{array}{l}32 \mathrm{a} . \\
33 .\end{array}$ &.. & V. iucúnda Meissn. & $4387 \mathrm{c}$ & 1641 & $70 \quad 8-7$ \\
\hline 33 a. & $"$ & $\begin{array}{l}\text { earnioliea Se. } \\
\text { v. berolinénsis Stgr }\end{array}$ & $\begin{array}{l}4388 \\
4388 \mathrm{f}\end{array}$ & $\begin{array}{l}164 l . / 165 \\
164.1\end{array}$ & $" 10,8 \mathrm{a}, \mathrm{b}$ \\
\hline 34. & $"$ & occitảnica Vill. & 4389 & 1651. & $\because 10,9$ \\
\hline $\begin{array}{l}34 \mathrm{a} . \\
\text { jo. }\end{array}$ & $"$ & ab. álbicans Stgr & 4389 a & 165 & \\
\hline c. & $"$. & $\begin{array}{l}\text { neta Spul. } \\
\text { ea Stgp }\end{array}$ & & 1651. & \\
\hline & & Stgp $P$ & t389 b & $165 \mathrm{l}$. & \\
\hline
\end{tabular}




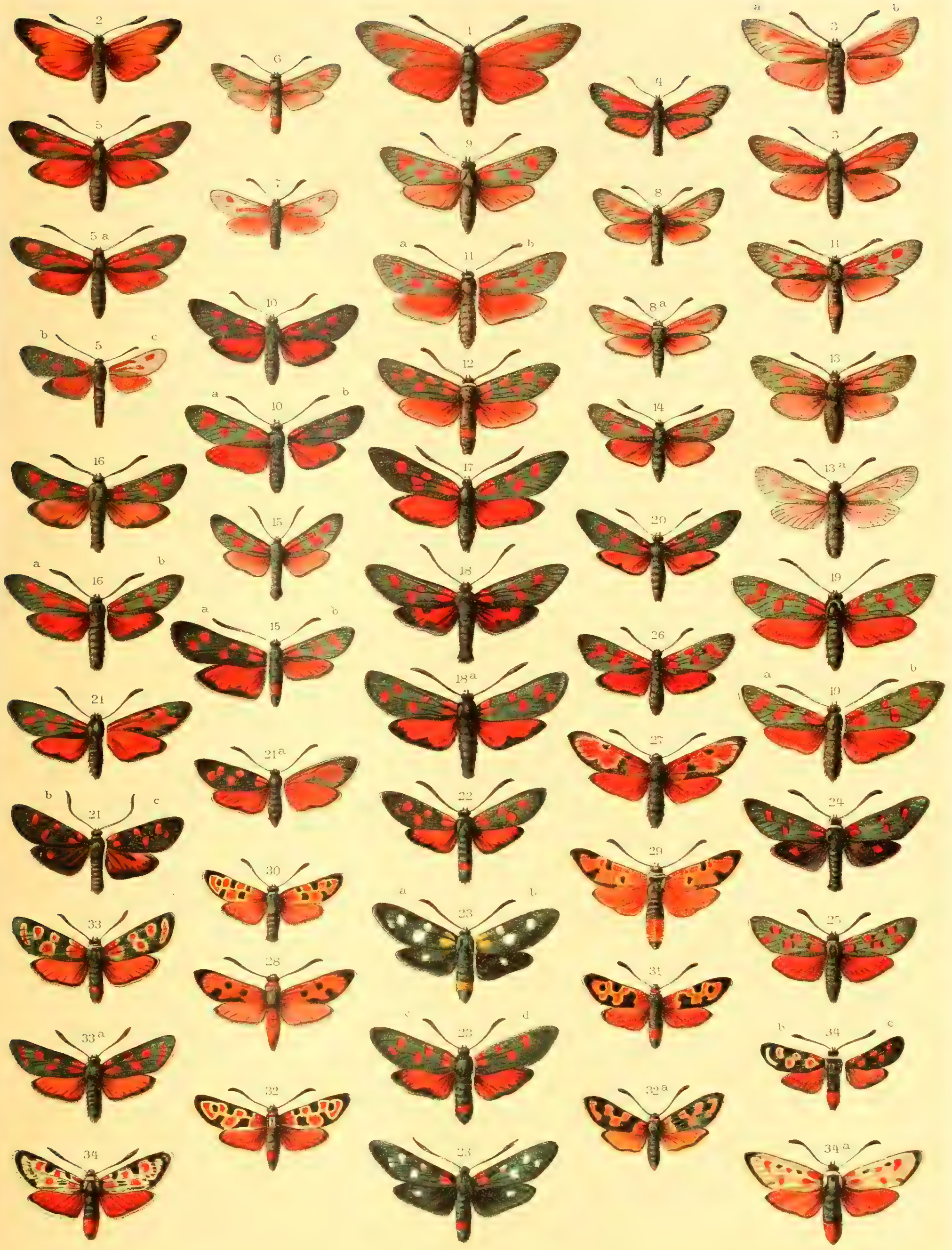






\section{TAFEL 78.}

\begin{tabular}{|c|c|c|c|c|}
\hline Nr. & Name & Katalog I & Seite des I. Ban & $\begin{array}{l}\text { Raupen-(Puppen-) } \\
\text { Abbildung }\end{array}$ \\
\hline 1. & Hebdomochóndra syrticola Stgr ơ & 2349 & $283 \mathrm{r}$. & \\
\hline $2 \mathrm{a}$. & $\begin{array}{r}\text { Acontíola laseivális Ld., } 2 / 1 \text { nat. Gr.; } \\
\text { 2. Umriß in nat. Gr. }\end{array}$ & 2383 & 2871. & \\
\hline 3. & Prothýmia coniéphala Stge $\sigma^{\top}$ & 2485 & $295 \mathrm{r}$. & \\
\hline 4. & Plúsia herrichi Stgr or & 2528 & $300 \mathrm{r}$. & \\
\hline 5. & $"$ beckéri Stgr v. itálica Stgr $q$ & $2533 a$ & $301 \mathrm{r}$. & \\
\hline 6. & $" \quad$ aurífera $\mathrm{Hb} . q$ & 2541 & 3021. & \\
\hline 7. & $" \quad$ intercaláris (Ev.) HS. mandarma $F r r$ 우 & 2558 & $303 \mathrm{r}$. & \\
\hline 8. & " diaséma B. 우 & 2575 & $305 \mathrm{r}$. & \\
\hline 9. & $" \quad$ excélsa Kretsehm. $\sigma^{7}$ & 2544 & $302 \mathrm{r}$. & \\
\hline 10. & Euelidia fortalítium Tauseh $\mathcal{F}^{7}$ & 2592 & 3081. & \\
\hline 11. & Aeanthólipes reguláris $\mathrm{Hb}$. 우 & 2602 & 3091. & \\
\hline 12. & Palpángula henkéi Stgr $\sigma^{7}$ & 2611 & $309 \mathrm{r}$. & \\
\hline 13. & cestis Mén. $\sigma^{7}$ & 2613 & $309 \mathrm{r}$. & \\
\hline 14. & Leueanítis rada B. $\sigma^{7}$ & 2622 & 3101. & \\
\hline 15. & " eáspica Stgr $\sigma^{7}$ & $2633 \mathrm{~b}$ & $310 \mathrm{r}$. & \\
\hline 16. & Palpángula ténera Stgr & 2639 & $309 / 10$ & \\
\hline 17. & Catócala oberthǘri Aust. $\sigma^{7}$ & 2671 & 3151. & \\
\hline 18. & $" \quad$ adúltera Mén. $\sigma^{7}$ & 2679 & 3161. & \\
\hline 19. & Apopéstes limbáta Stgr. 우 & 2722 & 3201. & \\
\hline 20. & Cucúllia clárior F. Fuehs $\sigma^{7}$ & & 2711. & \\
\hline 21. & Habrósyne derása L. 우 & 2834 & 3331. & Taf. $21,6 \mathrm{a}, \mathrm{b}$ \\
\hline 22. & Thyatíra batis L. $q$ & 2836 & $333 \mathrm{r}$. & $" 21,7 \mathrm{a}, \mathrm{b}$ \\
\hline 23. & Cymatóphora flavicórnis (L.) Cl. or $F \cdot \sigma^{7}$ & 2843 & 3341. & 21,9 \\
\hline 241. & $\begin{array}{c}\text { oculáris L. octogésima } H b \text {. } \\
\sigma^{7}, \text { r. Fl. } q\end{array}$ & 2844 & $334 \mathrm{r}$ & Taf. 21, 8 u. Ntr.-T. III, 1 \\
\hline 25. & dupláris L. $q$ & 2848 & 3351. & Taf. $21,10 a, b$ \\
\hline $26 \mathrm{r}$. & $\begin{array}{l}\text { dilúta F., 1. Übergang zu } \\
a b . \text { nubiláta Tutt }\end{array}$ & 2849 & 3351. & $" 21,12$ \\
\hline 27. & fluetuósa $\mathrm{Hb}$. 오 & 2846 & $334 / 35$ & Nachtr.-Taf. IV, 43 \\
\hline 28. & Polýploea rufieóllis F. $q$ & 2850 & $335 \mathrm{r}$. & Taf. 21,11 \\
\hline $\begin{array}{l}29 . \\
30 \mathrm{r} .\end{array}$ & " einérea Goeze flavicómis auct. $q$ & 2852 & $335 / 36$ & $" 21,13$ \\
\hline & céphala E & 28553 & 3361. & $" 21,14 \mathrm{a}, \mathrm{b}$ \\
\hline
\end{tabular}

Diese Tafel enthält Nachträge zu den Tafeln $48-52$ und 54 . 

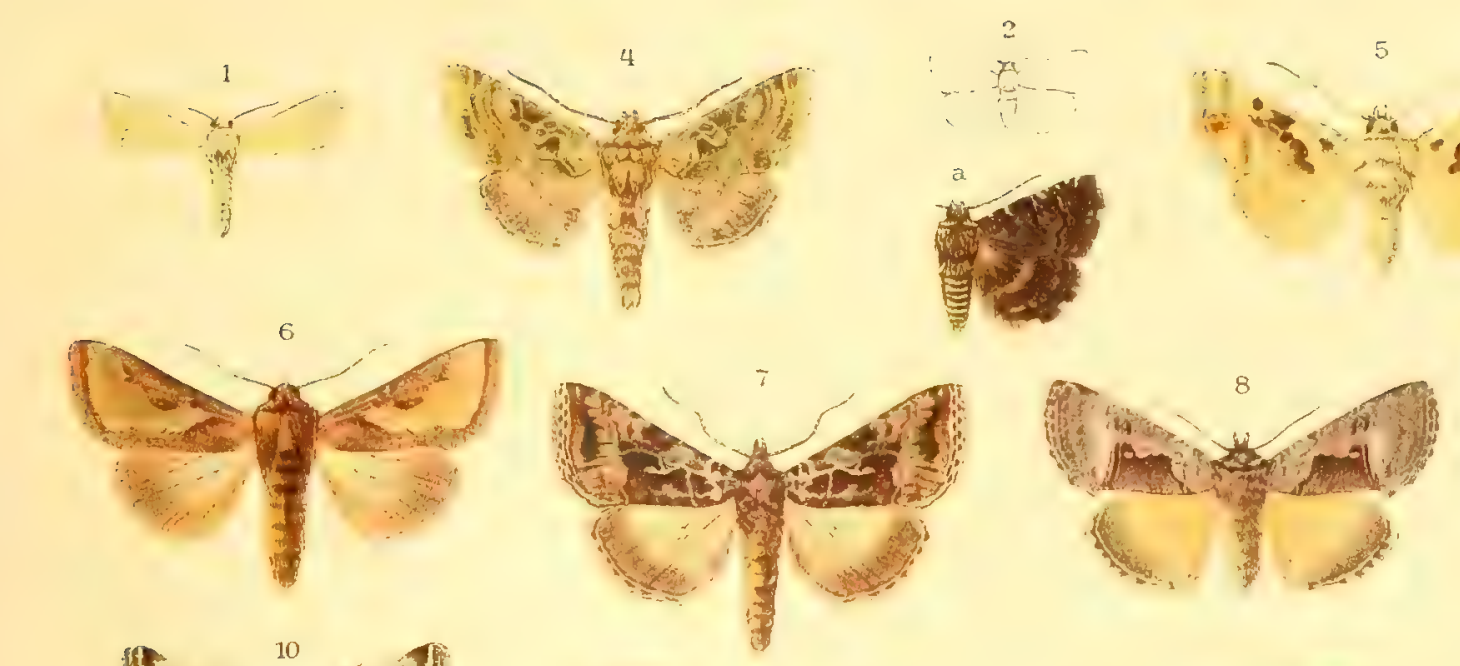

$$
(8 \times-48)
$$
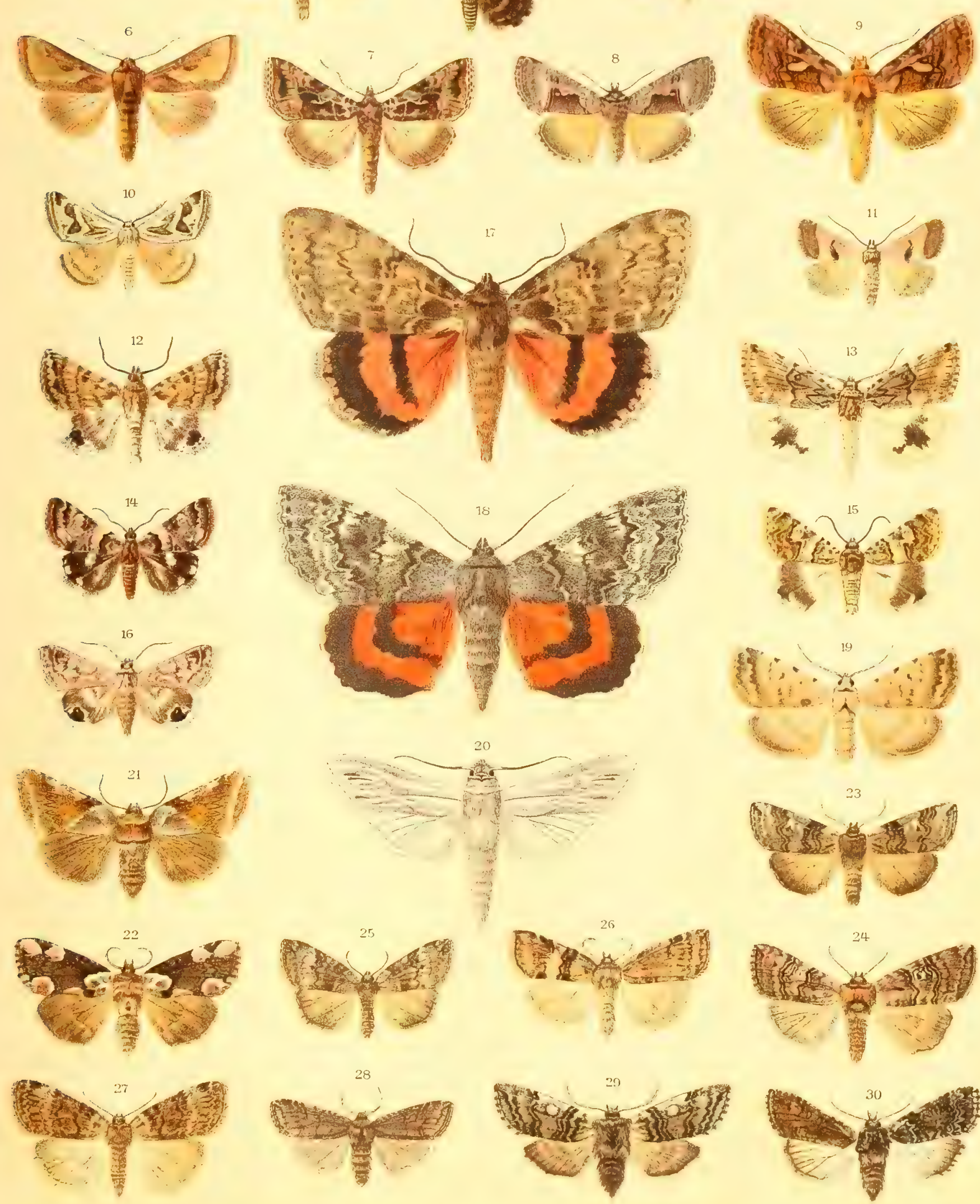
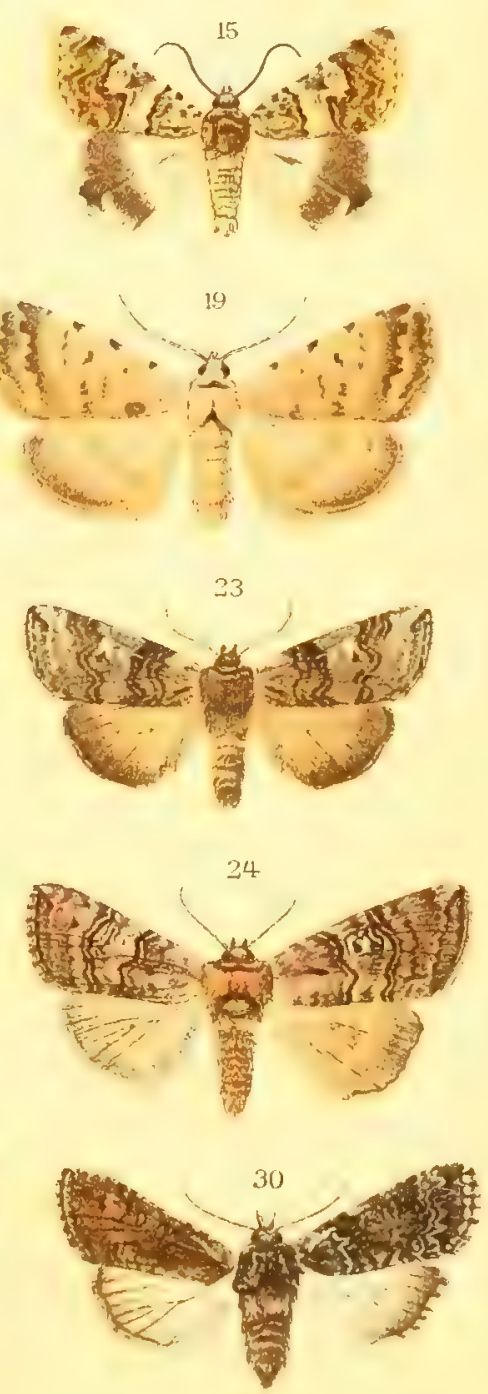




\section{TAFEL $\%$.}

\begin{tabular}{|c|c|c|c|c|c|}
\hline Nr. & Name & Katalog I & Seite des II. Bandes & Raup & $\begin{array}{l}\text { pen- (Puppen- } \\
\text { Abbildung }\end{array}$ \\
\hline 1. & Trochílium seoliifórme Bkh. & 4545 & 309 r. & Taf. & f. 9,5 \\
\hline 2. & \% sphecifóme Gerning & 4546 & $309 / 10$ & & \\
\hline 3. & mesilórme HS. $\sigma^{7}$ & 4549 & 3101. & & \\
\hline 4. & andrenifórme Lasp. & 4550 & 3101. & & \\
\hline 5. & cephifórme 0 & 4551 & 3101. & & \\
\hline 6. & tipulifórme Cl. & 4552 & 3101. & $"$ & 9,6 \\
\hline 7. & flavivéntre Stgr $\sigma^{7}$ & 4553 & 3101. & & \\
\hline 8. & rufibasále Bartel & & 3101. & & \\
\hline 9. & conopifórme Esp. & 4554 & $310 \mathrm{r}$. & & \\
\hline 10. & vespifórme $\mathbf{L}$, a $\sigma^{7}, \mathrm{~b}$ ㅇ & 4555 & $310 \mathrm{r}$. & $"$ & 9,7 \\
\hline 11. & myopifórme Bkh. & 4557 & $310 \mathrm{r}$ & $"$ & $9,8 \mathrm{a}, \mathrm{b}$ \\
\hline 12. & typhiifórme Bkh. & 4559 & $310 \mathrm{r}$. & & \\
\hline 13. & eruentátum Mn & 4560 & $310 / 11$ & & \\
\hline 14. & eulieifórme L. & 4563 & 3111 & $"$ & 9,9 \\
\hline 15. & uralénse Bart. & & 3111. & & \\
\hline 16. & stomoxy fórme $\mathrm{Hb}$. & 4564 & 3111. & & \\
\hline 17. & " formicifórme Esp. & 4566 & 3111. & $"$ & 9,10 \\
\hline 18. & Dipsosphécia iehneumonifórmis F. & 4573 & 3171. & & \\
\hline $\begin{array}{l}18 \text { a. } \\
19 .\end{array}$ & megillifórmis $\mathrm{Hb}$. & 4573 a & 3171 & & \\
\hline 19. & upocerifórmis Tr. & 4576 & 3171. & & \\
\hline 20. & $\begin{array}{l}\text { himmighofféni Stgr } \\
\text { Chamäesphécia masapifóemis } 0\end{array}$ & 4577 & 3171. & & \\
\hline 1. & & 4580 & $313 / 14$ & & \\
\hline 22 a. & $\begin{array}{l}\text { annelláta } \mathrm{Z} . \\
\text { v. eorilifórmis Ld. } \sigma^{7}\end{array}$ & $\begin{array}{l}4586 \\
4586 \text { a }\end{array}$ & $\begin{array}{l}3141 . \\
3141\end{array}$ & & \\
\hline 3. & empifórmis Esp. & 4587 & 3141 & & \\
\hline 4. & monspeliénsis Stgr & 4591 & 314 r. & & \\
\hline 5 & astatifórmis HS. & 4592 & $314 \mathrm{r}$. & & \\
\hline 6. & triannulifórmis FPr & 4593 & $314 \mathrm{r}$ & & \\
\hline & manni Ld. $\sigma^{7}$ & 4594 & $314 \mathrm{r}$. & & \\
\hline 8 & dolerifómis HS. 오 & 4595 & $314 r$. & & \\
\hline 9. & colpifórmis Stgre & 4596 & $314 \mathrm{r}$. & & \\
\hline & stelidifórmis Fre & 4598 & 3151. & & \\
\hline & rambúpi Stgr ণ্ & 4600 & 3151. & & \\
\hline & $\begin{array}{l}\text { bibionifórmis Esp. } \\
\text { museifórmis View. }\end{array}$ & 4604 & 3151. & & \\
\hline & $\begin{array}{l}\text { muscifópmis View. ㅇ } \\
\text { leucomelaéna } \mathrm{Z} \text {. }\end{array}$ & 4605 & $315 \mathrm{r}$. & & \\
\hline & $\begin{array}{l}\text { leucomelaéna } \mathrm{Z} . \\
\text { cópsiea Stgre }\end{array}$ & 4606 & $315 x$ & & \\
\hline 36. & $\begin{array}{l}\text { cópsiea Stgr } \\
\text { affínis Stgr o }\end{array}$ & 4607 & $315 \mathrm{r}$. & & \\
\hline 37. & $\begin{array}{l}\text { affinis Stgr } \sigma^{7} \\
\text { mysinifórmis Rbr } \sigma^{7}\end{array}$ & 4608 & $315 \mathrm{r}$. & & \\
\hline 8 & $\begin{array}{l}\text { mysiniformis Rbe o } \\
\text { aérifrons } Z \text {. O }\end{array}$ & 4609 & $315 \mathrm{r}$. & & \\
\hline 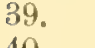 & leueopsifórmis Esp. $\sigma^{7}$ & $\begin{array}{l}4610 \\
4612\end{array}$ & $\begin{array}{l}315 r \\
315 / 16\end{array}$ & & \\
\hline 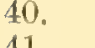 & alyssonifórmis HS. & 4614 & 3161. & & \\
\hline 4 & fenusifórmis Ld. 운 & 4616 & 3161. & & \\
\hline 4 & osmíifórmis HS. đ & 4617 & 3161. & & \\
\hline & $\begin{array}{l}\text { anthracifórmis } \mathrm{Rbr}^{*} \text { ) } \\
\text { dorylifórmis } 0 \text {. }\end{array}$ & 4619 & 3161. & & \\
\hline $\mathrm{a}$ & $\begin{array}{l}\text { dorylifórmis } 0 . \\
\text { v. eerifórmis Lue. } \sigma^{7}\end{array}$ & 4622 & 3161. & & \\
\hline 45. & $\begin{array}{l}\text { v. eerifórmis Lue. } \sigma^{7} \\
\text { englossifórmis Lue.**) }\end{array}$ & 4622 a & 3161. & & \\
\hline 6. & $\begin{array}{l}\text { englossiformis Lue. }{ }^{* *} \text { ) } \\
\text { oryssifórmis HS. }{ }^{* * *} \text { ) }\end{array}$ & $\begin{array}{l}4623 \\
4626\end{array}$ & $\begin{array}{ll}3161 . \\
3161 .\end{array}$ & & \\
\hline & chrysidifórmis Esp. & 4627 & 3161. & & \\
\hline & " minianifórmis Frp o & 4628 & $316 \mathrm{r}$. & & \\
\hline & " ehaleidifórmis Hb. ab. expléta Stg & gr 4629 & $316 r$ & & \\
\hline & sehmidtilópmis Frr & $4629 \mathrm{a}$ & $316 \mathrm{r}$ & & \\
\hline & foenifórmis HS. †) & 4631 & $316 \mathrm{r}$. & & \\
\hline & Weissmánnia agdistifórmis Stgr & 4603 & $317 \mathrm{r}$ & & \\
\hline & Bembéeia hylaeifórmis Lasp. $\sigma^{7}$ & 4632 & 3061. & $"$ & $9,11 \mathrm{a}, \mathrm{b}$ \\
\hline & Paranthréne tineifórmis Esp. & 4633 & $306 \mathrm{r}$. & & \\
\hline 5 & " V. brosifórmis $\mathrm{Hb}$ & $4634 \mathrm{a}$ & $306 \mathrm{r}$. & & \\
\hline & myrmosifórmis $\mathrm{H}$ & 4635 & $306 \mathrm{r}$ & & \\
\hline
\end{tabular}

*) Kopie nach Ann. S. Ent. Fr. 1832, pl. 7, fig. 7.

Kopie nach Oberthür, Etude XIII, pl. 8, fig. 90.

***) Die Segmente 4-6 des Hinterleibs sollten rotgelb sein, ebenso die Schulterdecken.

t) Kopie nach Herrich-Schäffer, Sesiid., Fig. 11. 


\section{\%}

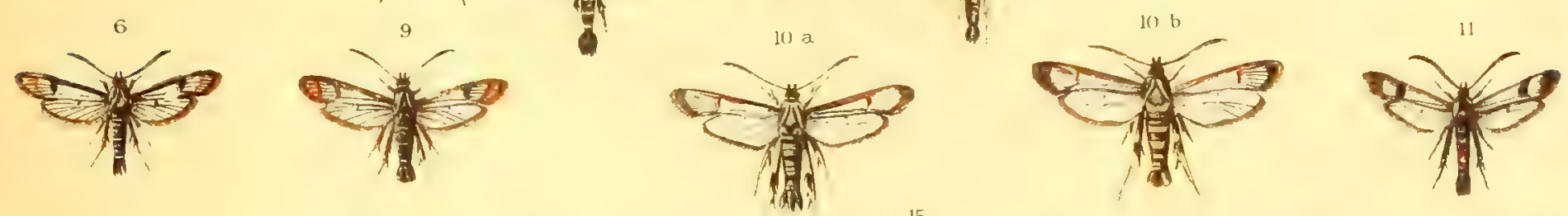

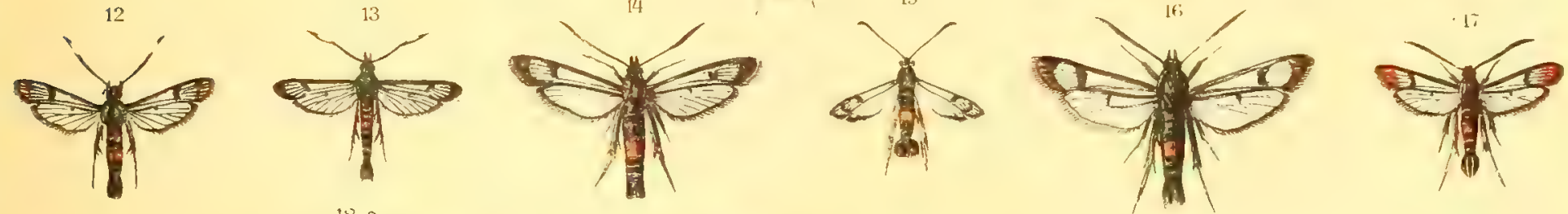

म

कौ

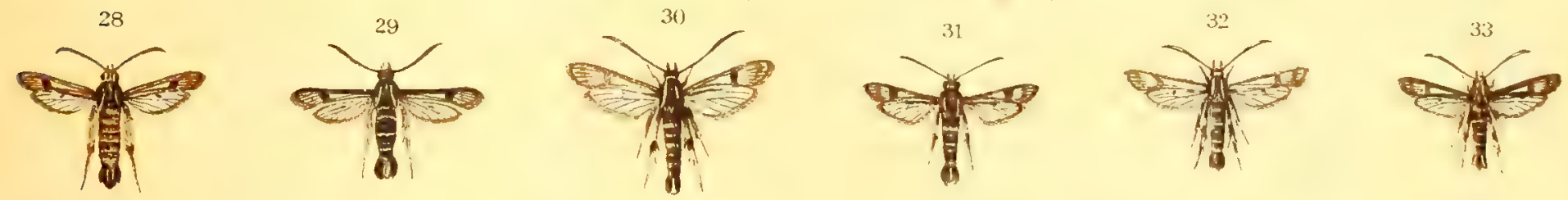

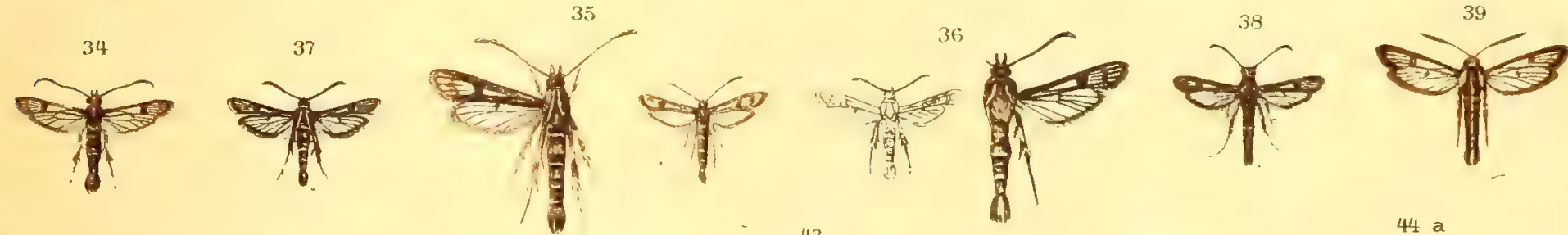

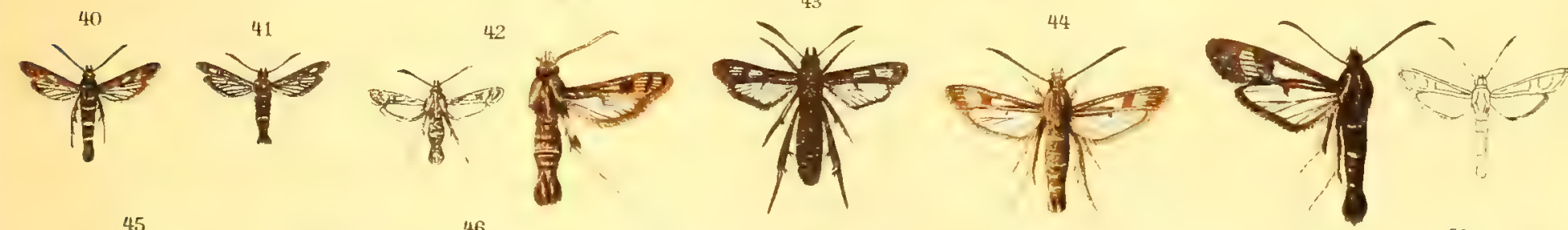

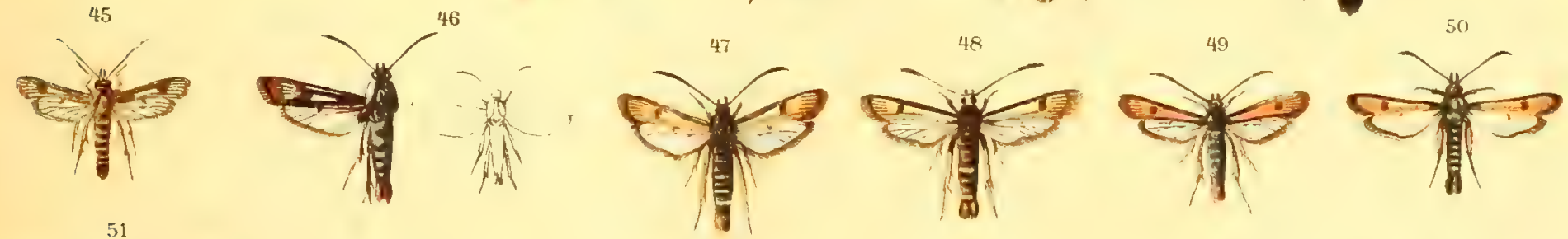

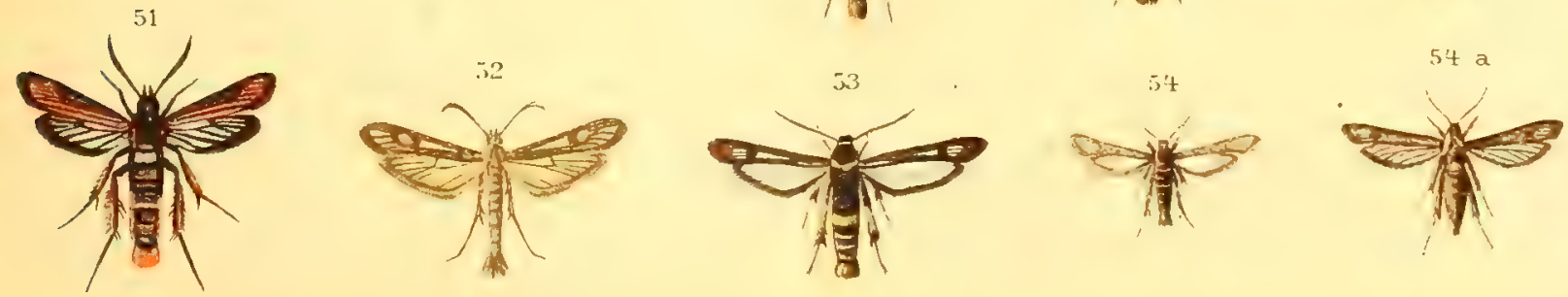






\section{TAFE L 80 .}

\begin{tabular}{|c|c|c|c|c|}
\hline \multicolumn{2}{|l|}{ Nr: } & Kat. I & Seite des II. Ban & $\begin{array}{l}\text { Raupen-(Puppen-) } \\
\text { Abbildung }\end{array}$ \\
\hline \multicolumn{2}{|c|}{1 a. Spilosóma lubrieipédum L. 1. 오, r. $\sigma^{7}$} & 4159 & $129 \mathrm{l}$. & Taf. $14,2 \mathrm{a}-\mathrm{c}$ \\
\hline \multicolumn{2}{|r|}{ b. $\quad " \quad$ v. zátima $\mathrm{Cr} \cdot \sigma^{n}$} & $4159 \mathrm{a}$ & 1291. & \\
\hline 2. & menthástri Esp. 우 & 4163 & $129 \mathrm{r}$. & $" 14,3 \mathrm{a}, \mathrm{b}$ \\
\hline 3. & urtieae Esp. 우 & 4164 & $129 / 30$ & $" 13,4 \mathrm{a}, \mathrm{b}$ \\
\hline \multicolumn{2}{|c|}{ 4. Hepiolus húmuli L. 1. $\sigma^{7}$, r. $q^{*}$ ) } & 4726 & $484 / 85$ & $\left\{\begin{array}{r}\text { Ei Taf. 50, } 32 \\
\quad 14,5 \mathrm{a}, \mathrm{b}\end{array}\right.$ \\
\hline $5 \mathrm{a}$. & "sylvinus $\mathrm{L} \cdot \mathrm{O}^{7}, \mathrm{~b} q$ & 4727 & 4851. & Taf. 14,6 \\
\hline 6. & fusconebulósus De Geer. & 4730 & $485 \mathrm{r}$. & \\
\hline 7. & earna Esp. & 4736 & $485 \mathrm{r}$ & \\
\hline $8 \mathrm{a}$ & lupulínus L. $\sigma^{7}, \mathrm{~J} q$ & 4738 & $485 / 86$ & $" 14,7 \mathrm{a}, \mathrm{b}$ \\
\hline 9. & ganna $\mathrm{Hb}$. & 4742 & 4861. & \\
\hline $10 \mathrm{a}$. & hecta L. $\sigma^{7}, \mathrm{~b}$ 우 & 4743 & $486 \mathrm{r}$ & $" 14,8 \mathrm{a}, \mathrm{b}$ \\
\hline \multicolumn{2}{|c|}{ 11. Cossus cossus L. $q$} & 4641 & 3021. & $\left\{\begin{array}{cc}\text { Ei Taf. } 50,33 \\
, \quad 14,9 \mathrm{a}, \mathrm{b}\end{array}\right.$ \\
\hline \multicolumn{2}{|c|}{ 12. " térebra F. } & 4650 & $302 \mathrm{r}$. & Taf. $14,10 \mathrm{a}, \mathrm{b}$ \\
\hline \multicolumn{2}{|c|}{ 13. Zeúzera pyrína L. 1. $\sigma^{7}, 1$, 早 } & 4718 & 3051. & $\left\{\begin{array}{r}\text { Ei Taf. } 50,34 \mathrm{a}, \mathrm{b} \\
" 14,11 \mathrm{a}-\mathrm{c}\end{array}\right.$ \\
\hline \multicolumn{2}{|c|}{ 14. Phragmatoéeia eastáneae Hb. $\sigma^{7}$} & 4713 & $304 \mathrm{r}$. & Taf. 14,12 u. Ntr.-T. X, 18 \\
\hline \multicolumn{2}{|c|}{ 15. Hypópta thrips $\mathrm{Hb}$. } & 4628 & 3031. & \\
\hline \multicolumn{2}{|r|}{ 16. $\quad$ eaestrum $\mathrm{Hb}$} & 4685 & 3031. & \\
\hline \multicolumn{2}{|c|}{ 17. Stýgia austrális Latr. $\sigma^{7}$} & 4705 & 3041. & \\
\hline \multicolumn{2}{|c|}{ 18. Dyspéssa úlula $\mathrm{Bkh}$. $\subsetneq$} & 4689 & 303 r. & Nachtr.-Taf. X, 19 \\
\hline \multicolumn{2}{|c|}{ 19. Cochlídion limacódes Hufn. 1. , r. } & 4440 & $169 / 70$ & $\left\{\begin{array}{l}\text { Ei Taf. } 50,35 \\
\quad 14,13 \mathrm{a}-\mathrm{c}\end{array}\right.$ \\
\hline \multicolumn{2}{|r|}{ 20. Heterogénea asélla Knoch 1. , r. $0^{7}$} & 4443 & $170 \mathrm{l}$. & Taf. $14,14 \mathrm{a}, \mathrm{b}$ \\
\hline \multicolumn{2}{|r|}{ 21. Pachytélia unícolor L. $\sigma^{7}$} & 4450 & 1741. & $" 15,1 \mathrm{a}, \mathrm{b}$ \\
\hline \multicolumn{2}{|c|}{ 22. $\quad$ villosélla 0} & 4451 & $174 \mathrm{r}$. & $\because \quad 15,2 \mathrm{a}, \mathrm{b}$ \\
\hline \multicolumn{2}{|c|}{ 23. Amícta febrétta Boyer o7 } & 4457 & 1751. & Nachtr.-Taf. X, 60 \\
\hline \multicolumn{2}{|c|}{ 24. $"$ eeksteini Ld. $\sigma^{7}$} & 4458 & $175 \mathrm{r}$. & $\mathrm{X}, 61$ \\
\hline \multicolumn{2}{|c|}{ 25. Psyehe vieiélla Schiff. О7 } & 4183 & 1781. & Taf. $15,3 \mathrm{a}, \mathrm{b}$ \\
\hline \multicolumn{2}{|c|}{ 26. Phalaerópteryx apiformis Rossi $\sigma^{7}$} & 4490 & 1791. & $" 15,4 \mathrm{a}, \mathrm{b}$ \\
\hline \multicolumn{2}{|c|}{ 27. $\quad$ graslinélla B. $\sigma^{7}$} & 4493 & $179 \mathrm{l}$. & $" 15,5 \mathrm{a}, \mathrm{b}$ \\
\hline 28. A & Acanthopsýche atra L. $\sigma^{7}$ & 4446 & $173 / 74$ & $" 15,6 \mathrm{a}, \mathrm{b}$ \\
\hline 29. $\mathrm{H}$ & Hyálina álbida Esp. $\sigma^{7}$ & 4463 & $175 / 76$ & \\
\hline 30. 0 & Oreopsýehe angustélla HS. & 4477 & 1771. & Nachtr.-Taf. 10,64 \\
\hline 31. $\mathrm{S}$ & Seióptera sehiffermilléri Stgr $\sigma^{7}$ & 4482 & 1781. & \\
\hline 32.0 & Oreopsýche museélla F. & 4472 & $177 \mathrm{l}$ & \\
\hline & plumífera 0 & 4478 & $177 \mathrm{r}$. & Taf. $15,11 \mathrm{a}, \mathrm{b}$ \\
\hline 34. $\mathrm{s}$ & Scioptera plumistrélla Hb. O & 4481 & $178 \mathrm{l}$. & \\
\hline
\end{tabular}

*) Auf der Tafel unrichtig bezeichnet. 

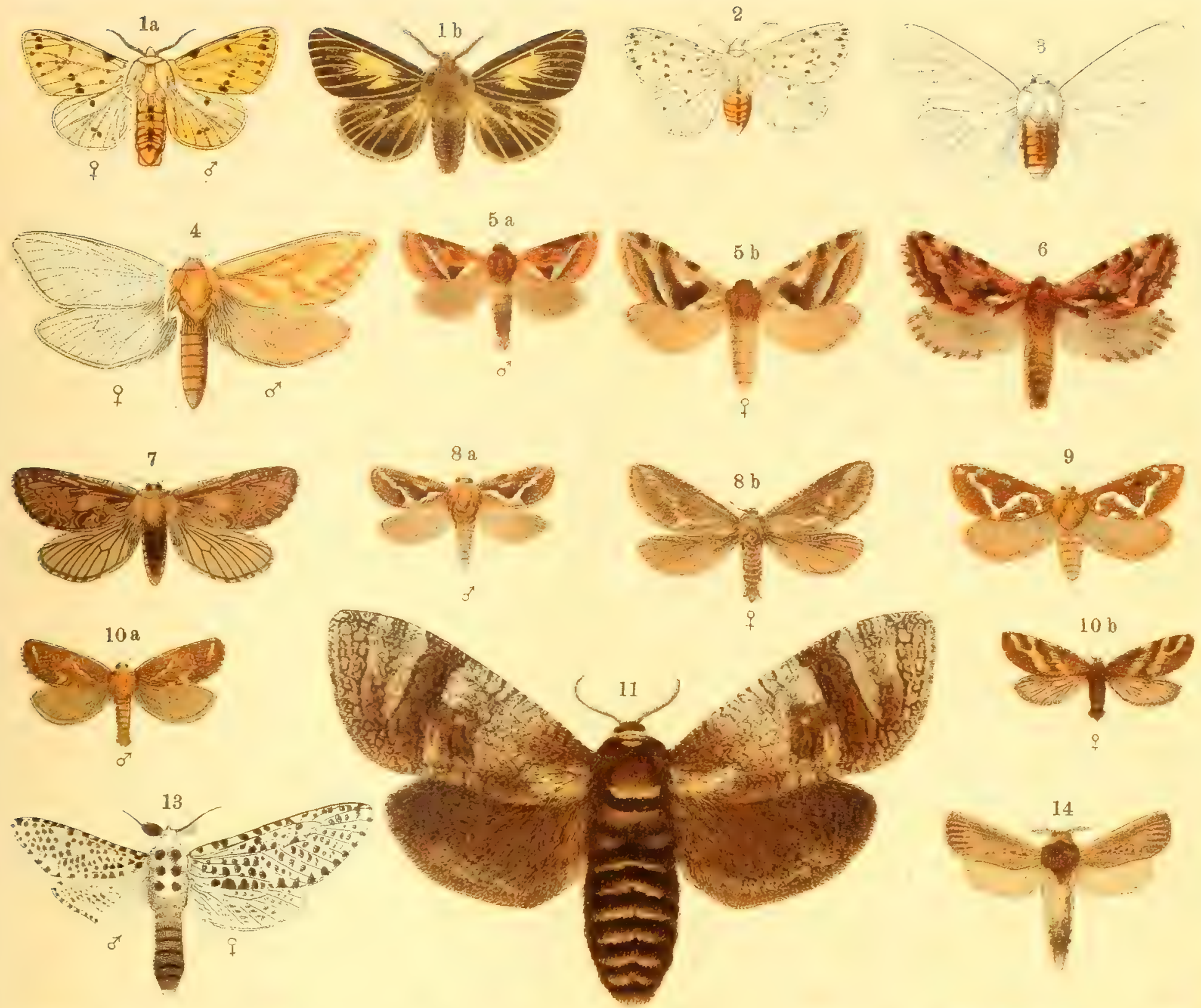

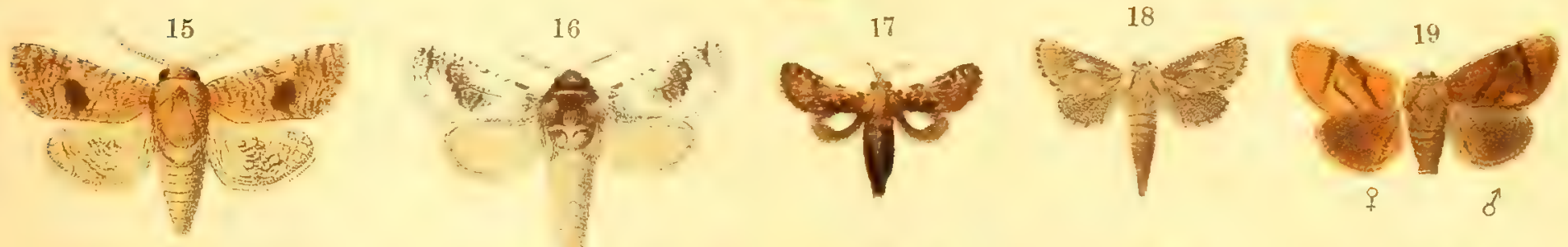
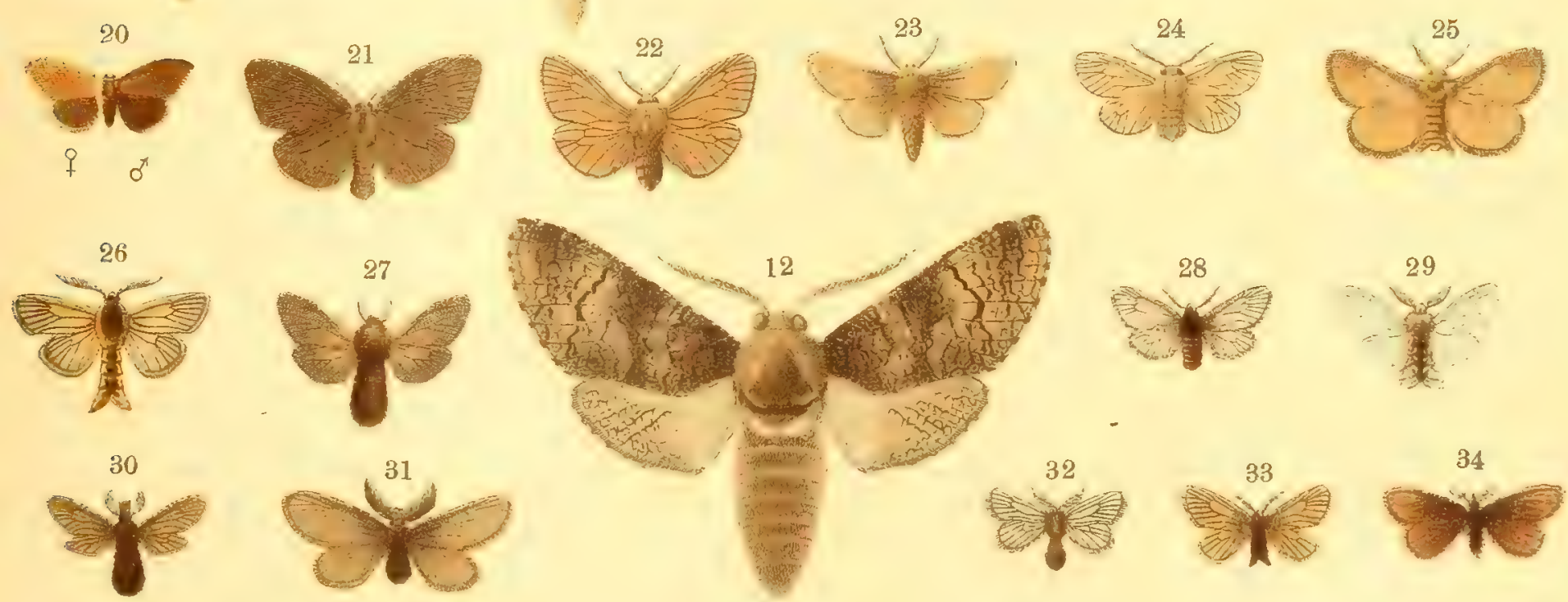




\section{TAFEL 81 .}

Nis.

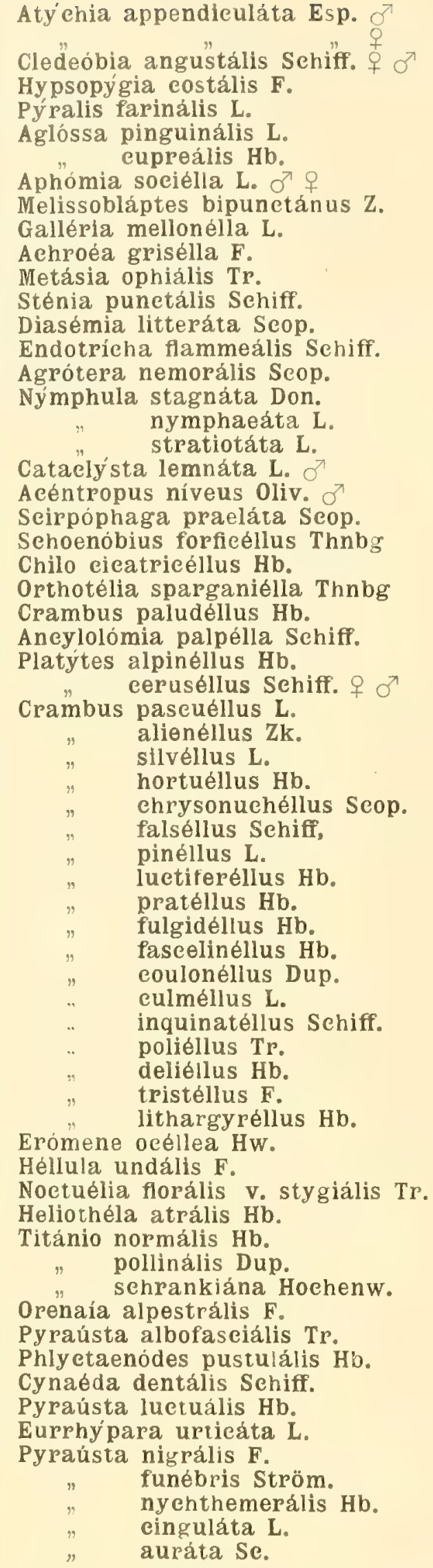

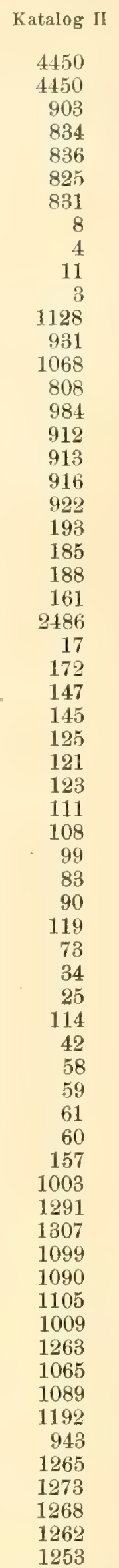

Seite des II. Bandes

Raupen- (Puppen-)
Abbildung

3011.

3011.

2211.

$218 \mathrm{r}$.

2191.

$218 \mathrm{r}$.

$218 \mathrm{r}$.

1901.

1901.

$190 \mathrm{x}$.

$189 \mathrm{r}$.

2311.

2221.

2291.

217 r.

$205 \mathrm{r}$.

2211.

221 r.

2211.

$221 \mathrm{r}$.

199 r.

$198 \mathrm{l}^{*}$.

1991.

$197 \mathrm{r}$.

4531.

1911.

1981.

1971.

$196 \mathrm{r}$.

1961.

1961.

1961.

$195 \mathrm{r}$.

$195 \mathrm{l}$.

1951.

1941.

$194 \mathrm{r}$.

$195 \mathrm{r}$.

$193 \mathrm{r}$.

$191 \mathrm{r}$.

$191 \mathrm{I}$.

$195 \mathrm{r}$.

1921.

$192 \mathrm{r}$.

1931.

1931.

1931.

1931.

2261.

$237 \mathrm{r}$.

2381.

$230 \mathrm{r}$.

2301.

$230 \mathrm{r}$.

$226 r$.

236 r.

$228 \mathrm{r}$.

2301.

2341.

222 r.

2371.

2371.

2371.

$236 \mathrm{r}$.

2361.
Nachtr.-Taf. IX, 51

IX, 50

IX, 49

IX, 27

IX, 26

IX, 28

IX, 25

IX, 65̃

IX, 48

IX, 52

IX, 53

IX, 54

IX, 34

IX, 33

$\mathrm{X}, 41$

IX, 29

IX, 32

IX, 30

IX, 31

IX, 68

X, ธ

IX, 67

IX, 57

$X, 6$
$X, 4$

Die Figuren 50 und 51 zur Hälfte in doppelter linearer Vergrößerung dargestellt. 


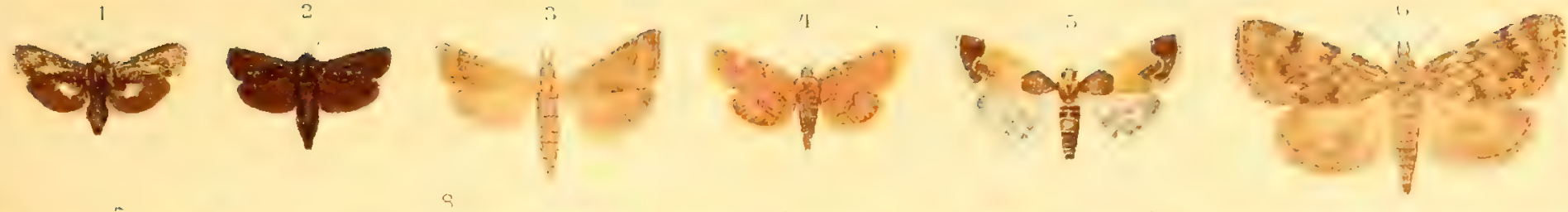

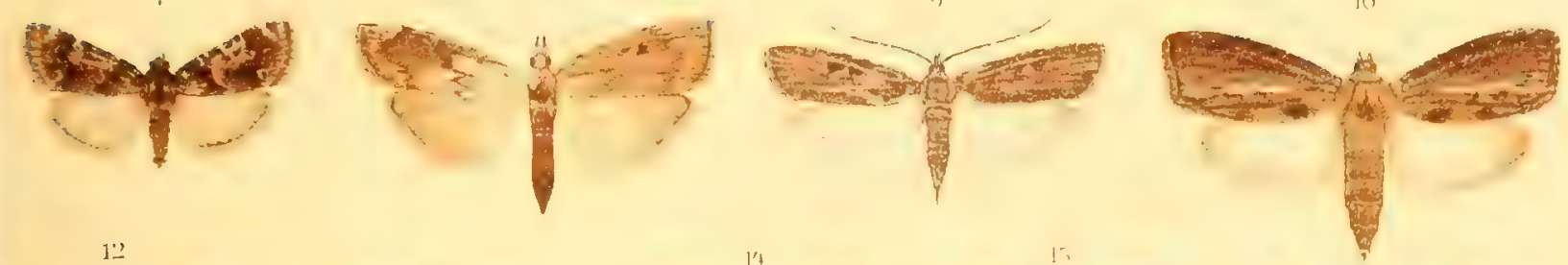

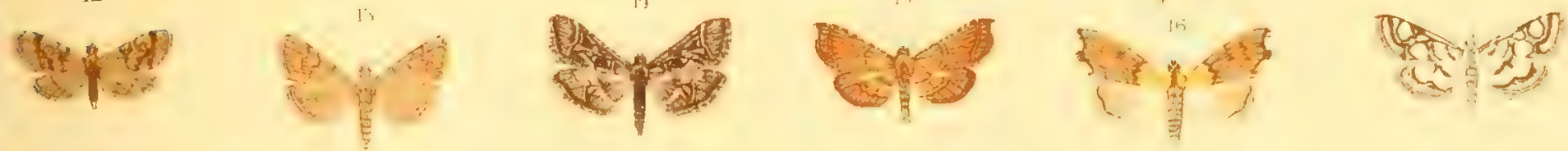

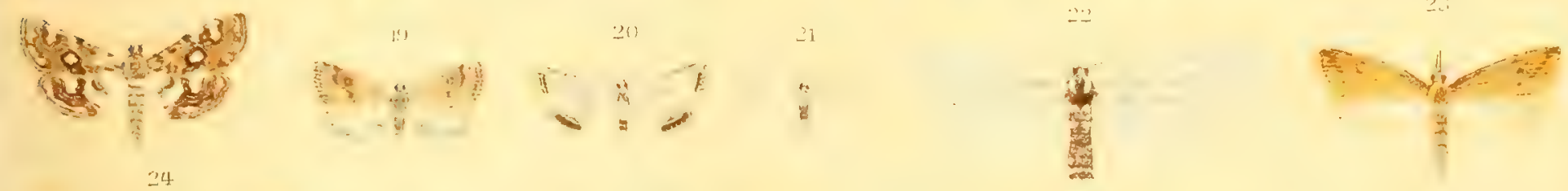

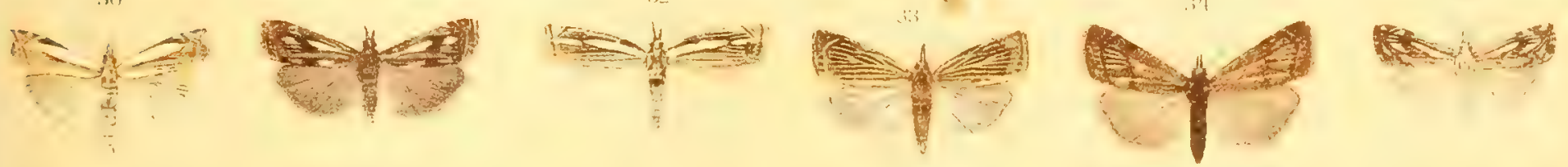
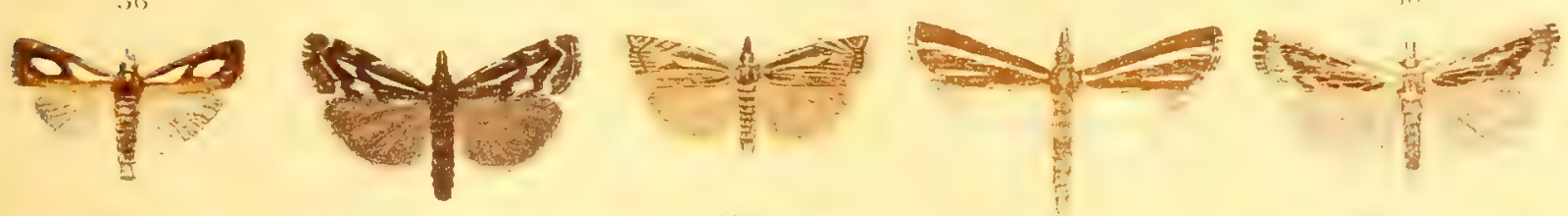

$\frac{1}{1-2 x}$

F d.

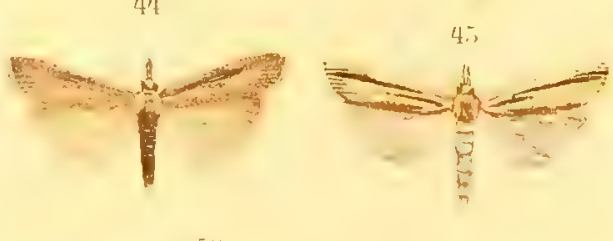

$\rightarrow$

$\sum+10+100$
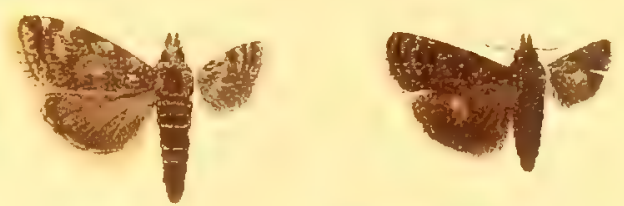

at?

expos

\section{but \\ 16)

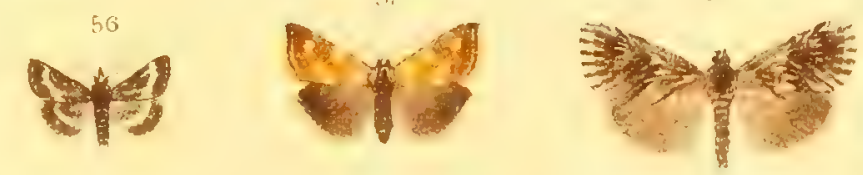 \\ 20}
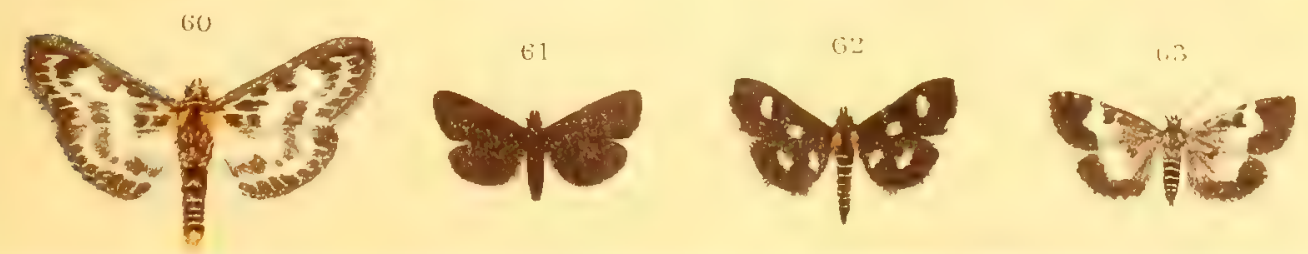

a बै। 




\section{TAFEL 82.}

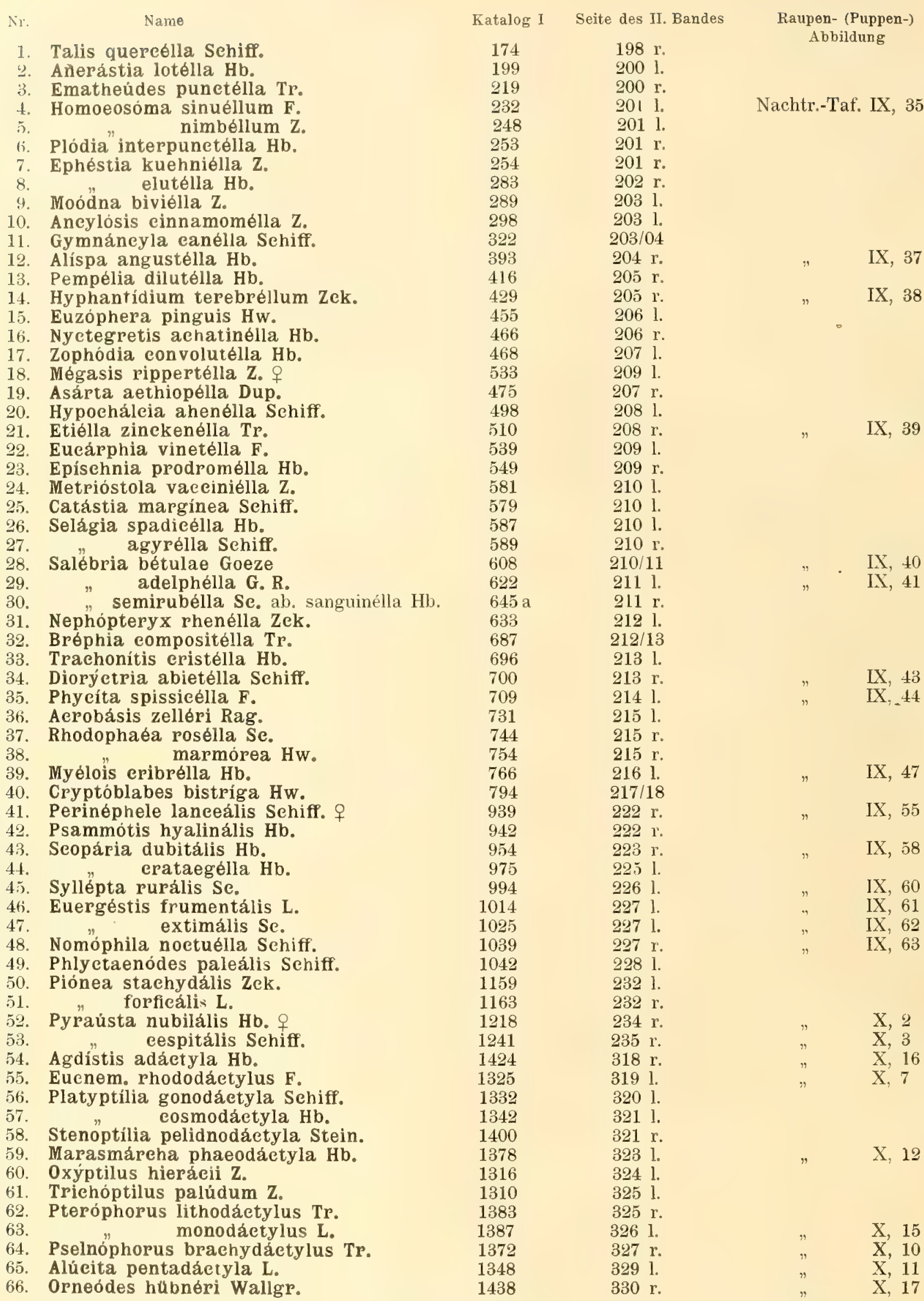

Die ganzen Tiere in natürl. Größe, die einzelnen Teile in doppelter linearer Vergrößerung. 


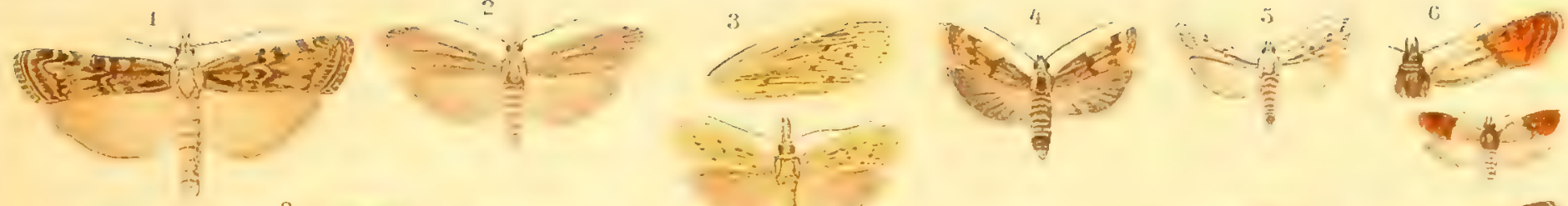

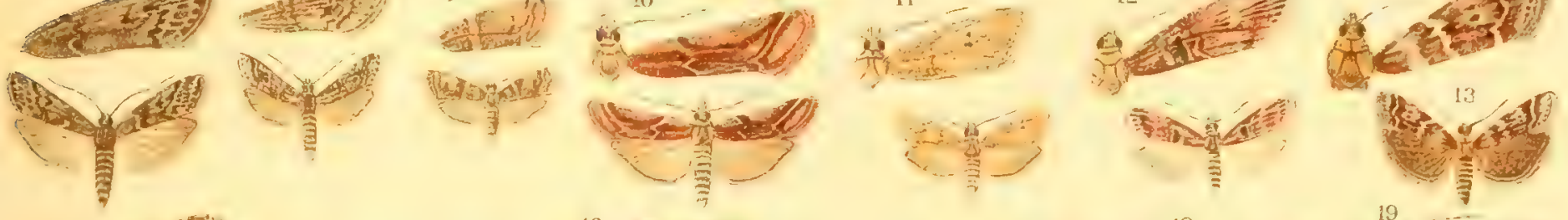

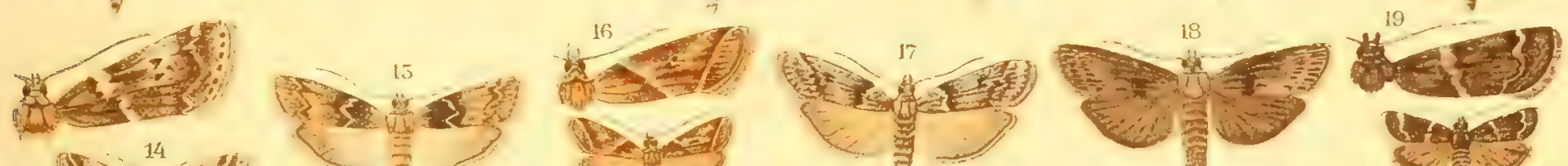

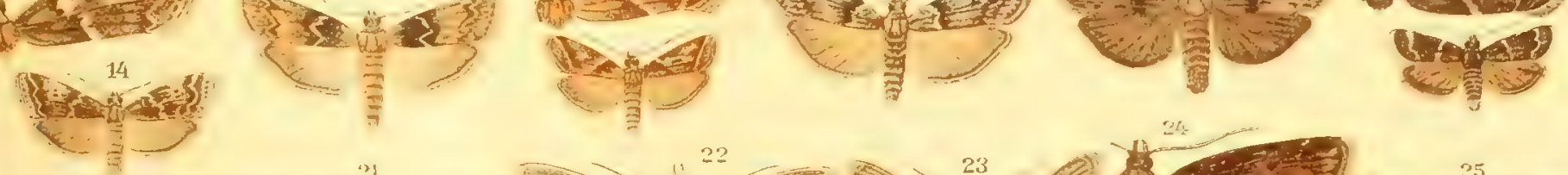

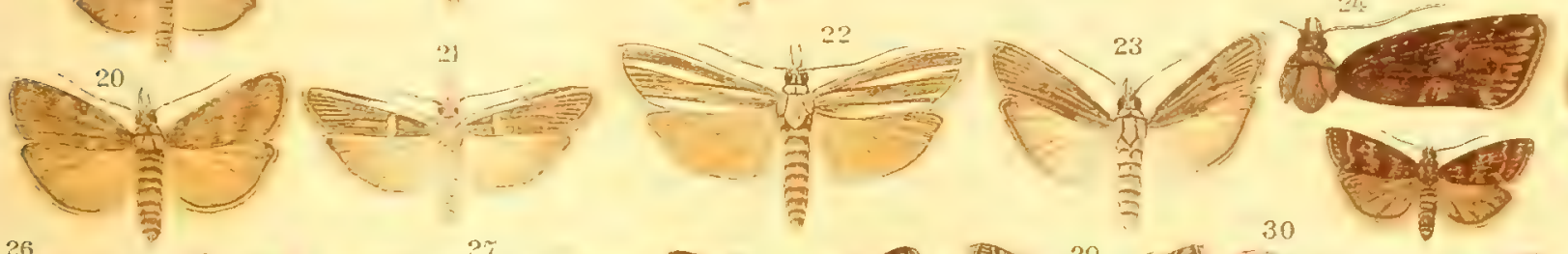

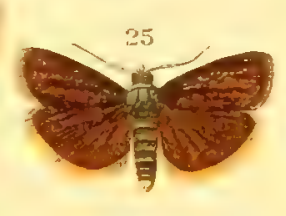

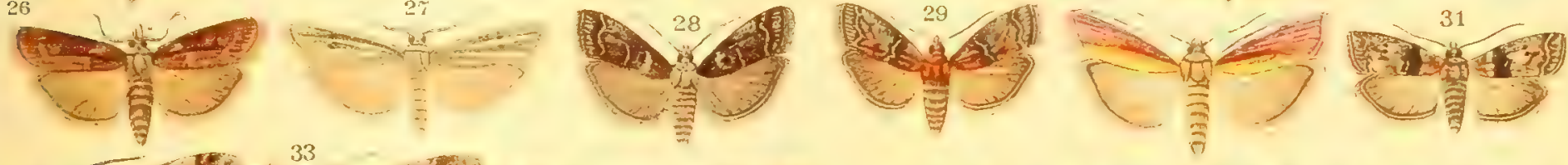

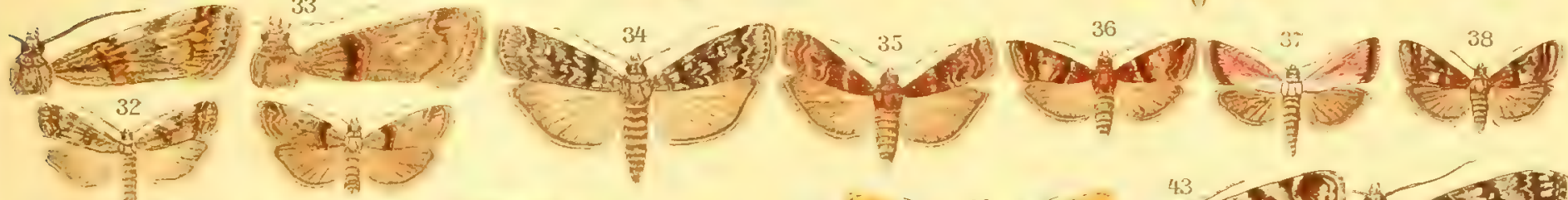

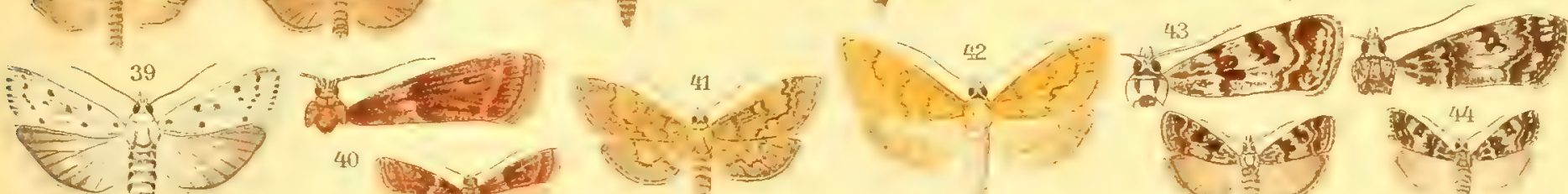

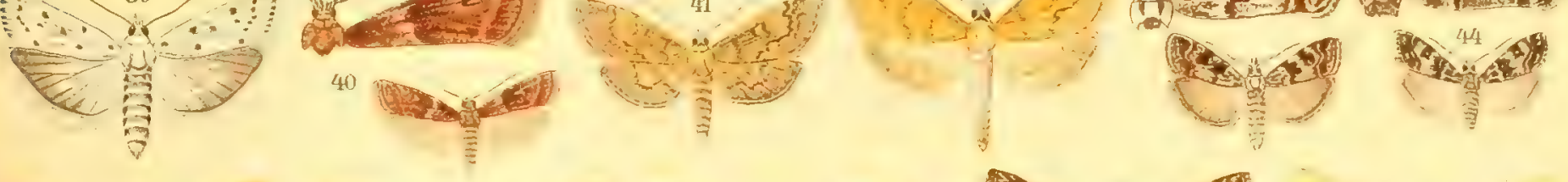
Wh2 ${ }^{45}$ 세
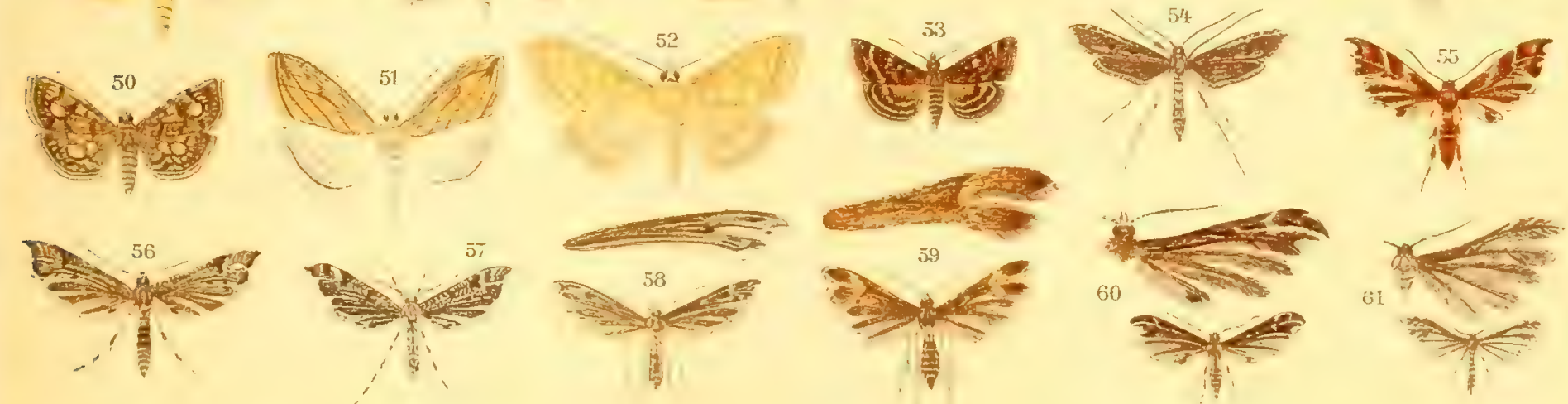

2. $=10$
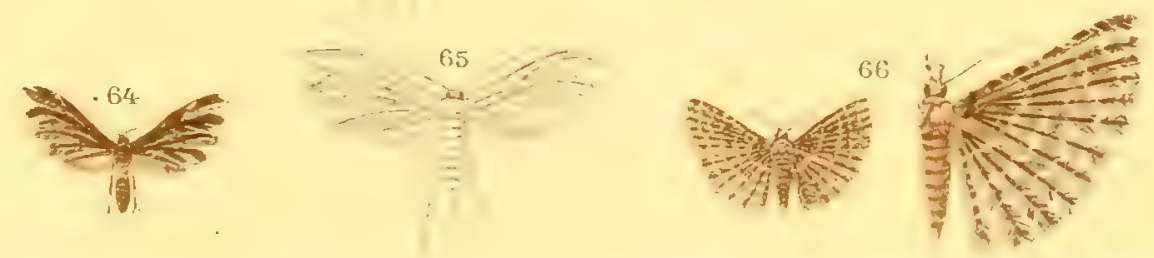




\section{TAFEL 83.}

\begin{tabular}{|c|c|c|}
\hline Name & Katalog II & Seite des II. Bandes \\
\hline Acálla emargána F. & 1440 & $240 \mathrm{r}$. \\
\hline " epistána $F$. & 1441 & $240 / 41$ \\
\hline v. fulvovittána Stph. & $1441 \mathrm{f}$. & $240 \mathrm{r}$. \\
\hline hastiána L. v. coronána Thnbg & 1446 a & 2411. \\
\hline $\begin{array}{l}\text { abietána } \mathrm{Hb} \text {. } \\
\text { maccána Tr. }\end{array}$ & 1448 & $241 \mathrm{r}$. \\
\hline $\begin{array}{l}\text { maccána Tr. } \\
\text { fimbriána Thnbg }\end{array}$ & 1449 & $241 \mathrm{r}$. \\
\hline $\begin{array}{l}\text { fimbriána Thnbg } \\
\text { mixtána Hb. }\end{array}$ & $\begin{array}{l}1450 \\
1451\end{array}$ & 2421. \\
\hline logiána Sehiff. v. germarána Froel. & . $1452 \mathrm{a}$ & $\begin{array}{l}2421 . \\
2421 .\end{array}$ \\
\hline hippophaëána Heyd. & 1453 & 2421. \\
\hline literána $\mathrm{L}$ & 1458 & $242 \mathrm{r}$. \\
\hline boseána F. g. aest. parisiána Gn. & $1457 \mathrm{a}$ & $242 \mathrm{r}$. \\
\hline boseána $F$. & 1457 & $242 / 43$ \\
\hline variegána Sehiff. & 1455 & 2431. \\
\hline niveána $\mathrm{F}$. & 1459 & $243 \mathrm{l}$. \\
\hline $\begin{array}{l}\text { sponsána } \mathrm{F} \text {. } \\
\text { rufána Sehiff. }\end{array}$ & 1464 & $243 \mathrm{r}$ \\
\hline $\begin{array}{l}\text { rufána Sehiff. } \\
\text { lipsiána Sehiff. } \sigma^{7}\end{array}$ & 1466 & $243 \mathrm{r}$. \\
\hline $\begin{array}{l}\text { lipsiána Sehiff. } \\
\text { sehalleriána L. }\end{array}$ & 1461 & $243 \mathrm{r}$. \\
\hline 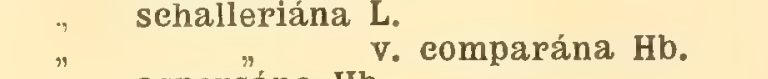 & $\begin{array}{l}1469 \\
1469 \mathrm{a}\end{array}$ & $243 \mathrm{r}$. \\
\hline aspersána $\mathrm{Hb}$. comparana $\mathrm{Hb}$. & $\begin{array}{l}1469 \mathrm{a} \\
1471\end{array}$ & $\begin{array}{l}243 \mathrm{r} . \\
244 \mathrm{l}\end{array}$ \\
\hline " ferrugána Tr. & 1473 & 2441. \\
\hline "holmiána L. & 1479 & $244 / 45$ \\
\hline$" \quad$ eontaminána Hb. & 1480 & 2451. \\
\hline Amphisa gerningána Sehiff. ๑, Fig. 26 우 & 1482 & 2451. \\
\hline prodromána Hb. $\sigma^{7}$, Fig. 28 q & 1484 & $245 \mathrm{r}$. \\
\hline Zelothérses alboeiliána HS. & 1489 & $245 \mathrm{r}$. \\
\hline Diehélia grotiána F. & 1494 & $246^{\circ} 1$. \\
\hline " gnomána Cl. & 1495 & 2461. \\
\hline Capua reticulána Hb. $\sigma^{7}$ & 1503 & 246 r. \\
\hline " favillaceána $\mathrm{Hb}$. & 1504 & $246 \mathrm{r}$. \\
\hline Sparganóthis pilleriána Sehiff. & 1505 & $246 \mathrm{r}$. \\
\hline Cacoécia podána Se. $0^{7}$, Fig. 36 우 & 1507 & 2471. \\
\hline$" \quad$ erataegána Hb. $\sigma^{7}$ & 1512 & $247 \mathrm{r}$. \\
\hline xylosteána L. の゙, Fig. 39 \& & 1513 & $247 \mathrm{r}$. \\
\hline rosána L. o7 & 1514 & $247 \mathrm{r}$. \\
\hline sorbiána $\mathrm{Hb} \cdot \mathrm{O}^{7}$ & 1515 & $247 \mathrm{r}$. \\
\hline histrionána Froel. o & 1523 & $248 \mathrm{l}$ \\
\hline museulána $\mathrm{Hb} . \sigma^{\top}$ & 1525 & 2481. \\
\hline unifasciána Dup. or & 1528 & $248 \mathrm{r}$. \\
\hline strigána $\mathrm{Hb} . \mathrm{O}^{7}$ & 1531 & $248 \mathrm{r}$. \\
\hline semialbána Gn. 우 & 1518 & $248 \mathrm{r}$. \\
\hline " lecheána Hb. $\sigma^{7}$ & 1533 & 2491. \\
\hline Pandémis eorylána F. O7 & 1 อ3 & 249 r. \\
\hline ribeána $\mathrm{Hb}_{\text {. }}$ ㅇ & 1540 & $249 \mathrm{r}$. \\
\hline "heparána Sehiff. $\sigma^{7}$ & 1547. & $249 \mathrm{l}$. \\
\hline Chrosis bifaseiána $\mathrm{Hb}$ & 1570 & 2501. \\
\hline Tortpix (Eúlia) oehreána Hb. ㅇ & 1549 & 2501. \\
\hline " politána $\mathrm{Hw}_{0} \mathrm{o}^{7}$ & 1553 & 2501. \\
\hline einetána Sehiff. & 1556 & $.250 \mathrm{l}$ \\
\hline rigána Sodoff. & 1558 & $250 \mathrm{r}$. \\
\hline ministrána $\mathrm{L}$. & 1562 & $250 \mathrm{r}$. \\
\hline Tortrix (Tortrix) forskaleána L. & 1564 & $250 \mathrm{r}$. \\
\hline " bergmanniána L. & 1568 & $250 \mathrm{r}$. \\
\hline eonwayána $F_{\text {. }}$ & 1569 & $250 \mathrm{r}$. \\
\hline loefflingiána L. & 1571 & $250 \mathrm{r}$. \\
\hline viridána L. & 1572 & $250 / 51$ \\
\hline forsterána F. $0^{7}$ & 1576 & 2511. \\
\hline viburniána $\mathrm{F}$. & 1578 & 2511. \\
\hline steineriána Hb. 우 & 1591 & $251 \mathrm{r}$. \\
\hline rusticána Tr. の , Fig. 66 q & 1597 & $251 \mathrm{r}$. \\
\hline diversána Hb. O & 1601 & $251 / 52$ \\
\hline $\begin{array}{c}\text { Cnephasia osseána Seop. } \\
\text { longána Hw. ơ, Taf. } 84 \text {, Fig. } 1\end{array}$ & $\begin{array}{l}1605 \\
1608\end{array}$ & $\begin{array}{l}2521 . \\
2521 .\end{array}$ \\
\hline
\end{tabular}

Nachtr-Taf. X, 21

Die Tiere sind alle in zweifacher linearer Vergrößerung dargestellt. 
nteos

4.
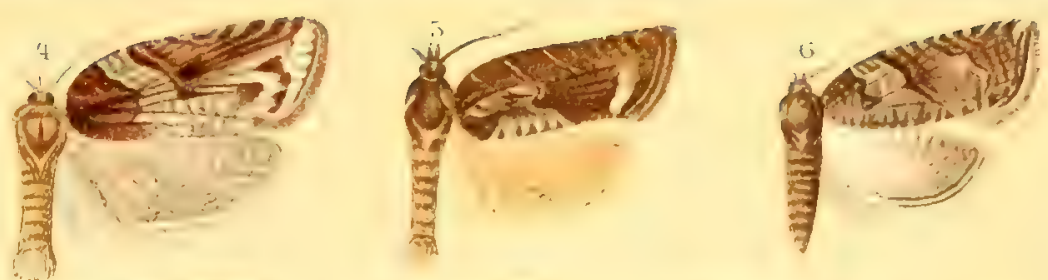

$\sqrt{2}(5)$

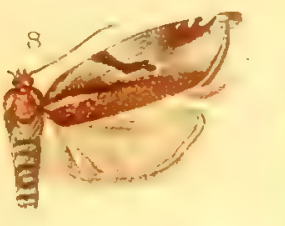

$\frac{2}{1+2}$

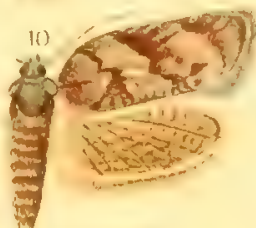

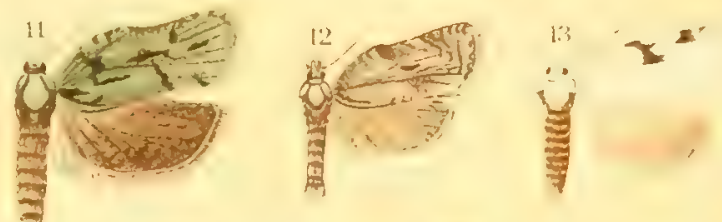

点我)
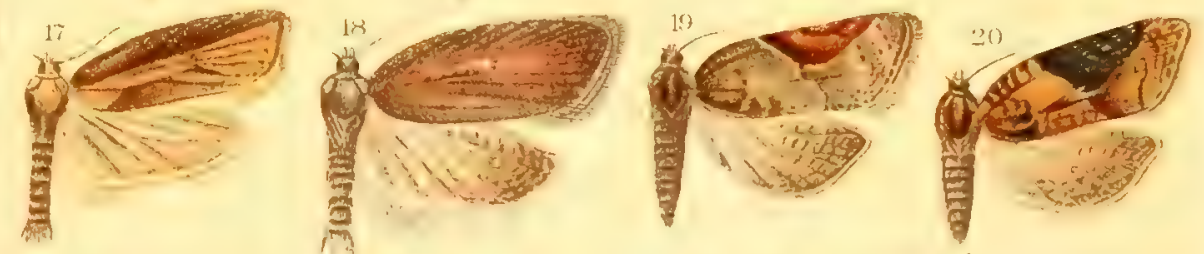

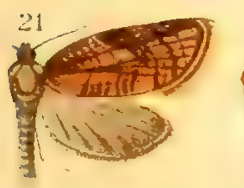
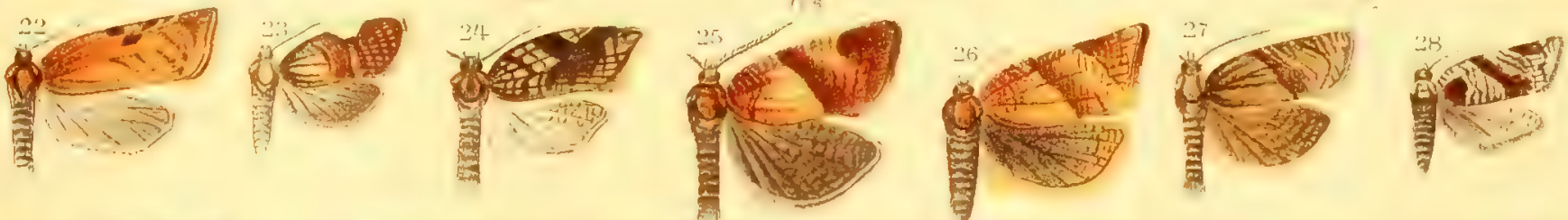

in
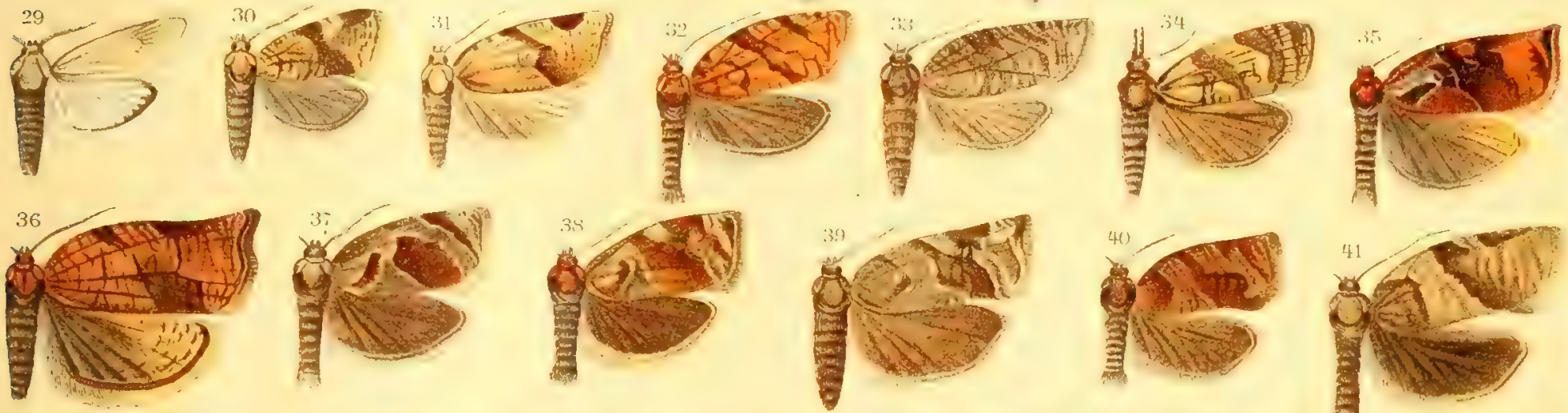

\section{西}

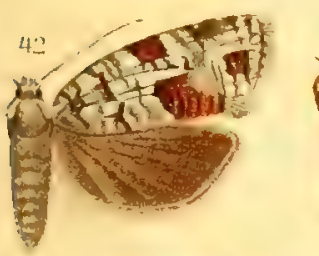
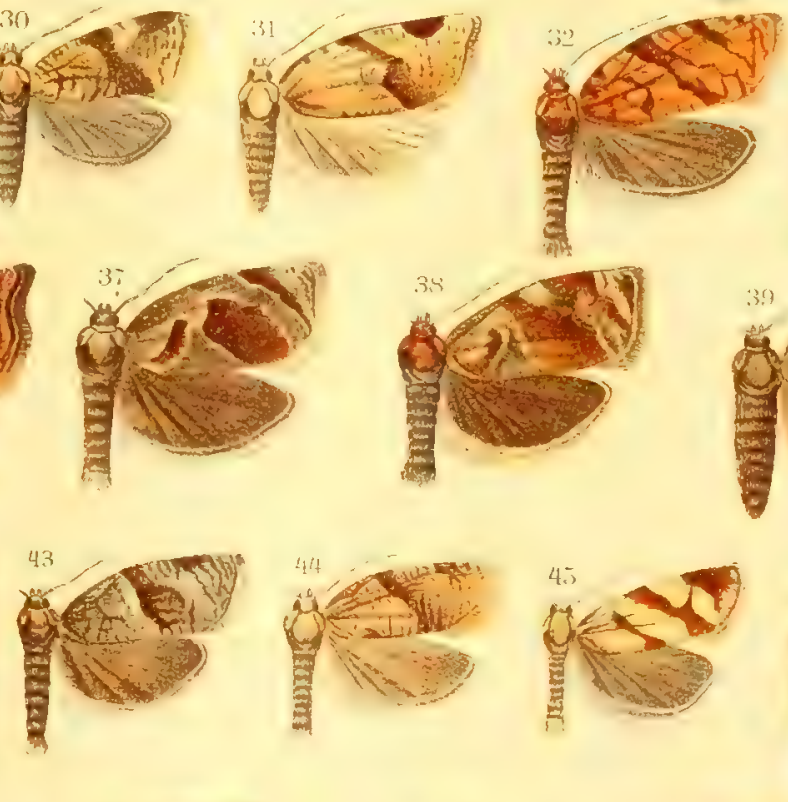

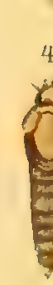
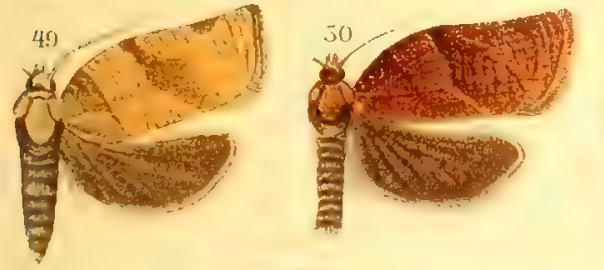

(3)
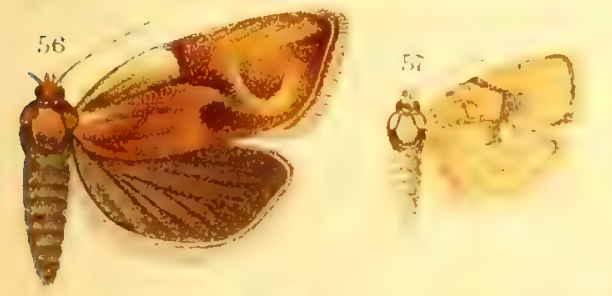

18
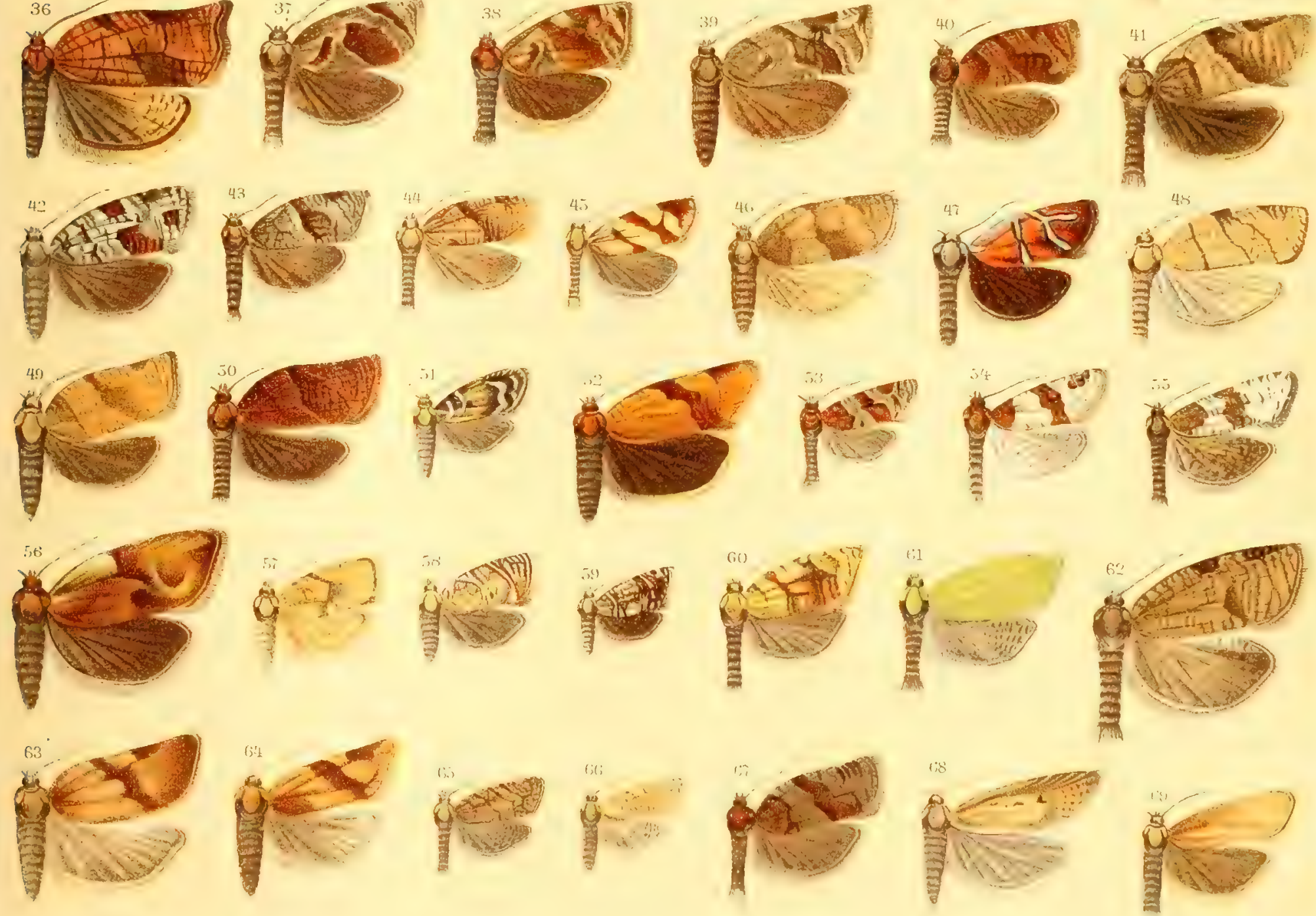




\section{TAFEL 84.}

1.

2.

3.

5 .

bia.

\%.

8.

a. Exapáte congelatélla Cl. $\sigma^{7}, \mathrm{~b}$. ㅇ

Anisotaénia ulmána $\mathrm{Hb}$.

hybridána $\mathrm{Hb}$. ㅇ

Lozópera francillána F. flagellána Dup.

Clýsia ambiguélla $\mathrm{Hb}$.

Phalónia $\mathrm{Hb}$. aleélla Schulzo kuhlweiniána F. R. badiána $\mathrm{Hb}$.

kindermanniána Tr.

richteriána F. R.

smeathmanniána $\mathrm{F}$.

epilinána $\mathrm{Z}$.

purpuratána HS.

zephyrána Tr.

pallidána Z.

sanguisorbána HS.

hybridélla $\mathrm{Hb}$.

phaleratána HS.

manniána F. R.

udána Gn.

mussehliána Tr.

dipoltélla $\mathrm{Hb}$.

fulvifasciána Const.

purána Gn.

Chlidónia hartmanniána $\mathrm{Cl}$.

Euxánthis parpeyssiána Dup.

cebrána Hb.

loeupletána $\mathrm{Hb}$.

lathoniána Hb.

perfusána Gn.

hamána L.

zoegána L.

angustána $\mathrm{Hb}$.

hilarána HS.

elongána F. R.

impurána Mn.

aureopurctána Rag.

aeneána $\mathrm{Hb}$.

Commóphila sehreibersiána Forst. rugosána $\mathrm{Hb}$. pulvillána HS.

Hysterósia sodaliána $\mathrm{Hb}$. maculosána $\mathrm{Hw}$. inopiána $\mathrm{HW}$.

Carposina scirrhosélla HS.

Evétria duplána $\mathrm{Hb}$.

$$
\text { posticána Zett. }
$$

pinivorána $\mathrm{Z}$.

turionána $\mathrm{Hb}$.

buoliána Sehiff.

tessulatána Stgr

margarotána HS.

resinélla L.

Argyróploce salicéila L. semifaseiána Hw. seriptána $\mathrm{Hb}$. capreána $\mathrm{Hb}$. corticána $\mathrm{Hb}$. betulaetána Hw. soropculána Zett. sauciána $\mathrm{Hb}$. variegána Hb. pruniána $\mathrm{Hb}$. ochroleueána Hb. sororiána HS.

$\begin{array}{ccc}\text { Katalog II } & \text { Seite des II. Bandes } & \text { Raupen-Abbildung } \\ 1608 & 252 \mathrm{l} & \\ 1627 \text { u. 1626 } & 253 \mathrm{l} . & \\ 1624 & 253 \mathrm{l} . & \\ 1622 & 252 \mathrm{r} . & \\ 1630 & 253 \mathrm{l} . & \\ 1632 & 253 \mathrm{l} . & \\ 1633 & 253 \mathrm{r} . & \\ 1638 & 253 \mathrm{r} . & \\ 1640 & 253 \mathrm{l} . & \text { Nachtr.Taf. X, } 23 \\ 1641 & 254 \mathrm{l} . & \end{array}$

0511

$25+1$.

2541.

$254 r$.

$25 \pm r$.

2551.

$25.5 \mathrm{r}$.

$255 \mathrm{r}$.

$250 \mathrm{r}$.

2561.

2561 .

2561.

$256 r$.

2571.

2571.

257 r.

2581.

2581.

$258 \mathrm{r}$.

$258 r$.

$258 \mathrm{r}$.

2591.

2591 .

2601.

2601.

2601.

$260 \mathrm{r}$.

$260 \mathrm{r}$.

$260 \mathrm{r}$.

$260 \mathrm{r}$.

2611.

2611.

2611.

2611.

2611.

2621.

2621.

2621.

2621.

2621.

2621.

$262 \mathrm{r}$.

$262 \mathrm{r}$.

$262 \mathrm{r}$.

$262 \mathrm{r}$.

2631.

$263 \mathrm{r}$.

$263 \mathrm{r}$.

$263 \mathrm{r}$.

$263 \mathrm{r}$.

$263 \mathrm{r}$

$263 \mathrm{r}$.

2641.

2641.

2641.

$264 \mathrm{r}$.

$264 \mathrm{r}$.

$26 \pm r$.

$264 \mathrm{r}$.

$264 \mathrm{r}$.

$264 \mathrm{r}$.

264 r.

2651.

2651.

2651 .

2651.
Nachtr.-Taf. X, 24

Nachtr.-Taf. X, 25

Die Tiere sind in zweifacher linearer Vergrößerung wiedergegeben. 
15

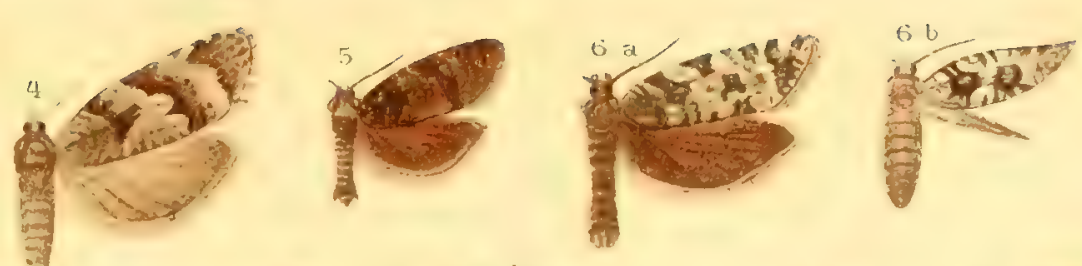

ins?
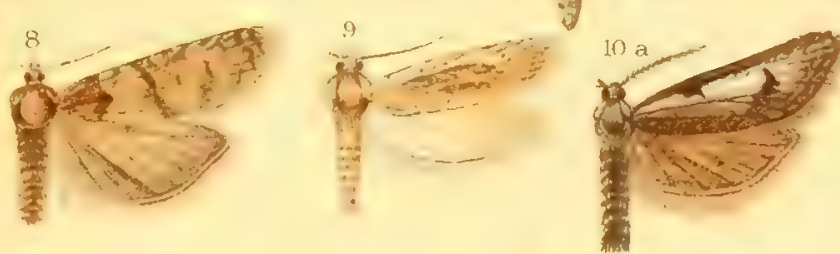

सing

(13)

82

4 is

Yin

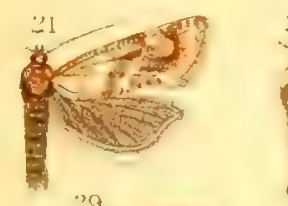

tis

2

34

$6-1$
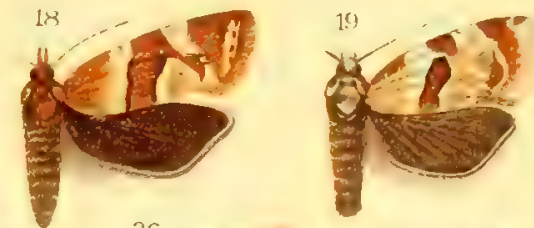

$\sqrt{3}$

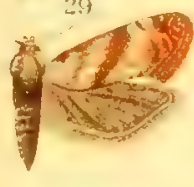

1

$\mathrm{C}^{26}$
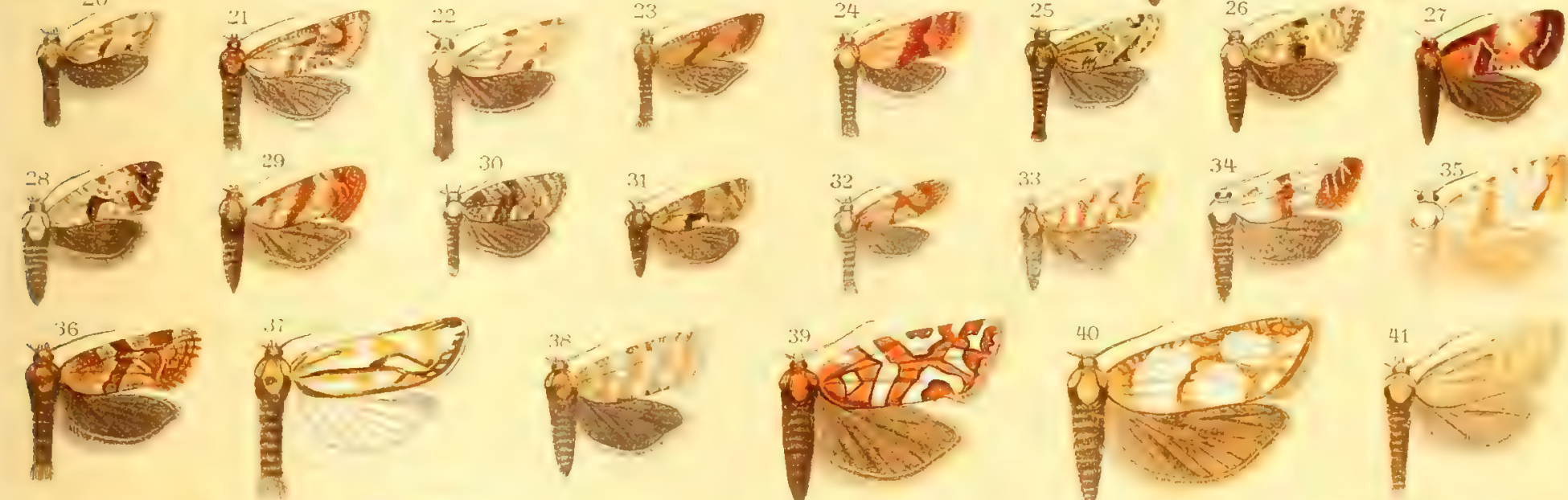

110
62

सोत्र

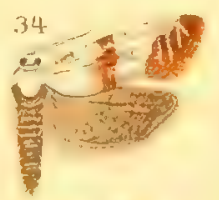

$y^{3.5}=7$

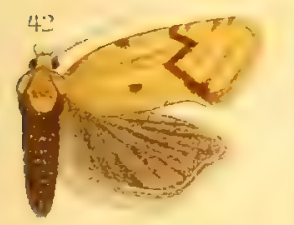

$$
\sum^{43}+23
$$

jof
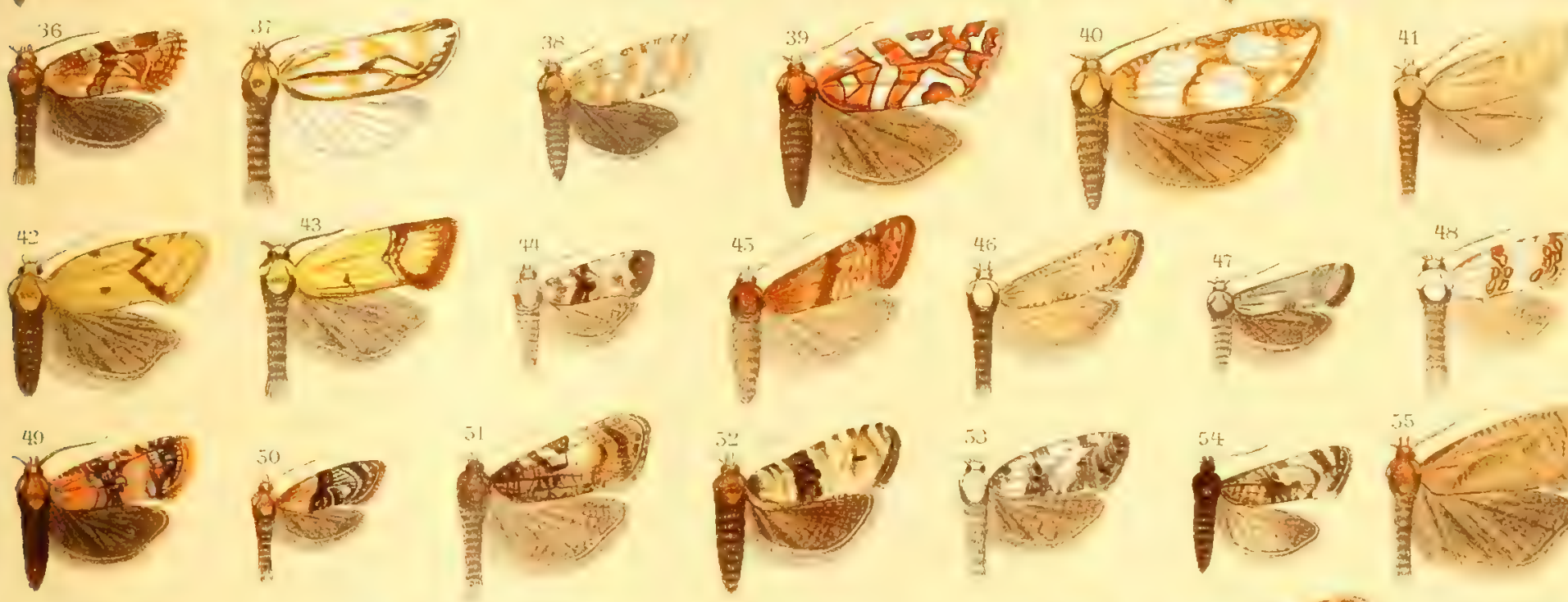

(sit
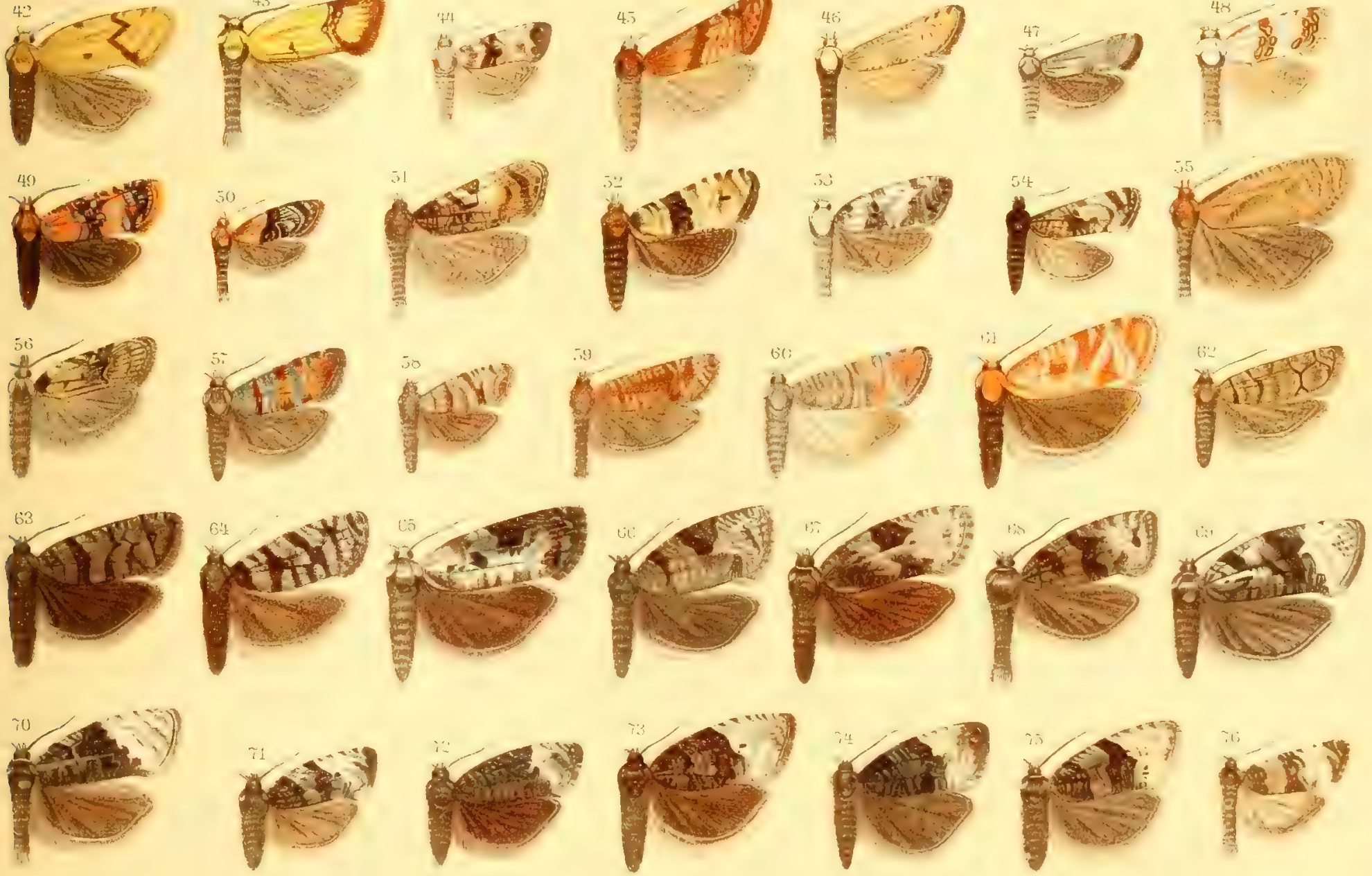




\section{TAFEL 85.}

\begin{tabular}{|c|c|c|c|c|c|c|c|}
\hline Nr. & & Name & Kat. II & $\begin{array}{l}\text { Seite d. } \\
\text { II. Bds }\end{array}$ & $\mathrm{Nr} . \quad$ Name & Kat. II & $\begin{array}{l}\text { Seite d. } \\
\text { II. Bds. }\end{array}$ \\
\hline 1. & Argyróploce & e lapideána HS. & 1891 & 2651. & 43. Rhopóbota naevána Hb. & 2281 & 2731. \\
\hline 2. & $n$ & oblongána Hw. & 1877 & 2651. & 44. Acróclita consequána HS. & 1966 & 2731. \\
\hline 3. & $"$ & lediána L. & 1880 & $265 \mathrm{r}$. & 45. Crocidoséma plebeiána Z. $\sigma^{7}$ & 1968 & 2731. \\
\hline 4. & $"$ & sehreberiána L. & 1856 & $265 \mathrm{r}$. & 46. Baetra laneөolána $\mathrm{Hb}$. & 2017 & 2731. \\
\hline 5. & " & nigrieostána Hw. & 1887 & 2661. & furfurána Hw. & 2020 & $273 \mathrm{r}$. \\
\hline 6. & $"$ & penthinána Gn. 우 & 1892 & 2661. & 48. Epibáctra sareptána HS. & 2021 & $273 \mathrm{r}$. \\
\hline 7. & " & siderána Tr. & 1904 & $266 \mathrm{r}$ & 49. Rhyaeiónia hastána $\mathrm{Hb}$. & 2016 & $273 / 74$ \\
\hline 8. & $"$ & mieána Hb. ㅇ & 1916 & $266 \mathrm{r}$ & 50. Pelatéa klugiána Frr & 2014 & 2741. \\
\hline 9. & $"$ & turfosána HS. & 1893 & $266 r$ & festivána $\mathrm{Hb}$. & 2015 & 2741. \\
\hline 10. & $"$ & sehulziána F. 우 & 1912 & $266 \mathrm{r}$ & 52. Epinótia profundána $\mathrm{F}$. & 1886 & $274 r$. \\
\hline 11. & $n$ & palustrána $\mathrm{Z} \cdot \mathrm{O}^{7}$ & 1910 & $266 \mathrm{r}$. & corticána $\mathrm{Hb}$. & 1978 & $274 \mathrm{r}$. \\
\hline 12. & $"$ & bipunetána F. & 1933 & 2671. & ratzeburgiána Rtzb. & 1983 & $274 / 75$ \\
\hline 13. & $"$ & hereyniána Tr. & 1941 & 2671. & quadrána Hb. & 1997 & $275 \mathrm{l}$ \\
\hline 14. & $"$ & urtieána Hb. & 1921 & 267 r. & nanána Tr. & 1984 & 2751. \\
\hline 15. & $"$ & umbrosána Frr & 1919 & $267 \mathrm{r}$ & pauperculána Stgr & 1976 & 2751. \\
\hline 16. & $"$ & Iaeunána Dup. & 1922 & $267 / 68$ & delitána F.R. & 1970 & 2751. \\
\hline 17. & $n$ & rivulána Se. & 1918 & 2681. & diniána Gn. & 1977 & 2751. \\
\hline 18. & $"$ & Purestrána Dup. & 1926 & 2681. & oppressána Tr. & 1975 & 2751. \\
\hline 19. & $"$ & branderiána $\mathrm{L}$. & 1902 & 2681. & simplána F.R. & 1965 & $275 \mathrm{r}$. \\
\hline 20. & " & antiquána $\mathrm{Hb}$. & 1945 & 2681. & rubiginosána HS. & 2000 & $275 \mathrm{r}$. \\
\hline 21. & $"$ & ericetána Westw. & 1944 & 2681. & vaceiniána $Z$. & 1987 & $275 \mathrm{r}$. \\
\hline 22. & " & striána Sehiff. & 1901 & 2681. & eruciána L. & 2003 & $275 \mathrm{r}$. \\
\hline 23. & $"$ & pufána Se. & 1899 & 268 r. & 65. (Pamplúsia) mereuriána $\mathrm{Hb}$. & 2001 & $275 / 76$ \\
\hline 24. & $"$ & arbutélla $\mathrm{Z}$. & 1897 & $268 \mathrm{r}$ & pauperána Dup. & 1971 & 2761. \\
\hline & Olethreútes & arcuélla $\mathrm{Cl}$. & 1896 & $268 \mathrm{r}$ & 67. (Gypsonóma) aceriána Dup. & 2008 & 2761. \\
\hline 26. & $"$ & boisduvaliána Dup. & 1917 & $268 \mathrm{r}$ & " inearnána Hw. & 2010 & 2761. \\
\hline 27. & $"$ & metallicána $\mathrm{Hb}$. & 1905 & 2691. & 69. (Cýdia) neglectána Dup. & 2011 & 2761. \\
\hline 28. & $"$ & stibiána Gn. & 1907 & 269 ]. & nigromaculána Hw. & 1972 & 2761 . \\
\hline 29. & Cymolómia $h$ & hartigiána Rtzb. & 1964 & 2691. & ramélla L. & 1974 & $276 \mathrm{r}$. \\
\hline 30. & $\because \quad 1$ & latifasciána $\mathrm{Hw}$. & 1965 & $269 \mathrm{l}$. & ustomaeulána Curt. & 1985 & $275 \mathrm{r}$. \\
\hline 31. & Áneylis acha & atána $\mathrm{F}$. & 1943 & $269 \mathrm{r}$. & nitidulána $\mathrm{Z}$. & 1989 & $276 \mathrm{r}$ \\
\hline 32. & lunde & dána $F$. & 2264 & $269 / 70$ & fractifasciána Hw. & 1992 & $276 / 77$ \\
\hline 33. & sieul & llána $\mathrm{Hb}$. & 2267 & $270 \mathrm{l}$ & trimaeulána Dup. & 2005 & 2771. \\
\hline 34. & mitte & terbacheriána Schiff. & 2277 & $270 \mathrm{r}$. & minutána $\mathrm{Hb}$. & 2007 & 2771. \\
\hline 35. & lactá & ána F. (pro laetána)*) & 2280 & $270 \mathrm{r}$ & obtusána Hw. & 2004 & 2771. \\
\hline 36. & selen & nána Gn. & 2269 & $270 / 71$ & 78. Semásia pupillána $\mathrm{Cl}$. & 2042 & $277 \mathrm{l}$. \\
\hline 37. & uneá & ána Hb. & 2272 & 2711. & lepneána Tr. & 2037 & $277 \mathrm{r}$. \\
\hline 38. & biapc & ceuána Stph. & 2273 & 2711 & aspidiseána Hb. & 2049 & 277 r. \\
\hline 39. & g) dimi & inutána Hw. & 2276 & 2711. & aemulána Schläg. & 2031 & $277 \mathrm{r}$. \\
\hline 40. I & Polyehrósis & euphorbiána Frr & 1947 & $211 \mathrm{r}$ & tripoliána Barr. & 2032 & $278 \mathrm{r}$ \\
\hline 41. & $n \quad 1$ & botrána Sehiff. & 1949 & $271 \mathrm{r}$ & ineána $\mathrm{Z}$. & 2046 & 2781. \\
\hline 42. & " & artemisiána $\mathrm{Z}$. & 1951 & $271 \mathrm{r}$ & & & \\
\hline
\end{tabular}

*) Die Raupe siehe Nachtr.-Taf. X, 3\%.

Diese Tíere sind in doppelter linearer Vergrößerung dargestellt. 


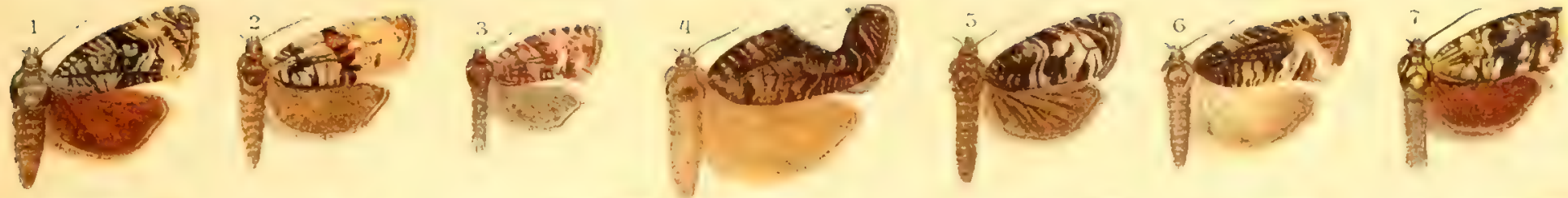

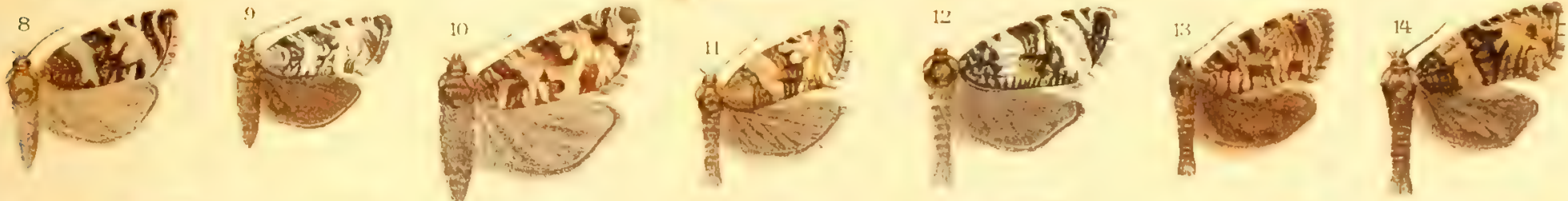
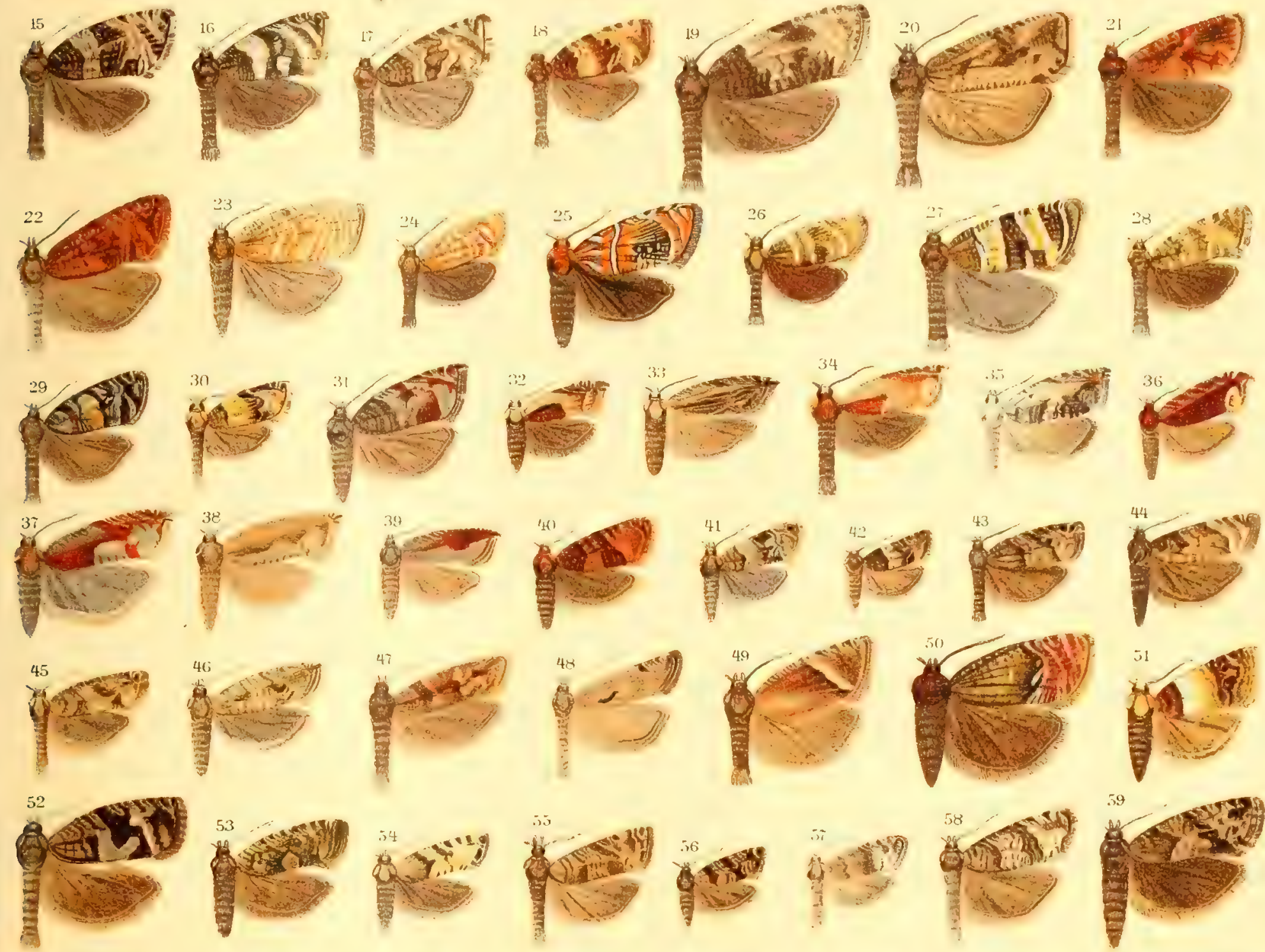

(1)
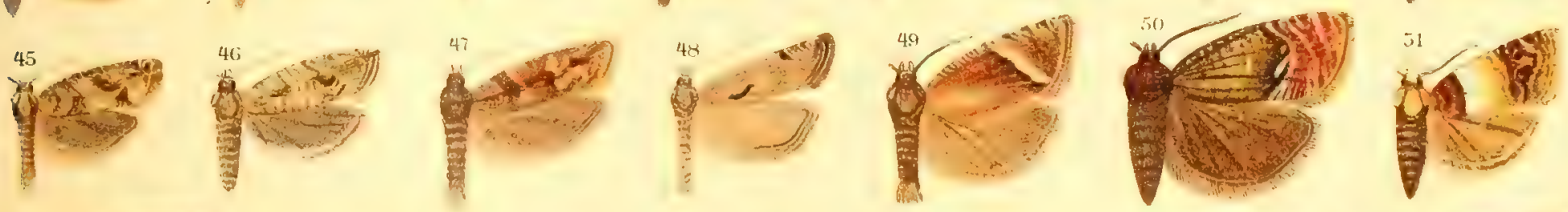

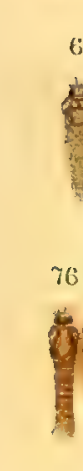

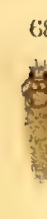

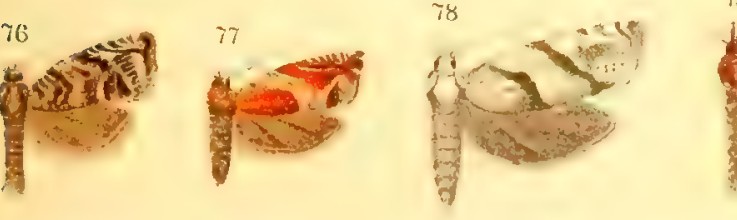

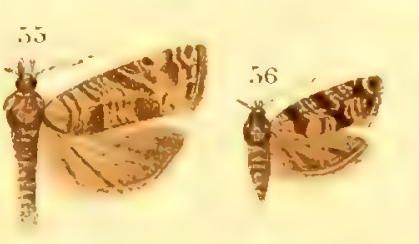
an

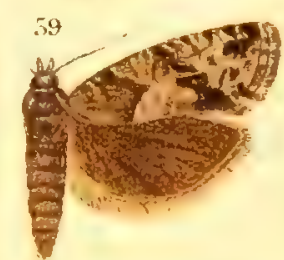
1 801 in 6in क्य $y=4$ gin

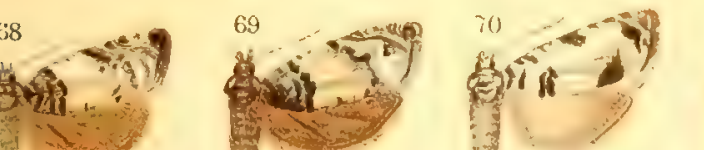
and

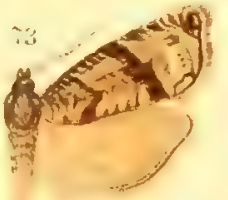
(4)

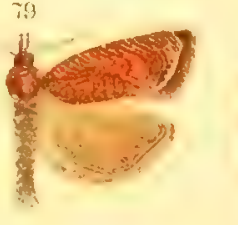
30

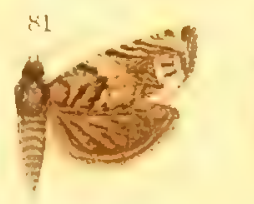
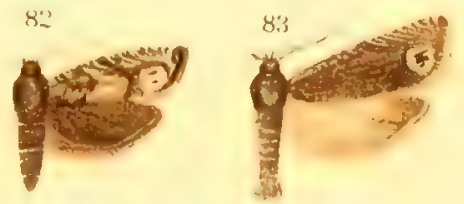




\section{TAFEL 86.}

\begin{tabular}{|c|c|c|c|c|c|c|c|c|}
\hline Nir. & Name & Kat. II & $\begin{array}{l}\text { Seite d. } \\
\text { II. Bds. }\end{array}$ & Nr. & & Name & Kat. II & $\begin{array}{l}\text { Scife d. } \\
\text { II. Bds. }\end{array}$ \\
\hline & Semásia candidulána Nolck. & 2080 & 2781. & 47. & Hemimé & gne sequána Hb. & 2282 & $286 \mathrm{r}$ \\
\hline 2. & messingiána F. R. & 2034 & $278 \mathrm{r}$ & 48. & $"$ & petiverélla L. & 2284 & $286 / 87$ \\
\hline 3. & hypericána Hb. & 2022 & $278 / 79$ & 49. & $"$ & agilána Tngstr. & 2289 & 2871. \\
\hline & Thiódia citrána $\mathrm{Hb}$. & 2035 & 2791. & 50. & $"$ & plumbagána Tr. & 2295 & $287 \mathrm{r}$. \\
\hline & Asthénia pygmaeána Hb. & 2012 & 2791. & 51. & $"$ & simpliciána Hw. & 2288 & 2881. \\
\hline & Tmetócera ocellána $\mathrm{F}$. & 2255 & $279 \mathrm{r}$ & 52. 1 & Lipópty & cha incursána HS. & 2310 & $288 \mathrm{r}$. \\
\hline & Notocélia uddmanniána L. & 2055 & $279 \mathrm{r}$ & 53. & $"$ & plumbána Se. *) & 2309 & $288 \mathrm{r}$. \\
\hline 8. & suffusána $Z$. & 2060 & $279 \mathrm{r}$ & 54. & Carpocá & ápsa pomonélla L. & 2257 & 2891. \\
\hline 9. & rosaecolána Dbld. & 2061 & $279 / 80$ & 55. & $"$ & splendána $\mathrm{Hb}$. & 2959 & $289 \mathrm{r}$. \\
\hline 10. & roborána Tr. & 2062 & 2801. & 56. & $"$ & amplána $\mathrm{Hb}$. & 2260 & $289 \mathrm{r}$. \\
\hline 11. & incarnatána $\mathrm{Hb}$. & 2063 & 2801. & 57.1 & Laspeyr & césia wooberiána Schiff.*) & 2157 & 2901. \\
\hline 12. & tetragonána Steph. & 2065 & 2801. & 58. & $"$ & albersána $\mathrm{Hb}$. & 2155 & 2901. \\
\hline 13. & Sphaeroéca obscurána Steph. & 2013 & 2801. & 59. & $"$ & funebrána Tr. & 58 แ. 2159 & 2901. \\
\hline 14. & Pygólopha lugubrána Tr. & 2066 & 2801. & 60. & $"$ & nigrieána Steph. & 2160 & 2901. \\
\hline & Epibléma grandaevána $\mathrm{Z} \cdot \sigma^{\top}$ & 2067 & $280 \mathrm{r}$ & 61. & $"$ & zebeána Rtzb. & 2163 & $290 \mathrm{r}$ \\
\hline 16. & hübneriána $\mathrm{Z}$. & 2073 & $280 \mathrm{r}$ & 62. & $"$ & servillána Dup. & 2173 & $290 \mathrm{r}$ \\
\hline 17. & infidána $\mathrm{Hb}$. & 2074 & $280 \mathrm{r}$. & 63. & $"$ & suceedána Froel. & 2171 & $290 / 91$ \\
\hline 18. & lacteána Tr. & 2081 & $281 \mathrm{l}$. & 64. & $"$ & eaecána Schläg. & 2169 & 2911. \\
\hline 19. & scopoliána Hw. & 2085 & $281 \mathrm{r}$. & 65. & $"$ & pactolána $\mathrm{Z} .^{*}$ ) & 2190 & 2911. \\
\hline 20. & fulvána Steph. & 2084 & $281 \mathrm{r}$ & 66. & $"$ & pinetána Schläg. & 2246 & $291 \mathrm{r}$. \\
\hline 21. & eaecimaeulána Hb. & 2093 & 2821. & 67. & $"$ & strobilélla L. & 2177 & $291 \mathrm{r}$. \\
\hline 22. & modieána ${ }^{-} \mathrm{Z}$. & 2097 & $282 \mathrm{r}$ & 68. & $"$ & compositélla F. & 2194 & $291 \mathrm{r}$. \\
\hline 23. & pflugiána Hw. & 2143 & $282 r$ & 69. & $"$ & leplastriána Curt. & 2196 & $291 \mathrm{r}$ \\
\hline 24. & trigeminána Steph. & 2103 & $282 \mathrm{r}:$ & 70 & $"$ & corollána Hb. & 2181 & $291 / 92$ \\
\hline 25. & brunniehiána Froel. & 2150 & 2831. & 71. & $"$ & cosmophorána Tr. & 2184 & 2921. \\
\hline 26. & foenélla L.: & 2154 & $283 \mathrm{r}$ & 72. & ". & seopariána HS. & 2182 & 2921. \\
\hline 27. & similána $\mathrm{Hb}$. & 2135 & $283 \mathrm{r}$ & 73 & $"$ & coniferána Rtzb. & 2187 & $292 \mathrm{r}$. \\
\hline 28. & erenána Hb. & 2133 & $283 \mathrm{r}$ & 74. & $"$ & duplieána Zett. & 2204 & $292 \mathrm{r}$. \\
\hline 29. & fusculána Z. & 2106 & $283 \mathrm{r}$ & 75 & $"$ & eoronillána $\mathrm{Z}$. & 2217 & 2931. \\
\hline 30. & kochiána HS. & 2107 & 2841. & 76. & $"$ & perlepidána $\mathrm{Hw} \cdot \sigma^{\top}$ & 2207 & 2931. \\
\hline 31. & graphána Tr. & 2105 & 2841. & 77. & $"$ & orobána Tr. & 2216 & $293 \mathrm{l}$ \\
\hline 32. & ustulána $\mathrm{Hb}$. & 2120 & 2841. & 78. & $"$ & ianthinána Dup. & 2224 & 2941. \\
\hline 33. & tripunctána F. & 2138 & $284 \mathrm{l}$. & 79. & $"$ & aurána $\mathrm{F}$. & 2222 & $29 \pm 1$ \\
\hline 34. & fuchsiána Rössl. & 2140 & $284 \mathrm{r}$. & 80. & Crobyló & phora inquinatána $\mathrm{Hb}$. & 2212 & $294 \mathrm{r}$. \\
\hline 35. & couleruána Dup. & 2116 & $284 \pi$ & 81. & Pamene & fimbriána $\mathrm{Hw}$. & 2225 & $294 \mathrm{r}$ \\
\hline 36. & demarniána F.R. & 2115 & $284 \mathrm{r}$. & 82. & $"$ & argyrána $\mathrm{Hb}$. ㅇ & 2226 & $294 / 95$ \\
\hline 37. & immundána F. R. & 2132 & $284 / 85$ & 83. & $"$ & gallicolána Z.*) & 2231 & 2951. \\
\hline 38. & nisélla $\mathrm{Cl}$. & 2119 & 2851. & 84. & $"$ & spiniána Dup. & 2240 & 2951. \\
\hline 39. & penkleriána F. R. *) & 2121 & 2851. & 85. & Carpocá & ápsa iuliána Curt. & 2237 & $289 \mathrm{r}$ \\
\hline 40. & tedélla $\mathrm{Cl}$. & 2111 & $285 \mathrm{r}$. & 86. & Pamene & populána $F$ & 2241 & $295 \mathrm{r}$. \\
\hline 41. & turbidána Tr. & 2153 & 2861. & 87. & $"$ & regiána $\mathrm{Z} .{ }^{*}$ ) & 2244 & $295 \mathrm{r}$. \\
\hline 42. & bilunána Hw. & 2128 & 2861. & 88. & $"$ & germarána Hb. (pro & & \\
\hline 43. & ophthalmicána $\mathrm{Hb} .^{*}$ ) & 2123 & 2861. & & & germmána) & 2252 & $295 / 96$ \\
\hline 44. & " solandr. v. trapezána F. & $2125 \mathrm{~b}$ & 2861. & 89. & $"$ & nitidána $\mathrm{F}$. & 2250 & 2961. \\
\hline 45. & solandriána $\mathrm{L}$. & 2125 & 2861. & 90. & $"$ & oehsenheimeriána Z. & 2247 & $296 \mathrm{r}$. \\
\hline 46. & semifuscána Steph. & 2126 & 2861. & 91. & $"$ & phediélla $\mathrm{Cl}$. & 2254 & $296 \mathrm{r}$. \\
\hline
\end{tabular}

*) Die Raupe von Fig. 26 ist Nachtr.-Taf X, 29; die von Fig. 39 ib. 27; die von Fig. 43 ib. 28; die von Fig. 53 ib. 36; die von Fig. 57 ib. 30; die von Fig. 65 ib. 31; die von Fig. 83 ib. 32 und die von Fig. 87 ib. 33 abgebildet.

Die Tiere sind sämtlich in doppelter linearer Vergrößerung dargestellt. 


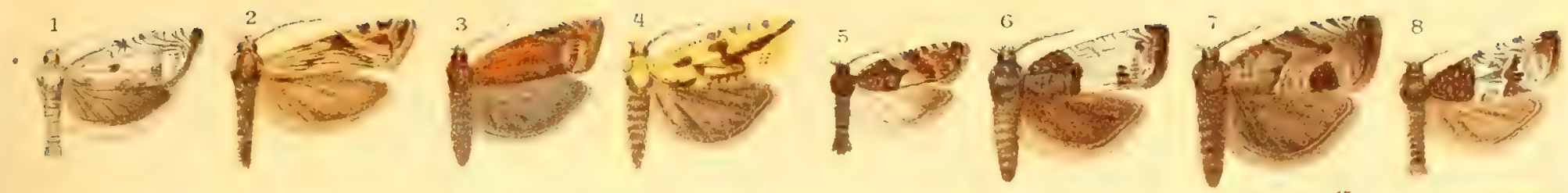

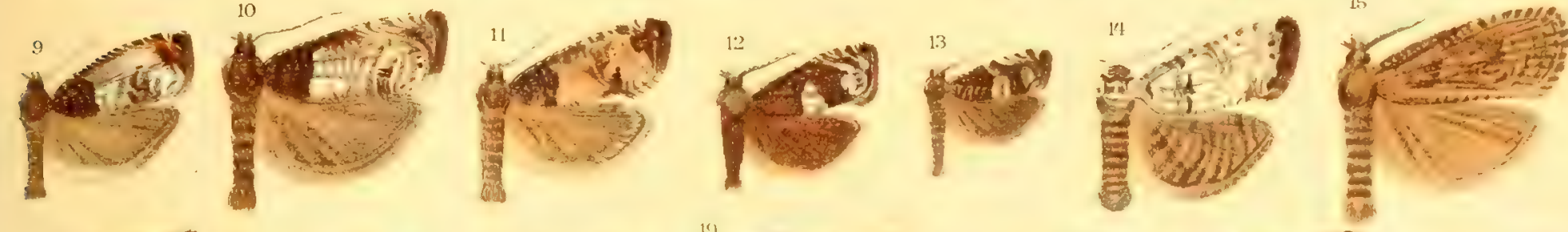

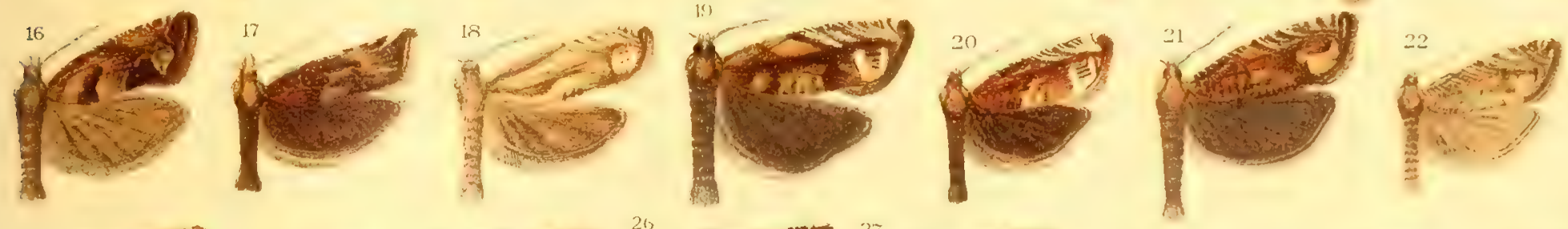

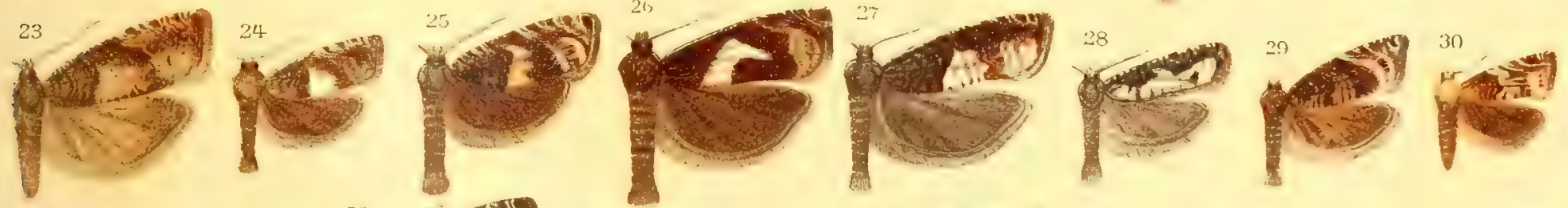

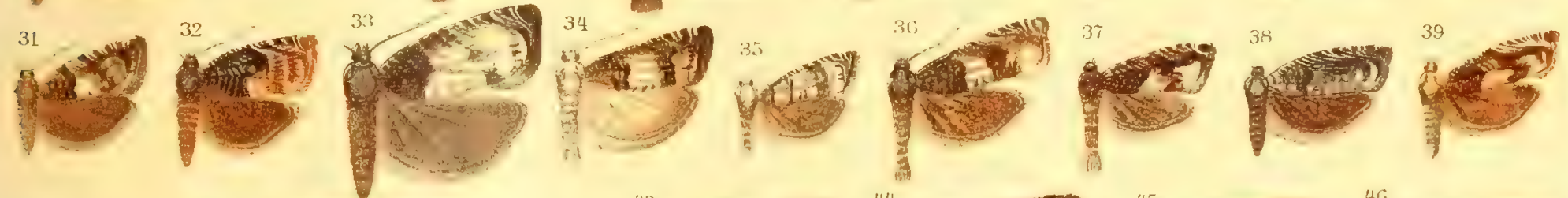
in 10 N 10

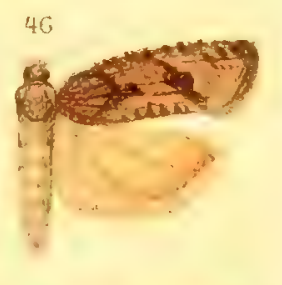

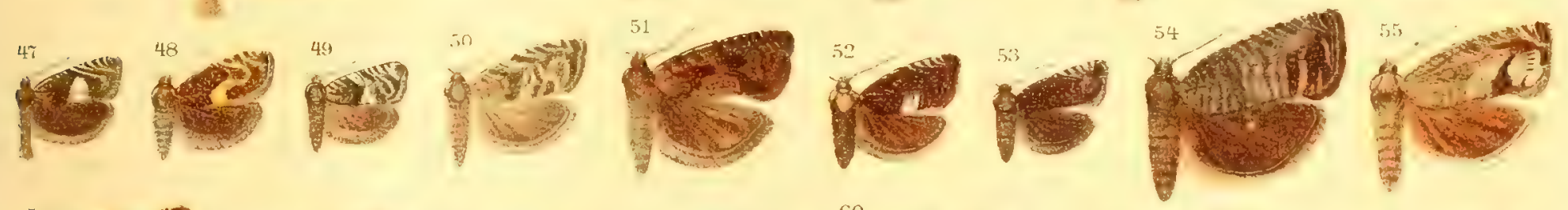

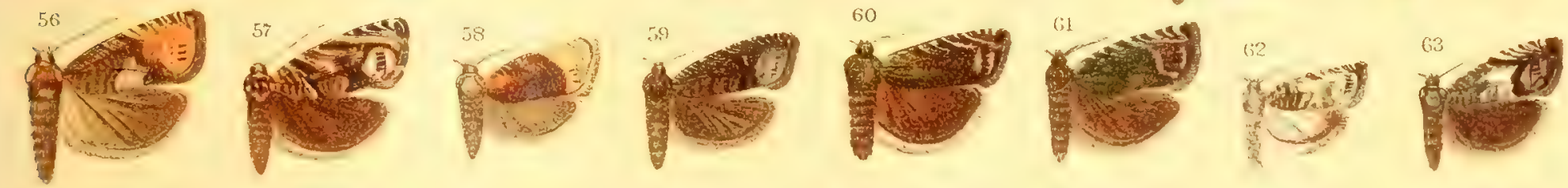
8.

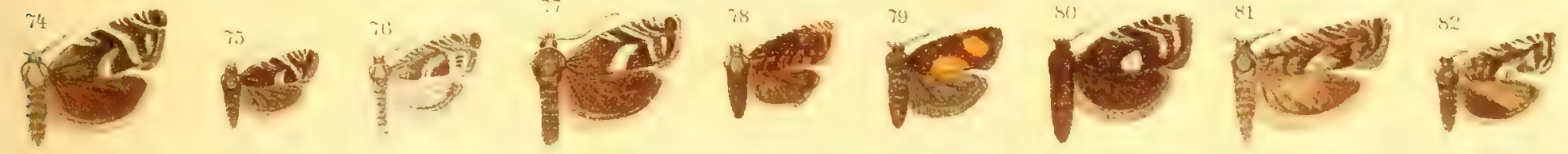

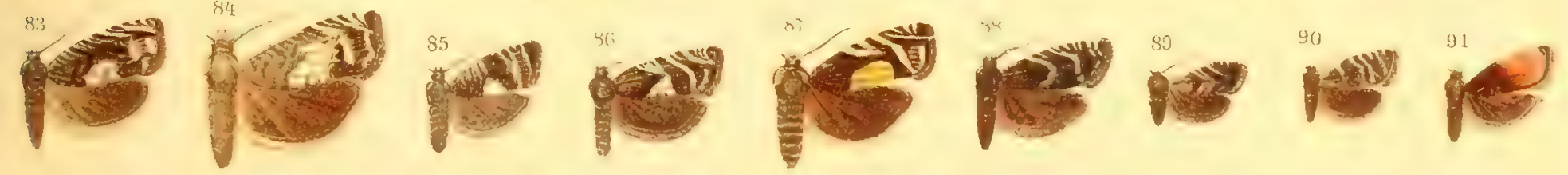






\section{TAFEL $8 \%$}

\begin{tabular}{|c|c|c|c|c|c|c|}
\hline Name & Kat. II & $\begin{array}{l}\text { Seite d. } \\
\text { II. Bds. }\end{array}$ & Nr. & Name & Kat. II & $\begin{array}{l}\text { Seite d. } \\
\text { II. Bds. }\end{array}$ \\
\hline 1. Choreútis myllerána F. & 2313 & $297 \mathrm{r}$ & 40. & Cedéstis farinatélla Dup. & 2436 & 4491. \\
\hline 2. Simaéthis pariána $\mathrm{Cl}$. & 2315 & $297 \mathrm{r}$ & 41. & Oeneróstoma piniariélla $\mathrm{Z} .{ }^{*}$ ) & 2437 & $449 \mathrm{r}$. \\
\hline fabriciána L. & 2318 & $297 \mathrm{r}$. & 42. & Eidophásia messingiélla $\mathrm{F}$. & 2438 & 4521. \\
\hline 1. Millièréia dolosána HS. & 2319 & 2981. & 43. & Plutélla hufnagéli $\mathrm{Z}$. & 2443 & $451 \mathrm{r}$. \\
\hline 5. Glyphípteryx bergstraesserélla F. & 2323 & $298 r$ & & maculipénnis Curt. & 2447 & 4521. \\
\hline thrasonélla Se. & 2326 & $298 \mathrm{r}$ & 45. & inearnatélla Steud. & 2450 & 4521. \\
\hline forsterélla $\mathrm{F}$. & 2334 & $299 \mathrm{I}$ & 46. & Ceróstoma vitéllum L. & 2451 & 4501. \\
\hline 8. Tinágma perdicéllum $\mathrm{Z}$. & 2337 & $299 \mathrm{r}$ & 47. & sequéllum $\mathrm{Cl}$. & 2453 & 4501. \\
\hline 9. Douglásia transversélla $\mathrm{Z}$. & 2339 & $299 \mathrm{r}$ & 48. & radiatéllum Don. & 2466 & $450 \mathrm{r}$ \\
\hline oenerostomélla Stt. & 2341 & $299 \mathrm{r}$ & 49. & parentheséllum L. & 2467 & $450 \mathrm{r}$. \\
\hline 11. Calántica albélla $\mathrm{Z}$. & 2344 & 4421. & 50. & sylvéllum L. & 2471 & $450 \mathrm{r}$. \\
\hline 12. (Procal.) Phreáleia eximiélla Rbl & 2346 & 4421. & 51. & persicéllum F. & 2474 & 4511. \\
\hline 13. Distágmos lederéri HS. & 2348 & 4431. & 52. & asperéllum L. & 2375 & 4511. \\
\hline 14. Woekéia asperipunetélla Brnd & 2349 & 4431 & 53. & horpidéllum Tr. & 2478 & 4511. \\
\hline 15. Seythrópia erataegélla L. & 2350 & $443 \mathrm{r}$ & 54. & xylostéllum L.*) & 2482 & 4511. \\
\hline 16. Herríchia excelsélla Stgr & 2352 & $443 \mathrm{r}$. & 55. & Theristis mueronélla Se. & 2484 & $451 \mathrm{r}$. \\
\hline 17. Hyponomeúta egregiéllus Dup. & 2353 & $444 \mathrm{l}$. & 56. & Reúttia subocéllea Stph. & 2875 & $380 \mathrm{r}$. \\
\hline stannéllus Thnbg & 2355 & 4441. & 57. & Metznéria paueipunetélla $\mathrm{Z}$. & 2487 & $371 / 72$ \\
\hline plumbéllus Sehiff. & 2357 & 4441 & 58. & neuropterélla $\mathrm{Z}$. & 2503 & 3721. \\
\hline malinéllus $\mathrm{Z}$. & 2363 & $444 \mathrm{r}$ & 59. & Chelária hübnerélla Don. & 2506 & 357 l. \\
\hline evonyméllus L. & 2366 & $444 \mathrm{r}$ & 60. & Psoricóptera gibbosélla Z. & 2507 & $3599 \mathrm{l}$. \\
\hline 22. Swammerdámia combinélla $\mathrm{Hb}$. & 2367 & $444 / 5$ & 61. & Platyédra vilélla $Z$. & 2509 & $359 / 60$ \\
\hline heroldélla Tr.*) & 2369 & 4451. & 62. & Bryótropha terrélla $\mathrm{Hb}$. & 2510 & 3701. \\
\hline 24. Prays curtiséllus Don. & 2380 & $442 \mathrm{r}$ & 63. & seneetélla $\mathrm{Z}$. & 2520 & $370 \mathrm{r}$. \\
\hline 25. Parádoxus osyridéllus Stt. & 2383 & $442 / 3$ & 64. & basaltinélla $\mathrm{Z}$. & 2535 & 3711. \\
\hline 26. Hofmánnia saxífragae Stt. & 2390 & $446 \mathrm{r}$ & $6 \check{0}$ & Geléchia pinguinélla Tr. & 2538 & 3601. \\
\hline 27. Zelléria phillyrélla Mill. & 2386 & 4461. & 66. & tragicélla Heyd. & 2548 & 3611. \\
\hline 28. Atemélia torquatélla $\mathrm{Z}$. & 2384 & 4421 & 67. & distinetélla $\mathrm{Z}$. & 2559 & $361 \%$ \\
\hline 29. Argyrésthia coniugélla $\mathrm{Z}$. & 2393 & $447 \mathrm{l}$ & 68. & flavicomélla $\mathrm{Z}$. & 2572 & 3621. \\
\hline spiniélla Z. & 2402 & $447 \cdot 1$ & 69. & ericetélla $\mathrm{Hb}$. & 2580 & $362 \mathrm{r}$. \\
\hline ephippiélla F. & 2404 & $447 x^{2}$ & 70. & mulinélla $\mathrm{Z}$. & 2586 & 3631. \\
\hline aurulentélla Stt. & 2412 & 4481 & 71. & interruptélla $\mathrm{Hb}$. & 2587 & $363 \mathrm{l}$. \\
\hline fundélla F. R. & 2415 & 4481 & 72. & galbanélla $\mathrm{Z}$. & 2594 & $363 \mathrm{r}$. \\
\hline cornélla F. & 2416 & 4481. & 73. & virgélla Thnbg & 2604 & $36+1$. \\
\hline goedartélla L. & 2420 & 448 l. & 74. & diffínis $\mathrm{Hw}$. & 2611 & 3641. \\
\hline andereggiélla Dup. & $24: 3$ & $448 r$ & 75. & sealélla se. & 2615 & $364 \mathrm{r}$. \\
\hline trifasciáta Stgr & 2424 & $448 \mathrm{r}$. & 76. & tessélla $\mathrm{Hb}$. & 2621 & $365 \mathrm{l}$. \\
\hline ceptélla Z. & 2429 & 4491 & 77. & eytisélla Tr. & 2624 & $365 \mathrm{l}$. \\
\hline ysselinélla Dup. & 2435 & 4491. & & & & \\
\hline
\end{tabular}

abgebildet.

*) Die Raupe von Fig. 23 ist Nachtr.-Taf. X, 37; die von Fig. 41 ebenda, Fig. 40; die von Fig. 54 ebenda, Fig. 42 Alle Arten sind in doppelter linearer Vergrößerung dargestellt. 


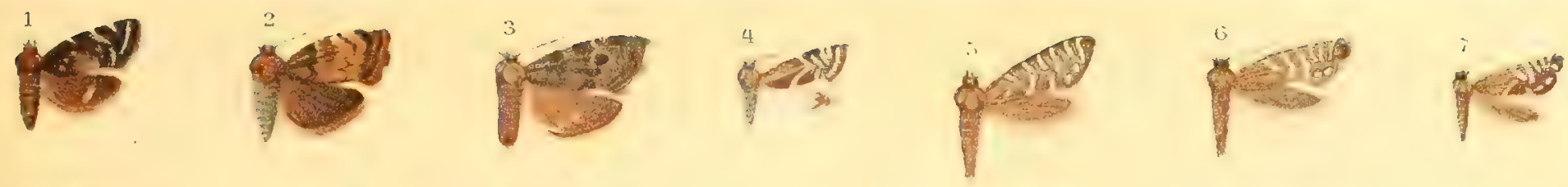
820

1504<smiles>C#CC=C</smiles>
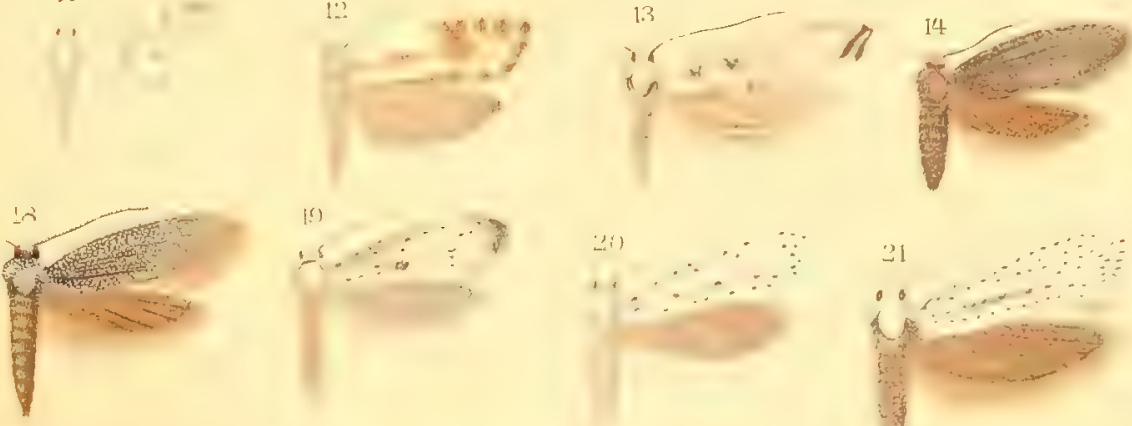

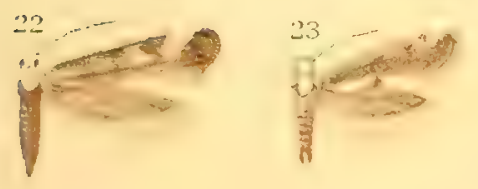
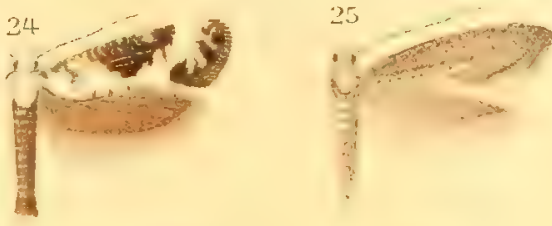

$$
\ddot{y} \geq
$$
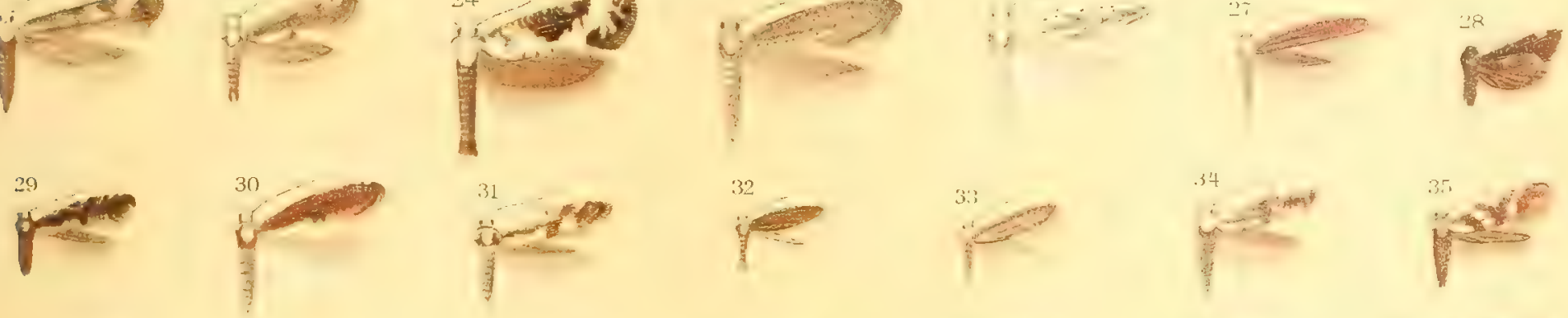

1

32

p?

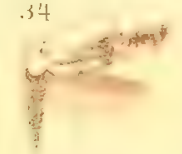

$x^{3 f}$

6

38

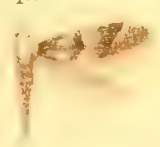

12

Pa
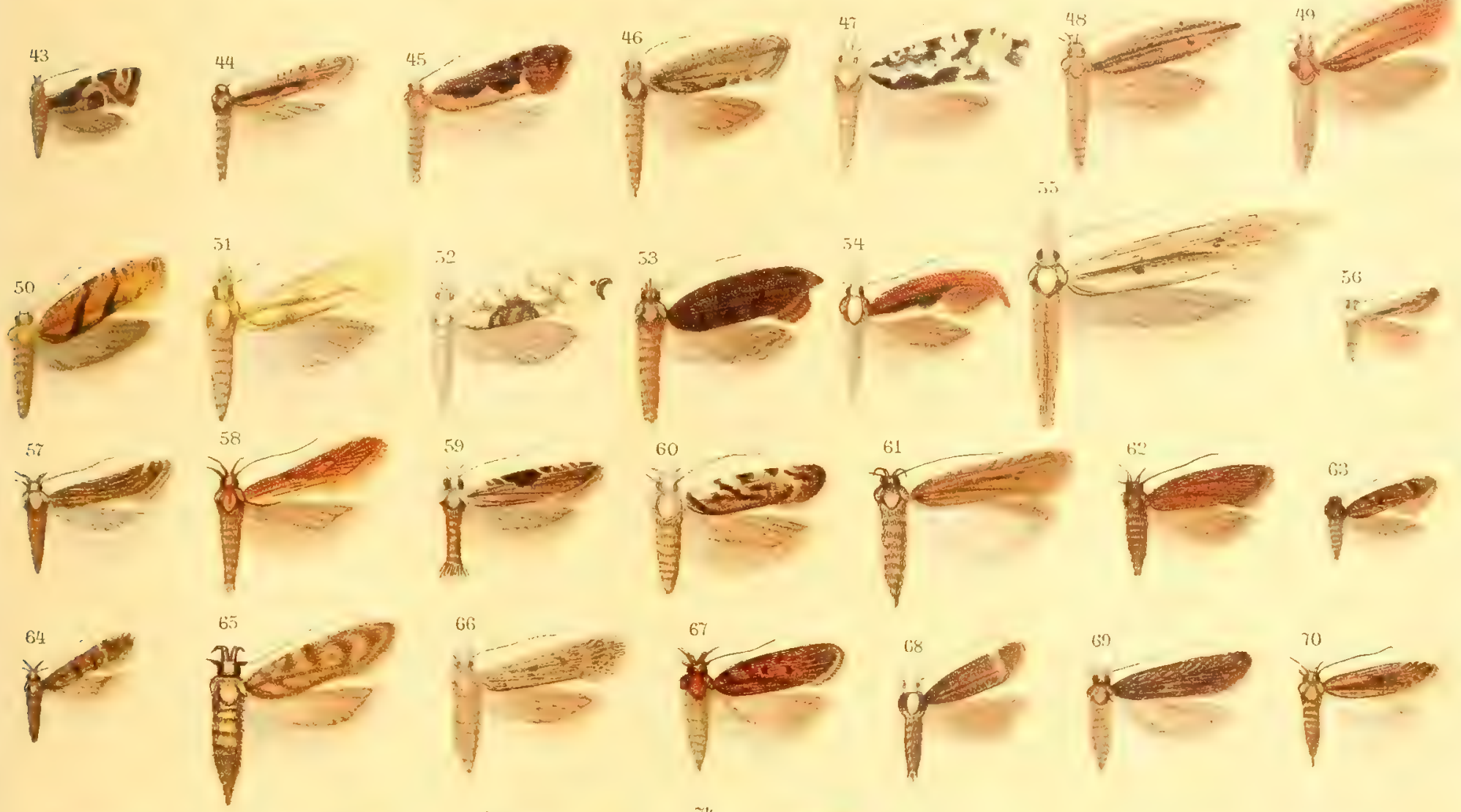

西
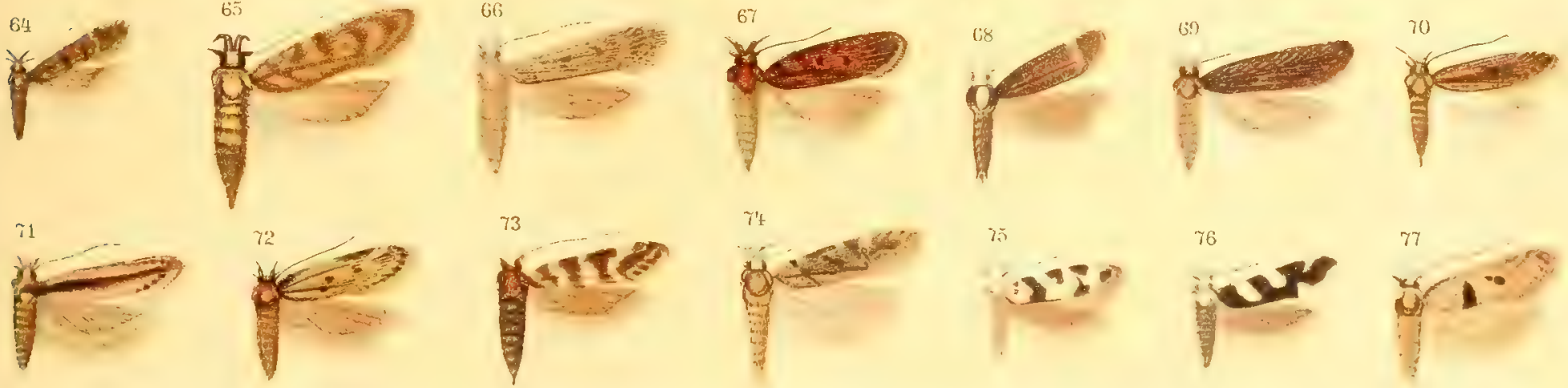




\section{TAFEL 88.}

\begin{tabular}{|c|c|c|}
\hline Name & Kat. Il & $\begin{array}{l}\text { Seite d. } \\
\text { II. Bds. }\end{array}$ \\
\hline Lita artemisiélla Tr. & 2639 & $365 / 66$ \\
\hline .. insulélla Hein. & 2668 & 3671. \\
\hline ." proclivélla Fuchs & 2640 & 3661. \\
\hline .. brahmiélla Heyd. & 2694 & 3681. \\
\hline " moritzélla $\mathrm{Hb}$. & 2695 & $368 \mathrm{r}$. \\
\hline "hübnéri Hw. & 2698 & $368 \mathrm{l}:$ \\
\hline " petrýi Hofm. & 2724 & $369 \mathrm{r}$. \\
\hline " tischeriélla $\mathrm{Z}$. & 2727 & $370 \mathrm{l}$ \\
\hline -Teleía seriptélla $\mathrm{Hb}^{*}$ ) & 2733 & 3581. \\
\hline , alburnélla Dup. & 2735 & 3581. \\
\hline " fugacélla $\mathrm{Z}$. & 2747 & $358 \mathrm{r}$. \\
\hline " proximélla $\mathrm{Hb}$. & 2752 & $358 \mathrm{r}$. \\
\hline Heríngia dodecélla L. & 2762 & $357 \mathrm{r}$. \\
\hline Alloclíta recisélla Stgr & 2769 & $357 \mathrm{l}$. \\
\hline Acómpsia einerélla $\mathrm{Cl}$. & 2771 & 3511. \\
\hline $\begin{array}{cc}\text { Tachyptília populélla } & \mathrm{Cl} . \\
& \text { populélla } \\
\mathrm{Cl}\end{array}$ & 2776 & 3551. \\
\hline subsequélla $\mathrm{Hb}$. & 2783 & $355 \mathrm{r}$. \\
\hline Acanthóphila alacélla Dup. & 2785 & 3561. \\
\hline Xystóphora pulveratélla HS. & 2787 & 3751. \\
\hline lueidélla Stph. & 2814 & $375 \mathrm{r}$. \\
\hline (Monoehróa) tenebrélla Hb. & 2818 & 3761. \\
\hline Anacámpsis coronillélla Tr. & 2829 & $373 \mathrm{r}$. \\
\hline (Sehützéia) anthyllidélla $\mathrm{Hb}$. & 2835 & 3741. \\
\hline taeniolélla $\mathrm{Z}$. & 2844 & 3741. \\
\hline Epithéetis mouffetélla Sehiff. & 2850 & 3561. \\
\hline nigricostélla Schiff. & 28 อ̃ & 3561. \\
\hline Aristotélia brizélla Tr. & 2861 & $377 \mathrm{r}$. \\
\hline decurtélla $\mathrm{Hb}$. & 2871 & 3781. \\
\hline Recurvária leueatélla $\mathrm{Cl}$. 우 & 2873 & $356 \mathrm{r}$. \\
\hline Ptocheuúsa inopélla $\mathrm{Z}$. & 2879 & $377 \mathrm{r}$. \\
\hline Stenoléchia álbiceps $\mathrm{Z}$. & 2886 & $357 \mathrm{r}$. \\
\hline Argyritis pietélla Z. & 2890 & $378 \mathrm{r}$. \\
\hline Chrysopóra eppelsheimi Stgr & 2895 & $376 \mathrm{r}$. \\
\hline hermannélla $\mathrm{F}$. & 2896 & 3771. \\
\hline Apódia bifractélla $\mathrm{Dgl}$. & 2898 & 3771. \\
\hline Sitotróga cerealélla 0livier & 2902 & 3731. \\
\hline Didactylóta kinkerélla Snell. & 2903 & 3791. \\
\hline
\end{tabular}

\begin{tabular}{|c|c|c|c|}
\hline $\mathrm{Nr}$. & Name & II & $\begin{array}{l}\text { Seite d. } \\
\text { II. Bds. }\end{array}$ \\
\hline 39. & Stomópteryx detersélla $\mathrm{Z}$. & 2906 & $376 \mathrm{r}$. \\
\hline 40. & Bráchmia puféseens Hw. & 2909 & 351 l. \\
\hline 41. & (Cladódes) demidiélla Sehiff. & 2917 & $351 \mathrm{r}$. \\
\hline 42. & Rhinósia denisélla $F$. & 2920 & $344 \mathrm{l}$. \\
\hline 43. & sordidélla $\mathrm{Hb}$. & 2923 & 344 l. \\
\hline 44. & Deuterogónia pudorína Wek. & 2908 & 3491. \\
\hline 45. & Eúteles kollarélla Costa & 2929 & $352 \mathrm{r}$. \\
\hline 46. & Paltodóra striatélla $\mathrm{Hb}$. & 2935 & $372 \mathrm{r}$. \\
\hline 47. & Mésophleps silacéllus $\mathrm{Hb}$. & 2944 & $371 \mathrm{r}$. \\
\hline 48. & Hypsólophus ustuléllus F. & 2951 & 354 \\
\hline 49. & limoséllus Sehläg. & 2953 & 354 \\
\hline 50. & Nothris marginélla $\mathrm{F}$. & 2960 & $353 \mathrm{r}$. \\
\hline 51. & lemniseélla $\mathrm{Z}$. & 2976 & 3541. \\
\hline 52. & Holeopógon bubuleéllus Stgr & 2979 & 3541. \\
\hline 53. & Holeóphora statíces Stgr & 2981 & 3541. \\
\hline 54. & Sophrónia semicostélla $\mathrm{Hb}$. & 2982 & 3521. \\
\hline 55. & Nothris verbascélla $\mathrm{Hb}$. & 2961 & $353 \mathrm{r}$. \\
\hline 56. & Sophrónia humerélla Sehiff. & 2988 & 352 \\
\hline 57. & Anársia spartiélla Sehrk $\sigma^{7}$ & 2996 & 3531. \\
\hline ๑8. & lineatélla Z. $\sigma^{\top}$ & 2999 & 3531. \\
\hline 59. & Megaeráspedus binotéllus F. R. & 3006 & 3791. \\
\hline 60. & Pterolónche inspérsa Stgr & 3014 & $380 \mathrm{l}$. \\
\hline 61. & Oecocécis guyonélla Gn., & 3016 & $380 \mathrm{r}$. \\
\hline 62. & Epidóla stigma Stgr & 3019 & $379 \mathrm{r}$. \\
\hline 63. & Symmóea signélla $\mathrm{Hb}$. & 3022 & $344 \mathrm{r}$. \\
\hline 64. & signatélla $\mathrm{HS}$. & 3030 & 3541. \\
\hline 65. & Oegocónia quadripúneta $\mathrm{Hw}$. & 3050 & 350 \\
\hline 66. & Endrósis laeteélla Sehiff. & 3051 & 349 \\
\hline 67. & Blastobásis phyeidélla $\mathrm{Z}$. & 3054 & 3501. \\
\hline 68. & Hypátima binotélla Thnbg & 3070 & 3501. \\
\hline $69 \mathrm{a}$ & Pleuróta rostrélla $\mathrm{Hb} . \sigma^{\top}, \mathrm{b} q$ & 3075 & 3411. \\
\hline 70. & sehlaegeriélla $\mathrm{Z}$. & 3099 & 341 \\
\hline 71. & bieostélla $\mathrm{Cl}$. & 3116 & 3421. \\
\hline 72. & Aplóta palpélla $\mathrm{Hw}$. & 3120 & 3421. \\
\hline 73. & Holoseólia forficélla $\mathrm{Hb}$. & 3121 & \\
\hline & Prótasis punctélla Costa & 3122 & 34 \\
\hline 75. & Topeútis barbélla $F$. & 3125 & 341 \\
\hline
\end{tabular}

Die Raupe von Fig. 9 ist Nachtr.-Taf. X, 44 abgebildet.

Alle Arten, außer Fig. 61, sind in doppelter linearer Vergrößerung dargestellt, Teile der Figuren $27,31,32$ und 35 noch stärker vergrößert. 


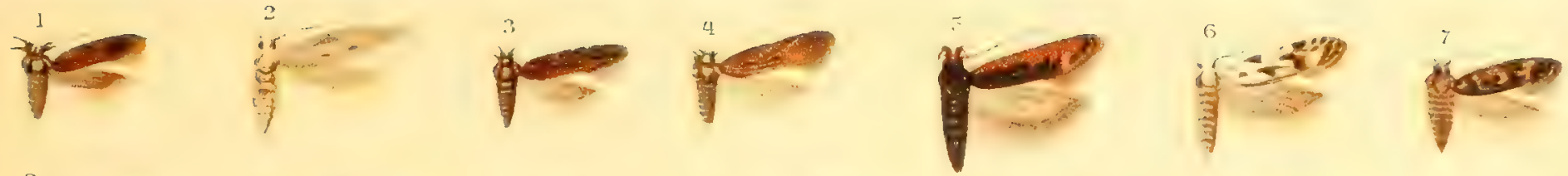

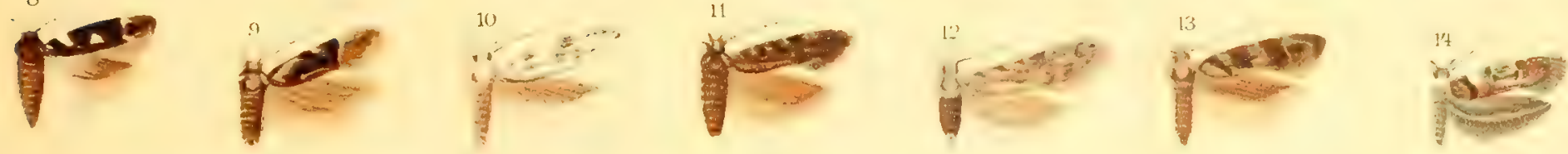

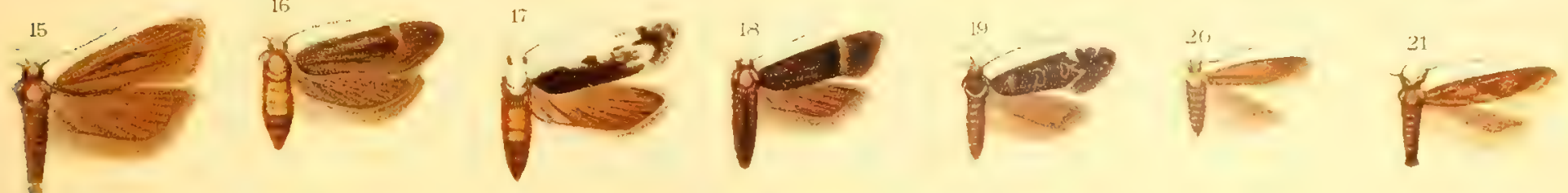

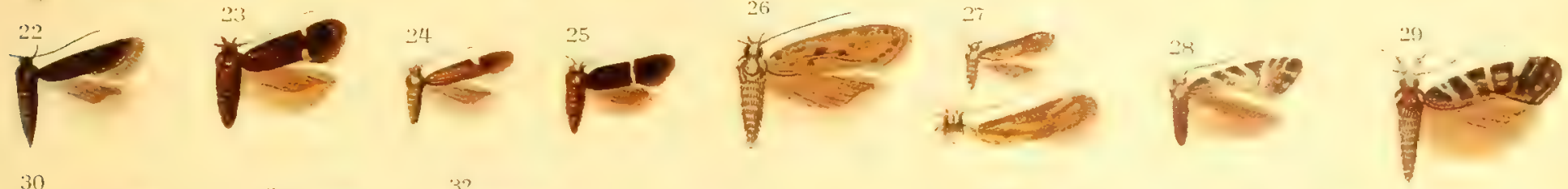

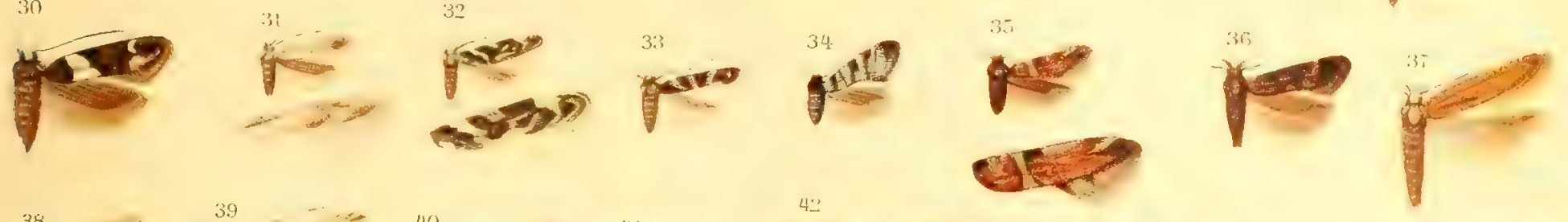
1 y

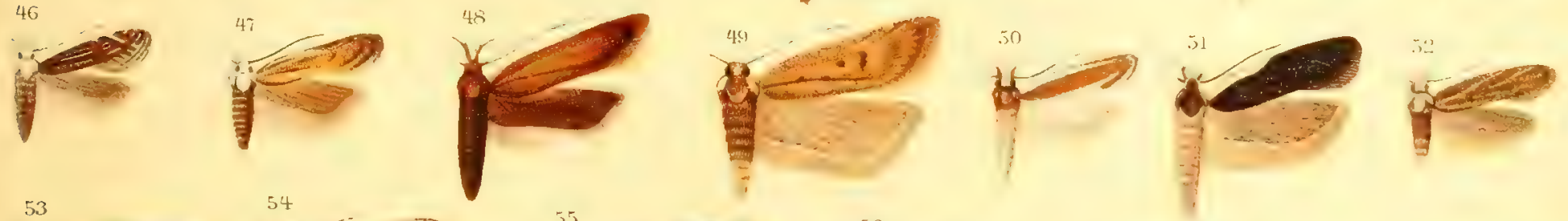

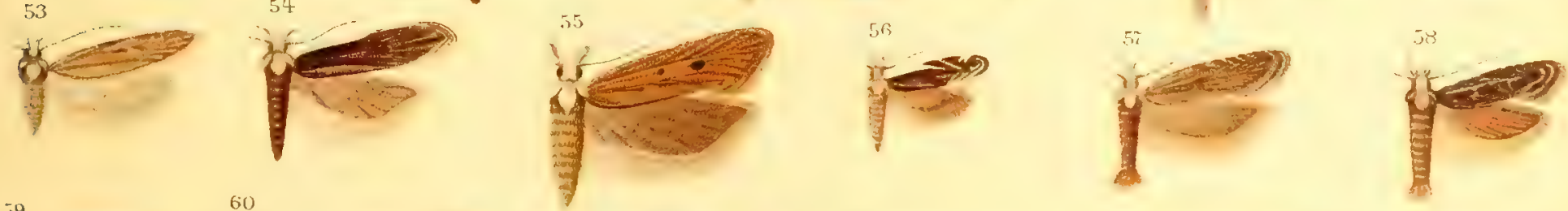

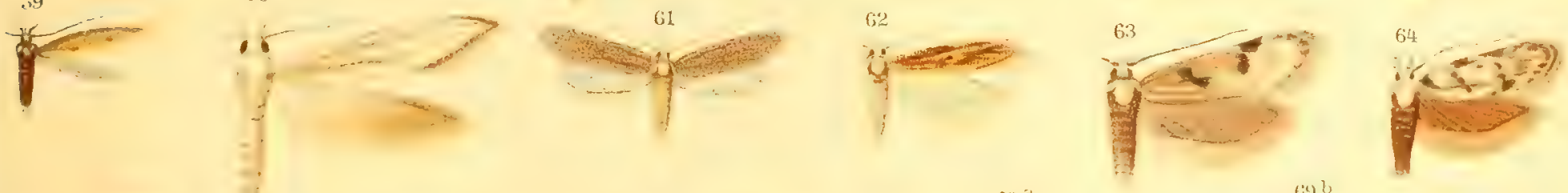
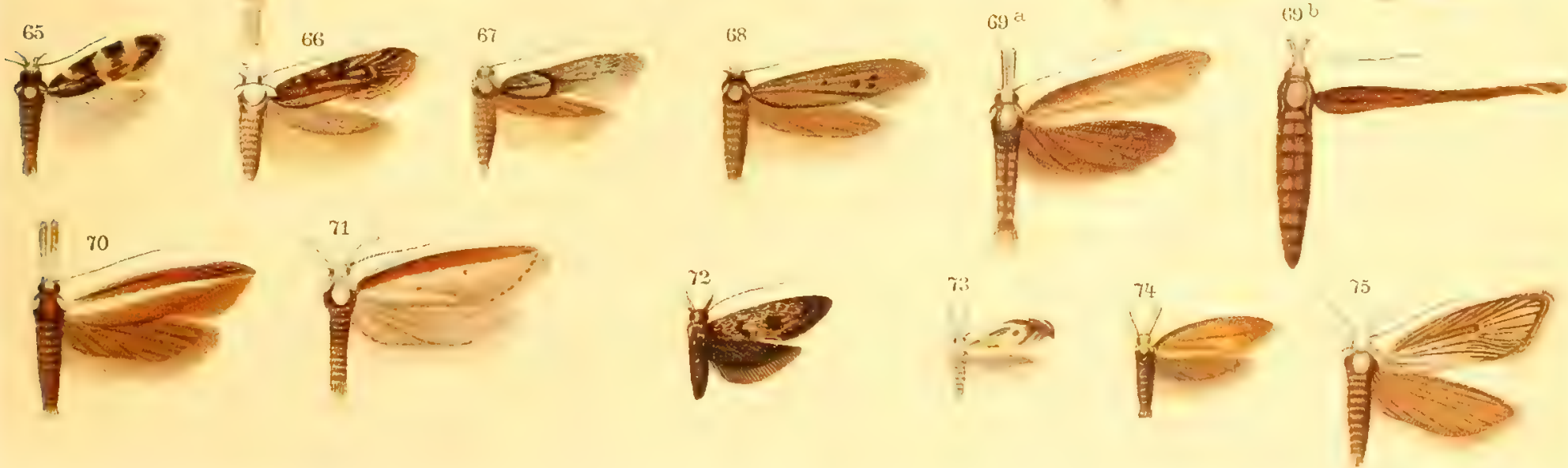




\section{TAFEL 89.}

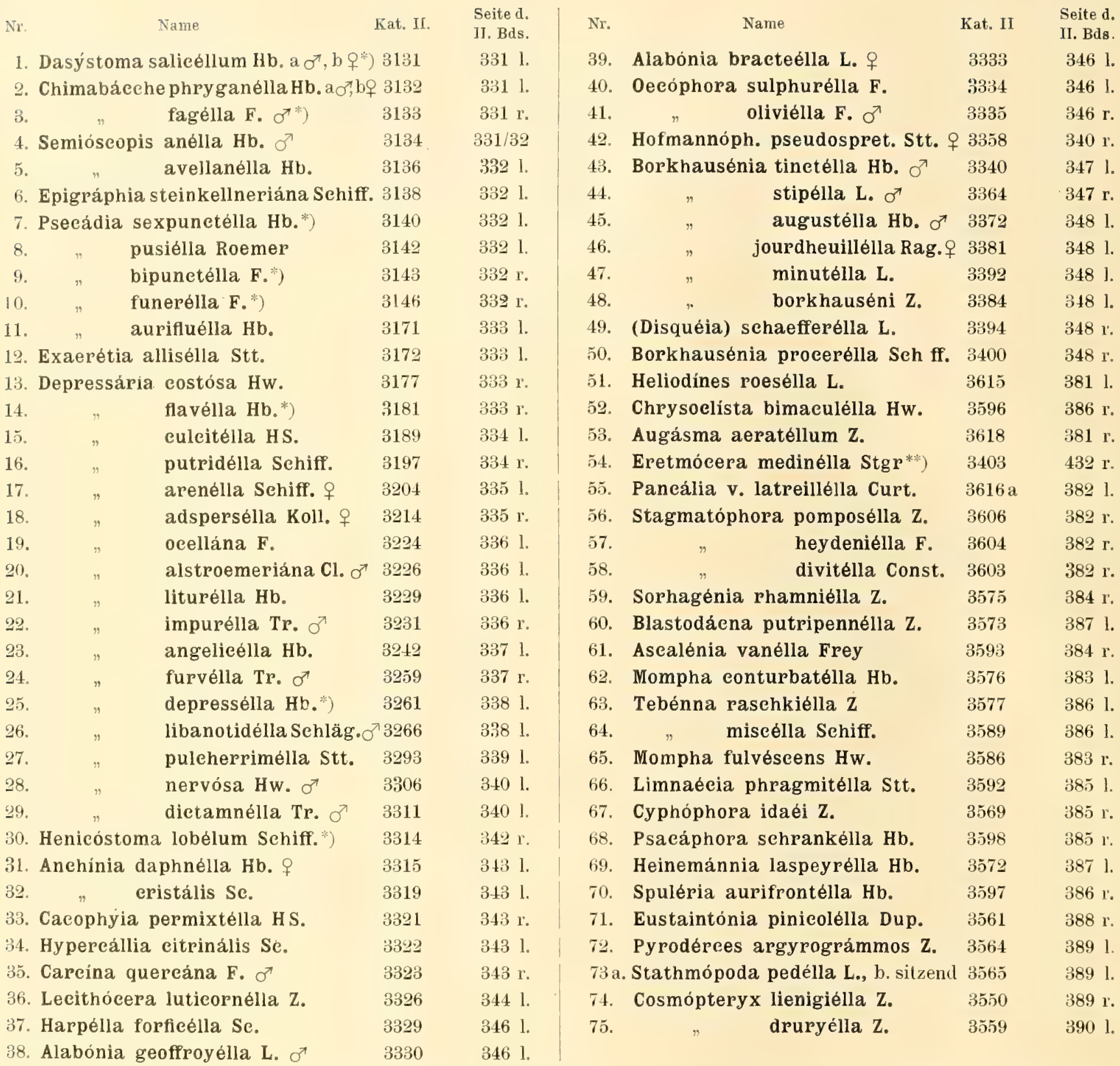

*) Die Raupe von Fig. 1 ist Nachtr.-Taf. X, 46; die von Fig. 3 ebenda 45; die von Fig. 7 ebenda 48; die von Fig. 9 ebenda 49; die von Fig. 10 ebenda 50; die von Fig. 14 ebenda 53; die von Fig. 25 ebenda 52 ; die von Fig. 30 ebenda 54 abgebildet.

**) Kopie nach Wlsghm, Tr. E. Soc. 1889, t. 6, f. 20 .

Die Tiere sind in doppelter linearer Vergrößerung wiedergegeben, die Figuren $7-11,37 \mathrm{u} .73 \mathrm{~b}$ indes in natürlicher Größe und Fig. 51 in dreifacher linearer Vergrößerung; die einzelnen Flügel sind noch stärker vergrößert. 


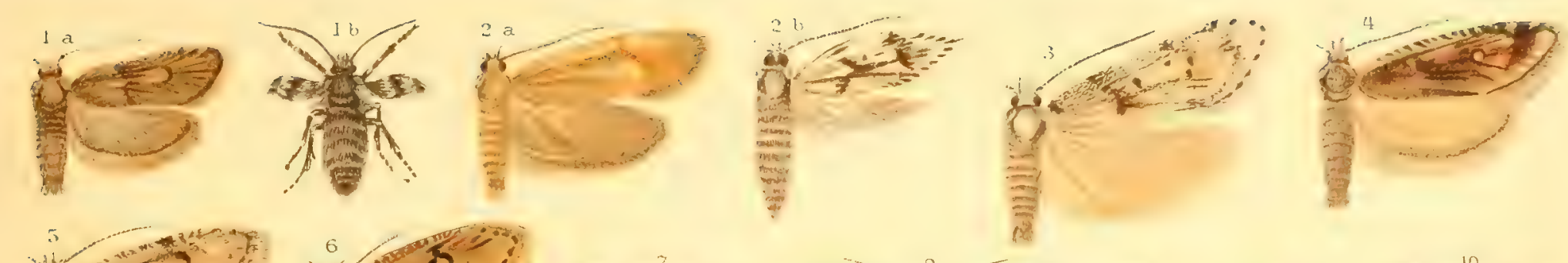

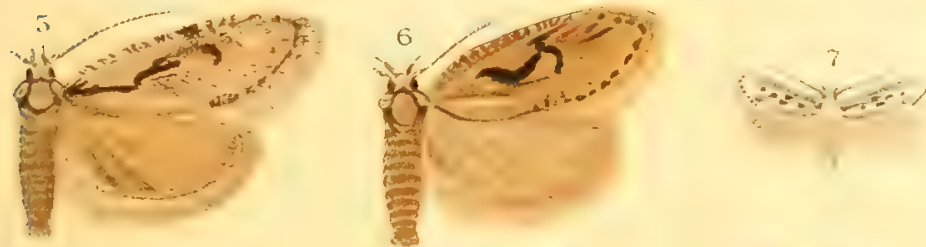

Hon

0 는

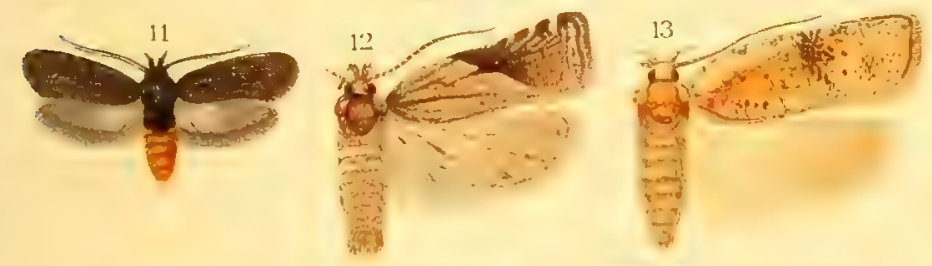
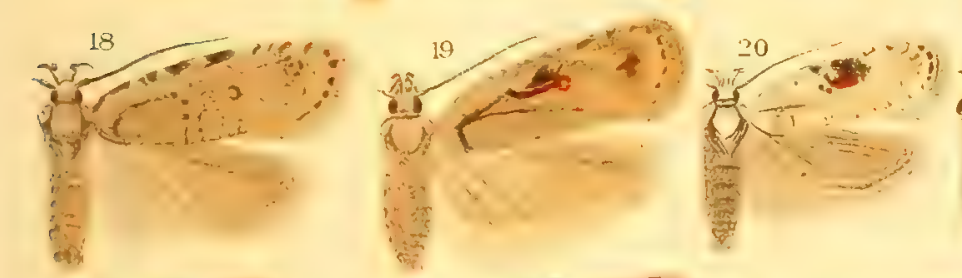

$3 \frac{14}{2}$

3
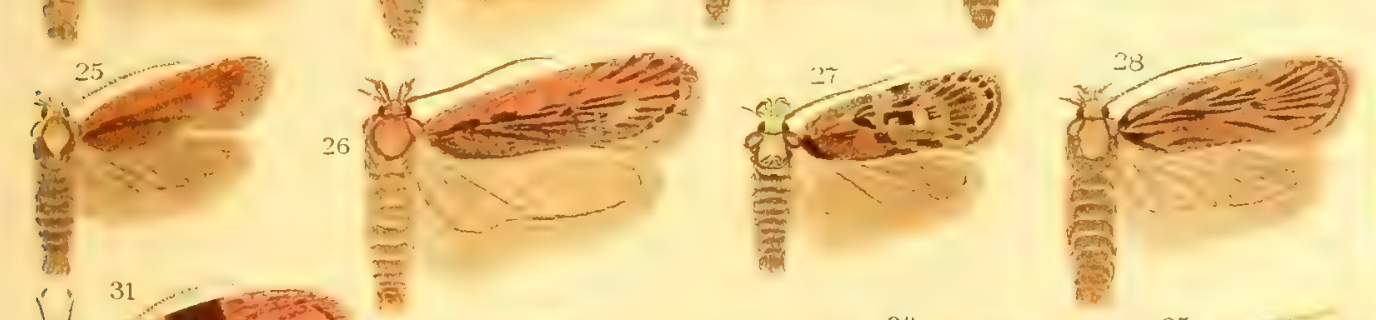

ijact

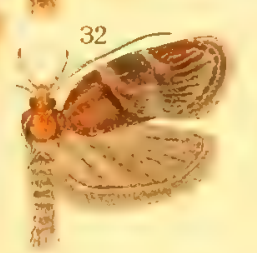

$i^{21}$

(2)
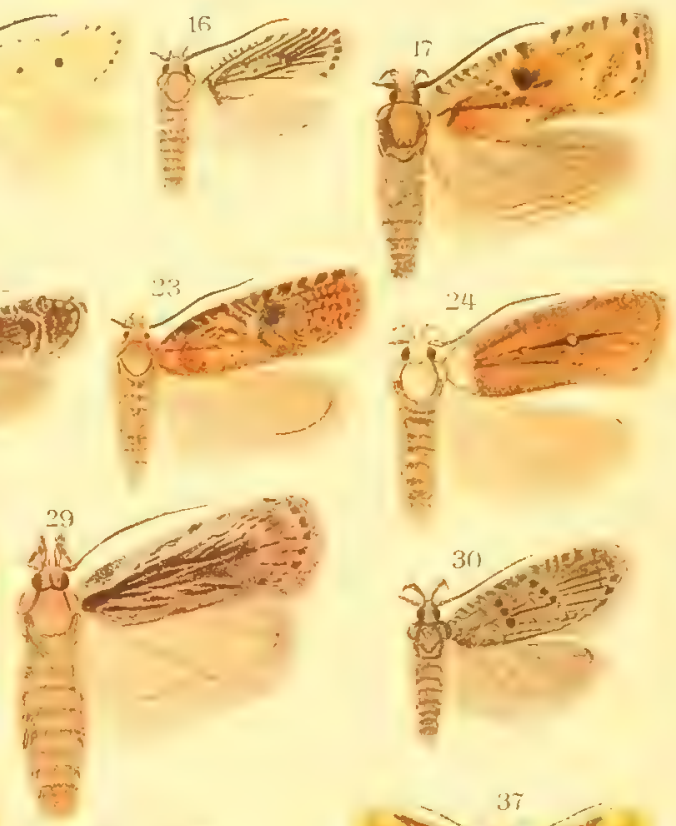

b $\cdots$

weref

$\frac{3+2}{7}$
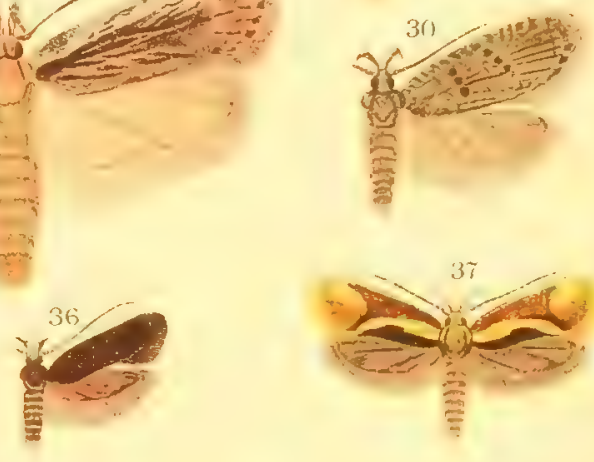

$\underbrace{38}_{2}$

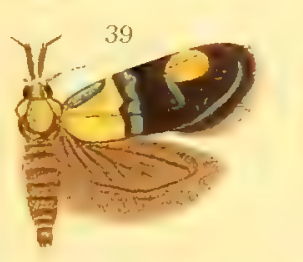

30
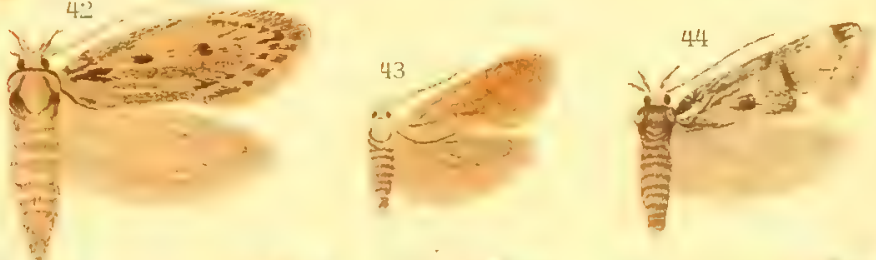

He

(

$\sqrt[53]{2}$
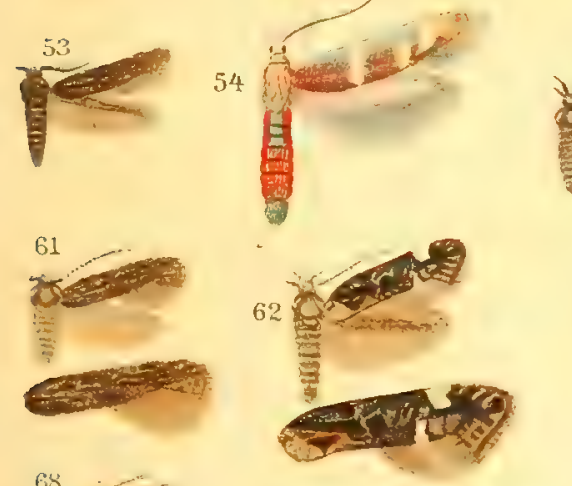

vel

deI

है.
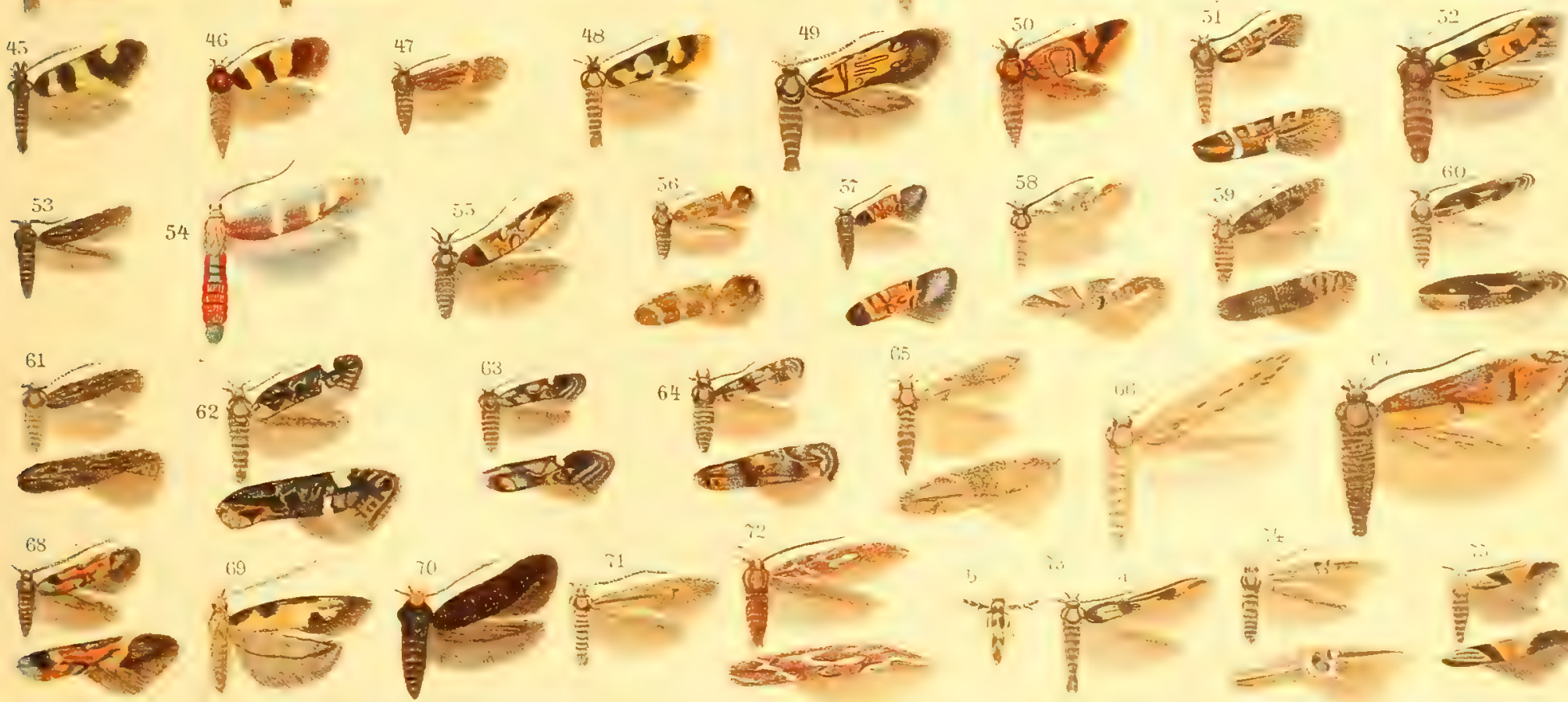

88
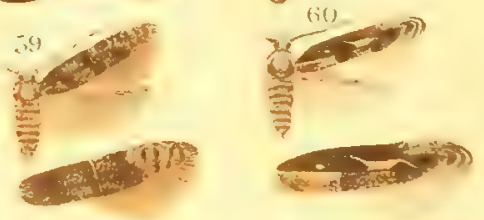




\section{TAFEL 90.}

\begin{tabular}{|c|c|c|c|c|c|c|}
\hline$x$ & Tame & Kat. II & $\begin{array}{l}\text { Scited. } \\
\text { II. Bus. }\end{array}$ & Name & Kat. II & $\begin{array}{l}\text { Seite d. } \\
\text { II. Bds. }\end{array}$ \\
\hline & Anýbia epilobiélla Roem. & 3594 & 3841. & 43. Bedéllia somnulentélla $\mathrm{Z}$. & $\$ 107$ & $418 \mathrm{r}$. \\
\hline & Asýehna modestélla Dup. & 3629 & 3911 & 14. Buceulátrix thoracélla Thnbg & 4238 & 419 \\
\hline & Coleóphora laricélla Hb. & 3633 & 3911. & 45. $\quad " \quad$ eidarélla $\mathrm{Z}$ & 1239 & 419 \\
\hline 4. & lutipennélla $Z$. & 3640 & $391 \mathrm{r}$. & erataégi $Z$. & 4242 & 419 \\
\hline 5. & albitarsélla Z. & 3672 & 393 r. & gnaphaliélla Tr. & 4267 & 420 \\
\hline h. & lixélla $\mathrm{Z}$. & 3685 & 3941 & " nigricomélla $\mathrm{Z}$. & 4270 & 420 \\
\hline 7. & squalorélla Z. & 3699 & 3951. & 49. Oenóphila v. flavum Hw. & 4621 & 421 \\
\hline$\therefore$. & woekeélla Z. & 3704 & 3951. & 50. Cemióstoma spartifoliélla $\mathrm{Hb}$. & 4228 & 423 \\
\hline 9. & gallipennélla $\mathrm{Hb}$. & 3753 & $397 \mathrm{r}$ & scitélla $\mathrm{Z}$. & 4236 & $423 / 9$ \\
\hline 10. & discordélla $\mathrm{Z}$. & 3716 & $395 \mathrm{r}$. & 52. Phylloenístis suffusélla $\mathrm{Z}$. & 4224 & 421 \\
\hline 11. & vibicélla $\mathrm{Hb}$. & 3788 & $399 \mathrm{l}$. & 53. Phyllobróstis hartmánni Stgr & 4223 & 422 \\
\hline 12. & palliatélla Zk. & 3794 & 4001 & 54. Lyonétia clerkélla L. & 4217 & 422 \\
\hline 13. & unipunetélla $\mathrm{Z}$. & 3803 & $400 \mathrm{r}$ & ab. padifoliélla $\mathrm{Hb}$. & $4219 \mathrm{a}$ & 422 \\
\hline 14. & auricélla $\mathrm{F}$. & 3809 & $400 \mathrm{r}$ & 56. 0póstega salaciélla Tr. & 4278 & 482 \\
\hline 15. & onosmélla Brahm & 3820 & 4011. & 57. Stephénsia brunnichiélla L. & 3920 & 424 \\
\hline 16. & gnaphálii Z. & 3871 & $403 \mathrm{r}$ & 58. Seirtópoda herpichiélla HS. & 4036 & 424 \\
\hline 17. & laripennélla Zett. & 3904 & 4051. & 59. Upodéla eisticolélla Stt. & 4035 & 425 \\
\hline 18. C & Goniodóma auroguttélla F.R. & 3914 & $405 \mathrm{r}$ & 60. Perittia obscurepunetélla Stt. & 3919 & 425 \\
\hline 19.0 & Gracilária alchimiélla Se. & 4040 & $406 \mathrm{l}$ & 61. Elachísta quadrélla $\mathrm{Hb}$. & 3921 & 425 \\
\hline & elongélla L. & 4056 & $407 \mathrm{l}$ & nobilélla Z. & 3928 & 426 \\
\hline 21. A & Aspilápteryx tringipennélla $\mathrm{Z}$. & 4059 & $407 \mathrm{r}$ & luticornélla $\mathrm{Z}$. & 3936 & 426 \\
\hline 22. 1 & Miepurápt. pavoniélla Z. & 4078 & 409 l. & nigrélla Hw. & 3950 & 427 \\
\hline 23. & $" \quad$ kollariélla $\mathrm{Z}$. & 4079 & 4091. & bedellélla Sire. & 3962 & 428 \\
\hline 24. & Coriseium brogniardéllum $\mathrm{F}$. & 4082 & $409 \mathrm{r}$. & pullicomélla $\mathrm{Z}$. & 3965 & 428 \\
\hline 25. & eueullipennéllum $\mathrm{Hb}$. & 4083 & $409 / 10$ & bifasciélla Tr. & 3974 & 429 \\
\hline 26. & Ornix gúttea $\mathrm{Hw}$. & 4086 & 4101 & megerlélla Stt. & 3978 & 429 \\
\hline & " anglicélla Stt. & 4097 & $411 \mathrm{l}$ & albidélla Tgstr. & 3994 & 430 \\
\hline 28. & " caudulatélla $\mathrm{Z}$. & 4106 & 4121. & disertélla HS. & 4003 & 430 \\
\hline 29.1 & Lithocollétis róboris $\mathrm{Z}$. & 4108 & 4131. & cerusélla Hb. & 4008 & 431 \\
\hline 30. & amyotélla Dup. & +109 & 4131. & rufocinérea $\mathrm{Hw}$. & 4013 & 431 \\
\hline 31. & genieulélla Rag. & 4112 & 4131. & dispilélla $\mathrm{Z}$. & 4019 & 431 \\
\hline 32. & strigulatélla $\mathrm{Z}$. & 4120 & $413 r$ & argentélla $\mathrm{Cl}$. & 4024 & $431 / 3$ \\
\hline 33. & fraxinélla $\mathrm{Z}$. & 4128 & 4141 & 75. Catapléctica profugélla Stt. & 3542 & 433 \\
\hline 34. & spinolélla Dup & 4129 & 4141. & 76. Mompha nodicolélla Fuehs & & 383 \\
\hline 35. & lantanélla Schrk & $415 ั 7$ & $415 \mathrm{r}$. & 77. Bucculátrix antispilélla Chrét. & - & 420 \\
\hline 36. & scitulélla $\mathrm{Z}$. & 4162 & 4161. & 78. Catapléctica fulviguttélla $\mathrm{Z}$. & 3547 & 433 \\
\hline 37. & quereifoliélla $\mathrm{Z}$. & 4164 & 4161. & 79. Phaulérnis dentélla $\mathrm{Z}$. & 3404 & 434 \\
\hline 38. & corylifoliélla $\mathrm{HW}$. & 4182 & 4171 & 80. Eperménia pontificélla $\mathrm{Hb}$. & 3408 & 434 \\
\hline 39. & froehliehiélla Z. & 4187 & 4171 & chaerophyllélla Goeze & 3416 & 435 \\
\hline 40. & trifasciélla $\mathrm{Hw}$. & 4196 & $417 r$ & 82. Oehromolópis ietélla $\mathrm{Hb}$. & 3508 & 435 \\
\hline 41. & pastorélla Z. & 4199 & 4181. & 83. Sehreckensteínia festaliélla $\mathrm{Hb}$. & 3405 & 433 \\
\hline 42. & comparélla $\mathrm{Z}$. & 4204 & $418 \mathrm{l}$. & 84. Amphisbátis ineongruélla Stt. & 3540 & 441 \\
\hline
\end{tabular}

Die Raupe von Fig. 3 ist Nachtr.-Taf. X, 55, die von Fig. 9 ebenda Fig. 57 abgebildet. Alle Falter sind in doppelter linearer Vergrößerung dargestellt. 

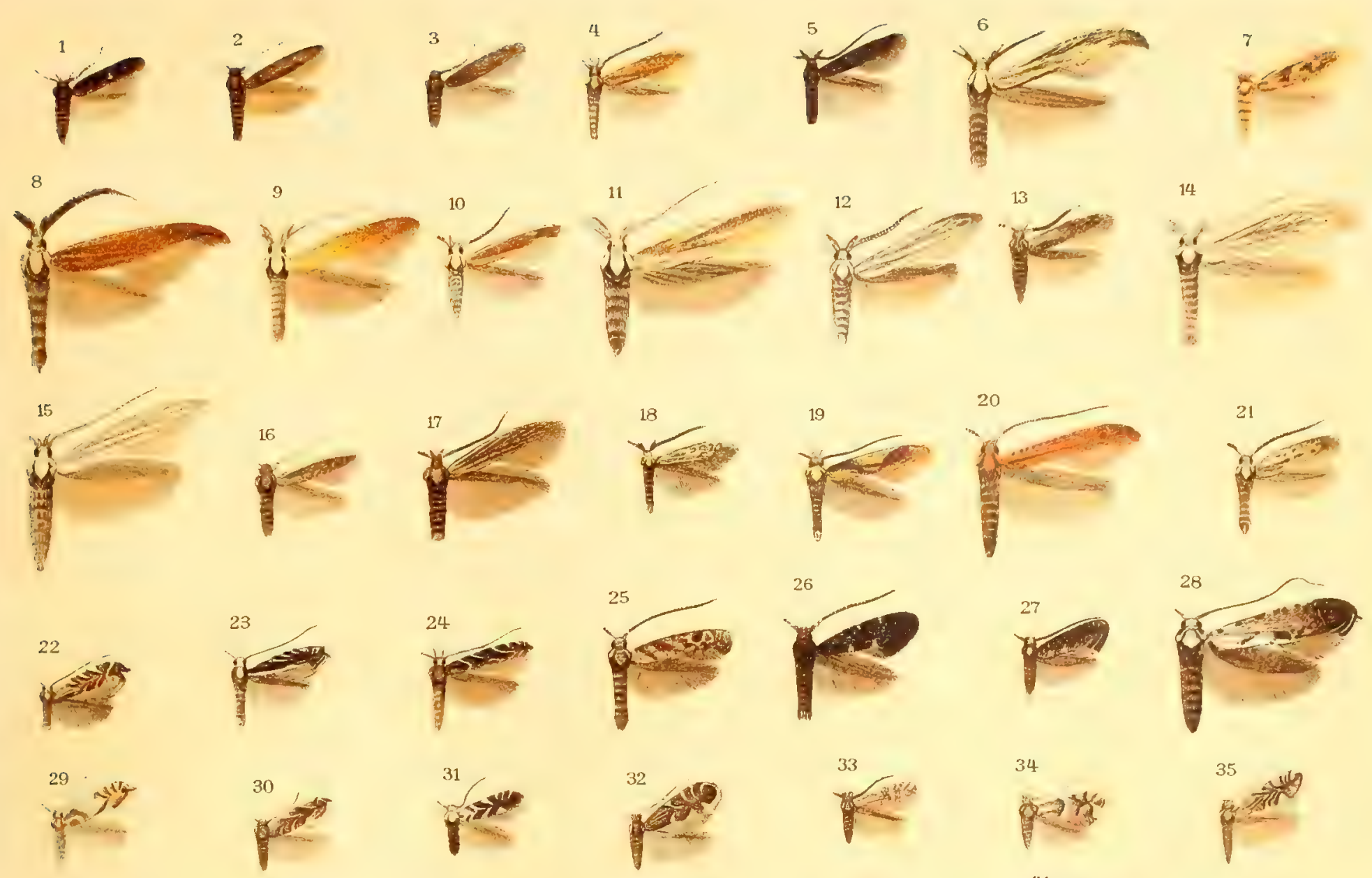

K

34

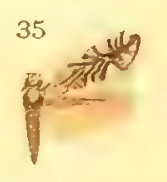

$y=0$
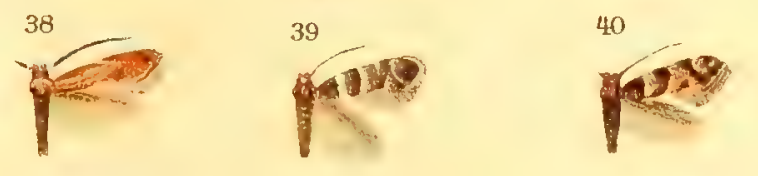

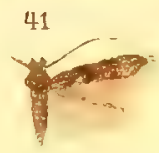

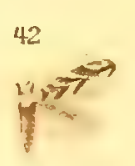

is
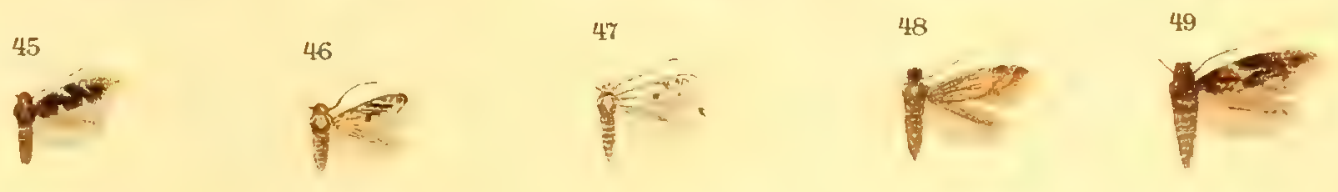

in " is
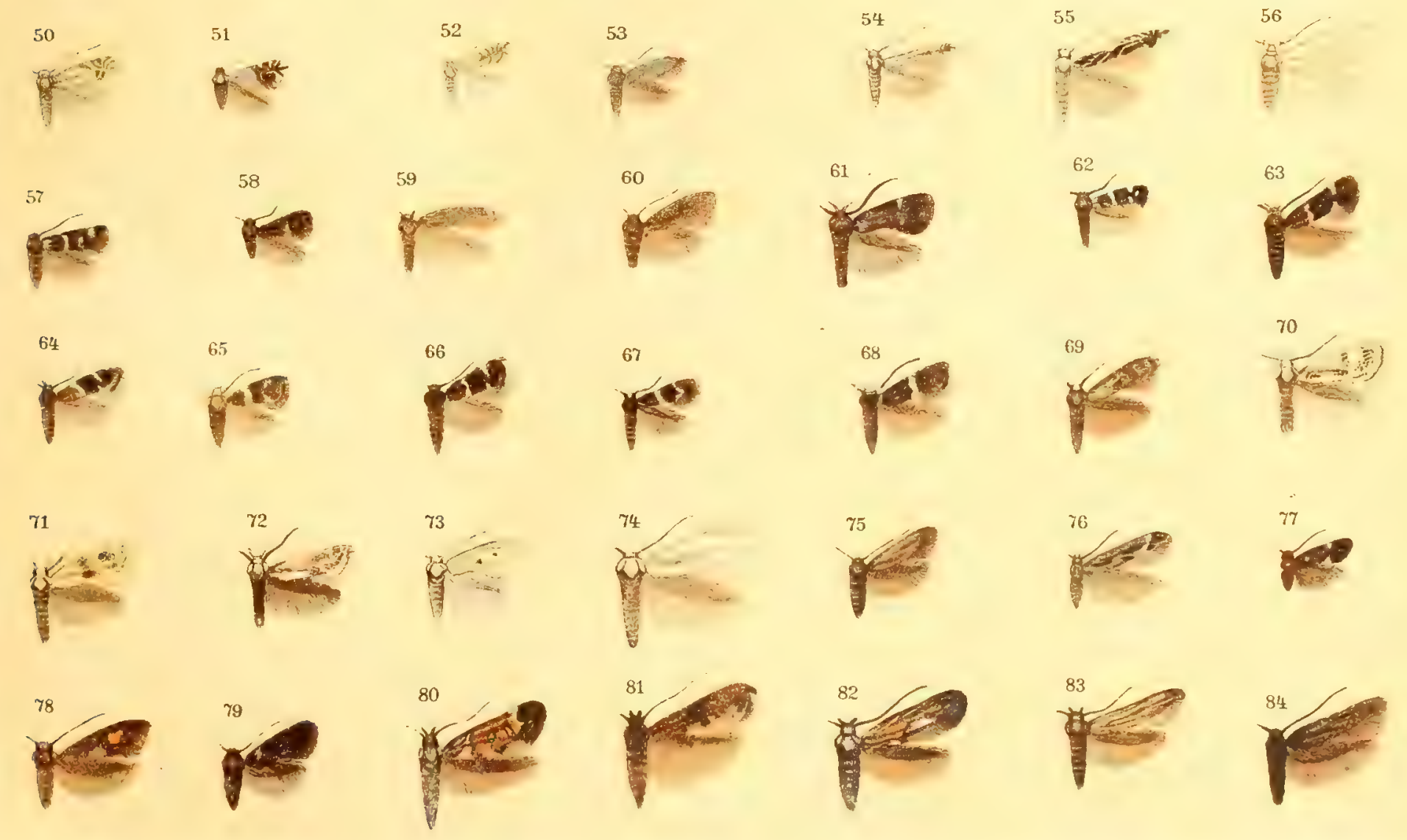




\section{TAFEL 91 .}

\begin{tabular}{|c|c|c|c|c|c|c|}
\hline Nr. & Name & Kat. II & $\begin{array}{l}\text { Seite d. } \\
\text { II. Bds. }\end{array}$ & Name & $\mathrm{K}$ at. II & $\begin{array}{l}\text { Seite d. } \\
\text { II. Bds. }\end{array}$ \\
\hline & Seythris fallacélla Sehleg. & 3426 & $436 \mathrm{r}$. & 40. Phyllopória bistrigélla $\mathrm{Hw}$. & 4620 & $464 \mathrm{r}$. \\
\hline 2. & senéseens Stt. & 3449 & $437 \mathrm{r}$. & 41. Ineurvária morósa $\mathrm{Z}$. & 4650 & 4651. \\
\hline 3. & laminélla HS. & 3477 & 4391. & praelatélla Sehiff. & 4655 & $465 \mathrm{r}$. \\
\hline 4. & euspidélla Schiff. & 3487 & 4391. & koepneriélla $\mathrm{Z}$. & 4673 & 4661. \\
\hline 5. & knochélla F. & 3492 & $439 \mathrm{r}$. & muscalélla F. & 4674 & $466 \mathrm{r}$. \\
\hline 6. & seopolélla Hb. & 3515 & $440 \mathrm{l}$. & 45. Nemóphora swammerdamélla L. & 4678 & $466 \mathrm{l}:$ \\
\hline 7. & inspersélla $\mathrm{Hb}$. & 3525 & $440 \mathrm{r}$. & 46. $" \quad$ pilélla $\mathrm{F}$. & 4686 & 4671. \\
\hline 8. & eieadélla $\mathrm{Z}$. & 3533 & $440 / 41$ & 47. Adéla viridélla Se. & 4713 & 4691. \\
\hline 9. & acanthélla God. & 3538 & $441 \mathrm{l}$. & degeerélla L.*) & 4721 & 4691. \\
\hline 10. & Ochsenhoiméria taupélla Sehiff. & 4467 & 3001. & rufimitrélla Se. & 4741 & 4701 \\
\hline & Acrolepia eariosélla Tr. & 4475 & 4531 . & 50. Nemotóis metállicus Poda. & 4691 & $467 / 68$ \\
\hline 12. & vesperélla $\mathrm{Z}$. & 4478 & $453 \mathrm{r}$. & eupriacéllus $\mathrm{Hb}$. & 4696 & 4681. \\
\hline & assectélla $\mathrm{Z}$. & 4480 & $453 \mathrm{r}$. & dumeriliéllus Dup. & 4710 & $468 \mathrm{r}$. \\
\hline 14. & Blabóphanes imélla $\mathrm{Hb}$. & 4529 & $463 \mathrm{r}$. & 53. Roesslerstámmia erxlebeniélla F. & $\$ 490$ & $4 \check{5} 4 \mathrm{r}$. \\
\hline & monachélla Hb. & 4536 & $463 \mathrm{r}$. & 54. Eriocóttis fuscanélla $\mathrm{Z}$. & 4646 & $467 \mathrm{l}$. \\
\hline 16. & Tinéola biselliélla Hummel & 4624 & 4621. & 55. Antispila treitsehkeélla F. R. & 3620 & $471 \mathrm{r}$. \\
\hline 17. & Mymecozéla ochraceélla Tngstr. & 4637 & 4631. & 56. Heliozéla stannélla F. R. & 3624 & 4721. \\
\hline 18. & Dysmásia parietariélla HS. & 4643 & 4581. & 57. Tischéria complanélla $\mathrm{Hb}$. & 4209 & $470 \mathrm{r}$. \\
\hline 19. & Triehóphaga Rag. tapetiélla L. & 4539 & 4581. & angusticolélla Dup. & 4216 & $471 \mathrm{l}$ \\
\hline 20. & Tínea areélla $\mathrm{F}$. & 4543 & 4591. & 59. Neptícula rufieapitélla Hw. & 4294 & 4731. \\
\hline & " parasitélla $\mathrm{Hb}$. & 4545 & $459 \mathrm{I}$. & oxyaeanthélla Stt. & 4312 & $474 / 75$ \\
\hline & " eloacélla Hw. & 4556 & $459 \mathrm{r}$. & áceris Frey & 4317 & 4751. \\
\hline & " eaprimulgélla HS. & 4558 & $459 \mathrm{r}$. & splendidissimélla HS. & 4328 & $475 \mathrm{r}$ \\
\hline & " fuseipunetélla $\mathrm{Hw}$. & 4583 & 4611. & ulmivora Fologne & 4341 & $476 \mathrm{r}$. \\
\hline & $"$ lapélla $\mathrm{Hb}$. & 4596 & 4611. & centifoliélla $\mathrm{Z}$. & 4352 & $477 \mathrm{l}$ \\
\hline & $"$ semifulvélla $\mathrm{Hw}$. & 4597 & $461 \mathrm{r}$. & plagicolélla Stt. & 4358 & $477 \mathrm{r}$. \\
\hline 27. & Ischnósia borreonélla Mill. & 4617 & 4621. & argentipedélla $\mathrm{Z}$. & 4369 & $478 \mathrm{l}$. \\
\hline 28. & Deuterotínea easanélla Ev. & 4632 & $462 \mathrm{l}$. & freyélla Heyd. & 4372 & $478 \mathrm{r}$. \\
\hline 29. & Moróphaga morélla Dup. & 4525 & $457 \mathrm{r}$. & agrimóniae Frey & 4379 & 4791. \\
\hline 30. & Ateliótum hungaricéllum $\mathrm{Z}$. & 4528 & 4581. & floslactélla Hw. & 4390 & $479 \mathrm{r}$ \\
\hline 31. & Seárdia boléti $F$. & 4520 & $457 \mathrm{r}$ & serieopéza $\mathrm{Z}$. & 4399 & 4801. \\
\hline 32. & Euplóeamus anthracinális Sc. & 4511 & 4571 & trimaculélla Hw. & 4404 & $480 \mathrm{r}$ \\
\hline & Narýcia monilifera Geoffr.*) & 4497 & $156 \mathrm{r}$. & 72. Trifúreula immundélla $\mathrm{Z}$. & 4287 & $48: 1$. \\
\hline 34. & Diplodóma marginepunctélla Stph. & 4499 & $456 \mathrm{r}$. & 73. Crinópteryx familiélla Peyer & 4749 & $467 \mathrm{r}$ \\
\hline 35. & Melasína lủgubris $\mathrm{Hb}$. & 4505 & 4561. & 74. Eriocránia v. fastuosélla $\mathrm{Z}$. & $4752 \mathrm{a}$ & 4831. \\
\hline & Penestoglóssa dardoinélla Mill. & 4502 & 4561 & purpurélla $\mathrm{Hw}$. & 4758 & 483 \\
\hline 37. & Lypúsa maurélla F. & 4495 & $455 \mathrm{r}$. & 76. Mieróptery $\mathrm{x}$ ammanélla $\mathrm{Hb}$. & 4766 & 484 \\
\hline & $\begin{array}{l}\text { Teichóbia verhuellélla Stt. } \\
\text { Meéssia vineulélla HS. }\end{array}$ & $\begin{array}{l}4496 \\
4611\end{array}$ & $\begin{array}{l}455 \mathrm{l} . \\
464 \mathrm{r} .\end{array}$ & calthélla L. & 4782 & 484 \\
\hline
\end{tabular}

*) Die Raupe von Fig. 33 ist Nachtr.-Taf. X, 59; die von Fig. 48 ebenda Fig. 58 abgebildet.

Die Arten sind in den Fig. 1-58 und 73-77 in doppelter, in den Fig. 59-72 in vierfacher linearer Vergrößerung dargestellt; unter Fig. 59 ist das Tier in $2 / 1$, unt. den Fig. 60-72 in 1/1 natürlicher Größe dargestellt. 

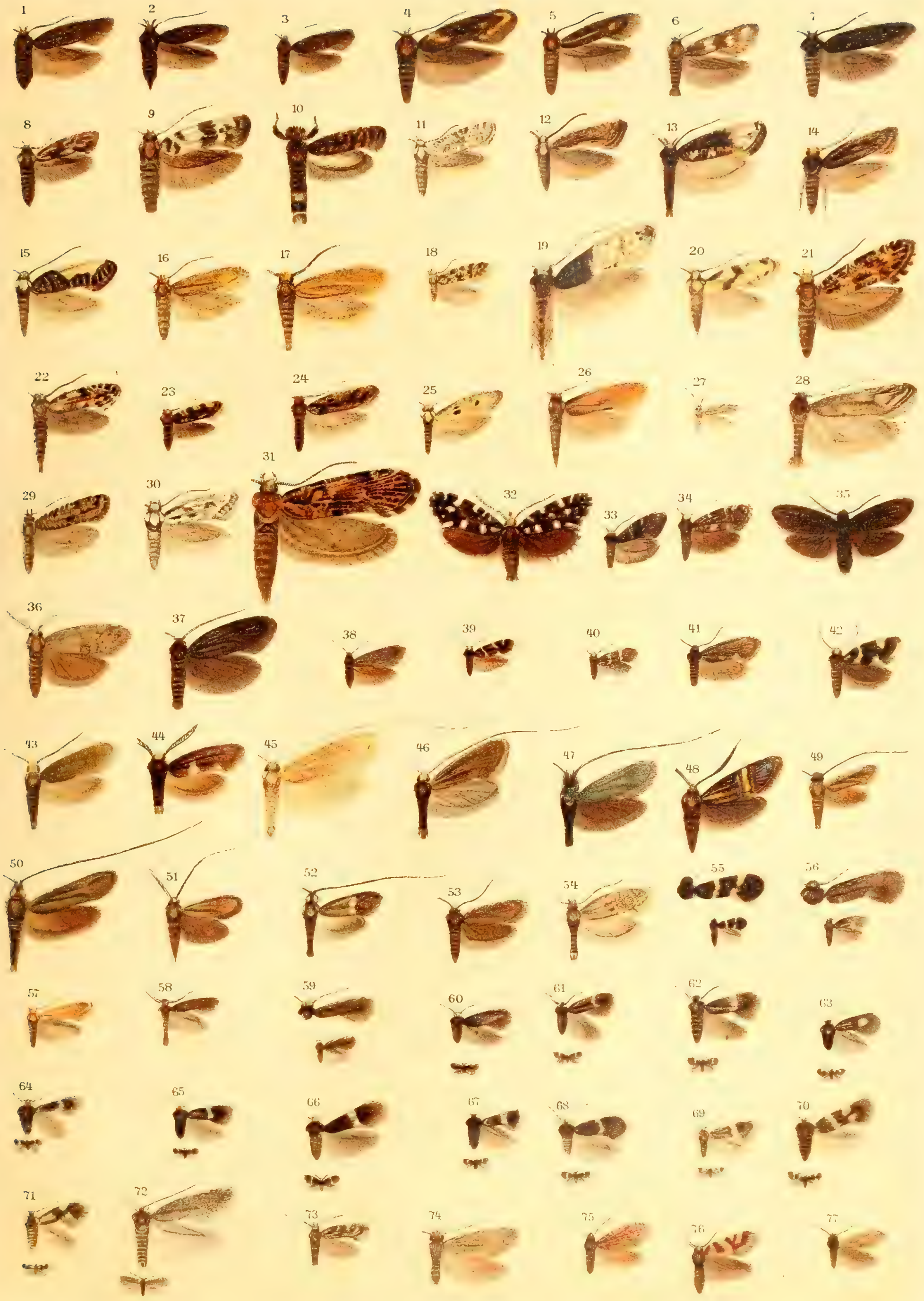




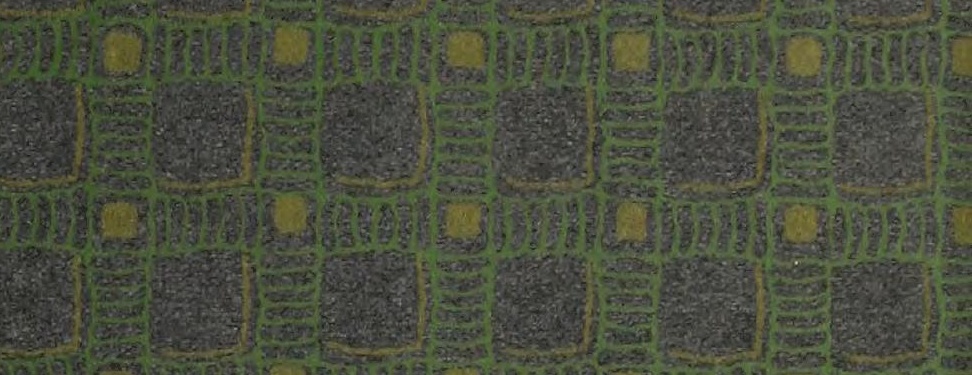
(20.

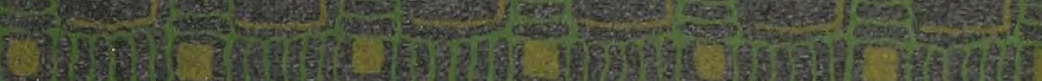
sor.

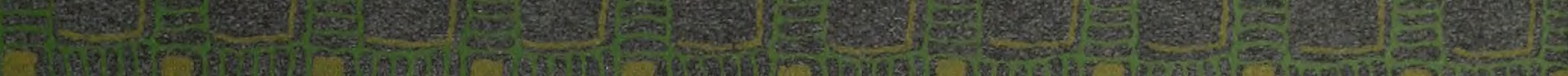
(2)

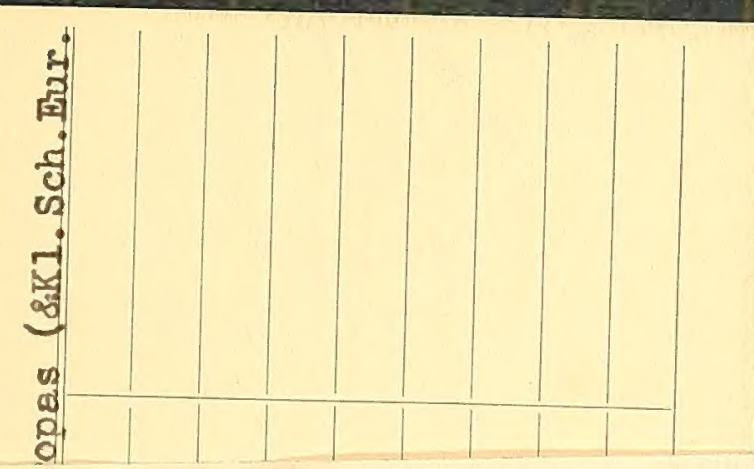

(1)

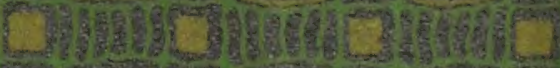

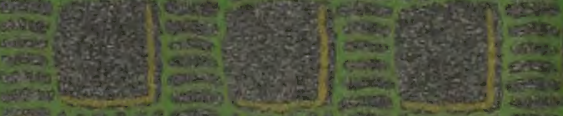
(3)

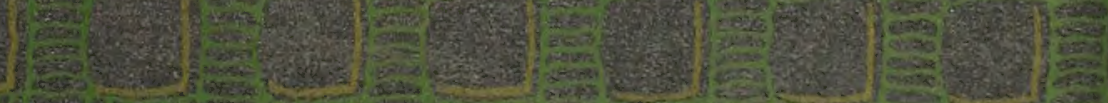
4 1.

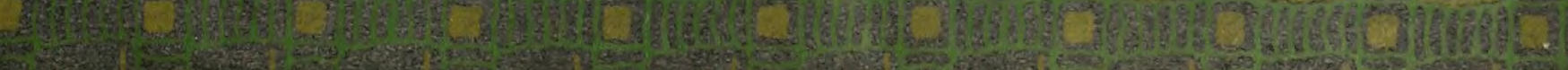


